\title{
Zur Entwicklung und Förderung rhythmischer Kompetenz in Bezug auf den Umgang mit Rhythmusnotation im Musikunterricht der Grundschule
}

Eine empirische Untersuchung

\author{
Dissertation \\ zur Erlangung des Grades eines Doktors der Philosophie \\ an der Hochschule für Musik Carl Maria von Weber Dresden \\ vorgelegt von Christin Werner \\ geb. am 23. November 1966 in Potsdam
}

Betreuer: $\quad$ Prof. Dr. Stefan Gies, Hochschule für Musik Carl Maria von Weber Dresden

Gutachter: $\quad$ 1. Prof. Dr. Stefan Gies, Hochschule für Musik Carl Maria von Weber Dresden

2. Prof. Dr. Frauke Heß, Universität Kassel

Die Disputation fand am 22.11.2016 unter Vorsitz von Prof. Dr. Matthias Herrmann statt. 


\section{Danksagung}

Dieses Forschungsprojekt wurde erst durch das Mitwirken vieler Menschen möglich. Deshalb spreche ich an dieser Stelle dafür meinen Dank aus.

Allen voran möchte ich mich bei meinem Betreuer, Professor Stefan Gies, für die stets ermutigende und konstruktive Unterstützung bedanken. Alle Anfragen wurden stets umgehend beantwortet, versehen mit einem guten Maß für das Machbare und mit einem aufmunterndem Schuss Humor.

Einen besonderen Dank möchte ich Professor Hans-Christian Jabusch für entscheidende Impulse in Bezug auf die Objektivierung des in dieser Studie zum Einsatz kommenden Testes aussprechen. Für die gewinnbringende Unterstützung in Bezug auf statistische Fragestellungen bedanke ich mich bei Juniorprofessor Friedrich Platz. Frau Professorin Frauke Heß danke ich für die sächliche Unterstützung im statistischen Bereich, ihre bestärkenden Rückmeldungen sowie ihre kritische Sicht auf Fragen zur Theorie. Herrn Professor Kaiser danke ich für den regen Gedankenaustausch zum Kompetenzbegriff.

Ein ganz besonderer Dank geht an die Kolleginnen Frau Grafe, Frau Heinrich, Frau KommerStahr, Frau Liebsch, Frau Kleinstück, Frau Schmidt, Frau Haufe und Frau Deke, die sowohl in den Untersuchungs- als auch in den Kontrollklassen an diesem Projekt mitgewirkt haben. Ebenso werden mir alle Schülerinnen und Schüler, die ich durch diese Studie sehr persönlich kennen lernen durfte, in ihrer Einmaligkeit in Erinnerung bleiben. Mein Dank gilt ebenfalls der Referentin Frau Preusche, die das Projekt von Anfang an befürwortet und die Schulbesuche der zweiten Erhebung ermöglicht hat.

Meiner Familie danke ich für die Rücksichtnahme ganz besonders in der Endphase der Promotion. Ein besonders großer Dank gilt meinem Vater für das mehrfache, kritische Lesen der Arbeit sowie meiner Schwester Katrin für das stets offene $\mathrm{Ohr}$ in methodischen Fragen. Meinem Ehemann gehört mein Dank für die vielen Stunden geduldigen Zuhörens und Diskutierens sowie die immerwährende Ermutigung zu diesem Projekt. 


\section{Inhaltsverzeichnis}

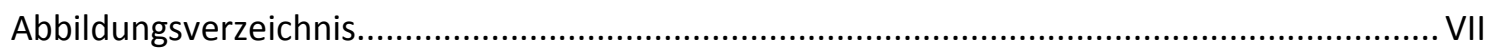

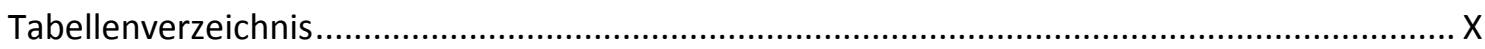

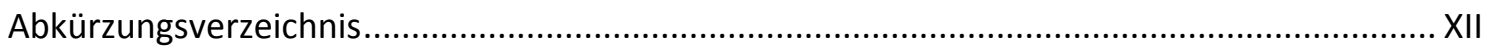

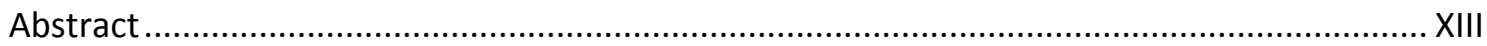

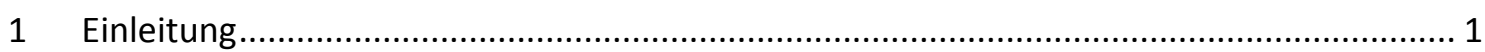

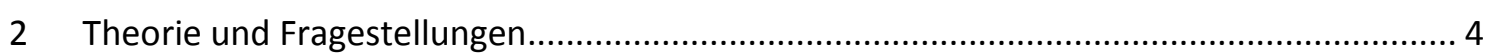

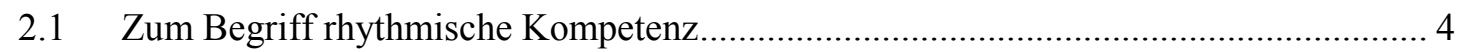

2.2 Lern- und Lehrvoraussetzungen zum Erwerb rhythmischer Kompetenz..................... 13

2.2.1 Stufe A Körperkoordination, Erfahrung von Metrum und Puls .......................... 15

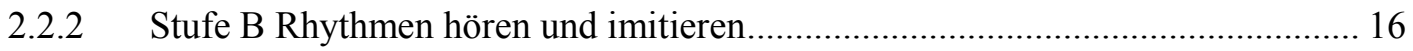

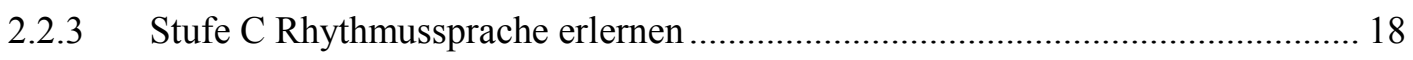

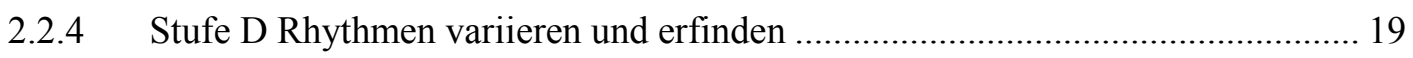

2.2.5 Stufe E Rhythmen lesen und F Rhythmen schreiben ........................................ 21

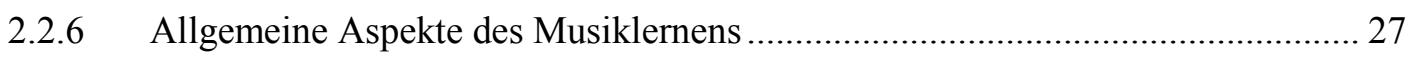

2.3 Didaktische Analyse des Lerngegenstandes Rhythmusnotation ................................. 29

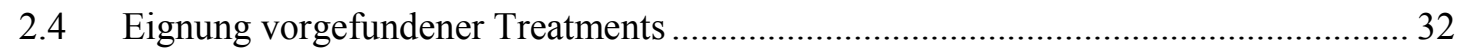

2.5 Tests zur Messung rhythmischer Kompetenz …...................................................... 33

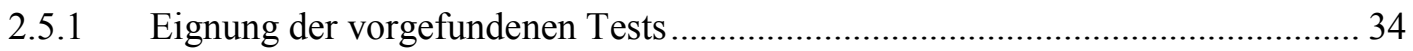

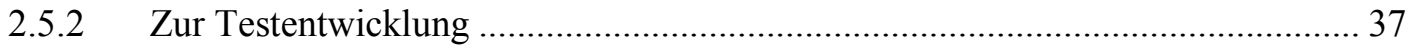

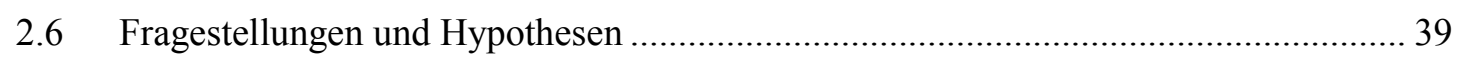

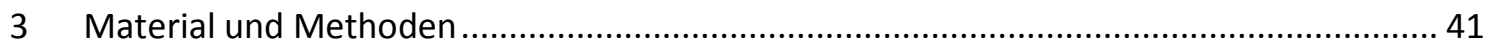

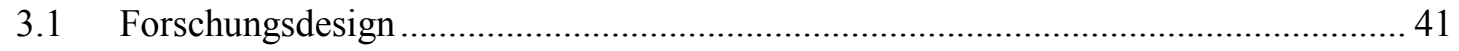

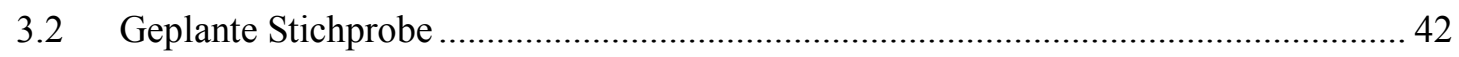

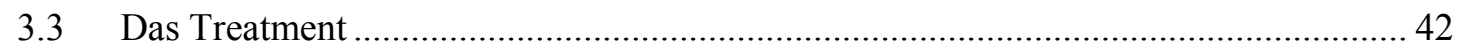

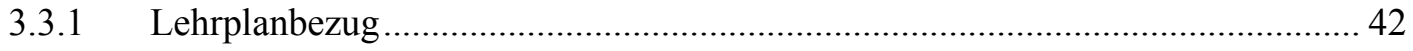

3.3.2 Aufbau und Inhalt des Rhythmus-Lehrgangs ................................................. 43

3.3.3 Ansteigende Schwierigkeit der Patterns ............................................................. 47

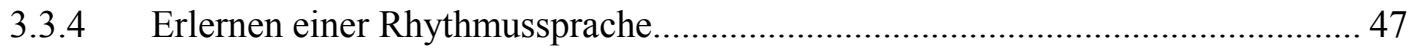


3.3.5 Ikonische Notation als Teil einer dreifachen Wissensdarbietung......................... 48

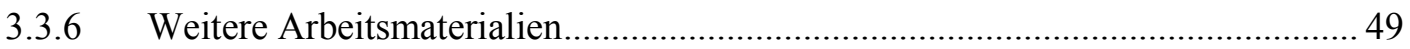

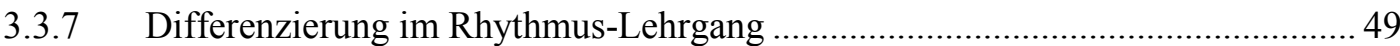

3.3.8 Zusammenfassende Darstellung der Gestaltung des Treatments .........................51

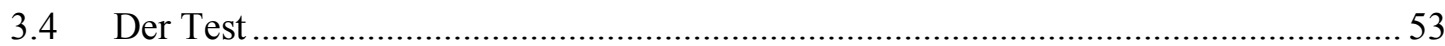

3.4.1 Operationalisierung der Stufen des Pyramidenmodells........................................ 54

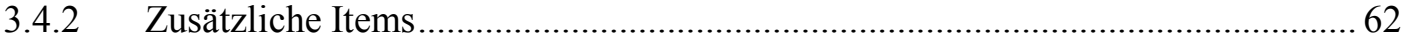

3.4.3 Zusammenfassende Darstellung des Tests ............................................................6 64

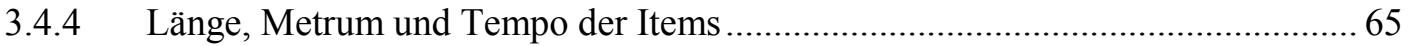

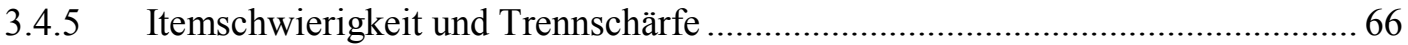

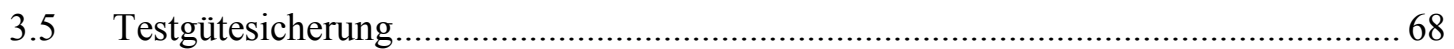

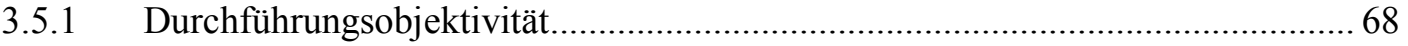

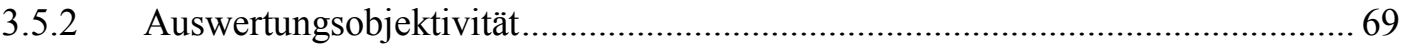

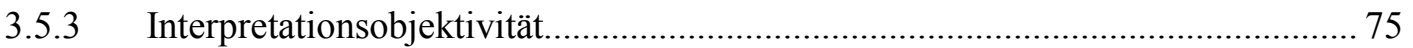

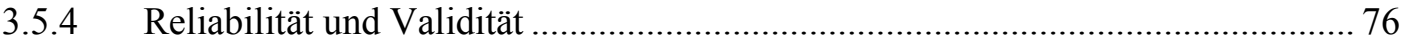

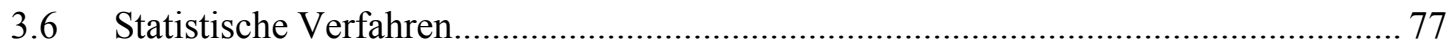

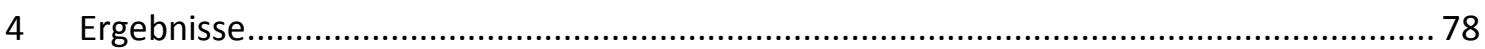

4.1 Dokumentation der Stichprobe und des Untersuchungszeitraums ............................... 78

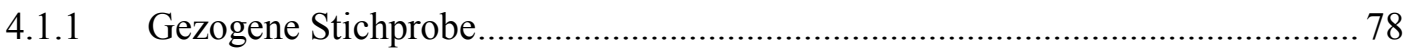

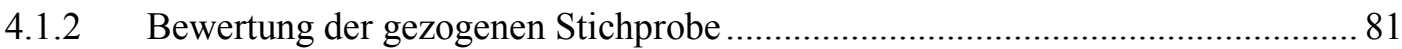

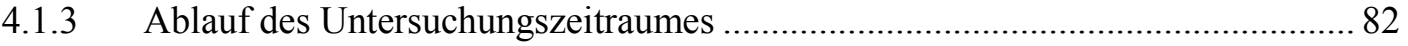

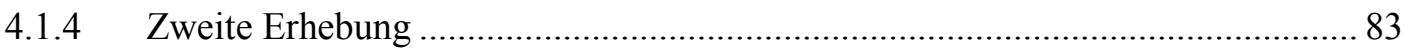

4.1.5 Zusammenarbeit mit den Lehrkräften ........................................................... 83

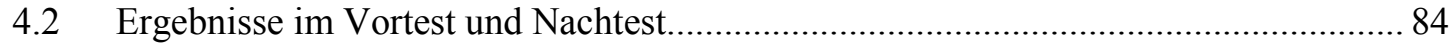

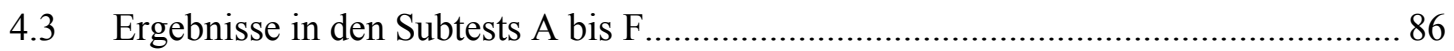

4.3.1 Ergebnisse im Subtest F Rhythmen musizieren und aufschreiben ....................... 86

4.3.2 Ergebnisse im Subtest A Metrum und Grundschlag darstellen ............................ 91

4.3.3 Ergebnisse im Subtest B Rhythmen nachspielen ............................................... 97

4.3.4 Ergebnisse im Subtest D Rhythmen bewusst abwandeln ................................. 103

4.3.5 Ergebnisse im Subtest E Rhythmen lesen und musizieren................................. 109 
4.3.6 Ergebnisse im Subtest C Rhythmussprache anwenden

4.4 Ergebnisse in der Gesamtpunktwertung des Testes ............................................... 123

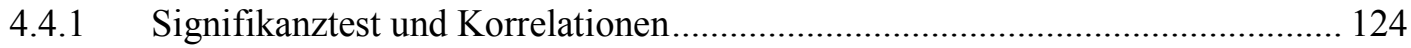

4.4.2 Individuenbezogene Ergebnisdarstellung................................................... 125

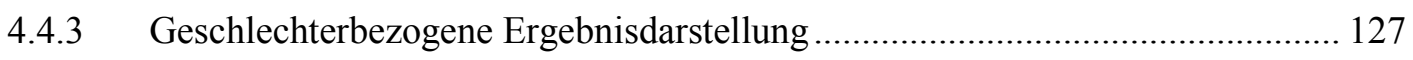

4.4.4 Ergebnisse im Nachtest getrennt nach einzelnen Klassen.................................. 127

4.4.5 Ergebnisse im Subtest F im Nachtest in Bezug auf alle Probanden................... 128

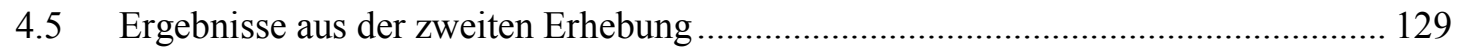

4.6 Ergebnisse in den zusätzlichen Subtests ……...................................................... 130

4.6.1 Untertest $\mathrm{f}$ Rhythmen musizieren und aufschreiben mit Zeichen ...................... 130

4.6.2 Untertest e Rhythmen lesen und musizieren nach Zeichen................................ 132

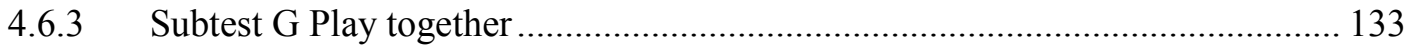

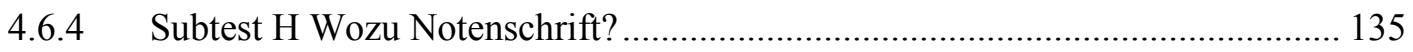

4.6.5 Subtest I Hörendes Vergleichen von Rhythmen ................................................. 135

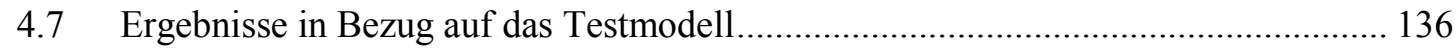

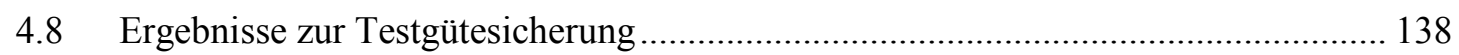

4.9 Ergebnisse in Bezug auf das Treatment ............................................................... 141

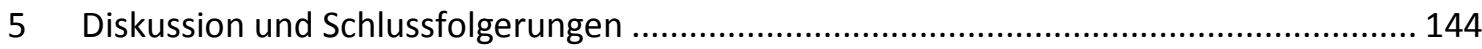

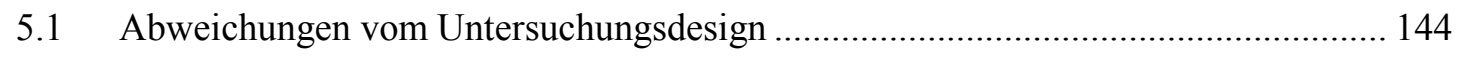

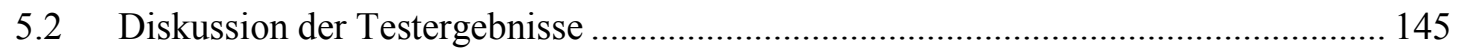

5.2.1 Stand der Entwicklung zu Beginn und am Ende der Klassenstufe 3 ................. 145

5.2.2 Ergebnisse aus den zusätzlichen Subtests G, H und I .................................... 154

5.2.3 Vergleich der Mittelwertunterschiede und Effektstärken................................... 156

5.3 Beurteilung von Testentwicklung, Testmodell und Testgüte ...................................... 158

5.4 Beurteilung der Treatmententwicklung ................................................................ 163

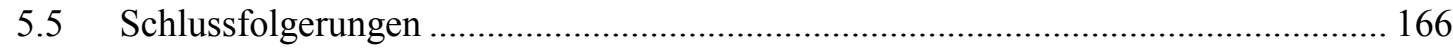

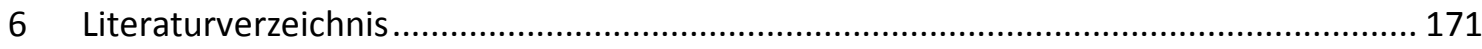

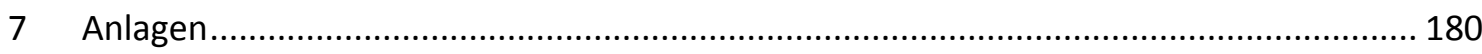

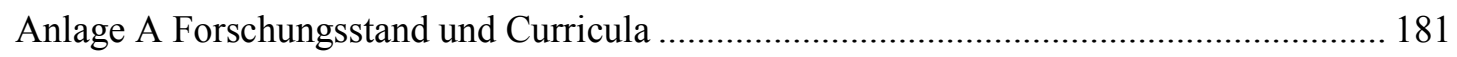

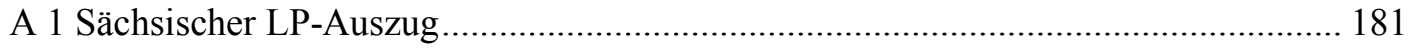


A 2 Relevante Items anderer Leistungstests...... 182

Anlage B Material und Methoden der Testgestaltung......................................................... 191

B 1 Testdauer mündlicher und schriftlicher Test .......................................................... 191

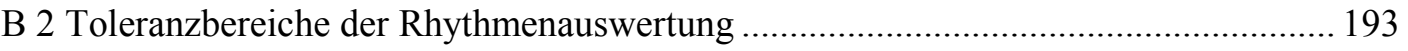

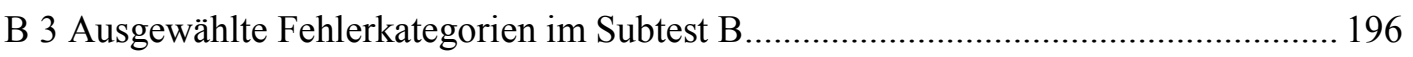

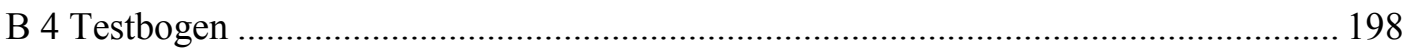

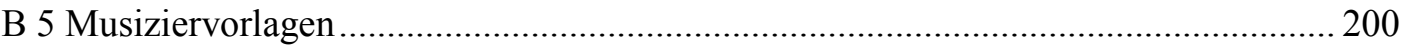

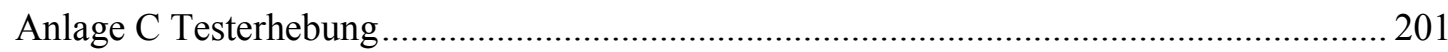

C 1 Teststärke (Power) und kritischer Wert t (Critical t) ....................................................2 201

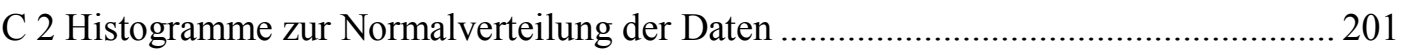

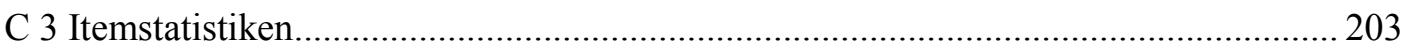

C 4 Interne Konsistenz und Reliabilität im Nachtest ..................................................... 205

C 5 Mittelwerte, t-Test-Werte, Effektstärken und Differenzen der MW im VT und NT . 207

C 6 Übersicht über Ergebnisse in den Untertests ec und fc im Vor- und Nachtest........... 209

Anlage D zur Treatmentgestaltung und - durchführung ................................................... 210

D 1 Ablauf des Untersuchungszeitraums und Hospitationstermine ................................. 210

D 2 Inhalte der einzelnen Stunden der Lernsequenzen 1 bis 5.....................................2 211

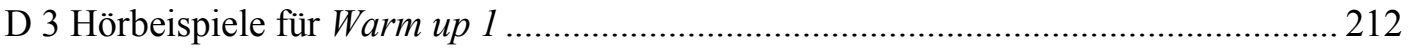

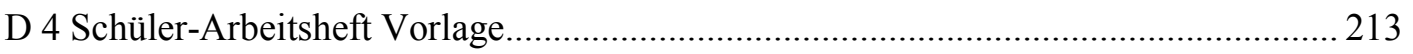

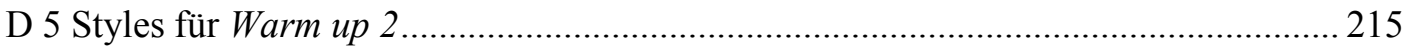

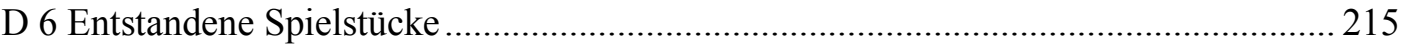

Anlage E Vorgegebene und gespielte Rhythmen im Test................................................... 218 


\section{Abbildungsverzeichnis}

Abb. 2-1: Komponenten des Kompetenzbegriffs nach Weinert ....................................................... 5

Abb. 2-2: Erweiterung des Kompetenzbegriffs in Bezug auf den fachbezogenen Konstruktteil............ 7

Abb. 2-3: Erweiterung des Kompetenzbegriffs in Bezug auf den überfachlichen Konstruktteil............ 8

Abb. 2-4: Gegenüberstellung Puls, Metrum und Rhythmus ................................................................ 9

Abb. 2-5: Stufen des Unterscheidenden Lernens bei Gordon ............................................................ 10

Abb. 2-6: Pyramide des Aufbauens rhythmischer Fähigkeiten bei Jank............................................. 10

Abb. 2-7: Erweiterter Kompetenzbegriffs in Bezug auf rhythmische Kompetenz ................................ 12

Abb. 2-8: Übersicht über die Silben verschiedener Rhythmussprachen ............................................... 18

Abb. 2-9: Rhythmussilben im geraden und ungeraden Metrum bei Gordon ........................................ 19

Abb. 2-10: Zugrundeliegender Rhythmus in den Studien Bamberger, Upitis und Hildebrandt ........... 22

Abb. 2-11: Typen der Rhythmusdarstellung nach Bamberger und Upitis .......................................... 22

Abb. 2-12: Typen der Rhythmusdarstellung nach Hildebrandt ........................................................ 23

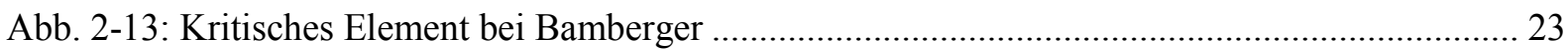

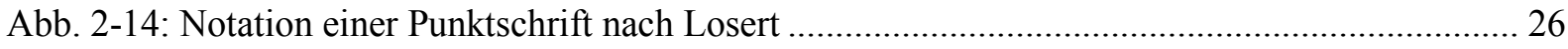

Abb. 3-1: Grafische Darstellung des Zweigruppen-Pretest-Posttest-Forschungsdesigns ...................... 41

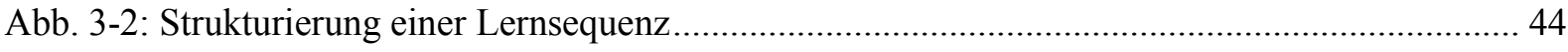

Abb. 3-3: Verteilung der Lernsequenzen auf den Untersuchungszeitraum............................................ 44

Abb. 3-4: Umsetzung der Stufen rhythmischer Kompetenz in den Bausteinen des Rhythmus-

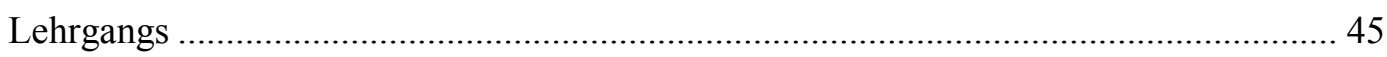

Abb. 3-5: Ansteigende Schwierigkeit der Patterns in den Lernsequenzen ......................................... 47

Abb. 3-6: Übertragung ausgewählter Symbole der Rhythmusnotation in die Strichnotation............... 48

Abb. 3-7: Tafelbild mit Rhythmus-Rahmen, Notenlaminaten sowie A2-Kopie................................... 49

Abb. 3-8: Umsetzung der Stufen rhythmischer Kompetenz in den fünf Lernsequenzen...................... 50

Abb. 3-9: Grafische Veranschaulichung der Aufgabenstellung im ST D ............................................. 59

Abb. 3-10: Kopie der Rückseite zweier Testbogen für ST F, VT und NT ........................................... 61

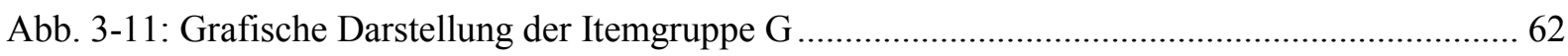

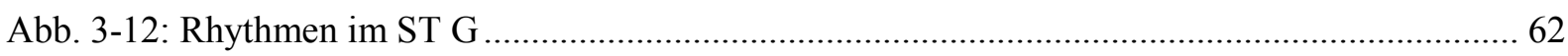

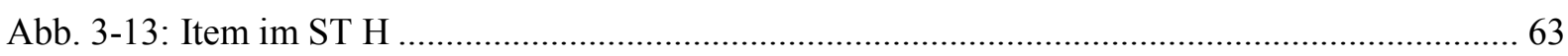

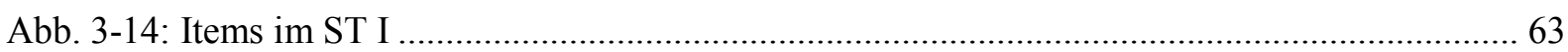

Abb. 3-15: Erwartete Mittelwerte und Standardabweichungen in den Subtests A-F............................. 67

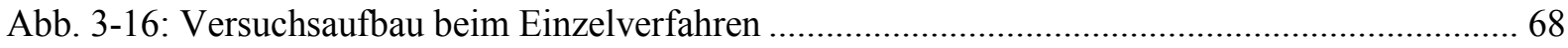

Abb. 3-17: Bildschirmfoto eines Rhythmus in der Sequenzer-Software ............................................... 69

Abb. 3-18: Sequenzeransichten für Item A 1, Takt 1 und 2 .............................................................. 71

Abb. 3-19: Positionswerte der Schläge in Item B 1 von elf Probanden ................................................ 71

Abb. 3-20: Toleranzbereiche für Positionen auf Hauptschlägen in Millisekunden .............................. 72

Abb. 3-21: Toleranzbereiche für Positionen auf Nebenschlägen in Millisekunden.............................. 72 
Abb. 3-22: Darstellung der drei Algorithmusfaktoren .....

Abb. 3-23: Beispiele für ausgewertete Rhythmen für Item B 1 …...................................................... 74

Abb. 3-24: Formeln zur Berechnung der Effektstärke d und der gepoolten Standardabweichung $\sigma \quad \ldots 77$

Abb. 4-1: Klassenstärken und Schulstandorte der involvierten Klassen............................................. 78

Abb. 4-2: Zuordnung der Klassen zu Lehrkräften........................................................................... 79

Abb. 4-3: Anzahl der Probanden zum Zeitpunkt des Nachtests........................................................... 80

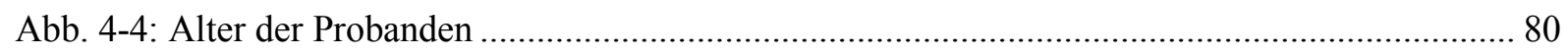

Abb. 4-5: Anteil an Jungen und Mädchen unter den Probanden.......................................................... 80

Abb. 4-6: Verteilung von Instrumentalschülern unter den Probanden.................................................... 81

Abb. 4-7: Zeitplan der Hauptuntersuchung im Schuljahr 2013/14 ..................................................... 82

Abb. 4-8: Ergebnisse in der Gesamtpunktwertung aller Probanden im VT ......................................... 84

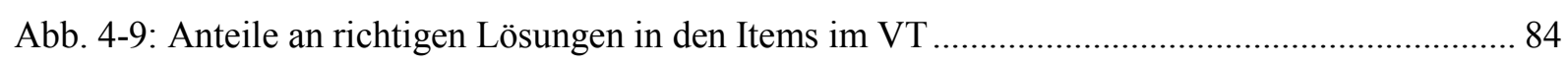

Abb. 4-10: Ergebnisse in der Gesamtpunktwertung aller Probanden im NT ....................................... 85

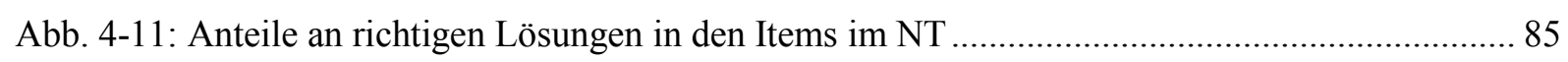

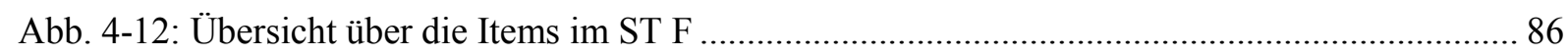

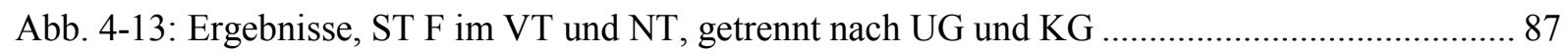

Abb. 4-15: Gesamtdarstellung aller Leistungen im ST F nach Lösungskategorien ............................... 89

Abb. 4-16: Rückseite des Testbogens aus ST F sowie UT f und fc ................................................. 90

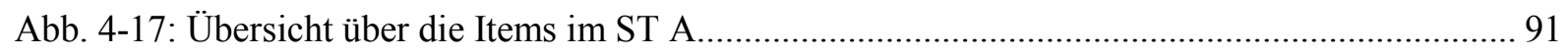

Abb. 4-18: Ergebnisse im ST A, VT und NT, getrennt nach UG und KG........................................ 92

Abb. 4-19: Melodie des Liedes Bruder Jacob ................................................................................. 93

Abb. 4-20: Melodie des Menuettes G-Dur von J. S. Bach .................................................................. 94

Abb. 4-21: Melodie des Liedes Around The World der Band ATC .................................................... 94

Abb. 4-22: Gesamtdarstellung aller Leistungen im ST A nach Lösungskategorien ............................. 95

Abb. 4-23: Angeleiteter bzw. selbstständiger Teil in Item A 4, getrennt nach UG und KG................. 96

Abb. 4-24: Taktgenaue Darstellung des richtigen Spielens im Item A 4, Gruppe 1:0 und 0:1 ........... 97

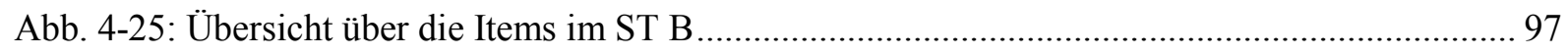

Abb. 4-26: Ergebnisse im ST B, VT und NT, getrennt nach UG und KG ......................................... 98

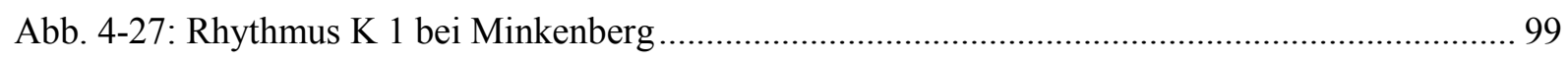

Abb. 4-28: Häufigkeit von Fehlern in der Rubrik Rhythmus an sich im ST B, VT und NT ............... 100

Abb. 4-29: Häufigkeit von Fehlern in der Rubrik Einsatz des Rhythmus im ST B, VT und NT ........ 100

Abb. 4-30: Häufigkeit von Fehlern in der Rubrik Tempo des Rhythmus im ST B, VT und NT ......... 101

Abb. 4-31: Gesamtdarstellung aller Leistungen im ST B nach Lösungskategorien............................. 102

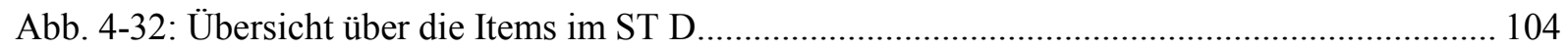

Abb. 4-33: Ergebnisse im ST D, VT und NT, getrennt nach UG und KG....................................... 104

Abb. 4-34: Häufigkeit von Fehlern in der Rubrik Aufgabenverständnis im ST D, VT und NT ......... 106

Abb. 4-35: Häufigkeit von Fehlern in der Rubrik Einsatz des Rhythmus im ST D, VT und NT ........ 107 
Abb. 4-36: Häufigkeit von Fehlern in der Rubrik Tempo des Rhythmus im ST D, VT und NT 107

Abb. 4-37: Gesamtdarstellung aller Leistungen im ST D nach Lösungskategorien . 108

Abb. 4-38: Vielfalt der gespielten Rhythmen im ST D, VT 109

Abb. 4-39: Vielfalt der gespielten Rhythmen im ST D, NT ..... 109

Abb. 4-40: Übersicht über die Items im ST E 109

Abb. 4-41: Ergebnisse im ST E, VT und NT, getrennt nach UG und KG 110

Abb. 4-42: Beispiele für Fehlerkategorie 51, 52 und 55 im ST E. 111

Abb. 4-43: Beispiele für Fehlerkategorie 60 im ST E. 112

Abb. 4-44: Häufigkeit von Fehlern in der Rubrik Rhythmus an sich im ST E, VT und NT. 113

Abb. 4-45: Häufigkeit von Fehlern in der Rubrik Tempo des Rhythmus im ST E, VT und NT 114

Abb. 4-46: Gesamtdarstellung aller Leistungen im ST E nach Lösungskategorien 115

Abb. 4-47: Einteilung der Tempobereiche in ST E. 116

Abb. 4-48: Häufigkeit der Wahl des individuellen Tempos im ST E im NT. 116

Abb. 4-49: Übersicht über die Items im Subtest C. 117

Abb. 4-50: Ergebnisse im ST C, VT und NT, getrennt nach UG und KG 118

Abb. 4-51: Häufigkeiten von Fehlern im UT ec, NT, getrennt nach UG und KG 119

Abb. 4-52: Ergebnisse im ST E und UT ec im NT, getrennt nach UG und KG 120

Abb. 4-53: Gesamtdarstellung aller Leistungen im UT ec nach Lösungskategorien 121

Abb. 4-54: Häufigkeit von Fehlern im UT fc im NT, getrennt nach UG und KG 122

Abb. 4-55: Ergebnisse im ST F und UT fc im NT, getrennt nach UG und KG. 122

Abb. 4-56: Gesamtdarstellung aller Leistungen im UT fc nach Lösungskategorien 123

Abb. 4-57: Ergebnisse in der Gesamtpunktwertung, VT und NT, Probanden der UG 126

Abb. 4-58: Ergebnisse in der Gesamtpunktwertung, VT und NT, Probanden der KG 126

Abb. 4-59: Differenzen aus VT und NT in der Gesamtpunktwertung, getrennt nach UG und KG.... 127

Abb. 4-60: Mittelwerte der Subtests im NT, getrennt nach Jungen und Mädchen 127

Abb. 4-61: Ergebnisse aller Schüler der HU im ST F im NT, getrennt nach UG und KG 128

Abb. 4-62: Mittelwerte aller Schüler der HU im ST F im NT, getrennt nach Klassen 129

Abb. 4-63: Ergebnisse in der 2. Erhebung, getrennt nach Klassenstufe 3 und 4 129

Abb. 4-64: Mittelwerte der einzelnen Klassen der 2. Erhebung, getrennt nach Klassenstufe 3 und 4130

Abb. 4-65: Ergebnisse im ST G, VT und NT, getrennt nach UG und KG... 134

Abb. 4-66: Mittelwerte der einzelnen Subtests im VT, getrennt nach UG und KG 137

Abb. 4-67: Mittelwerte der einzelnen Subtests im NT, getrennt nach UG und KG. 137

Abb. 4-68: Darstellung der Ergebnisse nach der Methode known groups 140

Abb. 5-1: Vorschlag für eine Veränderung des Modells zur Förderung rhythmischer Kompetenz ... 162 


\section{Tabellenverzeichnis}

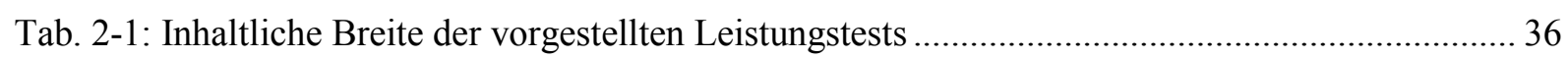

Tab. 3-1: Übereinstimmung der operationalisierten Test-Stufen mit dem Lehrplan............................. 43

Tab. 3-2: Umsetzung der Aspekte des Musiklernens im Rhythmus-Lehrgang.................................... 51

Tab. 3-3: Umsetzung der didaktischen Analyse im Rhythmus-Lehrgang............................................ 52

Tab. 3-4: Operationalisierung der Stufen rhythmischer Kompetenz................................................... 55

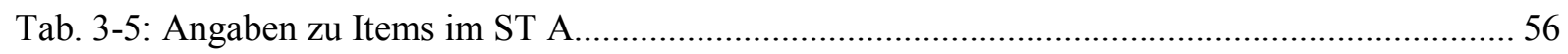

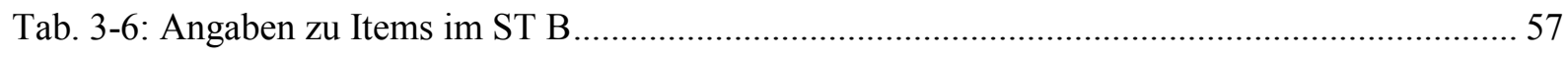

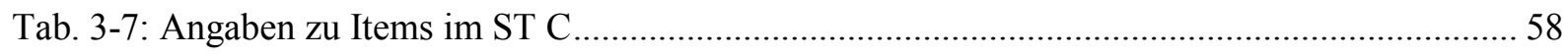

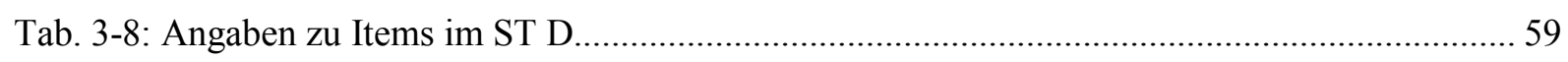

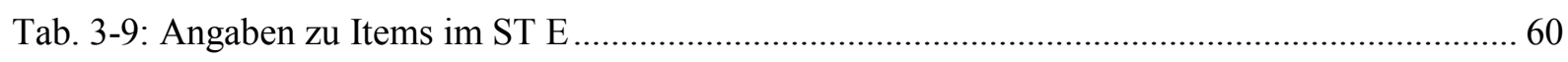

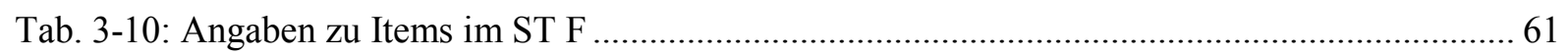

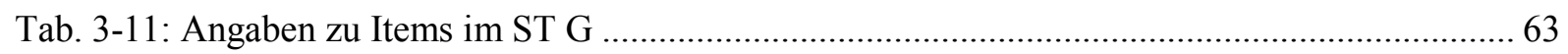

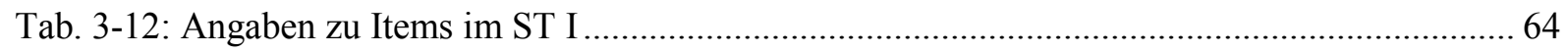

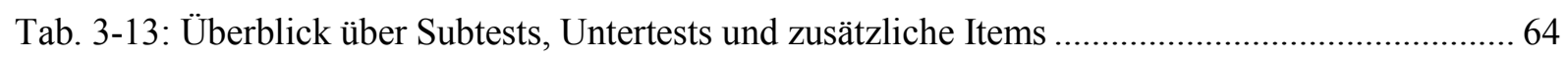

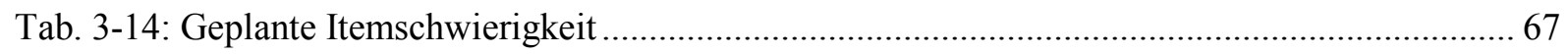

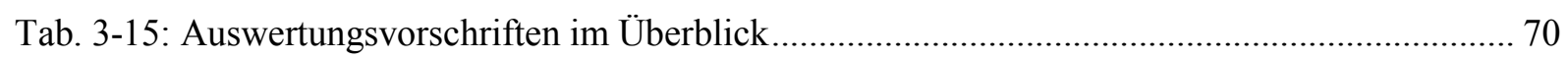

Tab. 4-1: Übersicht über ausgewählte Eckdaten zu Probanden ......................................................... 82

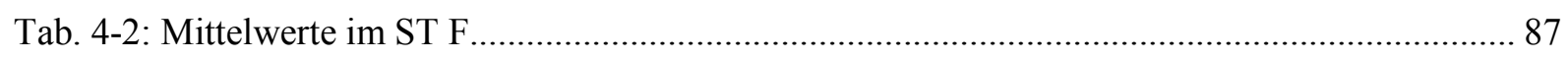

Tab. 4-3: Ergebnisse für t-Test und Effektstärke im ST F ……........................................................ 87

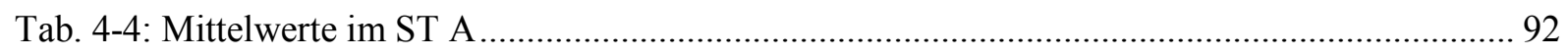

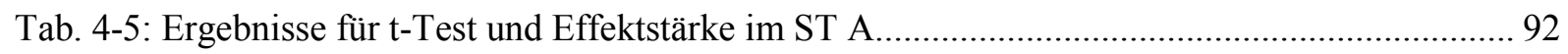

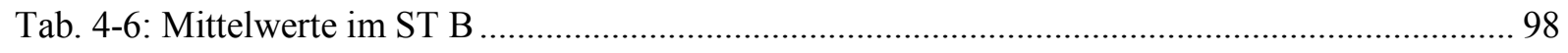

Tab. 4-7: Ergebnisse für t-Test und Effektstärke im ST B ................................................................ 98

Tab. 4-8: Anteil an korrekten Lösungen im ST B und im zusätzlichen Item b 6, VT und NT ............. 99

Tab. 4-9: Übersicht über häufige Ersatzrhythmen im ST B ............................................................ 103

Tab. 4-10: Notenbild für Item B 2 sowie Ersatzrhythmen 5b und 20 ............................................. 103

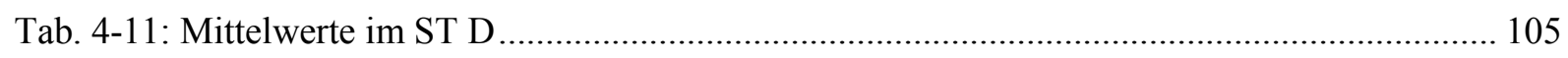

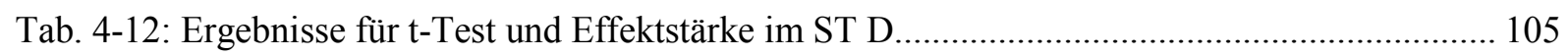

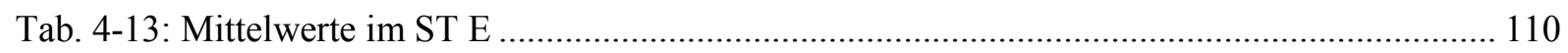

Tab. 4-14: Ergebnisse für t-Test und Effektstärke im ST E ................................................................ 111

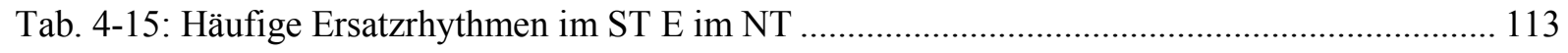

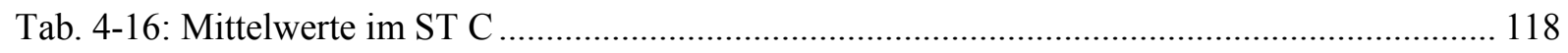

Tab. 4-17: Ergebnisse für t-Test und Effektstärke im ST C............................................................. 118

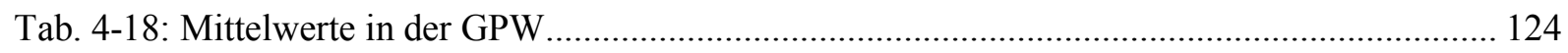

Tab. 4-19: Ergebnisse für t-Test und Effektstärke in der GPW ....................................................... 124

Tab. 4-20: Korrelationen zwischen den Subtests A bis F und GPW im NT ..................................... 125 
Tab. 4-21: Gruppierte Korrelationen der Subtests A bis F 125

Tab. 4-22: Ergebnisse der Gesamtpunktwertung im NT, getrennt nach Klassen. 128

Tab. 4-23: Gewählter Typus beim Aufschreiben von Rhythmen mit Zeichen im UT f im VT 131

Tab. 4-24: Anteil an richtigen Lösungen im UT f und ST F im VT 131

Tab. 4-25: Gewählter Typus beim Aufschreiben von Rhythmen mit Zeichen im UT f im NT 132

Tab. 4-26: Anteil an richtigen Lösungen im UT f und ST F im NT 132

Tab. 4-27: Anteil an richtigen Lösungen im UT e und ST E im VT. 133

Tab. 4-28: Anteil an richtigen Lösungen im UT e und ST E im NT. 133

Tab. 4-29: Korrelation von ST G zu den ST A - F sowie dem GPW im NT 134

Tab. 4-30: Anteil an richtigen Lösungen im ST I, VT und NT, getrennt nach UG und KG. 135

Tab. 4-31: Korrelation zwischen ST I und anderen Subtests sowie dem GPW im NT 136

Tab. 4-32: Vergleich der tatsächlichen Itemschwierigkeiten im NT mit dem Modell 136 


\section{Abkürzungsverzeichnis}

bpm

Schläge pro Minute (beats per minute)

Diff.

Differenz

GPW

Gesamtpunktwertung

HU

Hauptuntersuchung

Kat.

Kategorie

K

Kontrollklasse

KG

Kontrollgruppe

LK

Lehrkraft

LS

Lernsequenz

MU

Musikunterricht

MW

Mittelwert

ms

Millisekunden

NT

Nachtest

SD

Standardabweichung

SE

Standardfehler

ST

Subtest

$\mathrm{Tb}$.

UT

U

Untersuchungsklasse

UG

Untersuchungsgruppe

VT

Vortest 


\section{Abstract}

Die theoretische Fundierung rhythmischer Kompetenz wird an den Kompetenzbegriff Weinerts (2001) sowie das Pyramidenmodell Janks $\left(2013^{5}\right.$ ) angelehnt. Zur Messung rhythmischer Kompetenz wird ein kriteriumsorientierter Leistungstest entwickelt, der sechs Subtests enthält. Nach dem Zweigruppen-Pretest-Posttest-Forschungsdesign (Bortz \& Döring, 2006 ${ }^{4}$ ) wird mit 82 Probanden in der Untersuchungs- sowie 67 Probanden in der Kontrollgruppe (Alter acht bis neun Jahre) aus insgesamt 12 Schulklassen in Klassenstufe 3 gearbeitet. Die Untersuchungsgruppe erhält ein Treatment über 20 Unterrichtswochen hinweg, welches in den regulären Musikunterricht integriert wird. Es enthält Elemente der Music Learning Theory Gordons $\left(2012^{8}\right)$, des Konzeptes der natürlichen Differenzierung nach Krauthausen \& Scherer (2010), der Hinzunahme einer ikonischen Ebene nach Bruner (1974) sowie des Arbeitens in Stamm- und Expertengruppen nach Bovet \& Huwendiek (2008). Die Klassen der Kontrollgruppe erhalten regulären Musikunterricht. Der Entwicklungsstand rhythmischer Kompetenz wird zu Beginn sowie am Ende der Klassenstufe 3 gemessen. Die Leistungsunterschiede der beiden Gruppen im Nachtest dokumentieren in vier der sechs Subtests kleine, mittlere und große Effekte. Die Probanden der Untersuchungsgruppe erzielen beim Lesen von Rhythmen einen bedeutend höheren Anteil an korrekten Lösungen. Das Aufschreiben von Rhythmen erfolgt zu einem hohen Anteil im metrischen Typus.

\section{Abstract}

The theoretical foundation of rhythmical competence draws on Weinert's concept of competence (2001) as well as Jank's pyramid model $\left(2013^{5}\right)$. A criterion-oriented proficiency test containing six sub-tests is developed in order to measure rhythmical competence. Following the two-group pre-test-post-test research design (Bortz \& Döring, 2006 ${ }^{4}$ ), the work is focused on 82 subjects in the test group and 67 subjects in the control group (age eight to nine years), taken from a total of 12 classes. The test group receives coaching over 20 teaching weeks which is integrated into the regular music lessons. This coaching consists of elements of Gordon's music learning theory $\left(2012^{8}\right)$, Krauthausen \& Scherer's concept of natural differentiation (2010), the addition of an iconic stage according to Bruner's stages of representation (1974), as well as working in base groups and expert groups following the pedagogical guidelines of Bovet \& Huwendiek (2008). The classes in the control group have the regular music lessons. The stage of development of rhythmical competence is measured both at the beginning and at the end of year 3. In four of the six sub-tests, small, medium and large effect sizes indicate the differences in proficiency between the two groups in the post-test. The subjects in the test group achieve a significantly higher proportion of correct answers in their reading of rhythms. A high proportion of rhythms are written in metrical type. 



\section{Einleitung}

Der Umgang mit Notenschrift im Musikunterricht der Grundschule ist auf vielen Ebenen von Ambivalenz geprägt: unterrichtspraktisch, curricular und fachdidaktisch. Das Lesen von Notenschrift lässt sich nicht durch Vor- und Nachmachen vermitteln bzw. erlernen. Dies unterscheidet den Lerngegenstand Notenschrift gravierend von anderen wie z. B. Liedern, Mitspielsätzen oder Tänzen. Notenschrift ist ein Symbolsystem, welches helfen kann, notierte Musik zu reproduzieren oder erfundene Musik grafisch zu fixieren. Dafür braucht es einen anderen methodischen Zugang als für musizierpraktische Tätigkeiten. Anders als Lieder, Musikwerke und Tänze wirkt dieser Lerngegenstand nicht auf unsere ästhetischen Empfindungen, er dient lediglich der kognitiven Verständigung über notierbare Parameter von Musik. Dies verstellt den Zugang zu diesem Lerngegenstand in einem Fach, das von Freude am eigenen musikalischen Ausdruck, dem Entdecken der klanglichen Umwelt und der Ausbildung musikalischer Grundkompetenzen geprägt sein soll (Sächsisches Staatsministerium für Kultus, 2009, S. 2).

In den meisten Curricula ist für die dritte Klassenstufe eine Einführung in die Notenschrift, vornehmlich die Rhythmusnotation, vorgesehen. Während in Sachsen ein Umgang mit Rhythmusbausteinen gefordert wird, zählen die meisten anderen Curricula als konkrete Unterrichtsinhalte auf: Viertel- und Achtelnoten, halbe und ganze Noten sowie die dazugehörigen Pausenwerte. Methodische Hinweise auf traditionelle Übungs- und Denkmittel (Losert, 2011), wie z. B. die Rhythmussprache nach Kodály, werden nur in einem Curriculum der 15 Bundesländer gegeben (Ministerium für Bildung, Wissenschaft, Jugend und Kultur Rheinland-Pfalz, 2009) und scheinen ansonsten zum alten Eisen (Gies, 2001) zu gehören.

In der Fachdidaktik gehen die Meinungen über eine Verankerung der Einführung der Notenschrift im Musikunterricht der Grundschule weit auseinander. So wird das Erlernen der Notenschrift einerseits als Bildungsgut (Dartsch, 2010, S. 201), als Beitrag zu Literalität (Spychiger, 2004, S. 4) oder zur Musikalisierung (Fuchs, 2006, S. 45) proklamiert. Schütz befürwortet es im Interesse einer „,besseren intersubjektiven Verständigung im Musikunterricht“ (1997, S. 5), betont jedoch den Stellenwert einer angemessenen Art und Weise des Umgangs damit. Kritisch wird betont, dass im Bildungswesen der allgemeinbildenden Schulen dem Umgang mit Notenschrift der Status einer Kulturtechnik fehle (Küntzel, 2006, S. 61-62). Der goldene Mittelweg wird im gleichzeitigen Erlernen der Notenschrift und des Spiels auf einem Melodieinstrument gesehen (Bähr, 2007, S. 162-163; Maschke, 2008, S. 10; Weber, 2006³ , S. 274). Bildungsstandards für das Fach Musik werden erwünscht (Niessen, Lehmann-Wermser, Knigge \& Lehmann, 2008, S. 6; Knigge \& Lehmann-Wermser, 2008), der Diskurs darüber ist noch im Gange.

Der Forschungsstand zum Umgang mit Notenschrift im Musikunterricht der Grundschule bestätigt, dass die Altersgruppe der Dritt- und Viertklässler entwicklungspsychologisch gute Voraussetzungen besitzt für das Arbeiten mit Symbolsystemen an sich, als auch für den Umgang mit dem 
speziellen System der traditionellen Notenschrift. Sowohl die sich natürlich entwickelnden Fähigkeiten zum Erfinden von Notationsweisen für Musik (Bamberger, 1982, S. 19-30; Upitis, 1987, zitiert nach Gembris, $2013^{4}$, S. 242-246) als auch die kognitiven Voraussetzungen für das Verständnis von Regelsystemen sind gegeben (Zimmermann, 1985, zitiert nach Gembris, 2013 ${ }^{4}$, S. 212-213). Dennoch belegen Studien, dass die Lernerfolge hinter den Erwartungen eher zurückbleiben (Hasselhorn, 2015, S. 149-150, 163; McPherson, 2005, S. 14; Minkenberg, 1991, S. 229232; Wagner, 1970, S. 27-33; s. Anlage A 2).

In der Unterrichtspraxis beobachtet die Autorin (in der Funktion als Fachberaterin) verschiedene Herangehensweisen an den Umgang mit Notenschrift: Unterricht, der die Vermittlung von Wissen über die dazugehörige Praxis stellt und die Verbindung zu Klang und Spielaktion vermissen lässt (vgl. Schütz, 1996, S. 3; Weber, 2006³ , S. 265-266). Unterricht, der nur sporadisch und ohne Kontinuität in Bezug auf den Umgang mit Notenschrift agiert (vgl. Fuchs, 2010, S. 13). Unterricht, der Notation nur scheinbar verwendet und imitative Vermittlungswege wählt. Unterricht, der den Umgang mit Notation gar nicht mehr aufgreift, da die Lehrkräfte resigniert aufgegeben haben. Lehrer an weiterführenden Schulen beklagen die fehlenden Lernstände.

„Es bedarf erheblicher Anstrengungen im Bereich der Binnendifferenzierung und daraus folgernd eines großen methodischen Geschicks, die Kinder auf eine gemeinsame Basis hinzuführen ohne Einzelnen die Freude an der tätigen Auseinandersetzung im Fach Musik zu nehmen. [...] Darüber hinaus müssen in Teilbereichen fachspezifische Aspekte eingeführt werden, die sowohl von ihrer inhaltlichen Verständlichkeit wie von der Altersangemessenheit aus betrachtet, bereits in der Grundschule hätten gefestigt werden können. [...] So kollidiert in diesem Sinne z. B. das notwendige Erkennen, Lesen, Schreiben und musikalische Umsetzen von elementaren Notenwerten (Ganze, Halbe, Viertel, Achtel) mit ihrer Hörerfahrung von bereits sehr komplexen rhythmischen Pattern aus dem Bereich der Popmusik“ (Hortien, Kivi, Stange \& Urban, 2007, S. 8).

Es gibt aktuell keine statistischen Angaben zur Kompetenz im Umgang mit Notenschrift. Um die Aussagen zum Lernstand zu bestätigen, wurde im Rahmen dieser Studie eine sekundäre Erhebung mit 549 Dritt- und Viertklässlern zu diesem Schwerpunkt durchgeführt. Vier leichte Rhythmen wurden gemeinsam musiziert und anschließend mit Zeichen, Silben und / oder Noten aufgeschrieben. Die Ergebnisse bestätigten teilweise das Angemahnte (s. Abschnitt 4.5).

Zusammenfassend geben alle bisherigen Aussagen Hinweise auf ein offenes didaktischmethodisches Entwicklungsfeld. Der geschilderte Zustand ist bedenklich, da das optimale Zeitfenster für den Erwerb grundlegender musikalischer Fähigkeiten innerhalb der ersten neun bis zehn Lebensjahre liegt (Gembris, $2013^{4}$, S. 276; Gordon, 2012 ${ }^{8}$, S. 44-46).

Interessante Ansätze gibt es im Rahmen des sogenannten Aufbauenden Musikunterrichts $A M U$, der sich in Bezug auf das Rhythmuslernen auf die Music Learning Theory Gordons $\left(2012^{8}\right)$ stützt. Diese Theorie betrachtet das Lesen und Schreiben von Rhythmen als Ergebnis eines mehrstufigen Lern- und Abstraktionsprozesses. Dieser beginnt mit dem Erfahren von Metrum und Puls 
und gelangt über das Erarbeiten und Reflektieren eines Hörvokabulars an Rhythmen zum Lesen und Schreiben derselben. Wesentlicher Bestandteil dieser Theorie ist das Erlernen einer Rhythmussprache. Das Modell wurde im deutschsprachigen Raum von Jank für den Musikunterricht in der Sekundarstufe in die Pyramide des Aufbauens rhythmischer Fähigkeiten überführt (20135 , S. 129). Eine Übertragung für den Musikunterricht in der Grundschule fand durch Fuchs statt (2010, S. 1726). Die dazugehörigen Praxisvorschläge geben zahlreiche Anregungen zur Arbeit nach dieser Theorie, steigern jedoch zu schnell die Schwierigkeit der verwendeten Rhythmussilben und sind nicht auf die zielgerichtete Umsetzung in einer Klassenstufe ausgerichtet (ebd., S. 113-137).

Die vorliegende Arbeit verfolgt das Ziel, ein Konzept für ein didaktisch-methodisch abgerundetes Treatment zur systematischen Einführung in die Rhythmusnotation zu entwickeln und zu erproben. Es soll rhythmische Kompetenz im Allgemeinen sowie das Lesen und Schreiben von Rhythmusnotation im Besonderen fördern, die vom Aufbauenden Musikunterricht befürworteten Theorien von Gordon $\left(2012^{8}\right)$ bzw. Jank $\left(2013^{5}\right)$ aufnehmen sowie auf Grundlagen fußen, die bereits zum Wissensstand der Fachdidaktik und der allgemeinen Pädagogik zählen. Als notwenige Voraussetzung wird die Erhebung des Entwicklungstandes rhythmischer Kompetenz bei Schülern zu Beginn der Klassenstufe 3 gesehen. Der Erfolg des Treatments sollte messbar sein. Dazu wird ein theorie-adäquater Test benötigt, der am ehesten im Modell eines kriteriumsorientierten Leistungstests (Gembris, 2013 ${ }^{4}$, S. 106-107; Rost, 2007², S. 150-152) zu finden sein könnte. 


\section{Theorie und Fragestellungen}

In diesem Teil der Arbeit wird der Begriff rhythmische Kompetenz näher definiert. Dabei wird zuerst Bezug zum allgemeinen Kompetenzbegriff nach Weinert genommen, anschließend zum musikpädagogischen Kontext. Anschließend werden allgemeine und spezielle Lern- und Lehrvoraussetzungen in Bezug auf Erwerb und Förderung rhythmischer Kompetenz aus Sicht der Fachdidaktik betrachtet (s. Abschnitt 2.2). Mit Blick auf die Umsetzung dieser Lernprozesse in einer groBen heterogenen Lerngruppe wird der Umgang mit Rhythmusnotation einer didaktischen Analyse nach Klafki unterzogen (s. Abschnitt 2.3).

Anschließend werden vorhandene Treatments auf deren Eignung zur Förderung rhythmischer Kompetenz betrachtet (s. Abschnitt 2.4). Daran an schließt sich die Betrachtung vorhandener Testinstrumente zur Erhebung von Daten zum Entwicklungsstand und zum Lernzuwachs in Bezug auf rhythmische Kompetenz (s. Abschnitt 2.5). Mit den wissenschaftlichen Fragestellungen und Hypothesen wird das Kapitel abgeschlossen (s. Abschnitt 2.6).

\subsection{Zum Begriff rhythmische Kompetenz}

Der aktuell am häufigsten verwendete Kompetenzbegriff geht auf Weinert zurück und betrachtet die ,bei Individuen verfügbaren oder durch sie erlernbaren kognitiven Fähigkeiten und Fertigkeiten, um bestimmte Probleme zu lösen“ sowie die „damit verbundenen motivationalen, volitionalen und sozialen Bereitschaften, damit die Problemlösungen in variablen Situationen erfolgreich und verantwortungsvoll genutzt werden können“ (Weinert, 2001, S. 27). In Bezug auf die inhaltlichen Komponenten lässt sich ein zweiteiliges Konstrukt aus fachbezogenen und überfachlichen Komponenten erkennen (s. Abb. 2-1). Beide Komponenten werden beim Problemlösen vom Individuum herangezogen, wobei es abhängig vom fachlichen Kontext sinnvoll sein kann, den Terminus Problemlösen gegen die Begriffe Anforderungssituation oder Aufgabenstellungen auszutauschen (vgl. Knigge, 2014, S. 112-114). Wird die Anforderungssituation gemeistert, kann auf das Vorhandensein von Kompetenz geschlussfolgert werden.

Seit Beginn des Jahrtausends nimmt der Kompetenzbegriff, nicht zuletzt angestoßen durch die PISA-Debatte, immer mehr Raum im pädagogischen Diskurs ein (vgl. Knigge, 2014, S. 106-109). Für die Musikpädagogik ist dies nicht unproblematisch.

„Aus musikpädagogischer Perspektive ist diese Situation in mehrerlei Hinsicht herausfordernd, was bereits auf grundlegend terminologischer Ebene deutlich wird; denn ,Kompetenz ${ }^{‘}$ ist bislang weder ein ,Grundbegriff“ des Faches, noch kann er zu den historisch gewachsenen, einheimischen` Begriffen der Musikpädagogik gezählt werden“" (Knigge, 2014, S. 108).

Als einengend wird die Fokussierung auf kognitive Fähigkeiten und Fertigkeiten betrachtet. Niessen, Lehmann-Wermser, Knigge und Lehmann stellen dazu in ihren Betrachtungen eine deutlich 
weitergreifende Sichtweise anderer Pädagogen vor, bleiben aber in den weiteren Ausführungen sehr eng an der Formulierung von Weinert.

„Competent performance or effective action implies the mobilization of knowledge, cognitive and practical skills, as well as social and behavior components such as attitudes, emotions, and values and motivations. A competence - a holistic notion - is therefore not reducible to its cognitive dimension" (Rychen \& Salganik, 2003, zitiert nach Niessen et al., 2008, S. 4).

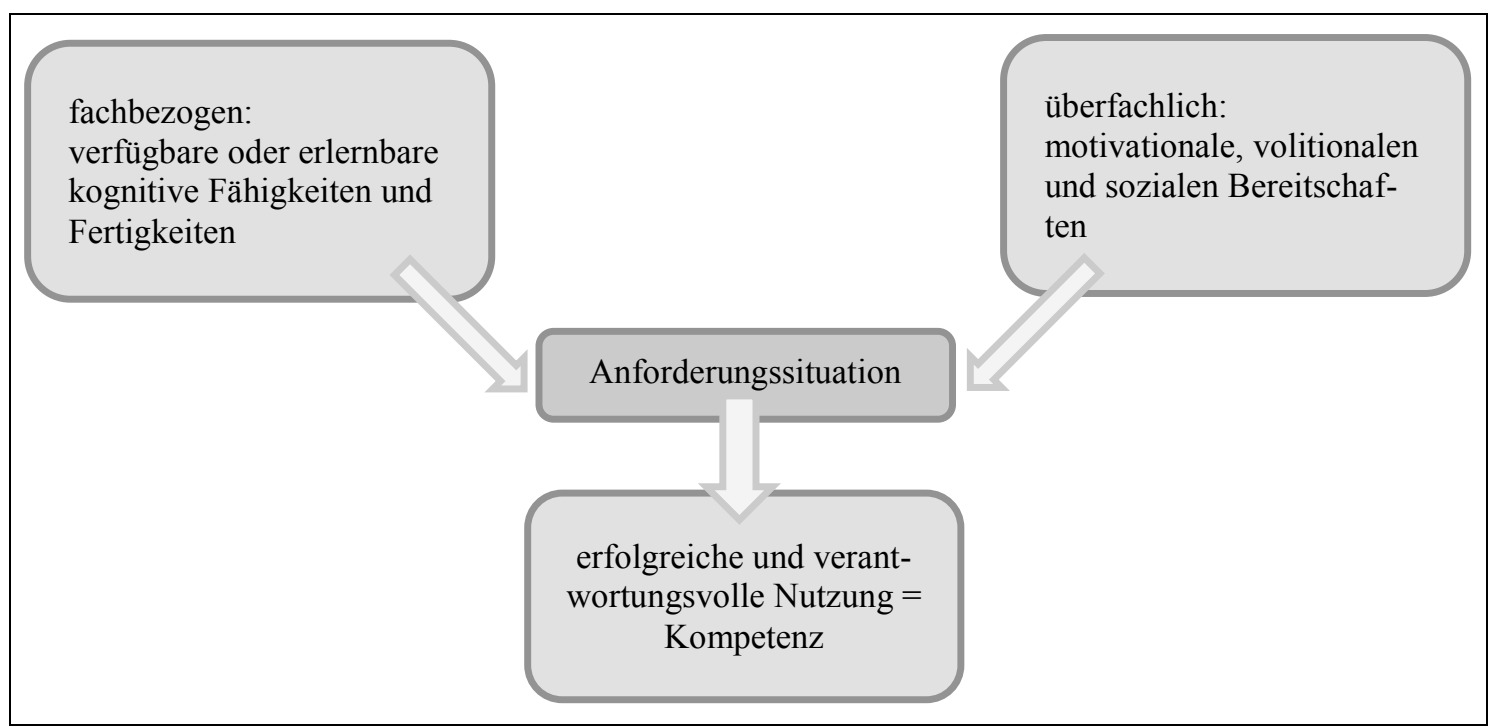

Abb. 2-1: Komponenten des Kompetenzbegriffs nach Weinert

Niessen et al. betonen ausdrücklich, dass der kognitive Aspekt eine bedeutende Rolle beim Aufbau musikalischer Kompetenz spielt und dass musikpraktisches Lernen an vorhandenes Wissen und an Erfahrung gebunden ist (ebd., S. 5), doch wird mit der alleinigen Nennung des Begriffes kognitiv in einem Fach, das stark von handelndem Vollzug, Emotion und Ästhetik geprägt ist, nur schwer eine Brücke geschlagen zu vormals getroffenen Untergliederungen in einen kognitiven, affektiven und psychomotorischen Bereich. Da der Wissenserwerb beim Musizieren auf Handlungsvollzug aufbaut, braucht es ein Zusammenspiel von sensomotorischen und kognitiven Fähigkeiten und Fertigkeiten.

„Das für musikalische Handlungskompetenz notwendige Handlungswissen ist implizites, prozedurales Wissen, das auf die Repräsentation genuin musikalischer Phänomene [...] gerichtet ist. Unterricht, der zuerst und vor allem musikalisches Lernen ermöglichen und unterstützen soll, muss sich an den lerntheoretischen Voraussetzungen zur Bildung eines musikalischen Handlungswissens orientieren. Dazu bedarf es der Bildung genuin (immanent) musikalischer (nicht: begrifflicher) Repräsentationen. [...] Wenn genuine Repräsentationen der musikalischen Phänomene nicht entwickelt werden, ist eine bewusste Wahrnehmung der musikalischen Gestalten - seien es komponierte Werke oder spontane Improvisationen - nicht möglich. Als Ausweg bleiben dann der Rückzug auf emotionale Empfindungen, assoziative Beschreibungen und lebensweltliche Verknüpfungen, die ihren pädagogischen Sinn aber erst entfalten können, wenn sie an musikalische Wahrnehmungen gebunden sind“" (Gruhn, 2003, S. 115-116). 
Die Schwierigkeit der Übernahme des Kompetenzbegriffs in die Musikpädagogik liegt deshalb möglicherweise in der Besonderheit des Erwerbs musikalischer Kompetenzen schlechthin. Wenn Musiklernen nur über den Vollzug musikalischer Prozesse gelingen kann, liegt es auf der Hand, dass der handelnde, sensomotorische Vollzug vor dem Erwerb erlernbarer kognitiver Fähigkeiten (Kompetenzbegriff Weinert) liegen muss bzw. mit ihm einhergeht.

Musikalische „Gegenstände als Handlungsergebnis [unterscheiden sich] von anderen Objekten gravierend: Sie existieren nur in ihrem Vollzug und können nur durch Aktivieren von Prozessen des Nachvollziehens erfaßt werden. Hierin gleichen sie der gesprochenen Sprache. Da aber Sprache von jedem Schriftkundigen auch über Lesen erfaßt werden kann, erfährt sie zusätzliche Stützung durch andere Prozesse der Informationsverarbeitung. Diese Möglichkeit bleibt in der Musik nur einem geringen Prozentsatz von Experten vorbehalten“ (Oerter, 2002 ${ }^{4}$, S. 253-254).

Ein weiteres Problem in Bezug auf den Kompetenzbegriff liegt darin, musikalische Kompetenz prinzipiell einer empirischen Überprüfung zugänglich zu machen. Kaiser betrachtet hierzu den Kompetenzbegriff in der Musikpädagogik in Abgrenzung zum Performanzbegriff nach Chomsky. Während unter Kompetenz die ideale Hervorbringung von Leistung nach einem zugrundeliegenden System von Regeln verstanden wird, entspricht Performanz der tatsächlichen Leistungserbringung durch ein konkretes Individuum (vgl. Kaiser, 2001, S. 5). Mit Blick auf ein Zweiebenenmodell aus Oberflächen- und Tiefenstruktur von Handlungen bezieht sich Kaiser zunächst auf die Überprüfung von Kompetenz in Sprachhandlungen. Dazu stellt er fest, dass diese nur aufgrund der „Analyse der Oberflächenstruktur rekonstruiert werden“ kann und eine „Rekonstruktion der Kompetenz aus den performativen Akten immer höchstens annähernd gelingen kann“(Kaiser, 2001, S. 6).

„Ein realer Sprecher realisiert immer nur ausschnitthaft sprachliche Kompetenz. Folglich kann aus den performativen Akten nur bedingt auf die Form einer darunter liegenden Kompetenz geschlossen werden“ (Kaiser, 2001, S. 6).

Daraus folgt, dass Kompetenz nicht direkt gemessen werden kann und ein vollständiges Abbild nicht möglich ist. Dieses Argument spricht jedoch durch die Betonung von performativen Akten ebenfalls, zumindest in Bezug auf rhythmische Kompetenz, für eine Erweiterung des Begriffes aus erlernbaren kognitiven Fähigkeiten auf handlungsbezogene und beobachtbare, also sensomotorische performative und Fähigkeiten und Fertigkeiten.

Kaiser wirft bezogen auf musikalische Kompetenz den Begriff der operativen Fähigkeiten auf (Kaiser, 2001, S. 6-7). Dieser beinhaltet einerseits konkretes Musizieren (z. B. das Singen eines Liedes) als auch die gleichzeitige Anwendung musikgenerativer Regeln (z. B. Wahrnehmen und gedächtnismäßiges Behalten-Können einer Folge von Tönen; vgl. ebd.). Hier schließt sich wiederum der Kreis: Sensomotorische Fähigkeiten und Fertigkeiten werden zum praktischen Vollzug von Musiken benötigt, gleichzeitig werden kognitive Fähigkeiten und Fertigkeiten zum Anwenden der jeweiligen musikgenerativen Regeln benötigt. Lange bevor generative Regeln von Musikaus- 
übenden erworben und angewendet werden können, kann das Kind schon in der Lage sein, einfache Lieder zu singen, Rhythmen zu klatschen oder im Takt mitzuwippen. Auch hier gibt es Parallelen zum Spracherwerb. Nach einem individuell verschieden langen Entwicklungszeitraum, in dem Kinder vorwiegend nachsprechen und allmählich Einwort- und Zweiwortsätze bilden, verfügen sie schlussendlich über eine Syntax, die es ihnen erlaubt, längere Sätze zu bilden (vgl. Gruhn, 2010, S. 55). Der Grad des Anwendens dieser generativen Regeln unterscheidet dann vorwiegend imitatorisch erworbene von selbstständig erbrachten Leistungen bzw. implizites von explizitem Regelwissen.

„In analoger Weise kann das Gehirn auch aus musikalischen Mustern, den Melodien und Rhythmen allgemeine Regeln ableiten, z.B. darüber, welchen Grundton es erwarten kann oder welche Töne in eine Melodie passen und welche nicht“ (Gruhn, 2010, S. 58).

Aus den bisher dargestellten Gründen wird für diese Studie der Kompetenzbegriff im fachbezogenen Konstruktteil um den Begriff sensomotorisch erweitert (s. Abb. 2-2). Der ebenfalls hinzugefügte Begriff operative Fähigkeiten betont die beobachtbaren und messbaren Handlungen, aus denen ein Rückschluss auf die kognitiven Fähigkeiten und Fertigkeiten gezogen werden kann.

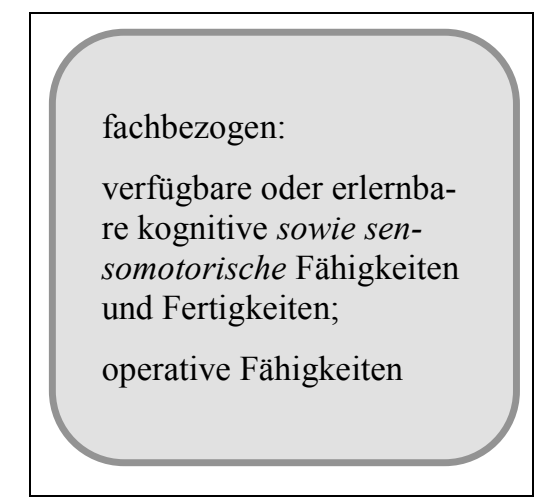

Abb. 2-2: Erweiterung des Kompetenzbegriffs in Bezug auf den fachbezogenen Konstruktteil

Im überfachlichen Konstruktteil wird die Anforderungssituation von den mentalen Dispositionen Motivation und Wille sowie den sozialen Bereitschaften geprägt. Diese überfachlichen Kompetenzanteile aus motivationalen, volitionalen und sozialen Bereitschaften stellen sich besonders für die empirische Schulleistungsforschung (in vielen Fächern) als schwer nachprüfbar dar (vgl. Knigge, 2014, S. 112). Möglicherweise ist dies der Tatsache geschuldet, dass in Bezug auf Motivation und Wille sowohl langfristige und prinzipielle Dispositionen mit einer gewissen Stabilität und Konstanz als auch kurzfristige Dispositionen, die tagesformabhängigen Schwankungen und momentanen Verfassungen unterliegen, in das Bewältigen einer Anforderungssituation hineinwirken. Die Gebundenheit an einen in der Zeit ablaufenden Musizierprozess erfordert aber auf jeden Fall vom Individuum, Bereitschaft zu zeigen, über eine festgelegte Zeitspanne hinweg, Freude am Mu- 
sizieren zu haben sowie Ausdauer und Konzentration zu erbringen und finden deshalb Erwähnung im erweiterten Kompetenzbegriff (s. Abb. 2-3).

„Auch der Kompetenzbegriff im Bildungsstandardkontext bezieht ausdrücklich motivational und volitionale Faktoren mit ein, auf unser Fach bezogen also etwa die Lust, sich mit verschiedenen Formen von Musik zu beschäftigen, und die Anstrengung, ein Instrumentalstück zu bewältigen“ (Knigge \& LehmannWermser, 2009, S. 4).

In Bezug auf die sozialen Bereitschaften sollte im Fach Musik nicht allein auf den solistischen sondern vor allem auf den im Ensemble der Schulklasse stattfindenden Musizierprozess das Augenmerk gelegt werden. Für diesen Musizierprozess sind Fähigkeiten wie u. a. Zuhören und Rücksichtnahme notwendig. Zuhören als Bedingung für das richtige Timing sowie Rücksichtnahme in Bezug auf das angemessene Einbringen des eigenen Spiels in das Ensemble. Einen gewinnbringenden Rahmen für Fähigkeiten in diesem sozialen Bereich kann man in den sogenannten Big Five sehen. Dieses Raster findet in der allgemeinen und pädagogischen Psychologie eine vergleichsweise große Akzeptanz (vgl. Gnahs, 2010, S. 24). Zu ihnen zählen die Dimensionen Neurotizismus / emotionale Stabilität, Extraversion / Offenheit und Entgegenkommen, Verträglichkeit, Gewissenhaftigkeit und Offenheit für neue Erfahrungen.

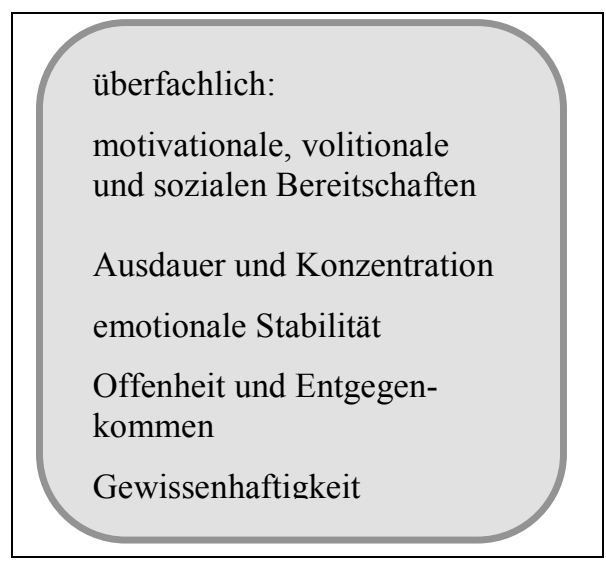

Abb. 2-3: Erweiterung des Kompetenzbegriffs in Bezug auf den überfachlichen Konstruktteil

Speziell drei Dimensionen könnten von größerem Einfluss beim gemeinsamen Musizieren im Ensemble sein. Die Dimension Neurotizismus / emotionale Stabilität bewegt sich zwischen den Polen ängstlich-selbstsicher, nervös-zufrieden sowie gestresst-entspannt. Damit hat sie großen Einfluss auf das Einbringen in den Musizierprozess. Ebenso liegt es nahe, dass gleiches auf die Dimension Extraversion / Offenheit und Entgegenkommen zutrifft. Hier werden die Pole aus den Eigenschaften unbekümmert-zögernd, zugewandt-abgewandt sowie entscheidungsfreudig-zurückgezogen gebildet. Ebenso ist die Dimension Gewissenhaftigkeit, deren Pole aus sorgfältig-nachlässig, wohlorganisiert-planlos sowie pflichtbewusst-lustbetont geprägt sind, von entscheidendem Einfluss (vgl. ebd., S. 24-25). Obwohl das Problem der Messbarkeit dieser Eigenschaften und Bereitschaften sich nicht aufheben lässt, sollen drei dieser Merkmale in den erweiterten Kompetenzbegriff aufgenom- 
men werden, um das Augenmerk auf diese in der Musikpädagogik wichtige Facette zu erweitern (s. Abb. 2-3).

Nachdem der Begriff der Kompetenz betrachtet wurde, soll nun das erweiternde Attribut rhythmisch näher definiert werden. Lexikalisch bezeichnet der Begriff Rhythmus ein „Ordnungs- und Gestaltungsprinzip der relativen Tondauern und Tondauerverhältnisse, aus dem sich Takt und Taktschwerpunkt ergeben“ (Hirsch, 1987, S. 393). Der Taktschwerpunkt ergibt sich dabei aus dem Metrum, welches wiederum aus einem fortwährenden und gleichmäßigem Puls (Grundschlag) gebildet wird (s. Abb. 2-4; in Anlehnung an die Grafik auf der Seite http://musik-ist-mehr.de/pulstakt-metrum/).

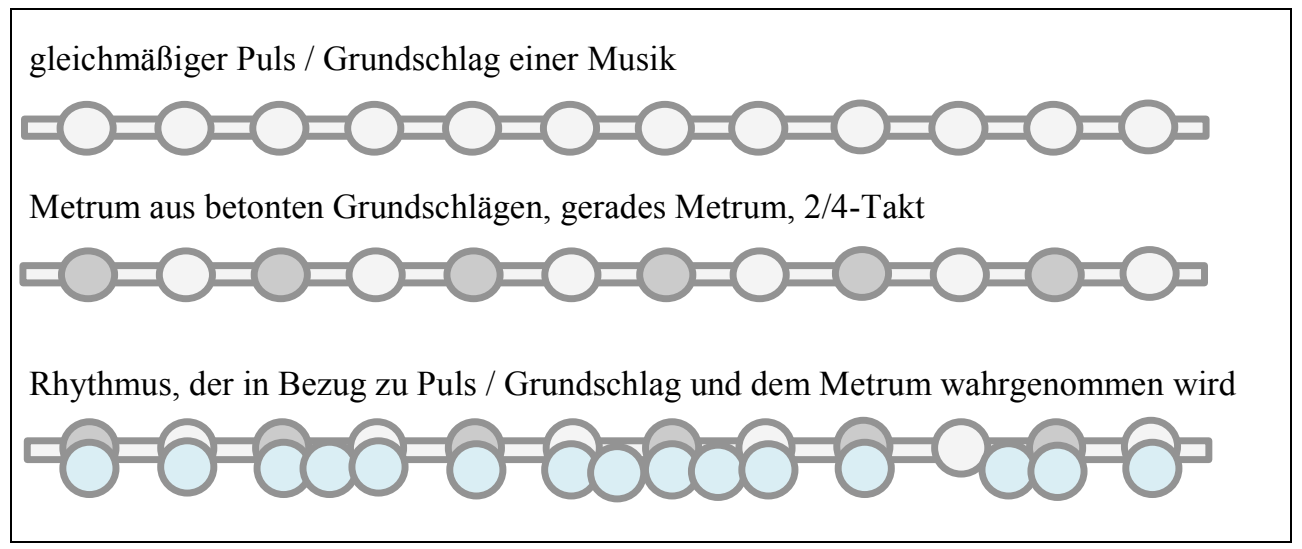

Abb. 2-4: Gegenüberstellung Puls, Metrum und Rhythmus

Das abgeleitete Adjektiv rhythmisch umfasst deshalb gleichermaßen die Parameter Rhythmus und Metrum. Während der rhythmische Aspekt ausdrücklich im Begriff rhythmische Kompetenz enthalten ist, wird der metrische Aspekt nur mitgedacht, jedoch nicht ausdrücklich benannt. Dies hat folgenden Grund: Während das Metrum musikalisch einzeln dargestellt werden kann, bedarf das Musizieren von Rhythmen immer eines mitschwingenden bzw. mitgedachten Metrums (vgl. Gordon, 2012 ${ }^{8}$, S. 173-174; Jank, 2007², S. 107). Rhythmus ist ohne Bezug zu einem Metrum nicht realisierbar. Deshalb darf davon ausgegangen werden, dass der Leser beim Begriff rhythmisch den Begriff metrisch konnotiert.

Gordon $\left(2012^{8}\right)$ betrachtet als wesentlichen Bestandteil rhythmischer Kompetenz im Konzept der Music Learning Theory den Erwerb eines Hör- und Aufführungsvokabulars rhythmischer Patterns (Stufe 1, s. Abb. 2-5). Diese Patterns werden in Verbindung mit Rhythmussilben gebracht (Stufe 2). Die empfohlenen Rhythmussilben gehören zur Gruppe der relativen Rhytmussprachen, welche in erster Linie den Bezug zum Puls / Grundschlag bewusst machen. Dies ermöglicht, bei vetrauten Patterns das Metrum bestimmen zu können (Stufe 3). Auf diesen drei Stufen aufbauend werden die Patterns mit der entsprechenden Notation in Beziehung gesetzt (Stufe 4). 
„Partial synthesis und symbolic association werden auf der Stufe composite synthesis kombiniert. Partial synthesis befähigt die Schüler, einer Serie vertrauter Patterns syntaktischen Zusammenhang zu geben sowie die innere Logik der Patterns und das Verhältnis der Patterns unterreinander zu verstehen. In Stufe symbolic association werden einzelne Patterns notationsbezogen auditiert. Die Stufe composite synthesis schließlich schafft eine Verbindung dieser Fähigkeiten und versetzt Schüler in die Lage, beim Lesen einer Serie vertrauter Patterns diese notationsbezogen zu auditieren und ihr syntaktische Bedeutung zu geben“ (Tappert-Süberkrüb, 1999, S. 87).

\begin{tabular}{|c|c|}
\hline Stufe & Inhalt \\
\hline Aural / Oral & $\begin{array}{l}\text { Erarbeitung eines Hör- und Aufführungsvokabulars } \\
\text { rhythmischer Patterns }\end{array}$ \\
\hline Verbal Association & Verbindung rhythmischer Patterns mit Rhythmussilben \\
\hline Partial Synthesis & Bestimmung des Metrums einer Serie vertrauter Patterns \\
\hline $\begin{array}{l}\text { Symbolic Association } \\
\text { reading - writing }\end{array}$ & Erlernen der Notation einzelner rhythmischer Patterns \\
\hline $\begin{array}{l}\text { Composite Synthesis } \\
\text { reading - writing }\end{array}$ & $\begin{array}{l}\text { notationsbezogene Audiation einer Serie vertrauter Patterns, } \\
\text { dieser Folge eine syntaktische Bedeutung geben }\end{array}$ \\
\hline
\end{tabular}

Abb. 2-5: Stufen des Unterscheidenden Lernens bei Gordon

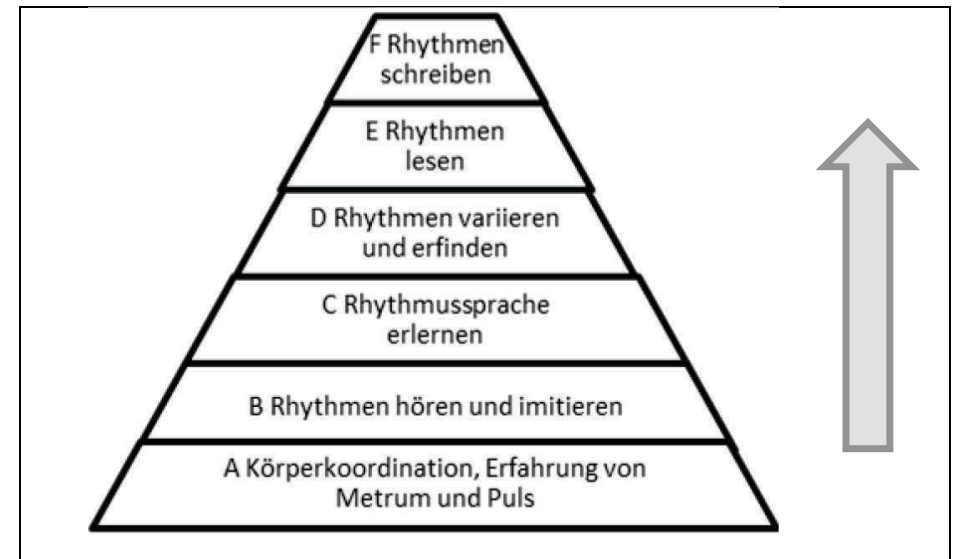

Abb. 2-6: Pyramide des Aufbauens rhythmischer Fähigkeiten bei Jank

Nach Jank und Gallus bildet rhythmische Kompetenz im Konzept des Aufbauenden Musikunterrichts eine von mehreren Dimensionen musikalischer Kompetenz (Jank, 2013 ${ }^{5}$, S. 124-131). Dabei wurde das Konzept Gordons variiert übernommen. In einer Pyramide wird die großräumige Abfolge der Lehrschritte verdeutlicht und diese mit den Buchstaben A bis F benannt (s. Abb. 2-6, in Anlehnung an Jank, 20135 , unter Mitarbeit von Gallus, S. 129). Das Bild der Pyramide versinnbildlicht ein Erreichen höherer und damit komplexer werdender Stufen.

Als Basis wird die Stufe A Körperkoordination, Erfahrung von Metrum und Puls eingefügt (ebd.). Ziel ist es, den eigenen Körper zu erklingender oder produzierter Musik bewegungsmäßig in Einklang (Koordination) zu bringen und sowohl Metrum als auch Puls / Grundschlag empfinden 
bzw. darstellen zu können. Gordon integriert diese Stufe in die Stufe Partial Synthesis, in der er nach einer großen Gruppe von Rhythmen im geraden Metrum, Rhythmen im ungeraden Metrum anbietet (vgl. Gordon, 2001).

Die Stufen B Rhythmen hören und imitieren und C Rhythmussprache erlernen entsprechen den Stufen Aural / Oral sowie Verbal Association bei Gordon. Ein Repertoire aus Patterns (Rhythmusbausteinen) wird in Zusammenhang mit dem gleichzeitigen Erlernen der Rhythmussprache (nach Gordon) erworben. Diese Patterns werden im Call-Response-Prinzip vermittelt, der Lehrer spielt vor, die Schülerinnen und Schüler spielen nach.

Die Stufe D Rhythmen variieren und erfinden fügen die Autoren zusätzlich ein. Sie findet bei Gordon keine direkte Entsprechung. Die dieser Position entsprechende Stufe Partial Synthesis umfasst bei Gordon die Befähigung, „das Metrum einer Serie vertrauter Patterns (welche in gewohnter oder ungewohnter Reihenfolge erklingen) zu bestimmen und dem Gehörten auf diese Weise syntaktische Bedeutung zu geben. Hierbei spielt das Auditieren ... der macrobeats eine signifikante Rolle“ (Tappert-Süberkrüb, 1999, S. 85). Bei Gordon finden sich Inhalte zum Rhythmenvariieren in der Stufe Skill Creativity / Improvisation-verbal, die jedoch zum unterscheidenden Lernen gehört. Im Kursheft Jump Right In ist in Rhythm Unit 7 eine Übung integriert, in welcher nur ein Schüler auf den Call des Lehrers eine Response gibt, die nicht dem Call des Lehrers gleicht (Gordon, 2001 ${ }^{4}$, S. 132-133). Diese Übung entspricht der Idee von Stufe D.

Die Stufen E Rhythmen lesen und F Rhythmen schreiben entsprechen in ihrer Position (in der Pyramide) den Stufen Symbolic Association reading - writing und Composite Synthesis reading writing bei Gordon. Dies suggeriert, dass das Schreiben von Rhythmen eine dem Lesen von Rhythmen nachfolgend zu erlernende bzw. schwerer erlernbare Fähigkeit sei. Auch bei Gordon erfolgt die Reihenfolge im Kursheft Jump Right In erst mit Lesen (Rhythm Unit 10), dann mit Schreiben (Rhythm Unit 13) (Gordon, 2001 , S. 137-139, 143-145).

Beide Modelle zusammenfassend betrachtend überwiegen die Gemeinsamkeiten, die im Erwerb eines Rhythmusrepertoires (Patterns) fußen, das Erlernen einer Rhythmussprache integrieren sowie im darauf aufbauenden Umgang mit dem Notenbild durch einen lesenden und schreibenden Zugang liegen. Insofern stellen die Abweichungen in der Darstellung von Jank und Gallus eher eine Implementierung der praktischen Handlungsvorschläge aus Gordons Kursheft Jump Right In dar. Die Stufen A bis F entsprechen im Konstrukt rhythmischer Kompetenz den Anforderungssituationen (s. Abb. 2-7, mittig).

Über diese sechs Stufen hinaus erachtet die Autorin auch das Zusammenspiel mit einem zweiten oder mehreren anderen Musizierenden als wichtigen Bestandteil rhythmischer Kompetenz. Dies entspricht einer häufig anzutreffenden Anwendungssituation im Musikunterricht der Grundschule, 
z. B. beim Umsetzen eines Mitspielsatzes. Es verlangt vom Spielenden die Konzentration sowohl auf das eigene rhythmische Spiel als auch auf das der Mitmusizierenden.

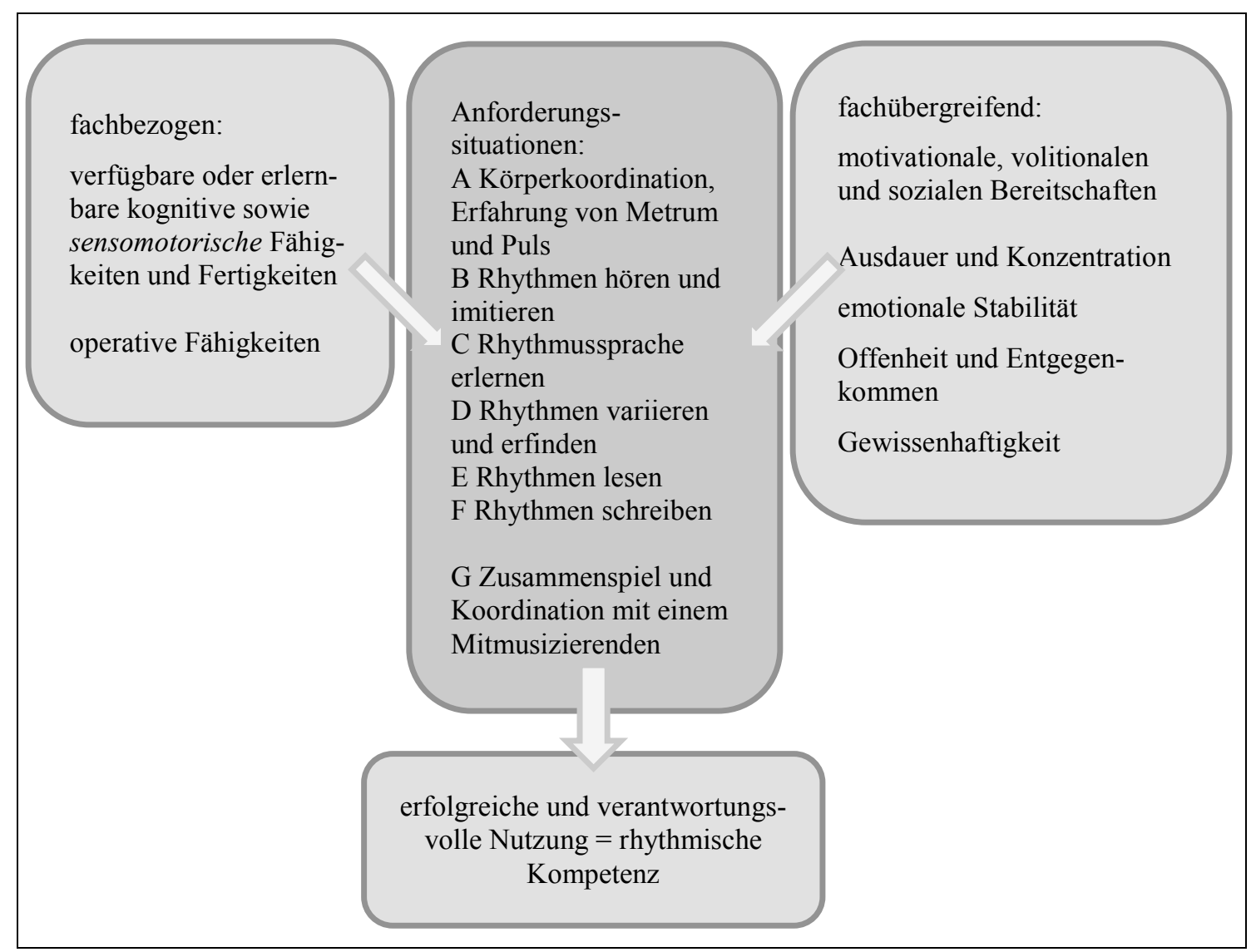

Abb. 2-7: Erweiterter Kompetenzbegriffs in Bezug auf rhythmische Kompetenz

Das gemeinsame Rhythmusspiel wird den bereits genannten Fähigkeiten in Bezug auf rhythmische Kompetenz als Stufe G hinzugefügt (s. Abb. 2-7, mittig). Erst das Musizieren im Ensemble (sozialer Aspekt) erschließt die Notwendigkeit des Aufbauens von Fähigkeiten in Bezug auf rhythmische Kompetenz in den genannten Stufen A bis F (vgl. Oerter \& Bruhn, 1998, S. 332). Damit umfassen die Anforderungssituationen in Bezug auf die rhythmische Kompetenz insgesamt sieben verschiedene Aufgabenstellungen.

Ausgehend von Weinerts Kompetenzbegriff kann der Begriff rhythmische Kompetenz für diese Studie zusammenfassend als die verfügbaren oder erlernbaren kognitiven sowie sensomotorischen Fähigkeiten, die sich als operative Fähigkeiten beim Musizieren beobachten lassen, als auch die motivationalen, volitionalen und sozialen Bereitschaften, die sich vor allem in Ausdauer und Konzentration sowie in der Freude am Musizieren zeigen, beschrieben werden. Für ein erfolgreiches Bewältigen der Anforderungssituation werden die positiv ausgeprägten überfachlichen Fähigkeiten emotionale Stabilität, Offenheit und Entgegenkommen sowie Gewissenhaftigkeit (als drei von insgesamt fünf Dimensionen der Big Five) als vorteilhaft erachtet (s. Abb. 2-7). Die Anforderungssituationen ergeben sich aus den einzelnen Stufen der Pyramide des Aufbauens rhythmischer Fähig- 
keiten nach Jank $\left(2013^{5}\right)$, die eng an die Stufen des Unterscheidenden Lernens nach Gordon $\left(2012^{8}\right)$ gebunden sind. Zusätzlich wird das Zusammenspiel des Individuums mit anderen Musizierenden als Anforderungssituation im Musikunterricht gesehen.

\subsection{Lern- und Lehrvoraussetzungen zum Erwerb rhythmischer Kompetenz}

In den ersten zehn Lebensjahren werden ,die grundlegenden musikalischen Fähigkeiten erworben, die notwendig sind, um sich in der Musikkultur im Wesentlichen zurechtzufinden und an ihr teilhaben zu können" (Gembris, 20134, S. 276). Diese Lernprozesse finden durch Akkulturation, das Hineinwachsen in die jeweilige Musikkultur, statt. Eine anregende soziale Umgebung ist dabei förderlich. Teilweise kann der Erwerb musikalischer Fähigkeiten durch formellen Unterricht beschleunigt werden (vgl. ebd.).

„Der Möglichkeit, durch Unterricht eine Beschleunigung in der Entwicklung einzelner musikalischer Fähigkeiten zu erzielen, sind dadurch Grenzen gesetzt, daß die Entwicklung musikalischer Fähigkeiten an allgemeine geistige, affektive und sensumotorische Reifungsprozesse gebunden ist“ (Gembris, 2013 S. 276).

Die für diese Studie interessierende Altersgruppe der Grundschulkinder in Klassenstufe 3 befindet sich nach Piaget in der Phase des konkret-operatorischen Denkens ${ }^{1}$. Dies ermöglicht beim Umgang mit Musik, mehrere Wahrnehmungs- und Handlungsaspekte gleichzeitig auszuführen.

„Eine neue Entwicklungsstufe wird nach Piaget mit etwa sieben bis elf Jahren erreicht... Das Kind lernt mehrere Wahrnehmungs- und Handlungsaspekte miteinander zu koordinieren, indem es eine Vorstellung von Invarianz entwickelt [...] Die Entwicklung des Invarianzbegriffs oder der Konservation ist auch für den musikalischen Bereich von Bedeutung, da sie allgemeine Prinzipien der geistigen Entwicklung darstellt und daher auch für die Wahrnehmung und Verarbeitung musikalischer Reize relevant ist. Die Fähigkeit zur gleichzeitigen Koordination von verschiedenen Wahrnehmungsaspekten ist beispielsweise deshalb wichtig, weil sinnerschließendes Musikhören die Fähigkeit verlangt, die Identität oder Ähnlichkeit des Melodischen, Rhythmischen oder Harmonischen bei gleichzeitiger Veränderung anderer musikalischer Parameter zu erkennen. Erst wenn sich das Invarianzprinzip herausgebildet hat, ist es möglich, eine rhythmisch oder harmonisch variierte Melodie als dieselbe Melodie wiederzuerkennen oder ein einheitliches Metrum über verschiedene rhythmische Gruppierungen hinweg zu ,erhalten ““ (Gembris, 20134, S. 233).

\footnotetext{
${ }^{1}$ Piagets Stufentheorie wurde in seiner Nachfolge stark kritisiert, vor allem wegen ihrer einseitigen Bezugnahme auf die kognitive Entwicklung des Menschen, aber auch wegen einer als rigide empfundenen Zuordnung der Stufen zu bestimmten Lebensaltern. Weitgehend unbestritten bleibt aber die Abfolge der von Piaget beschriebenen Stufen als universale anthropologische Konstante der menschlichen Entwicklung.
} 
Die individuellen Leistungsunterschiede beim Stand der Entwicklung musikalischer Fähigkeiten können stark schwanken. Dies wird sowohl in Bezug auf innere Bedingungen (musikalische Begabung) als auch äußere (Umweltfaktoren, Training) erforscht.

„Da derzeit noch nicht klar ist, wie genau Anlagen und Training beim Fertigkeitserwerb ineinandergreifen, gehen wir vereinfachend davon aus, dass bei günstigen Anlagen ... der Erfolg des Trainings maximal ist, während er andernfalls nicht zu den höchsten Ergebnissen führt“ (Oerter, 1998; 2005; Lehmann $2002^{4}$; Gruber \& Lehmann, 2007).

„Die Frage, ob eine theoretische Position der zugeschriebenen musikalischen Begabung oder nachgeordneten Trainingsfaktoren die Hauptrolle bei der Erklärung von Leistungsunterschieden zuspricht, ist deshalb nicht trivial, weil sie Konsequenzen für den Alltag hat. Schüler, denen von ihrer sozialen Umwelt (Lehrer, Eltern, usw.) ,Talent ${ }^{\star}$ zugeschrieben wird, werden anders behandelt und fühlen sich anders als solche, von denen weniger erwartet wird (z. B. Kingsbury, 1988; Eccles \& Wigfield, 2002). Dies kann den sog. Pygmalion-Effekt zur Folge haben, bei dem sich Schüler so verhalten, wie sie eingeschätzt werden. Lernende, die glauben, dass sie eine bestimmte Leistung erbringen bzw. ihren Erfolg sinnvoll erklären können (z. B. mit Fleiß oder Begabung), sind längerfristig erfolgreicher" (Lehmann \& Oerter, 2011², S. 118).

Gordon vertritt die Auffassung, dass sich das musikbezogene Begabungspotenzial eines Kindes „mit dem 9. Lebensjahr verfestigt. Bis dahin wird das angeborene Begabungspotenzial durch Umwelteinflüsse bei geeigneter Förderung positiv und bei ausbleibender Förderung negativ beeinflusst“ (Tappert-Süberkrüb, 1999, S. 78). Serafine (1988) weitet diesen Zeitraum bis zum elften Lebensjahr aus (zitiert nach Gembris, 20134, S. 257). Das Zeitfenster für eine geeignete Förderung rhythmischer Kompetenz liegt in beiden Fällen innerhalb des Schuljahres der Klassenstufe 3, welche in der Regel mit acht bis neun Jahren absolviert wird.

Neben der prinzipiellen Förderung musikalischer Fähigkeiten kommt in Bezug auf die Umwelteinflüsse vor allem dem regelmäßigen Üben und Wiederholen eine Schlüsselrolle zu.

„Selbst bekannte Künstler behaupten, dass kein Musiker ohne Übung Erfolg haben kann (Marsalis, 1995), was angesichts der Notwendigkeit gezielter Übung und Wiederholung bei sensomotorischen Fertigkeiten nicht überrascht. Bereits die frühe psychologische Forschung hat gezeigt, dass mit zunehmender Übung Bewegungen schneller und genauer werden (im Überblick Proctor \& Dutta, 1995)“ (Lehmann \& Oerter, $2011^{3}$, S. 118).

„Hirnphysiologisch könnte man Üben als zielgerichtete musikalische Betätigung definieren, die dem Erwerb, der Verfeinerung und dem Erhalt sensomotorischer, auditiver, visueller, struktureller und emotionaler Hirnrepräsentationen von Musik dient. [...] Das Erlernen der Bewegungen beim Musizieren geschieht in mehreren Schritten: zunächst wird unter Kontrolle der beteiligten Sinne - Gehör, Somatosensorik, z.T. Gesichtssinn - ein grober und noch fehlerhafter Entwurf des Bewegungsprogramms erstellt. Die Bewegungen sind unkoordiniert und unökonomisch, denn die Anzahl der beteiligten Muskeln, die Dauer der Muskelaktivität und die notwendige Muskelkraft sind noch nicht optimiert. Durch Einstudieren des Be- 
wegungsablaufes gelingt es in der zweiten Phase des Lernens, Ökonomie und Koordination zu verbessern und die Bewegungsgeschwindigkeit zu erhöhen“"(Altenmüller \& Jabusch, 2016, S. 52-53).

Den Zusammenhang von Übung und Leistung beschreiben Lehmann \& Oerter als eine mathematische Funktion.

„Zunächst wird mit relativ wenig Übung relativ viel Fortschritt erzielt, später muss für immer kleiner werdende Leistungsverbesserungen immer mehr Übung aufgewandt werden. Diese Erfahrung macht jeder Musiker, der ein neues Stück einübt“ (Lehmann \& Oerter, 2011³, S. 118).

Für die einzelnen Fähigkeiten in den sechs verschiedenen Stufen des Aufbauens rhythmischer Fähigkeiten sind verschiedene Voraussetzungen notwendig. Inwieweit diese Voraussetzungen in der interessierenden Altersstufe der Acht- bis Neunjährigen bereits ausgeprägt ist, soll im Folgenden betrachtet werden.

\subsubsection{Stufe A Körperkoordination, Erfahrung von Metrum und Puls}

Die Extraktion eines Grundpulses und das Empfinden des dazugehörigen Metrums ist Inhalt der Stufe A Körperkoordination, Erfahrung von Metrum und Puls. Fischinger und Kopiez haben zur Fähigkeit des Menschen, seine Körperbewegungen zu erklingender Musik zu koordinieren festgestellt, dass es sich dabei um eine komplexe Integrationsleistung von Wahrnehmung und Handlung handelt.

„Die Leichtigkeit, mit der es dem Menschen gelingt, sich zyklisch zu einem externen periodischen Pulsschlag zu bewegen..., lässt vermuten, dass es sich um eine angeborene Fähigkeit handelt. Doch bei genauerer Betrachtung der zugrunde liegenden Verarbeitungsprozesse stellt sich die Fähigkeit zur Synchronisation als eine sehr differenzierte und komplexe Integrationsleistung von Wahrnehmung und Handlung heraus, die im Detail nicht so einfach zu erklären ist“" (Fischinger \& Kopiez, 2011³ , S. 458).

Erwachsenen gelingt das Mitbewegen zu erklingender Musik auch bei Tempoänderungen des äußeren Pulses. Kinder müssen dies erst erlernen.

„In einer Studie mit 46 Kindern zwischen zwei und vier Jahren haben Eerola et al. (2006) festgestellt, dass sich Kinder in diesem Alter zumindest zeitweise durch Hüpfen, Kreisen oder Auf- und AbBewegungen zur Musik synchronisieren können. Sie scheinen jedoch noch nicht in der Lage zu sein, ihre Synchronisationsbewegungen bei Tempowechseln der dargebotenen Musik anzupassen. Die Fähigkeit zur Tempoanpassung ist demnach von der physiologischen Entwicklung abhängig (vgl. Bruhn, 2005)“ (Fischinger \& Kopiez, 2011³, S. 459-460).

Beim Synchronisieren zu gleichmäßigen Schlagfolgen (z. B. Schläge eines Metronoms) zeigt sich ein typisches Verhalten, welches in einer leichten zeitlichen Vorwegnahme des kommenden Schlages zeigt.

„Die Versuchspersonen klopfen nicht mit einer zeitlichen Verzögerung gegenüber dem vorgegebenen Signal, sondern kommen den Metronomschlägen zuvor. Zumeist werden die Tastendrücke ca. 30 bis 80 Millisekunden zu früh ausgeführt. Dieses Phänomen wird als ,negative Asynchronie‘ bezeichnet. Derzeit 
ist noch nicht genau geklärt, worauf dieses Phänomen beruht. Da die Asynchronie davon unabhängig zu sein scheint, ob mit der Hand oder dem Fuß geklopft wird, ist die Hypothese, dass es sich um einen mit der Leitung von Nervenimpulsen in Zusammenhang stehenden Effekt handelt, wohl auszuschließen (Nervenleitungshypothese)“(Auhagen, 2011³, S. 445).

Upitis stellte fest, dass die metrischen Fähigkeiten von Kindern bis zum zehnten Lebensjahr schwächer ausgebildet sind als die Fähigkeiten zum Nachmachen rhythmischer Figuren (vgl. Upitis, 1987, S. 54). Jungbluth und Hafen (1997) erforschten bei 161 Probanden im Alter von 5 bis 8,6 Jahren die Fähigkeit, in einem Musikbeispiel den Grundschlag mitzuklatschen und nach dem Anhalten der Musik dies noch fortzusetzen. Durch die Aufsummierung der Teilergebnisse ist ein konkreter Nachvollzug der Ergebnisse nicht nachvollziehbar, jedoch erkennbar, dass diese Aufgabe von einem großen Teil der Probanden erfolgreich bewältigt wird (s. Anlage A 2c).

\subsubsection{Stufe B Rhythmen hören und imitieren}

Der Inhalt der Stufe B Rhythmen hören und imitieren besteht im Nachspielen vorgespielter Rhythmen, wobei zwischen dem Vor- und Nachspielen keine Pause entstehen soll (Call-ResponsePrinzip). Prinzipiell erlangen Kinder bis zum siebten Lebensjahr die Fähigkeit, Rhythmen in den wesentlichen Grundzügen auszuführen. Dabei hängt der Fortschritt in der Entwicklung von der Schwierigkeit der rhythmischen Bewegung ab. Langsame Rhythmen sind schwieriger zu realisieren als schnelle. Ein Tempo zwischen 112 bpm und 136 bpm scheint Kindern in diesem Alter am leichtesten zu fallen (vgl. Bruhn, 2002 ${ }^{4}$, S. 292-293). Die Schwierigkeit der Aufgabe bzw. deren Komplexität betrachtend kann festgestellt werden: „Generell werden Rhythmen, die eine Zweiergliederung aufweisen, besser reproduziert als solche mit einer Dreiergliederung“ (Drake, 1993, zitiert nach Auhagen, $2011^{3}$, S. 445).

Die Rhythmuswahrnehmung ist durch zwei Prozesse gekennzeichnet. Zum einen findet eine Untergliederung der akustischen Reize (Rhythmusschläge) in Gruppen (Auhagen, 2011 ${ }^{3}$, S. 438), zum anderen eine Extraktion eines Grundpulses statt (ebd., 2011 ${ }^{3}$, S. 440). Für beide Prozesse scheint es einen bevorzugten Tempobereich zu geben, der etwa zwischen den Metronomwerten 60 bpm und 150 bpm liegt (ebd., 2011³, S. 441).

Rhythmische Figuren entstehen beim Hörenden durch das Bilden von chunks (Wahrnehmungseinheiten). Die Fähigkeit, immer größere Gruppen von Elementen zusammenzufassen, wächst im Umgang mit geeignetem Stimulusmaterial kontinuierlich über die Jahre hinweg (vgl. Bruhn, $2002^{4}$, S. 291). Interessanterweise werden selbst gleichförmige akustische Reize untergliedert.

„Präsentiert man Versuchspersonen eine Folge optischer oder akustischer Ereignisse mit objektiv gleichen Merkmalen wie gleicher Dauer und Intensität, so werden diese Folgen als untergliedert wahrgenommen. Bevorzugt wird hierbei eine Gruppenbildung zu je zwei oder drei Elementen“ (Auhagen, 2011², S. 438-439). 
Für das Musizieren von Rhythmen sind prinzipiell, und somit auch beim Nachspielen derselben, verschiedene Planungsleistungen erforderlich. Es erfordert die Reproduktion einer wenn auch kurzen Serie von Klangereignissen in der richtigen zeitlichen Abstufung und in einem festgelegten Tempo. Für diese Planungen ist die Größe des Arbeitsspeichers von Bedeutung. Diese hängt vom Alter, von Vorerfahrungen und vom konkreten Tempo des Rhythmusspiels ab (vgl. Palmer \& Pfordresher, 2003, zitiert nach Lehmann \& Oerter, $2011^{3}$, S. 113).

„Es zeigt sich, das jüngere Kinder über eine geringere Gedächtnisspanne als ältere und Erwachsene verfügen und daher auch über einen geringeren Planungsumfang. Allerdings kann Erfahrung in Form von Übung und Expertisesteigerung hier zum Gleichstand oder sogar zur Überlegenheit der Kinder führen. [...] Generell ist davon auszugehen, dass der Abruf aus dem Gedächtnis und das anschließende Programmieren der Bewegung versetzt, aber gleichzeitig, also kaskadierend erfolgt (Palmer, 2005). Die Planungsspanne umfasst etwa vier bis sieben Noten, je nach Tempo, wobei bei schneller Ausführung die Aktivierung auf einen engeren Bereich begrenzt ist“ (Lehmann \& Oerter, 2011³, S. 113-114).

Werden die Rhythmen komplexer, so scheint eine Vereinfachung durch den Hörenden bzw. Musizierenden zu erfolgen.

„Eine Tendenz zur Vereinfachung von rhythmischen Strukturen zeigt sich, wenn unregelmäßige Rhythmussequenzen reproduziert werden sollen. Nach Fraisse (1956) ist die oft auftretende vereinfachte motorische Reproduktion ein Abbild der Repräsentation im Gedächtnis. Rhythmische Vorgänge werden demnach den beiden Kategorien ,kurz' und ,lang ' zugeordnet. Kontinuierliche Kategorien, die Feinabstufungen erlauben, gibt es seiner Ansicht nach nicht. Dem widersprechen eine Reihe von Untersuchungen. Versuchspersonen sind durchaus in der Lage, rhythmische Muster mit mehr als zwei Intervalldauern nachzuahmen (Povel \& Essens, 1985). Franek u. a. (1991) ließen unregelmäßige rhythmische Sequenzen erfolgreich synchronisieren. Der Einfluß einer mehrjährigen Musikpraxis erwies sich als vorteilhaft“ (Beck, 2002 ${ }^{4}$, S. 460).

Der Mensch ist sowohl in der Lage, Unregelmäßigkeiten im rhythmischen Spiel genau zu erkennen, als auch wohlwollend darüber hinweg zu hören. Unregelmäßigkeiten in Folgen gleichabständiger Töne werden mit einer hohen Genauigkeit erkannt. Nicht exaktes Rhythmusspielen kann bis zu einem bestimmten Punkt als richtig und damit kategorial wahrgenommen werden.

„Kategoriale Wahrnehmung bedeutet, dass einer kontinuierlich sich verändernden Größe wie dem Frequenzabstand zwischen zwei Tönen kein Kontinuum einer Wahrnehmungsqualität ... entspricht, sondern vielmehr die wahrgenommene Qualität bis zu einem bestimmten Punkt gleich bleibt, um dann in eine andere Qualität umzuschlagen. Dementsprechend gibt es Klassen von zeitlichen Mustern, die jeweils als ein und derselbe Rhythmus wahrgenommen werden“ (Auhagen, 2011³, S. 441-442).

Eine Längsschnittuntersuchung mit mehr als 50 Probanden zum Musikerleben von Kindern im Alter von fünf bis zehn Jahren befasste sich u. a. mit dem Nachspielen von Rhythmen über eine Länge von jeweils acht Grundschlägen im Tempo von $68 \mathrm{bpm}$. Es konnte nachgewiesen werden, dass zwischen $6 \%$ bis $15 \%$ der Probanden im Alter von sieben bis acht Jahren drei von fünf dieser 
Rhythmen richtig nachspielen konnten (Minkenberg, 1991, S. 73, 128-129, 156-159); im Alter von acht bis zehn Jahren gelang dies bei vier von fünf dieser Rhythmen bereits zwischen $13 \%$ bis $64 \%$ der Probanden (s. Anlage A 2b).

\subsubsection{Stufe C Rhythmussprache erlernen}

Die Stufe $C$ Rhythmussprache erlernen wird bei Gordon und bei Jank im Zusammenhang mit dem Erwerb eines Rhythmusrepertoires (aus Pattern/ Rhythmusbausteinen) vermittelt. Das Erlernen einer Rhythmussprache stellt in der Musikpädagogik eine spezielle Lernhilfen dar: Rhythmussprachen fungieren als Denk- und Übungsmittel (Losert, 2011, S. 125). Zu den bekanntesten Rhythmussprache-Systemen zählen die von Kodály, Paris und Gordon (s. Abb. 2-8). Sie werden in relative und absolute Rhythmussprachen unterschieden (ebd., S. 160). Losert versteht unter absoluten Rhythmussprachen solche, die sich auf Relationen von Notenwerten beziehen (ebd., S. 158-163). Die Silbe ta hat dabei grundsätzlich die doppelte Länge der Silbe te bzw. ti. Eine absolute Rhythmussprache ist z.B. das System von Kodály. Relative Rhythmussprachen enthalten diese Tonlängen-Information nicht zwingend, da lediglich der Bezug zum Grundschlag von Bedeutung ist. Die Silbe ta kann alleinstehend sowohl eine Viertelnote repräsentieren als auch eine Achtelnote in der Verbindung mit der Silben te in z.B. ta-te. Der Vokal $a$ signalisiert immer das Musizieren auf dem Grundschlag (Onbeat), der Vokal $e$ das Musizieren auf dem Nebenschlag sowie der Konsonant $t$ die zwingende Spielaktion.

\begin{tabular}{|c|c|c|c|c|c|c|}
\hline & Viertel & Achtel & Halbe & Viertelpause & Synkope & $\frac{2}{4}+15$ \\
\hline Kodály & ta & ti-ti & ta-o & $\mathrm{sch} / \mathrm{pst}$ & $\begin{array}{l}\text { ti ta ti oder } \\
\text { Syn-ko- pe }\end{array}$ & ta sch ti-ti ta \\
\hline Paris & ta & ta-te & ta-a & $\mathrm{sa}$ & ta-tea- te & ta sa ta-te ta \\
\hline Yamaha & $\tan$ & taka & ta-han & $\mathrm{hm} / \mathrm{pst}$ & - & tan pst taka tan \\
\hline Ernst & ta & te-te & ta-o & $\mathrm{sa}$ & te-ta- te & ta sa te-te ta \\
\hline Gordon & $\mathrm{du}$ & du-de & $\mathrm{du}$ & - & du-de- de & $d u-d u-d e d u$ \\
\hline
\end{tabular}

Abb. 2-8: Übersicht über die Silben verschiedener Rhythmussprachen

Die Systeme von Paris und Gordon unterscheiden sich dahingehend, dass bei Paris zu überdauernde Taktzeiten mit einem Vokal substituiert werden (z. B. die halbe Note als $t a-a$ ). Dieser Vokal ohne Spielaktion fungiert als Platzhalter, es gibt sozusagen eine klangliche Stütze beim Aushalten von längeren Tondauern. Der Platzhalter signalisiert den Grundschlag, der erst abgewartet werden muss. Ebenso gibt es Silben für Pausen. Bei Gordon wird das Empfinden für diese auszuhaltenden Klänge oder Pausen vorausgesetzt und nicht in Sprache umgesetzt. Beispiele für einzelne Notenwerte und Takte sind in Abb. 2-8 dargestellt (vgl. Losert, 2011, S. 163; Volst, 2000, S. 22). Das Alleinstellungsmerkmal in Gordons Rhythmussprache besteht jedoch in einem Sachverhalt, welcher der Übersicht, wie sie z. B. bei Losert (2011) im vergleichenden Überblick gegeben wird, nicht zu entnehmen ist: Gordon verwendet je nach geradem bzw. ungeradem Metrum verschiedene 
Vokale in den Silben, bei gemischten Metren sogar verschiedene Konsonanten (2001, S. 116; s. Abb. 2-9). Im geraden Metrum werden die beiden Vokale $u$ und $e$ genutzt, im ungeraden Metrum die drei Vokale $u, a$ und $i$. In den gemischten Metren beginnen die unbetonten Microbeat-Silben auf dem Konsonanten $b$ statt $d$. Unabhängig vom Metrum werden alle unbetonten Sechzehntelschläge mit dem Vokal $a$ dargestellt.

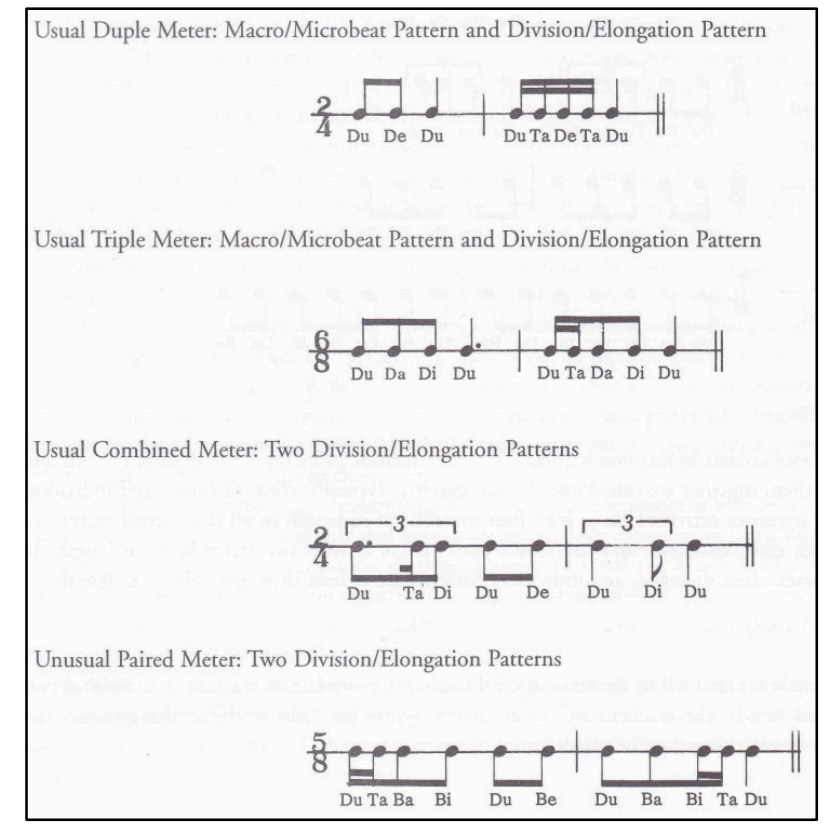

Abb. 2-9: Rhythmussilben im geraden und ungeraden Metrum bei Gordon

Forschungsergebnisse über den gewinnbringenden Einsatz des Erlernens einer Rhythmussprache in der Altersgruppe der acht- bis neunjährigen Grundschüler (oder auch anderer Altersgruppen) liegen nicht vor.

\subsubsection{Stufe D Rhythmen variieren und erfinden}

Die Stufe D Rhythmen variieren und erfinden wird bei Jank als Implementierung der praktischen Handlungsvorschläge Gordons integrierend eingefügt. Auf einen Call des Lehrers folgt eine Response, die nicht dem Call des Lehrers gleicht. Dieses Erfinden bzw. Variieren von Rhythmen stellt eine deutlich höhere Stufe als das Imitieren von Rhythmen auf Stufe B dar. Innerhalb von Bruchteilen einer Sekunde müssen der vorgespielte Rhythmus erkannt und der innere Spielbefehl für einen davon verschiedenen Rhythmus im Gehirn erzeugt werden. Wird zusätzlich verlangt, den erfundenen Rhythmus noch einmal zu wiederholen, muss dieser Rhythmus auch noch kurzzeitig gespeichert worden sein.

„Die Rückmeldung über das Produkt ist in der Improvisation immer zeitnah, da sie phänomenologisch eine Einheit von Erfindung, Verarbeitung und klanglicher Realisation [sic!] (Schramowski, 1973). Für den Spieler ist dabei die zu überblickende zeitliche Strecke wichtig, also wie weit zurück seine Erinnerung an 
gerade Gespieltes reicht bzw. wie weit voraus er planen kann (Kenny \& Gellrich, 2002)“ (Lehmann, 2011 $1^{3}$, S. 345).

Für den Prozess der Improvisation ist bekannt, dass der Spielende durchaus auch aus dem sogenannten Unterbewussten passende Ideen generiert oder bereits existierende Spielbausteine auswählt

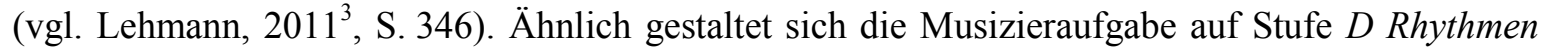
variieren und erfinden. Inwieweit die Patterns bewusst gewählt werden, lässt sich durch eine Aufforderung zum Wiederholen des Patterns möglicherweise rekonstruieren. Kann ein Pattern nicht wiederholt werden, liegt es nahe, dass es eher aus einem unbewusst verfügbaren (implizitem) Wissensschatz geschöpft wurde.

Für diese im Umfang relativ kleine Improvisationsaufgabe gelten dennoch ähnliche Bedingungen wie für anspruchsvollere Aufgaben: Dem Spielenden wird Orientierung durch die konkrete Aufgabe und das zu verwendende Instrument (Hände, Klanghölzer o. ä.) zuteil. Er benötigt eine Wissensbasis aus theoretischem und technischem Wissen (vgl. Andreas, 2002 ${ }^{4}$ a, S. 508). Im Falle des Abwandelns eines Patterns wären dies u. a. das Wissen über Patternbildung, das Erfassen der Anzahl der im geraden Metrum zur Verfügung stehenden Grundschläge sowie prinzipielles Wissen und Können in Bezug auf einen pünktlichen Spielbeginn und tempostabiles Rhythmusspiel.

Um Zeit zum Nachdenken für das ausgewählte Antwort-Pattern zu gewinnen, ist der rasche Zugriff auf diese Wissensbasis unerlässlich. Das Wissen über Patternbildung entspricht wiederum der Theorie der Bildung von Chunks (vgl. Andreas, 2002 a, S. 509), wie sie ebenfalls in Stufe B Rhythmen hören und imitieren benötigt wird. Um das Antwort-Pattern anschließend wiederholen zu können, ist eine Handlungskontrolle, ein sogenanntes Self-Monitoring notwendig (ebd.). Das Modell zur Entwicklung kreativen musikalischen Verhaltens bestätigt den Rückgriff auf eine vorhandene Wissensbasis.

„Die Entwicklung kreativen musikalischen Verhaltens erfolgt nach einem Modell von Swanwick \& Tillmann (Swanwick, 1991) in einer Spirale: Das Material, die Ausdrucksmöglichkeiten, die Formen und die Wertbezogenheit der Musik werden in dieser Reihenfolge vom Kind zunächst kennengelernt und erforscht, dann individuell ausgewählt und gestaltet“ (Andreas, 2002 ${ }^{4}$ b, S. 524).

In der Psychomotorikforschung geht man davon aus, dass dieser Zugriff auf vorhandenes Wissen über Abrufschemata realisiert werden kann.

„Die Schematheorie von Schmidt aus den 1970er Jahren (Schmidt \& Lee, 2005) enthält die bekannteste Erklärung: Eine Klasse von Bewegungen wird mit bestimmten Standardparametern (z. B. für Stärke, Geschwindigkeit, Gelenkstellung) gelernt, die zusammen ein Abrufschema bilden. Dieses wird mit einem Wiedererkennungsschema, einer Zielvorstellung verglichen, welches in einer bestimmten Situation aufgebaut wird“ (Lehmann \& Oerter, 2011³, S. 345).

Dieser Zugriff auf vorhandenes Wissen wird mitunter nicht wahrgenommen. Dies wird darauf zurückgeführt, dass ein wichtiges Merkmal kreativen Denkens in der Oszillation zwischen bewussten 
und nichtbewussten Ebenen der subjektiven kognitiven Struktur beruht (vgl. Andreas, $2002^{4}$ b, S. 525).

Forschungsergebnisse über den Stand der Fähigkeiten zum Variieren und Erfinden von Rhythmen in der Altersgruppe der acht- bis neunjährigen Grundschüler liegen nicht vor.

\subsubsection{Stufe E Rhythmen lesen und F Rhythmen schreiben}

Die Stufen E Rhythmen lesen und F Rhythmen schreiben bilden in den bereist vorgestellten Theoriemodellen die jeweils höchsten Stufen zum Aufbauen rhythmischer Fähigkeiten dar. Gordon stellt in seinem Theoriemodell das Lesen und Schreiben jeweils parallel auf die entsprechenden Stufen dar (s. Abb. 2-5), empfiehlt jedoch in seinem Kursheft Jump Right In zuerst Übungen zum Lesen, danach zum Schreiben. Jank und Gallus empfehlen ebenfalls die gleiche Abfolge (s. Abb. 2-6).

Anders als für den Prozess des Lese- und Schreiblehrgangs der eigenen Muttersprache im Fach Deutsch ist für die Einführung in die Notenschrift nicht durch Forschung nachwiesen, welche methodischen Wege erfolgreich eine Einführung in die Notenschrift der Rhythmusnotation ermöglichen. Es ist nicht erwiesen, ob dieser Lernprozess erfolgreicher verläuft, wenn er mit dem Lesen oder dem Schreiben beginnt. Bamberger wählt für ihre Studien in der Arbeit mit den Kindern folgenden Anlass: Eine Gruppe von Kindern erfindet ein Klassen-Spielstück in Form eines Rondos. Der Rhythmus, welcher als wiederkehrendes Thema fungieren soll, ist gefunden, doch die Stunde neigt sich dem Ende zu. Das Notieren des gewählten Rhythmus wird zum Zwecke des Nichtvergessens und des Wiederverwendens in der Folgestunde angetragen. Die Kinder lösen dies selbstständig, jeder auf seine Weise. Der Sinn des Notierens war für alle Beteiligten einleuchtend, die Motivation entsprechend hoch (vgl. Bamberger, 1991, S. 21-26).

Den Weg andersherum zu begehen, also erst das Lesen von Rhythmen zu erlernen, ist für Anfänger im Instrumentalspiel häufig Realität. Meist wird das Schreiben von Rhythmen gar nicht ausgeübt bzw. angeregt. Beispielhafte Instrumentalschulen für diesen Lernweg stellen die HolzweiBig-Klavierschule (Verlag Edition Peters), die Moser Klavierschule (Verlag Veritas), die Europäische Klavierschule (Verlag Schott) dar. Eine Ausnahme diesbezüglich macht z. B. die Klavierschule für Kinder-Musik wird lebendig - Rico lernt Klavier (Verlag Ricordi). In einem Begleitheft mit dem Titel Ricos Werkstatt werden parallel zu musiktheoretischen Übungen alle Sachverhalte auch notierend vertieft. Einige Instrumentalschulen beschreiten aber auch ganz andere Wege und ermuntern vor dem Erlernen des Lesens von Notationen zum experimentellen Erkunden des Instrumentes und von Klängen, die dem Instrument entlockt werden können (z. B. Zwei Hände- Zwölf Tasten, Verlag Schott). Das Erlernen des Lesens von Notation erfolgt zu einem späteren Zeitpunkt.

Für das Aufschreiben von Rhythmen belegen Studien unter dem Oberbegriff SymbolsystemAnsatz, dass zwischen dem zweiten und fünftem Lebensjahr der Grundstein für die Symbolisation, also den Erwerb von grundlegenden Fähigkeiten im Umgang mit den Symbolen einer Kultur durch 
Akkulturation gelegt wird (Gardner, 1993, zitiert nach Gembris, 20134 , S. 240). Bamberger (1982, 1991), Upitis (1987) und Hildebrandt (1987) können belegen, welche Formen Kinder ohne spezielle Unterweisung finden, um Rhythmen zu notieren. Ausgangspunkt ist ein einfacher Rhythmus, der einem englischsprachigen Abzählreim entnommen ist (s. Abb. 2-10).

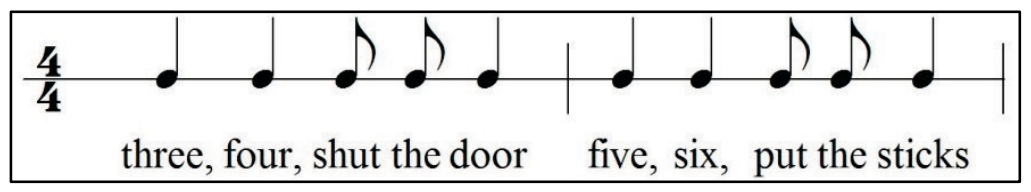

Abb. 2-10: Zugrundeliegender Rhythmus in den Studien Bamberger, Upitis und Hildebrandt

In den Studien können verschiedene Typen des Notierens von Rhythmen nachgewiesen werden. Dazu zählen auf der untersten Ebene die Typen Scribbles, Icon und No correspondence bzw. auf fortgeschrittenen Stufen die Typen Counting, Figural Types und Metric Types. Auf der untersten Ebene gelingt es Kindern noch nicht, die gehörten Schläge eines Rhythmus in eine Darstellungsform zu übertragen, die für andere nachspielbar wäre. Es werden z. B. Kringellinien (Scribbles) oder zum Rhythmusausführen notwendige Hände (Icon) dargestellt (Upitis, 1987, S. 50; s. Abb. 2-11).

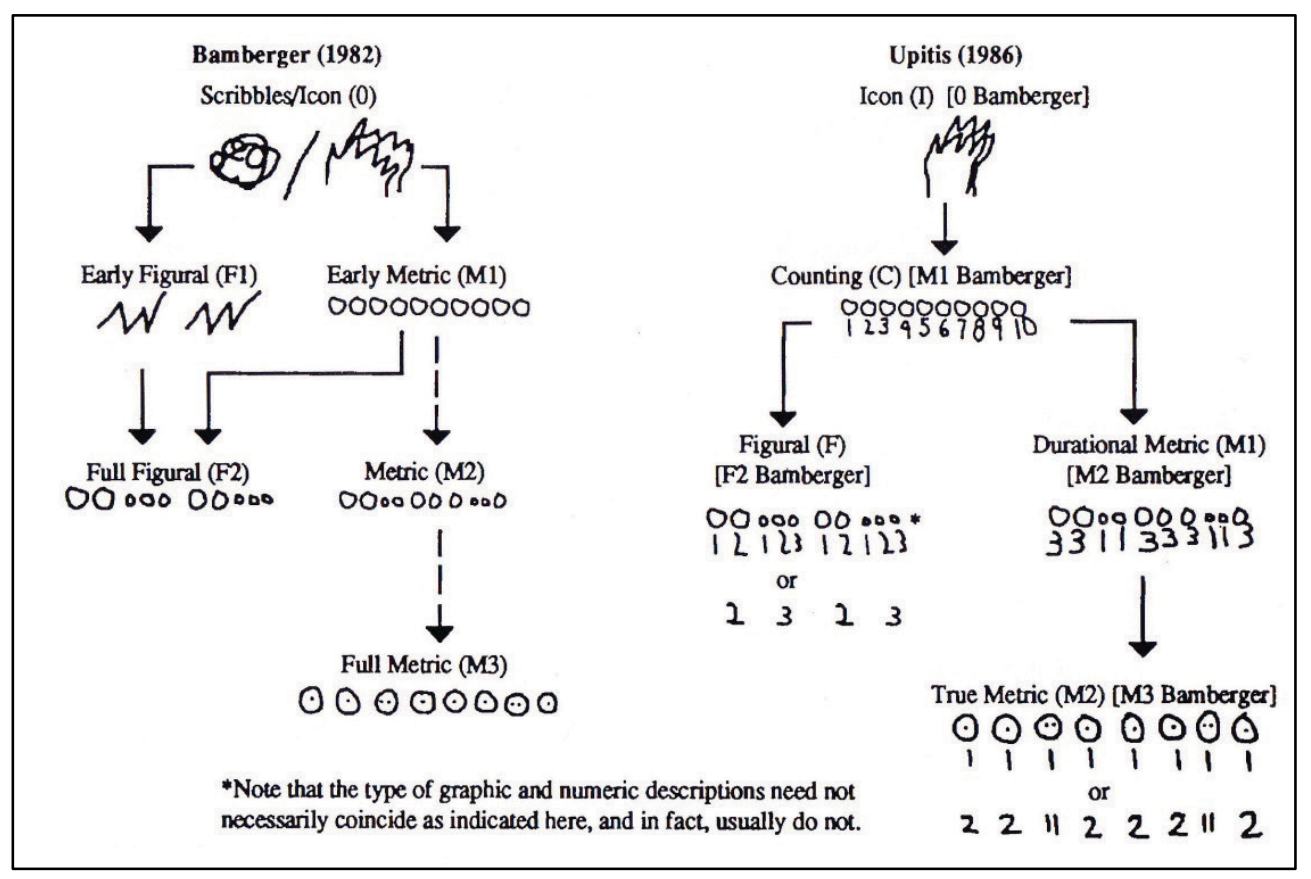

Abb. 2-11: Typen der Rhythmusdarstellung nach Bamberger und Upitis

Einige Kinder sind bereits in der Lage, die gehörten Schläge als abstrahierte Striche darzustellen, jedoch stimmt deren Anzahl nicht mit der tatsächlichen Anzahl der Schläge überein (No correspondence, s. Abb. 2-12, Type 1). Auf der Ebene Counting gelingt genau dies, die Anzahl der dar- 
gestellten Schläge (z. B. mit Strichen, Kreisen oder anderen Zeichen) entspricht den gehörten oder musizierten Schlägen (s. Abb. 2-11, Early Metric sowie Counting; s. Abb. 2-12, Type 2).

Auf der figuralen Ebene werden kurze und lange Schläge grafisch verschieden mit großen und kleinen Symbolen (z. B. Kreisen) oder bei nur einem Zeichen mit verschiedenen Abständen dargestellt. Jedoch wird bei einem Prozess des Gruppierens der erste lange Schlag nach mindestens zwei kurzen Schlägen, grafisch wie ein kurzer Schlag (s. Abb. 2-11, Full Figural bzw. Figural F) dargestellt, d. h. die Schläge auf door und sticks werden auch mit kleinen Kreisen dargestellt. Bei der Verwendung von Strichen werden die Schläge auf door und sticks der Gruppe der kurzen Schläge zugeordnet (s. Abb. 2-12, Type 3). Dies deutet darauf hin, dass diese Schläge von den Kindern in der Klangqualität den kurzen Schlägen zugeordnet werden. Bamberger bezeichnet dieses Phänomen als Clustering, den fehlerhaft dargestellten Schlag im Rhythmus als kritisches Element (Bamberger, 1991, S. 25-26; s. Abb. 2-13). Auch Upitis und Hildebrandt bezeichnen diese Phänomene, allerdings mit den Begriffen Figural boundary (Upitis, 1987, S. 54) bzw. Grouping (Hildebrandt, 1987, S. 90).

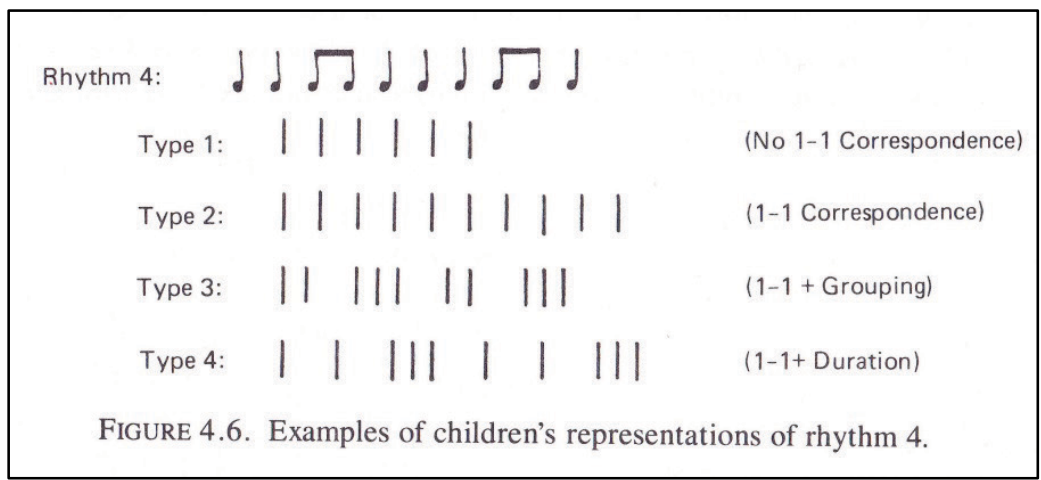

Abb. 2-12: Typen der Rhythmusdarstellung nach Hildebrandt

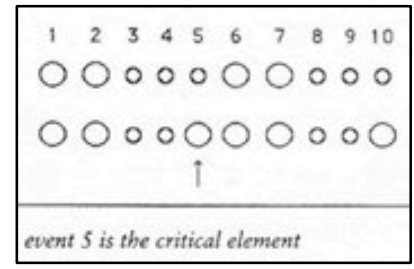

Abb. 2-13: Kritisches Element bei Bamberger

Erst auf der metrischen Ebene entsteht ein Abbild des Rhythmus, der für andere nachspielbar wäre. Kurze und lange Schläge werden korrekt dargestellt, entweder mit verschieden großen Symbolen (s. Abb. 2-11, Metric M2, Durational Metric M1), mit einer unterschiedlichen Anzahl von Schlägen in Kreisen, welche die Grundschlagzugehörigkeit andeuten (s. Abb. 2-11, Full Metric M3, True Metric M2), oder bei nur einem Zeichen mit einer exakten Darstellung der Länge des Schlages als 
korrekte Abstandsdarstellung (s. Abb. 2-12, Type 4). Eine tiefergehende Analyse der verschiedenen Typen der Darstellung von Rhythmen mit Zeichen lässt sich nach der Art der gewählten Zeichen in Form verschiedener Schemata nachweisen (Werner, 2016, S. 10-13).

In Bezug auf die Weiterentwicklung dieser vorhandenen Fähigkeiten zum Notieren von Rhythmen stellen Davidson und Scripp (1990) fest, dass ohne Unterweisung keine Weiterentwicklung stattfindet.

„Während die Entwicklung von Notationssystemen zwischen etwa vier bis acht Jahren schnell verläuft, findet danach ohne Unterricht kaum noch eine nennenswerte Entwicklung statt ... Ohne entsprechende Übung entwickeln sich musikalische Schreib- und Lesefähigkeiten auch bei Erwachsenen nicht weiter“ (zitiert nach Gembris, 20134, S. 245).

Damit scheint die interessierende Altersgruppe prädestiniert für eine Einführung in die kulturhistorisch entwickelte traditionelle Notenschrift. Ausgehend von einem beobachteten Ungleichgewicht (allerdings bei Konservatoriumsschülern) zwischen musikalischem Können und der Fähigkeit, einfache Melodien aufzuschreiben, betonen Davidson und Scripp die Notwendigkeit einer Unterweisung.

Es ist notwendig, ,alle Aspekte des musikalischen Wissens, auch die des Lesens und Schreibens von Musik, durch Übung zu entwickeln, um eine optimale Koordination zwischen den verschiedenen musikalischen Bereichen zu erreichen und eine optimale Leistungsfähigkeit zu entwickeln“ (Davidson und Scripp, 1990, zitiert nach Gembris, $2013^{4}$, S. 246).

In Bezug auf das Lesen von Rhythmen ist bekannt, dass auch hier Prozesse stattfinden, die einer Gruppenbildung in Form von Chunks gleichen:

„Beim Behalten von Musik werden nämlich nicht einzelne Informationen abgespeichert, sondern es wird Vorwissen über regelhafte Eigenschaften angewendet, um sinn- und bedeutungsvolle Chunks zu formen“ (Lehmann \& Chaffin, 2011 ${ }^{3}$, S. 358).

In Gordons Music Learning Theory bieten die erarbeiteten und verinnerlichten Patterns über jeweils zwei Grundschläge eine ideale Grundlage für diese Gruppenbildung. Das Üben des Notenlesens hat somit zum Ziel, nicht einzelne Noten wahrzunehmen, sondern Konstellationen bzw. Muster einzuprägen.

„Das Entziffern von Notentext ähnelt dem Lesenlernen von Wörtern, wobei eine Zuordnung von Mustern zu bestimmten Griffen oder Vorstellungen erfolgt, die nach und nach automatisiert werden (Rayner, 1998). [...] Das stille Lesen von Partituren ist bislang nur ungenügend erforscht, erfordert aber ein ausgeprägtes Training des inneren Hörens. Allen Tätigkeiten [Vomblattspiel und Vomblattsingen, d. V.] ist gemeinsam, dass notierte musikalische Strukturen wiedererkannt und dann in Bewegung oder auditorische Vorstellungen übersetzt werden müssen“ (Lehmann \& Chaffin, 2011³ , S. 358).

Beim Notenlesen unbekannter Musikstücke ist eine vorwärts- (auf das noch zu Spielende) sowie rückwärtsgerichtete (auf das bereits Gespielte) Orientierung notwendig. Dieser doppelseitig gerich- 
tete Prozess ermöglicht das Erkennen von bekannten Mustern und ermöglicht einen kontinuierlichen Informationsfluss. Die Menge der Informationen, die zeitgleich aufgenommen werden kann, wird als Wahrnehmungsspanne bezeichnet, die Menge der Informationen, die in die Musizierausführung umgesetzt werden kann, als Auge-Hand-Spanne (ebd., S. 366). Ein geübter Musiker kann durchschnittlich etwa sieben Töne wahrnehmen. Das entspricht der doppelten Menge eines Laien (Sloboda, 1985, S. 72, zitiert nach Lehmann 20024, S. 487).

Das Lesen von Notentext wird von Erfahrungen mit bestimmten Musiken geprägt und führt zu Erwartungshaltungen. Diese können unter Umständen bewirken, dass vom Musizierenden der Notentext gemäß den eigenen Erwartungen abgeändert wird (vgl. Lehmann $2002^{4}$, S. 488).

„Offensichtlich wurden viele Noten gar nicht richtig erfasst, sondern aus dem Kontext erschlossen. Dieser Interpolation genannte Prozess ist auch beim Textlesen belegt, bei dem häufig auftretende und kurze Worte wie bestimmte Artikel gar nicht angeblickt werden.“(Lehmann \& Chaffin, 2011³ , S. 368).

In einem dreistufigen Modell des Vomblattspielens wird in einem ersten Schritt in den Noten nach Informationen gesucht. Als hilfreich erweisen sich dabei Vorwissen und Erwartungen. Es werden Mustererkennungsprozesse aktiviert. Je weniger Erfahrungen ein Spieler besitzt, desto schwieriger gestaltet sich dieser Leseprozess. Rückschau und Vorausschau ermöglichen den Aufbau von Erwartungen (Lehmann \& Chaffin, $2011^{3}$, S. 368).

„Blattspiel-Experten unterscheiden sich von Anfängern durch eine effektive Blickbewegung und eine größere Augen-Hand-Spanne. Experten blicken in Notenzwischenräume und brauchen weniger Fixationen als Novizen. Dabei behalten sie selbst bei extrem kurzen Darbietungszeiten von 200 Millisekunden dennoch mehr Informationen. Der Grund dafür liegt in ihrer Fähigkeit, größere Chunks bilden zu können... Professionelle Musiker erkennen und enkodieren also musikalische Strukturen in größeren Sinneinheiten als Laien oder musikalische Anfänger, wobei ihnen musiktheoretisches Wissen und Erfahrungen behilflich sind“ (Lehmann \& Chaffin, 2011³, S. 366-367).

Für Kinder, die nicht gleichzeitig mit dem Instrumentalspiel beginnen, sondern das Lesen von Notentext im Rahmen einer allgemeineren musikalischen Unterweisung erlernen sollen, werden Anfang des 20. Jahrhunderts für das Schreiben und Lesen von Rhythmusnotation sogenannte Denkund Übungsmittel entwickelt. Sie entspringen dem Lehrprinzip des musikalisch Kindgemäßen (s. Abel-Struth, 2005², S. 369-373) und berücksichtigen, dass Rhythmusnotation per se nicht anschaulich, sondern ein historisch gewachsenes Symbolsystem ist. Zu ihnen zählen u. a. die Silbenschrift und die Punktnotation (Losert, 2011, S. 130-147). Letztere erfolgt mit den Symbolen Punkt, Strich und Null (ebd., S. 145; s. Abb. 2-14). Ziel ist es, im Rahmen der musikalischen Unterweisung, ausgehend vom Singen von Liedern deren Rhythmus grafisch zu fixieren. „Die Neuerung der Punktnotation gegenüber der Silbenschrift besteht in der Möglichkeit, einen Rhythmus simultan mit dem Erklingen zu notieren“ (ebd., S. 144). Ist die Punktschrift verinnerlicht, wird die traditionelle Rhythmusnotation abgeleitet. 


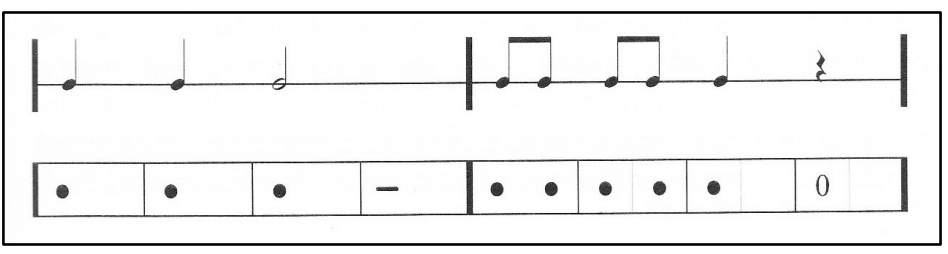

Abb. 2-14: Notation einer Punktschrift nach Losert

Das Unterstützen der Einführung in die Rhythmusnotation mit den o. g. Denk- und Übungsmitteln entspricht der Theorie einer dreifachen Darbietung eines Wissensbereichs nach Bruner. Dies erfolgt:

- durch geeignete Handlungen: enaktive Repräsentation - singen, musizieren

- durch Bilder oder Grafiken, die eine bestimmte Konzeption verdeutlichen: ikonische Repräsentation - Punktschrift verwenden

- durch eine Folge symbolischer Formsätze, die einem symbolischen System entstammen: symbolische Repräsentation - traditionelle Notenschrift verwenden

Damit wird die Idee vom Verlauf der geistigen Entwicklung von der enaktiven, über die ikonische hin zur symbolischen Darstellung der Welt umgesetzt (Bruner, 1974, S. 48-53; Klafki, 20076, S. 156-159; Seel, 2003, S. 61). Den geeigneten Handlungen (enaktive Repräsentation) entsprechen in diesem Kontext am besten Patternübungen in Form von Call-Responses. Als Bilder und Grafiken (ikonische Repräsentation) bieten sich Vorformen wie z. B. die Punktnotation an (Losert, 2011, S. 143-147). Die traditionelle Rhythmusnotation entspricht den symbolischen Formsätzen.

Der Forschungsstand zum Lesen und Schreiben von Rhythmen umfasst im Wesentlichen die bereits erwähnten Ergebnisse zum Notieren von Rhythmen mit Zeichen (siehe Bamberger 1982, 1991, Hildebrandt 1987, Upitis 1987). Zum Lesen und Musizieren von Rhythmen stellt McPherson mehrere Erkenntnisse vor. Das Augenmerk liegt dabei auf Schülern, die Instrumentalunterricht erhalten. So werden Zusammenhänge zwischen dem Beginn des Instrumentalunterrichts sowie bereichernden Aktivitäten wie Spielen nach Gehör und Improvisation sowie Qualität und Quantität des häuslichen Übens untersucht (vgl. McPherson \& Gabrielsson, 2002, S. 107). Dabei ist auffallend, dass das Vomblattspiel am stärksten vom Spielen nach Gehör und Improvisation sowie von der Quantität des häuslichen Übens beeinflusst wird (ebd., S. 108).

Des Weiteren werden bei Instrumentalspielern Zusammenhänge zwischen geübtem Instrumentalspiel, Vomblattspiel, Memorieren von Notenbildern, gehörmäßigen Nachspielen und Improvisation untersucht (vgl. McPherson, 2005, S. 11-12). Das im hiesigen Falle interessierende Lesen von Notenschrift ist bei jungen Instrumentalisten als durchaus unter den Erwartungen bleibend einzuschätzen. So erreichen die Probanden im Bereich Vomblattspiel nach der Watkins-Farnum Performance Scale in drei aufeinanderfolgenden Jahren im Durchschnitt weniger als die Hälfte der im 
Versuch erreichbaren Punkte (ebd., S. 14). In einer weiteren Studie wird festgestellt, dass junge Instrumentalmusiker (drei Jahre Unterricht im Instrumentalspiel) beim Vomblattspiel dreimal mehr Fehler beim Lesen eines Rhythmus machen als beim Lesen der Tonhöhe. Als Grund wird das Fehlen von notwendiger Audiationsfähigkeit angenommen, die scheinbar für musizierte rhythmische Folgen einen höheren Grad an Beherrschung benötigt als für musizierte tonale Folgen (vgl. McPherson \& Mills, 2006, S. 160).

\subsubsection{Allgemeine Aspekte des Musiklernens}

Die Förderung rhythmischer Kompetenz im Treatment soll sich neben den bereits erwähnten speziellen Lern- und Lehrvoraussetzungen an den aktuellen Erkenntnissen des Musiklernens orientieren. In der Fachdidaktik, insbesondere dem Konzept des Aufbauenden Musikunterrichts, ist das Musiklernen als ein Lernprozess gekennzeichnet, welcher auf den Erwerb der Fähigkeit zielt, musikalisch denken und sprechen zu können sowie musikalisch zu handeln. Musiklernen basiert auf (1) Handlung und Bewegung, dem (2) Zusammenspiel von imitatorischem Lernen und eigenem Anwenden, dem (3) Vorrang von musikalischem Können vor musikalischem Wissen, dem (4) Durchschreiten verschiedener Phasen und Stufen, (5) kontinuierlicher Anwendung sowie (6) Übung und (7) Reflexion.

(1) Musiklernen basiert auf Handlung und Bewegung. Der sinnliche Erfahrungsprozess muss am Anfang aller Lernprozesse stehen (Schütz, 1996, S. 3).

„Entsprechend [...] muss auch musikalisches Lernen zuerst als Vorgang verstanden werden, der zur Bildung von Vorstellungen (mentalen Repräsentationen) führt, damit man auditieren, d.h. gehörten und erzeugten Klängen eine musikalische Bedeutung geben kann. [...] Musikalisches Lernen stellt also die musikalische Handlung vor die Bildung der Begriffe, und diese vor die Einführung von symbolischen Zeichen. [...] Klangverbindungen und Tonbezüge, Pulsation und metrische Füllung erschließen sich nicht über Bild und Begriff, sondern über den körperlichen, d.h. stimmlichen und bewegungsmäßigen Vollzug““ (Gruhn, 2003, S. 109).

Musikunterricht ohne Handlung und Bewegung führt zu einem Lernen, dem nachgesagt wird, es vermittle Wissen über Musik, nicht aber musikalisches Wissen.

(2 a) Musiklernen basiert auf dem Zusammenspiel von imitatorischem Lernen und eigenem Anwenden. Musiklernen beginnt mit Imitation und sollte deshalb im Musikunterricht der Grundschule zuvorderst stehen.

„Die früheste Phase des Lernens, auch des musikalischen Lernens, ist die Imitation [...] Das Lernen durch Imitation bleibt während der gesamten Kindheit wichtig. So lässt sich in den ersten Schuljahren beobachten, dass Kinder oft unbewusst die Bewegungen, die Rhythmen und Töne der Lehrerin nachahmen, ohne eigens dazu aufgefordert zu werden. [...] Dies geschieht zum einen durch Vermittlung eines Repertoires an Liedern, Sprechversen, Bewegungsformen und ersten instrumentalen Fertigkeiten, zum anderen 
durch den Prozess des Vor- und Nachsingens elementarer tonaler und rhythmischer Bausteine" (Fuchs, 2010, S. 22-23).

„Auch aus gedächtnispsychologischer Sicht hat das Imitieren etwas Einfaches. Es ist nicht auf eine hohe Bewusstseinsstufe und auch nicht auf das Langzeitgedächtnis angewiesen, um eine Handlung zu generieren. Es kann via Imitation musiziert und gesungen werden, ohne zu audiieren... Das Lernen am Modell kann direkt als Reaktion auf eine Wahrnehmung erfolgen. Das Gehörte beansprucht das Arbeitsgedächtnis und innerhalb dessen die phonologische Schleife (vgl. dazu Hoffmann / Engelkamp 2013, 121 f.), in welcher auditives Material vorerst für ganz kurze Zeit gehalten werden kann. Es kreist hier in einer Kette von Neuronen, und wird dann entweder fallen gelassen oder aber tiefer verarbeitet. Die phonologische Schleife kann zwischen musikalischem und sprachlichem Material unterscheiden, das Prinzip ist aber dasselbe, sie erhält die Information durch kurzfristiges wiederholen (,rehearsel') - Kreisen in einer Kette von Neuronen - für einen Moment aufrecht. Von da besteht die Chance, dass Material in das Langzeitgedächtnis gelangt, d.h., gelernt wird“ (Spychiger, 2015, S. 55).

(2 b) Musikunterricht ist vom gemeinsamen Tun bestimmt. Eigenes und gemeinsames Lernen lassen sich schwer voneinander abgrenzen. Erst in der solistischen Aktion der Schülerinnen und Schüler wird dieser parallel verlaufende Lernprozess erfahrbar. Ihn dennoch immerwährend mitzudenken, ist Aufgabe der Lehrkraft.

„Wenn man es recht bedenkt, muss jeder das musikalische Lernen letztlich für sich allein vollziehen: die Intervall- und Rhythmuslehre und ihre klangliche Anwendung, das verständige Sprechen über musikalische Strukturen und Bedeutungen, das Singen, den Umgang mit Instrumenten, das Erfinden, die Bewegung zu Musik. Selbst was als besonders intensives gemeinsames Tun gilt - das Musizieren mit anderen - beruht mehr auf eigenem Tun und Lernen, als zumeist angenommen wird. Auch wenn gemeinsam gelernt und geübt wird, wird es in einmalig-individueller Weise ausgestaltet, erprobt und verantwortet, wird es individuell zusammengesetzt und unterliegt es den individuellen Bedingungen des Körpers, des Fühlens, des Denkens, der Kontrolle und der eigenen Erfolgseinschätzung“ (Richter, 2006, S. 78).

(3) Musiklernen basiert auf dem Vorrang von implizitem Wissen (musikalischem Können) vor explizitem Wissen (musikalischem Wissen). Diesem Vorrang liegen entwicklungspsychologische Erkenntnissen zugrunde (Gies, Jank \& Nimczik, 2001, S. 15-17).

„Durch Musikmachen im Unterricht entsteht [...] ein spezifisches Wissen, ein ästhetischer Erfahrungsschatz von Leiblichkeit, als körperbezogenes Wissen. Dieses Wissen, das durch unseren Körper gespeichert wird, ist jenes Können, das über das verfügbare ,Kopf'-Wissen hinausgeht. Es ist ein zentrales Moment ästhetischer Erfahrung“" (Schütz, 1997, S. 5).

(4) Musiklernen basiert auf sequentiell gestuften Lernschritten (Gies, Jank \& Nimczik, 2001, S. 17-18; Gruhn, 1999, S. 62). Gordon benennt in seiner Music Learning Theory $\left(2012^{8}\right)$ innerhalb der Skill Learning Sequence zwei verschiedene Phasen. Dazu zählen das unterschiedene und das schlussfolgernde Lernen. Ersteres ist durch Demonstration und Nachahmung, letzteres durch 
selbstständiges Übertragen des erworbenen Wissens geprägt. Die im Abschnitt 2.1 dargestellten Lernschritte zählen zur Phase des unterscheidenden Lernens.

(5) Musiklernen basiert auf kontinuierlicher Anwendung, weil sich dauerhafte Repräsentationen erst im Vollzug bilden (Gies, Jank \& Nimczik, 2001, S. 18; Gruhn, 2003, S. 110).

„Ein selbstkritischer Musikpädagoge weiß, dass das punktuelle 'Durchnehmen' von [...] Notenlehre kaum zu einem nachhaltigen Zuwachs an musikalischer Kompetenz führt. Denn diese benötigt zu ihrer Entstehung einen längerfristig angelegten Lehr- und Lernprozess, der Übung und Wiederholung mit einschließt und nach den Erkenntnissen der musikalischen Lernforschung organisiert ist“ (Fuchs, 2010, S. 13).

(6) Musiklernen basiert auf Übung (Gies, Jank \& Nimczik, 2001, S. 18). Ohne sie ist musikalisches Lernen nicht möglich. Von den zahlreichen Aspekten des Übens seien hier einige genannt: Aus neuronaler Sicht betrachtet Spitzer Üben als prozedurales Lernen (2002, S. 315). Altenmüller bezeichnet aus hirnphysiologischer Sicht Übung als multisensorisch-motorische Integrationsleistung (2006, S. 53-54). Dartsch betrachtet hinsichtlich des Übens entwicklungspsychologische Besonderheiten für ein spezielles Üben im Vorschul- und Grundschulalter (2006, S. 225-226). Ernst betont aus didaktischer Sicht, dass Üben und Lernen eine Einheit bilden (2006, S. 98). Den ästhetischen Aspekt betrachtend betont Jank dabei den Anspruch auf erfahrbare Qualität (2007², S. 106). Auch Gembris betont aus pädagogisch-psychologischer Sicht die Zusammengehörigkeit von Qualität und Effektivität des Übens (2013 , S. 160-162). Rüdiger betrachtet gruppendynamische Prozesse beim Üben im Ensemble, welche für kleine Gruppen bis hin zu Klassenverbänden ihre Bedeutung haben (2006, S. 156-159).

(7) Musiklernen basiert auf Reflexion. Dies ist zum einen in jedem Musizierakt involviert, über dessen Qualität sich die Musizierenden Rechenschaft geben, zum anderen in der Reflexion über das, was musiziert wird. „Der Ausführende ist in der Lage, mithilfe seines analytischen Denkvermögens [...] das Gespielte bewusst zu denken, zu gestalten und zu kontrollieren" (Schütz, 1996, S. 3). Gruhn (2003, S. 111) hebt hervor: „Im musikalischen Tun, also im Spielen von Musik sind Aktion und Reflexion nicht getrennt, sondern überlagern sich in zyklischen Schleifen“.

\subsection{Didaktische Analyse des Lerngegenstandes Rhythmusnotation}

Die bisher getroffenen Aussagen zu den allgemeinen Lehr- und Lernvoraussetzungen sowie den Aspekten des Musiklernens haben wesentlichen Einfluss auf konkrete Lehr- und Lernhandlungen in Bezug auf die Förderung rhythmischer Kompetenz einschließlich des Umgangs mit Notenschrift. Bezüge zum Lernen in der Sozialform eines großen Klassenverbundes von bis zu 28 Schülerinnen und Schülern und damit zur Diversität von Lernständen wurden dabei nicht betrachtet. Deshalb erscheint eine didaktische Analyse des Lerngegenstandes Rhythmusnotation nach den Prinzipien Klafkis $\left(1958 ; 2007^{6}\right)$ sinnvoll. 
Zwei Quellen werden dazu herangezogen: Zum einen die früheste Darstellung Klafkis aus dem Jahre 1958, welche in zahlreichen weiteren Schriften wiederholt publiziert wurde. Zum anderen die derzeit aktuellste Neuauflage des Werkes Neue Studien zur Bildungstheorie und Didaktik in der Fassung von 2007. Es werden insgesamt sieben Fragen zur Gegenwarts-, Zukunfts- und exemplarischen Bedeutung, zur Struktur des Inhalts, zur Erweisbarkeit und Überprüfbarkeit, zur unterrichtlichen Zugänglichkeit und zur Lehr-Lern-Prozessstruktur gestellt. ${ }^{2}$

Die Gegenwartsbedeutung beschreibt Klafki $\left(2007^{6}\right.$, S. 273) als „Frage nach den von Kindern und Jugendlichen erfahrenen und praktizierten Sinnbeziehungen und Bedeutungssetzungen in ihrer Alltagswelt“. Sie ließe sich in einem Musikunterricht, der seit Beginn der 90er Jahre von einer großen Hinwendung zu musizierpraktischen Tätigkeiten geprägt ist, schnell ableiten. Erschwingliche Instrumente für die Hand des Schülers sowie zahlreiche „Spiel-mit-Sätze“ zu Klassik und Pop ermöglichen ein ansprechendes Musizierangebot für Kinder im Musikunterricht der Grundschule. Doch die zu spielenden Rhythmen sind meist so leicht, dass sie durch Vor- und Nachmachen schnell erfasst und umgesetzt werden können. Das Notenbild ist real vorhanden, wird aber nicht genutzt. Eine Gegenwartsbedeutung ist erst dann gegeben, wenn in der Lehr- und Lerntätigkeit darauf geachtet wird, einen bewussten Umgang mit Notation zu initiieren. Dies sollte im Treatment durch gezielte Nutzung der Notenschrift in Form von selbstständigem Lesen und Schreiben derselben realisiert sein. Für die Schüler, für welche die Rhythmusnotation bereits Gegenwartsbedeutung besitzt, da sie außerschulisch Instrumentalunterricht erhalten, sollte das Treatment einen Differenzierungsansatz aufweisen.

Die Zukunftsbedeutung in Bezug auf den zunehmenden Gebrauch von Notationen in den weiterführenden Schulen der Sekundarstufe wurde bereits hervorgehoben. Klafki (1958, S. 457) fragt: „Worin liegt die Bedeutung des Themas für die Zukunft der Kinder?“ Für Schüler, welche Noten lesen und schreiben können, eröffnet sich das weite Feld des selbstständigen Arbeitens. Moderne Unterrichtsformen wie Gruppen- und Ensemblearbeit werden erst durch diesen selbstständigen Umgang mit Notation wirklich lebendig. Deshalb sollte das Treatment kontinuierliche Übungen zum Lesen und Schreiben von Rhythmen enthalten sowie Gruppenarbeit ermöglichen.

Die exemplarische Bedeutung des Lerngegenstandes hinterfragt Klafki (1958, S. 457) wie folgt: „Welches Urphänomen oder Grundprinzip, welches Gesetz, Kriterium, Problem, welche Methode,

\footnotetext{
${ }^{2}$ Die Fragen werden mit Blick auf das zum Einsatz kommende Treatment beantwortet. Die Auseinandersetzung mit diesen Fragen dient lediglich den oben angegebenen Zielen. Sie ist nicht davon geprägt, die in der späten Wirkungsphase vollzogene Hinwendung Klafkis zu einer kritisch konstruktivistischen Didaktik bildungstheoretisch zu hinterfragen, sondern um Bezug auf die Vermittlung des Lerngegenstandes Rhythmusnotation im Klassenunterricht der allgemeinbildenden Schule Bezug zu nehmen (vgl. Flämig, 2000, S. 4-8).
} 
Technik oder Haltung lässt sich in der Auseinandersetzung mit ihm ,exemplarisch“ erfassen?" Eine Antwort darauf könnte lauten, dass unsere Sprache und die Musik gleichermaßen von Rhythmus geprägt sind. Rhythmen werden grafisch fixiert, um sie zu einem späteren Zeitpunkt wiederholen zu können oder an andere weiterzureichen. Die Schüler erlernen damit eine Kulturtechnik, die ihnen ermöglicht, notierte Rhythmen lesen und musizieren bzw. eigene Rhythmen notieren zu können. Das Treatment sollte dazu befähigen.

Zur Struktur des Inhalts wirft Klafki $\left(2007^{6}\right.$, S. 280) verschiedene Fragen auf. Drei Fragen werden für die weiteren Überlegungen herangezogen: „Weist die Thematik eine Schichtung, etwa im Sinne von Oberflächen- und Tiefenstrukturen auf? Welches sind die notwendigen begrifflichen, kategorialen Voraussetzungen für die Auseinandersetzung mit dem Thema, und welche Verfahren für die Bewältigung des Themas müssen die Schüler sei es bereits mitbringen, sei es im Zusammenhang der Auseinandersetzung erwerben?“

Die über tausendjährige Geschichte der Entwicklung der traditionellen Notenschrift kann hier nicht erläutert werden. Für die Struktur der Rhythmusnotation ist wesentlich, die beiden Systeme Tonhöhe und Tondauer bewusst zu trennen. Dies entspricht dem Ansatz Gordons. Curricular vorgesehen ist für die Klassenstufe 3 der Umgang mit Halben Noten, Vierteln und Achteln, in einigen Bundesländern mitunter auch der ganzen Note. Gordons Music Learning Theorie legt nahe, dass Rhythmen nur dann gelesen und geschrieben werden können, wenn Schülerinnen und Schüler über ein Hörvokabular an Rhythmen verfügen, welche einem Metrum zugeordnet werden können, und eine Rhythmussprache anwenden können. Die Benennung der Notenwerte und deren Verhältnis untereinander (z. B. eine Halbe Note entspricht der Dauer zweier Viertelnoten) spielen eine untergeordnete Rolle. Dieser Abfolge Gordons soll die Methodik des Treatments in Bezug auf das Erlernen der Rhythmussprache folgen. Deshalb sollen bewusst nicht einzelne Notenwerte an sich betrachtet werden, sondern sogenannte Patterns (Lehrplan Sachsen: Rhythmusbausteine). Sie stellen das Herzstück der Music Learning Theory Gordons dar (Gordon, 2012 ${ }^{8}$, S. 217-224; Jank, 20135 , S. 124-131). Ziel soll der Erwerb eines großen Repertoires an Patterns / Rhythmusbausteinen sein. Mithilfe der Rhythmussprache sollen verschiedene Qualitäten dieser Patterns, aber auch Gemeinsamkeiten erfasst werden können. Die Schwierigkeit der Patterns soll allmählich ansteigen.

Zur Erweisbarkeit und Überprüfbarkeit fragt Klafki (20076, S. 280): „Wie, an welchen Fähigkeiten, welchen Erkenntnissen, welchen Handlungsformen, welchen ,Leistungen“ im weiten Sinne des Wortes soll sich zeigen und soll beurteilt werden, ob die angestrebten Lernprozesse bzw. Zwischenschritte als erfolgreich gelten können? “. Eine Evaluation des Lernzuwachses durch das Treatment soll durch Vor- und Nachtest gemäß dem Forschungsdesign durchgeführt werden.

Die unterrichtliche Zugänglichkeit hinterfragt Klafki (1958, S. 460) wie folgt: „Welches sind die besonderen Situationen in oder an denen die Struktur des jeweiligen Inhaltes den Kindern dieser Bildungsstufe, dieser Klasse interessant, fragwürdig, zugänglich, begreiflich, ,anschaulich` 
werden kann?““ Altersgemäße Rhythmicals, Abzählreime, „Spiel-mit-Sätze“ zu Klassik und Pop bieten in den Lehrwerken für das Fach Musik vielfältige Möglichkeiten, um Rhythmusnotation zugänglich und begreiflich werden zu lassen. Ebenfalls soll es Gelegenheiten geben, das Gelernte anzuwenden und zu „gebrauchen“. Da Rhythmusnotation per se nicht anschaulich ist, sondern ein historisch gewachsenes Symbolsystem, sollte dieser Wissensbereich auf dreifache Art angeboten werden:

- durch geeignete Handlungen (enaktive Repräsentation)

- durch Bilder oder Grafiken, die eine bestimmte Konzeption verdeutlichen (ikonische Repräsentation)

- durch eine Folge symbolischer Formsätze, die einem symbolischen System entstammen (symbolische Repräsentation)

Didaktisch wird damit die Idee vom Verlauf der geistigen Entwicklung von der enaktiven, über die ikonische hin zur symbolischen Darstellung der Welt umgesetzt (Bruner, 1974, S. 48-53; Klafki, 2007 ${ }^{6}$, S. 156-159; Seel, 2003, S. 61). Den geeigneten Handlungen entsprechen in diesem Kontext am besten Patternübungen in Form von Call-Responses (Gordon, 2001). Als Bilder und Grafiken bieten sich Vorformen für Notationen an (Losert, 2011, S. 130-147). Die Rhythmusnotation entspricht den symbolischen Formsätzen. Eine dieser Formen sollte Anwendung finden. Sowohl regelmäßige Call-Response-Übungen als auch eine Vorform für Notation sollen im Treatment Anwendung finden, um die dreifache Wissensdarbietung zu ermöglichen.

Die Lehr-Lern-Prozessstruktur betrachtend fragt Klafki (20076, S. 283): „Diese siebente Frage [...] richtet sich darauf, wie die durch die vorangehenden Fragen ermittelten Momente in eine sukzessive Abfolge eines Lehr-Lern-Prozesses ... übersetzt werden können. Die Frage richtet sich also auf die methodische Strukturierung bzw. die Strukturierung des Lehr-Lern-Prozesses“. In den aktuellen Lehrwerken werden die Unterrichtsinhalte, wie curricular gefordert (Sächsisches Staatsministerium für Kultus, 2009, S. 4), meist außermusikalischen Themen (Jahreszeiten, Feste im Jahreskreis, Freundschaft, Naturerscheinungen wie Wetter, Tag und Nacht u.v.m.) zugeordnet. Diese Strukturierung lässt den Fokus auf das Erlangen von Kompetenz im Umgang mit Notenschrift auBer Acht (vgl. Fuchs, 2010, S. 13). Da die im Lehrplan geforderte thematisch orientierte Strukturierung des Musikunterrichts nicht ohne weiteres aufgehoben werden kann, sollte das Treatment eine Idee von Schütz (1997, S. 5) aufgreifen: „Vielleicht bieten sich eingeschobene Phasen an, in denen man in kompakter Form und mit effektivsten Lernmethoden Wissensbestände aufbaut." Ihre Realisierung wird über eine Sequenzierung erfolgen (s. Abschnitt 3.3.2).

\subsection{Eignung vorgefundener Treatments}

Das zum Einsatz gelangende Treatment zur Entwicklung und Förderung rhythmischer Kompetenz soll den bisher dargestellten Erkenntnissen 
- in Bezug auf Aspekte des Musiklernens genügen,

- den didaktischen Prinzipien Klafkis folgen,

- Möglichkeiten zur Differenzierung und dreifachen Wissensdarbietung aufweisen,

- den Erwerb eines großen Repertoires an Patterns ermöglichen,

- das Erlernen einer Rhythmussprache sichern sowie

- Notenlese- und Notenschreibanlässe enthalten.

Ein infrage kommendes Treatment wurde im Rahmen der Evaluationsstudie zum „Musiklernen im Anfangsunterricht der Grundschule auf der Basis neuer Musiklerntheorien“ (Fuchs, 2004) entwickelt. Die Studie hatte zum Ziel, die Anbahnung und Förderung des Musiklernens im Zusammenwirken von Stimme, Bewegung und Instrumentalspiel auf der Blockflöte zu ermöglichen. Basierend auf der Music Learning Theory Gordons wurden Unterrichtsmaterialien für den Anfangsunterricht im Fach Musik erarbeitet. Diese wurden nach dem Prinzip des sequenziellen Lernens nach Gordon zusammengestellt (ebd., S. 4). Musiklehrkräfte und studentische Hilfskräfte arbeiteten mit fünf 2. Klassen über fünf Monate hinweg mit diesen Unterrichtsmaterialien. Der Einsatz dieses Treatments für die hier dargestellt Studie kam nicht infrage, da das Erlernen des Flötenspiels nicht beabsichtigt wurde.

Die Lehrmaterialien Rhythmus für Kids 1 (Filz \& Heidecker, 2008) sowie Notenlernen mit Trommelspaß (Herrmann, 2009) wurden ebenfalls zum Zwecke der Eignung für diese Studie genauer untersucht. Beide Werke beruhen auf kindgerechten und begeisternden Musikbeispielen und Musizieranregungen. Für den 20-wöchigen Untersuchungszeitraum hätten sie jedoch speziell aufbereitet werden müssen, um den Anforderungen an Differenzierung sowie dem Angebot an Notenlese- und Notenschreibanlässen zu genügen. Aus beiden Lehrmaterialien wurden Ideen und Anregungen adaptiert, wie z. B. der Einsatz eines Schülerheftes und eines Lehrerhandbuches inklusive einer CD mit Playbacks für alle Übungen sowie der Einsatz von Rhythmicals. Da die genannten Beispiele nicht allen Anforderungen genügen konnten, wurde ein Treatment entwickelt.

\subsection{Tests zur Messung rhythmischer Kompetenz}

Das Beobachtungsinteresse ist auf eine Kompetenz mit konkreten operativen Fähigkeiten in Bezug auf den Umgang mit Notenschrift gerichtet. Diese orientieren sich an den Stufen zum Aufbauen rhythmischer Fähigkeiten (Jank, 201355). Deshalb liegt es nahe, ausschließlich rezeptionsorientierte sowie musikalitätsbezogene Tests von vornherein als nur bedingt geeignet zu betrachten.

„So gibt es bei uns auch kaum allgemeingültige Normen oder Ziele musikalischer Entwicklung. Eine gewisse Ausnahme bilden allenfalls Musikalitätstests, welche akustisch-sensorische Hörfähigkeiten auf verschiedenen Altersstufen prüfen und diese dann mit bestimmten Erwartungswerten vergleichen... Allerdings werden Musikalitätstests in Deutschland und wohl auch in anderen europäischen Ländern außer in 
der Forschung praktisch nicht verwendet... Abgesehen davon beziehen Musikalitätstests sich lediglich auf begrenzte, rezeptive Aspekte musikalischer Fähigkeiten und berücksichtigen produktive oder kreative Fähigkeiten nicht“ (Gembris, 2013, S. 50-51).

Dem Charakter der Studie entsprechen am ehesten kriteriumsorientierte Leistungstests (Rost, 2007², S. 150-152; Kormann, 1985, S. 507) bzw. musikalische Leistungstest.

\begin{abstract}
„Bei den musikbezogenen Testverfahren sind verschiedene Arten zu unterscheiden: Begabungs- und Musikalitätstests wollen das von Lernerfahrungen unabhängige, angeborene Potential an musikalischen Fähigkeiten (aptitude) messen. Musikalische Leistungstests beziehen sich auf die Prüfung von musikalischen Fertigkeiten, die durch Unterricht erlernt wurden (achievement). Ferner gibt es Tests, die vokale und instrumentale Leistungen (performance) erfassen. Schließlich sind Testverfahren zu nennen, die musikalische Einstellungen, Urteile und Vorlieben (attitude, preference) prüfen“ (Gembris, 2013², S. 106107).
\end{abstract}

Ein geeigneter Test soll

- die sechs Stufen des „Aufbauens rhythmischer Fähigkeiten“ (Jank, 20135) operationalisieren und

- dem Konzept der Klassischen Testtheorie mit den Testgütekriterien Objektivität, Reliabilität und Validität (Bortz \& Döring, 2006 , S. 193-202) hinreichend genügen.

\title{
2.5.1 Eignung der vorgefundenen Tests
}

Fünf kriteriumsorientierte Leistungstests wurden auf ihre Eignung geprüft. In den 1970er Jahren führte Wagner mit 5000 sechs- bis elfjährigen Kindern eine Untersuchung zur Entwicklung der Musikalität mit Hilfe eines Musikleistungstests durch. In vier Untertests werden jeweils im Einzelverfahren Melodie-Auffassung, Rhythmus-Auffassung, Sinn für Klangqualität und MelodieErfindung geprüft (1970, S. 16-19). Im Untertest Rhythmus-Auffassung wird in drei Items das Hören von Unterschieden jeweils zweier Rhythmen sowie in vier Items das Nachklatschen von Rhythmen in verschiedenen Metren geprüft. Die Hörmodelle werden vom Band gegeben (ebd., S. 17). Das richtige Spielen der Rhythmen wird sofort bewertet und in einem Protokoll erfasst. Die Ergebnisse werden in richtig / falsch unterschieden. (Weitere Hinweise zu dieser und den folgenden Studien finden sich in Anlage A 2).

Eine Längsschnittuntersuchung zum Musikerleben von Kindern im Alter von fünf bis zehn Jahren mit mehr als 50 Probanden führte Minkenberg (1991) in den 1980er Jahren durch. Im Bereich rhythmisch-metrischer Kompetenz werden ebenfalls das Hören von Unterschieden sowie das Nachklatschen von Rhythmen (jeweils im Einzelverfahren) geprüft. Die Rhythmen sind wesentlich komplexer als bei Wagner. Minkenberg klatschte fünf Rhythmen über jeweils 8 Grundschläge im Tempo von 68 Schlägen pro Minute vor, zeichnete mit einem Kassettenrecorder auf und wertete die Ergebnisse im Nachhinein aus (ebd., S. 72). Die Ergebnisse sollten ursprünglich nur in richtig / falsch unterschieden werden. Da in einigen Altersstufen das Spielen der (recht langen) Rhythmen 
große Schwierigkeiten bereitete, wurden mitunter auch die Anzahl der richtig gespielten Zählzeiten erfasst (ebd., S. 73, 101, 128-129, 156-159).

Ein Musik-Screening für Kinder I + II wurde im Rahmen der Berlin-Studie, die unter der Leitung von Bastian (2000) stattfand, von Jungbluth und Hafen entwickelt (1997). (Es blieb bisher unveröffentlicht, wird jedoch auf Anfrage an Prof. Roland Hafen, Universität Vechta, an Interessenten versendet [Stand 2013].) Die Rubrik I ist für Kinder im Alter von 5 bis 8;6 Jahren gestaltet, die Rubrik II für Kinder von 8;7 bis 11 Jahren angepasst. Der Test untergliedert sich in ein Einzelund ein Gruppenverfahren. Im Einzelverfahren werden die Subtests Ein Lied lernen, Rhythmen nachspielen sowie Metrum ausführen getestet. Im Gruppenverfahren werden die Subtests Melodien vergleichen, Tonhöhen vergleichen: große und kleine Intervalle, rhythmische Figuren vergleichen, Tonlängen vergleichen sowie Metren vergleichen getestet. Im Subtest Rhythmen nachspielen werden jeweils zehn Rhythmen (Rubrik I: über vier Grundschläge; Rubrik II: über wechselnde Metren) von CD vorgespielt. Die Probanden spielen die Rhythmen auf einem Keyboard auf Ton a nach. Die Rhythmen werden sofort beim Testen von den jeweiligen Versuchsleitern in einem Testbogen bewertet. Richtig gespielte Rhythmen erhalten zwei Punkte, Rhythmen, die einen Ton zu wenig oder zu viel enthalten, erhalten nur einen Punkt. Im Subtest Metrum ausführen (Einzelverfahren) wird das Empfinden des Pulses bzw. Grundschlages eines ausgewählten Marsches getestet. Im Subtest Metrum vergleichen (Gruppenverfahren) wird das Vergleichen verschiedener Tempi getestet. Die Autoren geben für den Test an, dass die Items noch nicht in allen Details auf Validität, Reliabilität und Normwerte geprüft sind (Jungbluth \& Hafen, 1997, S. 1).

McPherson (2005) führte mit 124 Schülern die Langzeituntersuchung From child to musician: skill development during the beginning stages of learning an instrument durch. Die Schüler besuchten Klassen, in denen ein dem Klassenmusizieren ähnliches Modell unterrichtet wurde. Die Studie ist in die Subtests Spiel geübter Stücke, Vom-Blatt-Spiel, Spiel aus dem Gedächtnis, Spiel nach Gehör sowie Improvisieren unterteilt (McPherson, 2005, S. 9-10). Mit Hilfe der Watkins-Farnum Performance Scale (WFPS) wird die Fähigkeit des Notenlesens (Vom-Blatt-Spiel) im rhythmischmetrischen und tonalen Bereich getestet. Die Auswertung dieser Itemgruppe wird so gestaltet, dass der Versuchsleiter während des Testprozesses in einer Vorlage ankreuzt, wenn es in einem der 16 Takte der WFPS einen Fehler gibt (ebd., S. 11). Durch die Gebundenheit an ein Melodieinstrument kann der Ansatz dieser Untersuchung nicht übertragen werden.

Hasselhorn (2015) entwickelte für seine Studie zur Messbarkeit musikpraktischer Kompetenzen von Schülerinnen und Schülern ein computerbasiertes Setup, mit welchem er ausschließlich musikpraktische Kompetenzen in den Subtests Gesang, Instrumentales Musizieren sowie Rhythmusproduktion testete. Die Studie basiert auf den Ergebnissen von 445 Probanden der Jahrgangsstufe 9. Im Subtest Rhythmusproduktion spielen die Probanden auf einem speziell ausgestatteten Tablet mithilfe einer App, deren zweigeteiltes Display zwei unterschiedlich hohe Töne erzeugen lässt. Die Items 
bestehen aus insgesamt neun ein- bis zweitaktigen Patterns, davon fünf in binärem, vier in ternärem Metrum. Diese Patterns sollen von den Schülern jeweils zu achttaktigen Playbacks gespielt werden (ebd., S. 65). Dies findet unter Hinzunahme von Vorspiel (Demonstration) oder Notenbild bzw. beidem statt. Es findet eine digitale Aufzeichnung der Ergebnisse statt, die im Nachhinein von verschiedenen Ratern nach festgelegten Ratings bewertet wird (ebd., S. 97-100). Die Ergebnisse der Rater werden durch ein Verfahren der Inter-Rater-Korrelation (Intraklassenkorrelation, ICC) untereinander abgeglichen (ebd., S. 85-90). Die Entscheidung über richtig und falsch wird in die Verantwortung der einzelnen Rater gelegt:

„Korrekt bedeutet in diesem Zusammenhang allerdings nicht zwingend, dass die einzelnen Tonanfänge des gespielten Rhythmus' zu den mathematisch exakt bestimmbaren Zeitpunkten erklingen sollten, damit ein Leistung die höchste Bewertung erhält. Vielmehr sind kleinste Abweichungen von wenigen Millisekunden von diesen mathematisch bestimmbaren Punkten, das sogenannte Timing, das auch von musikalisch nicht ausgebildeten Hörern wahrgenommen wird, ausschlaggebend für eine besonders hohe Bewertung einer Leistung (Friberg \& Sundberg, 1995). Dabei sollten diese minimalen Abweichungen jedoch nicht zufällig sein, sondern sollten bewusst und kontrolliert eingebaut werden. So werden ,zu früh ' gespielte Töne als negativer wahrgenommen als ,zu spät ${ }^{\star}$ gespielte Töne (Frühauf et al., 2013)“ (ebd., S. 97).

In dieser Studie werden erstmalig Angaben zur Beurteilung von Rhythmen thematisiert, auch wenn sie nicht konkret zu einer Auswertungsanleitung für die Rater führten. Jedoch gibt es eine strukturierte Skala zur Bewertung der Schülerleistung, die zwischen 1 Der Schüler spielt den Rhythmus ganz richtig in nahezu oder völlig korrektem Tempo bis 6 Der Schüler spielt entweder gar nicht oder etwas, was nichts mit der Aufgabe zu tun hat (ebd., S. 84).

Tab. 2-1: Inhaltliche Breite der vorgestellten Leistungstests

\begin{tabular}{|c|c|c|c|c|c|c|c|c|c|c|}
\hline Testentwicklung von & 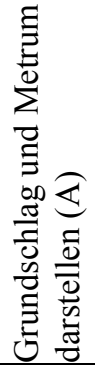 & 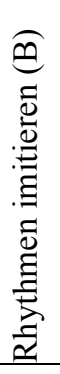 & 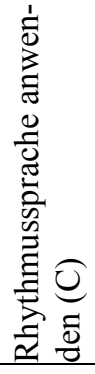 & 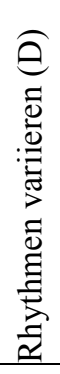 & 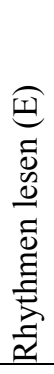 & 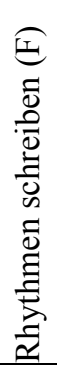 & 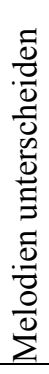 & 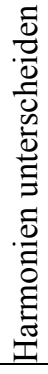 & 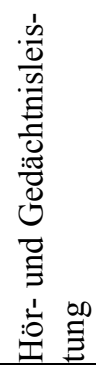 & 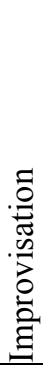 \\
\hline Wagner (1970) & - & $\mathrm{x}$ & - & $\mathrm{x}$ & - & - & $\mathrm{x}$ & $\mathrm{x}$ & $\mathrm{x}$ & $\mathrm{x}$ \\
\hline Minkenberg (1991) & - & $\mathrm{x}$ & - & - & - & - & $\mathrm{x}$ & $\mathrm{x}$ & $\mathrm{x}$ & - \\
\hline Jungbluth und Hafen (1997) & $\mathrm{x}$ & $\mathrm{x}$ & - & - & - & - & $\mathrm{x}$ & - & $\mathrm{x}$ & - \\
\hline McPherson (2005) & - & - & - & - & $\mathrm{x}$ & - & - & - & $\mathrm{x}$ & $\mathrm{x}$ \\
\hline Hasselhorn (2015) & $\mathrm{X}$ & $\mathrm{X}$ & - & - & $\mathrm{x}$ & - & - & - & - & - \\
\hline
\end{tabular}


Zusammenfassend wird für alle vorgestellten Tests festgestellt, dass für die Itemgruppen zum theoretischen Modell nach Gordon und Jank $\left(2013^{5}\right)$ in den vorgestellten Leistungstests zu wenig Bezüge hergestellt werden können (s. Tab. 2-1). Dennoch können den Tests viele Anregungen und Ideen entnommen werden.

\subsubsection{Zur Testentwicklung}

Die Suche nach einem geeigneten Testinstrument mündete in den Entschluss, selbst einen Test zu entwickeln. Anregungen aus den genannten Tests zum Nachspielen von Rhythmen (Stufe B), zum Erfassen von Metrum und Puls (Stufe A), zum Musizieren nach Notenvorlage (Stufe E) wurden aufgegriffen und weiterentwickelt. Für die Stufen C, D und F wurden eigene Ideen zur Operationalisierung entwickelt. Ebenso wurden die empfohlenen Tempi beim Rhythmennachspiel in der Erprobungsphase geprüft. Der Autorin war bewusst, dass auch diese eigene Testentwicklung der prinzipiellen Fehlerbehaftung nicht entrinnen werden würde, jedoch neue technische Möglichkeiten wie der Einsatz von Video, digitaler Aufzeichnung und millisekunden-genauer Messung erschlossen und erprobt werden könnten.

Die Testentwicklung war an vorderster Stelle durch pädagogisch-psychologische und ökonomische Erwägungen geprägt. Die zeitliche Belastbarkeit des Probanden Grundschulkind der Klassenstufe 3 am Nachmittag wurde auf maximal 20 Minuten eingeschätzt. Die Rahmenbedingungen der eigenen Forschungstätigkeit, die durch die hauptberufliche Tätigkeit als Grundschullehrerin, Fachberaterin und Lehrbeauftragte gekennzeichnet waren, begrenzten ebenfalls den zeitlichen Umfang. Das Forschungsdesign gab ein Zeitfenster von sechs Wochen für die nachmittäglich durchzuführenden Einzelverfahren vor. Alle Testaufgaben, die nicht zwingend eine unabhängige Einzelleistung der Probanden erforderten, sollten in einem Gruppenverfahren getestet werden (vgl. Jungbluth \& Hafen, 1997). Alle anderen Aufgaben wurden im Einzelverfahren getestet.

Der Testgestaltung liegt die Annahme zugrunde, dass jede Kompetenz durch entsprechende Items operationalisiert werden kann. Knigge stellt dazu fest: „Die Funktion von Testaufgaben besteht darin, Kompetenzen einer empirischen Überprüfung zugänglich zu machen“ (2010, S. 33). Es sollen musizierpraktische Aufgaben entwickelt werden, die realen Situationen des Musikunterrichts in der Grundschule entspringen könnten. Der Test soll ausschließlich kompetenzorientierte Items enthalten. Zusätzlich zu den inhaltlichen Ebenen der Theorie Gordons (in der Übertragung von Jank, 2013 ${ }^{5}$ ) haben die Ziele und Inhalte des sächsischen Lehrplans einen wesentlichen Einfluss auf die Operationalisierung (Sächsisches Ministerium für Kultus, 2009).

Jede der sechs Stufen der Pyramide des Aufbauens rhythmischer Fähigkeiten (Jank, 20135, S. 129) soll mit vier Items operationalisiert werden. Die Itemschwierigkeit der insgesamt 24 Items soll so gestaltet sein, dass das Pyramidenmodell mit seinen Stufen abgebildet wird, d.h. dass die Items in den höheren Stufen auch eine höhere Schwierigkeit aufweisen. Die klassische Testtheorie bevorzugt Items einer mittleren Schwierigkeit, da diese eine höhere Trennschärfe aufweisen kön- 
nen (Bühner, 2006, S. 98). Leichte und schwere Items decken wiederum gut die extremeren Leistungen ab und ermöglichen „auch in Randbereichen eines Merkmalsbereichs zu differenzieren“ (ebd., S. 99). Auch dies soll berücksichtigt werden. Damit die Items ihre Aufgabe erfüllen, Unterschiede zwischen den Ergebnissen der Probanden sichtbar zu machen, soll deren Trennschärfe bei Werten über 0.2 liegen. Liegt dieser Wert darunter, um „, 0 “ herum oder sogar im negativen Bereich, erfüllt das Item keine leistungsunterscheidende Funktion. „Ein Trennschärfenkoeffizient um 0 bringt zum Ausdruck, daß die Aufgaben von guten und schlechten Pbn [Probanden, d.V.] etwa gleich häufig richtig beantwortet wird; solche Aufgaben sind unbrauchbar (Lienert \& Raatz, $1998^{6}$, S. 78).“

Die Testgüte - Objektivität, Reliabilität und Validität - soll nach den allgemeinen Kriterien der Klassischen Testtheorie ausgerichtet sein. Mit der Testentwicklung sollen gleichzeitig Wege beschritten werden, die Auswertung dieser auf musizierpraktischen Kompetenzen beruhenden Leistungen objektiv zu gestalten. Das Hörempfinden ist prinzipiell stark subjektiv gefärbt. Hörer können auch dann rhythmische Strukturen erkennen, ,wenn die zeitlichen Längenverhältnisse zwischen den Tönen nicht mathematisch exakt gespielt werden, also beispielsweise punktierte Rhythmen nicht so gespielt werden, dass die punktierte Note exakt dreimal so lang ist, wie die nachfolgende Note, sondern Veränderungen der Längenverhältnisse auftreten“" (Auhagen, 2011 ${ }^{3}$, S. 441). Doch wo liegt die Grenze zwischen richtig und falsch? Um Leistungen aus zwei konkurrierenden Gruppen zu bewerten, muss es im Testverfahren klare Auswertungsregeln geben, um keine Gruppe willentlich oder unwissentlich zu bevorzugen. 


\subsection{Fragestellungen und Hypothesen}

Für die meisten Stufen des Aufbauens rhythmischer Fähigkeiten kann für die interessierende Altersgruppe kein konkreter Forschungsstand benannt werden (siehe z. B. Abschnitt 2.2.3, 2.2.4). Deshalb liegt eine wichtige Aufgabe dieser Studie darin, für die zwei Messzeitpunkte den Lernstand aller Probanden in diesen Stufen zu dokumentieren.

\section{Vortest}

(1) Welcher Stand der Entwicklung rhythmischer Kompetenz ist bei Grundschülern zu Beginn der Klassenstufe 3 zu verzeichnen?

\section{Nachtest}

(2) Welcher Stand der Entwicklung rhythmischer Kompetenz ist bei Grundschülern am Ende der Klassenstufe 3 zu verzeichnen?

Inwiefern das Treatment die Entwicklung rhythmischer Kompetenz fördern kann, lässt sich nur beurteilen, wenn die Ergebnisse beider Messzeitpunkte getrennt nach Untersuchungs- und Kontrollgruppe verglichen werden. Aus den Differenzen lässt sich eine Effektstärke für die Wirkung des Treatments berechnen.

\section{Evaluation}

(3) Welcher messbare Vorsprung beim Erwerb rhythmischer Kompetenz resultiert aus der Teilnahme am Treatment gegenüber der Teilnahme am regulären Musikunterricht?

Hypothese

$\mathrm{H}_{0}$ Durch die Teilnahme am Treatment, welches in den regulären Musikunterricht integriert ist, gewinnen Schüler der Klassenstufe 3 einen Lernzuwachs an rhythmischer Kompetenz mit einer eher kleinen Effektstärke $(d<0.5)$ gegenüber Schülern, welche regulären Musikunterricht erhalten.

$\mathrm{H}_{1}$ Durch die Teilnahme am Treatment, welches in den regulären Musikunterricht integriert ist, gewinnen Schüler der Klassenstufe 3 einen Lernzuwachs an rhythmischer Kompetenz mit einer mittleren Effektstärke ( $d \geq 0.5)$ gegenüber Schülern, welche nur regulären Musikunterricht erhalten.

$\overline{\mathrm{x}}_{\mathrm{UG}}>\overline{\mathrm{x}}_{\mathrm{KG}}$ und $\mathrm{d} \geq 0.5$

Eine Effektstärke von 0.5 wird angesetzt, da angeregt durch die Meta-Studie von Hattie davon ausgegangen wird, dass die Signifikanz von Unterschieden nur durch eine gleichzeitige Bestimmung der Effektstärken als aussagekräftig bezeichnet werden kann (Hattie, 2013, S. 9-12). Die Effekt- 
stärkeneinteilung bezieht sich auf Cohen, welcher $\mathrm{d}=0.2$ als kleinen Effekt, $\mathrm{d}=0.5$ als mittleren und $\mathrm{d}=0,8$ als großen bzw. starken Effekt bestimmt (vgl. Bortz \& Döring, 2006 ${ }^{4}$, S. 606).

Für das Treatment ist eine Übereinstimmung mit dem Pyramidenmodell Janks (2013², S. 129), den geschilderten Aspekten des Musiklernens sowie mit den didaktischen Prinzipien Klafkis nachzuweisen. Dies wird durch die Darstellung des Konzepts für ein Treatment zur Förderung rhythmischer Kompetenz in Abschnitt 3.3 realisiert. Das gelingende Umsetzen des Treatments muss durch den Einsatz eines geeigneten Tests erwiesen werden. Dieser Test soll inhaltlich ebenfalls an das Pyramidenmodell Janks angelehnt sein. Der Testaufbau soll die Schwierigkeitszunahme von Stufe zu Stufe widerspiegeln. Die Subtests und deren Items sollen allgemeine Testgütekriterien der Klassischen Testtheorie hinreichend erfüllen.

\section{Testaufbau}

(4) In welchem Maße bilden die erhobenen Daten das Modell der Pyramide des Aufbaus rhythmischer Fähigkeiten ab?

Die Itemschwierigkeiten des Leistungstestes (Nachtest) sollen auf jeder höheren Stufe ansteigen und folgender Gleichung entsprechen:

$$
\overline{\mathrm{X}}_{\mathrm{ST}_{-} \mathrm{A}}<\overline{\mathrm{x}}_{\mathrm{ST}_{-} \mathrm{B}}<\overline{\mathrm{x}}_{\mathrm{ST}_{-} \mathrm{C}}<\overline{\mathrm{x}}_{\mathrm{ST}_{-} \mathrm{D}}<\overline{\mathrm{x}}_{\mathrm{ST}_{-} \mathrm{E}}<\overline{\mathrm{X}}_{\mathrm{ST}_{-} \mathrm{F}}
$$

\section{Testgütekriterien}

(5) In welchem Maße hält der entwickelte Test die Gütekriterien ein?

Für den Nachweis einer zufriedenstellenden Reliabilität werden die Koeffizienten zur Testhalbierung sowie zur internen Konsistenz (Cronbach's Alpha) herangezogen. Die Kriteriumsvalidität wird über die Methode known groups sowie die Korrelation zu einer parallel geführten Itemgruppe belegt. 


\section{Material und Methoden}

In diesem Kapitel werden das Forschungsdesign, der notwenige Stichprobenumfang (s. Abschnitt 3.2), das Treatment (s. Abschnitt 3.3) sowie der Test dargestellt (s. Abschnitt 3.4). Treatment und Test wurden in einer Erprobungsphase getestet. Die dabei gesammelten Erfahrungen werden in die jeweiligen Unterkapitel integriert sowie in Abschnitt 3.3.8 dargestellt. Das Kapitel endet mit den Verfahren zur Testgütesicherung sowie zu verwendeten statistischen Verfahren (s. Abschnitt 3.5 und 3.6).

\subsection{Forschungsdesign}

Für die Studie wird ein Zweigruppen-Pretest-Posttest-Forschungsdesign gewählt (Bortz \& Döring, 2006 ${ }^{4}$, S. 116; Rost, 2007², S, 125-127). Mit ihm sollen sowohl zu zwei Messzeitpunkten Entwicklungsstände rhythmischer Kompetenz bei Schülerinnen und Schülern der Klassenstufe 3 gemessen werden, als auch Unterschiede beim Erwerb rhythmischer Kompetenz in einer Untersuchungsgruppe, die ein Treatment erhält sowie einer Kontrollgruppe, die nur regulären Musikunterricht erhält. Der Untersuchungszeitraum soll sich über 20 Unterrichtswochen erstrecken (s. Abb. 3-1). Eine Dauer von 20 Unterrichtswochen (halbes Unterrichtsjahr) erscheint als angemessener Zeitraum, um Veränderungen und Entwicklungen feststellen zu können, die auf gezielte didaktische Maßnahmen zurückgeführt werden können.

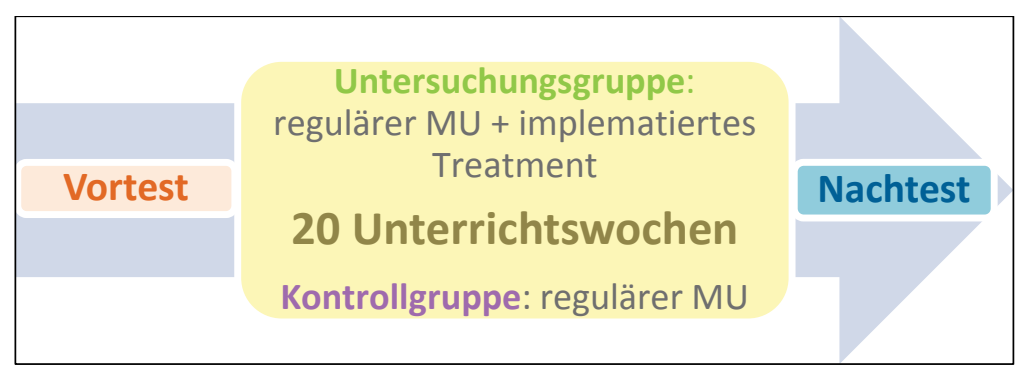

Abb. 3-1: Grafische Darstellung des Zweigruppen-Pretest-Posttest-Forschungsdesigns

Als zeitlicher Rahmen für den Vortest werden die Unterrichtswochen nach Schuljahresbeginn in der Klassenstufe 3 bis kurz nach den Herbstferien festgelegt. Dabei sollen zuerst die Tests in den Klassen der Untersuchungsgruppe stattfinden, damit die Erstauswertung der Daten für die Zusammenstellung der Lerngruppen im Rhythmus-Lehrgang genutzt werden kann. Der RhythmusLehrgang soll in den Unterrichtswochen nach den Herbstferien bis zu den Osterferien erteilt werden. Dieser Zeitraum entspricht 20 Unterrichtswochen. Anschließend soll der Nachtest stattfinden, wiederum beginnend mit den Probanden ${ }^{3}$ der Untersuchungsgruppe, um die Zeiträume zwischen

\footnotetext{
${ }^{3}$ Die Bezeichnung Probanden schließt männliche und weibliche Teilnehmer gleichermaßen ein.
} 
Vor- und Nachtest relativ stabil zu halten. Mit den involvierten Lehrkräften der Untersuchungsgruppe sollen drei Veranstaltungen stattfinden: Zwei Fortbildungen zum Treatment sowie eine Abschlussveranstaltung. Außerdem soll der Unterricht in den Klassen der Untersuchungsgruppe je zweimal hospitiert werden, um die Durchführung des Rhythmus-Lehrgangs zu begutachten.

\subsection{Geplante Stichprobe}

Eine zu messende Effektstärke $d$ von 0.5 (bei $\alpha$-Fehler: 0.05, $\beta$-Fehler: 0.80) erfordert laut a-prioriTeststärkenanalyse eine Stichprobe von insgesamt 102 Probanden. Diese müssen sich zu gleichen Teilen in die Untersuchungs- und eine Kontrollgruppe (UG / KG) aufteilen. Da es im Rahmen einer quasiexperimentellen Untersuchung nicht üblich ist, die Probanden durch Zufall zu gewinnen, werden Klumpenstichproben in Form bestehender Schulklassen gezogen (Bortz \& Döring, 2006 S. 54; Rost, 2005, S. 68). Sowohl die Lehrkräfte der Klassen als auch die einzelnen Probanden in den Klassen werden über persönliche Bereitschaftserklärungen (Lehrkräfte, Schulleiter, Eltern) gewonnen. Ein anderer Weg ist nach bestehendem Schulrecht im Freistaat Sachsen nicht möglich (vgl. Sächsisches Ministerium für Kultus, 2008, S. 2).

\subsection{Das Treatment}

Das eingesetzte Treatment zur Förderung rhythmischer Kompetenz im Musikunterricht der Klassenstufe 3 entspricht in seiner Konzeption dem Instructional Design. Dieses ist durch Analyse der individuellen Lernvoraussetzungen, Analyse des Lerngegenstandes, Sequenzierung und durch spezielle Lernhilfen geprägt (Helmke, 2009, S. 67-68). Die Analyse der individuellen Lernvoraussetzungen wird durch einen Vortest in der Erprobungsphase und in der Hauptuntersuchung realisiert (s. Kap. 4). Die Analyse des Lerngegenstands Einführung in den Umgang mit Rhythmusnotation ist bereits in den Abschnitten 2.2 Lern- und Lehrvoraussetzungen zum Erwerb sowie 2.3 Didaktische Analyse des Lerngegenstandes Rhythmusnotation erfolgt.

Im folgenden Abschnitt 3.3.1 wird die Analyse des Lerngegenstands durch eine Überprüfung einer Übereinstimmung mit den Lehrplanzielen des sächsischen Lehrplans vertieft. Die Sequenzierung wird in Abschnitt 3.3.2 Aufbau und Inhalt des Rhythmus-Lehrgangs sowie in Abschnitt 3.3.3 Ansteigende Schwierigkeit der Patterns betrachtet. Speziellen Lernhilfen werden in den Abschnitten 3.3.4 Erlernen einer Rhythmussprache, 3.3.5 Ikonische Notation als Teil einer dreifachen Wissensdarbietung sowie 3.3.6 Weitere Arbeitsmaterialien vorgestellt. Darüber hinaus wird die Bezugnahme zur Analyse der individuellen Lernvoraussetzungen in Abschnitt 3.3.7 Differenzierung im Rhythmus-Lehrgang thematisiert. Abschnitt 3.3.8 fasst alle Darstellungen zusammen.

\subsubsection{Lehrplanbezug}

Da die Studie im Freistaat Sachsen stattfand, wurden vor allem die konkreten Ziele und Inhalte des sächsischen Musiklehrplans in Bezug auf die Einführung in den Umgang mit Rhythmusnotation herangezogen (Sächsisches Staatsministerium für Kultus, 2009). Diese entsprechen in weiten Tei- 
len den Inhalten anderer Curricula deutscher Bundesländer. Den sechs Stufen (Jank, 20135, S. 129) werden die Lehrplanziele der Klassenstufen zugeordnet (s. Tab.3-1). Die Stufe CRhythmussprache erlernen weist (wie in fast allen Curricula anderer Bundesländer) keinen Lehrplanbezug auf. Für die Stufe D Rhythmen variieren und erfinden wird ein Lehrplanziel aus Klassenstufe 4 vorzeitig herangezogen. Die vollständigen Lehrplanauszüge finden sich in Anlage A 1.

Tab. 3-1: Übereinstimmung der operationalisierten Test-Stufen mit dem Lehrplan

\begin{tabular}{ll}
$\begin{array}{l}\text { Zu operationalisierende } \\
\text { Stufen des Aufbauens } \\
\text { rhythmischer Kompetenz }\end{array}$ & $\begin{array}{l}\text { Sächsischer Lehrplan Musik } \\
\text { (Sächsisches Staatsministerium für Kultus, 2009) }\end{array}$ \\
\hline $\begin{array}{l}\text { F Rhythmen schreiben \& } \\
\text { E Rhythmen lesen }\end{array}$ & $\begin{array}{l}\text { KI. 3: Notation von Rhythmusbausteinen ver- } \\
\text { wenden (S. 11) }\end{array}$ \\
$\begin{array}{l}\text { D Rhythmen variieren und } \\
\text { erfinden }\end{array}$ & $\begin{array}{l}\text { Kl. 4: eigene Rhythmen unter Verwendung viel- } \\
\text { fältiger Rhythmusbausteine erarbeiten (S. 16) }\end{array}$ \\
$\begin{array}{l}\text { C Rhythmussprache erler- } \\
\text { nen }\end{array}$ & keine Angabe \\
$\begin{array}{l}\text { B Rhythmen hören und } \\
\text { imitieren }\end{array}$ & $\begin{array}{l}\text { Kl. 3: vielfältige Rhythmusbausteine im 2er und } \\
\text { A Erfahrung von Metrum } \\
\text { und Puls }\end{array}$ \\
\hline
\end{tabular}

Die im Lehrplan geforderte thematisch orientierte Strukturierung des Musikunterrichts wird nicht aufgehoben. Der Rhythmus-Lehrgang wird in den thematisch strukturierten Unterricht integriert, ohne selbst dessen Thematik aufzugreifen. Die Inhalte der Lehrgangs-Gegenstände haben deshalb allgegenwärtige Themen wie z. B. Anfang, Geburtstag und Abzählreime.

\subsubsection{Aufbau und Inhalt des Rhythmus-Lehrgangs}

Die Inhalte der sechs Stufen des Aufbauens rhythmischer Fähigkeiten bzw. die Lehrplanziele werden im Rhythmus-Lehrgang in vier wiederkehrenden Bausteinen umgesetzt. Alle vier Bausteine bilden zusammen in einer Unterrichtsstunde einen Lernsequenzanteil (s. Abb. 3-2). Eine Musikstunde mit Lernsequenzanteil soll der Arbeit an den Inhalten des Rhythmus-Lehrgangs maximal 25 Minuten einräumen, um den Anteil der curricular vorgegebenen Zeitrichtwerte für den Lernbereich des Musizierens einzuhalten und anderen Lernbereichen wie Singen, Bewegen und Tanzen sowie Musikhören genug Raum zu lassen. Mehrere Lernsequenzanteile (ca. 4 bis 6) bilden eine Lernsequenz. Die Abfolge aus Lernsequenzanteil (Bausteine) sowie individuellem Stundenteil (Lieder, Tänze, Hörwerke) in der einzelnen Unterrichtsstunde wird jeder Lehrkraft freigestellt. Die individuellen Stundenteile können sowohl vor oder nach dem Lernsequenzanteil liegen oder ihn umrahmen.

Der Rhythmus-Lehrgang besteht aus fünf Lernsequenzen, die über eine Dauer von 20 Unterrichtswochen verteilt unterrichtet werden (s. Abb. 3-3). Dies entspricht etwa 38 Unter- 
richtsstunden. Die Sequenzierung folgt einer sachlogischen Schrittfolge vom Einfachen zum Komplexeren. Die Schwerpunkte der einzelnen Lernsequenzen liegen im Musizieren von Rhythmicals (LS 1,4) und Abzählreimen (LS 3) sowie in der Gestaltung und Präsentation von Spielstücken (LS 2, 5). Die konkreten Inhalte der 24 Stunden der fünf Lernsequenzen werden in Anlage D 2 dargestellt. Nicht alle 38 Stunden in diesem Zeitraum enthalten einen Lernsequenzanteil. Dies ermöglicht Spielraum zur Vertiefung anderer Unterrichtsinhalte, für Leistungskontrollen oder auch zum Ausgleich von Unterrichtsausfall.

\begin{tabular}{|c|c|c|c|c|}
\hline & Lernsequenz mit & ernsequenzanteilen & über vier Musikst & nden hinweg \\
\hline & Stunde 1 & Stunde 2 & Stunde 3 & Stunde 4 \\
\hline & $\begin{array}{l}\text { individueller } \\
\text { Stundenteil }\end{array}$ & & $\begin{array}{l}\text { individueller } \\
\text { Stundenteil }\end{array}$ & $\begin{array}{l}\text { individueller } \\
\text { Stundenteil }\end{array}$ \\
\hline $\begin{array}{l}\text { Gesamte Musik- } \\
\text { stunde à } 45 \text { Minu- } \\
\text { ten }\end{array}$ & $\begin{array}{l}\text { Lernsequenzanteil } \\
\text { mit Bausteinen } \\
\text { Warm up } 1 \\
\text { Warm up } 2 \\
\text { Workshop } \\
\text { Reflexion }\end{array}$ & $\begin{array}{l}\text { mit Bausteinen } \\
\text { Warm up } 1 \\
\text { Warm up } 2 \\
\text { Workshop } \\
\text { Reflexion }\end{array}$ & $\begin{array}{l}\text { Lernsequenzanteil } \\
\text { mit Bausteinen } \\
\text { Warm up } 1 \\
\text { Warm up } 2 \\
\text { Workshop }\end{array}$ & $\begin{array}{l}\text { Lernsequenzanteil } \\
\text { mit Bausteinen } \\
\text { Warm up } 1 \\
\text { Warm up } 2 \\
\text { Workshop } \\
\text { Reflexion }\end{array}$ \\
\hline & $\begin{array}{l}\text { individueller } \\
\text { Stundenteil }\end{array}$ & $\begin{array}{l}\text { individueller } \\
\text { Stundenteil }\end{array}$ & Reflexio & $\begin{array}{l}\text { individueller } \\
\text { Stundenteil }\end{array}$ \\
\hline
\end{tabular}

Abb. 3-2: Strukturierung einer Lernsequenz

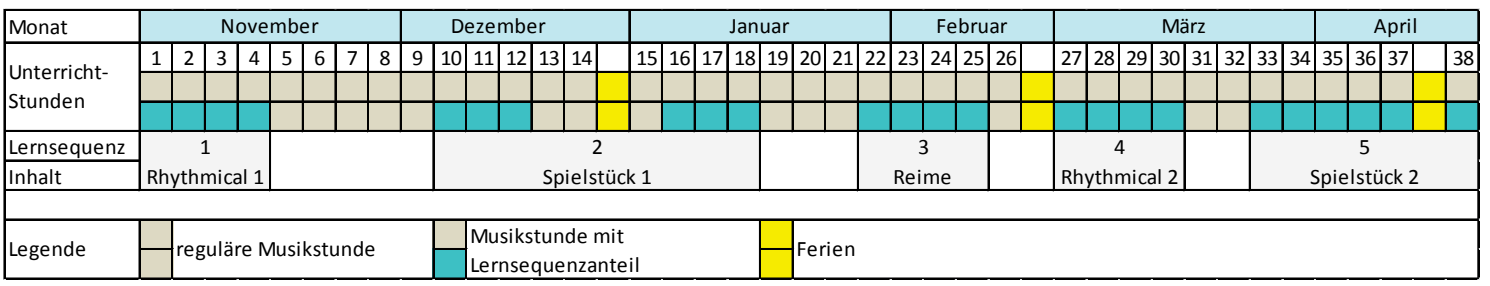

Abb. 3-3: Verteilung der Lernsequenzen auf den Untersuchungszeitraum

Über die Lehrgangsinhalte hinaus werden für die Klassen der Untersuchungsgruppe weitere Unterrichtsinhalte aus den Bereichen Singen, Bewegen und Tanzen sowie Musikhören festgelegt. Dies dient der besseren Vergleichbarkeit der Ergebnisse innerhalb der einzelnen Klassen der Untersuchungsgruppe sowie gegenüber der Kontrollgruppe.

\section{Baustein Warm up 1}

In jedem Warm up 1 wird die Erfahrung von Stufe A Erfahrung von Metrum und Puls ermöglicht (s. Abb. 3-4). Dazu dienen Übungen zum Erkennen und Darstellen von Macro- und Microbeats, wie sie bei Gordon empfohlen werden (Bluestine, 2000, S. 49-58; Gallus, 2003, S. 14-15; Gordon, 2012 ${ }^{8}$, S. 175-177). Zu einem erklingenden Hörwerk wird der Macrobeat mit den abgesenkten Fersen betont, welche auf der unbetonten Zählzeit zuvor vom Boden abgehoben werden müssen. Der 
Microbeat wird mit Patschen der Handinnenflächen seitlich auf die Oberschenkel dargestellt. Es werden jeweils zwei ca. 1-minütige Hörbeispiele aus Klassik und Pop angeboten. In zwei aufeinanderfolgenden Stunden werden dieselben Hörbeispiele verwendet. Es wird auf wechselnde Metrik, verschiedene Stilrichtungen sowie auf einen Bezug zum Jahreskreis geachtet (s. Anlage D 3).

Die Lehrkräfte werden aufgefordert, darauf Wert zu legen, die Imitationsbereitschaft von Grundschülern zu beachten. Dies kann, solange die Übungen noch neu sind, dazu genutzt werden, den Übungsablauf vertraut werden zu lassen. Später soll die Lehrkraft so lange wie möglich mit dem Mitbewegen warten, um die Schüler zum aktiven Hören und selbstständigen Finden der richtigen Körperbewegung anzuregen. Wichtig ist, diese Übung als ein Angebot zu betrachten, das vom Kind aus genutzt werden kann. Hinweise an einzelne Schüler sollen unterbleiben, lediglich mit Mimik und Gestik - vorrangig lobender Natur - kann gearbeitet werden. Es soll während der Übung nicht geredet werden.

\section{Baustein Warm up 2}

Das Warm up 2 beinhaltet die Patternarbeit. Mit diesem Baustein werden die Stufen B Rhythmen hören und imitieren und C Rhythmussprache erlernen umgesetzt. Die Schüler spielen die vorgeklatschten Rhythmen im Call-Response-Prinzip nach (s. Abb. 3-4). Wichtig ist nach Gordon der Impuls auf die Zählzeit 4 im Call-Takt. Nur dann ist es den Schülern möglich, pünktlich einzusetzen (Gordon, $2001^{4}$, S. 3). In Lernsequenz 1 sprechen Lehrkräfte und Schüler beim Klatschen die neutrale Silbe „bah“. Ab Lernsequenz 2 erlernen die Schüler eine Rhythmussprache. Einige Silben werden im Arbeitsheft schriftlich fixiert (s. Anlage D 4).

\begin{tabular}{|c|c|c|c|c|}
\hline $\begin{array}{l}\text { Stufen des Aufbau- } \\
\text { ens rhythmischer } \\
\text { Kompetenz }\end{array}$ & $\begin{array}{l}\text { Bausteine } \\
\text { Warm up } 1\end{array}$ & Warm up 2 & Workshop & Reflexion \\
\hline $\begin{array}{l}\text { F Rhythmen schreiben } \\
\text { \& E Rhythmen lesen }\end{array}$ & & \multirow{5}{*}{$\begin{array}{l}\text { Verbotener } \\
\text { Rhythmus } \\
\text { Call Response } \\
\text { Call Response } \\
\text { indirekt über } \\
\text { Call Response }\end{array}$} & \multirow{3}{*}{$\begin{array}{l}\text { nach E I S - } \\
\text { Prinzip } \\
\text { Erfinden von } \\
\text { Rhythmen für } \\
\text { Spielstücke } \\
\text { indirekt }\end{array}$} & \multirow{5}{*}{$\begin{array}{l}\text { reflektieren aus- } \\
\text { gewählter Aspekte, } \\
\text { je nach Inhalt des } \\
\text { Lernsequenz- } \\
\text { anteils }\end{array}$} \\
\hline $\begin{array}{l}\text { D Rhythmen variieren } \\
\text { und erfinden }\end{array}$ & & & & \\
\hline $\begin{array}{l}\text { C Rhythmussprache } \\
\text { erlernen }\end{array}$ & & & & \\
\hline $\begin{array}{l}\text { B Rhythmen hören } \\
\text { und imitieren }\end{array}$ & & & indirekt & \\
\hline $\begin{array}{l}\text { A Erfahrung von } \\
\text { Metrum und Puls }\end{array}$ & $\begin{array}{l}\text { Übung zu } \\
\text { Macro- und } \\
\text { Microbeat }\end{array}$ & & indirekt & \\
\hline
\end{tabular}

Abb. 3-4: Umsetzung der Stufen rhythmischer Kompetenz in den Bausteinen des Rhythmus-Lehrgangs

$\mathrm{Ab}$ Lernsequenz 3 wird das Spiel Verbotener Rhythmus eingeführt (Detterbeck \& SchmidtOberländer, 2011, S. 12). Dieser verbotene Rhythmus wird von einem ausgewählten Schüler an der 
Tafel angeheftet und von ihm mit der Klasse geübt. Wird anschließend im Call der Lehrkraft der verbotene Rhythmus vorgeklatscht, darf darauf keine Response gegeben werden. Anregende, der Popmusik ähnelnde Backgroundmusik unterstützt diese Übungsphase. Die Musik wurde für den Rhythmus-Lehrgang von der Autorin arrangiert und basiert auf dem Musiktitel It's good aus Gramß Boomwhackers-Spielheft (2007, S. 8). In jeder Lernsequenz wird ein anderer Style verwendet (s. Anlage D 4).

\section{Baustein Workshop}

Mit dem Baustein Workshop werden die Stufen D Rhythmen variieren und erfinden, E Rhythmen lesen sowie F Rhythmen schreiben umgesetzt (s. Abb. 3-4). Die Stufen B Rhythmen hören und imitieren und C Rhythmussprache erlernen werden indirekt gefördert: Stufe B insofern, da alle Workshop-Inhalt aus Rhythmusbausteinen (Pattern) bestehen. Stufe C dadurch, dass bei den Übungen der Spielstücke zur Koordination der Spielenden die Rhythmussprache mitgesprochen wird. Da sowohl die Rhythmicals, als auch die Spielstücke und Abzählreime stets zu Playbacks musiziert werden, findet auch eine Förderung der Stufe A Erfahrung von Metrum und Puls statt, zumindest für das gerade Metrum.

Inhalte dieses Bausteins bilden Rhythmicals, Spielstücke und das Musizieren von Abzählreimen. Zwei Rhythmicals bilden den Workshop-Inhalt der Lernsequenzen 1 und 4. Die Idee der Rhythmicals ist der Rhythmusarbeit von Filz entlehnt (Filz \& Heidecker, 2008, S. 52-55), welche in Unterrichtsmaterialien für die Primarstufe weite Verbreitung gefunden haben. Die verwendeten Rhythmicals wurden für das Treatment entworfen und mit einem Begleit-Playback versehen. Spielstücke werden in den Lernsequenzen 2 und 5 erfunden. Die zu spielenden Rhythmen werden von den Schülern unter Anleitung der Lehrkraft nach Vorgaben ausgewählt. Damit besteht die Möglichkeit, gelernte Patterns anzuwenden und zu festigen. Widerspiegelung im Lehrplan findet diese Form des Umgangs mit Rhythmusbausteinen ebenfalls erst in Klassenstufe 4. Die Spielstücke entstehen in jeder Klasse individuell (s. Anlage D 6). Von den Schülern werden sowohl die Patterns als auch die Umsetzung mit einem bestimmten Rhythmusinstrument festgelegt. Geübt wird in diesen Rhythmicals und Spielstücken vor allem das gleichzeitige und koordinierte Spielen der Gruppen. Dieser Umgang mit Rhythmusbausteinen findet im Lehrplan erst in Klasse 4 eine ausdrückliche Erwähnung (Sächsisches Staatsministerium für Kultus, 2009, S. 16).

Inhalt des Workshops in Lernsequenz 3 bilden Abzählreime (s. Anlage D 5). Sie entstammen der Alltagswelt der Kinder und sollen verdeutlichen, wie eng Sprache und Rhythmus verwoben sind. Als Begleit-Playback wird eine rap-ähnliche Hintergrundmusik verwendet.

Für alle Gruppen der Klasse soll ein Instrumentenkorb mit gleichem Inhalt bereitstehen. Eine Vorlage für die Spielstücke wird im Arbeitsheft vorgegeben (s. Anlage D 5). Musikalische Grundlage für Spielstück 1 und dessen arrangiertes Begleit-Playback bildet der Kanon in D-Dur von Pa- 
chelbel. Da die entsprechende Lernsequenz in die Weihnachtszeit fällt, bietet sich diese bekannte Melodie mit ihren wiederkehrenden Harmonien an. Das Begleit-Playback für das Spielstück 2 stellt eine kleine Improvisation über die Harmonien F-Dur, d-Moll, g-Moll und C-Dur dar.

\section{Baustein Reflexion}

In dieser Phase sollen die Schüler und Schülerinnen angeregt werden, über die Inhalte und die Lernwege bzw. über das gemeinsame Arbeiten in ihren Gruppen zu reflektieren (s. Abb. 3-4). Über Fragen wie z. B. „Was habe ich gelernt?“, „Wie habe ich gelernt?“ oder „Wie hat die Zusammenarbeit in der Gruppe geklappt?“ wird das Reflektieren über das eigene Handeln angeregt.

\subsubsection{Ansteigende Schwierigkeit der Patterns}

Das Erlernen und Spielen von Patterns / Rhythmusbausteinen hat im Rhythmus-Lehrgang höchste Priorität. Deshalb wird der Anstieg der Schwierigkeit und der Komplexität der Patterns bewusst geplant. Es werden drei Gruppen von Patterns unterschieden: Zu Gruppe 1 zählen Patterns, deren Hauptzählzeiten mit Viertel- und Achtelschlägen besetzt sind, jedoch keine Synkopen oder Pausen enthalten. Sie sind in allen Lernsequenzen enthalten. Gruppe 2 bilden Patterns, die bereits eine Viertelschlagpause am Patternende enthalten können. Sie sind vor allem in Lernsequenz 3 enthalten, da Abzählreime häufig mit einer Pause auf Schlag 4 enden. Gruppe 3 bilden Rhythmen, welche eine Viertelschlagpause in der Mitte des Patterns oder Synkopen enthalten. Sie werden erst in den Lernsequenzen 4 und 5 eingeführt bzw. verwendet (s. Abb. 3-5).

\begin{tabular}{|c|c|c|c|c|c|c|c|c|}
\hline \multicolumn{4}{|c|}{ Inhaltliche Progression der Pattern in den Lernsequenzen } & 1 & 2 & 3 & 4 & 5 \\
\hline \multicolumn{9}{|c|}{ Pattern aus Viertel- und Achtelnoten auf Hauptzählzeiten } \\
\hline$\frac{2}{4} \cdot \cdot \cdot \cdot \cdot 1$ & $\frac{2}{4} \cdot \cdot|\cdot \cdot|$ & ${ }_{2}^{2}+\cdots \cdot|\cdot|$ & $\frac{2}{4}+1.001 .0 .01$ & $\mathrm{x}$ & $\mathrm{x}$ & $\mathrm{x}$ & $\mathrm{x}$ & $x$ \\
\hline \multicolumn{9}{|c|}{ Weitere Pattern; Pattern mit Viertelschlagpause am Patternende } \\
\hline$\frac{2}{4}+0.01 \%$ & $\frac{2}{4} \cdot \cdots \cdot \cdot \cdot \cdot \mid$ & $\frac{2}{4}+8.0 \% \cdot \ln 1$ & $\frac{2}{4} \cdot \cdot|\cdot \geq|$ & & & $\mathrm{x}$ & $\mathrm{x}$ & $\mathrm{x}$ \\
\hline \multicolumn{9}{|c|}{ Pattern mit Viertelschlagpause am Patternanfang, mit Synkope sowie mit hlaber Note } \\
\hline$\frac{2}{4} \cdot,|, \cdot|$ & $\frac{2}{4} . D D D \mid d-1$ & $\left.\frac{2}{4} \supset J\right|_{0} \mid$ & & & & & $\mathrm{x}$ & $\mathrm{x}$ \\
\hline$\frac{2}{4} D D \cdot|d|$ & $\frac{2}{4} D D ! \cdot H$ & $\frac{2}{4}>\cdot 0 \cdot+!$ & $\frac{2}{4} \rho \mid \rho D \cdot$ & & & & & $\mathrm{x}$ \\
\hline
\end{tabular}

Abb. 3-5: Ansteigende Schwierigkeit der Patterns in den Lernsequenzen

\subsubsection{Erlernen einer Rhythmussprache}

Unabhängig von den Vorzügen und Nachteilen der einzelnen Rhythmussprachen (s. Abschnitt 2.2.3) ist vorwegnehmend festzuhalten, dass es zwischen dem Musikunterricht in der Grundschule und dem Instrumental- und Theorieunterricht in Musikschulen Überschneidungen gibt. Es muss damit gerechnet werden, dass einige Schüler der Klasse bereits eine Rhythmusspra- 
che an der städtischen Musikschule erlernen. Ebenfalls arbeiten Lehrer von Musikschulen über verschiedene Projekte (z. B. JeKI-Jedem Kind ein Instrument) zunehmend in der Grundschule. Sie haben sich bereits in ihrer pädagogischen Praxis auf eine Rhythmussprache festgelegt. Lehrende mit einer Ausbildung an ehemaligen Instituten für Lehrerbildung haben meist die Rhythmussprache nach Kodály mit einigen Varianten erlernt. Aber auch die Paris'sche Form wurde mitunter gelehrt. Da sowohl einige Schüler, aber auch die Lehrkräfte mitunter ihre Rhythmussprache mitbringen, wird die anfängliche Idee verworfen, wonach alle Schüler der Untersuchungsklassen die Rhythmussprache nach Gordon erlernen. Jede Lehrkraft entscheidet allein, welche Rhythmussprache zum Einsatz kommt.

\subsubsection{Ikonische Notation als Teil einer dreifachen Wissensdarbietung}

In Bezug auf die Stufen E Rhythmen lesen und $F$ Rhythmen schreiben war zu befürchten, sehr viele Schüler zu Beginn des Rhythmus-Lehrgangs zu überfordern. Der Umgang mit Rhythmusnotation bedeutet für die meisten Schüler Neuland. Abhilfe soll hier die Anwendung der Theorie Bruners (1974, S. 48-53) schaffen. Ausgehend von den enaktiven Repräsentationen, die bereits durch die Aktivitäten in den Stufen B und C gegeben sind, erfolgt die Notation von Rhythmen durch geeignete Zeichen (ikonische Repräsentation).

Solche Zeichen sind in der Musikpädagogik nicht neu und werden bei Losert als Denk- und Übungsmittel bezeichnet (2011, S. 130-147). Obwohl Losert unter dem diesem Schlagwort zahlreiche Formen für Notationen aufführt, wird keine dieser Formen ausgewählt. Obwohl anders beschrieben, erfüllen sie nicht den Anspruch, mit einfachen Schreibgeräten schlüssig notierbar zu sein (s. Abschnitt 2.2.5). Visuell ist diese Notationsform überzeugend und gut für die Reproduktion von Rhythmen geeignet. Das Malen eines gut sichtbaren Punktes verbraucht jedoch mehr Zeit als die reale Klangdauer. Für den Rhythmus-Lehrgang wird eine Notation entworfen, die sich leichter ausführen lässt. Sie arbeitet nur mit Strichen, senkrechten für Achtelschläge, und verschieden langen waagerechten Strichen für Viertelschläge bzw. für halbe Noten. Aufgrund des Bestehens aus nur einer Zeichenform wird sie als Strichnotation bezeichnet (s. Abb. 3-6).

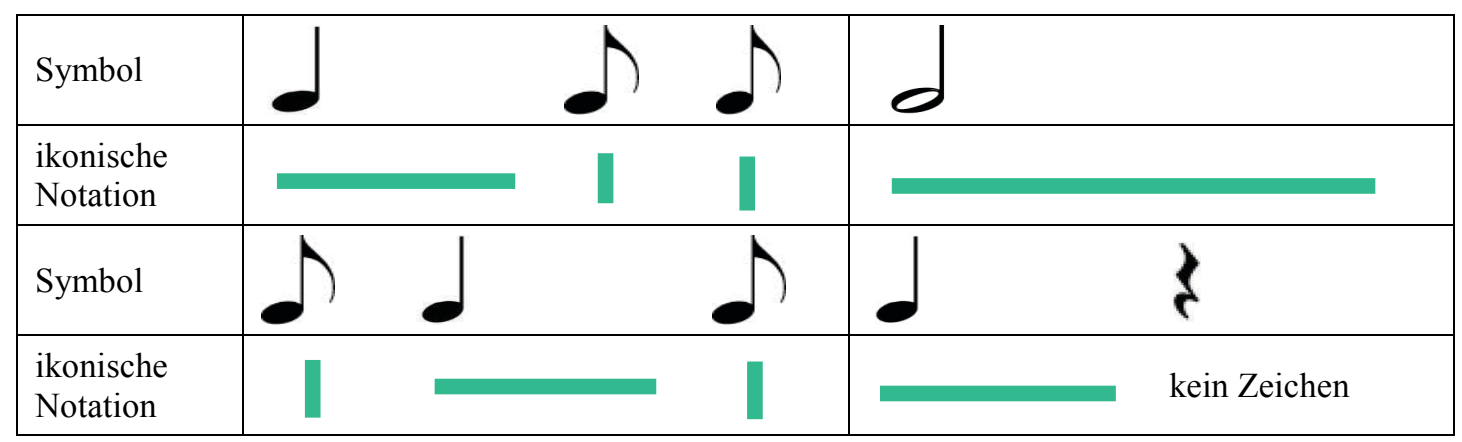

Abb. 3-6: Übertragung ausgewählter Symbole der Rhythmusnotation in die Strichnotation 


\subsubsection{Weitere Arbeitsmaterialien}

Um die Durchführung des Rhythmus-Lehrgangs zu vereinheitlichen, werden verschiedene Arbeitsmaterialien bereitgestellt:

- Schüler: Arbeitshefte

- Lehrer: A2-Kopien der Schüler-Arbeitsheft-Seiten zur Verwendung als Tafelbild (s. Abb. 3-7)

- Handreichung mit wichtigen Erläuterungen für jede Lernsequenz sowie dem konkreten Ablauf jedes Lernsequenzanteils sowie CDs mit Musiktiteln für alle Lernsequenzen

- Laminate für die Rhythmusnotation kombiniert mit einer adäquaten Balkenlänge sowie

- Pattern-Rahmen für die Übung Verbotener Rhythmus (beides s. Abb. 3-7).

Alle im Warm up 2 und im Workshop benötigten Playbacks werden durch die Versuchsleiterin an einem Yamaha CVP-503 arrangiert.

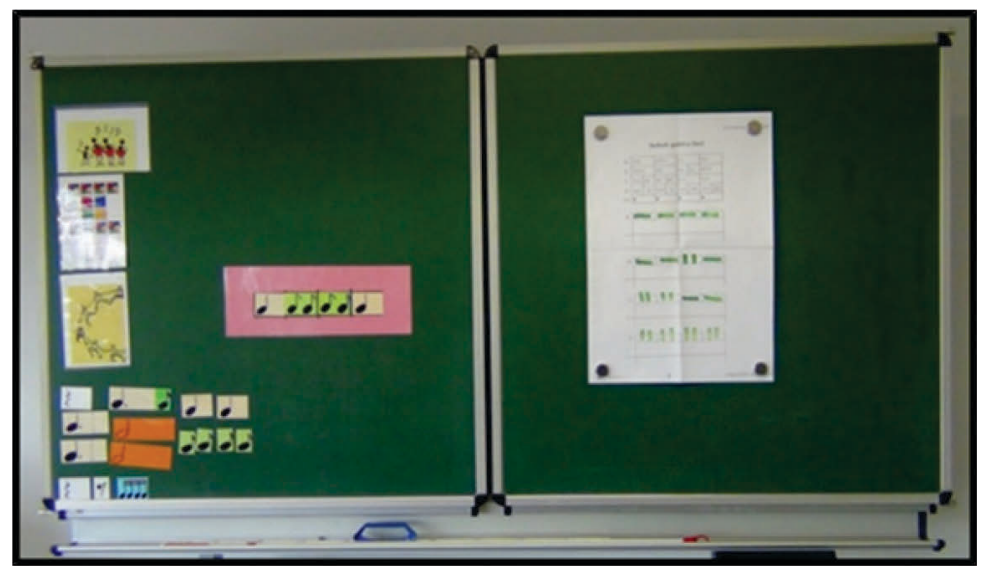

Abb. 3-7: Tafelbild mit Rhythmus-Rahmen, Notenlaminaten sowie A2-Kopie

\subsubsection{Differenzierung im Rhythmus-Lehrgang}

Der Vortest zeigt, dass bezogen auf diese Studie sowohl Schüler ohne nennenswerte Fähigkeiten und Kenntnisse in Rahmen rhythmischer Kompetenz mit Schülern zusammen lernen, die aufgrund ihrer Begabung oder zusätzlicher Förderung (außerunterrichtlicher Instrumentalunterricht o.ä.) schon als fortgeschritten gelten dürfen. Differenzierung ist deshalb ein Wesensmerkmal heutigen Unterrichts (vgl. Helmke, 2009, S. 245-246).

Gordons Theorie eines Lernprozess mit ansteigenden Levels besagt, erst bei Beherrschen des einen Levels, das Lernen auf dem nächsthöheren Level zu beginnen (Tappert-Süberkrüb, 1999, S. 75). In einem durch Leistungsheterogenität geprägten Klassenunterricht wäre dies nicht realisierbar, da der Klassenverband nicht aufgelöst werden kann. Alle Schüler einer Klasse müssten über Wochen hinweg gemeinsam auf der ersten Stufe verweilen. Den musikalisch weniger vorge- 
prägten Schülern würde dies ermöglichen, in Ruhe ein gutes Fundament im Hören und Empfinden von Metrum und Grundschlag aufzubauen. Schüler auf fortgeschrittenem Niveau würden zu einem Lernen ohne Aussicht auf die Weiterentwicklung der eigenen Fähigkeiten gezwungen sein. Dies würde die Motivation stark herabsetzen.

„Lernen kann nicht beliebig eingeleitet werden, sondern muß oberhalb des aktuellen Entwicklungsniveaus auf der Ebene ansetzen, auf der das Individuum eine musikalische Aufgabe mit Hilfe der sozialen Partner gerade bewältigen kann“ (Orter \& Bruhn, 1998, S. 332).

Um diesem Widerspruch gerecht zu werden, wird das Konzept der natürlichen Differenzierung für den Rhythmus-Lehrgang adaptiert (Krauthausen \& Scherer, 2010). In diesem Konzept erhalten alle Schüler das gleiche Lernangebot. Das Lernangebot im Konzept der natürlichen Differenzierung ist gekennzeichnet durch eine übergeordnete Problemfrage, eine angemessene Komplexität und Fragestellungen unterschiedlicher Schwierigkeitsgrade (ebd., S. 5-6). In diesem Konzept wird das zu bearbeitende Niveau nicht von der Lehrerin oder dem Lehrer bestimmt, sondern das Kind trifft diese Wahl.

Im Rhythmus-Lehrgang bedeutet dies, dass in (fast) jeder Lernsequenz die verschiedenen Stufen des Pyramidenmodells angeboten werden (s. Abb. 3-3). Dieses Lernangebot kann von jedem Schüler seinem Leistungsniveau entsprechend genutzt werden. Ein besonderes Augenmerk gilt dabei der Realisierung einer dreifachen Wissensdarbietung für Rhythmusnotation nach dem E I SPrinzip Bruners (s. Abschnitt 3.3.5).

\begin{tabular}{|l|c|c|c|c|c|}
\hline \multirow{2}{*}{$\begin{array}{l}\text { Stufen des Aufbauens rhythmischer } \\
\text { Kompetenz }\end{array}$} & \multicolumn{7}{|c|}{ Lernsequenz } \\
\cline { 2 - 7 } & $\mathbf{1}$ & $\mathbf{2}$ & $\mathbf{3}$ & $\mathbf{4}$ & $\mathbf{5}$ \\
\hline F Rhythmen musizieren und aufschreiben & $\mathrm{x} /-$ & $\mathrm{x} / \mathrm{x}$ & - & $\mathrm{x} / \mathrm{x}$ & $\mathrm{x} / \mathrm{x}$ \\
\hline E Rhythmen lesen und musizieren & $\mathrm{x} /-$ & $\mathrm{x} / \mathrm{x}$ & - & $\mathrm{x} / \mathrm{x}$ & $\mathrm{x} / \mathrm{x}$ \\
\hline D Rhythmen bewusst abwandeln & - & $\mathrm{x}$ & - & - & $\mathrm{x}$ \\
\hline C Rhythmussprache anwenden & $\mathrm{x}$ & $\mathrm{x}$ & $\mathrm{x}$ & $\mathrm{x}$ & $\mathrm{x}$ \\
\hline B Rhythmen nachspielen & $\mathrm{x}$ & $\mathrm{x}$ & $\mathrm{x}$ & $\mathrm{x}$ & $\mathrm{x}$ \\
\hline A Grundschlag und Metrum darstellen & $\mathrm{x}$ & $\mathrm{x}$ & $\mathrm{x}$ & $\mathrm{x}$ & $\mathrm{x}$ \\
\hline $\begin{array}{l}\mathrm{X}=\text { angeboten, - = nicht angeboten, } \mathrm{x} /-=\text { ikonisch, aber nicht symbolisch angeboten, } \\
\mathrm{x} / \mathrm{x}=\text { in beiden Formen angeboten }\end{array}$ &
\end{tabular}

Abb. 3-8: Umsetzung der Stufen rhythmischer Kompetenz in den fünf Lernsequenzen

Differenzierung in einem Musikunterricht, der von gemeinsamer Musizierpraxis gekennzeichnet sein soll, bedarf besonderer Wege. Während es in Fächern, in denen der Lernprozess durch zu Schreibendes in Gang gesetzt werden kann, möglich ist, differenzierte Aufgabenstellungen schriftlich und in Einzelarbeit zu lösen, ist dies im Rhythmus-Lehrgang aus zwei Gründen nicht gegeben: Zum einen kann rhythmische Kompetenz auf der unterscheidenden Ebene nur durch Unterweisung und Imitation erlernt werden. Zum anderen können bis zu 28 Schüler nicht verschiedene musikali- 
sche Tätigkeiten in einem Unterrichtsraum gleichzeitig umsetzen. Es ist nur möglich, Gleiches oder Ähnliches, jedoch tonal, rhythmisch bzw. harmonisch zueinander Passendes zu musizieren. An einer gemeinsamen musikalischen Aktion beteiligt zu sein und dennoch den eigenen Fähigkeiten entsprechend mitzuwirken, soll der Ansatz beim Differenzieren sein.

Deshalb wird in Kleingruppen gearbeitet. Die Rhythmicals und Spielstücke werden vierstimmig entworfen. In jeder vierköpfigen Stammgruppe können sich die Schüler je eine Stimme aussuchen. In der Erarbeitungsphase bietet es sich an, die jeweils gleichen Stimmgruppenschüler in sogenannten Expertengruppen zu bündeln. In der Festigungsphase kann jeder Schüler in seine Stammgruppe zurückgehen. Hier ist jeder verantwortlich für das Musizieren seiner Stimme. Diese Form der angeleiteten Gruppenarbeit in Stamm- und Expertengruppen (Bovet \& Huwendiek, 2008, S. 104) ist der Methode Gruppenpuzzle entlehnt. Eine hohe Aktivität jedes Schülers wird ermöglicht und gefordert. Die Stammgruppen-Mitglieder sollen auf diese Weise eine positive wechselseitige Abhängigkeit miteinander erfahren (Brüning \& Saum, 2007³, S. 144). Ebenso soll das Arbeiten in den auf Dauer angelegten Vierergruppen das selbstverantwortete gemeinsame Üben und Musizieren ermöglichen (Evelein, 2009, S. 6-8). Die Lehrkraft fungiert als Ensembleleiter.

Die für die Gruppenarbeit notwenige Bildung von Vierergruppen wird nach der Auswertung des Vortestes bewusst geplant und herbeigeführt. Da nicht alle Klassen eine durch vier teilbare Anzahl an Mitschülern aufweist, werden mitunter auch eine Fünfer- bzw. mehrere Dreiergruppen gebildet.

Jeder Schüler hat zusätzlich in der Stammgruppe eine besondere Aufgabe (Bochmann \& Kirchmann, 2006, S. 28): Die Schüler übernehmen eine Rolle als Chef, Streitschlichter, Schreiber oder Räumer. Diese Aufgabe wird am Anfang des Rhythmus-Lehrgangs festgelegt und über die 20 Unterrichtswochen hinweg beibehalten (s. Anlage D 4).

\subsubsection{Zusammenfassende Darstellung der Gestaltung des Treatments}

Die gewonnen Erkenntnisse aus den Aspekten des Musiklernens (s. Tab. 3-2) sowie aus der didaktischen Analyse des Lerngegenstandes (s. Tab. 3-3) werden für das Konzept des Treatments noch einmal zusammenfassend dargestellt.

Tab. 3-2: Umsetzung der Aspekte des Musiklernens im Rhythmus-Lehrgang

\begin{tabular}{|l|l|}
\hline Aspekt & Umsetzung im Konzept des Rhythmus-Lehrgangs \\
\hline $\begin{array}{l}\text { (1) Verbindung von } \\
\text { Handlung und Bewegung }\end{array}$ & $\begin{array}{l}\text { Die Bausteine Warm up 1, Warm up 2 und Workshop sind durch ganzkörper- } \\
\text { liche bzw. teilkörperliche Musizieraktionen gekennzeichnet. }\end{array}$ \\
\hline (2) imitatorisches Lernen & $\begin{array}{l}\text { Imitatorisches Lernen wird im Warm up 2 von der Lehrkraft, während des } \\
\text { Workshops in den Expertengruppen von fortgeschrittenen Schülern sowie bei } \\
\text { der Übung Verbotener Rhythmus von Mitschülern möglich. }\end{array}$ \\
\hline (2) eigenes Anwenden & $\begin{array}{l}\text { Solistische Aktionen in der eigenen Stammgruppe befördern das eigene An- } \\
\text { wenden. }\end{array}$ \\
\hline
\end{tabular}




\begin{tabular}{|l|l|}
\hline $\begin{array}{l}\text { (3) implizites Wissen vor } \\
\text { explizitem Wissen }\end{array}$ & $\begin{array}{l}\text { Durch Imitation wird zuerst ein körperbezogenes Wissen bzw. musikalisches } \\
\text { Handlungswissen zu Patterns / Rhythmusbausteinen aufgebaut. Dieses kör- } \\
\text { perbezogene Wissen kann nach dem Erlernen der Rhythmussprache und der } \\
\text { Strichnotation in ein explizites Wissen übergehen. }\end{array}$ \\
\hline $\begin{array}{l}\text { (4) gestufte, aufbauende } \\
\text { Lernschritte }\end{array}$ & $\begin{array}{l}\text { Gestufte Lernschritte werden durch einen gezielten Anstieg der Schwierigkeit } \\
\text { der Patterns / Rhythmusbausteine sowie durch eine insgesamt zunehmende } \\
\text { Komplexität der Inhalte in den fünf Lernsequenzen ermöglicht. }\end{array}$ \\
\hline $\begin{array}{l}\text { (5) kontinuierliches An- } \\
\text { wenden }\end{array}$ & $\begin{array}{l}\text { In den 24 Musikstunden mit einem Lernsequenzanteil wird nach einer festge- } \\
\text { legten Abfolge gleicher Bausteine kontinuierlich Können und Wissen ange- } \\
\text { wendet, gefestigt und erweitert. }\end{array}$ \\
\hline (6) Übung & $\begin{array}{l}\text { In jeder Lernsequenz wird das Lesen und Schreiben von Rhythmen geübt. } \\
\text { Durch Wechsel von Experten- und Stammgruppen wird auf ansteigendem } \\
\text { Niveau geübt. }\end{array}$ \\
\hline (7) Reflexion & $\begin{array}{l}\text { Über Fragen wie z. B. „Was habe ich gelernt?“ und „Wie habe ich gelernt?“ } \\
\text { wird das Reflektieren über das eigene musikalische Handeln angeregt. }\end{array}$ \\
\hline
\end{tabular}

Tab. 3-3: Umsetzung der didaktischen Analyse im Rhythmus-Lehrgang

\begin{tabular}{|c|c|}
\hline Analyse-Frage & Umsetzung im Konzept des Rhythmus-Lehrgangs \\
\hline Gegenwartsbedeutung & $\begin{array}{l}\text { - hohe Unterschiede in der Lernausgangslage durch Angebote zur } \\
\text { Differenzierung berücksichtigt } \\
\text { - konkrete Umsetzung durch Konzept der natürlichen Differenzierung }\end{array}$ \\
\hline Zukunftsbedeutung & $\begin{array}{l}\text { - Befähigung zum Lesen und Schreiben einfacher Rhythmen durch } \\
\text { dreifache Wissensdarbietung } \\
\text { - Befähigung zur Gruppenarbeit in Stamm- und Expertengruppen }\end{array}$ \\
\hline $\begin{array}{l}\text { Exemplarische } \\
\text { Bedeutung }\end{array}$ & - altersgerechte Inhalte wie Rhythmicals, Spielstücke und Abzählreime \\
\hline Struktur des Inhalts & - allmählich ansteigende Schwierigkeit der Patterns / Rhythmusbausteine \\
\hline $\begin{array}{l}\text { Erweisbarkeit und } \\
\text { Überprüfbarkeit }\end{array}$ & 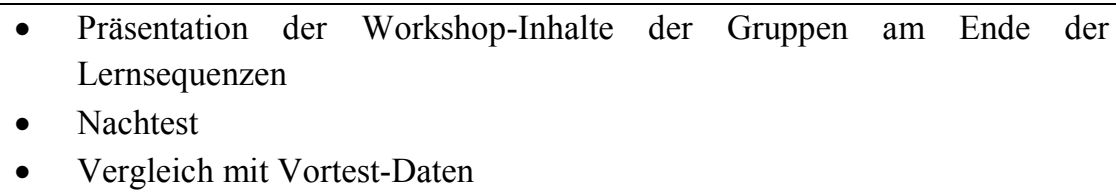 \\
\hline $\begin{array}{l}\text { Unterrichtliche } \\
\text { Zugänglichkeit }\end{array}$ & $\begin{array}{ll}\text { - } & \text { Rhythmussprache erlernen } \\
\text { - } & \text { Verwendung einer ikonischen Notation: Strichnotation } \\
\text { - } & \text { Spielstücke erfinden }\end{array}$ \\
\hline $\begin{array}{l}\text { Lehr-Lern- } \\
\text { Prozessstruktur }\end{array}$ & $\begin{array}{l}\text { - themenneutrales Treatment wird in den themenzentrierten regulären } \\
\text { Unterricht implementiert }\end{array}$ \\
\hline
\end{tabular}

\section{Erkenntnisse aus der Erprobungsphase}

Die Erprobungsfassung des Treatments fand im Schuljahr 2012/13 mit zwei 3. Klassen statt, die Musikunterricht bei der Autorin erhielten. Die wichtigsten Erfahrungen und deren Berücksichtigung für die Hauptuntersuchung werden folgend dargestellt. Dabei werden nur Details benannt, die aus Sicht der Autorin bzw. aus Gründen der Testergebnisse dieser Phase einer Änderung bedurften. 
Ursprünglich enthielt der Rhythmus-Lehrgang keine ausdrückliche Repräsentation der Stufe A Grundschlag und Metrum darstellen. Dies geschah aus Zeitgründen, da Sorge bestand, die anderen Lernbereiche zum Singen, Tanzen und Musikhören zu sehr zu verkürzen. Es wurde angenommen, dass diese Stufe sich auch sehr gut beim Bewegen und Tanzen fördern ließe. Die Testergebnisse der Erprobungsphase wiesen in diesem Subtest jedoch nur geringe Entwicklungssteigerungen aus. Deshalb erfolgte der Einbau einer Übung zum Darstellen von Grundschlag und Metrum, wie sie mit dem Warm up 1 realisiert wurde.

Die Call-Response-Übungen zur Förderung der Stufen B Rhythmen nachspielen und C Rhythmussprache anwenden wirkten auf Dauer sowohl auf die Schüler als auch auf die Autorin ermüdend. Es bedurfte eines spielerischen Ansatzes zum Erhalten der Motivation für diese Übung. Die Übung zum Verbotenen Rhythmus ab Lernsequenz 3 wurde deshalb eingebaut. In der Erprobungsphase wurde die Rhythmussprache nach Gordon verwendet. Die Schüler kamen nur bedingt mit dem unterschiedlichen Sprechen der Silben und dem davon abweichenden Schreiben der Notenwerte klar. Deshalb (und aus den in Abschnitt 3.3.4 dargelegten Gründen) wurde die Wahl der Rhythmussprache den involvierten Lehrkräften überlassen. Die Autorin wechselte im Folgejahr der Hauptuntersuchung auf die Rhythmussprache nach Paris.

Für die beiden Spielstücke in den Lernsequenzen 2 und 5 wurde den Gruppen bei der Suche nach geeigneten Rhythmen anfänglich viel Raum für Kreativität und selbstständiges Arbeiten eingeräumt. Diese Arbeitsweise benötigte eine lange Nachbereitungszeit, da jede Gruppe ihre Ideen vorstellen wollte. Diese Phase musste aus Gründen der begrenzten Gesamtlänge von maximal 25 Minuten pro Lernsequenzanteil gestrafft werden. Die Suche nach geeigneten Rhythmen sollte in der Hauptuntersuchung mit der gesamten Klasse stattfinden.

Analog zu Lehrwerken wurde zuerst als Form der ikonischen Notation mit Balken gearbeitet (Patho \& Schnabel, 2006, S. 23). Dies gelang den Schülern aufgrund der Malgeräte nicht überzeugend. Als Form der ikonischen Notation wurde deshalb die Strichnotation entwickelt und eingesetzt.

\subsection{Der Test}

Im Schuljahr 2012/13 wurde ein erstes Testmodell mit 37 Probanden aus zwei dritten Klassen mit insgesamt 52 Schülerinnen und Schülern erprobt. Etliche Items mussten aufgrund ihrer statistischen Eigenschaften (Itemschwierigkeit, Trennschärfe) verworfen bzw. überarbeitet werden. In der Erprobungsphase wurde der Test in einem sogenannten face-to-face-Verfahren durchgeführt. Dieses Verfahren ermöglichte einen direkten Kontakt zu jedem Kind mit seinen individuellen Besonderheiten. Durch den unmittelbaren Augenkontakt konnten sehr feine Signale der Kinder in Bezug auf das Verstehen der Aufgabenstellungen, Befürchtungen beim Lösen der Items sowie beim Einlassen auf Tempi und vieles mehr wahrgenommen werden. 
In der Hauptuntersuchung kommt dieser Test in einer Videofassung zum Einsatz. Dabei werden einige Erfahrungen aus der Erprobungsphase genutzt. Die wichtigsten methodischen Erkenntnisse liegen darin, Aufgabenstellungen nicht nur verbal zu erklären, sondern praktisch zu demonstrieren oder grafisch zu veranschaulichen. Dies ist notwendig, weil die Probanden noch nicht über einen Fachwortschatz verfügen. Begriffe wie Metrum und Grundschlag sind den meisten Probanden nicht vertraut. Die Demonstration verbal schwer zu transportierender Aufgabenstellungen übernimmt im Video ein Kind. Auch die Methode des Rhythmusnachspielens im Call-Response-Prinzip der Stufe B kann aus einem Zusammenspiel von Versuchsleiterin (Autorin) und dem Kind im Video für die Probanden nachvollziehbar demonstriert werden. Um die Durchführung der rhythmusbezogenen Subtests metrisch zu stabilisieren, stellt die Verwendung eines eigens dafür entwickelten Playbacks eine gute Unterstützung dar.

Es kommt somit ein Test mit einem Einzelverfahren (mündlicher Test) und einem Gruppenverfahren (schriftlicher Test) zum Einsatz. Der mündliche Test wird mit einem 15-minütigen Video (Stufen A, B und D) und einem sich anschließenden dreiminütigen Ergänzungsteil (Stufe E und C) mit jedem Probanden einzeln absolviert. Der Testablauf jedes Probanden wird zum Zwecke der späteren Auswertung digital als Audiospur aufgezeichnet. Der schriftliche Test wird mit einem Testbogen (Stufen F und C) über ca. 30 Minuten jeweils mit der gesamten Klasse realisiert.

\subsubsection{Operationalisierung der Stufen des Pyramidenmodells}

Für das Testmodell wird das sechsstufige Pyramidenmodell (Jank, 2013 ${ }^{5}$, S. 129) herangezogen (s. Abb. 2-6). Der Intention der Operationalisierung folgend werden die Bezeichnungen der Stufen nach Jank leicht abgewandelt und sprachlich angepasst (s. Tab. 3-4):

- Stufe A Körperkoordination, Erfahrung von Metrum und Puls wird umbenannt in A Grundschlag und Metrum darstellen, da hier nur die Testung genau dieser beiden Aspekte und dies auch nur teilkörperlich beim Musizieren infrage kommt.

- Stufe B Rhythmen hören und imitieren wird verkürzt auf B Rhythmen nachspielen, da das Hören als immanenter und notwendiger Vorgang vorausgesetzt werden darf.

- Stufe C Rhythmussprache erlernen wird aus testorientierter Sicht umbenannt in C Rhythmussprache anwenden.

- Stufe D Rhythmen variieren und erfinden wird verändert zu D Rhythmen bewusst abwandeln. Dies leitet sich aus Erfahrungen der Erprobungsphase ab, in der sich zeigte, dass ein Gelingen dieser Itemgruppe nur über das Verändern eines vorgegeben Rhythmus gelang. Damit trifft der Begriff Abwandeln eher zu als die Begriffe Variieren bzw. Erfinden.

- Die Stufe E Rhythmen lesen wurde testorientiert umbenannt in E Rhythmen lesen und musizieren. Damit wurde die enge Anknüpfung an den real erklingenden Rhythmus und die dazugehörige musizierpraktische Tätigkeit betont. 
- Die Stufe F Rhythmen schreiben wird aus den denselben Gründen wie Stufe E umbenannt in F Rhythmen musizieren und aufschreiben.

Jede der sechs Stufen wird in einem Subtest (mit je vier Items) bzw. einer Subskala (im Rahmen der statistischen Bewertung) gleichmäßig abgebildet (Bühner, 2006, S. 48). Ausführliche Erläuterungen finden sich in den nachfolgenden Unterkapiteln zu den einzelnen Subtests. Übersichten über die Dauern der Itemgruppen im mündlichen bzw. im schriftlichen Teil wurden in Anlage B 1 dargestellt.

Tab. 3-4: Operationalisierung der Stufen rhythmischer Kompetenz

\begin{tabular}{|c|c|c|}
\hline $\begin{array}{l}\text { Zu operationalisierende } \\
\text { Stufen }\end{array}$ & 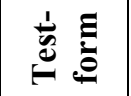 & Operationalisierung \\
\hline $\begin{array}{l}\text { F Rhythmen musizieren } \\
\text { und aufschreiben }\end{array}$ & 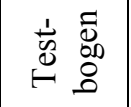 & $\begin{array}{l}\text { vier gemeinsam musizierte Rhythmen aufschreiben können, Tempo } \\
86 \text { bpm }\end{array}$ \\
\hline $\begin{array}{l}\text { E Rhythmen lesen und } \\
\text { musizieren }\end{array}$ & 过 & $\begin{array}{l}\text { vier notierte Rhythmen lesen und musizieren können; } \\
\text { freie Tempowahl }\end{array}$ \\
\hline $\begin{array}{l}\text { D Rhythmen bewusst } \\
\text { abwandeln }\end{array}$ & $\ddot{\Xi}: \frac{8}{\delta}$ & $\begin{array}{l}\text { auf einen Call einen davon verschiedenen Rhythmus spontan spie- } \\
\text { len und nach einem Takt Pause wiederholen können, zwei Auffor- } \\
\text { derungen; Tempo } 86 \text { bpm }\end{array}$ \\
\hline $\begin{array}{l}\text { C Rhythmussprache } \\
\text { anwenden }\end{array}$ & 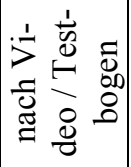 & $\begin{array}{l}\text { C1: zwei Rhythmen lesen, musizieren und Rhythmussilben dazu } \\
\text { sprechen können (zu Subtest E), freie Tempowahl; C2: zwei } \\
\text { Rhythmen musizieren und mit Rhythmussilben aufschreiben kön- } \\
\text { nen (zu Subtests F), Tempo } 86 \text { bpm }\end{array}$ \\
\hline B Rhythmen imitieren & 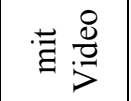 & $\begin{array}{l}\text { vier Rhythmen einem Call nachspielen können; } \\
\text { Tempo } 86 \text { bpm }\end{array}$ \\
\hline $\begin{array}{l}\text { A Grundschlag und Met- } \\
\text { rum darstellen }\end{array}$ & $\cdot \stackrel{\Xi}{\Xi}$ & $\begin{array}{l}\text { einmal Grundschlag und dreimal Metrum darstellen können (gera- } \\
\text { des und ungerades Metrum); Tempo 96, } 106 \text { bzw. } 132 \text { bpm }\end{array}$ \\
\hline
\end{tabular}

Im Test werden drei verschiedene Aufgabenformate verwendet: Die Items der Subtests A und B sind geschlossen, es gibt nur jeweils eine richtige Lösung. Die Items der Subtests $\mathrm{C}^{1}, \mathrm{C}^{2}$ sowie E und F sind halboffen. Es gibt Spielraum in den Lösungsformaten, da verschiedene Rhythmussprachen, verschiedene Bezüge zum Metrum beim Notieren bzw. individuelle Musiziertempi zulässig sind. Die Items des Subtests D sind als offenes Aufgabenformat gestaltet. Welche Rhythmen gespielt werden, ist dem Können und der Kreativität der Probanden freigestellt.

Neben den 24 Items, mit denen die sechs Stufen rhythmischer Kompetenz erfasst werden, sind weitere Items enthalten, welche die Probanden ermutigen oder ihnen das Hineinkommen in ein bestimmtes Aufgabenformat erleichtern sollen. Des Weiteren gibt es auch zusätzliche Itemgruppen, welche anderen Fragestellungen dienen (s. Abschnitt 3.4.2). Die 24 Items der Punktwertung werden fortlaufend mit Großbuchstaben gezählt, die sonstigen Items mit Kleinbuchstaben in kursivem Druck. 


\section{Subtest $A$ Metrum und Grundschlag darstellen}

Mit diesem Subtest wird der Videotest eröffnet. Zuerst sollen die Probanden ein Start-Item lösen. Dieses soll in den Subtest einstimmten und eventuelle Blockaden lösen. Dieses Item a 5 geht nicht in die Punktwertung ein. Inhalt dieses Items ist das Klatschen des Rhythmus des Liedes Bruder Jacob.

Die Wertung beginnt erst mit dem Spielen des Grundschlags auf Klanghölzern zum selben Lied (Item A 1). Anschließend wird das Lied noch ein drittes Mal genutzt, um auf einem Triangel im geraden Metrum jeweils den ersten und dritten Grundschlag im Takt zu betonen (Item A 2). Als nächstes musizieren die Probanden auf dem Triangel das Metrum zum Menuett G-Dur von J. S. Bach jeweils auf dem ersten Grundschlag im Takt (Item A 3). Abschließend musizieren die Probanden auf dem Triangel das Metrum zu einem Popsong im 4/4-Takt jeweils auf dem ersten Grundschlag im Takt (Item A 4; s. Tab. 3-5).

Das Tempo für Item a 5, A 1 und A 2 liegt bei 106, für Item A 3 bei 96 und für Item A 4 bei 132 Schlägen pro Minute. Die Hörbeispiele für Item a 5 bis A 3 wurden von der Autorin erstellt, deshalb konnte und wurde nach der Erprobungsphase leicht Einfluss auf das Tempo genommen. Alle Tempi liegen in einem für die Wahrnehmung eines Grundpulses bevorzugten Tempobereich (vgl. Auhagen, $2011^{3}$, S. 441).

Im Video demonstriert ein Kind für die Items a 5, A 1 und A 2 das Klatschen des Rhythmus, das Spielen des Grundschlages sowie das Musizieren des Metrums zu diesem Lied (jeweils am Beispiel der Takte 5 und 6, Notenbild s. Abb. 4-19). Die Idee des Demonstrierens beruht auf der in der Erprobungsphase gewonnenen Erkenntnis, dass die in diesem Subtest notwendigen Begriffe wie Rhythmus, Grundschlag und Metrum nicht zum Wissenstand der meisten Probanden dieser Altersgruppe gehören und nicht ad hoc erklärt werden können.

Tab. 3-5: Angaben zu Items im ST A

\begin{tabular}{c|c|c|l|c}
\hline Item & Werk & $\begin{array}{c}\text { Tempo } \\
\text { (bpm) }\end{array}$ & Aufgabe & Wertung \\
\hline \multirow{2nnnn}{*}{ a 5 } & \multirow{2}{*}{ L 1 } & 106 & Rhythmus klatschen \\
\cline { 1 - 1 } Jacob“ & 106 & Gruder & ohne \\
\cline { 1 - 3 } A 2 & & 106 & $\begin{array}{l}\text { gerades Metrum im 4/4-Takt auf Triangel } \\
\text { spielen (Betonung der Zählzeiten 1 und 3) }\end{array}$ & 1 \\
\hline A 3 & $\begin{array}{c}\text { J.S. Bach: „Me- } \\
\text { nuett G-Dur“ }\end{array}$ & 96 & $\begin{array}{l}\text { ungerades Metrum im 3/4-Takt auf Trian- } \\
\text { gel spielen }\end{array}$ & 1 \\
\hline A 4 & $\begin{array}{c}\text { ATC: „Around } \\
\text { The World“ }\end{array}$ & 132 & $\begin{array}{l}\text { gerades Metrum im 4/4-Takt auf Triangel } \\
\text { spielen (Betonung der Zählzeit 1) }\end{array}$ & 1 \\
\hline
\end{tabular}

Nach der Demonstration schließt sich das Spiel des Probanden an, welches in zwei Teilen verläuft. Im ersten Teil (8 Takte) unterstützt die Versuchsleiterin im Video das Spiel der Probanden durch 
pantomimische Bewegungen. Diese pantomimischen Bewegungen ermöglichen den Probanden das Mitspielen auf der Basis von Imitation und sollen die Musizieraufgabe intuitiv erfahrbar werden lassen. Dabei wird die Fähigkeit zum Imitieren genutzt, wie sie bei Fuchs (2010) und Spychiger (2015) näher beschrieben werden (s. Abschnitt 2.2.6). In Takt 8 erfolgt die Aufforderung, nun selbstständig zu musizieren (Takt 9-16). Das Spielen des Metrums in Item A 3 und 4 wird grafisch veranschaulicht.

Alle Musikstücke für Subtest A bestehen aus zwei nahezu gleichen Teilen von jeweils 8 Takten. Der erste Teil der Items (Takt 1 bis 8 ) wird nicht gewertet, sondern nur das selbstständige Spielen im jeweils zweiten Teil (Takt 9 bis 16). Hierbei darf nur in einem Takt ein Fehler auftreten. Abweichungen im Schlag des Pulses bzw. Metrums im Rahmen einer Sechzehntelnote (+/ -) zum Grundschlag sind zugelassen (Fehlertoleranzen s. Abschnitt 3.5.2).

\section{Subtest B Rhythmen nachspielen}

In diesem Subtest sollen die Probanden vorgespielte Rhythmen korrekt nachspielen. Dazu wird das Call-Response-Prinzip eingesetzt. Schon in der Demonstration dieser Aufgabe im Video sehen die Probanden, dass die Testleiterin mit dem Kind jeden Rhythmus zweimal spielt. Fünf verschiedene Rhythmen über vier Grundschläge werden jeweils zweimal vor- und nachgespielt. Ein doppelt so langer Takt über 8 Grundschläge schließt als zusätzliches Item diese Itemgruppe ab. Dieser Takt wird nicht wiederholt angeboten. Im Hintergrund wird ein Playback im Tempo 86 bpm verwendet.

Tab. 3-6: Angaben zu Items im ST B

\begin{tabular}{|c|c|c|c|c|}
\hline Item & $\begin{array}{l}\text { Tempo } \\
\text { (bpm) }\end{array}$ & Rhyth & & Wertung \\
\hline b 5 & \multirow{6}{*}{86} & $5 a$ & $\frac{2}{4} \cdot \sigma^{\prime}|\cdot| \cdot \mid$ & ohne \\
\hline B 1 & & 1a & $2 \cdot \downarrow|\cdot \cdot \cdot|$ & 1 \\
\hline B 2 & & $2 \mathrm{a}$ & $\frac{2}{4} \cdot D|D \cdot ?|$ & 1 \\
\hline B 3 & & $3 a$ & $\frac{2}{4} \cdot \cdots \cdot \cdot \cdot \mid$ & 1 \\
\hline B 4 & & $4 a$ & $\frac{2}{4} \partial \cdot \partial \cdot \cdot \mid$ & 1 \\
\hline b 6 & & $1 a+3 a$ & $\mid \cdots \cdot$ & ohne \\
\hline
\end{tabular}

Der erste Rhythmus (b 5) stellt wieder ein sogenanntes Start-Item dar (s. Tab. 3-6), das in der Punktwertung nicht zählt. Es verschafft den Probanden die Möglichkeit, sich auf die CallResponse-Übung einzustellen. Die Wertungs-Items werden in ihrem erwarteten Schwierigkeitsniveau in der Folge B 1 leicht, B 2 schwer, B 3 leicht, B 4 schwer gereiht. Dies soll einen Wechsel von Anspannung und Entspannung ermöglichen. Item B 1 enthält einen sogenannten Stadionrhythmus (Rhythmus 1a). Item B 3 ist mit Rhythmus 3a ohne jede nennenswerte Schwierigkeit 
aus einfachen Vierteln und Achtelpaaren gebildet. Die Rhythmen B 2 und B 4 sind von erhöhter Schwierigkeit. Item B 2 enthält eine punktierte Viertelnote und eine Pause auf Zählzeit 4. Item B 4 enthält eine Synkope. Während die Synkope in Liedern und Werken der Grundschule häufig anzutreffen ist, stellt Item B 2 eine große Herausforderung für die Probanden dar.

Als richtig wird ein Rhythmus gewertet, wenn er in der zweiten Response die erlaubten Fehlerabweichungen einhält (Fehlertoleranzen s. Abschnitt 3.5.2). Den vier Wertungsitems (B 1 bis B 4) folgt eine Rhythmuskombination aus den Rhythmen 1a und 3a (Item b 6). Sie dient dem Vergleich zu Daten aus der Studie von Minkenberg, dort als K1 bezeichnet (Minkenberg, 1991, S. 72).

\section{Subtest C Rhythmussprache anwenden}

Dieser Subtest entstand erst nach der Erprobungsphase. Grund dafür waren Bedenken zur Benachteiligung der Kontrollgruppe, die laut Lehrplan keine Rhythmussprache erlernen würde. Diese Stufe nicht zu repräsentieren, hätte aber bedeutet, das Theoriemodell (Gordon, 2012 ${ }^{8}$, Jank, 20135 ) nicht vollständig zu repräsentieren. Die im Nachhinein entwickelten Items werden an Subtest E und Subtest $\mathrm{F}$ angelagert.

Tab. 3-7: Angaben zu Items im ST C

\begin{tabular}{|c|c|c|c|}
\hline Item & $\begin{array}{c}\text { Tempo } \\
\text { (bpm) }\end{array}$ & Rhythmen & Wertung \\
\hline $\mathrm{C}^{1} 1$ & - & 1a $\quad \frac{2}{4} \cdot \cdot|\cdot \downarrow|$ & 1 \\
\hline $\mathrm{C}^{1} 2$ & - & 6a $\quad \frac{2}{4} \cdot \sqcap \cdot \sqcap \cdot$ & 1 \\
\hline $\mathrm{C}^{2} 3$ & \multirow{2}{*}{86} & 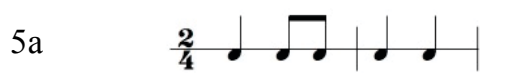 & 1 \\
\hline $\mathrm{C}^{2} 4$ & & 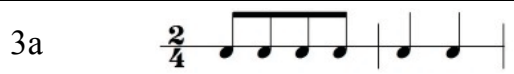 & 1 \\
\hline
\end{tabular}

Getestet werden zwei verschiedene Umgangsweisen mit einer Rhythmussprache: Zum einen, in welchem Maße Rhythmussprache unterstützend zum Lesen und Musizieren von Notenschrift genutzt werden kann (Losert, 2011, S. 159; Itemgruppe $C^{1}$ ). Zum anderen interessiert die Fähigkeit, musizierte Rhythmen in Rhythmussprache umwandeln und aufschreiben zu können (Gordon, $2012^{8}$, S. 85; Itemgruppe $C^{2}$ ).

Durch die Anbindung an die Subtests E und F kann über 8 Items verfügt werden. Mithilfe einer Itemanalyse (s. Anlage C 3) werden jeweils zwei Items ausgewählt. Sie sollen keinen Rhythmus doppelt repräsentieren, der geplanten Itemschwierigkeit entsprechen (s. Tab. 3-14) und die besten Werte in Bezug auf die Trennschärfe aufweisen (s. S. 64 sowie Abschnitt 4.3.6). Die Auswahl fällt auf die Items ec 2 und ec 3 sowie fc 1 und fc 3 (s. Tab. 3-7). Sie werden umbenannt in $C^{1} 1, C^{1} 2$, $\mathrm{C}^{2} 3$ und $\mathrm{C}^{2} 4$. 


\section{Subtest D Rhythmen bewusst abwandeln}

Für diesen Subtest wird eine Spielform gewählt, die am ehesten mit dem Begriff Call-Answer zu bezeichnen ist. Im Video wird ein Call gespielt (bestehend aus vier Viertelschlägen), der sofort im nächsten Takt beantwortet werden soll. Zwei Bedingungen werden gestellt: Die Antwort darf nicht dem Call gleichen. Außerdem muss nach einem Takt Pause, in welchem die Versuchsleiterin leise von 1 bis 4 zählt, die Antwort wiederholt werden können. Diese Aufgabenstellung soll zweimal gelöst werden. Die zweite Antwort darf der ersten nicht gleichen. Das Playback (86 bpm) wird wieder verwendet. Die Aufgabenstellung wird im Video grafisch veranschaulicht (s. Abb. 3-9). Gewertet werden die beiden Antwort-Rhythmen sowie die jeweiligen Wiederholungen (s. Tab. 3-8). Die gespielten Rhythmen werden aufgezeichnet und nach den Kriterien von Subtest B ausgewertet (s. Abschnitt 3.5.2).

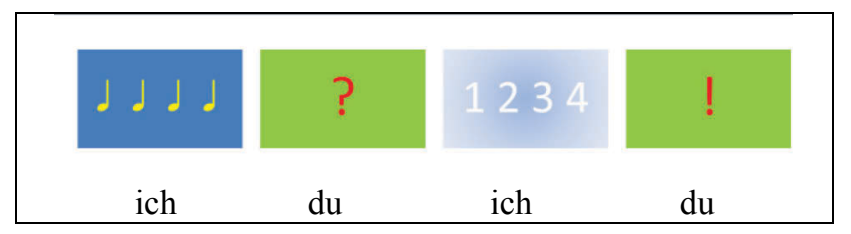

Abb. 3-9: Grafische Veranschaulichung der Aufgabenstellung im ST D

Tab. 3-8: Angaben zu Items im ST D

\begin{tabular}{|c|c|c|c|}
\hline Item & $\begin{array}{c}\text { Tempo } \\
\text { (bpm) }\end{array}$ & Aufgabe & Wertung \\
\hline D 1 & \multirow{4}{*}{86} & Antwort 1 geben & 1 \\
\hline D 2 & & Antwort 1 wiederholen & 1 \\
\hline D 3 & & Antwort 2 geben & 1 \\
\hline D 4 & & Antwort 2 wiederholen & 1 \\
\hline
\end{tabular}

\section{Subtest E Rhythmen lesen und musizieren}

Dieser Subtest folgt unmittelbar auf den Videotest mit den Subtests A, B und D. Die Probanden sollen nach verschiedenen Notationsformen musizieren. Insgesamt besteht dieser Subtest aus den drei Untertests e Rhythmen lesen und musizieren nach Zeichen, E Rhythmen lesen und musizieren nach Noten sowie ec Rhythmen lesen und musizieren nach Noten und sprechen von Rhythmussilben. Die musizierten Rhythmen werden aufgezeichnet und nach den Kriterien von Subtest B ausgewertet (s. Abschnitt 3.5.2).

Im Untertest e Rhythmen lesen und musizieren nach Zeichen können die Probanden zwischen Strich- oder Balkendarstellung (s. Tab. 3-9) wählen, im Subtest E Rhythmen lesen und musizieren nach Noten zwischen gedruckten bzw. handgeschriebenen Noten. Im Untertest ec Rhythmen lesen und musizieren nach Noten und sprechen von Rhythmussilben spielen sie nach derselben Notenvor- 
lage wie bereits im Subtest E. Vor den einzelnen Untertests werden in einem kurzen Gespräch jeweils die Musiziervorlagen mit Zeichen sowie mit Noten zur Auswahl gestellt. Es wird besprochen, was die Zeichen bedeuten könnten bzw. welche Unterschiede die Notationsformen aufweisen (Fähnchen / Achtelbalken).

Den ersten Untertest $e$ Rhythmen lesen und musizieren nach Zeichen absolvieren alle Probanden, es erfolgt keine Punktwertung. Der Subtest E Rhythmen lesen und musizieren nach Noten darf abgewählt werden, wenn der Proband nicht über Notenkenntnisse verfügt. Hier wird jeder richtig gespielte Rhythmus gewertet. Der Untertest ec Rhythmen lesen und musizieren nach Noten und sprechen von Rhythmussilben kann nur erfolgen, wenn Subtest E absolviert wird und eine Rhythmussprache bekannt ist. Zwei dieser Items aus dem Untertest ec werden im Nachhinein durch eine Itemanalyse für Subtest $\mathrm{C}$ gewertet.

Tab. 3-9: Angaben zu Items im ST E

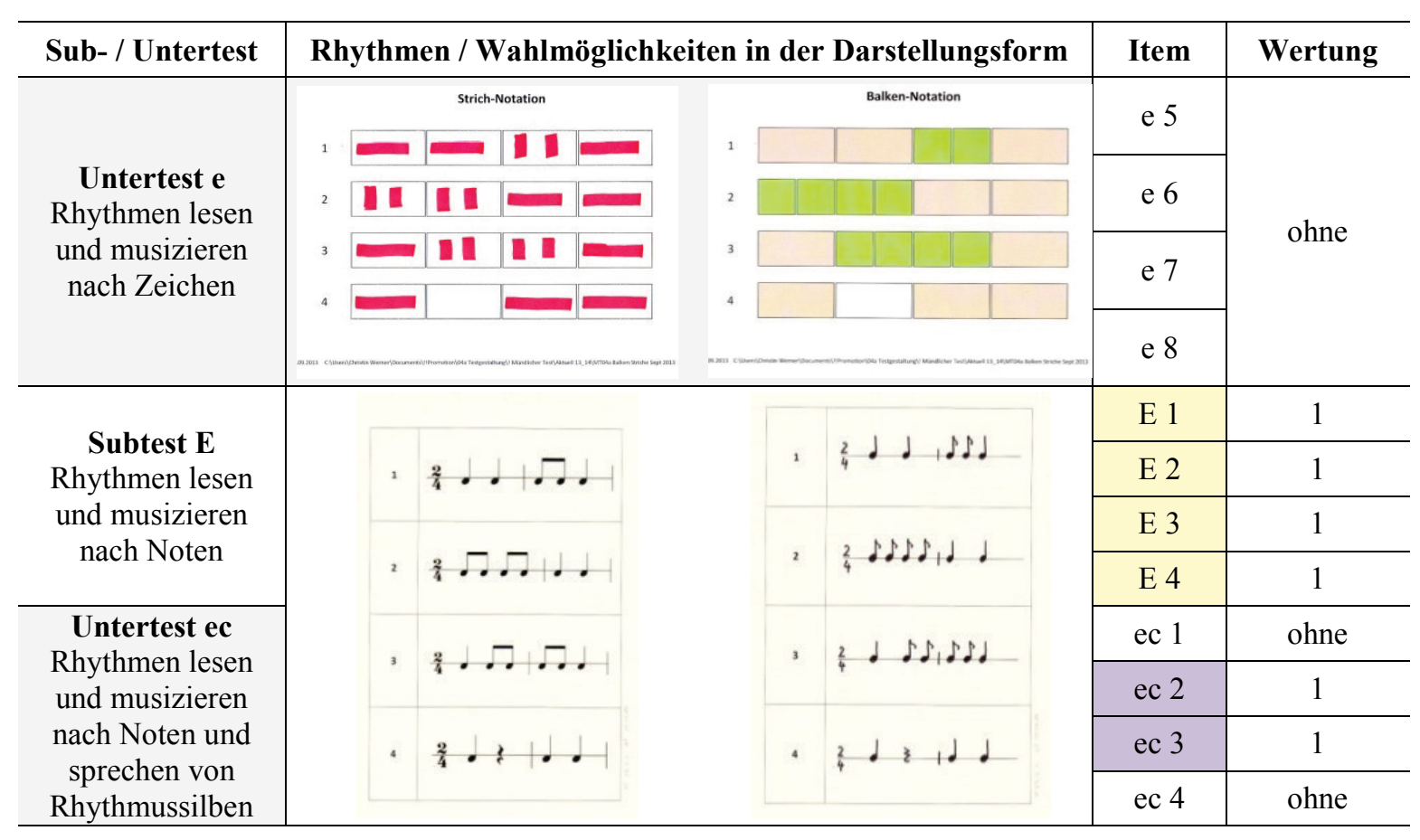

\section{Subtest F Rhythmen musizieren und aufschreiben}

Dieser Subtest wird mit der ganzen Schulklasse als schriftlicher Test realisiert. Dabei wird zu Beginn in einem Gespräch geklärt, was ein Rhythmus ist. Dies mündet in das Vorklatschen von Rhythmusbeispielen durch einzelne Schüler der Klasse. Anschließend wird gefragt, wie ein Rhythmus mit eigenen Zeichen notiert werden könnte. Die Schüler benennen verschiedene Möglichkeiten (ohne Veranschaulichung an der Tafel). Danach wird der Rhythmus 6a (s. Anlage E) gemeinsam zum Playback nach der Methode des Call Response gespielt. Anschließend wird am Tafelbild erläutert, wie der Testbogen (s. Anlage B 4) auszufüllen ist, welche Teile Pflicht (hell) 
und welche Teile Kür (dunkel) sind. Die Schülerinnen und Schüler verwenden für diesen Teil des Testes Füller, Bleistift und einen bereitgestellten roten Textmarker. Höraufgabe und Playback werden von einer CD abgespielt. Als Sichtschutz werden ca. 30 Zentimeter hohe Pappwände verwendet. Alle Ergebnisse werden auf dem Testbogen festgehalten.

Von allen Schülern und Schülerinnen wird im Untertest $f$ eine Notation mit selbst erfundenen Zeichen erbeten (s. Abb. 3-10). Die Notation mit Silben einer Rhythmussprache im Untertest fc bzw. mit traditionellen Noten im Subtest Fist fakultativ. Gewertet werden alle richtig notierten Rhythmen mit Noten (s. Tab. 3-10). Abweichungen in Bezug auf das gewählte Metrum sind zulässig.
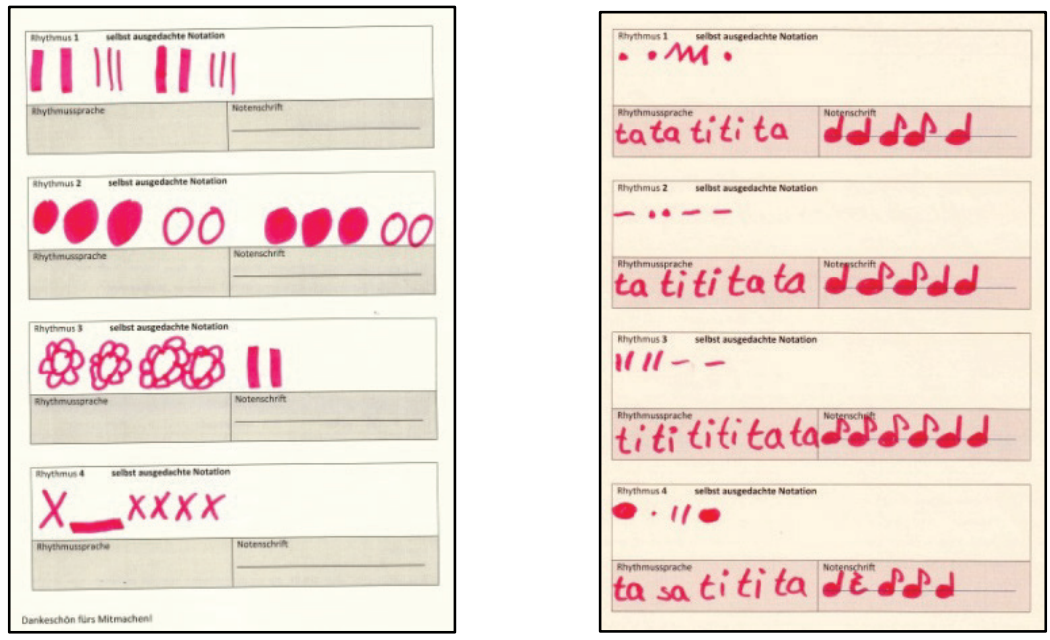

Abb. 3-10: Kopie der Rückseite zweier Testbogen für ST F, VT und NT

Tab. 3-10: Angaben zu Items im ST F

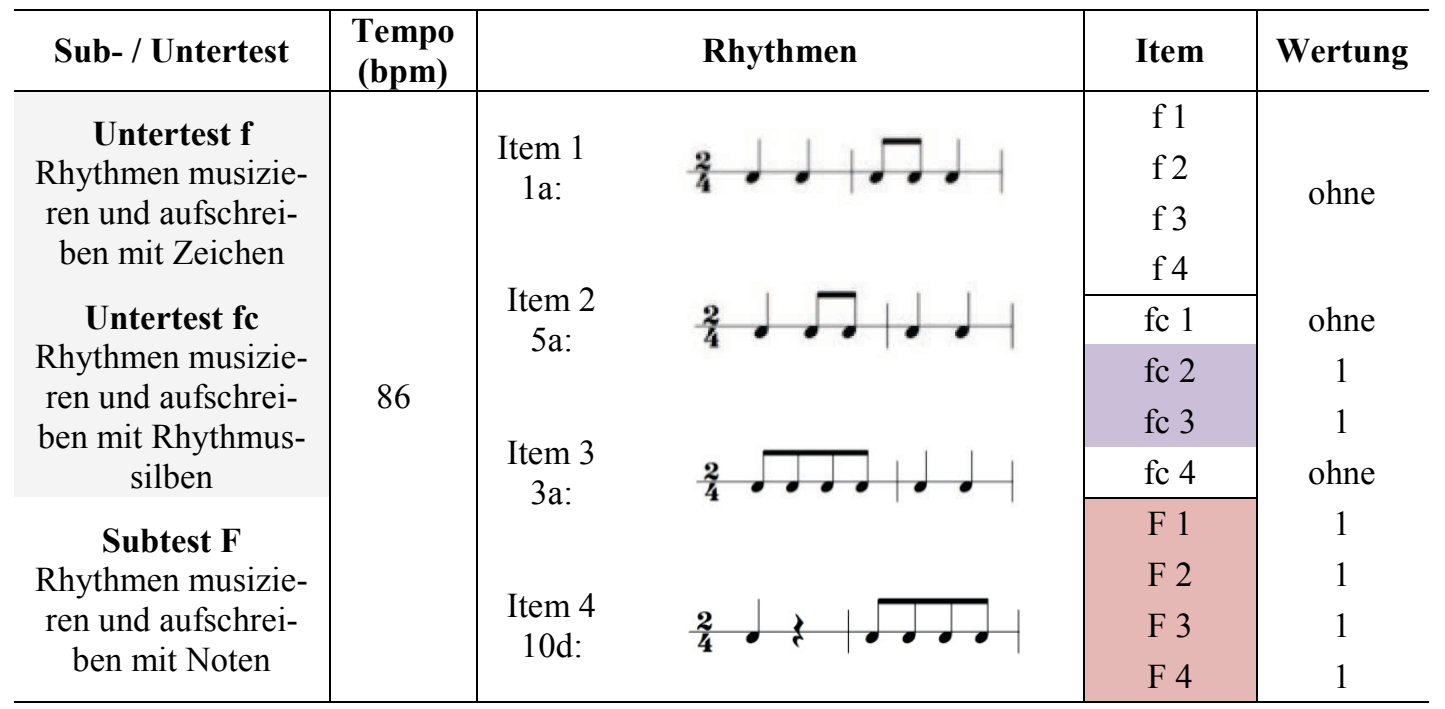




\subsubsection{Zusätzliche Items}

In den Subtests B, E und F werden bereits zusätzliche Items (b 6; e 5 bis e 8 sowie f 5 bis f 8) erwähnt. Sie dienen verschiedenen Zwecken. So soll zum Beispiel Item b 6 einen Vergleich zu vorhandenen Daten aus der Studie von Minkenberg (1991) liefern. Die Items e 5 bis e 8 sowie f 5 bis f 8, deren Inhalt der Umgang mit ikonischer Notation ist, dienen einer Rückmeldung zum Umgang mit ikonischer Notation mit und ohne Förderung durch das Treatment.

Um die Fragen zur Validität zu beantworten, wird der Subtest G zusätzlich in das Video eingefügt. Mit Subtest H wird die Sicht von Schülerinnen und Schülern auf die Bedeutung von Notenschrift in ihrem eigenen Musikvollzug erfragt. Subtest I stellt eine Ergänzung in Form von rezeptionsorientierten Aufgaben zu den musizierpraktischen Items dar.

\section{Subtest G Play together}

Zusätzlich gibt es die Itemgruppe G Play together. Sie ist am Ende des Videos eingebettet. Darin wird der Proband zum Musizieren im zweistimmigen Rhythmusspiel aufgefordert. Dies entspricht einer häufig anzutreffenden Situation im Musikunterricht. Die Erklärung der Aufgabenstellung wird mit grafischer Anschauung erleichtert (s. Abb. 3-11). Der Proband soll in Item 1 und 2 den Rhythmus 1a acht Takte lang allein spielen (gelbe Taktspur). Im fünften Takt setzt das Kind im Video mit Rhythmus 5a ein (blaue Taktspur, s. auch Abb. 3-12). Mit dem Probanden ist dessen Rhythmus zuvor über eine Videoanweisung geübt worden. In Item G 3 und G 4 beginnt das Kind im Video seinen Rhythmus 5a zu spielen (blaue Taktspur). Der Proband soll erst in Takt 5 mit Rhythmus 1a einsetzen. Dabei hilft die Versuchsleiterin (im Video) beim pünktlichen Einsatz und zählt im Takt zuvor deutlich bis 4 (rote Ziffern). Beide Itemgruppen werden mit dem bereits erwähnten Playback gestützt. Vor Item G 3 und G 4 wird der Rhythmus mit dem Probanden nochmals geübt. Diese Items gehen nicht in die Punktwertung ein (s. Tab. 3-11).

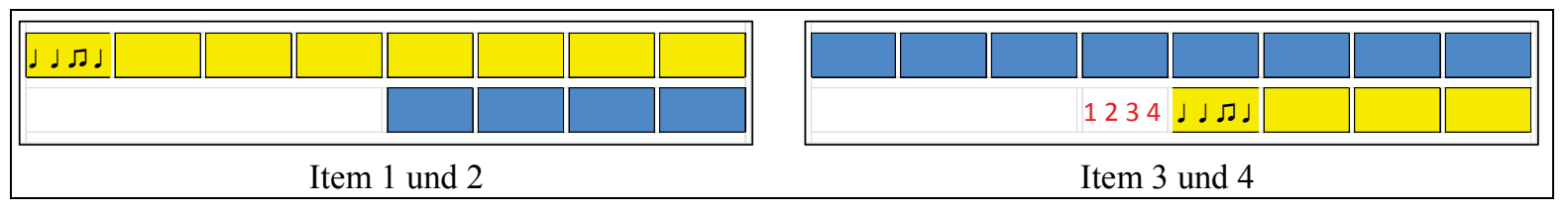

Abb. 3-11: Grafische Darstellung der Itemgruppe G

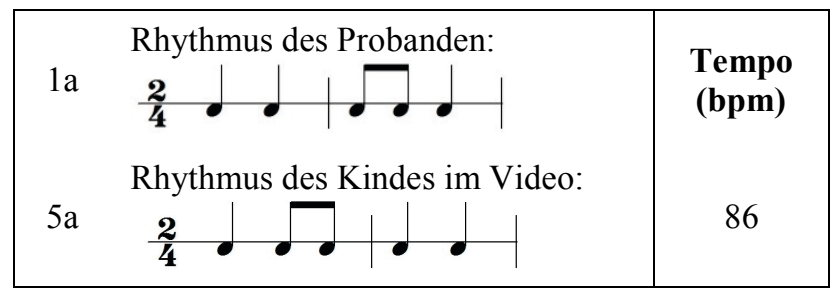

Abb. 3-12: Rhythmen im ST G 
Tab. 3-11: Angaben zu Items im ST G

\begin{tabular}{c|c|c}
\hline Item & Aufgabe & Wertung \\
\hline G 1 & $\begin{array}{c}\text { Rhythmus allein vier Takte lang korrekt spielen } \\
\text { G 2 }\end{array}$ & $\begin{array}{c}\text { Rhythmus im Duett vier Takte lang korrekt } \\
\text { weiterspielen }\end{array}$ \\
\cline { 1 - 2 } G 3 & pünktlich im Takt 5 einsetzen & \\
\cline { 1 - 2 } G 4 & $\begin{array}{c}\text { Rhythmus im Duett vier Takte lang korrekt } \\
\text { spielen }\end{array}$ & \\
\hline
\end{tabular}

\section{Subtest H Wozu kann Notenschrift wichtig sein?}

Im schriftlichen Test erhalten die Probanden neben der Notationsaufgabe im Subtests F eine weitere Aufgabe. Sie sollen die Frage beantworten: „Wozu kann die Notenschrift für einen Grundschüler, z. B. dich, wichtig sein?“ (s. Abb. 3-13; Testbogenausschnitt einer Probandin mit Klavierunterricht, 3. Unterrichtsjahr). Erwartet wird eine Antwort mit Musikbezug sowie mit einem Ich-Bezug.

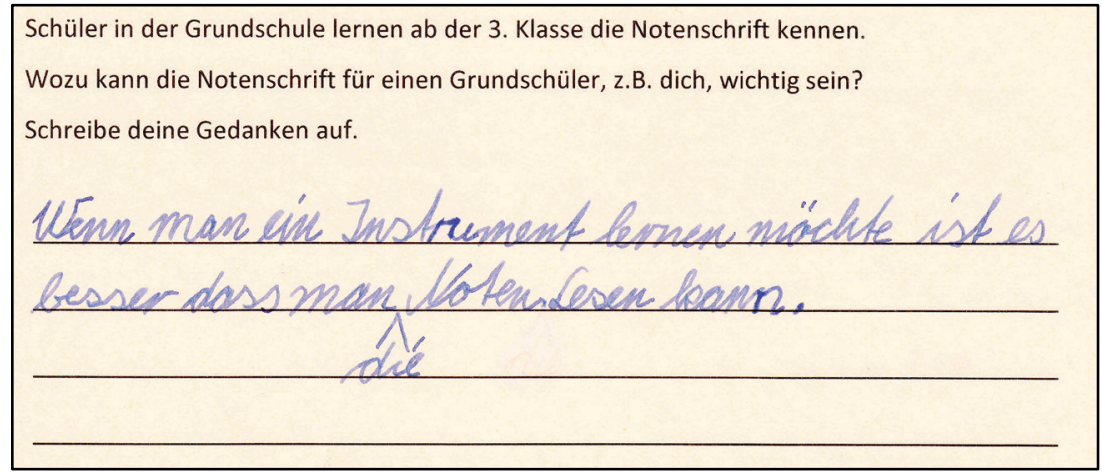

Abb. 3-13: Item im ST H

\section{Subtest I Hörendes Vergleichen von Rhythmen}

Am Ende des Testes werden den Probanden fünf Rhythmuspaare von einer CD vorgespielt. Sie

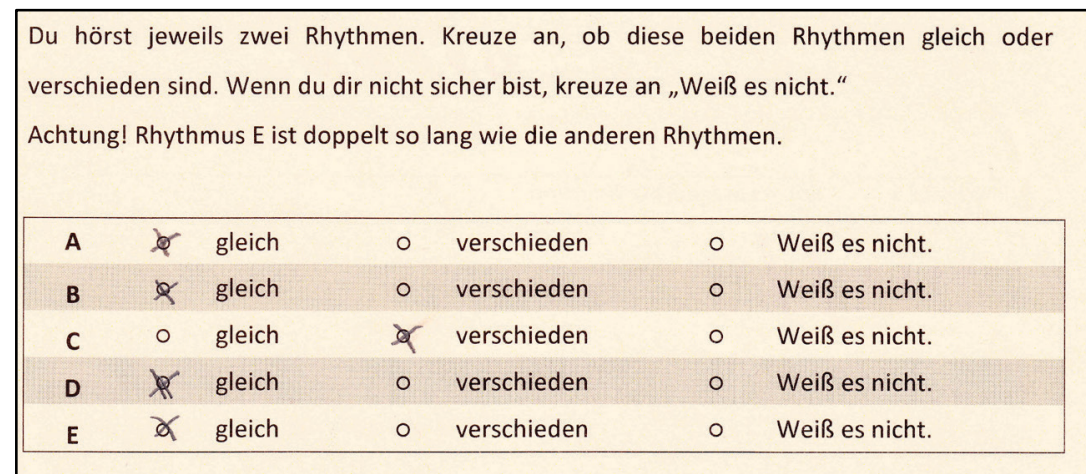

Abb. 3-14: Items im ST I 
sollen deren Gleichheit oder Verschiedenheit wahrnehmen und Entsprechendes im Testbogen ankreuzen (Itemgruppe I, s. Tab. 3-12). Dazu stehen die Optionen gleich, verschieden oder Weiß es nicht. zur Verfügung (s. Abb. 3-14; Testbogenausschnitt). Die Aufgabenstellung wird an einem nicht dazugehörigen Beispiel demonstriert. Sowohl für die Demonstration als auch für die eigentlichen Items, die von einer CD abgespielt werden, kommt das o.g. Playback zum Einsatz. Die Ergebnisse dieser Aufgabe werden in Korrelation zu den arithmetischen Mittelwerten der Gesamtpunktwertung der Probanden gesetzt.

Tab. 3-12: Angaben zu Items im ST I

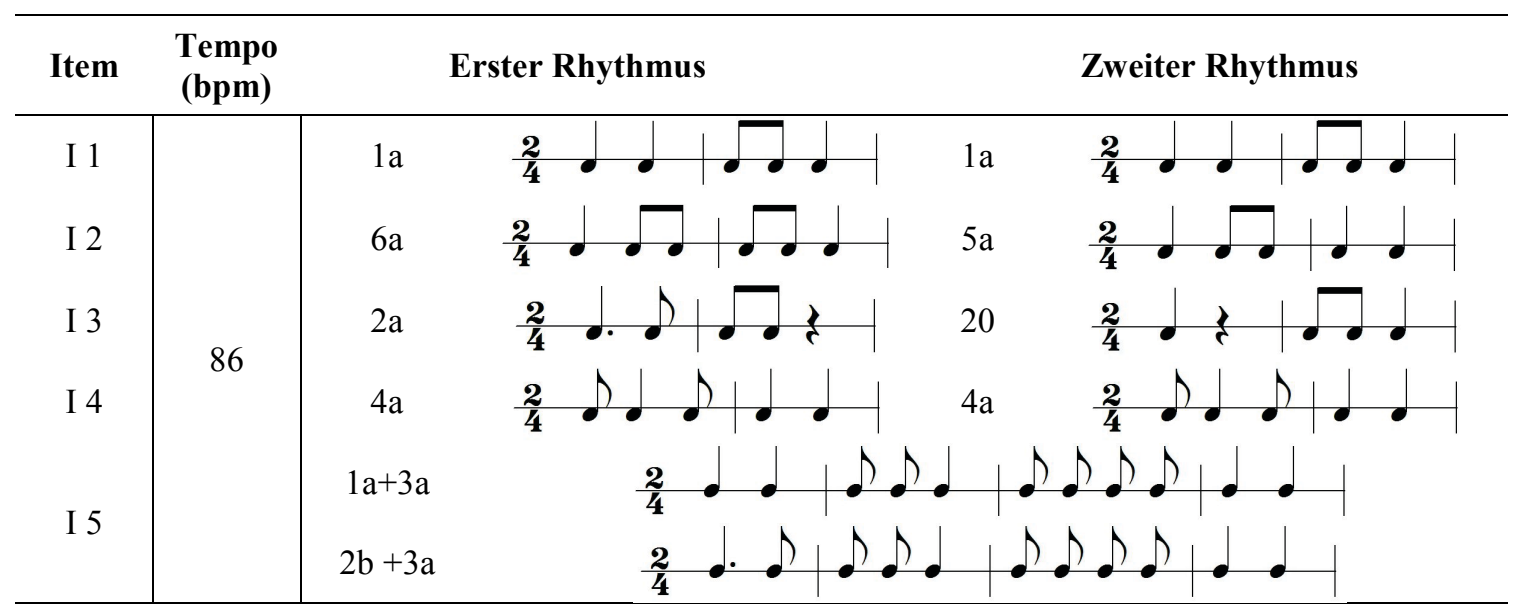

\subsubsection{Zusammenfassende Darstellung des Tests}

Tab. 3-13: Überblick über Subtests, Untertests und zusätzliche Items

\begin{tabular}{|c|c|c|}
\hline Subtests & \multicolumn{2}{|c|}{ Untertests; zusätzliches Item } \\
\hline \multicolumn{3}{|l|}{ I Hörendes Vergleichen von Rhythmen } \\
\hline \multicolumn{3}{|l|}{ H Wozu Notenschrift? } \\
\hline \multicolumn{3}{|l|}{ G Play together } \\
\hline F Rhythmen musizieren und aufschreiben mit Noten & f mit Zeichen & $\begin{array}{l}\text { fe mit Rhythmus- } \\
\text { silben }\end{array}$ \\
\hline E Rhythmen lesen und musizieren nach Noten & e nach Zeichen & $\begin{array}{l}\text { ec nach Noten und } \\
\text { Rhythmussilben }\end{array}$ \\
\hline \multicolumn{3}{|l|}{ D Rhythmen variieren } \\
\hline \multicolumn{3}{|l|}{$\begin{array}{l}\text { C Rhythmussprache anwenden: } \\
\text { wird mit den Untertests ec und fc realisiert }\end{array}$} \\
\hline B Rhythmen hören und imitieren & Item b 6 & \\
\hline A Grundschlag und Metrum darstellen & & \\
\hline
\end{tabular}

Der gesamte Test besteht aus den sechs Subtests in Analogie zu den entsprechenden Stufen der Pyramide des Aufbauens rhythmischer Fähigkeiten. Dabei werden die Subtests E und F durch jeweils zwei Untertest erweitert (s. Tab. 3-13). Aus den Untertests ec und fc wird der Subtest C gene- 
riert. Die Untertests e und f sollen zusätzlich Erkenntnisse zum Umgang mit ikonischer Notation im Rahmen der dreifachen Wissensdarbietung ermöglichen.

Das zusätzliche Item b 6 dient dem Vergleich mit einem Item aus der Studie von Minkenberg (1991). Des Weiteren sollen die Subtests G und I die Testgüte in Bezug auf die Validität bzw. die Entscheidung für einen kriteriumsorientierten Test stützen helfen. Der Subtest H wird zur Abfrage nach dem Sinn und Zweck von Notation aus der Sicht der Probanden eingefügt.

\subsubsection{Länge, Metrum und Tempo der Items}

Für diesen Test werden in den rhythmenbezogenen Items der Subtests B bis F stets Rhythmen über eine Länge von vier Grundschlägen gewählt. Dies weicht mitunter von den Entscheidungen der in Abschnitt 2.5.1 genannten Studien ab (s. Anlage A 2). Ein Teil der Items bei Wagner (1970), alle Items im Screening I von Jungbluth und Hafen (1997) sowie ein Teil der Items bei Hasselhorn (2015) werden über vier Grundschläge gestaltet. Minkenberg und Hasselhorn verwenden auch doppelt so lange Items. Die Zielgruppe von Hasselhorn sind Schüler der neunten Klassenstufe, dies kann der hier gewählten Probandengruppe nicht gleich gesetzt werden. Minkenberg bezieht sich bei seiner Entscheidung für das Nachspielen längerer Rhythmen auf Davidson und Colley (1987, S. 107 ff., zitiert bei Minkenberg, 1991, S. 36). In der Altersgruppe der 8 bis 10 Jahre alten Probanden aus der Studie Minkenberg liegt die Itemschwierigkeit für drei der insgesamt fünf Items bei Werten zwischen 0 und 0.28 (ebd., S. 229-232). Dies entspricht nicht den Vorstellungen der Autorin zur Itemschwierigkeit (Abschnitt 3.4.5). Bei Gordon (2001) werden in den Call-ResponseÜbungen zu Beginn der Ausbildung nach der Music Learning Theory Rhythmen/ Patterns mit einer Länge von vier Grundschlägen empfohlen. Dieser Empfehlung wird entsprochen.

Um zu einer endgültigen Entscheidung zu gelangen, wurden in der Erprobungsphase sowohl kurze als auch längere Rhythmen getestet. Die längeren Rhythmen wiesen eine deutlich höhere Itemschwierigkeit als die kurzen Rhythmen auf. So wurden für die endgültige Testfassung vier kurze Rhythmen gewählt. Aus Interesse eines Vergleiches wurde der erste Rhythmus von Minkenberg als zusätzliches Item b 6 in den Subtest B eingefügt.

Bezüglich des Wechselns des Metrums gibt es in den erwähnten Tests verschiedene Herangehensweisen. Bei Wagner (1970) sowie bei Jungbluth und Hafen (Screening II, 1997) wird das Metrum gewechselt (s. Anlage A 2). Minkenberg (1991) behält in seinen fünf Items das einmal gewählte Metrum bei. Ebenso gilt es als erwiesen, dass Rhythmen in Zweiergliederung besser als Rhythmen in Dreiergliederung reproduziert werden (Drake, 1993, zitiert nach Auhagen, 201133, S. 445). Deshalb werden nur Rhythmen im geraden Metrum verwendet und das Metrum nicht gewechselt. Dies gilt für alle Rhythmen im Subtest B, D, E und F.

Als Tempo für die Items im Subtest B (mit Call-Response-Prinzip) werden in der Erprobungsphase 72 Schläge pro Minute gewählt. Diese Entscheidung wird in Anlehnung an Minkenberg ge- 
troffen. Dieser wählt ein Tempo von „MM = 68“ (Minkenberg, 1991, S. 72). Obwohl er sich auf mehrere Autoren bezieht, verwendet er nicht deren empfohlenen Tempobereich. Dieser liegt zwischen „MM = 112 und $\mathrm{MM}=136$ “ (Christensen, 1938, S. 44, zitiert nach Minkenberg, 1991,

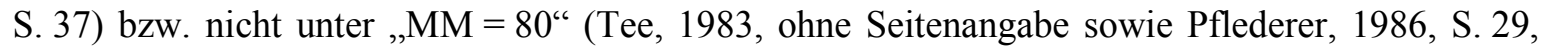
beide zitiert nach ebd.). Als die Vorbereitung der hier verwendeten Videofassung beginnt, wird das gewählte Tempo noch einmal geprüft. Dazu wurde mit der Hälfte der Probanden das bisherige Tempo von $72 \mathrm{bpm}$ sowie ein höheres Tempo von $92 \mathrm{bpm}$ nacheinander ausprobiert. Etwa die Hälfte der Probanden empfand das schnellere Tempo angenehmer. Auf einige Probanden wirkte das höhere Tempo im Zusammenhang mit der Reproduktionsaufgabe jedoch stressfördernd. Für die Endfassung des Videotests wird das Tempo auf $86 \mathrm{bpm}$ eingerichtet. Es wird in den Subtests B und D sowie beim gemeinsamen Musizieren im Subtest F genutzt. Im Subtest E und Untertest ec dürfen die Probanden das Tempo frei wählen.

Die wesentlichste Änderung zwischen Erprobungsphase und Hauptuntersuchung besteht in der Hinzunahme eines popmusikähnlichen Playbacks in der Videotestfassung. Dies hat seine Ursache in folgender Erfahrung: Die Versuchsleiterin verwendete zur Einhaltung eines stets gleich bleibenden Tempos in der Face-to-face-Verfahren (Erprobungsphase) für alle Calls ein In-Ear-Metronom. Wenn allerdings ein Kind zu langsam spielte, konnte der folgende Takt erst frühestens eine Zählzeit später beginnen. Damit wurde der eigentlich gewünschte dialogische Call-Response-Wechsel nicht mehr umgesetzt. Für den Videotest wird mit einem grundschlagbetonten, popähnlichen Playback (Yamaha-Style J-PopHit1) dafür gesorgt, dass die nachzuspielenden Takte ihr Zeitfenster akustisch gut erkennbar erhielten. Wenn nun ein Kind verzögert spielt, beginnt der nächste Call trotzdem pünktlich. Durch den gleichbleibenden Beat wirkt dies nicht störend. Dieses Playback wird für das Rhythmusnachspielen und -abwandeln in den Subtests B bzw. D sowie für das gemeinsame Musizieren der Rhythmen im Subtest F genutzt. Ebenfalls wird es in den zusätzlichen Subtests G Play together sowie I Hörendes Vergleichen von Rhythmen genutzt.

\subsubsection{Itemschwierigkeit und Trennschärfe}

\section{Itemschwierigkeit}

Die Itemschwierigkeit entspricht bei dichotomen Auswertungen (richtig / falsch) der Schwierigkeit eines Items für alle teilnehmenden Probanden.

$$
p=\frac{N_{R}}{N}\left(\mathrm{p}=\text { Schwierigkeitsindex, } \mathrm{N}_{\mathrm{R}}=\text { Anzahl der „Richtiglöser“, } \mathrm{N}=\text { Anzahl der Probanden }\right)
$$

Sie soll in Bezug auf die 24 Items so gestaltet sein, dass das Pyramidenmodell (Jank, 20135) mit seinen Stufen abgebildet wird, d.h. dass die Items in den höheren Stufen auch eine höhere Schwierigkeit aufweisen. Damit soll auch der Mittelwert der einzelnen Subtests von Stufe zu Stufe abnehmen (s. Tab. 3-14). 
Die klassische Testtheorie bevorzugt Items einer mittleren Schwierigkeit, da diese eine höhere Trennschärfe aufweisen können (Bühner, 2006, S. 98). Leichte und schwere Items decken wiederum die extremeren Leistungen gut ab und ermöglichen ,auch in Randbereichen eines Merkmalsbereichs zu differenzieren“ (ebd., S. 99). Die Schwierigkeitsindizes wurden in drei Gruppen untergliedert:

- $\quad$ schwere Items: $\quad \mathrm{p}=0.01$ bis 0.32

- $\quad$ mittelschwere Items: $\quad \mathrm{p}=0.33$ bis 0.65

- leichte Items: $\quad \mathrm{p}=0.66$ bis 1

Aus der Summe dieser einzelnen Werte wird der erwartete Mittelwert der Subtests vorausbestimmt. Dazu wird jeweils der niedrigste mögliche Wert pro Item $(0.0,0.33,0.66)$ für jeden Subtest angesetzt (s. Tab. 3-14). Es werden Mittelwerte zwischen 2.31 und 0.66 in den einzelnen Subtests erwartet.

Tab. 3-14: Geplante Itemschwierigkeit

\begin{tabular}{lllllc}
\hline $\begin{array}{l}\text { Operationalisierte Stufen des Aufbau- } \\
\text { ens rhythmischer Kompetenz }\end{array}$ & \multicolumn{4}{c}{$\begin{array}{c}\text { Schwierigkeit der je- } \\
\text { weiligen Items }\end{array}$} & $\begin{array}{c}\text { Möglicher Mittelwert } \overline{\boldsymbol{x}} \\
\text { der Subtests }\end{array}$ \\
\hline F Rhythmen musizieren und aufschreiben & 0.33 & 0.33 & 0.01 & 0.01 & 0.68 \\
E Rhythmen lesen und musizieren & 0.33 & 0.33 & 0.33 & 0.01 & 1.00 \\
D Rhythmen bewusst abwandeln & 0.33 & 0.33 & 0.33 & 0.33 & 1.32 \\
C Rhythmussprache anwenden & 0.66 & 0.33 & 0.33 & 0.33 & 1.65 \\
B Rhythmen nachspielen & 0.66 & 0.66 & 0.33 & 0.33 & 1.98 \\
A Grundschlag und Metrum darstellen & 0.66 & 0.66 & 0.66 & 0.33 & 2.31 \\
\hline
\end{tabular}

Die erwarteten Mittelwerte und deren Standardabweichungen werden grafisch veranschaulicht (s. Abb. 3-15). Subtest A weist dabei den höchsten Mittelwert, Subtest F den niedrigsten auf. Dies verdeutlicht eine allmählich zunehmende Itemschwierigkeit, die sich in abnehmenden Mittelwerten darstellt. Die Fehlerbalken (Standardabweichungen) markieren die erwarteten Differenzen der Leistungen in der Kontrollgruppe (niedriger) als auch in der Untersuchungsgruppe (höher).

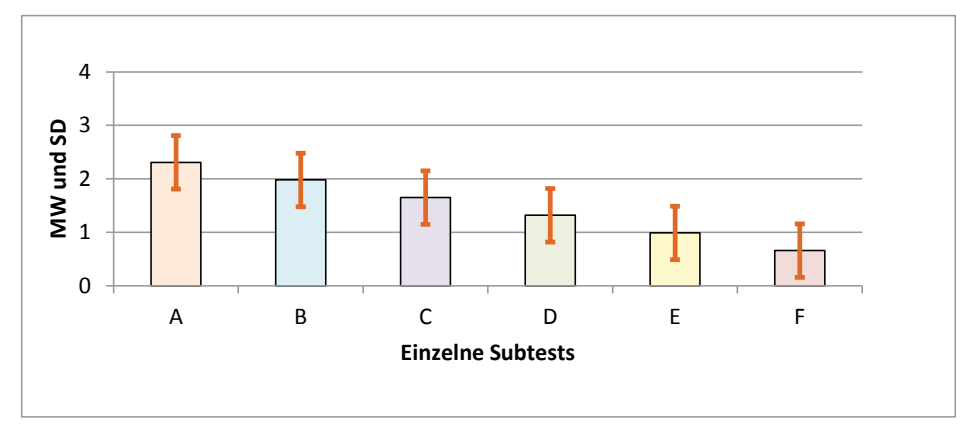

Abb. 3-15: Erwartete Mittelwerte und Standardabweichungen in den Subtests A-F 


\section{Trennschärfe}

Die Trennschärfe ist die Korrelation eines Items mit dem Gesamtergebnis eines Tests $\left(\mathrm{r}_{\mathrm{i}(\mathrm{t}-\mathrm{i})}\right)$. Die mögliche Spannweite liegt zwischen 1 und -1. Sie sollte jedoch bei Werten über 0.2 liegen. Liegt sich dieser Wert darunter, erfüllt das Item keine leistungsunterscheidende Funktion. „Ein Trennschärfenkoeffizient um 0 bringt zum Ausdruck, daß die Aufgabe von guten und schlechten Pbn [Probanden, d. V.] etwa gleich häufig richtig beantwortet wird; solche Aufgaben sind unbrauchbar“ (Lienert \& Raatz, 1998 6 , S. 78). Dieser Trennschärfenwert kann nicht bewusst geplant werden. Es können nur Items verworfen oder verändert werden, was nach der Erprobungsphase auch realisiert wurde. Eine besonders hohe Relevanz hat die Betrachtung des Trennschärfenwertes für die Itemgenerierung im Subtest $\mathrm{C}$, da dort die Items erst nach der Testdurchführung aus den Subtests E und $\mathrm{F}$ ausgewählt werden (Untertests ec und fc mit Rhythmussprache).

\subsection{Testgütesicherung}

In den folgenden Abschnitten werden die zur Anwendung kommenden Kriterien zur Testgütesicherung vorgestellt. Die Sicherstellung der Objektivität wird in die Abschnitte Durchführungsobjektivität, Auswertungs- sowie Interpretationsobjektivität unterteilt (s. Abschnitt 3.5.2 und 3.5.3). Es folgen die Angaben zur Sicherstellung von Reliabilität und Validität (s. Abschnitt 3.5.4).

\subsubsection{Durchführungsobjektivität}

Alle mündlichen und schriftlichen Tests werden stets von der Versuchsleiterin erhoben. Somit müssen keine weiteren Versuchsleiter instruiert werden. Die Testdurchführung der mündlichen Tests findet stets in einem dafür geeigneten Raum an der jeweiligen Schule statt. Der Aufbau der Geräte sowie die Position von Proband und Versuchsleiterin werden so ähnlich wie möglich gehalten. Die Subtests A, B und D sowie der zusätzliche Subtest G werden mithilfe eines Videos geprüft. Hier ist ein hoher Grad an Durchführungsobjektivität gegeben, da alle Probanden die Anweisungen durch das gleiche Medium erhalten.

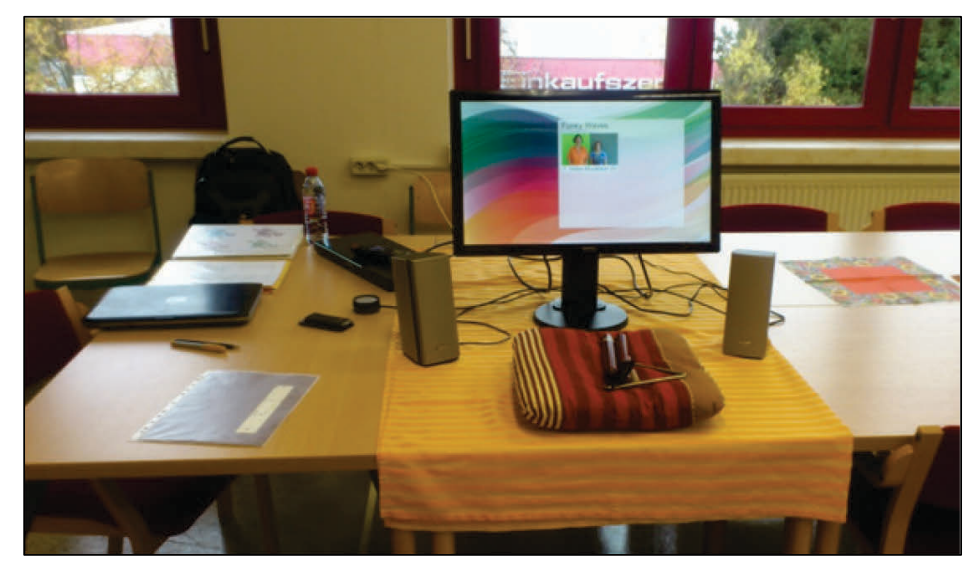

Abb. 3-16: Versuchsaufbau beim Einzelverfahren 
Die Subtests E und $C^{1}$ finden ohne Video statt, um keinen Zeitdruck aufzubauen. Es werden Musiziervorlagen verwendet (s. Anlage B 5). Die Konstanz aller weiteren Medien (Monitor, Lautsprecher, Instrumente, Versuchsaufbauten u.v.m. s. Abb. 3-16) wird ebenfalls gewährleistet. Über die gesamte Testdauer von ca. 18 Minuten findet eine Audioaufzeichnung statt (s. Anlage B 1).

Die Subtests F und $\mathrm{C}^{2}$ sowie die zusätzlichen Subtests H und I werden in einem Gruppenverfahren (schriftlich) geprüft. Es werden Testbogen, spezielle Stifte, Schablonen für die Aufgabenfelder, Sichtschutz zwischen den Banknachbarn sowie eine CD mit dem zu verwendenden Playback für die Call-Response-Aufgabe sowie für die zusätzliche Itemgruppe I verwendet. Die Ergebnisse werden von jedem Probanden auf einem Testbogen notiert.

\subsubsection{Auswertungsobjektivität}

Die Auswertung soll künstlich dichotome Ergebnisse (richtig = 1 und falsch $=0$ ) erzeugen. Dazu werden die sechs Subtests in Bezug auf die Auswertung drei Rubriken zugeordnet. Die erste Rubrik umfasst die Auswertung des Darstellens von Grundschlag und Metrum im Subtest A. Die zweite Rubrik umfasst die Auswertung der musizierten Rhythmen in den Subtests B, D und E einschließlich der Items aus dem Untertest ec (Subtest $C^{1}$ ). Die dritte Rubrik umfasst die notierten Rhythmen im Subtest $\mathrm{F}$ sowie die Items aus dem Untertest fc (Subtest $\mathrm{C}^{2}$ ).

Die musizierten Leistungen in der ersten und zweiten Rubrik (Subtests A bis E) unterliegen dem subjektiven Hörempfinden des Beurteilenden (vgl. Auhagen, $2011^{3}$, S. 441). Die Auswertungsobjektivität ist jedoch davon gekennzeichnet, dass nur die Versuchsleiterin die Leistungen der Probanden analysiert und bewertet. Für eine höchstmöglich objektive Bewertung der Ergebnisse aus der ersten und zweiten Rubrik wird folgendes Verfahren angewandt: Die digitalen AudioAufzeichnungen aller musizierpraktischen Handlungen der Probanden werden in einem Sequenzerprogramm analysiert. Dort können die einzelnen Schläge der Musizieraktionen erkannt und vermessen werden. In Abb. 3-17 sind aus Subtest B ein Call und das Response des Start-Items b 5 zu erkennen. Die Response setzt pünktlich ein (roter Pfeil), die anderen Schläge werden verspätet gespielt.

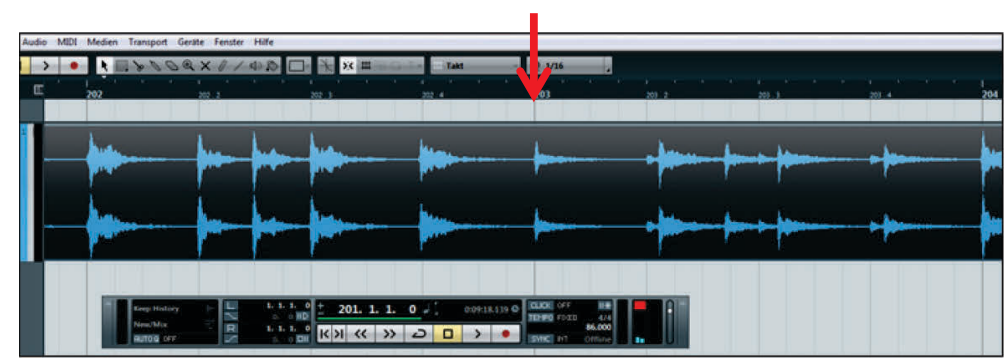

Abb. 3-17: Bildschirmfoto eines Rhythmus in der Sequenzer-Software 
Die vereinfachten Auswertungsvorschriften werden zusammenfassend vorgestellt (s. Tab. 3-15). Neben der Zuordnung zur entsprechenden Rubrik in Bezug auf die Auswertung werden die inhaltlichen und formalen bzw. temporelevanten Anforderungen und Toleranzspielräume dargestellt. Eine vertiefende Ausführung findet sich in den folgenden Unterabschnitten.

Zusätzlich zu den als richtig gewerteten Ergebnissen werden alle fehlerhaften Lösungen in verschiedene Lösungskategorien eingestuft. Diese umfassen die Kategorien Lösung fast richtig mit leichten Abstrichen, Lösung fehlerhaft sowie Lösung stark fehlerhaft. Je nach Auswertungs-Rubrik werden verschiedene Anforderungen für diese Einstufung aufgestellt. Sie werden in den folgenden Unterabschnitten erläutert.

Tab. 3-15: Auswertungsvorschriften im Überblick

\begin{tabular}{|c|c|c|c|}
\hline Rubrik & $\begin{array}{l}\text { Operationalisierte Stufen } \\
\text { des Aufbauens rhyth- } \\
\text { mischer Kompetenz }\end{array}$ & $\begin{array}{l}\text { Punktvergabe, wenn im ent- } \\
\text { sprechenden Toleranzraum ... }\end{array}$ & Toleranzraum \\
\hline \multirow{2}{*}{3} & $\begin{array}{l}\text { F Rhythmen musizieren } \\
\text { und aufschreiben }\end{array}$ & $\begin{array}{l}\text { musizierte Rhythmen richtig } \\
\text { notiert }\end{array}$ & \multirow{2}{*}{$\begin{array}{l}\text { Bezug zum Metrum frei } \\
\text { wählbar }\end{array}$} \\
\hline & $\begin{array}{l}\mathbf{C}^{2}(\mathbf{f c}) \text { Rhythmussprache } \\
\text { anwenden }\end{array}$ & $\begin{array}{l}\text { Rhythmen mithilfe von Rhyth- } \\
\text { mussilben richtig notiert }\end{array}$ & \\
\hline \multirow{4}{*}{2} & $\begin{array}{l}\text { E Rhythmen lesen und } \\
\text { musizieren }\end{array}$ & $\begin{array}{l}\text { notierte Rhythmen richtig musi- } \\
\text { ziert }\end{array}$ & \multirow{2}{*}{$\begin{array}{l}+/-1 / 32 \text { zu den Positionen } \\
\text { der Hauptschläge im Takt im } \\
\text { individuell gewählten Tempo }\end{array}$} \\
\hline & $\begin{array}{l}\mathbf{C}^{1}(\mathbf{e c}) \text { Rhythmussprache } \\
\text { anwenden }\end{array}$ & $\begin{array}{l}\text { Rhythmen und Rhythmussilben } \\
\text { richtig musiziert }\end{array}$ & \\
\hline & $\begin{array}{l}\text { D Rhythmen bewusst } \\
\text { abwandeln }\end{array}$ & $\begin{array}{l}\text { spontan abgewandelte Rhythmen } \\
\text { richtig gespielt / wiederholt }\end{array}$ & \multirow{2}{*}{$\begin{array}{l}+/-1 / 32 \text { zu den Positionen } \\
\text { der Hauptschläge im Takt im } \\
\text { Tempo } 86 \text { bpm }\end{array}$} \\
\hline & B Rhythmen imitieren & $\begin{array}{l}\text { Rhythmen in der zweiten } \\
\text { Response richtig gespielt }\end{array}$ & \\
\hline 1 & $\begin{array}{l}\text { A Grundschlag und Met- } \\
\text { rum darstellen }\end{array}$ & $\begin{array}{l}\text { Grundschlag bzw. Metrum richtig } \\
\text { musiziert und maximal ein Fehler } \\
\text { in einem der acht Takte }\end{array}$ & $\begin{array}{l}\text { +/- 1/16 zu den Positionen } \\
\text { der Grundschläge / des Met- } \\
\text { rums im Tempo der jeweili- } \\
\text { gen Musikstücke }\end{array}$ \\
\hline
\end{tabular}

\section{Erste Rubrik - Subtest A}

Für die Ergebnisse aus der ersten Rubrik müssen die verschiedenen Musikstücke zuerst auf das jeweilige Tempo im Sequenzerprogramm eingestellt werden. Die Messbarkeit der Fehler gestaltet sich hier schwierig. Mitunter überlagern sich Musiktitel und Spielaktionen der Probanden und sind schlecht voneinander zu unterscheiden (s. Abb. 3-18). Im linken Bild sind die einzelnen Schläge für die Darstellung des Grundschlages sehr gut erkennbar, im rechten Bild sind sie nur teilweise erkennbar. Dort muss hörend geprüft werden. Deshalb werden für diese Rubrik größere Toleranzbereiche für Tempoabweichungen der Positionen der Grundschläge bzw. der Metrumschläge gewährt (s. Tab. 3-15). 

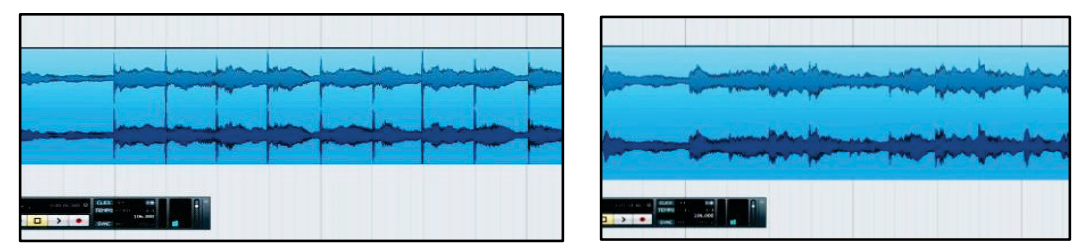

Abb. 3-18: Sequenzeransichten für Item A 1, Takt 1 und 2

Als richtig gewertet werden alle Takte, in denen Grundschlag bzw. Metrum im angegebenen Toleranzbereich von +/- eines Sechzehntelschlages zu den Positionen der Grundschläge sowie in Bezug auf das Tempo des jeweiligen Musikstückes korrekt musiziert werden. Es darf maximal ein Fehler in einem der acht Takte auftreten. Diese Lösungen gehören in Bezug auf die Lösungskategorien zur Kategorie Lösung korrekt. Zur Kategorie Lösung fast richtig mit leichten Abstrichen gehören Musizierleistungen, in denen fünf bis sechs Takte richtig musiziert werden. Zur Kategorie Lösung fehlerhaft gehören Musizierleistungen, in denen nur drei bis vier Takte richtig musiziert werden. Zur Kategorie Lösung stark fehlerhaft gehören Musizierleistungen, in denen null bis zwei Takte richtig musiziert werden oder gar nicht musiziert wird.

\section{Zweite Rubrik - Subtest B, D und E}

Für die Ergebnisse aus der zweiten Rubrik gibt es im Sequenzerprogramm keine Darstellungsprobleme. Die Positionen der einzelnen Schläge der musizierten Rhythmen können millisekundengenau im Editor der Tonspur des Sequenzerprogramms vermessen werden. Die gewonnenen Positionswerte jedes Schlages eines Rhythmus werden in ein Datenblatt eingegeben (s. Abb. 3-19). In dem abgebildeten Ausschnitt dieses Datenblattes sind die Positionswerte von elf ausgewählten Probanden dargestellt. Das Datenblatt wird in einer Auswertungsmatrix mit einem Algorithmus verlinkt. Dadurch können Abweichungen der Positionen der Schläge im Takt geprüft werden. Es werden neben dem Toleranzbereich ein erweiterter Toleranzbereich und ein sogenannter Extrembereich festgelegt (s. Abb. 3-20, s. Anlage B 2).

\begin{tabular}{|r|c|c|c|c|c|}
\hline & \multicolumn{6}{|c|}{ Item B 1 } \\
\hline Proband & 1 & 2 & 3 & $3 u$ & 4 \\
\hline 4 & -41 & 653 & 1382 & 1703 & 2063 \\
74 & -50 & 628 & 1277 & 1636 & 1982 \\
221 & 62 & 622 & 1272 & 1599 & 1920 \\
193 & 23 & 779 & 1537 & 1847 & 2188 \\
207 & 114 & 774 & 1371 & 1703 & 2007 \\
212 & 14 & 738 & 1334 & 1724 & 2064 \\
55 & 34 & 620 & 1247 & 1629 & 2122 \\
187 & 5 & 736 & 1484 & 1780 & 2099 \\
\hline 209 & -14 & 817 & 1324 & 1642 & 2028 \\
\hline 227 & -139 & 552 & 907 & 1196 & 1831 \\
\hline 177 & 543 & 1199 & 1714 & 2130 & 2456 \\
\hline
\end{tabular}

Abb. 3-19: Positionswerte der Schläge in Item B 1 von elf Probanden 
Für den ersten Schlag im Takt kann dies folgendes bedeuten: Wird dieser Schlag im Toleranzbereich zwischen - 88 und +93 Millisekunden zur exakten Position gespielt, gilt er als richtig. Früher oder später gespielte Schläge fallen in den erweiterten Toleranzbereich. Positionen vor - 176 oder nach +186 werden dem Extrembereich zugeordnet. Die Unterscheidung in erweiterten Toleranzbereich und Extrembereich spielt für die Entscheidung richtig oder falsch keine Rolle, da alle Ergebnisse bereits im erweiterten Toleranzbereich als falsch gewertet werden. In der differenzierten Fehleranalyse hilft diese Zuordnung jedoch, zwischen leicht schwankendem, unregelmäßigem und stark schwankendem Tempo zu unterscheiden (s. Anlage B 2) und die Zuordnung zu den Lösungskategorien festzulegen.

\begin{tabular}{|c|c|c|c|c|c|c|c|c|c|}
\hline Bereiche & \multicolumn{2}{|c|}{ Extrembereich } & $\begin{array}{c}\text { erweiterter } \\
\text { Bereich }\end{array}$ & \multicolumn{3}{|c|}{ Toleranzbereich } & $\begin{array}{c}\text { erweiterter } \\
\text { Bereich }\end{array}$ & \multicolumn{2}{|c|}{ Extrembereich } \\
\hline Toleranz & & & - & -88 & $\begin{array}{l}\text { exakte } \\
\text { Position }\end{array}$ & 93 & + & & \\
\hline \multirow{4}{*}{$\begin{array}{c}\text { Toleranzspiel- } \\
\text { räume }\end{array}$} & \multicolumn{2}{|c|}{ unter $-264-264$ bis -17} & -176 bis -89 & -88 & 0 & 93 & 94 bis 186 & 187 bis 2 & über 279 \\
\hline & \multirow{3}{*}{\multicolumn{2}{|c|}{ hythmus nicht bewertba }} & 518 bis 605 & 606 & 694 & 787 & 788 bis 880 & \multirow{3}{*}{\multicolumn{2}{|c|}{$\begin{array}{c}\text { Rhythmus nicht } \\
\text { bewertbar }\end{array}$}} \\
\hline & & & 1212 bis 1299 & 1300 & 1388 & 1481 & 1482 bis 1574 & & \\
\hline & & & 1906 bis 1993 & 1994 & 2082 & 2175 & 2176 bis 2268 & & \\
\hline Kategorie Einsatz & 111 & 101 & 100 & \multicolumn{3}{|c|}{0} & 200 & 202 & 222 \\
\hline Kategorie Tempo & - & 60 & $10,11,30,31,33$ & \multicolumn{3}{|c|}{0} & $20,22,30,32,33$ & 60 & - \\
\hline
\end{tabular}

Abb. 3-20: Toleranzbereiche für Positionen auf Hauptschlägen in Millisekunden

\begin{tabular}{|c|c|c|c|c|c|c|c|}
\hline Bereiche & Extrembereich & $\begin{array}{l}\text { erweiterter } \\
\text { Bereich }\end{array}$ & \multicolumn{3}{|c|}{ Toleranzbereich } & \multirow{2}{*}{$\begin{array}{c}\begin{array}{c}\text { erweiterter } \\
\text { Bereich }\end{array} \\
+\end{array}$} & \multirow[t]{2}{*}{ Extrembereich } \\
\hline Toleranz & & - & -85 & $\begin{array}{l}\text { exakte } \\
\text { Position }\end{array}$ & 90 & & \\
\hline \multirow{4}{*}{$\begin{array}{c}\text { Toleranzspiel- } \\
\text { räume }\end{array}$} & \multirow{4}{*}{$\begin{array}{c}\text { Rhythmus nicht } \\
\text { bewertbar }\end{array}$} & 177 bis 261 & 262 & 347 & 437 & 438 bis 527 & \multirow{4}{*}{$\begin{array}{c}\text { Rhythmus nich } \\
\text { bewertbar }\end{array}$} \\
\hline & & 871 bis 955 & 956 & 1041 & 1131 & 1042 bis 1221 & \\
\hline & & 1565 bis 1655 & 1650 & 1735 & 1825 & 1735 bis 1915 & \\
\hline & & 2259 bis 2343 & 2344 & 2429 & 2519 & 2430 bis 2609 & \\
\hline Kategorie Einsatz & 101 & 100 & & 0 & & 200 & 202 \\
\hline Kategorie Tempo & 60 & $10,11,30,31,33$ & & 0 & & $20,22,30,32,33$ & 60 \\
\hline
\end{tabular}

Abb. 3-21: Toleranzbereiche für Positionen auf Nebenschlägen in Millisekunden

Die Abweichungen auf den Positionen werden verschieden bewertet. Fehler auf Hauptschlägen (s. Abb. 3-20) fallen weniger ins Gewicht als Fehler auf Nebenschlägen (s. Abb. 3-21). Deshalb erhalten Abweichungen auf Nebenschlägen einen leicht geringeren Toleranzbereich. Als richtig gewertet werden Werte zwischen - 85 und + 90 Millisekunden zur exakten Position (oder den entsprechenden anderen exakten Positionen). Analog wird zwischen einem erweiterten Toleranzbereich und einem Extrembereich zum Zwecke einer differenzierten Fehleranalyse und der Zuordnung zu Lösungskategorien unterschieden.

Allein mit diesen Positionswerten ist es noch nicht möglich, den gehörmäßig getroffenen Entscheidungen über richtig und falsch ausreichend nahe zu kommen. Mittels Versuch und Irrtum 
wurden weitere Faktoren in den Algorithmus aufgenommen (s. Abb. 3-22). Zu den bereits unterschiedlich stark gewichteten Abweichungen auf den Positionen der Haupt- und Nebenschläge (senkrechte rote Pfeile) zählen nun ebenfalls die Dauern der verschiedenen Schlaglängen dazu (Interonset intervals; s. Auhagen, 201133, S. 447). Dies ist mit den waagerechten blauen Pfeilen zwischen Position 0 und 347 sowie 0 und 694 angedeutet. Zusätzlich werden die Differenzen dieser Dauern gleicher Schlaglängen zueinander ins Verhältnis gesetzt. Dies ist als waagerechtes lilafarbenes Pfeilpaar mit einem roten Doppelpunkt dargestellt. Je nach Länge des Schlages (Achtel-, Viertel- oder anderer Schlag) dürfen sich diese Differenzen auch verschieden unterscheiden (s. Anlage B 2). Die drei Algorithmusfaktoren bestehen somit aus der Position des Schlages bezogen auf die jeweilige Zählzeit (Haupt- bzw. Nebenschläge), der Dauer des Schlages sowie den Differenzen der Dauern zwischen zwei Schlägen gleicher Länge. Anwendungsbeispiele bezogen auf die elf Fälle aus Abbildung 3-19 finden sich ebenfalls in Anlage B 2.

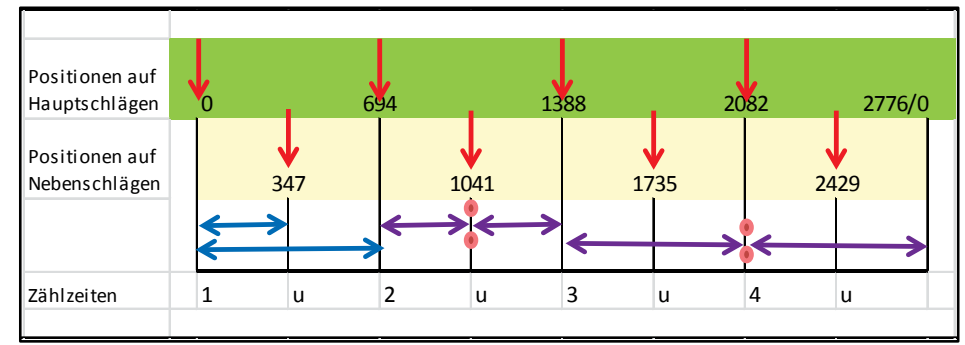

Abb. 3-22: Darstellung der drei Algorithmusfaktoren

Die Toleranzbereiche aller drei Faktoren wurden im Laufe des Auswertungsverfahrens immer wieder verändert und angepasst, bis eine relativ zufriedenstellende Übereinstimmung mit den gehörmäßig getroffenen Entscheidungen gefunden wurde. Dabei bestand eine kleine, aber wirkungsvolle Änderung darin, die Toleranzbereiche für die Positionen der Haupt- und Nebenschläge nicht symmetrisch, sondern leicht asymmetrisch zu verschieben. Diese Verschiebung wird um fünf Millisekunden zugunsten eines verspäteten Spielens gelegt (vgl. Frühauf, Kopiez \& Platz, 2013) und führte deshalb zu einer geringeren Toleranz im Minusbereich gegenüber dem Plusbereich $(-88 /+93 \mathrm{~ms}$ sowie $-85 /+90 \mathrm{~ms})$.

In der verlinkten Auswertungsmatrix werden abweichende Werte in Bezug auf die drei Algorithmusfaktoren in bedingte Formatierungen überführt. Bei Über- bzw. Unterschreitung der Toleranzbereiche zeigen die entsprechenden Zellen durch verschiedene Farben eine Toleranzbereichsüberschreitung an. So kann abgelesen werden, ob ein Rhythmus als richtig oder falsch zu werten ist (s. Abb. 3-23). Von den elf ausgewählten Lösungen für Item B 1 kann nur die erste Lösung (Proband 4) als richtig gewertet werden. In allen anderen Fällen gibt es zu große Abweichungen von den Positionen (Fall 74 bis 177, jedoch nicht Fall 212), zu lange oder kurze Dauern der Schläge (Fall 74 bis 177, jedoch nicht Fall 187) sowie zu große Differenzen zwischen den Dauern der 
Schläge gleicher Länge (Fall 55, 209, 227, 177). In vielen Fällen treten die Fehler kombiniert auf. Zusätzlich kann auch die Zuordnung zum erweiterten Toleranzbereich oder zum Extrembereich erkannt werden. (Beispiele für Item B 1 und deren Zuordnung zu Fehlerkategorien sind in Anlage B 2 dargestellt).

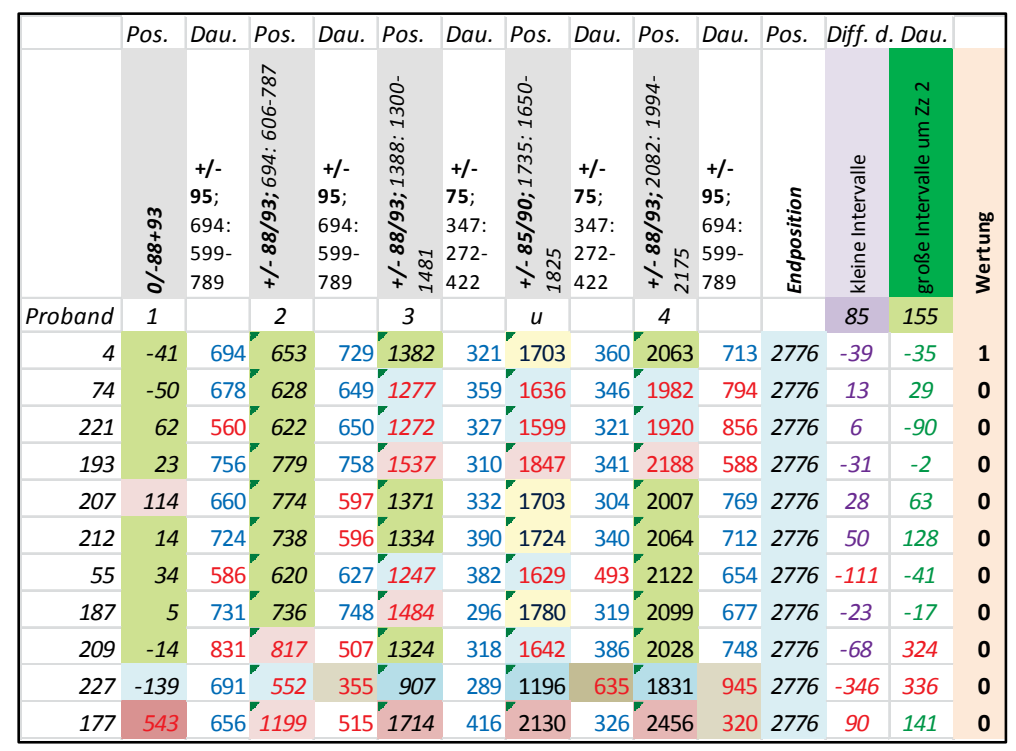

Abb. 3-23: Beispiele für ausgewertete Rhythmen für Item B 1

Im Subtest D werden aufgrund der Vielfalt der abgewandelten Rhythmen für weitere 14 Rhythmen Auswertungsmatrizes entwickelt. Damit kann der Vielfalt der abgewandelten Rhythmen und der gleichzeitig zu garantierenden Auswertungsobjektivität Rechnung getragen werden. Im Subtest E (sowie in den Untertests e und ec) musiziert jeder Proband in einem von ihm selbst gewählten Tempo. Deshalb wird in der Auswertung zuerst dieses Tempo ermittelt und anschließend als zusätzlicher Faktor in den Algorithmus eingefügt.

Der Algorithmus für die Analyse der musizierten Rhythmen in der zweiten Rubrik dient in erster Linie dem Überprüfen von als richtig oder falsch zu wertenden Lösungen. Durch die bedingten Formatierungen und die Zuordnung in den Toleranzbereich, den erweiterter Toleranzbereich sowie den Extrembereich kann im Bereich der fehlerhaften Lösungen weiter differenziert werden. Es entstehen u. a. die Fehlerkategorien zu schnell oder zu langsam, leicht schwankend bzw. stark unregelmäßig gespielt. Diese differenzierte Fehleranalyse lässt Rückschlüsse auf die Entwicklung und Förderung rhythmischer Kompetenz ziehen und ermöglicht eine Zuordnung auf die abgestuften Lösungskategorien.

Zur Kategorie Lösung korrekt werden alle Rhythmen gezählt, die an sich richtig und im angegebenen Toleranzbereich musiziert werden. Zur Kategorie Lösung fast richtig mit leichten Abstrichen gehören Musizierleistungen, in denen die Rhythmen an sich richtig und teilweise im erweiterten Toleranzbereich musiziert werden. Zur Kategorie Lösung fehlerhaft gehören Musizierleistun- 
gen, in denen die Rhythmen an sich richtig und überwiegend im erweiterten Toleranzbereich musiziert werden, einige Werte dürfen im Extrembereich liegen. Zur Kategorie Lösung stark fehlerhaft gehören Musizierleistungen, in denen die Rhythmen an sich richtig, jedoch überwiegend im Extrembereich musiziert werden, die Rhythmen an sich nicht richtig sind bzw. in denen kein Rhythmus musiziert wird. Im Subtest D kommt zusätzlich zu diesen Bedingungen die Erfüllung der Aufgabenstellung des Abwandelns und Wiederholens des abgewandelten Rhythmus hinzu.

\section{Dritte Rubrik - Subtest F}

Am eindeutigsten können die schriftlich fixierten Ergebnisse in der dritten Rubrik aus Subtest F mit den Untertests $\mathrm{f}$ und $\mathrm{fc}$ ausgewertet werden (s. Abb. 4-16, Abb. 3-10). Da es für alle Lösungen geschlossene bzw. halboffene Aufgabenformate gibt, können die Ergebnisse eindeutig zugeordnet werden. Toleranz besteht dahingehend, die Rhythmen im 2/4, 4/4 oder auch 2/2-Takt zu notieren.

In Bezug auf die Lösungskategorien werden in der dritten Rubrik die Typen des Darstellens von Rhythmen zugrunde gelegt (s. Abschnitt 2.2.5). Zur Kategorie Lösung korrekt zählen alle notierten Rhythmen, die im metrischen Typus notiert werden. Zur Kategorie Lösung fast richtig mit leichten Abstrichen gehören alle notierten Rhythmen im figuralen Typus, zur Kategorie Lösung fehlerhaft im zählenden Typus sowie zur Kategorie Lösung stark fehlerhaft alle notierten Rhythmen im nicht übereinstimmenden Typus bzw. auch fehlende Lösungen.

\subsubsection{Interpretationsobjektivität}

Die Interpretationsobjektivität sieht vor, dass ein Versuchsleiter sich bei der Interpretation der Testwerte nicht von seinen individuellen Deutungen leiten lassen soll, vielmehr Vergleichswerte und Normen aus Testhandbüchern herangezogen werden sollen (vgl. Bortz \& Döring, 2006 S. 195). Für die zu erhebenden Daten liegen solche Vergleichswerte kaum vor. Dennoch sollen die in Abschnitt 2.5.1 vorgestellten Tests zu Vergleichszwecken herangezogen werden. Wagner (1970) stellt fest: „Auffallend ist die fast durchweg bessere Leistung der Mädchen im Vergleich zu den Buben. Der Niveauunterschied liegt bei $10 \%$ oder, anders ausgedrückt, die Mädchen sind 1 bis 1 1 2 Jahre den Buben in der Entwicklung voraus" (Wagner, 1970, S. 27). Es interessiert, ob in dieser Untersuchung ein Nachweis erbracht werden kann, dass Mädchen in der hier dazustellenden Studie ebenfalls durchweg bessere Leistungen erbringen würden.

Aus Minkenbergs Studie wird der Rhythmus K 1 (1991, S. 72) ausgewählt und dieser Studie als Item b 6 im Subtest B hinzugefügt. Von Interesse ist, ob längere oder kürzere Rhythmen besser reproduziert werden können. Zum Test von Jungbluth und Hafen (1997) sind Vergleiche nicht möglich. Die Itemgruppe, welche Subtest B am meisten entspricht, enthält nur Gesamtpunktwerte und keine gesonderte Auflistung für einzelne Items. So können die Ergebnisse für einzelne Rhythmen, die sowohl bei Jungbluth \& Hafen als auch in dieser Untersuchung enthalten sind, nicht verglichen werden. Zum Test von McPherson (2005) können aufgrund des Umgangs mit Melodiein- 
strumenten keine Bezüge aufgestellt werden. $\mathrm{Zu}$ den Probanden aus der Studie von Hasselhorn (2015) sind Bezüge ebenfalls nicht möglich, da auch hier keine Werte für einzelne Items angegeben wurden.

\subsubsection{Reliabilität und Validität}

Die Prüfung der Reliabilität des entwickelten Testes erfolgt über die Testhalbierung und die Berechnung der internen Konsistenz. Bei der Testhalbierung wird die Reliabilität als Korrelation zweier Testhälften erfasst. Der Test wird dazu in zwei äquivalente Hälften unterteilt und die aus diesen beiden Teilen pro Testperson vorliegenden Messwerte miteinander korreliert (vgl. Bortz \& Döring, 2006 4 , S. 198). Die Halbierung erfolgt auf Basis der Analysedaten. Es werden aufgrund der ermittelten Itemschwierigkeit Item-Paare gebildet. Das schwerste und leichteste Item sowie die beiden mittelschweren Items werden je in eine Testhälfte geordnet. Im Subtest $C$ wird außerdem berücksichtigt, dass in jeder Testhälfte ein Item aus $C^{1}$ und $C^{2}$ enthalten ist. Im Subtest $D$ soll in jeder Testhälfte ein abgewandelter Rhythmus und eine Wiederholung enthalten sein (s. Anlage C 4).

Die interne Konsistenz wird mit dem Cronbach's-Alpha-Koeffizient dargestellt. „Interne Konsistenzschätzungen stellen eine Erweiterung der Testhalbierungsmethode dar, und zwar nach der Überlegung, dass sich ein Test nicht nur in Testhälften, sondern in so viele ,kleinste‘ Teile zerlegen lässt, wie er Items enthält. [...] Die Korrelationen zwischen den Items spiegeln dann die ,wahre‘ Varianz wider“" (Bortz \& Döring, 2006 ${ }^{4}$, S. 198).

Die Inhaltsvalidität eines Tests ist gegeben, wenn „der Inhalt der Testitems das zu messende Konstrukt in seinen wichtigsten Aspekten erschöpfend erfasst“ (Bortz \& Döring, 2006 ${ }^{4}$, S. 200). Streng genommen handelt es sich „nicht um ein Testgütekriterium, sondern nur um eine Zielvorgabe, die bei der Testkonstruktion bedacht werden sollte“" (ebd., S. 201). Die Entwicklung der Items wird eng an die Theorie Gordons, die Pyramide des Aufbauens rhythmischer Fähigkeiten (Jank) und vergleichbare Inhalte verschiedener Curricula deutscher Bundesländer (insbesondere des sächsischen Lehrplans) gebunden und soll damit diesem Kriterium bestmöglich genügen.

Die Kriteriumsvalidität wird durch zwei Methoden geprüft. Die Prüfweise der known groups (Atteslander, 20104 , S. 228; Bortz \& Döring, 2006 ${ }^{4}$, S. 201) soll zeigen, dass Probanden, von denen mit Sicherheit anzunehmen ist, dass sie die Merkmale der interessierenden Kompetenz sehr gut erfüllen müssten, deutlich bessere Ergebnisse erbringen als andere Probanden. Die zweite Methode prüft Korrelationen zwischen dem zusätzlichen Subtest G sowie den Subtests A bis F. Es wird davon ausgegangen, dass eine hohe rhythmische Kompetenz zu weiterer musikalischer Kompetenz außerhalb des sechsstufigen Theoriemodells führen müsste. 


\subsection{Statistische Verfahren}

Für die Auswertung der erhobenen Daten werden verschiedene statistische Verfahren angewendet. $\mathrm{Zu}$ ihnen zählen vor allem die Berechnung von arithmetischen Mittelwerten und deren Differenzen, von Effektstärken sowie einer einfachen Varianzanalyse. Arithmetische Mittelwerte werden für einzelne Items, die Subtests, den Gesamttest und für die Itemschwierigkeit berechnet. Es werden Korrelationen zwischen den Mittelwerten einzelner Subtests und dem Gesamtpunktwert berechnet. Außerdem werden Sonderformen von Korrelationen wie die Trennschärfe eines Items $\left(r_{i(t-i)}\right)$, die Testhalbierungsreliabilität $\left(r_{1 / 2 t / 2}\right)$ sowie die interne Konsistenz berechnet.

Für alle Subtests und die Gesamtpunktwertung wird der Signifikanztest mit dem t-Test gerechnet und anschließend mit dem kritischen Wert $t$ (Bortz \& Döring, 2006 ${ }^{4}$, S. 497) verglichen (Varianzanalyse). Für die Berechnung der Effektstärke $d$ wird nach der Formel in Abb. 3-24 gerechnet. Dabei wird die Differenz D $\left(D=\bar{x}_{U}-\bar{x}_{K}\right)$, welche aus den Mittelwerten der Nachtest- und Vortestergebnisse berechnet wird, durch die gepoolte Standardabweichung $\sigma$ dividiert. Mit der gepoolten Standardabweichung muss gerechnet werden, da Untersuchungs- und Kontrollgruppe nicht gleich groß sind (Platz, Kopiez \& Lehmann, 2012, S. 168). Sie ist ebenfalls in Abb. 3-24 dargestellt. Die Effektstärkeneinteilung wird nach Cohen mit $d=0.2$ als kleinen Effekt, $d=0.5$ als mittleren und $d=0,8$ als großen Effekt vorgenommen (vgl. Bortz \& Döring, 2006 ${ }^{4}$, S. 606).

$$
\begin{array}{|c|c|}
\hline \boldsymbol{d}=\frac{\boldsymbol{D}}{\boldsymbol{\sigma}} & \sigma=\sqrt{\frac{\left(n_{A}-1\right) * \sigma_{A}^{2}+\left(n_{B}-1\right) * \sigma_{B}^{2}}{\left(n_{A}-1\right)+\left(n_{B}-1\right)}} \\
\hline
\end{array}
$$

Abb. 3-24: Formeln zur Berechnung der Effektstärke d und der gepoolten Standardabweichung $\sigma$

Für die Rohdaten im Nachtest wird die Normalverteilung für alle Probanden aus beiden Gruppen mit dem Qui-Quadrat-Test über Kreuztabellen gerechnet. 


\section{Ergebnisse}

Dieses Kapitel beginnt mit der Dokumentation der gezogenen Stichprobe sowie der Durchführung der Hauptuntersuchung. Die darauf folgenden Ergebnisse im Abschnitt 4.2 beziehen sich zuerst auf alle Probanden unabhängig ihrer Zugehörigkeit zur Untersuchungs- oder Kontrollgruppe. Mit ihnen werden das Ausgangsniveau zu Beginn der Klassenstufe 3 sowie das Endniveau nach Abschluss des Untersuchungszeitraums am Ende von Klassenstufe 3 dokumentiert.

Die Ergebnisse zu den einzelnen Subtests getrennt nach Untersuchungs- und Kontrollgruppe werden im Abschnitt 4.3 in der Chronologie der Erhebung dargestellt. Im Abschnitt 4.4 werden Darstellungen zur Gesamtpunktwertung unter verschiedenen Gesichtspunkten aufgeführt. Die Ergebnisse der zweiten Erhebung, deren Notwenigkeit sich erst im Verlaufe der Studie erkennen ließ (s. Abschnitt 4.1.4), werden in Abschnitt 4.5 dokumentiert. Nachfolgend werden die Ergebnisse in den zusätzlichen Items und Subtests aufgeführt (s. Abschnitt 4.6). Ergebnisse zum Testmodell werden im Abschnitt 4.7 sowie zu den Testgütekriterien im Abschnitt 4.8 dokumentiert.

\subsection{Dokumentation der Stichprobe und des Untersuchungszeitraums}

Die Dokumentation gliedert sich in die Abschnitte zur Stichprobe sowie zu deren kritischen Bewertung (s. Abschnitt 4.1.2). Es folgt die Darstellung des Ablaufes des Untersuchungszeitraumes und besonderer Vorkommnisse darin (s. Abschnitt 4.1.3). Diese führen zur Planung einer zweiten Erhebung (s. Abschnitt 4.1.4). Abschließend werden einige Eckpunkte der Zusammenarbeit mit den involvierten Lehrkräften dokumentiert (s. Abschnitt 4.1.5).

\subsubsection{Gezogene Stichprobe}

Die teilnehmenden 12 Klassen wurden in Städten und Gemeinden mit bis zu 5.000 (hellgrau), 16.000 (mittelgrau) bzw. 40.000 Einwohnern (dunkelgrau) gewonnen (s. Abb. 4-1). Dies entspricht einem eher ländlichen und kleinstädtischen Lebensraum. Migrationshintergrund für mehr als drei Schüler pro Klasse bestand nur in den Klassen K 5 und K 6, vornehmlich durch Zuzug aus russischsprachigen Ländern.

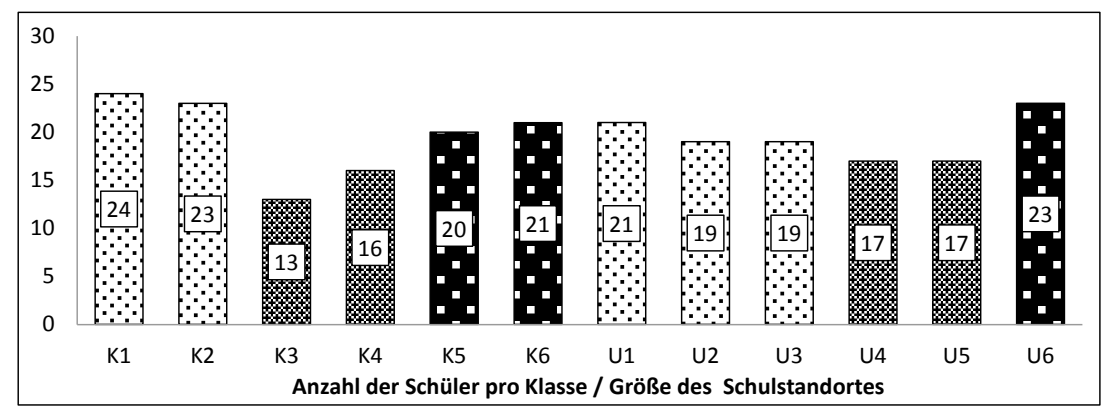

Abb. 4-1: Klassenstärken und Schulstandorte der involvierten Klassen 
Auffallend war die geringe Klassenstärke der Klassen K 3 und K 4. Diese wurde hervorgerufen durch Umschulung mehrerer Schüler in sogenannte Klassen zur speziellen Förderung einer diagnostizierten Lese-Rechtschreib-Schwäche bzw. durch den Wegzug von einigen Schülern. Die Klassenstärke war z.T. auch eine Folge der Darstellung: Schüler, die nach Beginn des Untersuchungszeitraumes neu in die Klassen kamen, wurden nicht aufgeführt. Die Klasse K 3 umfasste zum Zeitpunkt des Nachtestes die dargestellten neun Schüler sowie zwei Schüler, die neu hinzugekommen waren.

Die Studie startete mit 233 Schülerinnen und Schülern in 12 Klassen sowie acht Lehrkräften für das Fach Musik. Jeweils zwei Kontrollklassen wurden von derselben Lehrkraft (LK) unterrichtet. Die Klassen K 1 und K 2 durch die LK-A, Klassen K 3 und 4 durch LK-B, Klassen K 5 und 6 durch LK-C. In der Untersuchungsgruppe erhielten nur die Klassen U 4 und U 5 bei einer Lehrkraft Unterricht (s. Abb. 4-2). Etwa in der Mitte des Untersuchungszeitraums erkrankte die Lehrkraft LK-H. Das Treatment konnte in dieser Klasse nicht fortgesetzt werden. Um die Teststärke nicht zu gefährden, kam es zu einem Austausch dieser Gruppe. Die im gleichen Zeitraum bei der Autorin unterrichteten zwei dritten Klassen kamen dafür in Frage. Da sie ebenfalls den Vortest durchlaufen hatten und das Treatment erhielten, stellte dies kein größeres Problem dar. Da die neu hinzugekommene Klasse U 7 mehr Probanden als die Klasse U 6 enthielt, erhöhte sich die Probandenzahl leicht. Eine ca. sechs Wochen dauernde Erkrankung der Lehrkraft LK-E gab es im Dezember und Januar. Die in diesem Zeitraum liegenden Musikstunden wurden durch die Autorin vertreten (s. Anlage D 1). In einigen Klassen kam es schon kurz nach Schulstart zu Rückstellungen von Schülern nach Klasse 2 sowie zu Weg- und Zuzügen, so dass sich die Gesamtzahlen der Schülerinnen und Schüler bis zum Ende der Untersuchung geringfügig änderten.

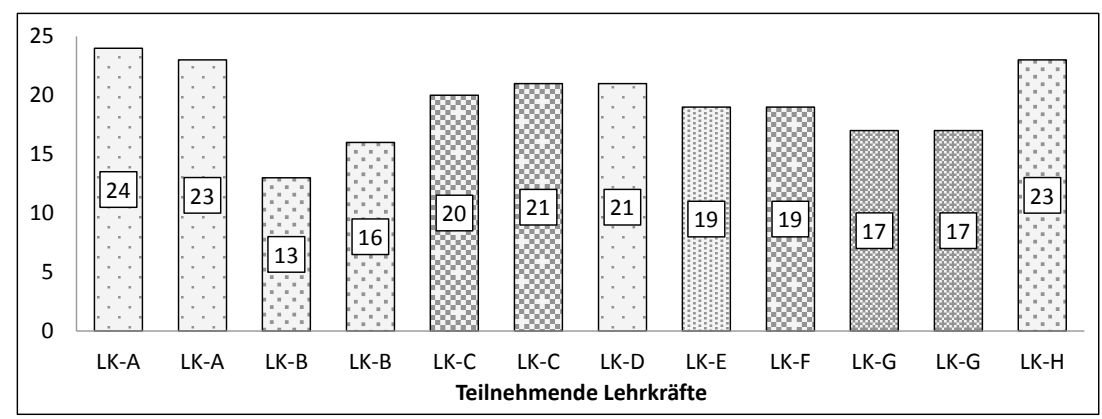

Abb. 4-2: Zuordnung der Klassen zu Lehrkräften

Die Gesamtgröße der Kontrollgruppe betrug 117, die der Untersuchungsgruppe 116 Schülerinnen und Schüler. In der Untersuchungsgruppe konnten 82, in der Kontrollgruppe 67 Probanden gewonnen werden (s. Abb. 4-3). Von den insgesamt 149 Probanden wurden komplette Datensätze erhoben. Nur im Subtest F kam es im Vor- und Nachtest in verschiedenen Klassen zu einem krankheitsbedingten Ausfall bei vier ( 3 x UG, 1 x KG) bzw. drei Probanden (3 x UG). Die fehlenden 
Ergebnisse wurden auf 0 gesetzt, auch wenn dies im Vergleich zu Lasten der Untersuchungsgruppe ging.

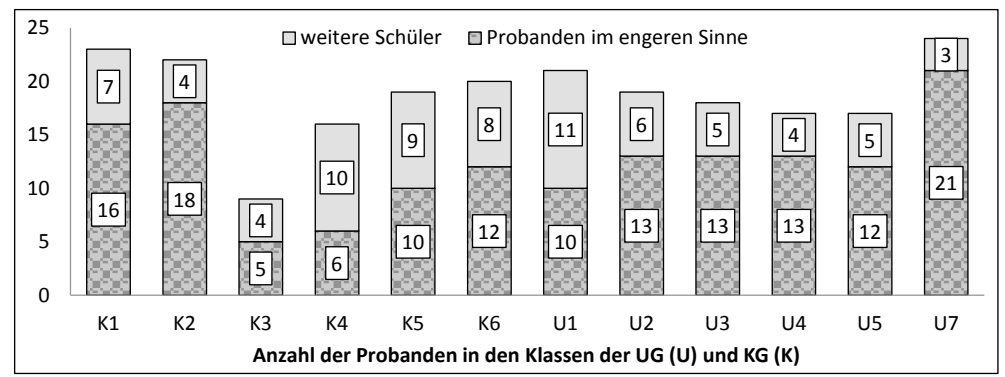

Abb. 4-3: Anzahl der Probanden zum Zeitpunkt des Nachtests

Das Alter der meisten Probanden lag zu Beginn des Untersuchungszeitraumes zwischen 8 Jahren, 3 Monaten und 9 Jahren, 2 Monaten (s. Abb. 4-4). Die jüngste Probandin war 7 Jahre, 04 Monate alt. Sie wurde auf Wunsch der Eltern ein Jahr früher eingeschult. Zwei Schüler waren deutlich über 10 Jahre alt, sie wurden aus Migrationsgründen in niedrigere Klassenstufen eingeordnet. Einige andere Schüler wiederholten bereits ein Schuljahr und waren deshalb etwas älter als der Durchschnitt. Dies traf häufiger auf Probanden aus der Kontrollgruppe als aus der Untersuchungsgruppe zu.

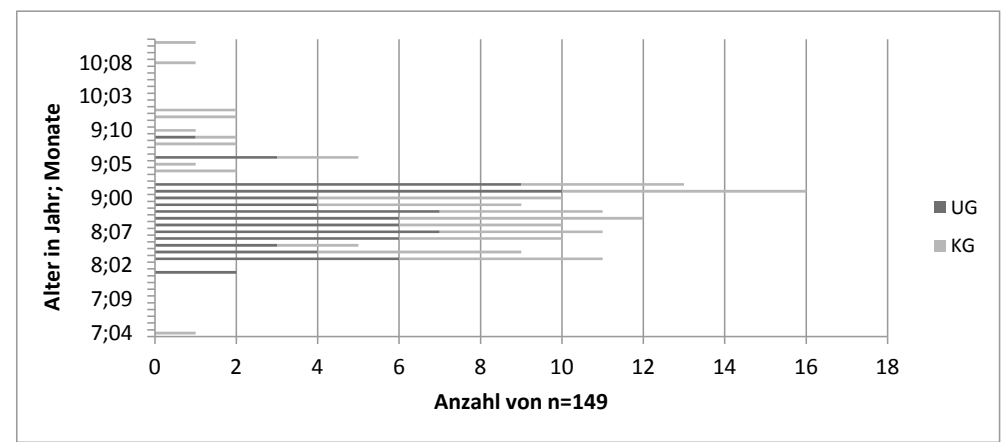

Abb. 4-4: Alter der Probanden

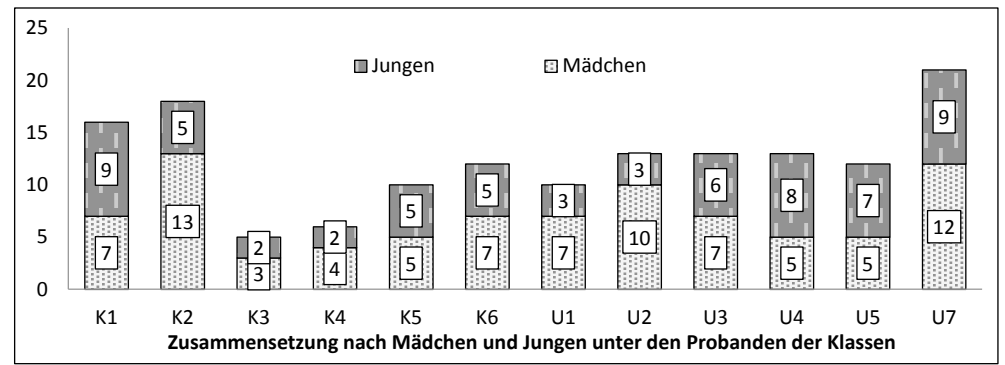

Abb. 4-5: Anteil an Jungen und Mädchen unter den Probanden 
Insgesamt lernten 123 Mädchen und 110 Jungen in den zwölf Klassen. Diese nahezu gleichmäßige Verteilung war unter den 149 Probanden mit 85 Mädchen und 64 Jungen nicht ganz gegeben (s. Abb. 4-5).

Instrumentalunterricht erhielten 35 Schülerinnen und Schüler im zweiten bzw. dritten Unterrichtsjahr, weitere 13 Schülerinnen und Schüler im ersten Unterrichtsjahr. Den früher einmal begonnenen Unterricht hatten zehn Schüler bereits beendet. Die involvierten Instrumentalschüler verteilten sich sehr verschieden in den einzelnen Klassen (s. Abb. 4-6), jedoch annähernd gleich auf Untersuchungs- und Kontrollgruppe (UG: $22 \%$; KG: $21 \%$ ). Von den zuerst genannten 35 Schülerinnen und Schüler im zweiten bzw. dritten Unterrichtsjahr gehören drei nicht zur Gruppe der Probanden. Sie entstammen den Klassen K 3 und K 4.

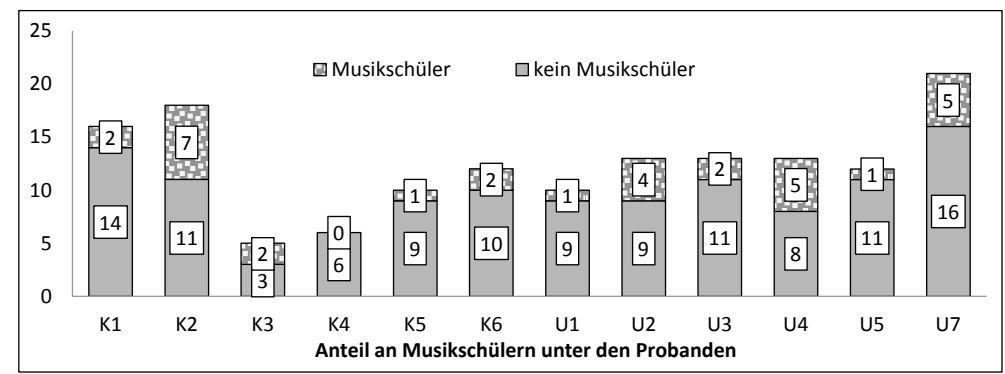

Abb. 4-6: Verteilung von Instrumentalschülern unter den Probanden

\subsubsection{Bewertung der gezogenen Stichprobe}

Die gezogene Stichprobe von 149 Probanden aus den insgesamt 12 teilnehmenden Klassen mit 233 Schülerinnen und Schülern war groß genug, um eine Effektstärke von $d$ größer oder gleich 0.5 zu ermitteln. Mit den 82 Probanden in der Untersuchungs- und den 67 Probanden in der Kontrollgruppe konnte eine Teststärke (Power: $1-\beta$ ) von 0.92 erzielt werden (s. Anlage C 1). Dieser Wert darf als zufriedenstellend betrachtet werden (Bortz \& Döring, 2006 ${ }^{4}$, S. 501). Die Itemschwierigkeiten und Trennschärfen der Items lagen im anvisierten Bereich.

Dennoch wurde die gezogene Stichprobe in ihrer Zusammensetzung als teilweise unbefriedigend betrachtet. Der Anteil der Probanden zur Klassenstärke schwankte in den einzelnen Klassen zwischen $38 \%$ und $88 \%$ (s. Tab. 4-1). Der Anteil an Musikschülern lag zwischen $7 \%$ und $41 \%$. Damit bestanden sehr ungleiche Verhältnisse zwischen den Probandengruppen der einzelnen Klassen. Aufgrund der bestehenden Rechtslage war die Auswahl der Probanden jedoch nicht anders handhabbar (s. Abschnitt 3.2).

Als missglückt wurde die Probandenwerbung in den Klassen K 3 und K 4 gewertet. Sie weisen die niedrigsten prozentualen Probandenzahlen (38\% und $45 \%$ ) auf (s. Tab. 4-1). Nur in diesen beiden Klassen zählten jeweils ein bzw. zwei Schülerinnen und Schüler mit außerschulischem Instrumentalunterricht nicht zur Gruppe der Probanden. Dies war in keiner anderen Klasse der Fall. 
Diese Tatsache war möglicherweise eine Folge der fehlenden Präsenz beim Elternabend in diesen beiden Klassen. Durch eine Häufung von elf Elternabenden an vier Abenden einer Woche und einem Planungsfehler der Fahrtroute an einem dieser Tage, konnte der Besuch der Elternabende bei den Klassen K 3 und K 4 nicht realisiert werden (s. Anlage D 1). Die Elternabende wurden zum Zwecke der Erläuterung des Forschungsvorhabens durchgeführt.

Tab. 4-1: Übersicht über ausgewählte Eckdaten zu Probanden

\begin{tabular}{cccccc}
\hline Klasse & $\begin{array}{c}\text { Anzahl } \\
\text { Schüler pro } \\
\text { Klasse } \\
\text { absolut }\end{array}$ & $\begin{array}{c}\text { Anzahl } \\
\text { Probanden } \\
\text { absolut }\end{array}$ & $\begin{array}{c}\text { Anteil } \\
\text { Probanden } \\
\text { in Prozent }\end{array}$ & $\begin{array}{c}\text { Anzahl } \\
\text { Musik- } \\
\text { schüler } \\
\text { absolut }\end{array}$ & $\begin{array}{c}\text { Anteil } \\
\text { Musikschüler } \\
\text { in Prozent }\end{array}$ \\
\hline U1 & 20 & 10 & $50 \%$ & 7 & $35 \%$ \\
U2 & 18 & 13 & $72 \%$ & 7 & $39 \%$ \\
U3 & 17 & 13 & $76 \%$ & 3 & $18 \%$ \\
U4 & 16 & 13 & $81 \%$ & 6 & $38 \%$ \\
U5 & 15 & 12 & $80 \%$ & 1 & $7 \%$ \\
U7 & 24 & 21 & $88 \%$ & 6 & $25 \%$ \\
K1 & 23 & 16 & $70 \%$ & 5 & $22 \%$ \\
K2 & 22 & 18 & $82 \%$ & 9 & $41 \%$ \\
K3 & 11 & 5 & $45 \%$ & 4 & $36 \%$ \\
K4 & 16 & 6 & $38 \%$ & 3 & $19 \%$ \\
K5 & 19 & 10 & $53 \%$ & 5 & $23 \%$ \\
K6 & 20 & 12 & $60 \%$ & 3 & $15 \%$ \\
\hline MW & 18,4 & 12,4 & $66 \%$ & 4,9 & $26 \%$ \\
\hline
\end{tabular}

\subsubsection{Ablauf des Untersuchungszeitraumes}

Der Vortest fand in den Untersuchungsklassen vor den Herbstferien des Schuljahres 2013/14 statt (s. Abb. 4-7). In den Herbstferien konnte eine erste Auswertung stattfinden und relativ schnell ein Überblick über den Stand der Leistungen gewonnen werden. Den Lehrkräften der Klassen in der Untersuchungsgruppe wurden Hinweise zur Zusammenstellung der Lerngruppen (s. Abschnitt 3.3.7) gegeben. Nach den Ferien fand der Vortest in den Klassen der Kontrollgruppe statt. Im Nachtest wurden wiederum zuerst die Probanden aus den Klassen der Untersuchungsgruppe getestet, um zwischen den Testterminen in etwa gleiche Abstände zu erhalten.

\begin{tabular}{|c|c|c|c|c|c|c|c|c|c|c|c|c|c|c|c|c|c|c|c|c|}
\hline 35 & 36 & 37 & 38 & 39 & 40 & 41 & 42 & 43 & 44 & \multicolumn{2}{|r|}{ Kalenderwochen 45 - 15} & 16 & 17 & 18 & 19 & 20 & 21 & 22 & 23 & 24 \\
\hline & & \multicolumn{19}{|c|}{$\begin{array}{l}\text { Eltern- } \\
\text { abende }\end{array}$} \\
\hline \multicolumn{6}{|c|}{$\begin{array}{l}\text { Vortest: Schriftlicher Test in } \\
\text { allen Klassen }\end{array}$} & & & \multirow{3}{*}{\multicolumn{2}{|c|}{ 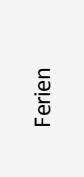 }} & & & & \multirow{3}{*}{$\begin{array}{l}\frac{0}{\frac{d}{d}} \\
\frac{\mathrm{U}}{4}\end{array}$} & \multicolumn{7}{|c|}{$\begin{array}{l}\text { Nachtest: Schriftlicher Test in } \\
\text { allen Klassen }\end{array}$} \\
\hline & & \multicolumn{6}{|c|}{$\begin{array}{l}\text { Vortest: Mündlicher Test in } \\
\text { Untersuchungsklassen }\end{array}$} & & & 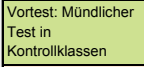 & & & & \multicolumn{7}{|c|}{$\begin{array}{l}\text { Nachtest: Mündlicher Test in } \\
\text { allen Klassen }\end{array}$} \\
\hline & & & & & & & & & & \multicolumn{3}{|c|}{ Treatment in den Untersuchungsklassen } & & & & & & & & \\
\hline
\end{tabular}

Abb. 4-7: Zeitplan der Hauptuntersuchung im Schuljahr 2013/14 
Im Untersuchungszeitraum erhielten die Untersuchungs- und Kontrollklassen die regulären zwei Stunden Musikunterricht. Das Treatment wurde in den regulären Unterricht implementiert. Die Klassen U 4 und U 5 erhielten den Musikunterricht als Doppelstunde. Die Klassen K 1 und K 2 erhielten zusätzlich 14-tägig eine Stunde Unterricht im Flötenspiel. Dies wurde ohne vorherige Absprache realisiert, fand aber hochmotiviert durch die Lehrkraft LK-A statt. Dennoch wurde eine Beeinträchtigung des Forschungsdesigns befürchtet, da die Bedingung - nur regulärer Musikunterricht - in der Kontrollgruppe für 34 der 62 Probanden nicht mehr zutraf. Deshalb wurde eine zweite Erhebung zusätzlich geplant und durchgeführt.

\subsubsection{Zweite Erhebung}

Schulrechtlich war es nicht möglich, nach Abschluss des Untersuchungszeitraums weitere Schulklassen zur Mitwirkung in der Kontrollgruppe aufzufordern. Deshalb wurde ein gesonderter Antrag auf eine zweite Erhebung gestellt. Ziel war es, die Leistungen der Probanden der Kontrollgruppe zumindest in Bezug auf den Subtest F mit einer größeren Probandengruppe zu vergleichen. So wurde der schriftliche Test als Messinstrument verwendet, wie er auch in der Hauptuntersuchung für Subtest F zur Anwendung kam. Die zweite Erhebung fand von Mai bis Juni 2015 an sieben Grundschulen mit den jeweiligen dritten und vierten Klassen statt $(n=549)$. Dieser Zeitpunkt kurz vor dem Ende des Schuljahres entsprach in etwa dem Zeitpunkt des Nachtestes der Hauptuntersuchung im Schuljahr davor. Die beteiligten Schulen wurden von der zuständigen Referentin der Sächsischen Bildungsagentur Regionalstelle Bautzen ausgewählt. Sie lagen ähnlich den involvierten Schulklassen der Hauptuntersuchung im ländlichen und kleinstädtischen Lebensraum.

\subsubsection{Zusammenarbeit mit den Lehrkräften}

Mit den involvierten Lehrkräften der Untersuchungsgruppe fand am 26.09.13 eine erste Fortbildung zum Kennenlernen der Methodik des Rhythmus-Lehrgangs sowie seiner Begleitmaterialien statt. Eine zweite Fortbildung fand am 07.11.13 zum Kennenlernen der weiteren Unterrichtsinhalte aus den Bereichen Singen, Bewegen und Tanzen sowie Musikhören statt. In einer Abschlussveranstaltung am 26.06.14 wurden die vorläufigen Ergebnisse der Evaluation vorgestellt sowie ein Feedback zur Durchführung des Treatments erbeten. Von den Untersuchungsklassen kamen alle Lehrkräfte, von den Kontrollklassen eine Lehrkraft (LK-B) zur Veranstaltung.

Die Lehrkräfte konnten jederzeit um Rat bitten. Die Versuchsleiterin führte parallel den Rhythmus-Lehrgang um 14 Tage vorversetzt in ihren 3. Klassen durch. Bei allen Lehrkräften der Untersuchungsklassen wurden ein bis zwei Hospitationen durchgeführt (s. Anlage D 1). Dies ermöglichte einen Abgleich von Innen- und Außensicht auf den erteilten Unterricht und ließ eine gewisse Kontrolle der Durchführung des Treatments zu. Es bestand für alle Lehrkräfte das Angebot, im Gegenzug auch im Musikunterricht der Versuchsleiterin zu hospitieren. Wahrgenommen wurde dies von den Lehrkräften LK-C und LK-E (s. Abb. 4-2). 


\subsection{Ergebnisse im Vortest und Nachtest}

Das Gefälle zwischen den individuell erzielten Punkten in der Gesamtpunktwertung im Vortest ist gravierend: 25 Probanden können im Vortest keinen Punkt erzielen (s. Abb. 4-8). Ein Proband aus der Untersuchungsgruppe sowie eine Probandin aus der Kontrollgruppe erzielen dagegen 20 bzw. 19 von 24 Punkten.

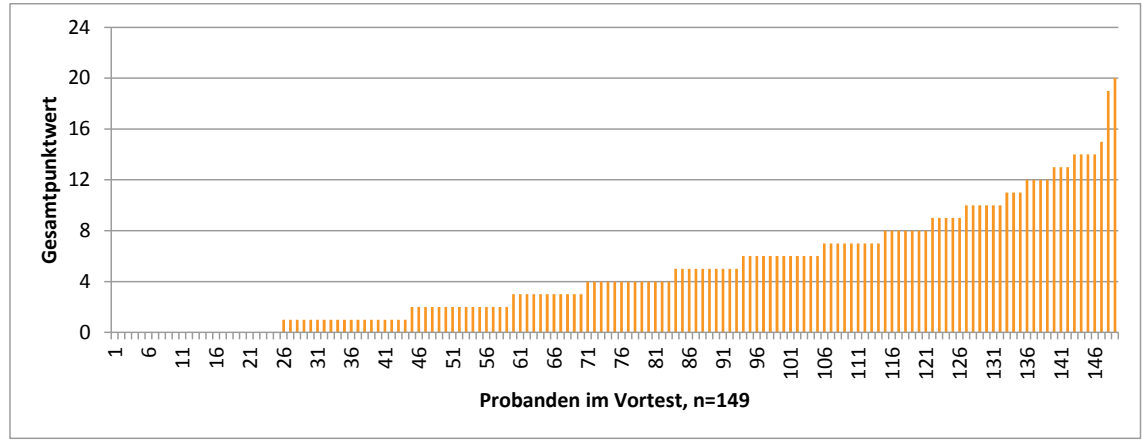

Abb. 4-8: Ergebnisse in der Gesamtpunktwertung aller Probanden im VT
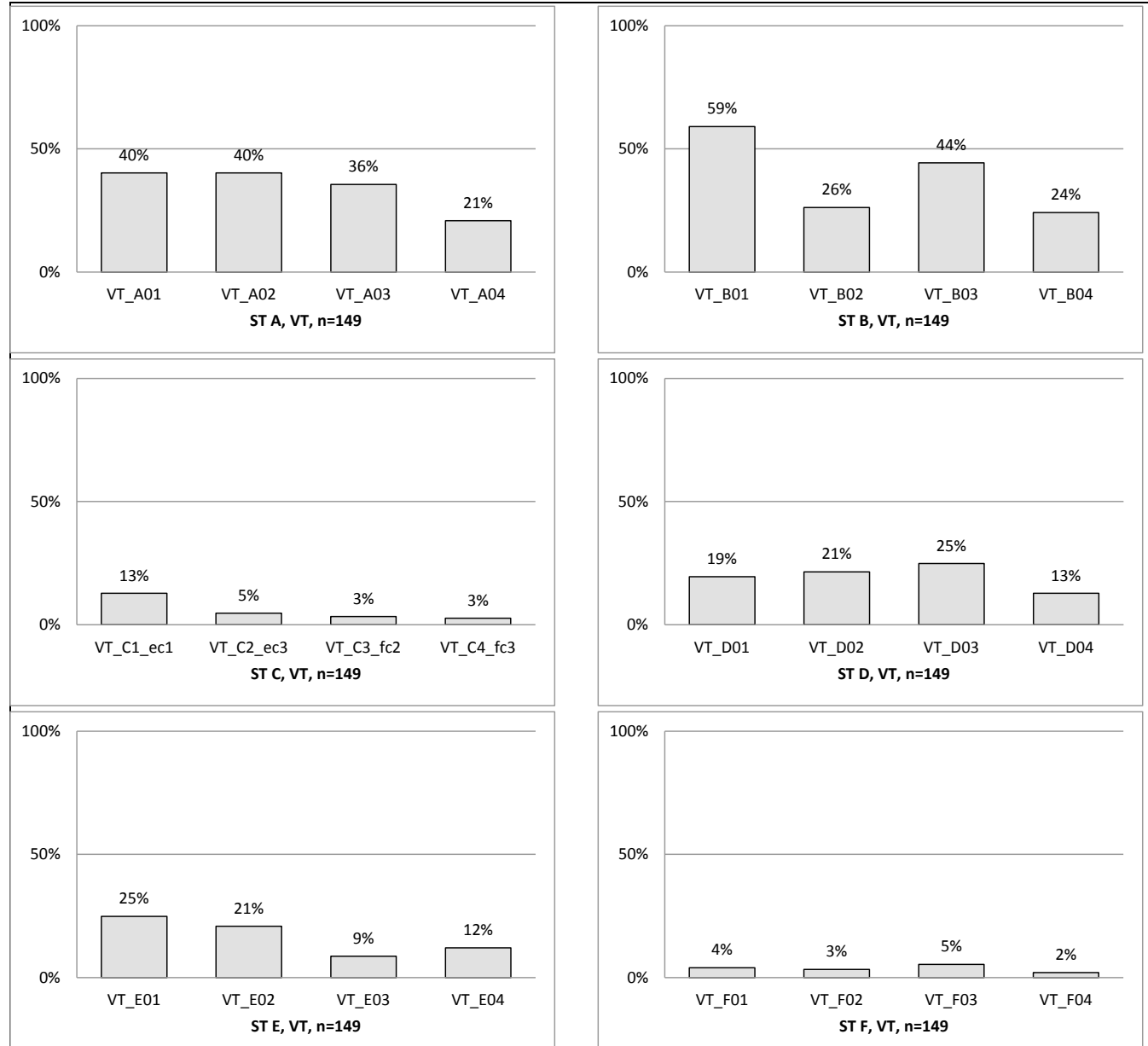

Abb. 4-9: Anteile an richtigen Lösungen in den Items im VT 
Der Mittelwert in Bezug auf die Gesamtpunktwertung liegt im Vortest bei etwa 4 von 24 Punkten. Alle erreichbaren Punktwerte sind relativ gleichmäßig vertreten (s. auch Anlage C 2). In den Subtests $\mathrm{A}$ und $\mathrm{B}$ sowie D und $\mathrm{E}$ können schon deutlich erkennbare Anteile an richtigen Lösungen pro Item verzeichnet werden (s. Abb. 4-9). Für die Subtests C und F trifft das nicht zu, hier werden nur sehr wenige richtige Lösungen erzielt.

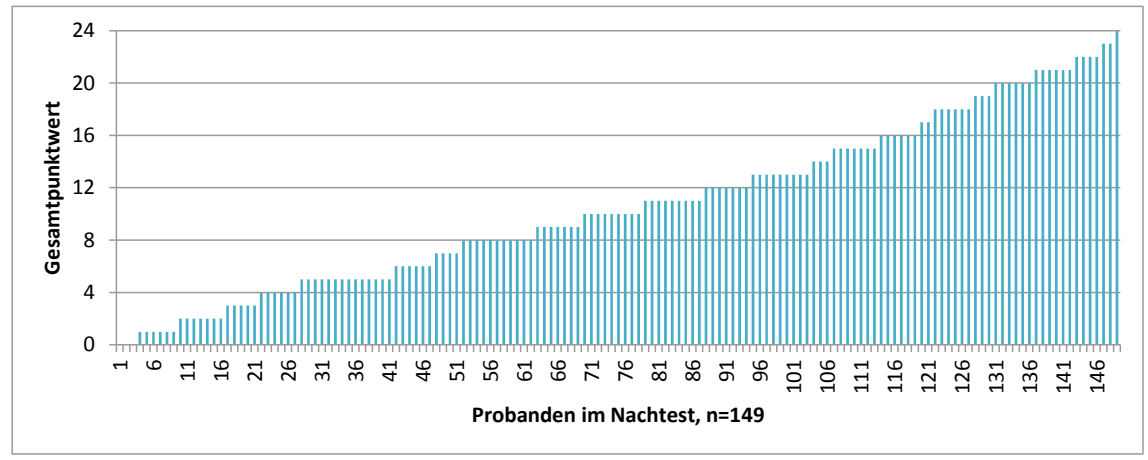

Abb. 4-10: Ergebnisse in der Gesamtpunktwertung aller Probanden im NT

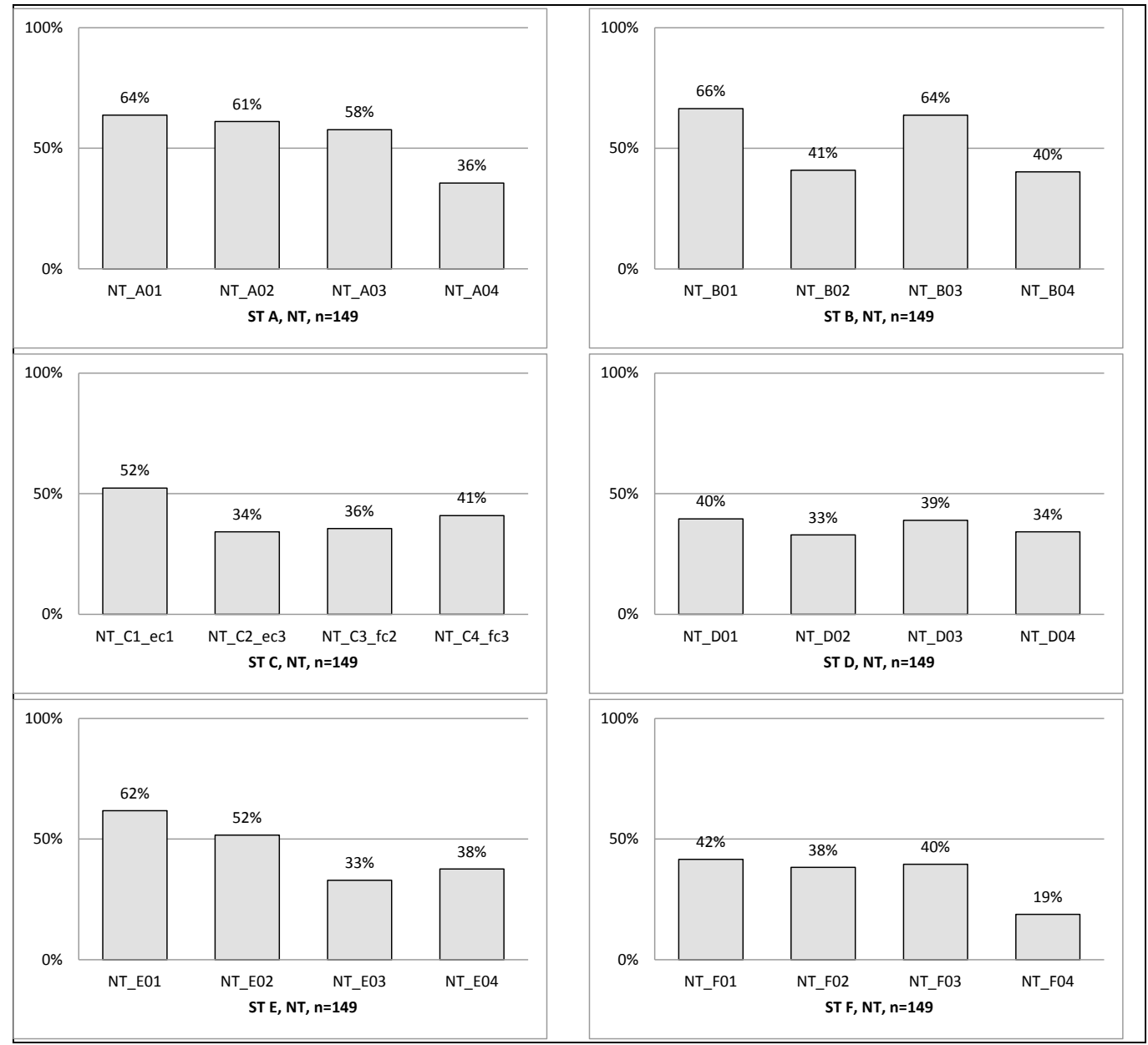

Abb. 4-11: Anteile an richtigen Lösungen in den Items im NT 
Im Nachtest ist das Gefälle zwischen den individuell erzielten Werten in der Gesamtpunktwertung deutlich ausgeglichener (s. Abb. 4-10). Zwar können drei Probanden immer noch keinen Punkt erzielen, doch erreichen 19 Probanden 20 bis 24 Punkte. Unter diesen 19 Probanden sind fünf Schüler aus der Untersuchungsgruppe, die keinen außerschulischen Instrumentalunterricht erhielten. Für die Anteile an richtigen Lösungen in den einzelnen Items ist auffallend, dass sich die Ergebnisse zueinander angeglichen haben (s. Abb. 4-11).

\subsection{Ergebnisse in den Subtests A bis F}

Die Ergebnisse zu den einzelnen Subtests werden in der Chronologie der Erhebung dargestellt. Diese begann mit dem schriftlichen Subtest F. Anschließend wurden die Subtests A, B und D im Videotest erhoben. Nach dem Videotest fand die Durchführung des Subtests E statt. Subtest C wird rein rechnerisch aus den Untertests von Subtest F und E generiert. Deshalb wird dieser Subtest zuletzt aufgeführt. In den Darstellungen wird jeweils die Zugehörigkeit zur Untersuchungs- oder Kontrollgruppe berücksichtigt.

Für jeden Subtest werden zuerst die Anteile an richtigen Lösungen pro Item des Subtests, die Mittelwerte des Subtests sowie die Berechnung des t-Tests und der Effektstärke dargestellt. Im Anschluss werden Analysen häufiger Fehler und deren mögliche Ursachen dargestellt. Wenn diese Fehleranalyse große Unterschiede zwischen Untersuchungs- und Kontrollgruppe aufzeigt, wird sie auch getrennt dargestellt. Im Unterabschnitt Abgestufte Ergebnisdarstellung werden alle Ergebnisse, die erzielt wurden in verschiedenen Lösungskategorien dargestellt. In einigen Subtests werden Erscheinungen innerhalb der Fülle der Ergebnisse vertiefend betrachtet. Sie werden jeweils zum Abschluss der Darstellungen des entsprechenden Subtests aufgeführt.

\subsubsection{Ergebnisse im Subtest F Rhythmen musizieren und aufschreiben}

In die Gesamtpunktwertung des Testes geht nur der Testteil F Rhythmen musizieren und aufschreiben mit Noten ein. Getestet wird das Aufschreiben von vier Rhythmen, die zuvor im CallResponse-Prinzip gemeinsam musiziert werden (s. Abb. 4-12).

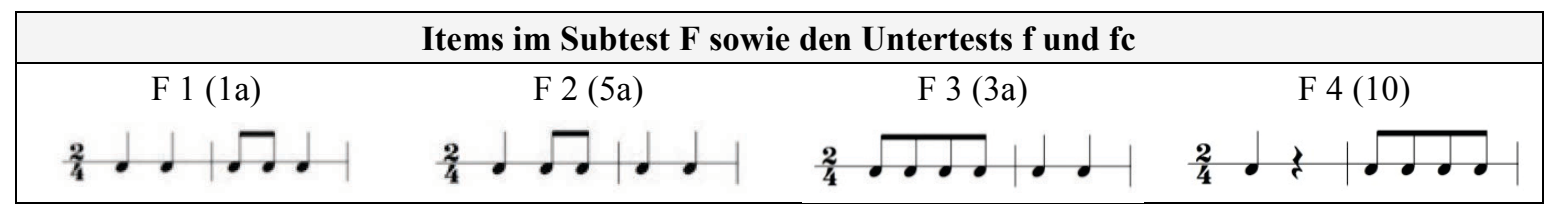

Abb. 4-12: Übersicht über die Items im ST F

Im Vortest erzielen bis zu $7 \%$ der Probanden richtige Ergebnisse in den vier Items (s. Abb. 4-13). Diese Probanden entstammen zum größten Teil der Gruppe der Schülerinnen und Schüler mit auBerschulischem Instrumentalunterricht. 
Betrachtet man gezielt die Gruppe der insgesamt 32 Schülerinnen und Schüler, die mindestens ein bis zwei Jahre Instrumentalunterricht erhalten, so gelingt etwa zwei Dritteln keines der vier Items (23 Schüler; UG: 11; KG: 12). Vier Schülern gelingt je ein Item (UG: 3; KG: 1), zwei Schülern je zwei Items (UG: 2; KG: 0), drei Schülern je drei Items (UG: 2; KG: 1) sowie keinem Schüler alle vier Items.
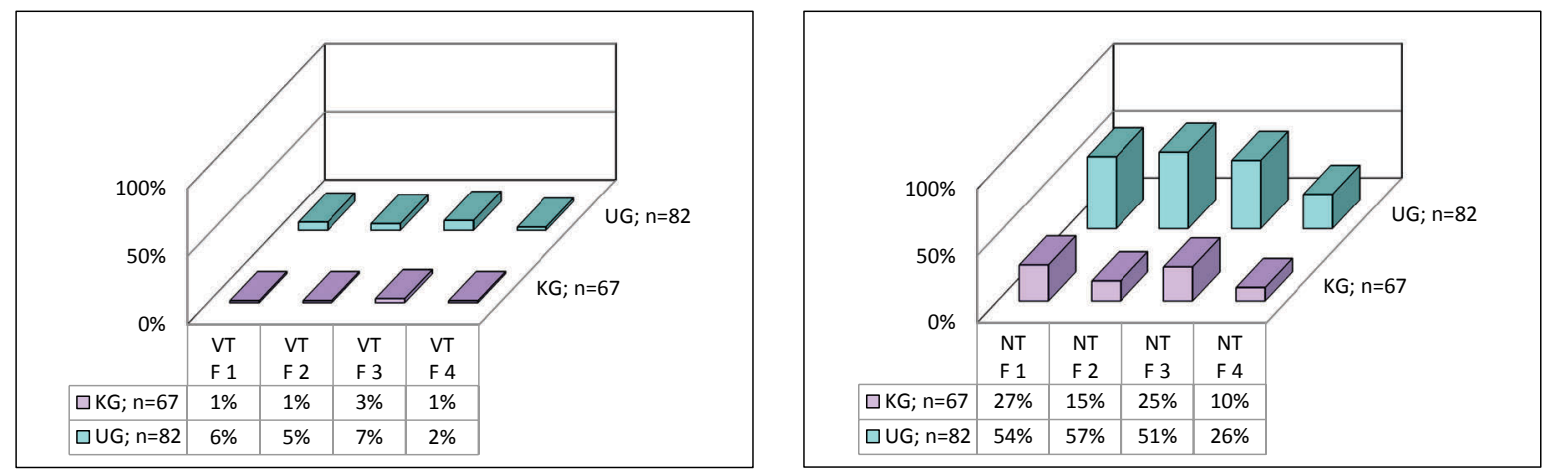

Abb. 4-13: Ergebnisse, ST F im VT und NT, getrennt nach UG und KG

Im Nachtest erreichen bis zu $57 \%$ der Probanden der Untersuchungsgruppe richtige Ergebnisse. Item F 4 mit der Pause auf Schlag 2 ist das schwerste Item. Die Ergebnisse der Probanden der Untersuchungsgruppe zeigen im Nachtest einen gewaltigen Vorsprung. Die Ergebnisse der Probanden der Kontrollgruppe für sich genommen weisen ebenfalls große Fortschritte gegenüber dem Vortest auf. In der Gruppe der 32 Instrumentalschüler gelingt im Nachtest sieben Schülern keines der vier Items (UG: 4; KG: 3). Vier Schülern gelingen je ein Item (UG: 0; KG: 4), drei Schülern je zwei Items (UG: 0; KG: 3), acht Schülern je drei Items (UG: 5; KG: 3) sowie zehn Schülern alle vier Items (UG: 9; KG: 1).

Tab. 4-2: Mittelwerte im ST F

\begin{tabular}{lllccccc}
\hline \multirow{2}{*}{ ST } & $\begin{array}{l}\text { UG } \\
\text { KG }\end{array}$ & N & $\begin{array}{c}\text { MW im } \\
\text { VT }\end{array}$ & $\begin{array}{c}\text { MW im } \\
\text { NT }\end{array}$ & $\begin{array}{c}\text { Diff. } \\
\text { VT/NT }\end{array}$ & $\begin{array}{c}\text { SD der } \\
\text { Diff. }\end{array}$ & $\begin{array}{c}\text { SE der } \\
\text { Diff. }\end{array}$ \\
\hline \multirow{2}{*}{ F } & UG & 82 & 0.21 & 1.88 & 1.67 & 1.46 & 0.16 \\
& KG & 67 & 0.07 & 0.78 & 0.70 & 0.94 & 0.11 \\
\hline
\end{tabular}

Tab. 4-3: Ergebnisse für t-Test und Effektstärke im ST F

\begin{tabular}{ccccccc}
\hline ST & $\mathbf{t}$ & df & $\mathbf{p}$ (2seit.) & $\begin{array}{c}\text { Diff. der } \\
\text { MW }\end{array}$ & $\begin{array}{c}\text { SE der Diff. } \\
\text { der MW }\end{array}$ & d [95\% CI] \\
\hline F & 4.70 & 147 & $<0.001$ & 0.97 & 0.21 & $0.77[0.56,1.38]$ \\
\hline
\end{tabular}

Die Mittelwerte im Nachtest steigen in beiden Gruppen an (s. Tab. 4-2). Die Differenzen der Mittelwerte aus Vor- und Nachtest unterscheiden sich zwischen Untersuchungs- und Kontrollgruppe 
enorm. Die berechnete Effektstärke $d$ weist dies mit einem Wert von 0.77 aus, einem nahezu großen Effekt (s. Tab. 4-3).

\section{Fehlerquellen im Subtest F}

Da im Vortest von Subtest F nur wenige richtige Lösungen erzielt werden, können in der Fehleranalyse keine Hauptfehlerquellen erkannt werden. In der Fülle der deutlich gestiegenen Anzahl der Lösungen im Nachtest kristallisieren sich nun auch deutlich gehäufte Fehlerquellen heraus, die sich in den beiden Gruppen unterscheiden und deshalb getrennt aufgeführt werden (s. Abb. 4-14).

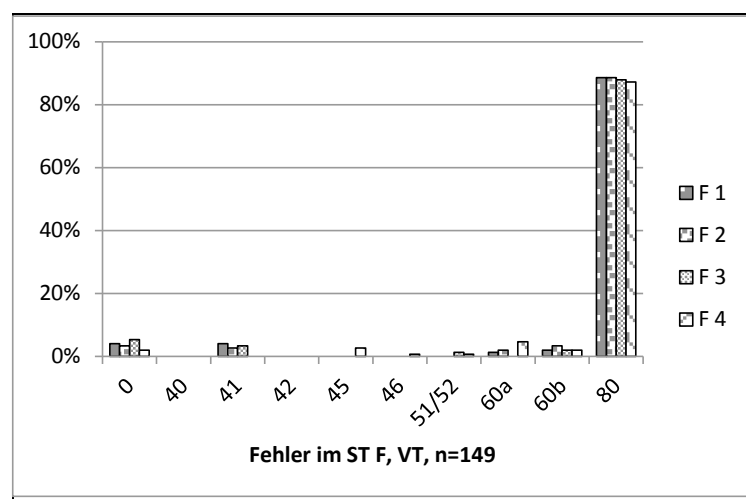

0 richtige Darstellung (metrischer Typus)

40 Symbole für Achtel- und Viertelschläge durchgehend vertauscht

41 Symbole für Achtel- und Viertelschläge einmalig vertauscht (figuraler Typus)

42 zwei Achtelschläge in einer Note dargestellt 45 fehlendes Pausenzeichen in Item 4

46 Notenköpfe nicht ausgemalt

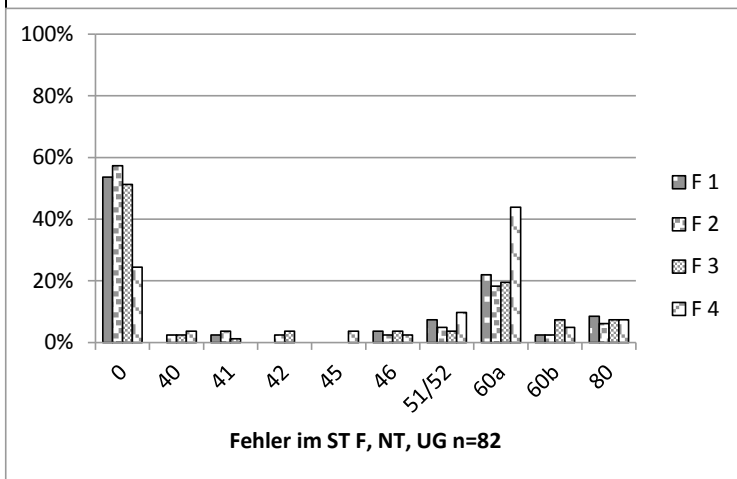

51 Symbol zu wenig notiert 52 Symbol zu viel notiert 60a fehlerhafte Darstellung ohne spezielle

Fehlerkategorie (Anzahl der Symbole korrekt)

60b fehlerhafte Darstellung ohne spezielle

Fehlerkategorie (Anzahl der Symbole nicht korrekt)

$\mathbf{8 0}$ keine Lösung angeboten

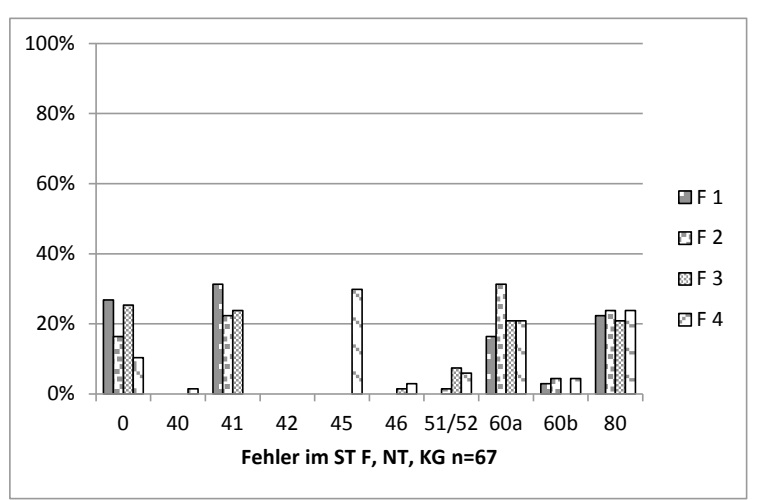

Abb. 4-14: Häufigkeit von Fehlern im ST F, VT und NT, getrennt nach UG und KG

Dazu zählen das fehlerhafte Anwenden von Notensymbolen (Kat. 40-46) wie z. B. das Vertauschen der Symbole für Achtel- und Viertelschlag (Kat. 40, 41) oder das Nicht-Ausmalen von Notenköpfen (Kat. 46). Ebenfalls wird beobachtet, dass zu viele bzw. zu wenige Schläge wahrgenommen werden, was zu einem fehlerhaften Notenbild führt (Kat. 51-52). Zusätzlich gibt es eine unspezifische Fehlerkategorie 60, bei der die vorher benannten Fehlerquellen nicht zutreffen. Sie wird unterschieden in die Kategorien 60 a und b, je nachdem ob die Anzahl der Symbole der Anzahl der 
Schläge entspricht oder nicht. In der Untersuchungsgruppe dominiert keine Fehlerquelle. In der Kontrollgruppe fallen besonders das einmalige Vertauschen der Symbole für Achtel- und Viertelschlag (Kat. 41) sowie die fehlerhafte Darstellung der Pause in Item 4 auf (Kat. 45) auf. Das einmalige Vertauschen der Symbole für Achtel- und Viertelschlag wird im übernächsten Abschnitt genauer betrachtet.

\section{Abgestufte Ergebnisdarstellung}

Alle Lösungen werden nun in abgestuften Kategorien von Lösung korrekt bis Lösung stark fehlerhaft dargestellt (s. Abschnitt 3.5.2, dritte Rubrik). Dabei entspricht die Zuordnung in die vier Lösungskategorien den vier Typen des Notierens von Rhythmen. Den Lösungskategorien werden die Fehlerkategorien (s. Abb. 4-14) je nach Typus zugeordnet (s. Abb. 4-15). Im Vortest dominiert die Kategorie Lösung stark fehlerhaft bzw. keine Lösung mit einem Anteil von etwa 90 \% der Lösungen. Die Anteile der Ergebnisse in den drei anderen Kategorien liegen zusammen bei etwa $10 \%$. Auf die Kategorie Lösung korrekt entfallen lediglich zwischen $2 \%$ bis 5 \% der Lösungen.

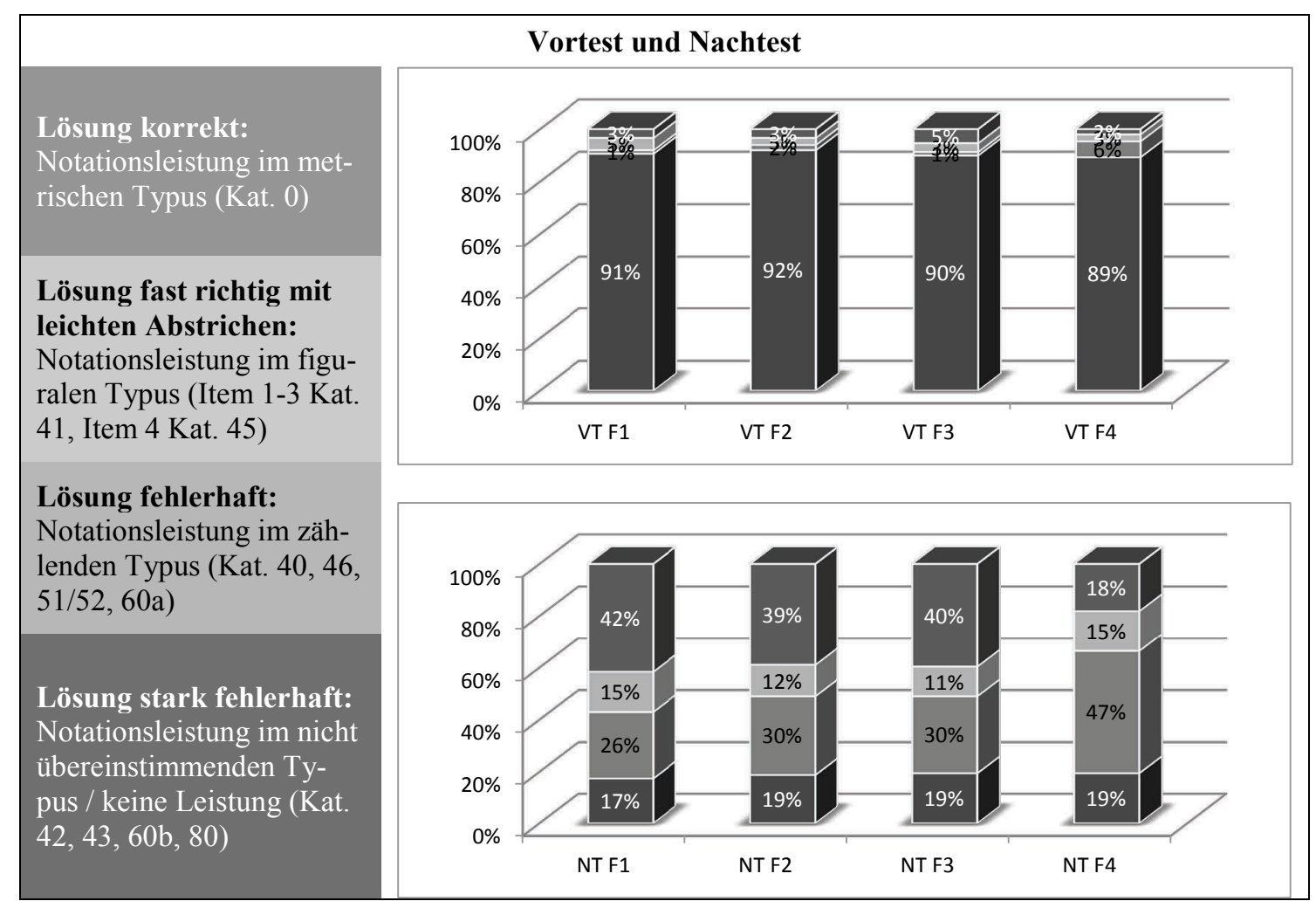

Abb. 4-15: Gesamtdarstellung aller Leistungen im ST F nach Lösungskategorien

An den Ergebnissen des Nachtestes lässt sich der enorme Leistungszuwachs innerhalb von 20 Unterrichtswochen in Bezug auf die Zuordnung zu den vier Lösungskategorien sehr gut ablesen. Die Gruppe der Probanden, die die Items im metrischen Typus (Lösung korrekt) sowie im figuralen Typus (Lösung fast richtig mit leichten Abstrichen) aufschreiben können, umfasst für die Items F 1 bis F 3 einen Anteil von mehr als 50 \%. In Item F 4 sinkt dieser Anteil auf unter 50 \%. Es schlägt 
die Tatsache zu Buche, dass das Symbol für die Viertelpause vielen Probanden nicht vertraut ist. Im zählenden Typus (Lösung fehlerhaft) notieren zwischen $26 \%$ bis $47 \%$ der Probanden. Etwa ein Achtel der Probanden notiert im nicht übereinstimmenden Typus oder erbringt noch keine Lösungen (Lösung stark fehlerhaft).

\section{Einmaliges Vertauschen der Notensymbole}

Das besonders in der Kontrollgruppe beobachtete einmalige Vertauschen der Symbole für Achtelund Viertelschlag (Kat. 41) tritt besonders gehäuft in den Items F 1, F 2 und F 3 auf (s. Abb. 4-14). Dieses Vertauschen findet immer an derselben Stelle im Rhythmus statt, nämlich stets bei der ersten Viertelnote nach zwei Achtelnoten. Dieses Phänomen wurde bereits von Bamberger beobachtet, speziell für den Rhythmus in Item F 1. Bamberger bezeichnet diese Art der Notation (dort allerdings mit Zeichen) als figuralen Typus, die konkrete Fehlerstelle als kritisches Element (1991, S. 25, S. 46; s. Abb. 2-13). In Item F 4 fehlt diese Konstellation. Hier tritt gehäuft der Fehler auf, die Pause nicht richtig darzustellen.

Auf der Kopie der Testbogenrückseite lässt sich dieses Phänomen des einmaligen Vertauschens der Symbole für Achtel- und Viertelschlag (Kat. 41) gut beobachten (s. Abb. 4-16, Proband aus Klasse K 1). In diesem Fallbeispiel wird der letzte Schlag des Rhythmus in Item F 1 in allen drei Darstellungsformen als kurzer Schlag notiert. Am Ende dieses Rhythmus hat dies musikalisch gesehen kaum Auswirkungen.

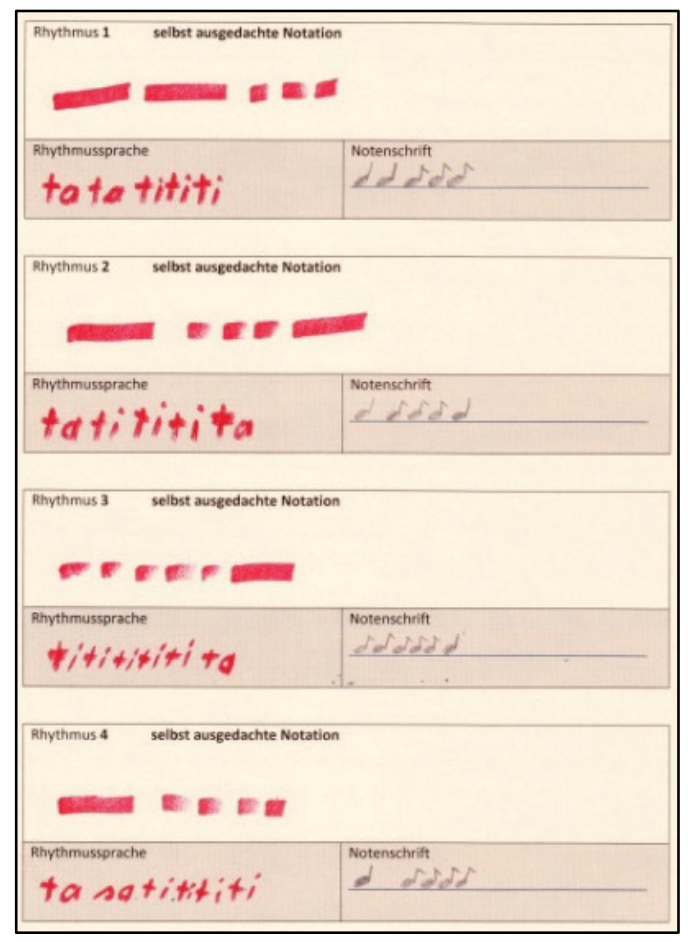

Abb. 4-16: Rückseite des Testbogens aus ST F sowie UT $\mathrm{f}$ und fc 
Im Rhythmus in Item F 2 stellt sich dieser Sachverhalt jedoch anders dar. Der vierte Schlag, der den Wert einer Viertelnote hat, wird in allen drei Darstellungsformen wiederum als kurzer Schlag notiert. Nun entsteht ein Rhythmus mit Synkope. Gleiches gilt für den Rhythmus in Item F 3, hier allerdings auf Schlag 5. Alle drei Lösungen aus gehören zur besagten Fehlerkategorie 41 und in die Lösungskategorie fast richtig mit leichten Abstrichen - figuraler Typus.

Der Rhythmus in Item F 4 enthält kein kritisches Element. Der Proband stellt den ersten Schlag im Rhythmus mit einem Zeichen für einen langen Schlag dar. Beim Notieren mit Rhythmussilben wird der gleiche Schlag als Viertelschlag $(t a)$ mit nachfolgender Viertelpause ( $s a$ ) dargestellt. Beim Notieren mit Noten scheint das Zeichen für Pause nicht bekannt zu sein, es wird nicht notiert. Trotz der verschiedenen Darstellungsformen werden die Lösungen für die Untertests $\mathrm{f}$ und $\mathrm{fc}$ als korrekt gewertet. Dies lässt der Toleranzspielraum zu (s. Tab. 3-15). Die Lösung mit Noten in Item F 4 gilt als fehlerhaft nach Kategorie 45 und gehört in die Lösungskategorie fehlerhaft - zählender Typus.

\subsubsection{Ergebnisse im Subtest A Metrum und Grundschlag darstellen}

Getestet wird das Darstellen des Grundschlages sowie verschiedener Metren zu erklingenden Musikbeispielen (s. Abb. 4-17).

\begin{tabular}{|cccc|}
\hline \multicolumn{4}{|c|}{ Items im Subtest A } \\
\hline Grundschlag & gerades Metrum (2/4) & ungerades Metrum (3/4) & gerades Metrum (4/4) \\
Bruder Jacob & Bruder Jacob & Menuett G-Dur & Around The World \\
& & J. S. Bach & ATC \\
\hline
\end{tabular}

Abb. 4-17: Übersicht über die Items im ST A

In diesem Subtest sind im Vortest bereits gute Entwicklungsstände erkennbar. Bereits $40 \%$ der Probanden sind in der Lage, Grundschlag und gerades Metrum im Lied Bruder Jacob darzustellen. Das ungerade Metrum in einem Menuett zu betonen, gelingt etwa einem Drittel der Probanden. Das gerade Metrum zu einem Popsong zu spielen, gelingt nur etwa 20 \% der Probanden (s. Abb. 4-18). Nennenswerte Unterschiede zwischen den Ergebnissen der Probanden der Untersuchungs- und Kontrollgruppe im Vortest sind nur bei Item A 3 festzustellen. Hier erzielt die Untersuchungsgruppe deutlich höhere Werte.

Betrachtet man gezielt die Gruppe der insgesamt 32 Schülerinnen und Schüler, die mindestens ein bis zwei Jahre Instrumentalunterricht erhalten, so gelingt zwei Schülern keines der vier Items (UG: 0; KG: 2), sieben Schülern je ein Item (UG: 2; KG: 5), sechs Schülern je zwei Items (UG: 4; KG: 2), neun Schülern je drei Items (UG: 7; KG: 2) sowie acht Schülern alle vier Items (UG: 4; KG: 4).

Im Nachtest erreichen die Probanden in den ersten drei Items im Schnitt einen Anteil von etwa $60 \%$ an richtigen Ergebnissen (s. Abb. 4-18). In Item A 4 gelingt dies nur etwa einem Drittel der Probanden. Leichte Leistungsvorsprünge für die Untersuchungsgruppe sind zu erkennen. In der 
Gruppe der 32 Instrumentalschüler gelingt im Nachtest einem Schüler keines der vier Items (UG: 0; KG: 1), zwei Schülern je ein Item (UG: 0; KG: 2), sechs Schülern je zwei Items (UG: 3; KG: 3), elf Schülern je drei Items (UG: 7; KG: 4) sowie zwölf Schülern alle vier Items (UG: 8; KG: 4).
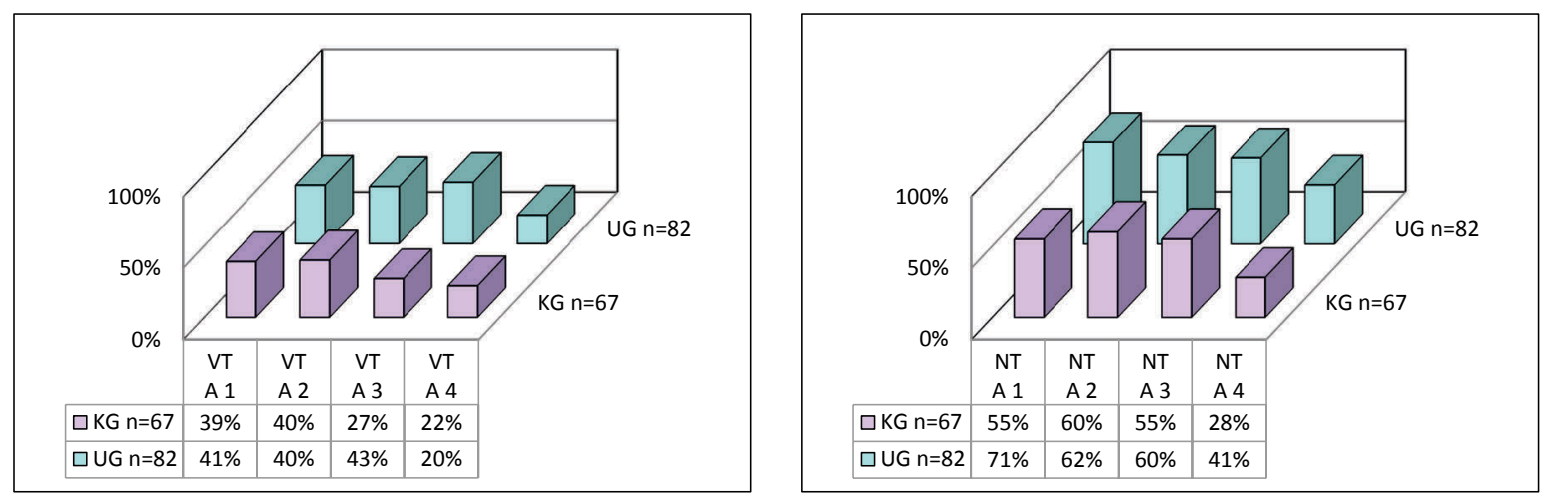

Abb. 4-18: Ergebnisse im ST A, VT und NT, getrennt nach UG und KG

Die Mittelwerte für den Subtest A steigen im Nachtest in beiden Gruppen an (s. Tab. 4-4), jedoch liegt die berechnete Effektstärke $d$ mit einem Wert von 0.17 noch unterhalb der Grenze eines kleinen Effektes (s. Tab. 4-5).

Tab. 4-4: Mittelwerte im ST A

\begin{tabular}{llllllll}
\hline \multirow{2}{*}{ ST } & $\begin{array}{llll}\text { UG } \\
\text { KG }\end{array}$ & N & $\begin{array}{l}\text { MW im } \\
\text { VT }\end{array}$ & $\begin{array}{l}\text { MW im } \\
\text { NT }\end{array}$ & $\begin{array}{l}\text { Diff. } \\
\text { VT/NT }\end{array}$ & $\begin{array}{l}\text { SD der } \\
\text { Diff. }\end{array}$ & $\begin{array}{l}\text { SE der } \\
\text { Diff. }\end{array}$ \\
\hline \multirow{2}{*}{ A } & UG & 82 & 1.44 & 2.34 & 0.90 & 1.19 & 0.13 \\
& KG & 67 & 1.28 & 1.99 & 0.70 & 1.17 & 0.14 \\
\hline
\end{tabular}

Tab. 4-5: Ergebnisse für t-Test und Effektstärke im ST A

\begin{tabular}{ccclccc}
\hline ST & $\mathbf{t}$ & df & p (2seit.) & $\begin{array}{c}\text { Diff. der } \\
\text { MW }\end{array}$ & $\begin{array}{c}\text { SE der Diff. } \\
\text { der MW }\end{array}$ & d [95\% CI] \\
\hline A & 1.03 & 147 & 0.303 & 0.20 & 0.20 & $0.17[-0.18,0.59]$ \\
\hline
\end{tabular}

\section{Fehleranalyse im Subtest A}

Im Subtest A wird keine Unterscheidung zwischen den Fehlern wie z. B. Schlag zu früh / zu spät vorgenommen. Grund ist die deutlich schlechtere Messbarkeit der Fehler (s. Abb. 3-18). Daher rühren auch die größeren Toleranzbereiche für Tempoabweichungen der Positionen der Grundschläge bzw. der Metrumschläge (s. Tab. 3-15). Dennoch werden zu jedem Item kurze Anmerkungen in Bezug auf die Fehlerquellen gegeben. 
Item A 1 testet das Spielen des Grundschlages zum Lied Bruder Jacob (s. Abb. 4-19). Die Handhabung der Klanghölzer bereitet keine Probleme. Trotz vorgelagerter Demonstration und pantomimischer Unterstützung für die Takte 1 bis 8 ist erkennbar, dass das Spielen des Grundschlages für viele Probanden ungewohnt ist. Die meisten Fehler entstehen in Takt 3 und 4, in Takt 7 und 8 sowie analog in Takt 11/12 und 15/16. In diesen Takten ist der Grundschlag auf Zählzeit 4 (anders als in den Takten 1 und 2, in denen Rhythmus und Grundschlag identisch sind) nicht in der Melodie enthalten. Es wird häufig kein Grundschlag gespielt. Das Spielen des Grundschlages zu den Achtelnoten in Takt 5 und 6 sowie in Takt 13 und 14 bereitet ebenfalls Probleme, jedoch deutlich seltener.

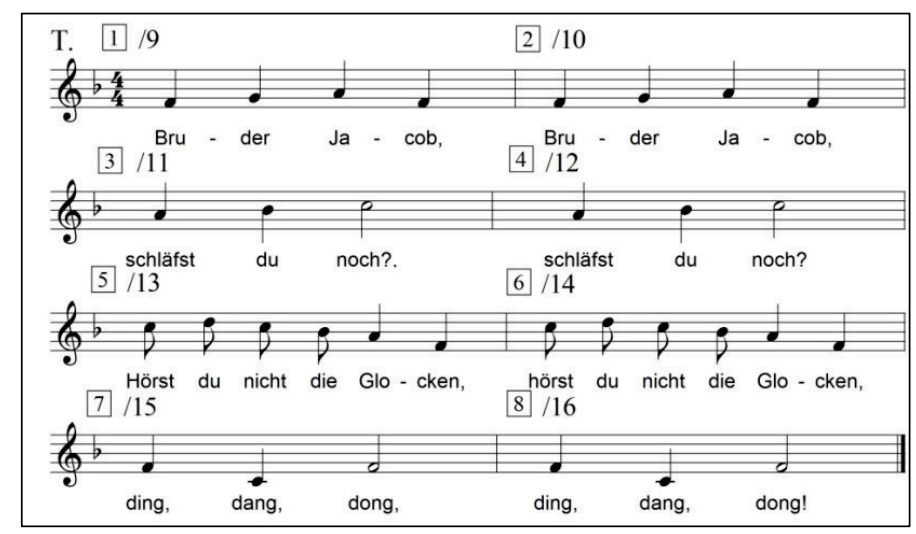

Abb. 4-19: Melodie des Liedes Bruder Jacob

Item A 2 testet das Spielen des Metrums zum Lied Bruder Jacob. In jedem Takt werden Schlag 1 und Schlag 3 betont. Das Spielen des Triangels bereitet den Probanden größere Probleme. Einigen Probanden kann angesehen werden, dass sie nicht recht wissen, ob sie ihre Aufmerksamkeit auf die pantomimische Unterstützung im Video oder auf das Treffen der Schenkel des Triangels legen sollen. Als besonders schwierig gestaltet sich zudem der erste Takt. Während sich im Item A 1 das Spielen des Grundschlages im Rhythmus des Liedes (Takt 1 und 2) exakt wiederfindet, muss im Item A 2 von Anfang an in Schlägen doppelter Länge musiziert werden. Nach einem anfänglichen Erwachen gelingen die weiteren Takte gut. Eine deutliche Herausforderung stellt das Spielen des Metrums zu den Achtelschlägen der Melodie in den Takten 13 und 14 dar. Einmal aus dem Takt geraten, kommen die Probanden bis zum Ende des Liedes nicht wieder hinein.

Item A 3 testet das Spielen des Metrums zum Menuett G-Dur von J. S. Bach. Viele Probanden geben kund, dieses Stück bereits zu kennen und musizieren freudig. Betont wird Schlag 1 in jedem Takt (s. Abb. 4-20). Schwierig gestaltet sich in diesem Item wiederum der erste Takt. Einige Probanden erwarten wie in den vorangegangenen Items eine Demonstration durch das Kind im Video, obwohl ausdrücklich betont wird, dass es bei diesem Item nach einem kurzen Vorspiel gleich losgehen würde. So wird der Einsatz häufig verpasst. Dennoch gelingt das angeleitete Spielen des 
ungeraden Metrums mit pantomimischer Unterstützung insgesamt recht gut. Das selbstständige Spielen ohne Unterstützung gelingt von Takt zu Takt weniger Probanden, in Takt 12 noch $47 \%$ aller Probanden, in Takt 16 nur noch $14 \%$ aller Probanden.

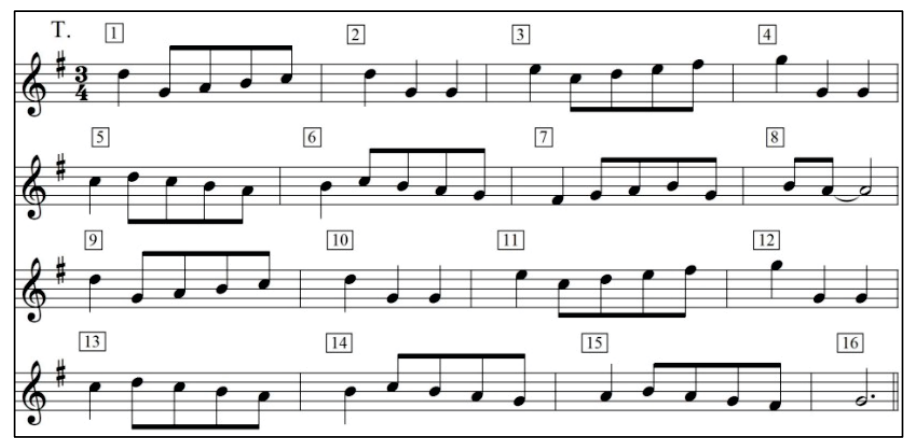

Abb. 4-20: Melodie des Menuettes G-Dur von J. S. Bach

Item A 4 testet das Spielen des Metrums zum Lied Around The World der Band ATC (s. Abb. 4-21). In jedem Takt wird Schlag 1 betont. Auch dieses Stück ist vielen Probanden bekannt. Sie freuen sich sichtlich über ein Beispiel aus der Popmusik. In diesem Item gelingt vielen Probanden der Einsatz wiederum nicht richtig. Ein Grund hierfür wird nicht ersichtlich, möglicherweise ist bei diesem Musikbeispiel das Vorspiel mit 16 Takten zu lang. Mit pantomimischer Unterstützung finden sich die Probanden jedoch gut in die Aufgabe hinein. Das Spielen des Metrums ohne Unterstützung bis zum Schluss weiterzuführen, gelingt nur etwa $20 \%$ der Probanden.

Im Nachtest sind die Probanden in allen Items dieses Subtests deutlich geschickter. Dennoch ist Item A 4 immer noch das schwerste Item. Obwohl sich in diesem Musikbeispiel trotz des relativ schnellen Tempos von 132 bpm die absolute zeitliche Spanne zwischen den Metrumschlägen gegenüber Item A 3 nicht erhöht (die Zeitspanne zwischen den Metrumschlägen beträgt in Item A 3 ca. 1890 bpm, in Item A 4 ca. 1790 bpm), fällt hier die Anzahl der korrekten Lösungen deutlich geringer aus. Auffallend ist bei einem Teil der Probanden, dass sie mit ihrer Mimik betonen, die pantomimische Unterstützung im Video nicht zu brauchen. Sie schauen betont zur Seite.

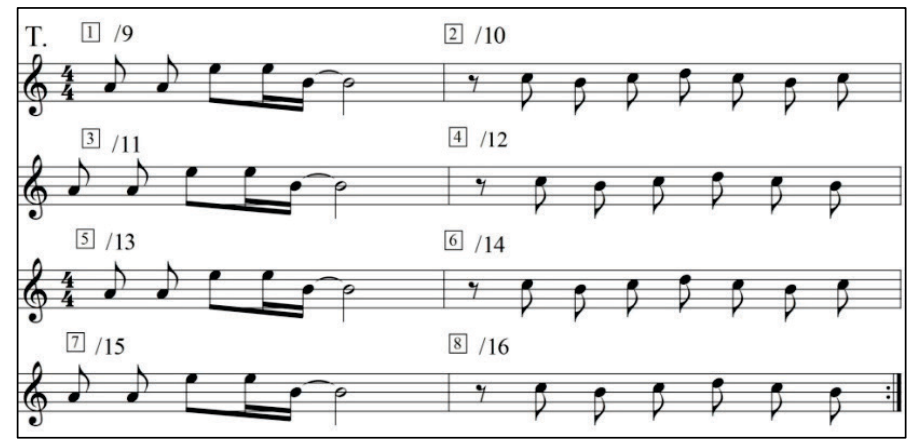

Abb. 4-21: Melodie des Liedes Around The World der Band ATC 


\section{Abgestufte Ergebnisdarstellung}

Alle Lösungen werden in abgestuften Kategorien von Lösung korrekt bis Lösung stark fehlerhaft dargestellt. Hierbei muss im Unterschied zu den anderen musizierbezogenen Subtests ein gravierender Unterschied gemacht werden: Da die Abweichung der Schlagpositionen nur relativ grob analysiert werden können (s. Abschnitt 3.5.2; Erste Rubrik), werden hier für die vier Kategorien Abstufungen in quantitativer und nicht in qualitativer Sicht vorgenommen. In die Kategorie Lösung korrekt werden alle als richtig gewerteten Ergebnisse, in den mindestens sieben Takte korrekt musiziert werden. In die Kategorie Lösung fast richtig mit leichten Abstrichen werden Lösungen eingestuft in denen fünf oder sechs Takte korrekt musiziert werden usf. (s. Abb. 4-22).

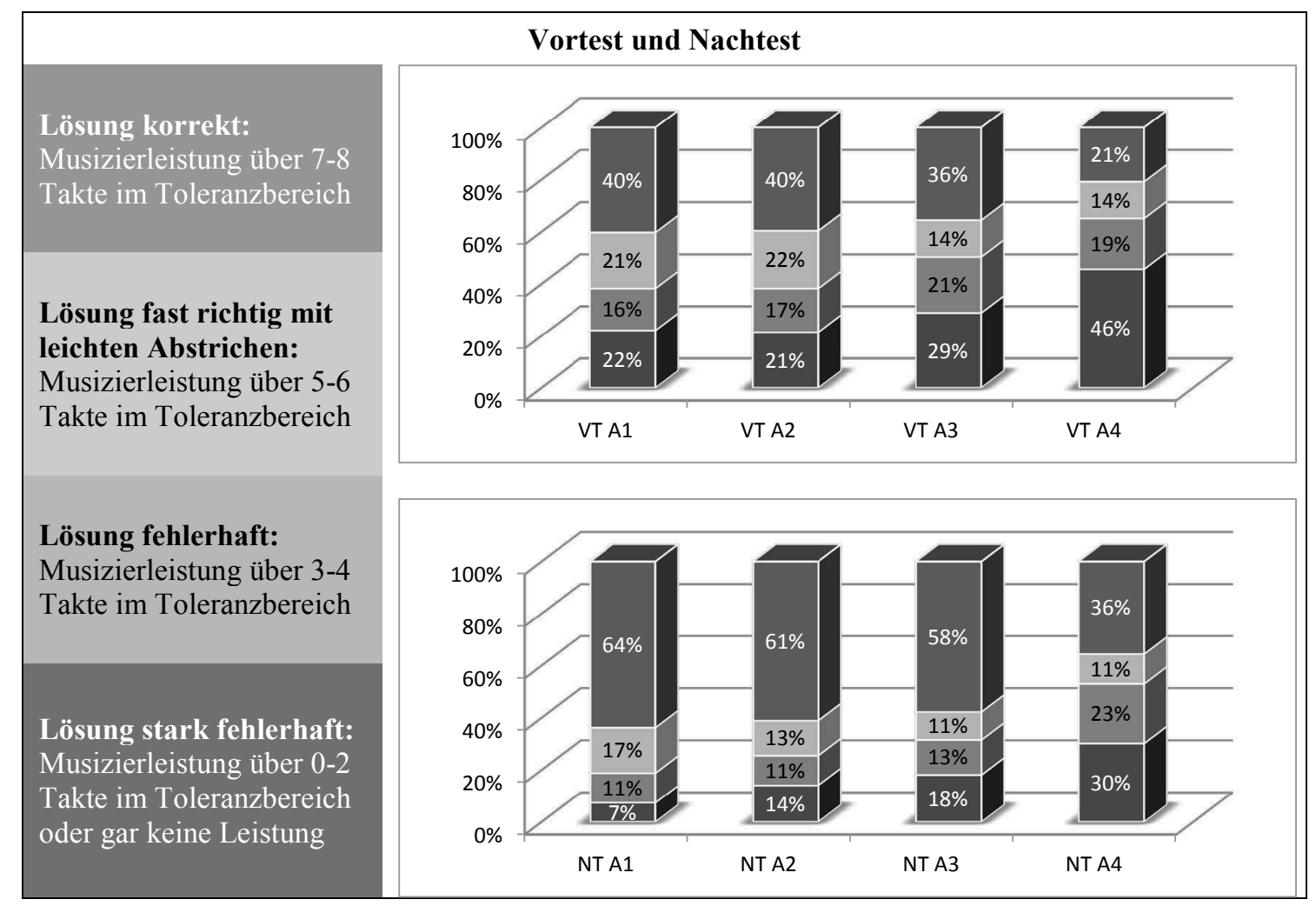

Abb. 4-22: Gesamtdarstellung aller Leistungen im ST A nach Lösungskategorien

$\mathrm{Zu}$ der schon recht großen Gruppe der Probanden (Anteile zwischen $21 \%$ bis $40 \%$ ), welche im Vortest bereits jeweils das gesamte Item (einschließlich eines erlaubten Fehlertaktes) meistern und deren Ergebnisse damit zur Kategorie Lösung korrekt zählen, kommen noch weitere Probanden zu Anteilen zwischen $14 \%$ und $22 \%$ hinzu, die bereits mehr als die Hälfte der Aufgabe bewältigen und deren Ergebnisse zur Kategorie Lösung fast richtig mit leichten Abstrichen zählen (s. Abb. 4-22). Item A 4 fällt den Probanden bei weitem am schwersten, obwohl es sich hier um ein Musikbeispiel aus dem Popbereich handelt. Interessanterweise bleibt bei diesem Item der Anteil in den Kategorien Lösung richtig mit leichten Abstrichen sowie Lösung fehlerhaft gegenüber den 
Items A 1 bis A 3 relativ stabil. Jedoch steigt sprunghaft der Anteil an Lösungen in der Kategorie Lösung stark fehlerhaft bzw. keine Leistung.

Im Nachtest liegen die Ergebnisse in den ersten beiden Kategorien Lösung korrekt und Lösung richtig mit leichten Abstrichen für die Items A 1 bis A 3 bei Anteilen zwischen insgesamt $70 \%$ bis $80 \%$. Ausnahme bildet immer noch Item A 4 mit nur knapp 50 \%. Das Spielen des Grundschlages im geraden Metrum fällt den Probanden etwas leichter als das Spielen des Metrums. Innerhalb der angebotenen Metren fällt das Spielen im ungeraden Metrum (3/4-Takt) schwerer als im geraden Metrum im 2/4-Takt und leichter als im geraden Metrum im 4/4-Takt.

\section{Nachschwingkraft}

In allen Items des Subtests A wird eine Beobachtung gemacht, die mit dem Begriff Nachschwingkraft bezeichnet werden soll. Aufgabe der Probanden ist es, in den Takten 1 bis 8 der pantomimischen Unterstützung im Video zu folgen und jeweils ab Takt 9 Grundschlag bzw. Metrum ohne diese Unterstützung weiter zu musizieren. Während dies vielen Probanden zu Beginn des Items noch gut gelingt, entsteht der Eindruck, als ob Takt für Takt eine leitende Vorstellungskraft für das $\mathrm{zu}$ Musizierende schwindet und in eine hilflose Beliebigkeit entgleitet. Stellvertretend für alle anderen Items wird dies für Item A 4 differenzierter dargestellt.

Dazu werden alle Ergebnisse in vier verschiedene Gruppen eingeteilt: Angeleiteter und selbstständiger Teil richtig: Gruppe 1:1, Angeleiteter und selbstständiger Teil falsch: Gruppe 0:0, Angeleiteter Teil richtig, selbstständiger Teil falsch: Gruppe 1:0 sowie Angeleiteter Teil falsch, selbstständiger Teil richtig: Gruppe 0:1 (s. Abb. 4-23). Im Vortest bilden die Gruppe 0:0 sowie die Gruppe 1:0 den größten Anteil. Deutlich erkennbar ist im Nachtest die Abnahme der Ergebnisse in Gruppe 0:0 sowie eine deutliche Zunahme in den Gruppen 1:0 und 1:1.
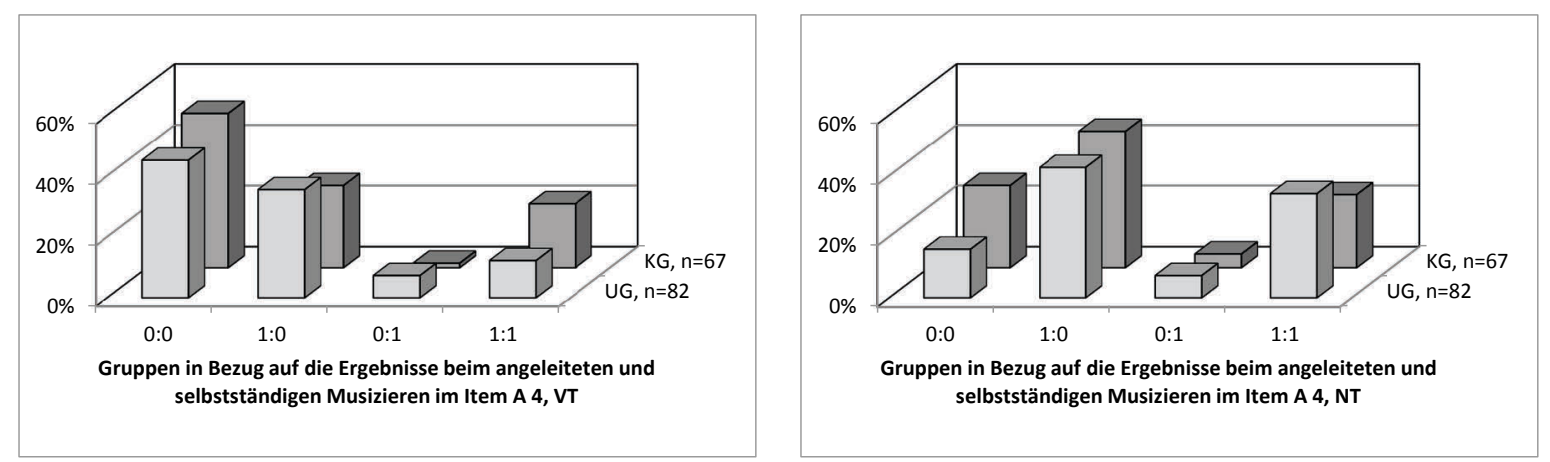

Abb. 4-23: Angeleiteter bzw. selbstständiger Teil in Item A 4, getrennt nach UG und KG

Die Zunahme des Anteils in der Gruppe 1:0 lässt erkennen, dass die pantomimische Unterstützung besser als im Vortest genutzt werden kann. Der Anteil in der Gruppe 1:1 steigt deutlich an, in der Untersuchungsgruppe stärker als in der Kontrollgruppe. 
Um das Phänomen der Nachschwingkraft besser verdeutlichen zu können, wird für die im Nachtest deutlich angestiegene Gruppe 1:0 (65 Probanden zu annähernd gleichen Teilen aus Untersuchungs- und Kontrollgruppe) jeder Takt der beiden Teile (angeleitetes / selbstständiges Musizieren) von Item A 4 gesondert dargestellt (s. Abb. 4-24, links). Während die angeleiteten Takte zu über $90 \%$ richtig musiziert werden, nimmt dies ab Takt 10 (Takt 2 beim selbstständigen Musizieren) rapide ab. Diese Beobachtung kann in den Items A 1 bis A 3 in abgeschwächter Form ebenfalls gemacht werden.
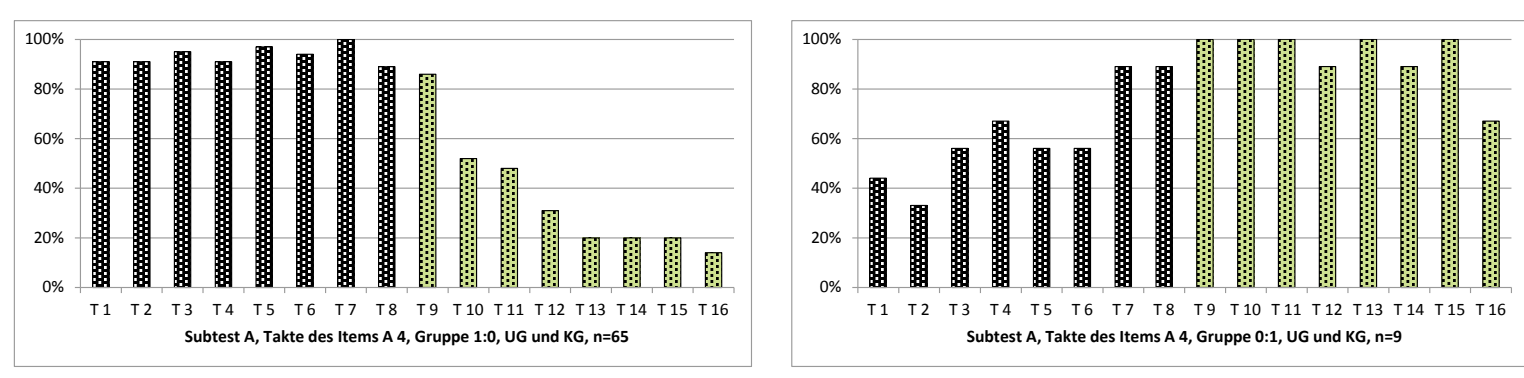

Abb. 4-24: Taktgenaue Darstellung des richtigen Spielens im Item A 4, Gruppe 1:0 und 0:1

Im umgekehrten Falle kann beobachtet werden, dass in der anteilmäßig eher kleinen Gruppe 0:1 (9 Probanden) sich ein umgekehrtes Bild ergibt. Takt für Takt finden mehr Probanden in das angeleitete Musizieren hinein (s. Abb. 4-24, rechts). Etwa ab Takt 7 wird eine Übereinstimmung gefunden. Die Takte 9 und 10 können zu $100 \%$ richtig gespielt werden. In den Takten 12 und 14 sinkt die Leistung leicht ab, kann aber in den nachfolgenden Takten 13 und 15 wieder zu $100 \%$ zurück gelangen. Der letzte Takt missrät einigen Probanden, möglicherweise ist die Konzentration aufgebraucht.

\subsubsection{Ergebnisse im Subtest B Rhythmen nachspielen}

Getestet wird das korrekte Nachspielen von insgesamt vier Rhythmen als Wertungsitems (s. Abb. 4-25).

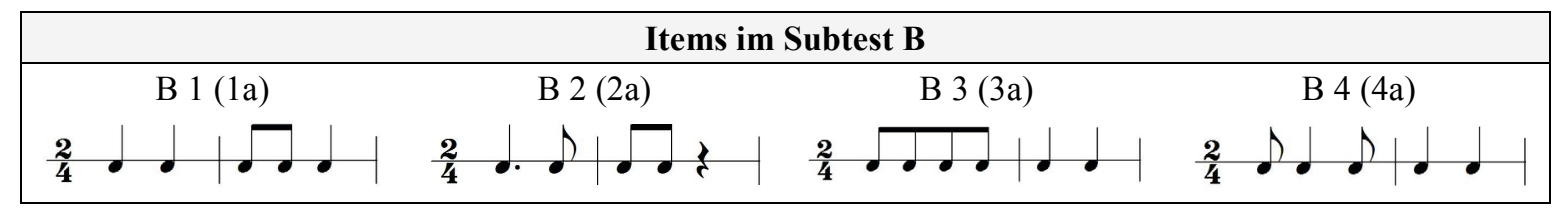

Abb. 4-25: Übersicht über die Items im ST B

Im Vortest sind bereits gute Entwicklungsstände erkennbar. Die leichten Items B 1 und B 3, die nur aus einfachen Kombinationen aus Viertel- und Achtelschlägen bestehen (s. Tab. 3-6), werden von etwa der Hälfte der Probanden richtig gespielt. Die beiden schwereren Items B 2 und B 4 gelingen nur etwa einem Viertel der Probanden. 
Betrachtet man gezielt die Gruppe der insgesamt 32 Schülerinnen und Schüler, die mindestens ein bis zwei Jahre Instrumentalunterricht erhalten, so gelingt vier Schülern keines der vier Items (UG: 2; KG: 2), sechs Schülern je ein Item (UG: 3; KG: 3), zehn Schülern je zwei Items (UG: 6; KG: 4), drei Schülern je drei Items (UG: 2; KG: 1) sowie neun Schülern alle vier Items (UG: 5; KG: 4).
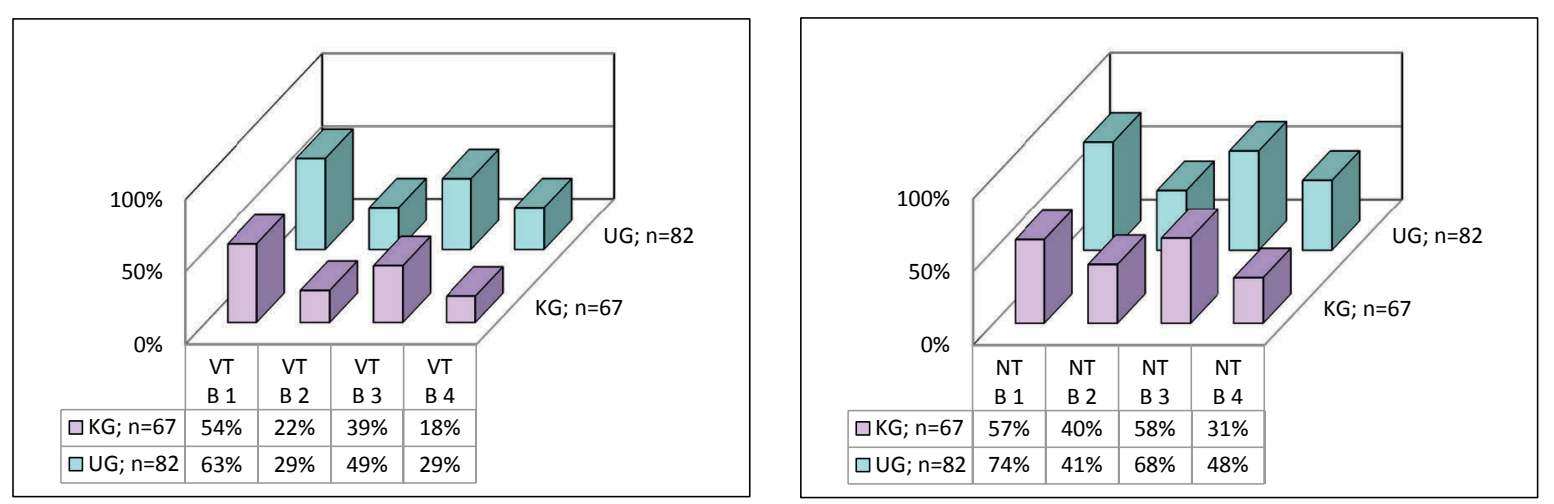

Abb. 4-26: Ergebnisse im ST B, VT und NT, getrennt nach UG und KG

Im Nachtest steigen die Anteile beim Gelingen der Rhythmen in Item B 1 und B 3 auf etwa 65 \%, in Item B 2 und B 4 auf etwa 40 \% der Probanden an (s. Abb. 4-26). In der Gruppe der 32 Instrumentalschüler gelingt im Nachtest einem Schüler keines der vier Items (UG: 1; KG: 0), vier Schülern je ein Item (UG: 1; KG: 3), sieben Schülern je zwei Items (UG: 3; KG: 4), zehn Schülern je drei Items (UG: 5; KG: 5) sowie zehn Schülern alle vier Items (UG: 8; KG: 2).

Die Mittelwerte im Nachtest steigen in beiden Gruppen an (s. Tab. 4-6). Die berechnete Effektstärke $d$ liegt mit einem Wert von 0.06 noch weit unterhalb der Grenze eines kleinen Effektes (s. Tab. 4-7).

Tab. 4-6: Mittelwerte im ST B

\begin{tabular}{llllllll}
\hline \multirow{2}{*}{ ST } & $\begin{array}{llll}\text { UG } \\
\text { KG }\end{array}$ & N & $\begin{array}{l}\text { MW im } \\
\text { VT }\end{array}$ & $\begin{array}{l}\text { MW im } \\
\text { NT }\end{array}$ & $\begin{array}{l}\text { Diff. } \\
\text { VT/NT }\end{array}$ & $\begin{array}{l}\text { SD der } \\
\text { Diff. }\end{array}$ & $\begin{array}{l}\text { SE der } \\
\text { Diff. }\end{array}$ \\
\hline \multirow{2}{*}{ B } & UG & 82 & 1.71 & 2.32 & 0.61 & 1.23 & 0.14 \\
& KG & 67 & 1.33 & 1.87 & 0.54 & 1.16 & 0.14 \\
\hline
\end{tabular}

Tab. 4-7: Ergebnisse für t-Test und Effektstärke im ST B

\begin{tabular}{ccclccc}
\hline ST & $\mathbf{t}$ & df & p (2seit.) & $\begin{array}{c}\text { Diff. der } \\
\text { MW }\end{array}$ & $\begin{array}{c}\text { SE der Diff. } \\
\text { der MW }\end{array}$ & d [95\% CI] \\
\hline B & 0.37 & 147 & 0.715 & 0.07 & 0.20 & $0.06[-0.32,0.46]$ \\
\hline
\end{tabular}




\section{Zusätzliches Item b 6: Rhythmus über acht Grundschläge}

Dieses Item soll überprüfen, inwieweit längere Rhythmen besser als kürzere Rhythmen zu reproduzieren sind (s. Abschnitt 3.5, Interpretationsobjektivität). Dazu wird den bereits dargestellten Ergebnissen von Subtest B mit Rhythmen über vier Grundschläge, das zusätzliche Item $b 6$ mit Rhythmen über acht Grundschläge parallel gestellt. Item $b 6$ stellt eine Kombination aus Item B 1 und B 3 dar (s. Abb. 4-27).

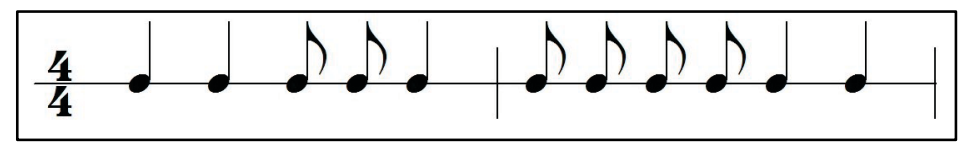

Abb. 4-27: Rhythmus K 1 bei Minkenberg

Bei Minkenberg können $54 \%$ der insgesamt 38 Probanden ( 8 bis 10 Jahre alt) diesen Rhythmus im Tempo 68 bpm fehlerfrei spielen (1991, S. 229-232; s. Anlage A 2 b). In dieser Studie gelingt dies im Nachtest bei Tempo 86 bpm nur $20 \%$ der Probanden in der Untersuchungsgruppe sowie $15 \%$ der Probanden in der Kontrollgruppe (s. Tab. 4-8). Das Nachspielen eines Rhythmus über acht Grundschläge fällt den Probanden deutlich schwerer als bei Rhythmen über vier Grundschläge.

Tab. 4-8: Anteil an korrekten Lösungen im ST B und im zusätzlichen Item b 6, VT und NT

\begin{tabular}{|c|c|c|c|c|c|c|c|c|c|c|}
\hline & \multicolumn{5}{|c|}{ Vortest } & \multicolumn{5}{|c|}{ Nachtest } \\
\hline & B 1 & B 2 & $\overline{\mathrm{B}} 3$ & B 4 & b 6 & B 1 & B 2 & $\overline{\mathrm{B}} 3$ & B 4 & b 6 \\
\hline $\mathrm{KG}, \mathrm{n}=67$ & $54 \%$ & $22 \%$ & $39 \%$ & $18 \%$ & $9 \%$ & $57 \%$ & $40 \%$ & $58 \%$ & $31 \%$ & $15 \%$ \\
\hline $\mathrm{UG}, \mathrm{n}=82$ & $63 \%$ & $29 \%$ & $49 \%$ & $29 \%$ & $10 \%$ & $74 \%$ & $41 \%$ & $68 \%$ & $48 \%$ & $20 \%$ \\
\hline
\end{tabular}

\section{Fehlerquellen im Subtest B}

Für Subtest B werden Fehler in drei Rubriken analysiert: Rhythmus an sich, Einsatz des Rhythmus sowie Tempo des Rhythmus (s. Abschnitt 3.5.2). In der Rubrik Rhythmus an sich werden im Vortest verschiedene Fehler beobachtet (s. Abb. 4-28). Zu ihnen zählt das Nichtspielen des letzten Schlages des Rhythmus (Kat. 51). Es tritt nur sehr selten auf. Das fehlerhafte Spielen von Rhythmen, deren Schläge den einzelnen Positionen nicht sinnvoll zugeordnet werden können, tritt etwas häufiger auf (Kat. 61). Diese Rhythmen wirken entstellt. Des Weiteren werden zum Teil Rhythmen gespielt, die eindeutig falsch sind, aber einen anderen Rhythmus gut erkennen lassen (Kat. 62). Auf sie wird im Abschnitt Response mit einem anderen Rhythmus Bezug genommen. Werden Rhythmen extrem zu früh / zu spät gespielt und überlagern dadurch den vorausgehenden bzw. nachfolgenden Call, kann keine Wertung erfolgen (Kat. 70). Außerdem gibt es im Vortest einen Fall, in denen keine Rhythmus-Response gegeben wird (Kat. 80). Im Nachtest verschwinden die Kategorien 51, 62, 70 und 80 fast vollständig. Unterschiede zwischen Untersuchungs- und Kontrollgruppe belaufen sich lediglich 
auf die etwas größere Sicherheit der Untersuchungsgruppe beim Spielen des Items B 4, der eine Synkope enthält.

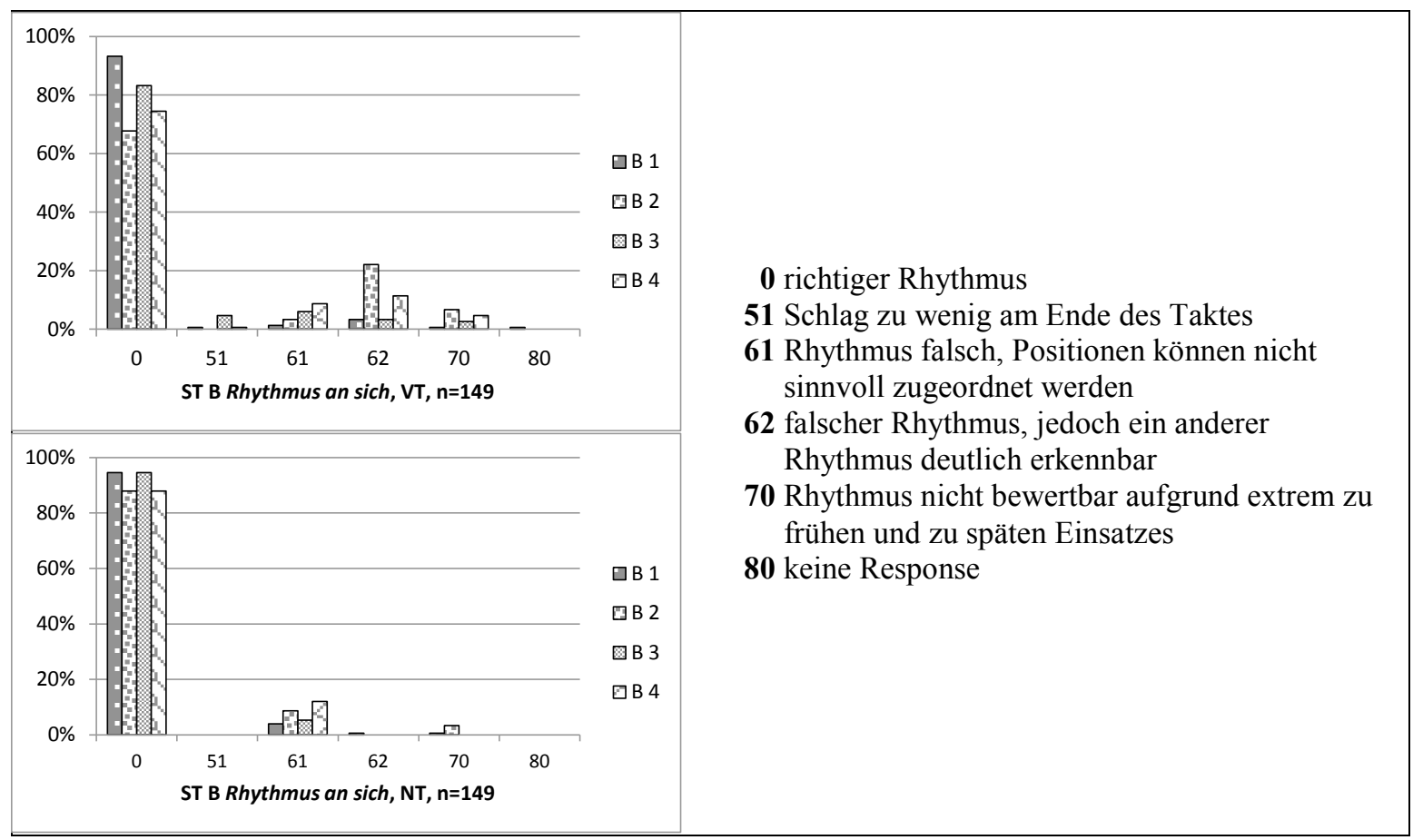

Abb. 4-28: Häufigkeit von Fehlern in der Rubrik Rhythmus an sich im ST B, VT und NT

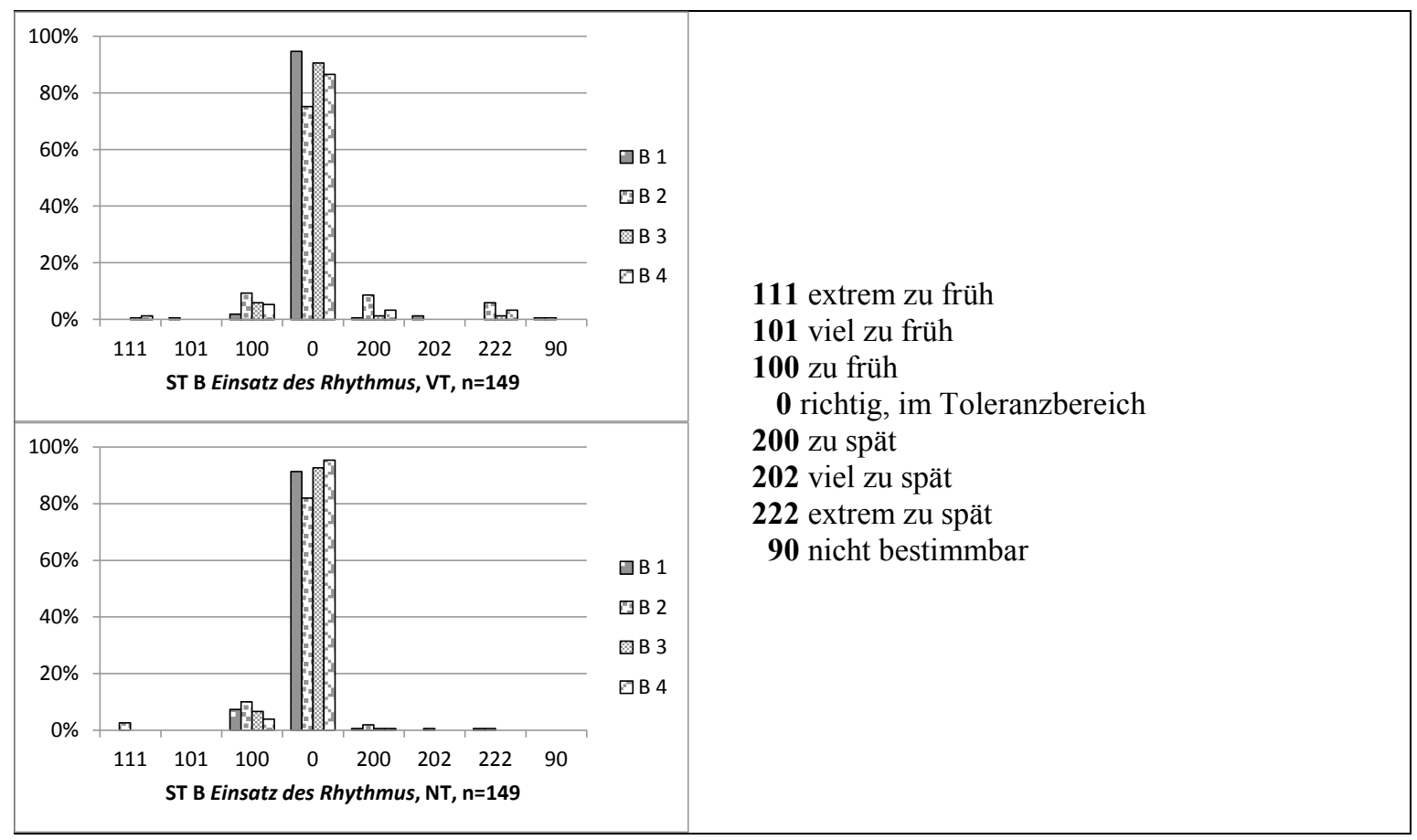

Abb. 4-29: Häufigkeit von Fehlern in der Rubrik Einsatz des Rhythmus im ST B, VT und NT

In der Rubrik Einsatz des Rhythmus liegen im Vortest etwa 80 \% der Lösungen in der Kategorie 0 richtig, im Toleranzbereich (s. Abb. 4-29). Lediglich Item B 2 bildet mit nur $75 \%$ eine deutliche 
Ausnahme. Bei einem Anteil von $2 \%$ bis $10 \%$ der Lösungen beginnt die Rhythmus-Response zu früh bzw. zu spät (Kat. 100, 200), bei etwa $1 \%$ viel zu früh bzw. viel zu spät sowie bis zu $6 \%$ extrem zu früh bzw. spät (Kat. 111, 222). Zu frühe und zu späte Einsätze halten sich in etwa die Waage. Je eine Lösung in Item B 1 und B 2 ist in Bezug auf den Einsatz des Rhythmus nicht bestimmbar (Kat. 90). Im Nachtest liegt der Anteil an Lösungen in der Kategorie 0 richtig, im Toleranzbereich leicht über $90 \%$, lediglich in Item B 2 bei nur $80 \%$. Der Anteil der zu frühen und zu späten Einsätze hält sich nicht mehr die Waage, deutlich mehr Responses weisen zu frühe als zu späte Einsätze auf. Viel zu frühe bzw. viel zu späte Einsätze treten vereinzelt in Item B 1 und B 2 auf. Wesentliche Unterschiede zwischen Untersuchungs- und Kontrollgruppe sind nicht auszumachen.

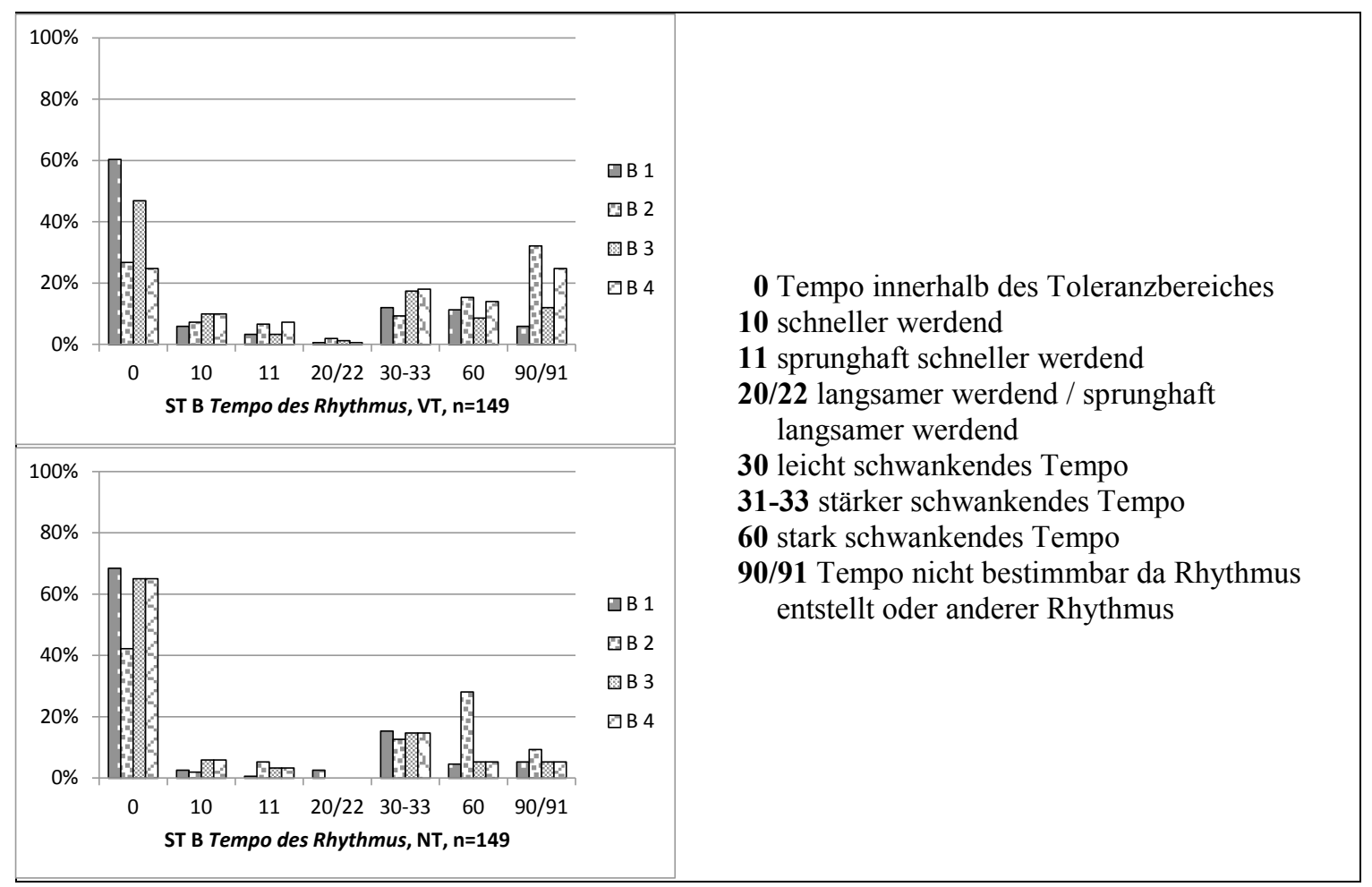

Abb. 4-30: Häufigkeit von Fehlern in der Rubrik Tempo des Rhythmus im ST B, VT und NT

In der Rubrik Tempo des Rhythmus werden im Vortest je nach Item zwischen $25 \%$ und $60 \%$ der Responses im richtigen Tempo gespielt (Kat. 0 ; s. Abb. 4-30). Ein erheblicher Teil der Rhythmen wird accelerierend bzw. ritardierend gespielt (Kat. 10, 11, 20/22). Das schneller werdende Spielen ist deutlich häufiger als das langsamer werdende Spielen zu beobachten. Bei einem nahezu ebenso großen Anteil an Lösungen ist ein Spielen in leichter oder stärker schwankendem Tempo zu beobachten (Kat. 30-33). Ein weiterer Anteil der Lösungen schwankt sehr stark im Tempo (Kat. 60). Bei einem Anteil von $5 \%$ bis $30 \%$ der Lösungen kann das Tempo nicht bestimmt werden, da der Rhythmus entstellt oder ein ganz anderer Rhythmus gespielt wird (Kat. 90, 91). Im Nachtest steigen die im Tempo richtig gespielten Responses je nach Item auf $40 \%$ bis $70 \%$ an. Das schneller 
werdende Spielen ist auch im Nachtest ebenfalls häufiger als das langsamer werdende Spielen zu beobachten. Die anderen Temposchwierigkeiten, insbesondere in der Kategorie 60 sowie 90 / 91 nehmen stark ab. Wesentliche Unterschiede zwischen Untersuchungs- und Kontrollgruppe sind nicht zu beobachten.

\section{Abgestufte Ergebnisdarstellung}

Alle Lösungen werden in abgestuften Kategorien von korrekt bis stark fehlerhaft bzw. keine Lösung dargestellt (s. Abschnitt 3.5.2; Zweite Rubrik). In dieser Darstellung lässt sich wie schon zuvor in den Fehlernanalysen erkennen, dass die Schwierigkeit der Items sowohl im Vor- als auch im Nachtest der Reihenfolge B 1 und B 3 sowie B 4 bzw. B 2 entspricht (s. Abb. 4-31). Für die beiden Items B 2 und B 4 lässt sich beobachten, dass zwar der Anteil der Lösungen in der Kategorie Lösung korrekt für Item B 2 besser ausfällt, jedoch in der Kategorie Lösung fast richtig mit leichten Abstrichen ein deutlich größerer Anteil für Item B 4 zu erkennen ist. Dies spricht dafür, dass Item B 4 mit der Synkope doch etwas leichter als Item B 2 mit der punktierten Viertelnote und Pause auf Zählzeit 4 gelingt. Im Nachtest bleibt die Schwierigkeit der Items unverändert, jedoch steigen die Anteile in den Kategorien Lösung korrekt sowie Lösung fast richtig mit leichten Abstrichen deutlich an. Erfreulicherweise sind im Nachtest alle prozentualen Anteile in der Kategorie Lösung stark fehlerhaft deutlich unter $15 \%$.

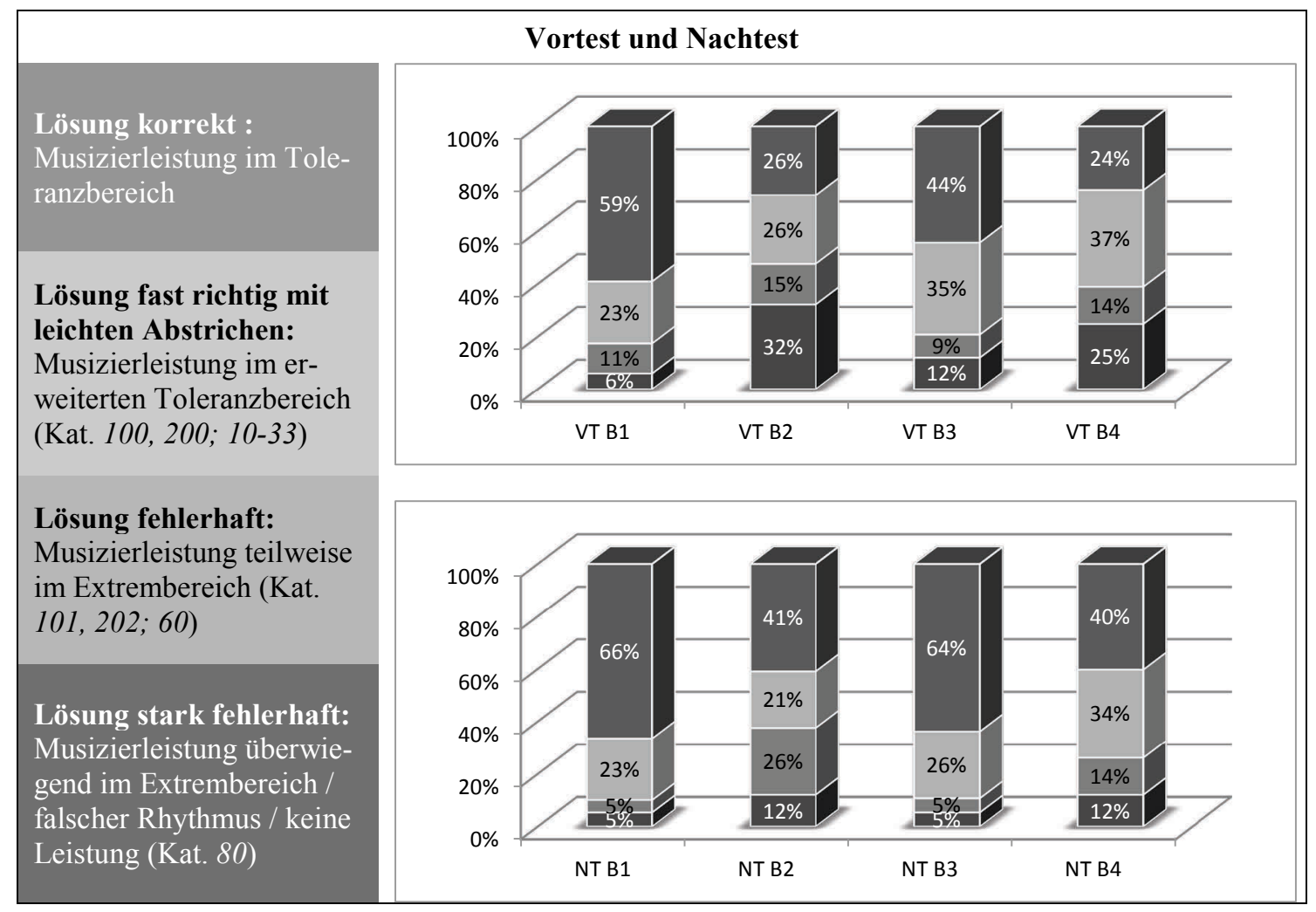

Abb. 4-31: Gesamtdarstellung aller Leistungen im ST B nach Lösungskategorien 


\section{Response mit einem anderen Rhythmus}

Die in der Rubrik Rhythmus an sich dargestellte Fehlerhäufung in der Kategorie 62 falscher Rhythmus, jedoch ein anderer Rhythmus deutlich erkennbar (s. Abb. 4-30) wird besonders häufig bei Item B 2 mit insgesamt 29 Fällen verzeichnet (s. Tab. 4-9; Notenbildübersicht s. Anlage E). Die ersatzweise für Item B 2 gespielten Rhythmen 5b und 20 weisen sowohl in der Anzahl der Schläge als auch in ihrer Struktur eine hohe Ähnlichkeit zum eigentlichen Rhythmus auf. Bei Rhythmus 5b wird die Punktierung verkürzt, bei Rhythmus 20 verlängert. Der auf die Punktierung folgende Achtelschlag wird auf den Grundschlag davor bzw. danach verlegt, der Rhythmus wird sozusagen geglättet (s. Tab. 4-10). Die anderen ersatzweise für Item B 2 gespielten Rhythmen scheinen eher unbedacht gespielt zu sein. Im Nachtest wird Kategorie 62 nur noch ein einziges Mal bei einem Probanden aus der Untersuchungsgruppe beobachtet.

Tab. 4-9: Übersicht über häufige Ersatzrhythmen im ST B

\begin{tabular}{|c|c|c|c|c|}
\hline & Item B 1 & Item B 2 & Item B 3 & Item B 4 \\
\hline Rhythmus & $1 \mathrm{a}$ & $2 a$ & $3 a$ & $4 a$ \\
\hline Notenbild & $\frac{2}{4} \cdot \cdot|\cdot \cdot|$ & $\frac{2}{4} \cdot \rho \mid \sigma ?$ & \multicolumn{2}{|c|}{$\frac{2}{4} \cdot \cdots \cdot|\cdot \cdot| \frac{2}{4} \partial \cdot \partial|\cdot \downarrow|$} \\
\hline $\begin{array}{l}\text { Anzahl der Erset- } \\
\text { zungen }\end{array}$ & $\begin{array}{c}4 \text { Fälle } \\
\text { (Rhythmen 5a, 5b, } \\
\text { 7a, 8a) }\end{array}$ & $\begin{array}{c}9 \text { x Rhythmus } 5 \mathrm{~b} \text {; } \\
20 \text { weitere Fälle } \\
\text { (Rhythmen 1a, 5a, } \\
\text { 6a, 6b, 7a, 8a, 20, } \\
\text { 21, 28, 29) }\end{array}$ & $\begin{array}{l}5 \text { x Rhythmus 5a; } \\
1 \text { x Rhythmus 1a }\end{array}$ & $\begin{array}{c}10 \text { Fälle } \\
\text { (Rhythmen } 2 \mathrm{a}, 3 \mathrm{a}, \\
\text { 3b, 4c, 6a, 7a, 8a, } \\
11,24)\end{array}$ \\
\hline Ersatzrhythmus & & $5 b$ & $5 a$ & \\
\hline Notenbild & & $\frac{2}{4} \cdot \sigma \mid \cdot ?$ & $\frac{2}{4} \cdot \neg|\cdot \cdot|$ & \\
\hline
\end{tabular}

Tab. 4-10: Notenbild für Item B 2 sowie Ersatzrhythmen 5b und 20

\begin{tabular}{|c|c|c|c|c|c|}
\hline \multicolumn{6}{|c|}{ Rhythmusnummer und Notenbild } \\
\hline $2 \mathrm{a}$ & D? & $5 b$ & $\frac{2}{4} \cdot \sigma \cdot d ?$ & 20 & . \\
\hline
\end{tabular}

\subsubsection{Ergebnisse im Subtest D Rhythmen bewusst abwandeln}

Getestet wird das Abwandeln des Rhythmus 7a sowie die Wiederholung des eigenen abgewandelten Rhythmus nach einem Takt Pause (s. Abb. 4-32). Das Abwandeln eines Rhythmus gelingt im Vortest in den Items D 1 und D 3 zwischen einem Viertel und einem Fünftel der Probanden (s. Abb. 4-33), das richtige Wiederholen (Item D 2, D 4) nur zwischen einem Fünftel und einem Sechstel der Probanden. Innerhalb der beiden Gruppen gestalten sich die Ergebnisse recht ausgeglichen, bei den Items D 1 und D 2 liegt die Untersuchungsgruppe etwas im Vorsprung, bei Item D 3 und D 4 die Kontrollgruppe. 


\begin{tabular}{|c|c|c|c|}
\hline \multicolumn{4}{|c|}{ Items im Subtest D } \\
\hline & Call aus Rhythmus 7 a & $\frac{2}{4} \cdot \cdot|\cdot \cdot|$ & \\
\hline D 1 & D 2 & D 3 & D 4 \\
\hline $\begin{array}{l}\text { erster abgewandelter } \\
\text { Rhythmus }\end{array}$ & $\begin{array}{c}\text { Wiederholung } \\
\text { des ersten abgewandel- } \\
\text { ten Rhythmus }\end{array}$ & $\begin{array}{c}\text { zweiter abgewandelter } \\
\text { Rhythmus }\end{array}$ & $\begin{array}{l}\text { Wiederholung } \\
\text { des zweiten abgewan- } \\
\text { delten Rhythmus }\end{array}$ \\
\hline
\end{tabular}

Abb. 4-32: Übersicht über die Items im ST D

Betrachtet man gezielt die Gruppe der insgesamt 32 Schülerinnen und Schüler, die mindestens ein bis zwei Jahre Instrumentalunterricht erhalten, so gelingt acht Schülern keines der vier Items (UG: 2; KG: 6), zehn Schülern je ein Item (UG: 9; KG: 1), acht Schülern je zwei Items (UG: 3; KG: 5), drei Schülern je drei Items (UG: 1; KG: 2) sowie drei Schülern alle vier Items (UG: 3; KG: 0).

Im Nachtest gelingen die Items D 1 und D 3 knapp $40 \%$ der Probanden, die Items D 2 und D 4 etwa einem Drittel der Probanden. In der Untersuchungsgruppe sind die Ergebnisse für Item D 1 bis D 4 auf stabilem Niveau, ihnen gelingt das korrekte Wiederholen in gleichem Maße wie das Abwandeln des Rhythmus. In der Kontrollgruppe fällt auf, dass sich die Werte der Items D 1 bis D 4 unausgeglichen darstellen. Die Items D 1 und D 3 werden gut gemeistert, die Items D 2 und D 4 fallen ab. Den erfundenen Rhythmus zu wiederholen fällt diesen Probanden deutlich schwerer, als einen Rhythmus abzuwandeln. In der Gruppe der 32 Instrumentalschüler gelingt im Nachtest zwei Schülern keines der vier Items (UG: 0; KG: 2), sieben Schülern je ein Item (UG: 3; KG: 4), 13 Schülern je zwei Items (UG: 9; KG: 4), sechs Schülern je drei Items (UG: 4; KG: 2) sowie vier Schülern alle vier Items (UG: 2; KG: 2).
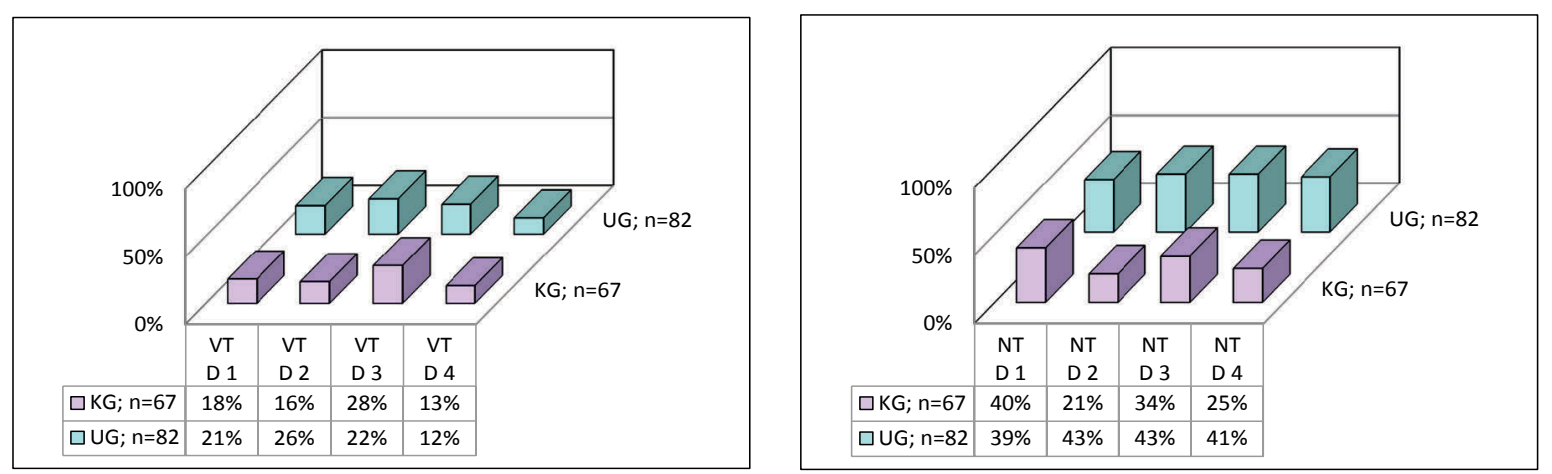

Abb. 4-33: Ergebnisse im ST D, VT und NT, getrennt nach UG und KG

Die Mittelwerte im Nachtest steigen in beiden Gruppen an, wobei dies in der Untersuchungsgruppe deutlich stärker der Fall ist (s. Tab. 4-11). Dieser Vorsprung zeigt sich in einem mittleren Effekt mit einem Wert von $d=0.31$ (s. Tab. 4-12). 
Ergebnisse

Tab. 4-11: Mittelwerte im ST D

\begin{tabular}{lllccccc}
\hline \multirow{2}{*}{ ST } & $\begin{array}{l}\text { UG } \\
\text { KG }\end{array}$ & \multirow{2}{*}{ N } & $\begin{array}{c}\text { MW im } \\
\text { VT }\end{array}$ & $\begin{array}{c}\text { MW im } \\
\text { NT }\end{array}$ & $\begin{array}{c}\text { Diff. } \\
\text { VT/NT }\end{array}$ & $\begin{array}{c}\text { SD der } \\
\text { Diff. }\end{array}$ & $\begin{array}{c}\text { SE der } \\
\text { Diff. }\end{array}$ \\
\hline \multirow{2}{*}{$\mathrm{D}$} & UG & 82 & 0.80 & 1.66 & 0.85 & 1.36 & 0.15 \\
& KG & 67 & 0.76 & 1.21 & 0.45 & 1.25 & 0.15 \\
\hline
\end{tabular}

Tab. 4-12: Ergebnisse für t-Test und Effektstärke im ST D

\begin{tabular}{ccclccc}
\hline ST & $\mathbf{t}$ & df & p (2seit.) & $\begin{array}{c}\text { Diff. der } \\
\text { MW }\end{array}$ & $\begin{array}{c}\text { SE der Diff. } \\
\text { der MW }\end{array}$ & d [95\% CI] \\
\hline $\mathrm{D}$ & 1.88 & 147 & 0.715 & 0.07 & 0.20 & $0.06[-0.32,0.46]$ \\
\hline
\end{tabular}

\section{Fehlerquellen im Subtest D}

Die Fehlerquellen im Subtest D werden in den Rubriken Aufgabenverständnis, Einsatz und Tempo des Rhythmus analysiert. In der Rubrik Aufgabenverständnis kann festgestellt werden, dass die gestellte Aufgabe des Abwandelns eines Rhythmus im Vortest im Item D 1 von über $80 \%$ der Probanden, im Item D 3 von etwa $60 \%$ der Probanden richtig umgesetzt wird (s. Abb. 4-34). In neun Fällen werden Rhythmen gespielt, die im Rahmen eines Grundschlagbezuges nicht identifizierbar sind, jedoch die Aufgabenstellung erfüllen. (Nicht als eigene Kategorie erfasst.) Weiterhin gibt es Lösungen, die um einen Grundschlag zu lang gestaltet sind (Kat. 52). Häufig wird in Item D 1 oder D 3 statt eines abgewandelten Rhythmus der Call-Rhythmus (Kat. 74) nachgespielt bzw. in Item D 3 der Rhythmus aus Item D 1 noch einmal angeboten (Kat. 75). In wenigen Fällen wird gar keine Lösungen angeboten (Kat. 80). Die gestellte Aufgabe des Wiederholens des abgewandelten Rhythmus wird im Vortest im Item D 2 von etwa 45 \% der Probanden, im Item D 4 von etwa $25 \%$ der Probanden prinzipiell richtig umgesetzt (Kat. 0.2, s. Abb. 4-34). Der häufigste Fehler ist das Spielen eines ganz neuen Rhythmus statt des abgewandelten Rhythmus (Kat. 76). Mitunter wird auch ein Rhythmus gespielt, der in Item D 1 bzw. D 3 den Kategorien 74 und 75 entspricht und damit nicht zulässig ist (Kat. 77). In wenigen Fällen wird gar keine Lösungen angeboten (Kat. 80.2).

Alle Fehlerquellen sind auch im Nachtest zu beobachten. Die Aufgabe des Abwandelns eines Rhythmus wird nun im Item D 1 von bis zu $90 \%$, im Item D 3 von bis zu $80 \%$ der Probanden richtig umgesetzt (s. Abb. 4-34). Nur in vier Fällen werden Rhythmen gespielt, die im Rahmen eines Grundschlagbezuges nicht identifizierbar sind. (Nicht als eigene Kategorie erfasst.) Weiterhin gibt es wenige Lösungen, die um einen Grundschlag zu lang gestaltet sind (Kat. 52). Nur noch selten wird in Item D 1 oder D 3 statt eines abgewandelten Rhythmus der Call-Rhythmus (Kat. 74) nachgespielt bzw. in Item D 3 der Rhythmus aus Item D 1 noch einmal angeboten (Kat. 75). Die gestellte Aufgabe des Wiederholens des abgewandelten Rhythmus wird im Nachtest im Item D 2 
von über $60 \%$ der Probanden der Untersuchungsgruppe sowie von etwa $45 \%$ der Probanden der Kontrollgruppe prinzipiell richtig umgesetzt (Kat. 0.2). Mehr als ein Drittel dieser Lösungen wird in Bezug auf die Rubriken Einsatz des Rhythmus bzw. Tempo des Rhythmus nicht im Toleranzbereich gespielt. Das Spielen eines ganz neuen Rhythmus statt des abgewandelten Rhythmus (Kat. 76) sinkt bei den Lösungen der Probanden der Untersuchungsgruppe auf etwa 30 \%, bei den Lösungen der Probanden der Kontrollgruppe bleibt der Anteil relativ ähnlich zum Vortest zwischen $40 \%$ und $50 \%$. Nur noch selten wird ein Rhythmus gespielt, der in Item D 1 bzw. D 3 den Kategorien 74 und 75 entspricht und damit nicht zulässig ist (Kat. 77). Sehr wenige Lösungen entfallen auf die Kategorie 80 kein Rhythmus gespielt.

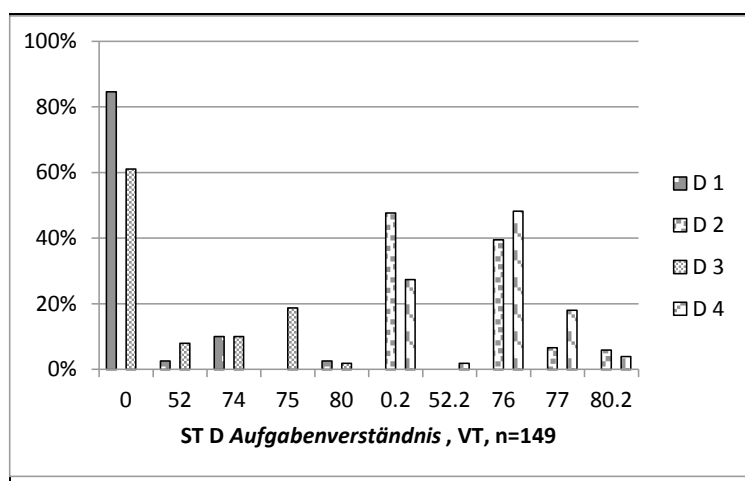
D 1 und D 3
0 ein anderer Rhythmus als Rhythmus $7 \mathrm{a}$
52 Rhythmus zu lang (über fünf Grundschläge)
74 Rhythmus gleicht dem Call (7a)
75 Rhythmus im Item D3 gleicht dem Rhythmus in Item D1
80 kein Rhythmus gespielt

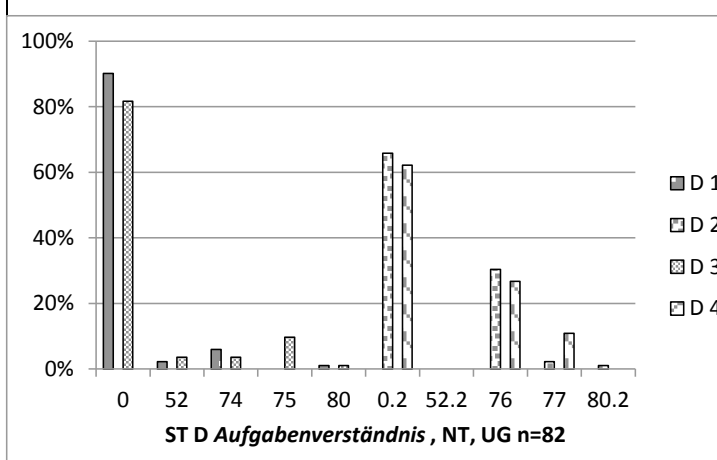

D 2 und D 4

0.2 korrekte Wiederholung

76 Wechsel auf einen anderen Rhythmus

77 korrekte Wiederholung eines ,unzulässigen“ Rhythmus (Kat. 74, 75)

52.2 Rhythmus zu lang

80.2 kein Rhythmus gespielt

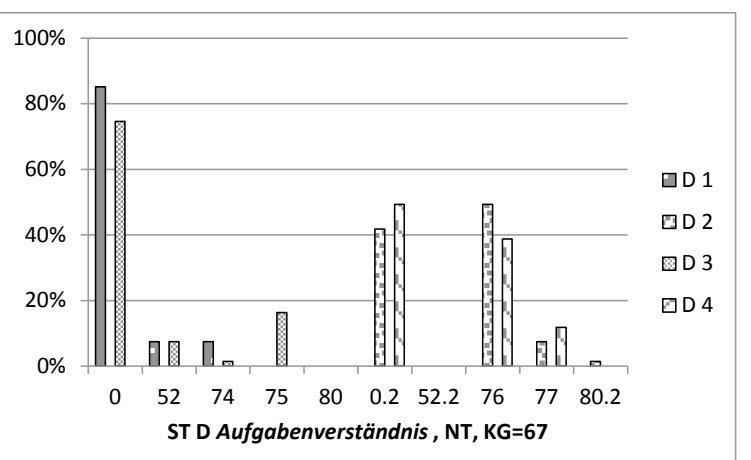

Abb. 4-34: Häufigkeit von Fehlern in der Rubrik Aufgabenverständnis im ST D, VT und NT

Ein erheblicher Anteil der Lösungen, welche die Aufgabenstellung richtig umsetzen, wird in Bezug auf die Rubriken Einsatz des Rhythmus (s. Abb. 4-35) bzw. Tempo des Rhythmus (s. Abb. 4-36) im erweiterten Toleranzbereich oder auch im Extrembereich gespielt. Hierbei überwiegen deutlich das zu späte Einsetzen sowie stark schwankendes Tempo. Auch im Nachtest wird noch ein erheblicher Anteil der Lösungen, welche die Aufgabenstellung richtig umsetzen, in Bezug auf die Rubriken Einsatz des Rhythmus (s. Abb. 4-35) bzw. Tempo des Rhythmus (s. Abb. 4-36) nicht im Toleranz- 
bereich gespielt. Es überwiegen ebenfalls deutlich das zu späte Einsetzen sowie das schwankende (Kat. 30-33) bzw. stark schwankendes Tempo (Kat. 60). Der Anteil an richtigen Lösungen hat jedoch deutlich zugenommen.

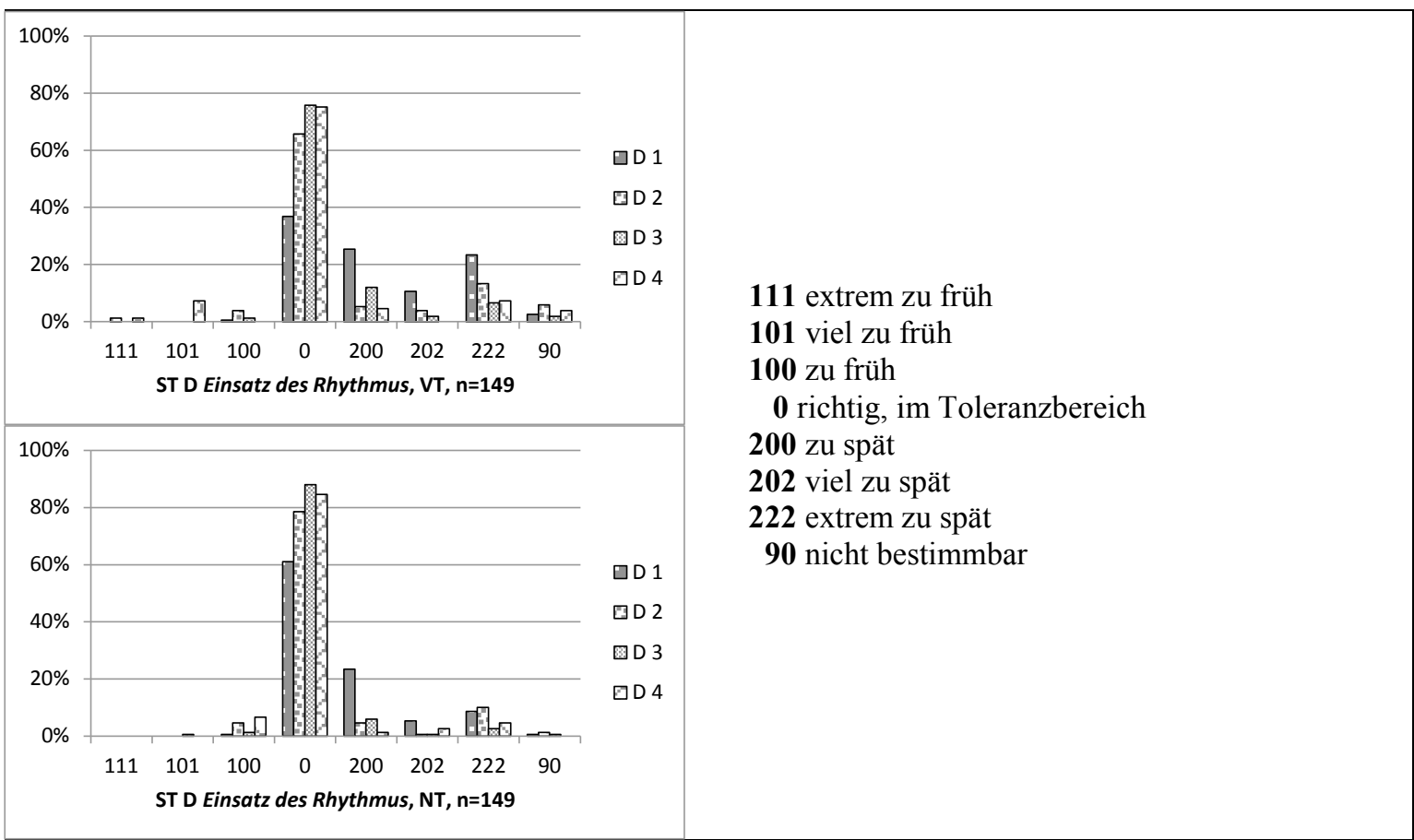

Abb. 4-35: Häufigkeit von Fehlern in der Rubrik Einsatz des Rhythmus im ST D, VT und NT

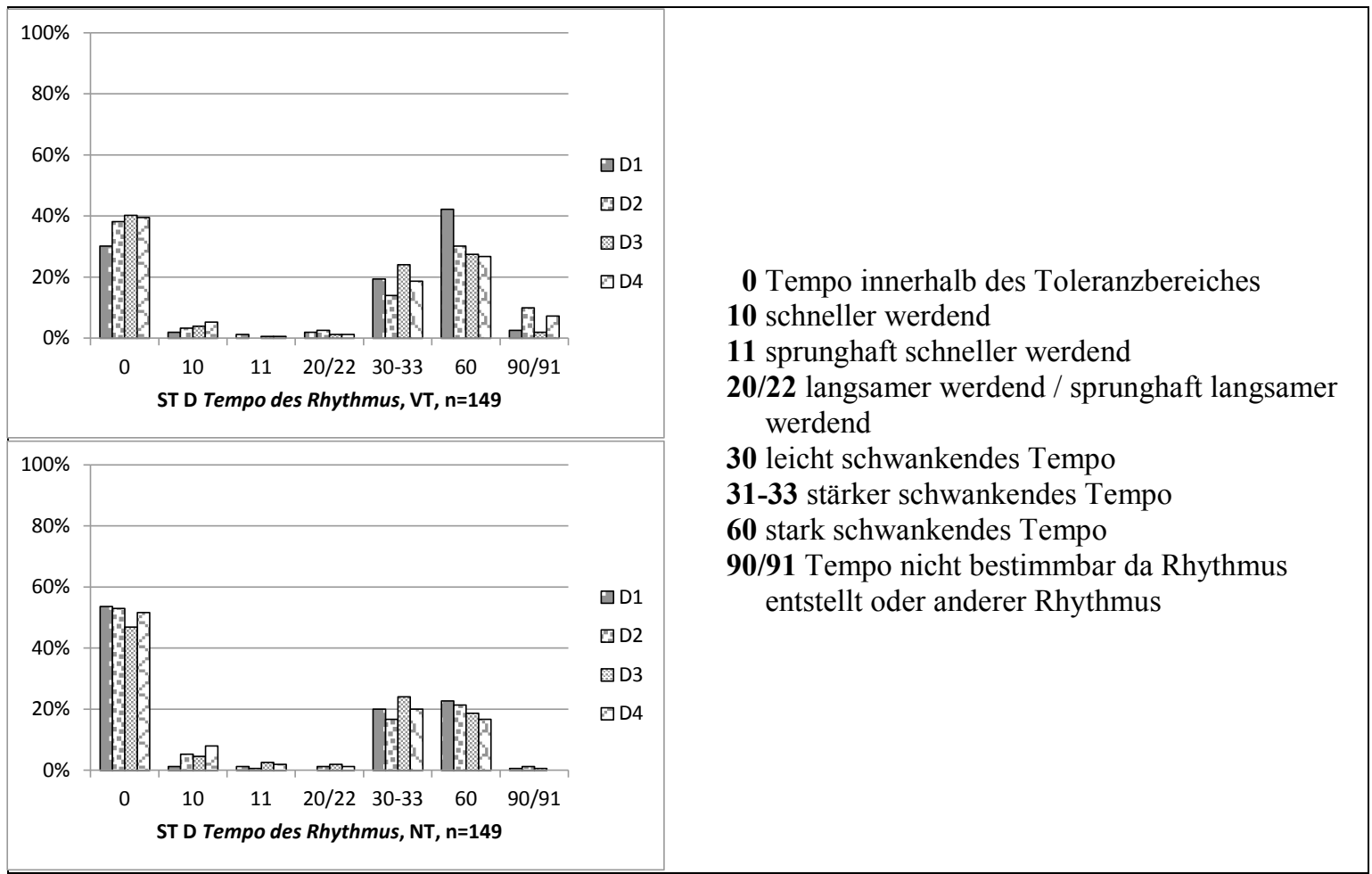

Abb. 4-36: Häufigkeit von Fehlern in der Rubrik Tempo des Rhythmus im ST D, VT und NT 


\section{Abgestufte Ergebnisdarstellung}

Ein erheblicher Teil der in Bezug auf das Aufgabenverständnis richtigen Lösungen muss in Bezug auf die Rubriken Einsatz des Rhythmus bzw. Tempo des Rhythmus im Vortest dem erweiterten Toleranzbereich oder Extrembereich zugeordnet werden. Auf die Kategorie Lösung korrekt entfallen deshalb nur durchschnittlich ein Fünftel der Lösungen (s. Abb. 4-37). Auf die Kategorie Lösung richtig mit leichten Abstrichen entfallen noch weitere $10 \%$ bis $25 \%$ der Lösungen. Die restlichen Lösungen entfallen je nach Erfüllung der Anforderungen für die Toleranzbereiche auf die Kategorien Lösung fehlerhaft bzw. stark fehlerhaft. Die neun Fälle, deren zugrundeliegender Rhythmus nicht identifiziert werden kann, werden automatisch der Kategorie Lösung stark fehlerhaft zugeordnet.

Die Lösungen, die in der Rubrik Aufgabenverständnis als fehlerhaft gewertet wurden (Kategorie 52 / 52.2) werden der Lösungskategorie Lösung fehlerhaft zugeordnet, wenn die Analyse des Rhythmus den Anforderungen des erweiterten Toleranzbereichs genügt. Alle Lösungen in den Kategorien 74, 75 sowie 80 (Item D 1 und D 3) sowie 76, 77 sowie 80.2 (Item D 2 und D 4) werden der Lösungskategorie Lösung stark fehlerhaft zuordnet, da die Aufgabenstellung nicht erfüllt wurde. Diese hat besonders bei den Items D 2 und D 4 mit über $50 \%$ sehr hohe Anteile. Grund ist das fehlende Vermögen, den gleichen Rhythmus noch einmal zu spielen und stattdessen mit einem anderen Rhythmus aufzuwarten.

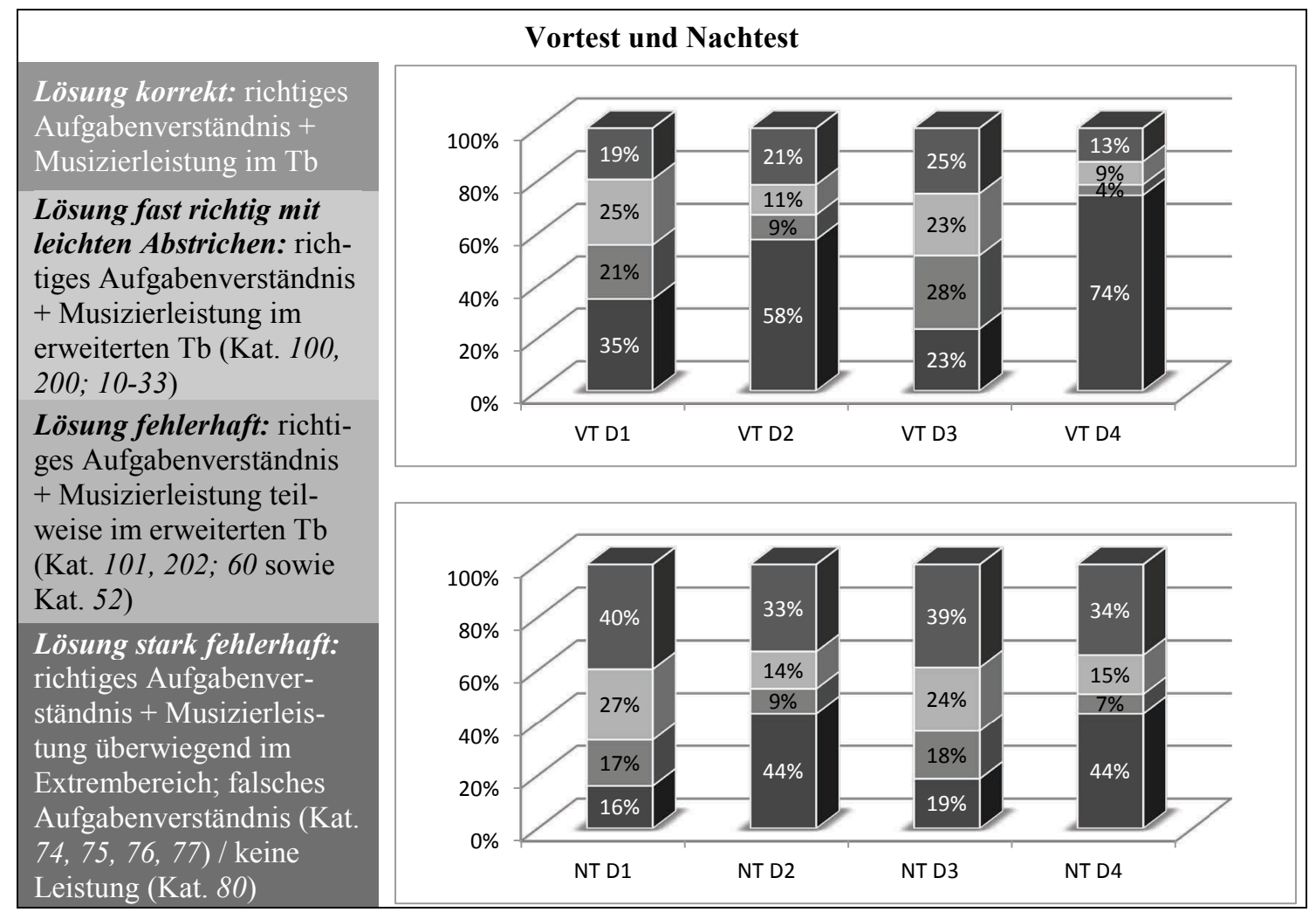

Abb. 4-37: Gesamtdarstellung aller Leistungen im ST D nach Lösungskategorien 
Auch im Nachtest wird ein erheblicher Teil der richtigen Lösungen in der Rubrik Aufgabenverständnis in Bezug auf die Rubriken Einsatz bzw. Tempo des Rhythmus fehlerhaft musiziert. Dennoch entfallen auf die Kategorie Lösung korrekt im Nachtest bereits die Hälfte der Lösungen, auf die Kategorie Lösung richtig mit leichten Abstrichen entfallen weitere $14 \%$ bis $27 \%$.

\section{Vielfalt der abgewandelten Rhythmen}

In Bezug auf die Vielfalt der abgewandelten Rhythmen werden nur die korrekt gespielten Rhythmen aus der Kategorie 0 ein anderer Rhythmus als Rhythmus 7a, Einsatz und Tempo richtig berücksichtigt. Im Vortest werden insgesamt 18 verschiedene Rhythmen (s. Abb. 4-38; Übersicht mit Notenbild der Rhythmen s. Anlage E.), im Nachtest bereits 29 angeboten (s. Abb. 4-39).

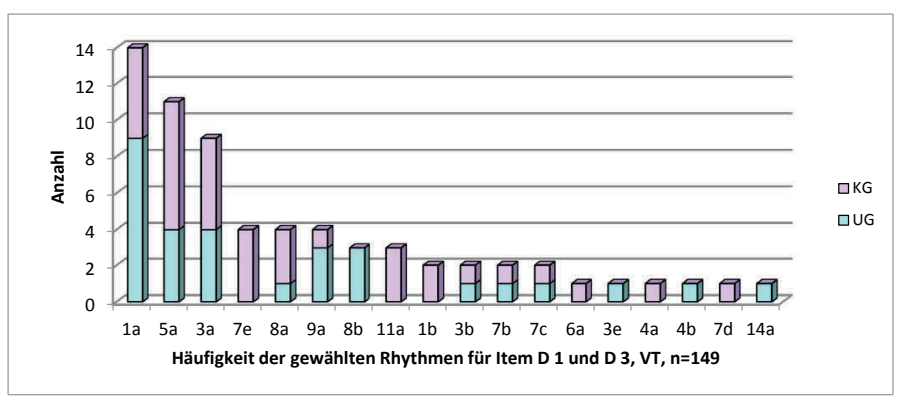

Abb. 4-38: Vielfalt der gespielten Rhythmen im ST D, VT

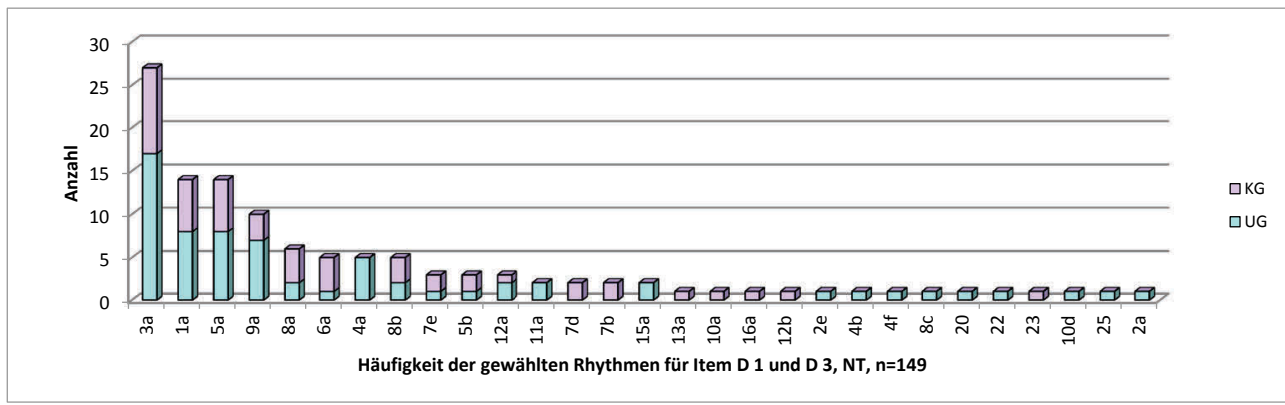

Abb. 4-39: Vielfalt der gespielten Rhythmen im ST D, NT

\subsubsection{Ergebnisse im Subtest E Rhythmen lesen und musizieren}

In die Gesamtpunktwertung des Testes geht nur der Testteil E Rhythmen lesen und musizieren nach Noten ein. Getestet wird das Musizieren von vier Rhythmen nach Notenvorlage (s. Abb. 4-40).

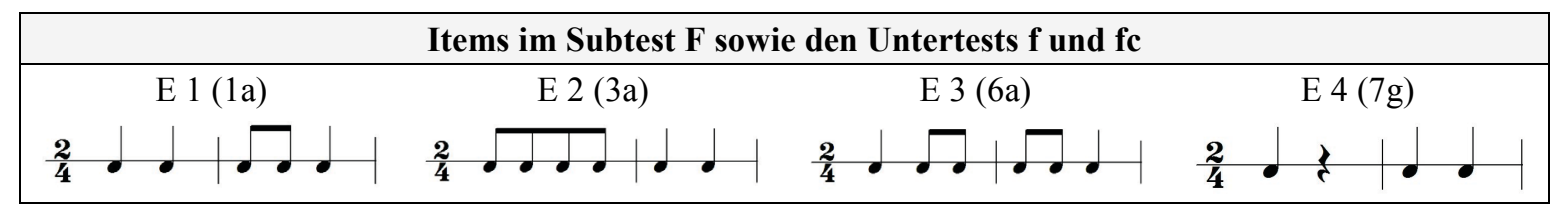

Abb. 4-40: Übersicht über die Items im ST E 
Das Musizieren der Rhythmen nach Notenvorlage gelingt erstaunlich vielen Probanden (s. Abb. 4-41). Bereits im Vortest können bis zu einem Drittel der Probanden der Kontrollgruppe zumindest Item E 1 richtig lösen. In der Untersuchungsgruppe ist das nur für knapp ein Sechstel der Probanden der Fall. Der Vorsprung in den Leistungen der Probanden der Kontrollgruppe im Vortest geht vor allem auf die Probanden aus den Klasse K 1 und K 2 zurück (11 x 0 Punkte, 10 x 1 Punkte, 5 x 2 Punkte, 6 x 3Punkte sowie 2 x 4 Punkte).

Der größte Anteil an richtigen Lösungen wird in der Gruppe der Schülerinnen und Schüler mit außerschulischem Instrumentalunterricht erbracht. Betrachtet man gezielt diese Gruppe der insgesamt 32 Schülerinnen und Schüler, die mindestens ein bis zwei Jahre Instrumentalunterricht erhalten, so gelingt in dieser Gruppe zwar auch acht Schülern keines der vier Items (UG: 3; KG: 5). Jedoch jeweils sechs Schülern gelingen je ein Item (UG: 4; KG: 2), zwei Items (UG: 4; KG: 2), drei Items (UG: 4; KG: 2) sowie bereits alle vier Items (UG: 3; KG: 3).
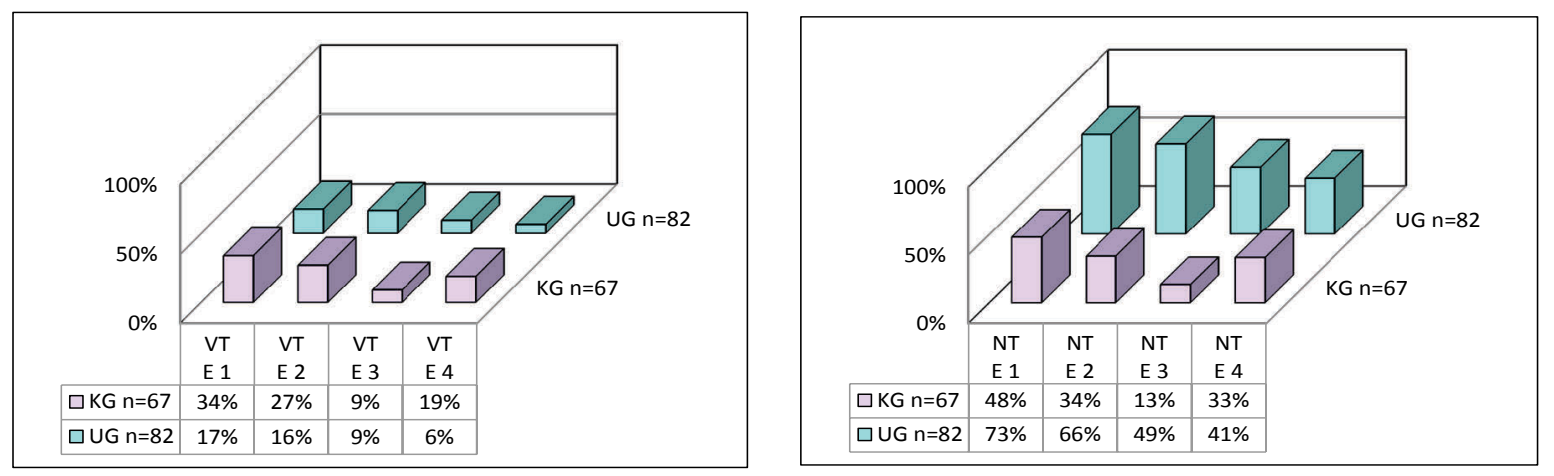

Abb. 4-41: Ergebnisse im ST E, VT und NT, getrennt nach UG und KG

Im Nachtest werden die Items von ca. einem Drittel der Probanden der Kontrollgruppe richtig gelöst, jedoch bereitet das Spielen von Item E 3 größere Schwierigkeiten. In der Untersuchungsgruppe liegt der Anteil an Probanden, welche die Rhythmen lesen und musizieren können bei etwa $50 \%$. Die Items nehmen in ihrer Reihenfolge kontinuierlich an Schwierigkeit zu .In der Gruppe der 32 Instrumentalschüler gelingt im Nachtest zwei Schülern keines der vier Items (UG: 1; KG: 1) sowie einem Schüler nur ein Item (UG: 1; KG: 1). Vier Schülern gelingen je zwei Items (UG: 3; KG: 1), 14 Schülern je drei Items (UG: 5; KG: 9) sowie elf Schülern bereits alle vier Items (UG: 9; KG: 2).

Tab. 4-13: Mittelwerte im ST E

\begin{tabular}{cccccccc}
\hline \multirow{2}{*}{ ST } & UG & N & $\begin{array}{c}\text { MW im } \\
\text { VT }\end{array}$ & $\begin{array}{c}\text { MW im } \\
\text { NT }\end{array}$ & $\begin{array}{c}\text { Diff. } \\
\text { VT/NT }\end{array}$ & $\begin{array}{c}\text { SD der } \\
\text { Diff. }\end{array}$ & $\begin{array}{c}\text { SE der } \\
\text { Diff. }\end{array}$ \\
\hline \multirow{2}{*}{ E } & UG & 82 & 0.48 & 2.29 & 1.82 & 1.40 & 0.15 \\
& KG & 67 & 0.90 & 1.28 & 0.39 & 1.17 & 0.14 \\
\hline
\end{tabular}


Tab. 4-14: Ergebnisse für t-Test und Effektstärke im ST E

\begin{tabular}{ccccccc}
\hline ST & $\mathbf{t}$ & df & $\mathbf{p}$ (2seit.) & $\begin{array}{c}\text { Diff. der } \\
\mathbf{M W}\end{array}$ & $\begin{array}{c}\text { SE der Diff. } \\
\text { der MW }\end{array}$ & $\mathbf{d}[\mathbf{9 5 \%} \mathbf{C I}]$ \\
\hline $\mathrm{E}$ & 6.68 & 147 & $<0.001$ & 1.43 & 0.21 & $1.10[1.01,1.85]$ \\
\hline
\end{tabular}

Die Mittelwerte steigen im Nachtest in beiden Gruppen an, wobei dies in der Untersuchungsgruppe etwa um das Vierfache stärker ausfällt (s. Tab. 4-13). Dieser Vorsprung zeigt sich in einem großen Effekt mit einem Wert von $d=1.10$ (s. Tab. 4-14).

\section{Fehlerquellen im Subtest E beim Musizieren nach Noten}

Die Fehlerkategorien im Subtest E werden nur in den Rubriken Rhythmus an sich sowie Tempo des Rhythmus betrachtet. Die Rubrik Einsatz des Rhythmus entfällt, da die Probanden den Einsatz Rhythmus selbst bestimmen dürfen. Die Rubrik Rhythmus an sich umfasst die Kategorien: zu viele / zu wenige Schläge (Kat. 51, 52), Achtelpaare um einen Schlag erweitert (Kat. 55), Rhythmus erkennbar, mit starken Temposchwankungen (Kat. 60), Rhythmus falsch ohne spezielle Fehlerkategorie (Kat. 61), Rhythmus falsch, jedoch anderer Rhythmen gut erkennbar (Kat. 62) sowie keinen Rhythmus gespielt (Kat. 80).

Die Kategorien 51 und 52 erschließen sich aus ihrer Bezeichnung heraus. Es wird beim Lesen und Musizieren des Rhythmus ein Schlag hinzugefügt bzw. weggelassen. In der Kategorie 55 wird den Achtelpaaren jeweils ein dritter Achtelschlag hinzugefügt. Damit entstehen Dreiergruppen aus Achtelschlägen, die mitunter auch triolierend gespielt werden. Beispiel für die Items E 2 und E 3 aus der Sequenzersoftware werden dargestellt (s. Abb. 4-42).

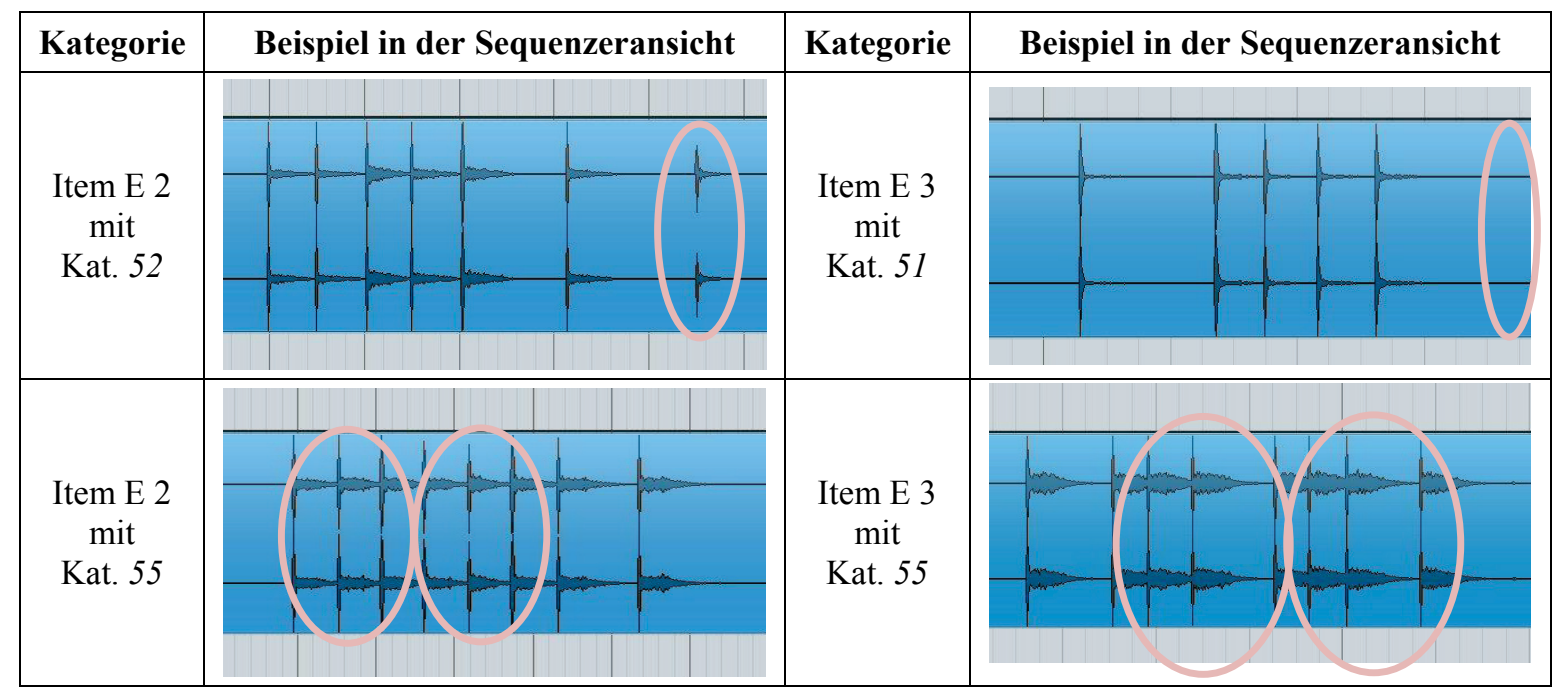

Abb. 4-42: Beispiele für Fehlerkategorie 51, 52 und 55 im ST E

Die Kategorie 60 Rhythmus erkennbar, mit starken Temposchwankungen stellt sich im Subtest E deutlich anders als in den Subtests B und D dar. Zudem stellen sie sich in den vier Items verschie- 
den dar. In den Items E 1, E 2 und E 3 ist zwischen dem jeweils zweiten Achtelschlag (eines Paares von Achtelschlägen) und dem folgenden Schlag auf dem nächsten Grundschlag ein zu großer Abstand zu beobachten (s. Abb. 4-43, oben links und oben rechts). Diese Abstände weisen trotz der starken Temposchwankungen eine gewisse Regelmäßigkeit auf. Sie müssten treffender mit regelmäßig starke Temposchwankungen bezeichnet werden. In Item E 4 lassen sich bei den Probanden zwei verschiedene Schlagbilder für die Kategorie 60 beobachten: Die Pause auf Zählzeit 2 wird stark überdehnt oder stark verkürzt (s. Abb. 4-43, unten links und unten rechts).

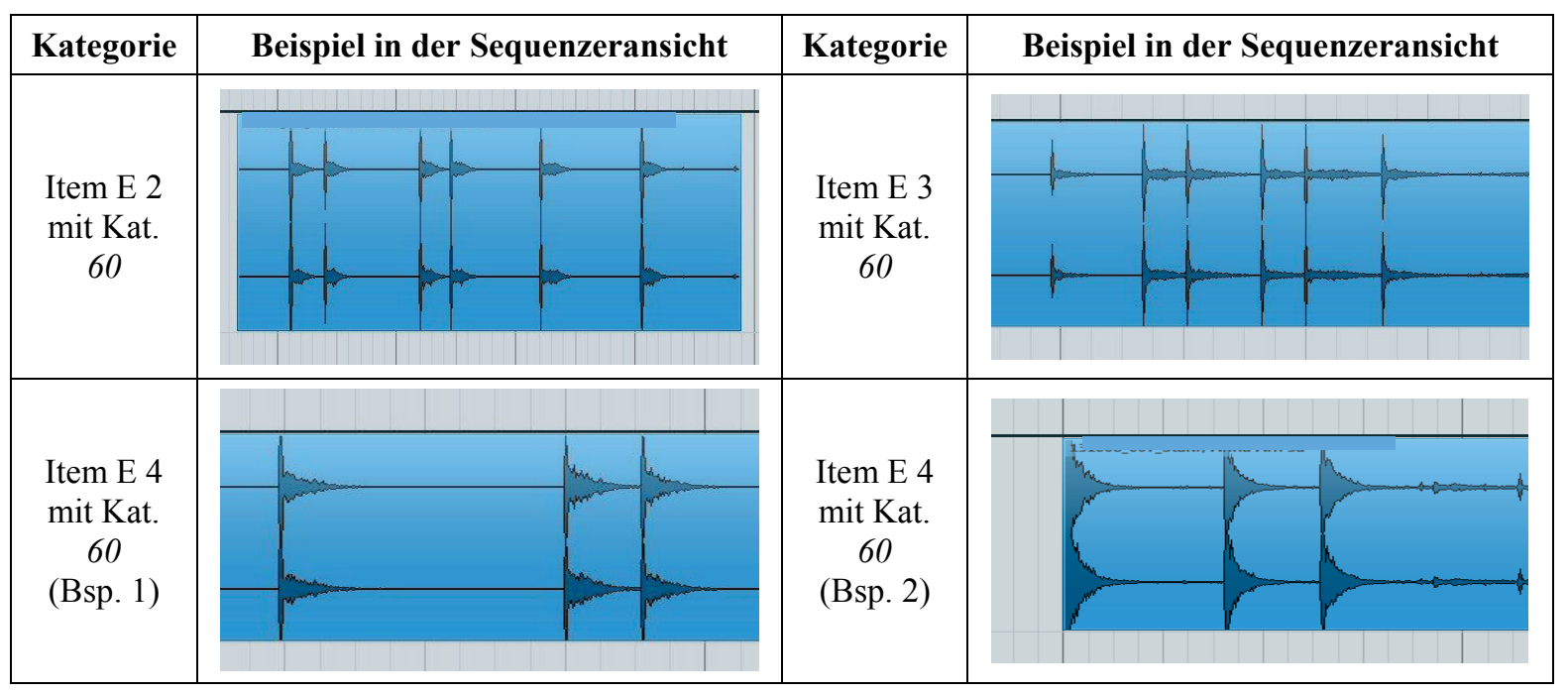

Abb. 4-43: Beispiele für Fehlerkategorie 60 im ST E

Der Kategorie 61 Rhythmus falsch ohne spezielle Fehlerkategorie werden alle Lösungen zugeordnet, die den bisherigen bzw. der folgenden Kategorie nicht entsprechen. In der Kategorie 62 Rhythmus falsch, jedoch anderer Rhythmen gut erkennbar werden Rhythmen erfasst, die dem Notenbild nicht entsprechen, aber in ihrer Struktur einen anderen Rhythmus klar erkennen lassen. Die Kategorie 80 keinen Rhythmus gespielt erklärt sich selbst.

In der Rubrik Rhythmus an sich nimmt im Vortest die Kategorie 80 keinen Rhythmus gespielt erwartungsgemäß den größten Raum mit einem Anteil von über $50 \%$ in allen Items ein. Im Nachtest ist eine starke Abnahme dieser Kategorie zu verzeichnen (s. Abb. 4-44). Diese korreliert mit der Zunahme in der Kategorie 0 richtiger Rhythmus. Die Kategorien 51, 52, 55, 61 sowie 62 sind im Nachtest mit Anteilen zwischen $1 \%$ und $11 \%$ relativ schwach vertreten. Eine Übersicht über Ersatzrhythmen für die Kategorie 62 Rhythmus falsch, jedoch ein anderer Rhythmus gut erkennbar wird gegeben (s. Tab. 4-15). Die Unterschiede zwischen Untersuchungs- und Kontrollgruppe im Nachtest belaufen sich hauptsächlich auf eine insgesamt stärkere Ausprägung in der Kategorie 0 richtiger Rhythmus und eine schwächere Ausprägung in den Kategorien 60 und 61 zugunsten der Untersuchungsgruppe. 


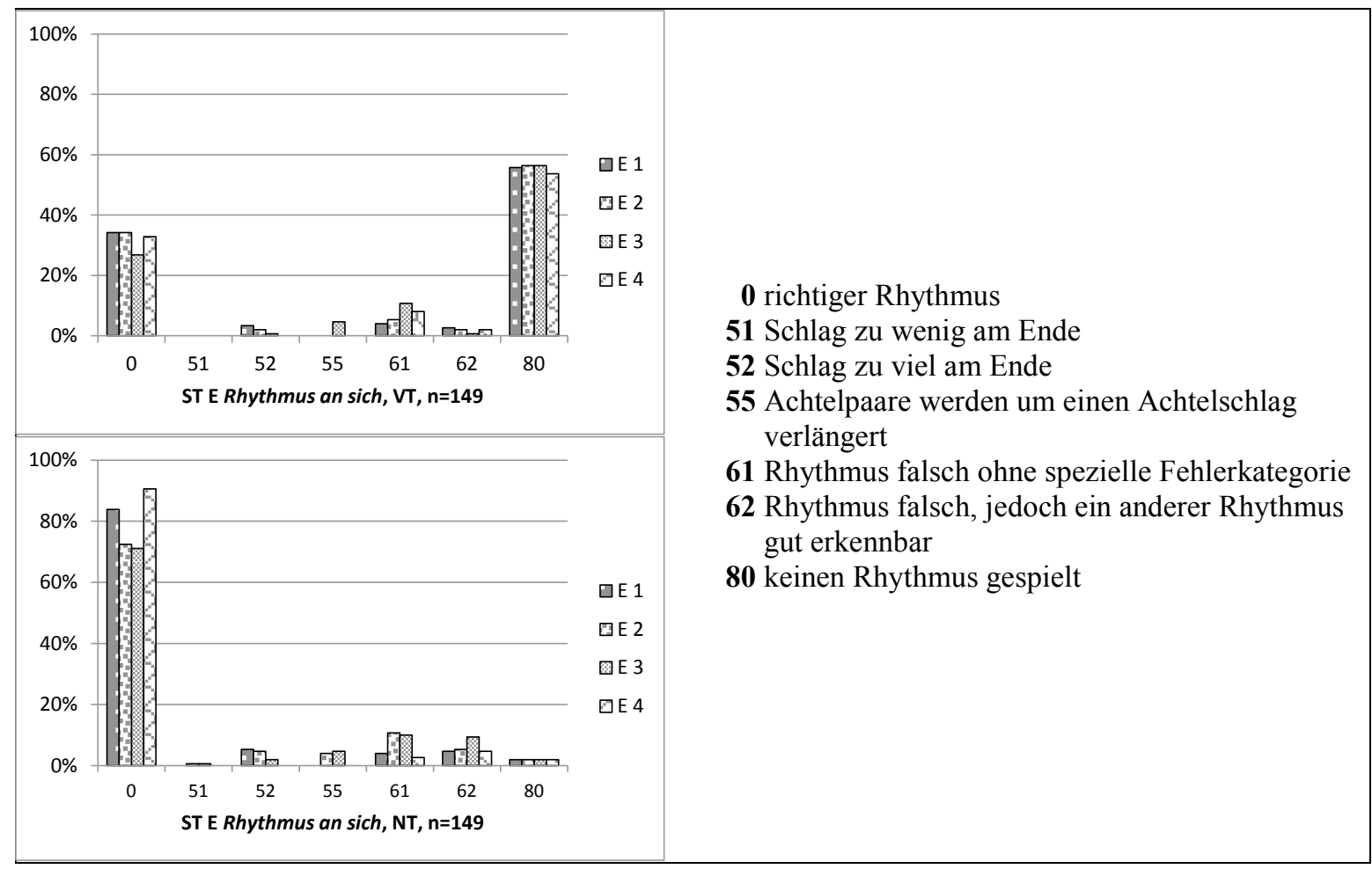

Abb. 4-44: Häufigkeit von Fehlern in der Rubrik Rhythmus an sich im ST E, VT und NT

Tab. 4-15: Häufige Ersatzrhythmen im ST E im NT

\begin{tabular}{|c|c|c|c|c|}
\hline & Item E1 & Item E2 & Item E3 & Item E4 \\
\hline Rhythmus & $1 \mathrm{a}$ & $3 a$ & $6 a$ & $7 \mathrm{~g}$ \\
\hline Notenbild & $\frac{2}{4} \cdot|\cdot| \cdot \mid$ & $\frac{2}{4} \cdot \cdots \cdot \cdot \cdot \cdot \mid$ & $\frac{2}{4} \cdot \sqcap|\curvearrowleft \cdot|$ & $\frac{2}{4} \cdot !|\cdot|$ \\
\hline $\begin{array}{l}\text { Anzahl des } \\
\text { dominanten } \\
\text { Rhythmus }\end{array}$ & keine Beobachtung & 3 x Rhythmus $8 \mathrm{a}$ & 13 x Rhythmus 5a & 5 x Rhythmus 20 \\
\hline $\begin{array}{l}\text { Ersatzrhytmus } \\
\text { Notenbild }\end{array}$ & $\begin{array}{l}\text { kein dominanter } \\
\text { Rhythmus }\end{array}$ & $\frac{2}{4} \cdot \cdot|\cdot| \cdot \mid$ & $\frac{2}{4} \cdot \neg|\cdot \cdot|$ & $\frac{2}{4} \cdot|\cdot| \cdot \mid$ \\
\hline $\begin{array}{l}\text { Ersetzungen } \\
\text { insgesamt }\end{array}$ & $7 x$ & $8 x$ & $14 x$ & $9 x$ \\
\hline
\end{tabular}

Ein großer Teil der an sich richtig gespielten Rhythmen hält der genauen Tempoanalyse nicht stand. In der Rubrik Tempo des Rhythmus werden ähnliche Tempofehler wie in Subtest B und D beobachtet. Dazu zählen besonders die Kategorien mit leicht schwankenden (Kat. 30-32) bis stark schwankendem Tempi (Kat. 60). Die Kategorien 90 und 91 enthalten Rhythmen, deren Tempo aufgrund vorgelagerter Fehler nicht bestimmt werden kann (s. Abb. 4-45).

In der Kontrollgruppe ist die Kategorie 60 die häufigste Fehlerquelle. Am prozentualen Anteil pro Item lässt sich sehr gut ablesen, wie schwer die einzelnen Rhythmen zu musizieren waren. Wieder fällt besonders Item E 3 auf (s. Abb. 4-41). Interessanterweise ändert sich dies im Subtest C Rhythmussprache anwenden (s. Abb. 4-52). 


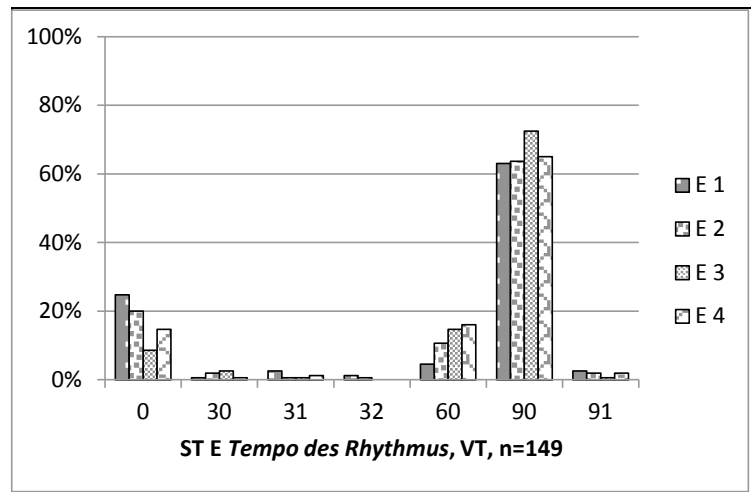

0 Tempo innerhalb des Toleranzbereiches

30 leicht schwankendes Tempo

35 Tempo aufgrund eines Temposprungs über $15 \mathrm{bpm}$ als fehlerhaft gewertet

31 einige Werte im negativ erweiterten 60 stark schwankendes Tempo

Toleranzbereich

32 einige Werte im positiv erweiterten

90 Tempo nicht bestimmbar aufgrund Kat. 51, 52, 61,80

Toleranzbereich

91 Tempo nicht bestimmbar, da anderer Rhythmus gespielt (zu Kat. 62)
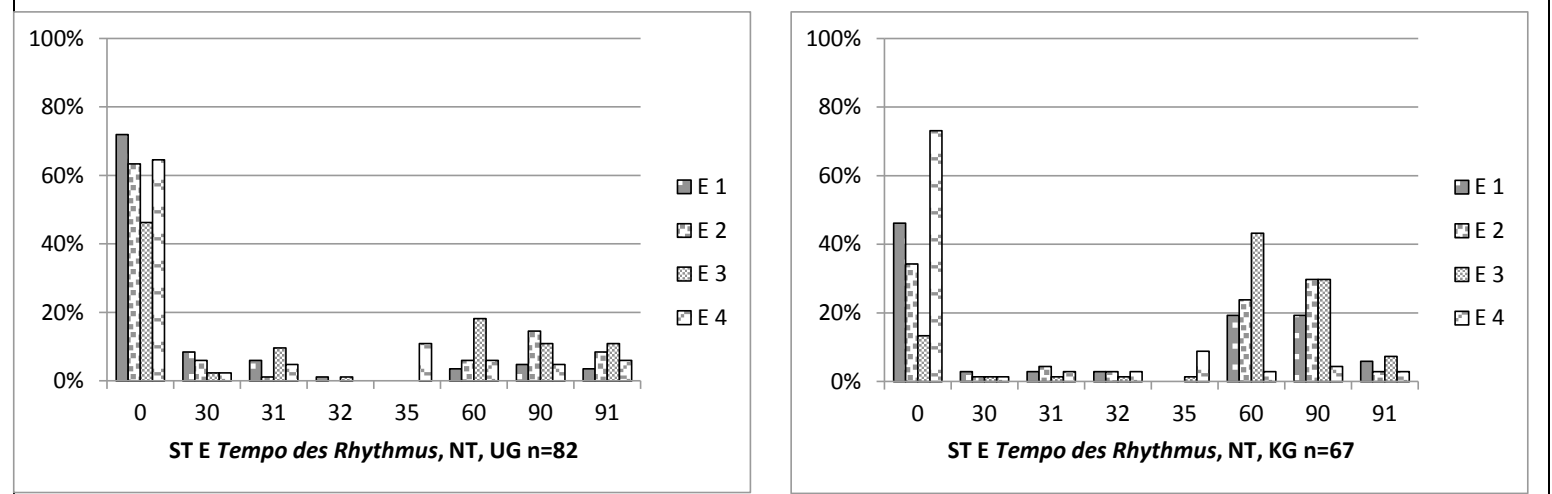

Abb. 4-45: Häufigkeit von Fehlern in der Rubrik Tempo des Rhythmus im ST E, VT und NT

\section{Abgestufte Ergebnisdarstellung}

Alle Lösungen werden in abgestuften Kategorien von Lösung korrekt bis Lösung stark fehlerhaft bzw. keine Lösung dargestellt. In dieser Darstellung lässt sich erkennen, dass im Vortest über 60 \% der Lösung in die Kategorie Lösung stark fehlerhaft bzw. keine Lösung eingestuft werden muss. Aus den vorangehenden Abbildungen im Unterabschnitt Fehlernanalyse ist ersichtlich, dass dies auf die vielen Probanden zurückzuführen ist, die keine Lösungen anbieten (s. Abb. 4-46). In der Kategorie Lösung korrekt findet sich ein beachtlicher Anteil zwischen $9 \%$ bis $25 \%$ der Lösungen.

Im Nachtest bieten nur noch drei Probanden keine Lösung an (Kat. 80). Alle anderen Lösungen in der Kategorie Lösung stark fehlerhaft gehen auf die Fehlerkategorien 61 und 62 zurück. In der Kategorie Lösung korrekt findet sich ein beachtlicher angewachsener Anteil zwischen $30 \%$ bis 60 \% der Lösungen. Erstaunlicherweise ist der Rhythmus in Item E 3, welcher nur aus Viertel- und Achtelschlägen ohne Schwerpunktverlagerung besteht, schwerer zu spielen als der Rhythmus in Item E 4 mit einer Viertelpause. 


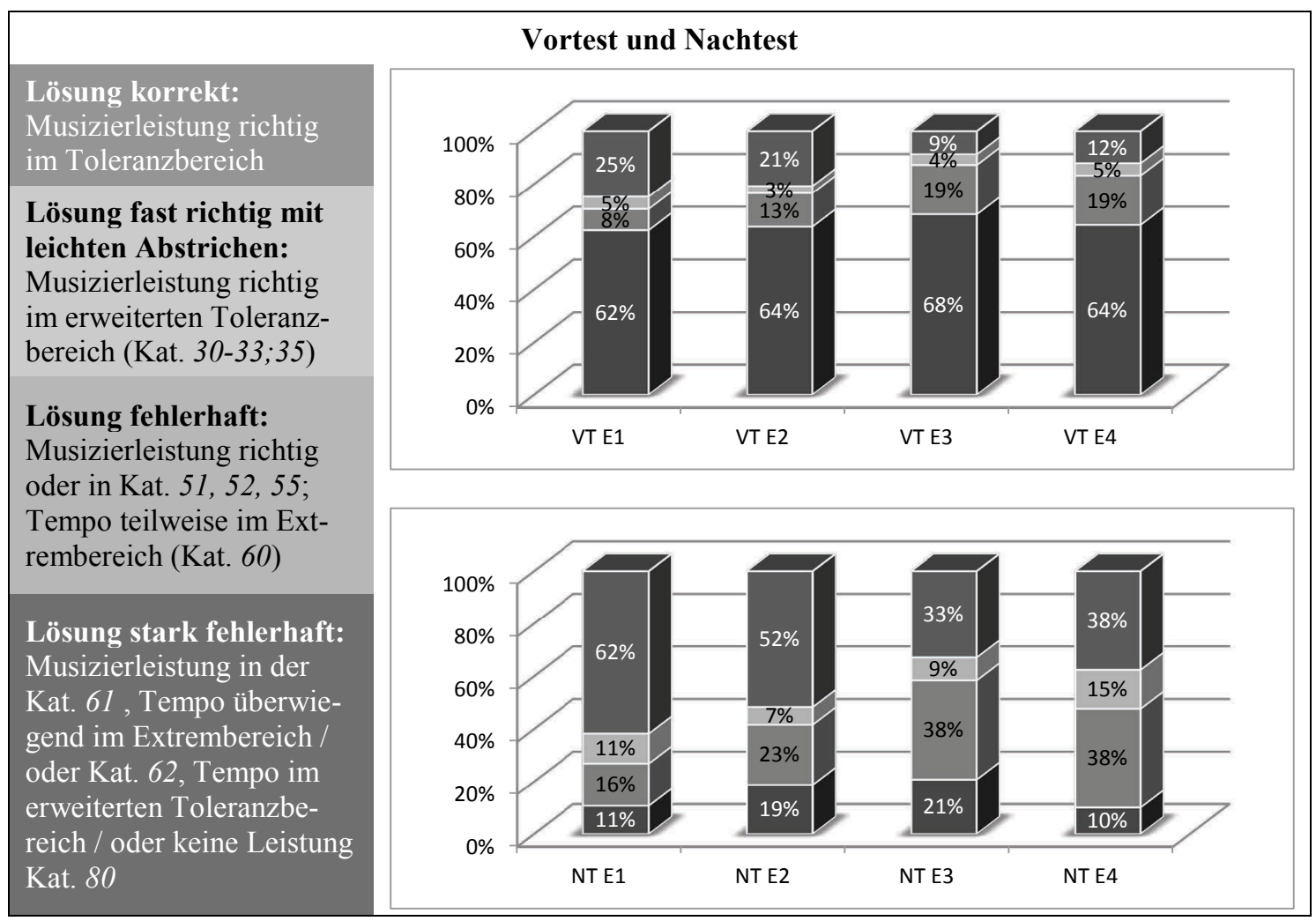

Abb. 4-46: Gesamtdarstellung aller Leistungen im ST E nach Lösungskategorien

\section{Zur Wahl des individuellen Musiziertempos}

In diesem Subtest dürfen die Probanden sowohl des Einsatz des Rhythmus als auch dessen Tempo selbst bestimmen. Um die Bandbreite bei der Wahl des individuellen Musiziertempos grafisch darstellen zu können, werden die gewählten Tempi der richtig gespielten Rhythmen in verschiedene Tempo-Bereiche untergliedert. Ausgangspunkt bildet das im Videotest im Subtest B und D genutzte Playback mit einem Tempo von 86 bpm. Dieses Tempo wird zum Mittelpunkt der Skala erklärt. Die anderen Bereiche entstehen durch Addition bzw. Subtraktion mit einem Wert von 15 bpm. So entstehen sechs Tempobereiche.

Die beiden Bereiche neben dem Mittelpunkt von 86 bpm werden gekoppelt zum mittelschnellen Bereich (s. Abb. 4-47). Die anderen Bereiche tragen die Bezeichnungen langsam und sehr langsam bzw. schnell und sehr schnell. Diese Einteilung liegt in guter Relation zu den gebräuchlichen Tempobezeichnungen und deren ungefähren Tempoempfehlungen. Zum Vergleich werden die Angaben des Metronomherstellers Firma Wittner herangezogen.

Im Vortest bewegt sich das individuelle Musiziertempo der Probanden zwischen 28 bpm und $123 \mathrm{bpm}$. Im Nachtest bewegt sich das individuelle Musiziertempo der Probanden zwischen 37 bpm und 133 bpm. Die meisten richtigen Leistungen werden im mittleren Tempobereich erbracht (s. Abb. 4-48). 


\begin{tabular}{|c|c|c|c|c|c|c|c|}
\hline & & & & $>86$ & $\mathrm{pm}<$ & & \\
\hline \multirow{2}{*}{$\begin{array}{c}\text { Test- } \\
\text { Auswertung }\end{array}$} & bpm: & $37-55$ & $56-70$ & $71-85$ & $86-100$ & $101-115$ & 116-133 \\
\hline & Bezeichnung: & sehr langsam & langsam & \multicolumn{2}{|c|}{ mittelschnell } & schnell & sehrschnell \\
\hline \multirow{2}{*}{$\begin{array}{l}\text { Metronom } \\
\text { Wittner }\end{array}$} & Bezeichnung: & largo & largh./adagio & \multicolumn{2}{|c|}{ andante } & moderato & allegro \\
\hline & bpm: & $40-60$ & $60-76$ & \multicolumn{2}{|c|}{$76-108$} & $108-120$ & $120-168$ \\
\hline
\end{tabular}

Abb. 4-47: Einteilung der Tempobereiche in ST E

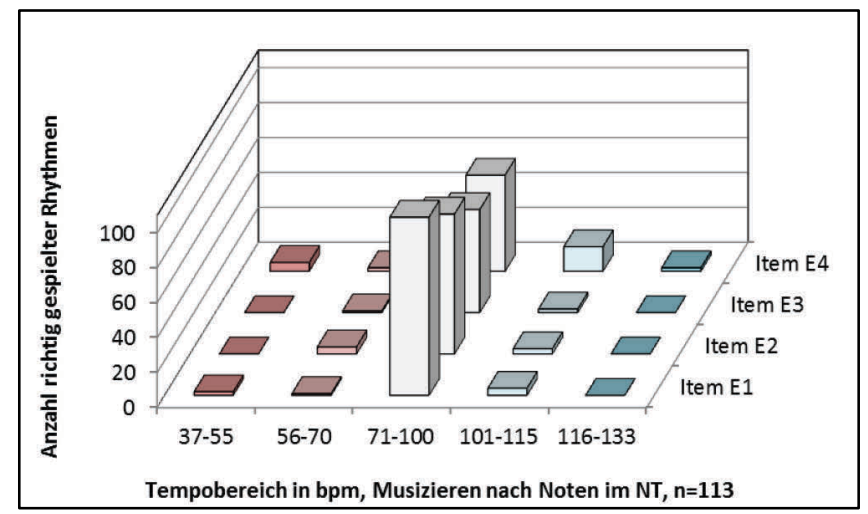

Abb. 4-48: Häufigkeit der Wahl des individuellen Tempos im ST E im NT

Es fällt auf, dass im Item E 4 die Verteilung über die verschiedenen Tempobereiche unregelmäßiger als in den anderen Items ausgeprägt ist (s. Abb. 4-48). Es gibt die meisten sehr langsam und die meisten schnell gespielten Ergebnisse. Deshalb werden die Temposchwankungen bei den 113 Probanden, die mindestens zwei Rhythmen richtig gespielt haben, genauer betrachtet. Dazu werden die Differenzen der Temposchwankungen berechnet. Durchschnittlich schwankt das Tempo im Nachtest um 8 bpm. Diese Schwankungen umfassen zum größten Teil Tempoanstiege, aber auch -verluste. Die Extremwerte für Temposchwankungen liegen zwischen - 29 und +31 bpm. In der Musizierpraxis ergäbe solch eine Spielweise für aufeinanderfolgende Rhythmen kein akzeptables Musizierergebnis. Deshalb wird folgende Entscheidung getroffen: Zwei Rhythmen, die für sich genommen richtig gespielt werden, jedoch extreme Temposchwankungen aufweisen, sollen nicht gleichzeitig als richtig gewertet werden.

Ausgehend von den berechneten Mittelwerten der Schwankungen von $8 \mathrm{bpm}$ wird festgelegt, dass Rhythmen, die um mehr als 15 bpm im Verhältnis zum vorhergehenden (richtig gespielten) Rhythmus schneller oder langsamer gespielt werden, nicht mehr als richtig gewertet und der Fehlerkategorie 35 zugeordnet werden (s. Abb. 4-45). Dies führt im Nachtest bei Item E 3 zu einer Abwertung und bei Item E 4 zu 12 Abwertungen (UG: 7; KG: 6). Die Anzahl der Abwertungen für Item $\mathrm{E} 4$ liegt in Übereinstimmung mit der eingangs erwähnten Beobachtung, dass bei diesem Item die Spannbreite der Verteilung über die verschiedenen Tempobereiche am unregelmäßigsten ist. 
Ergebnisse

\subsubsection{Ergebnisse im Subtest C Rhythmussprache anwenden}

Im Untersuchungszeitraum erlernten alle Schülerinnen und Schüler der Untersuchungsgruppe sowie die Klassen K 1 bis K 4 eine Rhythmussprache (100 \% der Probanden der Untersuchungsgruppe sowie $70 \%$ der Probanden der Kontrollgruppe). Nur die Klassen K 5 und K 6 hatten keine Rhythmussprache erlernt (30\% der Probanden der Kontrollgruppe). Zwei Probanden aus der Klasse K 5 erlernten jedoch im Instrumentalunterricht die Sprache nach Ernst bzw. die Tatak-Sprache (Giordani \& Brodesser, 2009). Die Klasse U 7 erlernte die Sprache nach Paris, alle anderen Klassen die Sprache nach Kodály.

Der Subtest $\mathrm{C}$ wird rechnerisch aus dem Untertest ec Rhythmen lesen und musizieren und sprechen von Rhythmussilben sowie dem Untertest $f_{c}$ Rhythmen musizieren und aufschreiben mit Rhythmussilben generiert. Kein Rhythmus soll doppelt repräsentiert werden (d.h. nicht gleichzeitig ec 1 und fc 1 oder ec 2 und fc 2). In einer Itemanalyse werden Itemschwierigkeit und Trennschärfe für die Items berechnet (s. Anlage C 3 a). Sie sollen dem geplanten Wert für die Itemschwierigkeit $p$ (s. Tab. 3-14) nahekommen sowie eine möglichst hohe Trennschärfe $\left(r_{i(t-i)}\right)$ aufweisen (s. Abschnitt 3.4.5). Die Auswahl fällt auf die Items ec 1 und ec 3 sowie fc 2 und fc 3 (s. Abb. 4-49). Die Items werden in der genannten Reihenfolge umbenannt in Item C 1, C 2, C 3 und C 4. Die Itemstatistiken finden sich in Anlage C 3 b, die vollständigen Ergebnisse aller Items aus den Untertests ec und fe für Vor- und Nachtest in Anlage C 6.

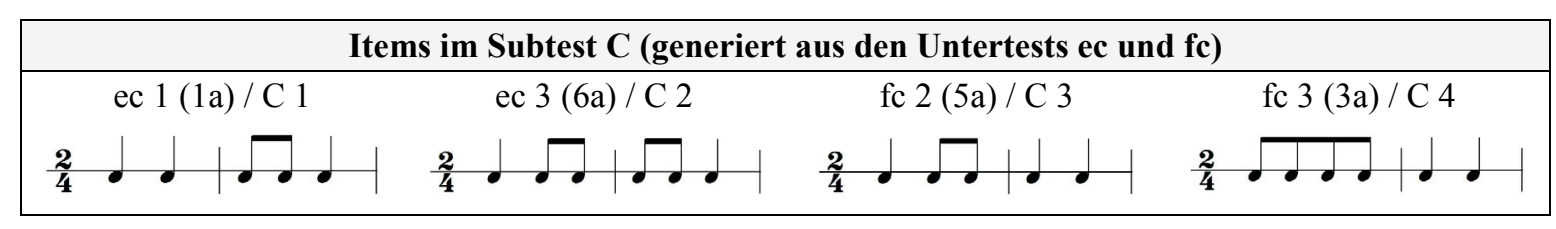

Abb. 4-49: Übersicht über die Items im Subtest C

Für Subtest C werden im Vortest kaum Ergebnisse erwartet. Bis zu 7 \% der Probanden können richtige Lösungen beim Anwenden einer Rhythmussprache erbringen, für Item C 1 sogar 19 \% der Probanden der Kontrollgruppe (s. Abb. 4-50). Die Probanden der Kontrollgruppe liegen leicht im Vorsprung. Item C 1 wird besonders von den Probanden aus den Klassen K 1 und K 2, welche zu diesem Zeitpunkt schon eine Rhythmussprache erlernt haben, richtig musiziert und mit Silben gesprochen.

Betrachtet man gezielt die Gruppe der insgesamt 32 Schülerinnen und Schüler, die mindestens ein bis zwei Jahre Instrumentalunterricht erhalten, so gelingt auch in dieser Gruppe drei Vierteln der Probanden (22 Schülern; UG: 13, KG: 9) keines der vier Items. Drei Schülern gelingt ein Item (UG: 1; KG: 2), fünf Schülern je zwei Items (UG: 1; KG: 4), keinem Schüler je drei Items (UG: 0; KG: 0) sowie zwei Schülern bereits alle vier Items (UG: 2; KG: 0). Die zehn Instrumentalschüler, 
welche in den Items erfolgreich Leistungen erbringen, besuchen zusätzlich zum Instrumentalunterricht den Theorieunterricht ihrer Musikschule.
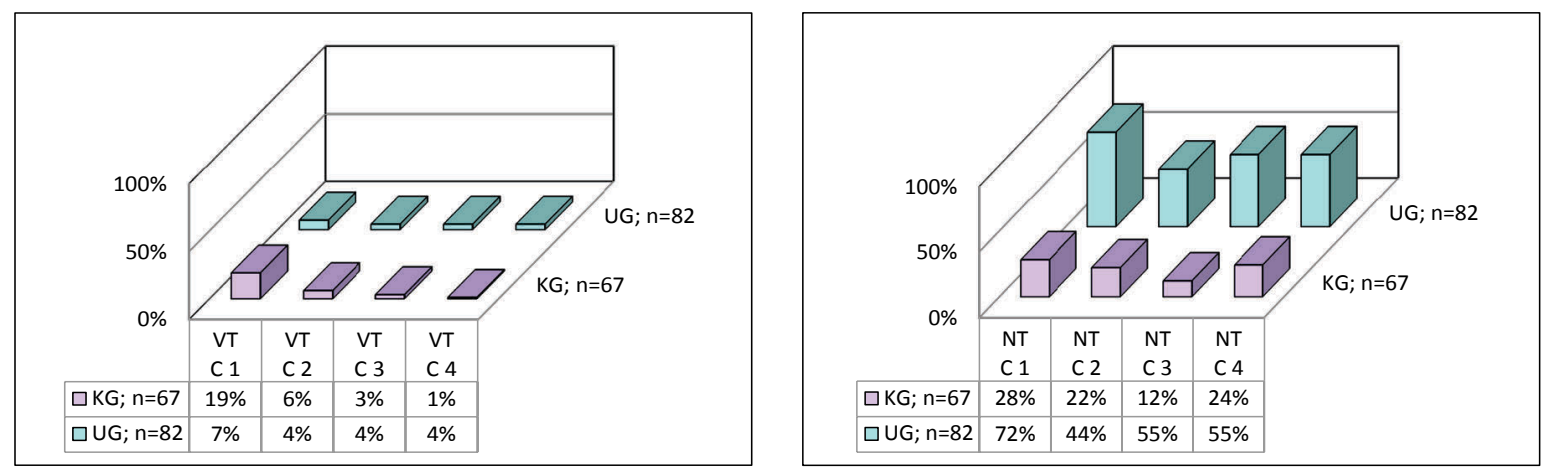

Abb. 4-50: Ergebnisse im ST C, VT und NT, getrennt nach UG und KG

Im Nachtest können relativ viele Probanden richtige Ergebnisse erbringen. In der Untersuchungsgruppe gelingt Item C 1 beachtlichen $72 \%$ der Probanden. Die anderen Items werden von etwa der Hälfte der Probanden bewältigt. In der Kontrollgruppe kann fast durchweg ein Viertel der Probanden die Aufgaben bewältigen. Item C 3 gelingt nur $12 \%$ der Probanden. In der Gruppe der 32 Instrumentalschüler gelingt im Nachtest vier der Probanden keines der vier Items (UG: 2, KG: 2). Fünf Schülern gelingt ein Item (UG: 1; KG: 4), fünf Schülern je zwei Items (UG: 3; KG: 2), sechs Schülern je drei Items (UG: 2; KG: 4) sowie 12 Schülern bereits alle vier Items (UG: 10; KG: 2).

Die Mittelwerte im Nachtest steigen in beiden Gruppen an, wobei dies in der Untersuchungsgruppe etwa um das Vierfache stärker ausfällt (s. Tab. 4-16). Dieser Vorsprung zeigt sich in einem großen Effekt mit einem Wert von $d=1.15$ (s. Tab. 4-17).

Tab. 4-16: Mittelwerte im ST C

\begin{tabular}{llllcccc}
\hline \multirow{2}{*}{ ST } & $\begin{array}{l}\text { UG } \\
\text { KG }\end{array}$ & N & $\begin{array}{c}\text { MW im } \\
\text { VT }\end{array}$ & $\begin{array}{c}\text { MW im } \\
\text { NT }\end{array}$ & $\begin{array}{c}\text { Diff. } \\
\text { VT/NT }\end{array}$ & $\begin{array}{c}\text { SD der } \\
\text { Diff. }\end{array}$ & $\begin{array}{c}\text { SE der } \\
\text { Diff. }\end{array}$ \\
\hline \multirow{2}{*}{$\mathrm{C}$} & UG & 82 & 0.18 & 2.26 & 2.07 & 1.46 & 0.16 \\
& KG & 67 & 0.30 & 0.87 & 0.57 & 1.08 & 0.13 \\
\hline
\end{tabular}

Tab. 4-17: Ergebnisse für t-Test und Effektstärke im ST C

\begin{tabular}{ccccccc}
\hline ST & $\mathbf{t}$ & df & p (2seit.) & $\begin{array}{c}\text { Diff. der } \\
\text { MW }\end{array}$ & $\begin{array}{c}\text { SE der Diff. } \\
\text { der MW }\end{array}$ & d [95\% CI] \\
\hline C & 7.01 & 147 & $<0.001$ & 1.51 & 0.22 & $1.15[1.08,1.93]$ \\
\hline
\end{tabular}


Ergebnisse

\section{Fehlerquellen im Untertest ec Rhythmen lesen und musizieren und sprechen von Rhythmussilben}

Das Mitsprechen der Rhythmussilben beim Musizieren der Rhythmen wurde unabhängig von der Rubrik Tempo des Rhythmus betrachtet. Es sind alle Lösungen analysiert in Bezug auf das Anwenden der Silben. Dabei muss in einigen Kategorien die Rubrik Rhythmus an sich gleichzeitige betrachtet werden (Kat. 496 / 497). Die hohe Anzahl der Lösungen in der Kategorie 0 Silben richtig zugeordnet, beinhaltet deshalb auch Lösungen, deren Tempo nicht korrekt ist. Zu den Fehlerquellen beim Mitsprechen der Rhythmussilben beim Musizieren der Rhythmen zählen folgende Konstellationen (s. Abb. 4-51): Die Silben für Achtel- und Viertelschläge werden nicht zugeordnet (Kat. 491), die Silbe für Pause wird nicht gesprochen oder ist nicht bekannt (nur Item ec 4; Kat. 492), die Silben für einen Achtelschlag werden verdoppelt gesprochen (Kat. 493), statt Silben werden die Wörter lang / kurz gesprochen (Kat. 494), statt Silben werden Zahlenkombinationen gezählt (Kat. 495), die Silben des Items werden richtig gesprochen, der Rhythmus aber nicht richtig gespielt (Kat. 496) bzw. es wird ein falscher, jedoch gut erkennbarer anderer Rhythmus gespielt und dessen Silben richtig gesprochen (Kat. 497). Der Kategorie 80 werden alle fehlenden Leistungen zugeordnet, in denen keine Rhythmussprache anwendbar ist.

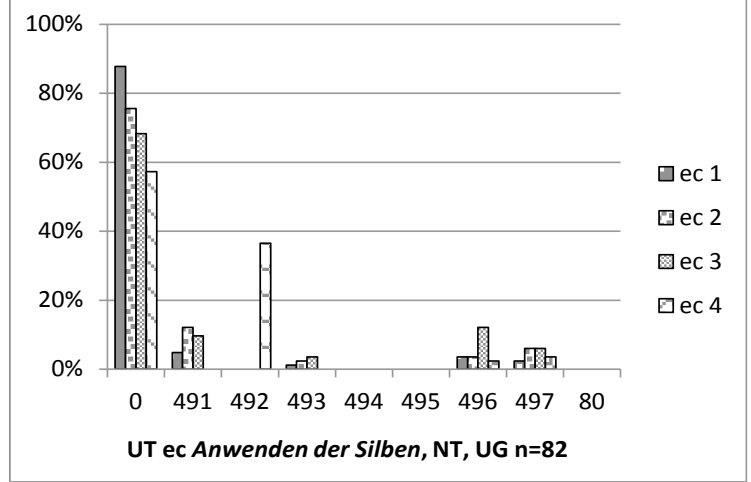

0 Silben richtig zugeordnet

491 Silben für Achtel- und Viertelschläge nicht richtig zugeordnet

492 Silbe für Pause nicht gesprochen / bekannt 493 Silben für Achtelschlag verdoppelt gesprochen 494 statt Silben Wörter lang / kurz gesprochen

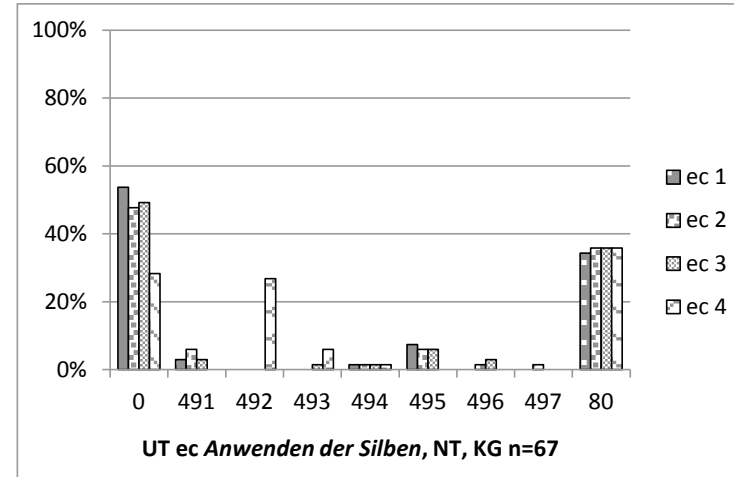

495 statt Silben Zahlenkombinationen gezählt 496 Silben des Items richtig gesprochen, Rhythmus nicht richtig gespielt

497 anderen Rhythmus gespielt (Kat. 62) und dessen Silben des anderen Rhythmus richtig gesprochen

$\mathbf{8 0}$ keine Rhythmussprache anwendbar

Abb. 4-51: Häufigkeiten von Fehlern im UT ec, NT, getrennt nach UG und KG

Die bereits im Subtest E dargestellten Fehlerquellen in den Rubriken Rhythmus an sich und Tempo des Rhythmus finden sich auch zu annähernd gleichen Anteilen im Untertest ec. Aufgrund dessen werden sie nicht separat für den Untertest ec aufgeführt. 


\section{Einflüsse des Erlernens einer Rhythmussprache auf das Musizieren von Rhythmen nach Noten}

Um dies beurteilen zu können, werden die im Nachtest aus dem Untertest ec Rhythmen lesen und musizieren und sprechen von Rhythmussilben erbrachten Lösungen (nicht nur die durch Itemanalyse extrahierten Items ec 1 und ec 3) mit den Lösungen aus dem parallel geführten Subtest E Rhythmen lesen und musizieren nach Noten verglichen (Rhythmen s. Abb. 4-40).

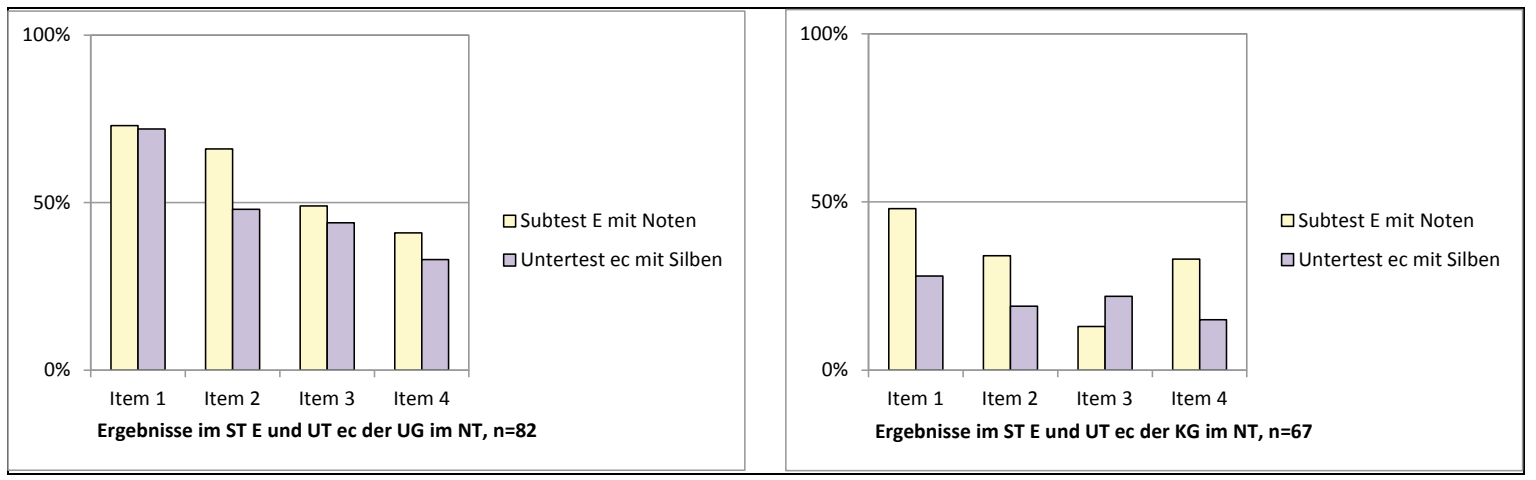

Abb. 4-52: Ergebnisse im ST E und UT ec im NT, getrennt nach UG und KG

Der prozentuale Anteil der Probanden, welcher die Silben der Rhythmussprache für Viertel- und Achtelnoten beim Musizieren richtig sprechen kann, ist in beiden Gruppen recht hoch (UG: 77 \%; KG: 50 \%). Die Silbe für Pause in Item ec 4 ist bei deutlich weniger Probanden anwendungsbereit (UG: $57 \%$; KG: $28 \%$ ). Für beide Gruppen wird beim Vergleich beobachtet, dass das Mitsprechen der Rhythmussilben kaum Steigerungen in der Anzahl der richtigen Lösungen bringt (s. Abb. 4-52). Ausnahme bildet in der Kontrollgruppe Item ec 3. Das ausdrückliche laute Mitsprechen der Rhythmussilben scheint eher ein Hindernis denn ein Hilfsmittel für das gelingende Musizieren der Rhythmen. Es werden jedoch auch etliche Probanden in beiden Gruppen beobachtet, die im vorgelagerten Subtest E von allein leise oder tonlos (nur die Lippen bewegend) Rhythmussilben mitsprechen. Die ausdrückliche Aufforderung zum lauten Mitsprechen, wirkt dann eher irritierend auf sie. Rhythmussilben können möglicherweise besser helfen, notierte Rhythmen sicher wiederzugeben, wenn sie nicht ausdrücklich laut gesprochen werden.

\section{Abgestufte Ergebnisdarstellung}

Alle Lösungen werden in abgestuften Kategorien von korrekt bis stark fehlerhaft bzw. keine Lösung dargestellt (s. Abschnitt 3.5.2; Zweite Rubrik). In dieser Darstellung lässt sich erkennen, dass im Vortest der größte Anteil der Probanden (ca. 80 \%) keine Lösung anbietet (s. Abb. 4-53). Der Anteil in der Kategorie Lösung korrekt liegt zwischen $4 \%$ und $13 \%$.

Im Nachtest werden zwischen $25 \%$ und $52 \%$ korrekte Lösungen erbracht. Zusätzlich entsprechen weitere $14 \%$ bis $23 \%$ der Lösungen in weiten Teilen den Toleranzbereichen. Erstaunlicherweise wird der Rhythmus in Item ec 3, welcher im Subtest E der am schwersten zu spielende 
Rhythmus ist (dort Item E 3), in diesem Untertest ähnlich gut wie der Rhythmus in Item ec 2 gespielt. In diesem Item zahlt sich das laute Mitsprechen der Rhythmussilben aus. Am schwersten zu spielen und sprechen ist nun der Rhythmus in Item ec 4. Die Ursache liegt in der fehlenden Kenntnis der Rhythmussilbe für die Pause.

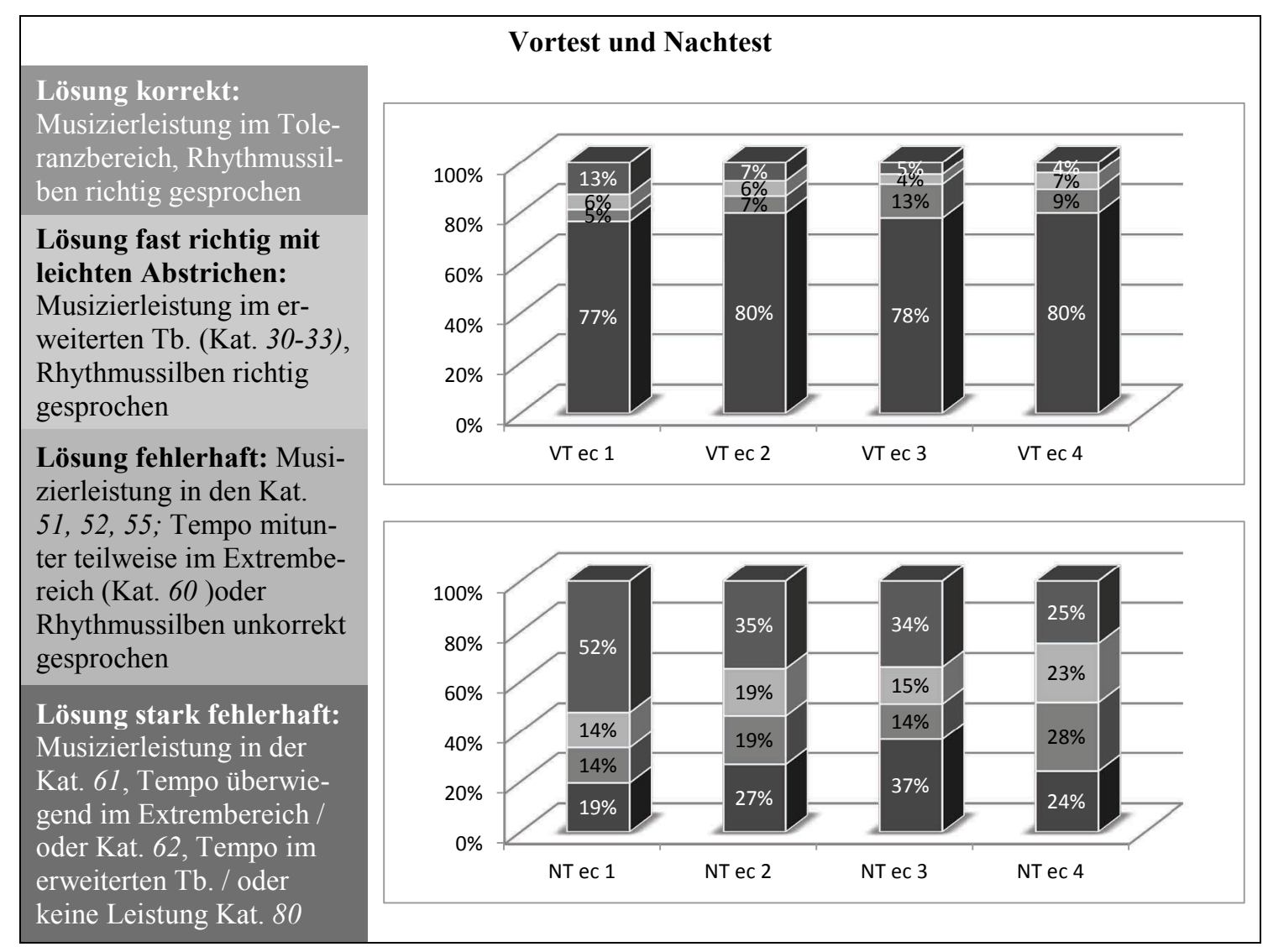

Abb. 4-53: Gesamtdarstellung aller Leistungen im UT ec nach Lösungskategorien

\section{Fehlerquellen im Untertest fc Rhythmen musizieren und aufschreiben mit Silben einer}

\section{Rhythmussprache}

Das bereits im Subtest F dargestellte einmalige Vertauschen der Symbole für Achtel- und Viertelschlag tritt auch im Untertest fc als einmaliges Vertauschen der Silben für Achtel- und Viertelschlag (Kat. 41) gehäuft auf (s. Abb. 4-54). Dies trifft besonders für die Probanden der Kontrollgruppe zu. Die Silben sind erlernt, können aber an der entscheidenden Stelle im Rhythmus - dem kritischen Element (Bamberger, 1991, S. 25, S. 46) - nicht wirksam werden. Die Lösungen bleiben damit dem figuralen Typus verhaftet. Ebenfalls ist bei vielen Probanden die Silbe für die Pause in Item fc 4 nicht vertraut (Kat. 45). 


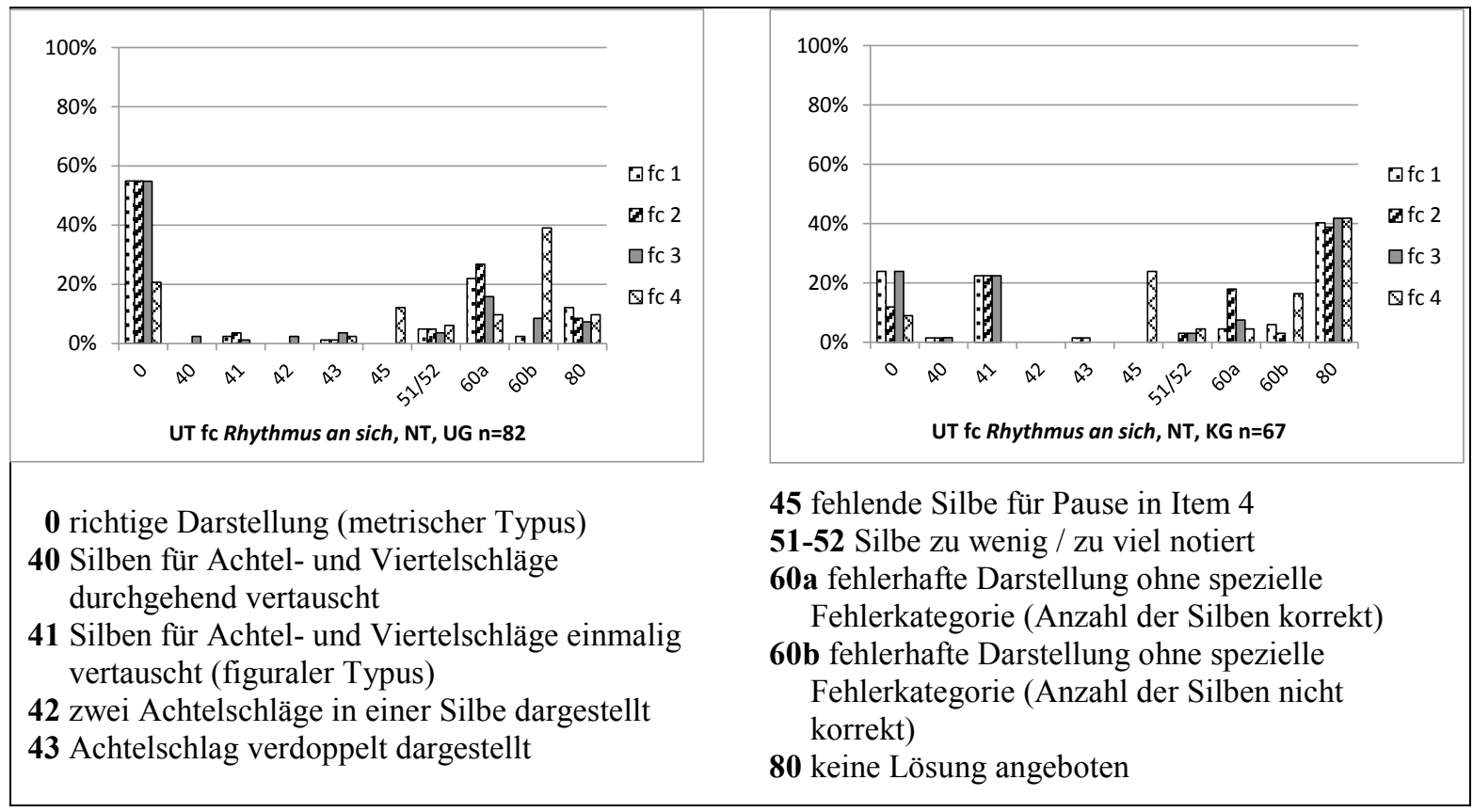

Abb. 4-54: Häufigkeit von Fehlern im UT fc im NT, getrennt nach UG und KG

\section{Einflüsse des Erlernens einer Rhythmussprache auf das Notieren von Rhythmen}

Um dies einschätzen zu können, werden die im Nachtest des Untertests $f_{c}$ Rhythmen musizieren und aufschreiben mit Silben einer Rhythmussprache erbrachten Lösungen mit den Lösungen aus dem parallel geführten Subtest $F$ Rhythmen musizieren und aufschreiben mit Noten verglichen (Rhythmen s. Abb. 4-12). Der prozentuale Anteil der Probanden, welcher die Silben der Rhythmussprache für Viertel- und Achtelnote beim Musizieren richtig notieren kann, ist in beiden Gruppen recht hoch (UG: 91 \%; KG: 60 \%). Die Silbe für Pause in Item ec 4 ist bei deutlich weniger Probanden anwendungsbereit (UG: 74 \%; KG: 30 \%). Für beide Gruppen wird bei einem Vergleich beobachtet, dass beim Notieren von Rhythmen mit Silben einer Rhythmussprache kaum Steigerungen in der Anzahl der richtigen Ergebnisse auftreten (s. Abb. 4-55). Ausnahme bilden lediglich in der Untersuchungsgruppe die Items fc 1 und fc 3.

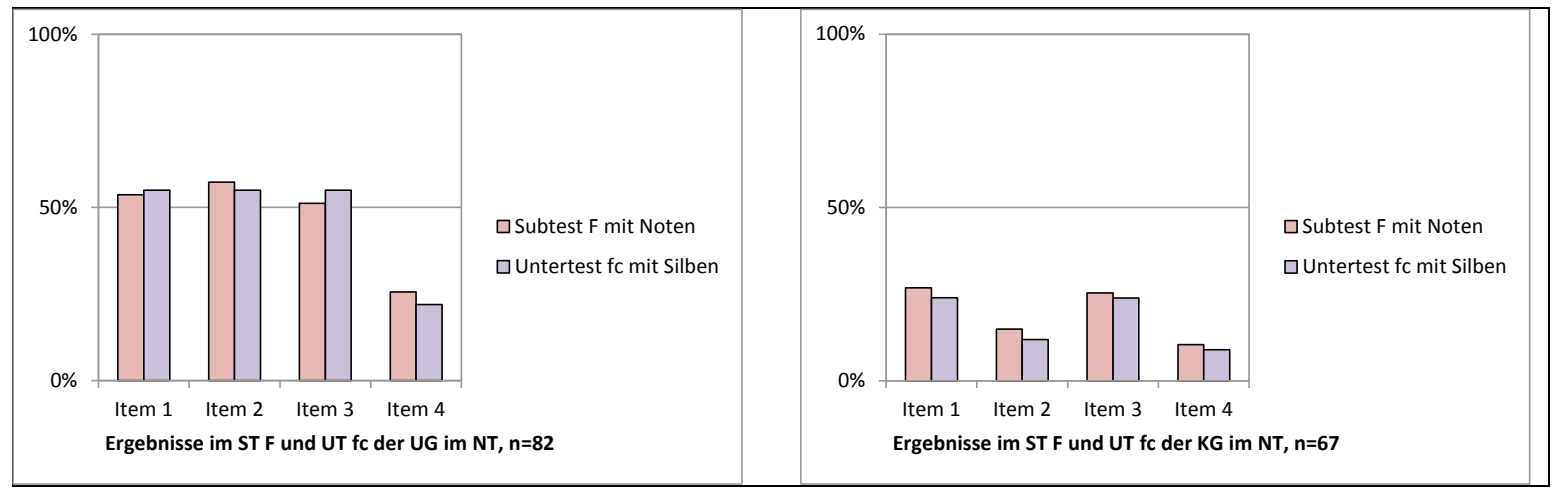

Abb. 4-55: Ergebnisse im ST F und UT fc im NT, getrennt nach UG und KG 


\section{Abgestufte Ergebnisdarstellung}

Alle Lösungen aus dem Untertest fc werden in abgestuften Kategorien von korrekt bis stark fehlerhaft bzw. keine Lösung dargestellt (s. Abschnitt 3.5.2). In dieser Darstellung lässt sich erkennen, dass nicht einmal ein Zehntel der Probanden in der Lage ist, Rhythmussilben prinzipiell bzw. korrekt anzuwenden (s. Abb. 4-56). Der Anteil in der Kategorie Lösung korrekt liegt zwischen 3 \% und $7 \%$.

Im Nachtest sind über ein Drittel der Probanden in der Lage, Rhythmussilben korrekt anzuwenden, mit Ausnahme von Item fc 4, welches eine Pause enthält. Die Silbe für die Pause ist vielen Probanden trotz schon vorhandener Kenntnisse der Silben für Viertel- und Achtelschläge nicht vertraut. Zusammen mit den Lösungen in der Kategorie Lösung richtig mit leichten Abstrichen (figuraler Typus) kann gesagt werden, dass über die Hälfte der Probanden schon korrekt und nachvollziehbar Rhythmen mit Rhythmussprache aufschreiben können, die aus Kombinationen aus Viertel- und Achtelschlägen bestehen. Für etwa ein Drittel der Probanden trifft dies auch auf einen Rhythmus mit einer Pause auf einem Viertelschlag zu. Weitere $16 \%$ bis $32 \%$ der Probanden können die Anzahl der musizierten Schläge korrekt darstellen (zählender Typus).

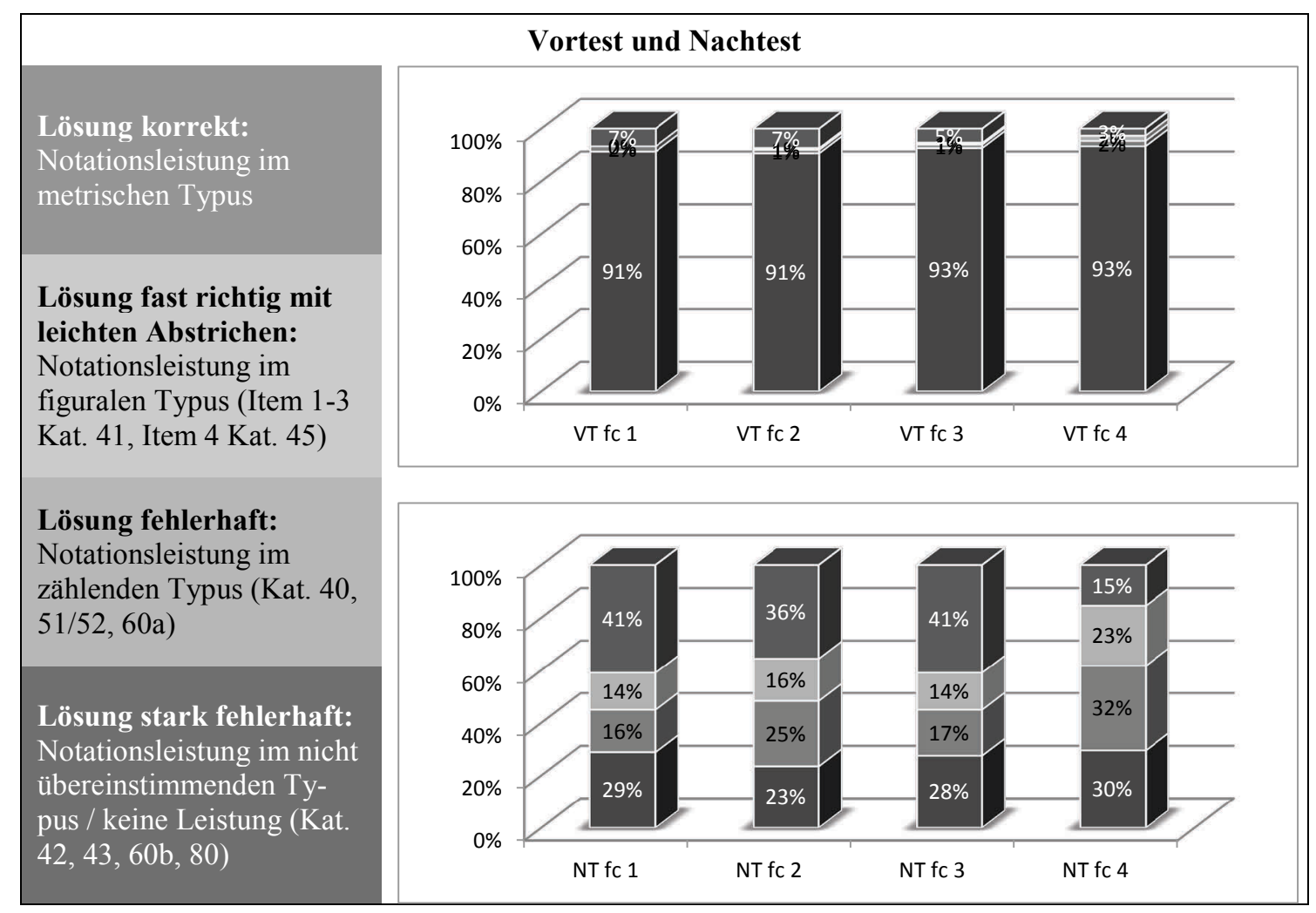

Abb. 4-56: Gesamtdarstellung aller Leistungen im UT fc nach Lösungskategorien

\subsection{Ergebnisse in der Gesamtpunktwertung des Testes}

In diesem Unterkapitel werden die Ergebnisse der Gesamtpunktwertung aus den Subtests A bis F dargestellt. Zuerst wird der Signifikanztest für den Mittelwert dieser Gesamtpunktwertung in Form 
des t-Testes und der Berechnung der Effektstärke $d$ angegeben (s. Abschnitt 4.4.1). Die Darstellung von Korrelationen zwischen den einzelnen Subtests und der Gesamtpunktwertung schließt sich an. In Bezug auf die Gesamtpunktwertung erfolgt eine individuen- und eine geschlechterbezogene Darstellung (s. Abschnitt 4.4.2 und 4.4.3). Zusätzlich werden die Mittelwerte der Gesamtpunktwertung bezogen auf die Probanden der einzelnen Klassen der Untersuchungs- und Kontrollgruppe vorgestellt (s. Abschnitt 4.4.4). Diese Mittelwerte werden für den Subtest F erweitert auf die Ergebnisse aller Schülerinnen und Schüler der jeweiligen Klassen (s. Abschnitt 4.4.5), um über vergleichbare Werte für die Darstellungen der Ergebnisse aus der 2. Erhebung (s. Abschnitt 4.5) zu verfügen.

\subsubsection{Signifikanztest und Korrelationen}

Die Summen aller Wertungspunkte aus den sechs Subtests werden zusammengefasst in einer Gesamtpunktwertung (GPW) aufgeführt. Beide Gruppen starten im Vortest mit einem relativ ähnlichen Mittelwert in der Gesamtpunktwertung (s. Tab. 4-18). Die Probanden der Untersuchungsgruppe erzielen im Nachtest einen enormen Vorsprung. Die Differenz der Mittelwerte der Gesamtpunktewertung aus Vor- und Nachtest fällt bei ihnen mehr als doppelt so hoch wie in der Kontrollgruppe aus. Die berechnete Effektstärke $d$ weist mit einem Wert von 1.36 einen sehr großen Effekt aus (s. Tab. 4-19).

Tab. 4-18: Mittelwerte in der GPW

\begin{tabular}{cccccccc}
\hline & UG & \multirow{2}{*}{ N } & $\begin{array}{c}\text { MW im } \\
\text { VT }\end{array}$ & $\begin{array}{c}\text { MW im } \\
\text { NT }\end{array}$ & $\begin{array}{c}\text { Diff. } \\
\text { VT/NT }\end{array}$ & $\begin{array}{c}\text { SD der } \\
\text { Diff. }\end{array}$ & $\begin{array}{c}\text { SE der } \\
\text { Diff. }\end{array}$ \\
\hline \multirow{2}{*}{ GPW } & UG & 82 & 4.01 & 12.74 & 8.73 & 4.50 & 0.50 \\
& KG & 67 & 3.88 & 7.99 & 4.10 & 3.09 & 0.38 \\
\hline
\end{tabular}

Tab. 4-19: Ergebnisse für t-Test und Effektstärke in der GPW

\begin{tabular}{ccccccc}
\hline & $\mathbf{t}$ & df & p (2seit.) & $\begin{array}{c}\text { Diff. der } \\
\text { MW }\end{array}$ & $\begin{array}{c}\text { SE der Diff. } \\
\text { der MW }\end{array}$ & d [95\% CI] \\
\hline GPW & 7.15 & 147 & $<0.001$ & 4.63 & 0.65 & $1.36[3.35,5.91]$ \\
\hline
\end{tabular}

Zwischen den Mittelwerten der sechs Subtests sowie dem Mittelwert des Gesamtpunktwertes bestehen starke Zusammenhänge. Die insgesamt als hoch einzustufenden Korrelationswerte (nach Pearson) liegen zwischen $r=.68$ und $r=.84$ (s. Tab. 4-20). Der Subtest C Rhythmussprache anwenden korreliert am höchsten mit dem Gesamtpunktwert. Das Erlernen einer Rhythmussprache hat demzufolge die größten Auswirkungen auf die Entwicklung rhythmischer Kompetenz. Innerhalb der einzelnen Subtests weisen die Subtests A, B und D sowie die Subtests C, E und F zueinander jeweils die größten Korrelationen auf. Die schwächste Korrelation besteht mit $r=.29$ zwischen den Subtests D Rhythmen abwandeln und F Rhythmen musizieren und aufschreiben. 
Ergebnisse

Tab. 4-20: Korrelationen zwischen den Subtests A bis F und GPW im NT

\begin{tabular}{ccccccc}
\hline Subtest & A & B & C & D & E & F \\
\hline B & .59 & & & & & \\
C & .46 & .47 & & & & \\
D & .49 & .48 & .42 & & & \\
E & .46 & .43 & .69 & .48 & & \\
F & .35 & .38 & .73 & .29 & .56 & \\
GPW & .74 & .73 & .84 & .68 & .80 & .74 \\
\hline
\end{tabular}

Tab. 4-21: Gruppierte Korrelationen der Subtests A bis F

\begin{tabular}{cccccc}
\hline Subtest & A & B & D & C & E \\
\hline B & .59 & & & & \\
D & .49 & .48 & & & \\
C & .46 & .47 & .42 & & \\
E & .46 & .43 & .48 & .69 & \\
F & .35 & .38 & .29 & .73 & .56 \\
\hline
\end{tabular}

Für die Subtests C, E und F liegt das vermutlich an der engen Beziehung zueinander, da Subtest C aus den Untertests ec und fc der Subtests E und F generiert wurde. Für die Subtests A, B und D liegt es möglicherweise an ihren höheren Ausgangswerten im Vortest. Hier zeigen die Probanden insgesamt schon deutlich erkennbare Fähigkeiten (s. Abb. 4-9). Diese besondere Konstellation zwischen den jeweils drei Subtests wird noch deutlicher, wenn die Subtests C und D ihre Positionen in der Tabelle tauschen und der jeweils höchste Korrelationswert grau unterlegt wird (s. Tab. 4-21).

\subsubsection{Individuenbezogene Ergebnisdarstellung}

Die Gesamtpunktwertung im Vor- und Nachtest soll durch eine individuenbezogene Ergebnisdarstellung ergänzt werden, da sie anders, als Mittelwerte, die Sicht auf die einzelnen Individuen ermöglicht. Dazu werden die Gesamtpunktwerte aus dem Vortest sowie der Zugewinn an Punkten aus dem Nachtest für alle Probanden gekoppelt dargestellt (s. Abb. 4-57). Somit können die Differenzen aus Vor- und Nachtest abgebildet werden. Für die Untersuchungsgruppe lässt sich ablesen:

- In der Gruppe Vortest 0 Punkte (laufende Nummer 1 bis 13) werden Zuwächse von 1 bis 12 Punkten gemessen,

- in der Gruppe Vortest 1 Punkt (laufende Nummer 14 bis 23) von 4 bis 17 Punkten usf.

- Der größte Teil der Probanden steigert seine Leistungen um 5 bis 11 Punkte.

- Die maximale Differenz zwischen Vor- und Nachtest beträgt 17 Wertungspunkte (laufende Nummer 23 und 46).

- Eine Probandin verschlechtert ihre Leistungen um vier Punkte (laufende Nummer 67) 
- Ein Proband erlangt keinen Zuwachs (laufende Nummer 82).

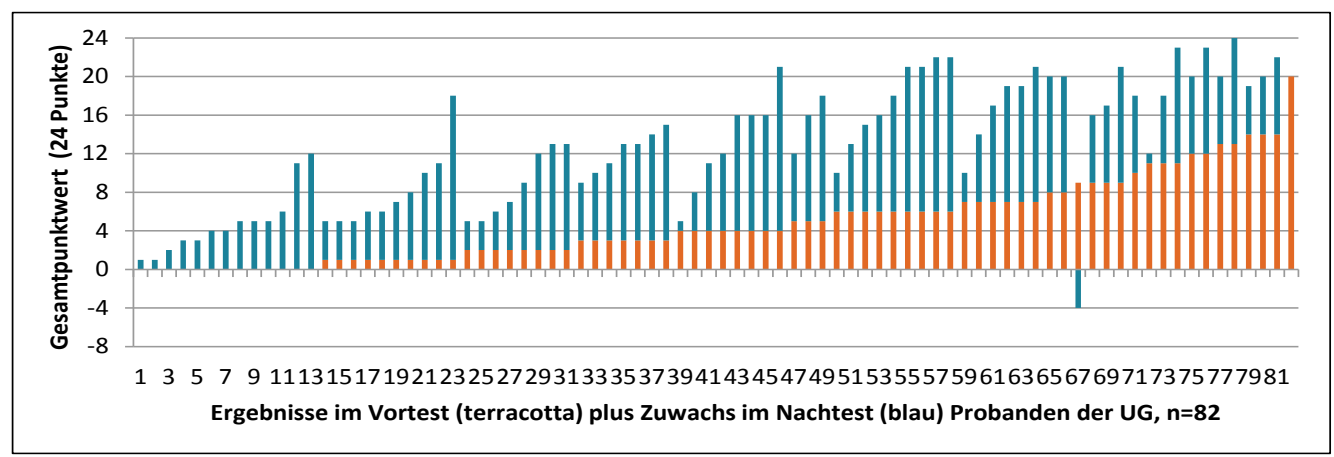

Abb. 4-57: Ergebnisse in der Gesamtpunktwertung, VT und NT, Probanden der UG

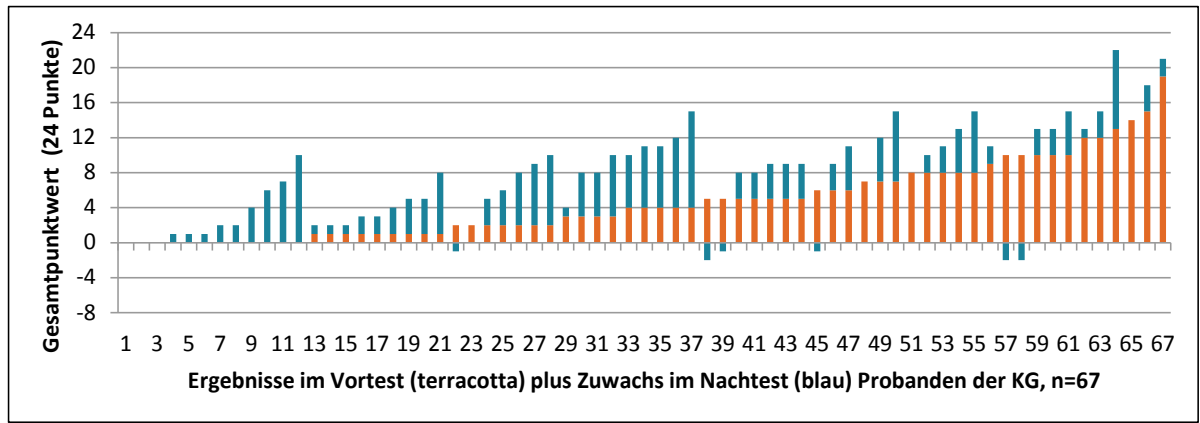

Abb. 4-58: Ergebnisse in der Gesamtpunktwertung, VT und NT, Probanden der KG

Für die Kontrollgruppe ließ sich folgendes ablesen (s. Abb. 4-58):

- In der Gruppe Vortest 0 Punkte (laufende Nummer 1 bis 12) werden Zuwächse von 0 bis 10 Punkten gemessen,

- $\quad$ in der Gruppe Vortest 1 Punkt (laufende Nummer 13 bis 21) von 1 bis 7 Punkten usf.

- Der größte Teil der Probanden steigert seine Leistungen um 1 bis 6 Punkte.

- Die maximale Differenz zwischen Vor- und Nachtest beträgt 11 Wertungspunkte (laufende Nummer 37).

- Drei Probanden verschlechtern ihre Leistungen um einen Punkt, weitere drei Probanden um zwei Punkte. Drei Probanden erlangen keinen Zuwachs (laufende Nummer 1 bis 3; 23, 48, 51 sowie 65).

Die über alle Ausgangspositionen hinweg größeren Entwicklungssprünge bei den Probanden der Untersuchungsgruppe lassen sich zusammenfassend darstellen (s. Abb. 4-59). Die Plots geben die minimal und maximal erreichten Differenzen an und stellen den Medianwert (zentraler Mittelwert) aller erreichten Differenzen dar. 


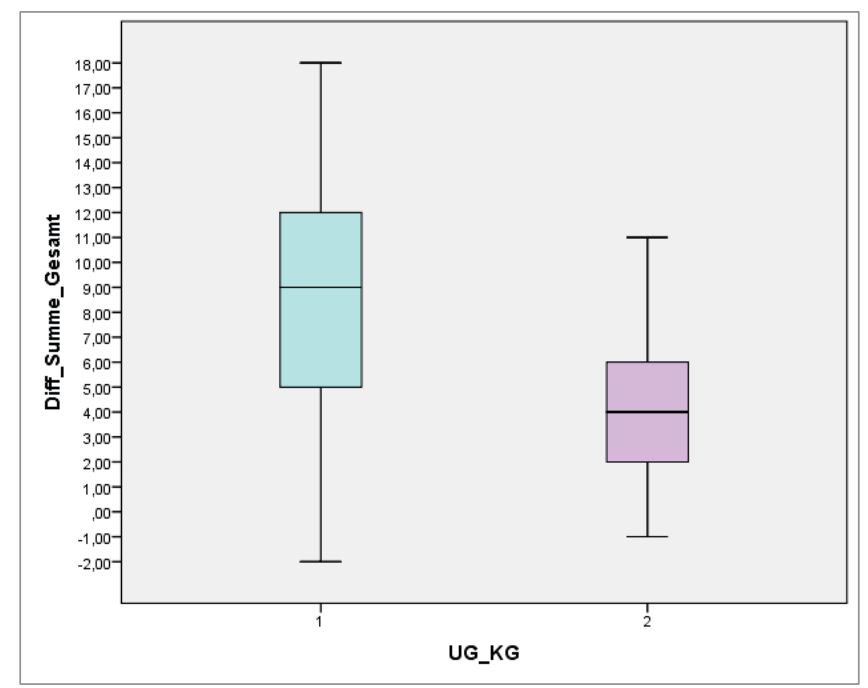

Abb. 4-59: Differenzen aus VT und NT in der Gesamtpunktwertung, getrennt nach UG und KG

\subsubsection{Geschlechterbezogene Ergebnisdarstellung}

In Bezug auf die Forschungsergebnisse von Wagner (1970; s. Abschnitt 3.5, Interpretationsobjektivität) soll überprüft werden, ob auch in dieser Studie die Mädchen den Jungen in den Leistungen voraus sind (s. Abb. 4-60). Die Mittelwerte der Subtests unterscheiden sich im Subtest E gar nicht sowie in den anderen Subtest um 0.03 bis maximal 0.29 Punkte. Die Gesamtpunktwerte betragen 10.1 Punkte für die Jungen sowie 11.0 Punkte für die Mädchen. Die Differenz von 0.9 Punkten entspricht bei 24 Punkten in der Gesamtwertung einem Unterschied von etwa $3 \%$. Damit fällt die Differenz zwischen den Leistungen der Mädchen und der Jungen deutlich niedriger als in Wagners Studie aus.

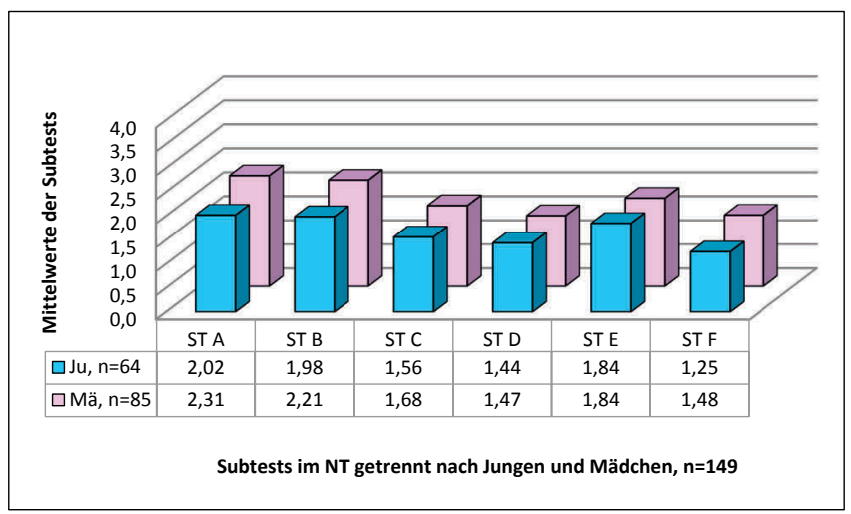

Abb. 4-60: Mittelwerte der Subtests im NT, getrennt nach Jungen und Mädchen

\subsubsection{Ergebnisse im Nachtest getrennt nach einzelnen Klassen}

Um eventuelle Einflüsse durch den zusätzlich erteilten Unterricht im Flötenspiel sowie durch die Erteilung des Musikunterrichts in Doppelstunden bewerten zu können (s. Abschnitt 4.1.2), werden die Mittelwerte der Gesamtpunktwertung sowie deren Standardabweichung und Standardfehler, der 
Median sowie die minimalen und maximalen Punktwerte aufgeführt (s. Tab. 4-22). Für die Klasse $\mathrm{K} 2$ ist erkennbar, dass sie in der Kontrollgruppe die besten Werte erreicht und mit den arithmetischen Mittelwerten, Medianen und der Standardabweichung des Mittelwertes in das Leistungsspektrum der Untersuchungsgruppe hineinreicht. Klasse K 1 folgt auf Klasse K 2. Dies lässt vorab darauf schließen, dass die zusätzliche Förderung erfolgreich wirkte.

Tab. 4-22: Ergebnisse der Gesamtpunktwertung im NT, getrennt nach Klassen

\begin{tabular}{ccccccc}
\hline Klasse & $\begin{array}{c}\text { arithm. } \\
\text { MW des } \\
\text { GPW }\end{array}$ & $\begin{array}{c}\text { Median } \\
\text { des } \\
\text { GPW }\end{array}$ & $\begin{array}{c}\text { SD des } \\
\text { arithm. } \\
\text { MW des } \\
\text { GPW }\end{array}$ & $\begin{array}{c}\text { mittl. } \\
\text { Fehler } \\
\text { der SD }\end{array}$ & $\begin{array}{c}\text { minima- } \\
\text { ler } \\
\text { GPW }\end{array}$ & $\begin{array}{c}\text { maxi- } \\
\text { maler } \\
\text { GPWF }\end{array}$ \\
\hline U 1 & 13.9 & 16 & 6.49 & 2.05 & 2 & 21 \\
U 2 & 16.1 & 17 & 5.48 & 1.52 & 5 & 22 \\
U 3 & 12.1 & 12 & 6.68 & 1.85 & 0 & 23 \\
U 4 & 12.7 & 12 & 5.51 & 1.53 & 4 & 22 \\
U 5 & 10.2 & 10 & 5.75 & 1.66 & 3 & 21 \\
U 7 & 11.1 & 8 & 6.94 & 1.51 & 1 & 24 \\
K 1 & 8.5 & 8 & 5.19 & 1.30 & 2 & 21 \\
K 2 & 10.2 & 11 & 5.37 & 1.27 & 2 & 20 \\
K 3 & 8.4 & 9 & 4.67 & 2.09 & 1 & 14 \\
K 4 & 6.2 & 5.5 & 3.76 & 1.54 & 2 & 11 \\
K 5 & 7.3 & 7.5 & 5.42 & 1.71 & 0 & 17 \\
K 6 & 5.5 & 6 & 3.80 & 1.10 & 0 & 12 \\
\hline
\end{tabular}

\subsubsection{Ergebnisse im Subtest F im Nachtest in Bezug auf alle Probanden}

Um die dargestellten Ergebnisse der Probanden der zweiten Erhebung (s. Abschnitt 4.5) mit den Ergebnissen der Hauptuntersuchung vergleichen zu können, werden in diesem Abschnitt die Ergebnisse aus dem Subtest F der Hauptuntersuchung anders dargestellt.

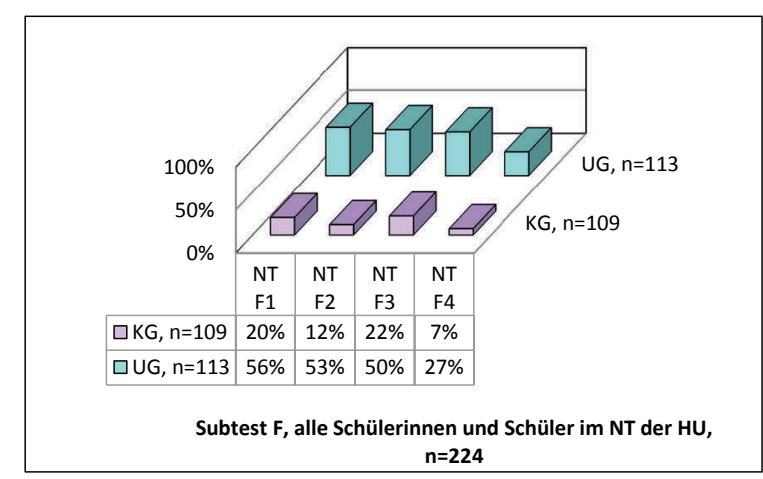

Abb. 4-61: Ergebnisse aller Schüler der HU im ST F im NT, getrennt nach UG und KG

Es werden die Lösungen aller zum Zeitpunkt des Nachtestes involvierten über 200 Schülerinnen und Schüler der Hauptuntersuchung aus dem schriftlichen Test (Subtest F) herangezogen. Dazu 
zählen also auch Schüler und Schülerinnen, die nicht zu den Probanden zählen. Es werden die prozentualen Anteile der richtigen Ergebnisse in Item F 1 bis F 4 (s. Abb. 4-61) sowie die arithmetischen Mittelwerte der einzelnen Klassen im Subtest F (s. Abb. 4-62) dargestellt.

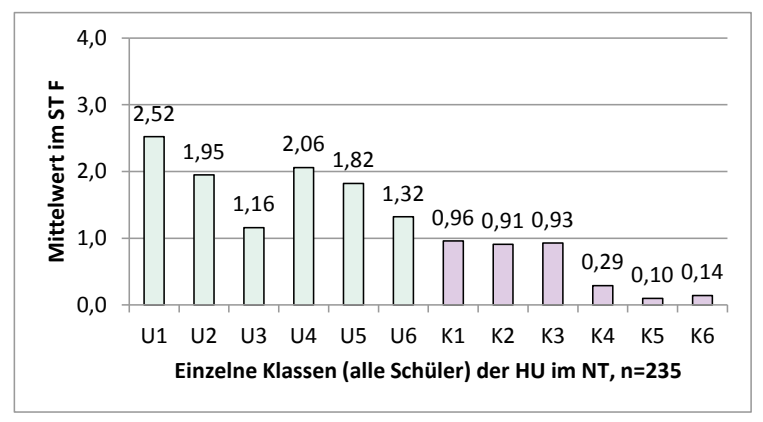

Abb. 4-62: Mittelwerte aller Schüler der HU im ST F im NT, getrennt nach Klassen

\subsection{Ergebnisse aus der zweiten Erhebung}

Die zweite Erhebung (s. Abschnitt 4.1.4) fand von Mai bis Juni 2015 an sieben Grundschulen mit den jeweiligen dritten und vierten Klassen statt $(n=549)$ statt. Folgend werden die Ergebnisse zum in Anwendung gekommenen Subtest $F$ Rhythmen musizieren und aufschreiben mit Noten für die beteiligten Probanden der Klassenstufe $3(n=266)$ und 4 aufgeführt $(n=283)$. In Klassenstufe 3 (KS 3 ) erbringen bis zu $10 \%$ der Probanden richtige Ergebnisse (Item F 3). Item F 3 fällt den Probanden leichter, Item F 4 eher schwerer. In Klassenstufe 4 (KS 4) erbringen bis zu 14 \% der Probanden richtige Ergebnisse (Item F 4; s. Abb. 4-63). Hier gelingt jedes Folge-Item besser als das vorangegangene und damit Item $\mathrm{F} 4$ am besten.

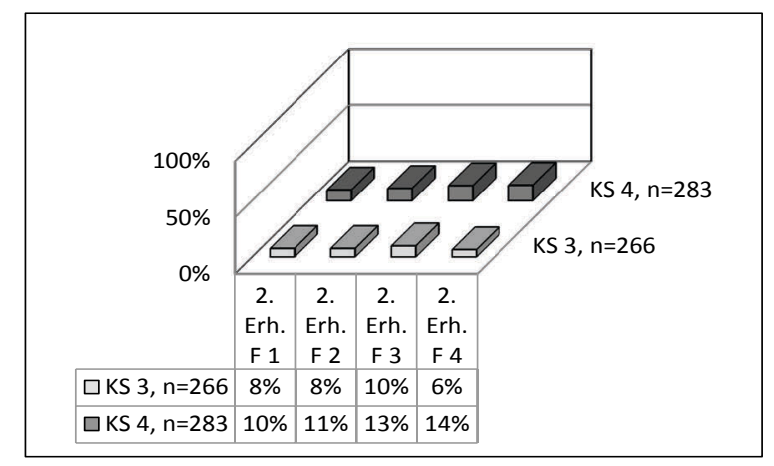

Abb. 4-63: Ergebnisse in der 2. Erhebung, getrennt nach Klassenstufe 3 und 4

Die Ergebnisse werden anschließend getrennt nach einzelnen Klassen mit dem geplanten Mittelwert für Subtest F in Höhe von 0.66 aufgeführt (s. Tab. 3-14). Dabei fällt auf, dass in drei Klassen der Klassenstufe 3 der Mittelwert erreicht bzw. überboten wird (s. Abb. 4-64, links: Klasse 2_3b; 5_3a und 5_3b). Diese Klassen haben im Musikunterricht die Rhythmussprache nach Kodály erlernt. In Klassen, deren Ergebnisse unterhalb dieses Wertes liegen, werden richtige Lösungen fast 
ausschließlich von einzelnen Schülern mit außerschulischem Instrumentalunterricht erbracht. Gleiches lässt sich für Klassenstufe 4 beobachten, hier liegen vier Klassen über dem geplanten Mittelwert (s. Abb. 4-64, rechts: Klasse 1_4a, 3_4, 5_4a und 5_4b).
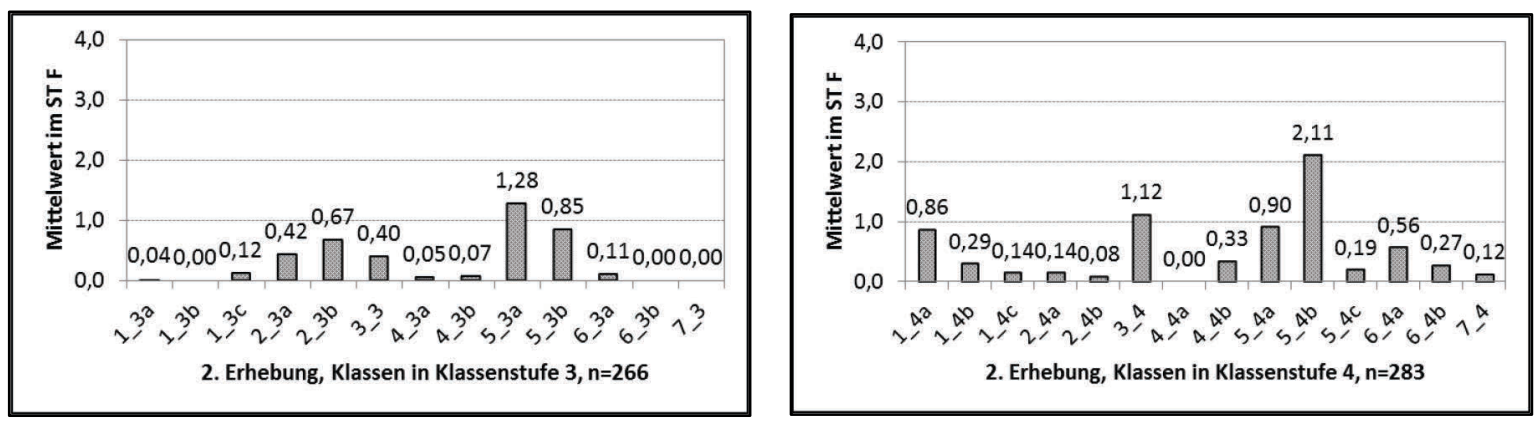

Abb. 4-64: Mittelwerte der einzelnen Klassen der 2. Erhebung, getrennt nach Klassenstufe 3 und 4

\subsection{Ergebnisse in den zusätzlichen Subtests}

In diesem Abschnitt werden die Ergebnisse der beiden Untertests e und f, welche sich auf den Umgang mit ikonischer Notation beziehen, vorgestellt und in Bezug zu den Ergebnissen der Subtests E und F gesetzt. Auch hier folgt die Darstellung der beiden Untertests der Chronologie der Erhebung, also beginnend mit Untertest $\mathrm{f}$ und fortführend mit Untertest e. Des Weiteren werden die Ergebnisse der zusätzlichen Subtests G, H und I sowie die Ergebnisse dargestellt.

\subsubsection{Untertest f Rhythmen musizieren und aufschreiben mit Zeichen}

Dieser Untertest testet die Fähigkeiten des Aufschreibens von Rhythmen mithilfe ikonischer Notation. Etwa 30 verschiedene Zeichen werden dafür von den Probanden gewählt. Dazu zählen vor allem senkrechte und waagerechte Striche, Balken, Punkte und Kreise. Es werden aber auch Wellenlinien, Buchstaben, Zahlen oder gegenständliche Darstellungen wie Sterne, Hände, Bäume u. a. gewählt. Neben den erwarteten Darstellungen mit verschiedenen Zeichen oder verschiedenen Abständen für kurze und lange Schläge werden auch Zahlengruppen, Darstellungen mit horizontalen und vertikalen Ebenen gewählt.

Alle von Bamberger, Upitis und Hildebrandt aufgeführten Typen - nicht übereinstimmend, zählend, figural und metrisch - können nachgewiesen werden. Für die genaue Splittung in diese vier Typen werden ausnahmsweise die Lösungen aller Schülerinnen und Schüler hinzugezogen, auch derjenigen, die nicht am restlichen Test teilgenommen haben. Diese große Gruppe bietet einen repräsentativen Querschnittsblick auf die Gruppe der Schülerinnen und Schüler in Klassenstufe 3. Dazu zählen im Vortest insgesamt 228 Schüler und Schülerinnen. Im Vortest bietet etwa ein Zehntel der Schülerinnen und Schüler der Untersuchungsgruppe keine Lösungen mit Zeichen an (s. Tab. 4-23). Sie arbeiten in den Lösungsfeldern mit Rhythmussprache und Notenschrift. Die Typen nicht übereinstimmend, zählend, figural werden je etwa zu einem Viertel von den Schülerinnen und Schüler als Lösungsansatz gewählt. Nur zwischen $5 \%$ bis $18 \%$ der Schülerinnen und 
Schüler sind bereits in der Lage, die Rhythmen der Items f 1 bis f 4 im metrischen Typus aufzuschreiben. In der Kontrollgruppe stellen sich die Ergebnisse ähnlich dar. Jedoch ist der Anteil der Schülerinnen und Schüler, die bereits im zählenden Typus darstellen können größer als beim nicht übereinstimmenden Typus.

Tab. 4-23: Gewählter Typus beim Aufschreiben von Rhythmen mit Zeichen im UT f im VT

\begin{tabular}{|c|c|c|c|c|c|c|c|c|}
\hline \multirow{2}{*}{ Typus } & \multicolumn{2}{|c|}{ UG, $n=114$} & \multirow[b]{2}{*}{ Item 3} & \multirow[b]{2}{*}{ Item 4} & \multicolumn{2}{|c|}{ KG, $n=114$} & \multirow[b]{2}{*}{ Item 3} & \multirow[b]{2}{*}{ Item 4} \\
\hline & Item 1 & Item 2 & & & Item 1 & Item 2 & & \\
\hline keine Lösung & $13 \%$ & $11 \%$ & $11 \%$ & $11 \%$ & $12 \%$ & $15 \%$ & $11 \%$ & $10 \%$ \\
\hline nicht übereinstimmend & $26 \%$ & $28 \%$ & $25 \%$ & $20 \%$ & $11 \%$ & $13 \%$ & $15 \%$ & $12 \%$ \\
\hline zählend & $18 \%$ & $38 \%$ & $29 \%$ & $28 \%$ & $29 \%$ & $39 \%$ & $34 \%$ & $41 \%$ \\
\hline figural & $36 \%$ & $18 \%$ & $19 \%$ & $23 \%$ & $39 \%$ & $25 \%$ & $27 \%$ & $28 \%$ \\
\hline metrisch & $7 \%$ & $5 \%$ & $16 \%$ & $18 \%$ & $9 \%$ & $8 \%$ & $13 \%$ & $9 \%$ \\
\hline
\end{tabular}

Der Anteil der Schülerinnen und Schüler, die in der Lage sind, diese Rhythmen auch mit Noten richtig aufzuschreiben, sinkt in der Untersuchungsgruppe auf Werte zwischen $2 \%$ bis $6 \%$ sowie in der Kontrollgruppe auf zwischen $1 \%$ bis $3 \%$. Dieses Anteilsverhältnis zeigt sich auch bei den Lösungen der 149 Probanden (s. Tab. 4-24). Während der Anteil an Lösungen im metrischen Typus zwischen $9 \%$ und $18 \%$ liegt, beträgt der Anteil an korrekten Lösungen mit Noten nur zwischen $1 \%$ und $7 \%$. Es kann festgestellt werden, dass mit dem Angebot einer ikonischen Notation deutlich höhere Leistungen erbracht werden können. Werden die Lösungen im Subtest F fast ausschließlich von Probanden mit außerschulischem Instrumentalunterricht erbracht (s. Abschnitt 4.3.1), können die Lösungen im Untertest $\mathrm{f}$ auch von anderen Schülerinnen und Schülern ohne diese Unterweisung erbracht werden.

Tab. 4-24: Anteil an richtigen Lösungen im UT f und ST F im VT

\begin{tabular}{|c|c|c|c|c|c|c|c|c|}
\hline \multirow{2}{*}{$\begin{array}{l}\text { Rhythmen musizieren und } \\
\text { aufschreiben }\end{array}$} & \multicolumn{2}{|c|}{$\mathbf{U G}, \mathbf{n}=82$} & \multirow[b]{2}{*}{$\begin{array}{c}\text { Item } \\
3\end{array}$} & \multirow[b]{2}{*}{$\begin{array}{c}\text { Item } \\
4\end{array}$} & \multicolumn{2}{|c|}{$K G, n=67$} & \multirow[b]{2}{*}{$\begin{array}{c}\text { Item } \\
3\end{array}$} & \multirow[b]{2}{*}{$\begin{array}{c}\text { Item } \\
4\end{array}$} \\
\hline & $\begin{array}{c}\text { Item } \\
1\end{array}$ & $\begin{array}{c}\text { Item } \\
2\end{array}$ & & & $\begin{array}{c}\text { Item } \\
1\end{array}$ & $\begin{array}{c}\text { Item } \\
2\end{array}$ & & \\
\hline UT f: mit Zeichen & $10 \%$ & $7 \%$ & $18 \%$ & $18 \%$ & $12 \%$ & $9 \%$ & $12 \%$ & $10 \%$ \\
\hline ST F: mit Noten & $6 \%$ & $5 \%$ & $7 \%$ & $2 \%$ & $1 \%$ & $1 \%$ & $3 \%$ & $1 \%$ \\
\hline
\end{tabular}

Im Nachtest bietet etwa ein Zehntel der Schülerinnen und Schüler der Untersuchungsgruppe keine Lösungen mit Zeichen an (s. Tab. 4-25). Sie arbeiten in den Lösungsfeldern mit Rhythmussprache und Notenschrift. Die Typen nicht übereinstimmend, zählend, figural werden anteilig deutlich weniger als zu einem Viertel der Lösungen als Lösungsansatz gewählt. Dafür steigt der Anteil an Schülerinnen und Schülern, welche die Rhythmen der Items f 1 bis f 4 im metrischen Typus aufschreiben können, auf fast $50 \%$. In der Kontrollgruppe stellen sich die Ergebnisse anders dar. Ebenfalls ein Zehntel der Schülerinnen und Schüler bietet keine Lösungen mit Zeichen an. Sie arbeiten in den Lösungsfeldern mit Rhythmussprache und Notenschrift. Der Typus nicht überein- 
stimmend umfasst etwa ein Sechstel der Lösungen, die Typen zählend und figural je etwa ein Viertel. Nur zwischen bis knapp 30 \% der Lösungen sind dem metrischen Typus zuzuordnen.

Tab. 4-25: Gewählter Typus beim Aufschreiben von Rhythmen mit Zeichen im UT f im NT

\begin{tabular}{|c|c|c|c|c|c|c|c|c|}
\hline \multirow{2}{*}{ Typus } & \multicolumn{2}{|c|}{$\mathrm{UG}, \mathrm{n}=113$} & \multirow[b]{2}{*}{ Item 3} & \multirow[b]{2}{*}{ Item 4} & \multicolumn{2}{|c|}{ KG, $n=119$} & \multirow[b]{2}{*}{ Item 3} & \multirow[b]{2}{*}{ Item 4} \\
\hline & Item 1 & Item 2 & & & Item 1 & Item 2 & & \\
\hline keine Lösung & $11 \%$ & $7 \%$ & $6 \%$ & $10 \%$ & $9 \%$ & $8 \%$ & $8 \%$ & $11 \%$ \\
\hline nicht übereinstimmend & $12 \%$ & $10 \%$ & $26 \%$ & $16 \%$ & $17 \%$ & $15 \%$ & $11 \%$ & $12 \%$ \\
\hline zählend & $18 \%$ & $27 \%$ & $19 \%$ & $32 \%$ & $22 \%$ & $36 \%$ & $27 \%$ & $20 \%$ \\
\hline figural & $12 \%$ & $9 \%$ & $7 \%$ & $12 \%$ & $29 \%$ & $25 \%$ & $30 \%$ & $29 \%$ \\
\hline metrisch & $47 \%$ & $47 \%$ & $42 \%$ & $30 \%$ & $23 \%$ & $16 \%$ & $24 \%$ & $28 \%$ \\
\hline
\end{tabular}

Betrachtet man den Anteil der Schülerinnen und Schüler, die bereits in der Lage sind, diese Rhythmen auch mit Noten richtig aufzuschreiben, so ist in beiden Gruppen zu verzeichnen, dass in etwa die gleichen Anteile an Leistungen, die im metrischen Typus mit Zeichen erbracht werden, auch mit Noten erzielt werden können. Dieses ausgeglichene Verhältnis zeigt sich sowohl den Lösungen aller 231 Schülerinnen und Schülern (keine Darstellung in Tabellenform) als auch der 149 Probanden, die alle Testteile absolvierten (s. Tab. 4-26). Lediglich in Item 4 sind die Anteile an richtigen Lösungen beim Aufschreiben von Rhythmen mit Zeichen in der Kontrollgruppe besser als mit Noten. Mit dem Angebot einer ikonischen Notation können anders als im Vortest keine höheren Leistungen als mit Noten erbracht werden. Die Differenzen der Mittelwerte aus Vor- und Nachtest von Untersuchungs- und Kontrollgruppe entsprechen im Untertest $\mathrm{f}$ einem kleinen Effekt in Höhe von $d=0.44$ (s. Anlage C 4 e-g).

Tab. 4-26: Anteil an richtigen Lösungen im UT f und ST F im NT

\begin{tabular}{|c|c|c|c|c|c|c|c|c|}
\hline \multirow{2}{*}{$\begin{array}{l}\text { Rhythmen musizieren und } \\
\text { aufschreiben }\end{array}$} & \multicolumn{2}{|c|}{$\mathrm{UG}, \mathrm{n}=\mathbf{8 2}$} & \multirow[b]{2}{*}{$\begin{array}{c}\text { Item } \\
3 \\
\end{array}$} & \multirow[b]{2}{*}{$\begin{array}{c}\text { Item } \\
4 \\
\end{array}$} & \multicolumn{2}{|c|}{$K G, n=67$} & \multirow[b]{2}{*}{$\begin{array}{c}\text { Item } \\
3 \\
\end{array}$} & \multirow[b]{2}{*}{$\begin{array}{c}\text { Item } \\
4\end{array}$} \\
\hline & $\begin{array}{c}\text { Item } \\
1 \\
\end{array}$ & $\begin{array}{c}\text { Item } \\
2\end{array}$ & & & $\begin{array}{c}\text { Item } \\
1 \\
\end{array}$ & $\begin{array}{c}\text { Item } \\
2 \\
\end{array}$ & & \\
\hline UT f: mit Zeichen & $51 \%$ & $51 \%$ & $46 \%$ & $32 \%$ & $30 \%$ & $18 \%$ & $28 \%$ & $31 \%$ \\
\hline ST F: mit Noten & $54 \%$ & $57 \%$ & $51 \%$ & $26 \%$ & $27 \%$ & $15 \%$ & $25 \%$ & $10 \%$ \\
\hline
\end{tabular}

\subsubsection{Untertest e Rhythmen lesen und musizieren nach Zeichen}

Dieser Untertest testet Fähigkeiten des Lesens und Musizierens von Rhythmen nach ikonischer Notation. Zur Auswahl stehen zwei Notationsvorlagen mit Strichen bzw. mit Balken (s. Tab. 3-9). Diese werden im Vortest zu etwa gleichen Teilen genutzt. Im Nachtest nutzen die Probanden der Untersuchungsgruppe vorrangig die Vorlage mit Strichnotation. In der Kontrollgruppe werden die Vorlagen wie im Vortest zu annähernd gleichen Anteilen genutzt.

Richtige Ergebnisse im Untertest e werden in beiden Gruppen bemerkenswerter Weise auch von Probanden erbracht, die keinen außerschulischen Instrumentalunterricht erhalten. In der Untersu- 
chungsgruppe sind im Vortest mit dem Angebot der ikonischen Notation in allen Items deutlich höhere Musizierleistungen gegenüber dem Musizieren nach Noten möglich (s. Tab. 4-27). Können im Subtest E nur zwischen $6 \%$ und $17 \%$ der Lösungen als richtig bewertet werden, so sind dies im Untertest e bemerkenswerte $12 \%$ bis $39 \%$. In der Kontrollgruppe zeigt sich ein gänzlich anderes Bild. Die Klassen K 1 und K 2 hatten zum Zeitpunkt des Vortestes bereits eine Einführung in die Notenschrift erhalten. Sie erbringen bereits beachtliche Leistungen in den Items E 1 und E 2. Während im Untertest e nur $10 \%$ bis $19 \%$ richtige Lösungen erbracht werden können, sind es im Subtests E zwischen $9 \%$ und $34 \%$.

Tab. 4-27: Anteil an richtigen Lösungen im UT e und ST E im VT

\begin{tabular}{ccccccccc}
\hline \multirow{2}{*}{$\begin{array}{c}\text { Rhythmen lesen und musi- } \\
\text { zieren }\end{array}$} & \multicolumn{3}{c}{ UG, $\mathbf{n = 8 2}$} & \multicolumn{3}{c}{ KG, $\mathbf{n = 6 7}$} \\
& 1 & 2 & 3 & 4 & 1 & 2 & 3 & 4 \\
\hline UT e: nach Zeichen & $39 \%$ & $21 \%$ & $15 \%$ & $12 \%$ & $19 \%$ & $19 \%$ & $10 \%$ & $21 \%$ \\
ST E: nach Noten & $17 \%$ & $16 \%$ & $9 \%$ & $6 \%$ & $34 \%$ & $27 \%$ & $9 \%$ & $19 \%$ \\
\hline
\end{tabular}

Im Nachtest gelingt auch den Probanden der Untersuchungsgruppe das Musizieren nach Noten besser als nach ikonischer Notation (s. Tab. 4-28). Lediglich in Item e 1 und e 4 gelingt das Musizieren nach ikonischer Notation etwas besser. In der Kontrollgruppe gibt es insgesamt keine so starke Weiterentwicklung der Fähigkeiten. Dennoch gibt es auch hier den Trend, dass sich die Ergebnisse im Untertest e sowie im Subtest E angeglichener darstellen. Auffallend ist im Untertest e ein deutlich höherer Anteil an richtigen Lösungen im Item e 3. Die Differenzen der Mittelwerte aus Vor- und Nachtest von Untersuchungs- und Kontrollgruppe entsprechen im Untertest e einem mittleren Effekt in Höhe von $d=0.73$ (s. Anlage C 4 e-g).

Tab. 4-28: Anteil an richtigen Lösungen im UT e und ST E im NT

\begin{tabular}{ccccccccc}
\hline $\begin{array}{c}\text { Rhythmen lesen und musi- } \\
\text { zieren }\end{array}$ & \multicolumn{4}{c}{ UG, $\mathbf{n = 8 2}$} & \multicolumn{4}{c}{ KG, $\mathbf{n = 6 7}$} \\
& Item & Item & Item & Item & Item & Item & Item & Item \\
& 1 & 2 & 3 & 4 & 1 & 2 & 3 & 4 \\
\hline UT e: nach Zeichen & $80 \%$ & $57 \%$ & $48 \%$ & $48 \%$ & $30 \%$ & $31 \%$ & $28 \%$ & $22 \%$ \\
ST E: nach Noten & $73 \%$ & $66 \%$ & $49 \%$ & $41 \%$ & $48 \%$ & $34 \%$ & $13 \%$ & $33 \%$ \\
\hline
\end{tabular}

\subsubsection{Subtest G Play together}

Die im Subtest G gewonnenen Daten dienen als Beleg für die Kriteriumsvalidität. Mit ihnen soll gezeigt werden, dass Probanden, welche in den Subtest A bis F gute Ergebnisse erzielen, auch bessere Leistungen im zweistimmigen Musizieren von Rhythmen erreichen (s. Abschnitt 3.5.4). Deshalb werden Korrelationen zwischen diesem Subtest und den einzelnen Subtests A bis F sowie dem Gesamtpunktwert des Testes berechnet. 
Item G 1, in welchem das mehrfache Spielen des Rhythmus 1a getestet wird, gelingt im Vortest etwa einem Achtel, im Nachtest schon etwa einem Drittel der Probanden (s. Abb. 4-65). Damit fällt es den Probanden relativ leicht, in einem zweistimmig angelegten Musizierteil mit der eigenen Stimme zuerst einzusetzen. Diesen Rhythmus im Item G 2 im Zusammenspiel fortzusetzen, wenn das Kind im Video seinen (anderen) Rhythmus spielt, gelingt im Vor- und Nachtest nur äußerst wenigen Probanden. Kommt die zweite Stimme hinzu, ist die Irritation meist groß, es wird der Rhythmus des Kindes im Video übernommen. Wenn andersherum das Kind im Video bereits spielt, gelingt den Probanden das pünktliche Einsetzen im Item G 3 ausgesprochen gut. Danach jedoch den eigenen Rhythmus im Item G 4 weiterzuspielen, während auch das Kind im Video seinen Rhythmus weitermusiziert, gelingt im Vortest nur $5 \%$ der Probanden, im Nachtest immerhin schon etwa $12 \%$ der Probanden. Wiederum verfallen viele Probanden in das Imitieren des Rhythmus des Kindes im Video. Die Leistungen der Probanden der Untersuchungsgruppe fallen im Nachtest etwas besser aus. Die Leistungsdifferenz kann mit einem Wert von $d=0.18$ beziffert werden, dies entspricht knapp einem kleinem Effekt (s. Anlage C 5 f-g).
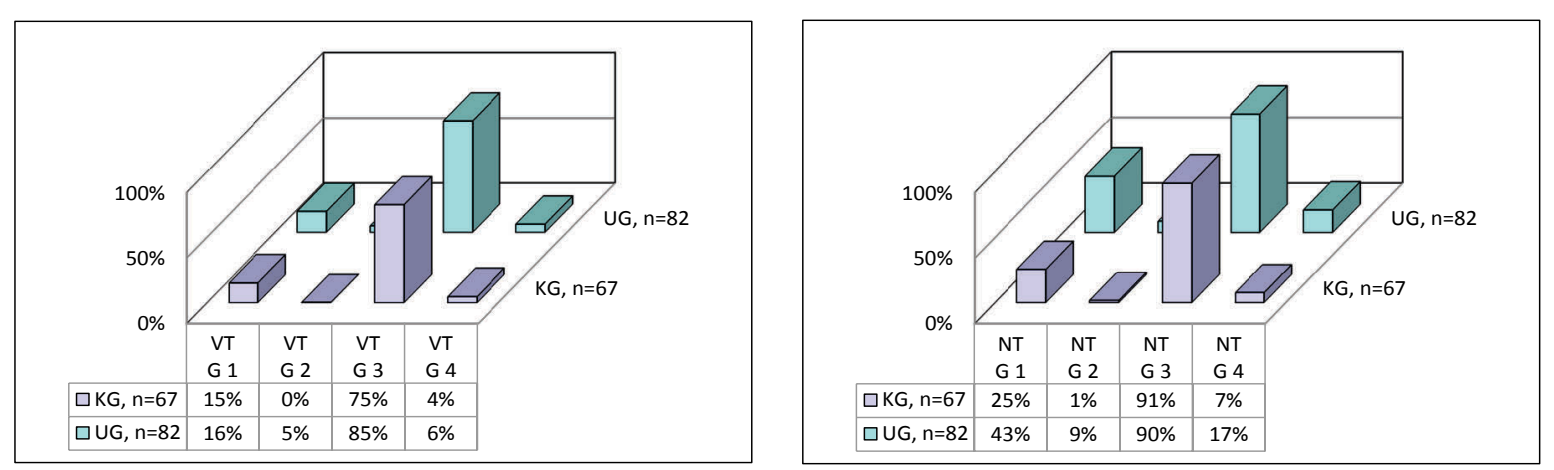

Abb. 4-65: Ergebnisse im ST G, VT und NT, getrennt nach UG und KG

Auch wenn die einzelnen Leistungen besonders in den Items G 2 und G 4 nicht überdurchschnittlich stark entwickelt sind, zeigt doch der Mittelwert des Subtests G hohe Korrelationen zu den Mittelwerten der anderen sechs Subtests und des Gesamtpunktwerts. Besonders hohe Korrelationen bestehen zwischen Subtest G und den Subtests D Rhythmen bewusst abwandeln, E Rhythmen lesen und musizieren sowie B Rhythmen nachspielen. Die höchsten Korrelationen bestehen zum Gesamtpunktwert (s. Tab. 4-29).

Tab. 4-29: Korrelation von ST G zu den ST A - F sowie dem GPW im NT

\begin{tabular}{cccccccc}
\hline Subtest & A & B & C & D & E & F & GPW \\
\hline G & .40 & .50 & .45 & .56 & .54 & .35 & .61 \\
\hline
\end{tabular}




\subsubsection{Subtest H Wozu Notenschrift?}

Die Frage „Wozu kann die Notenschrift für einen Grundschüler, z. B. dich, wichtig sein?“ beantworten die Probanden aus beiden Gruppen im Nachtest nahezu zu 100 \%. Einen Bezug zwischen Notenkenntnissen und deren Verwendung in musikalischen Zusammenhängen können 74 \% der Probanden der Untersuchungs- sowie $55 \%$ der Probanden der Kontrollgruppe benennen. Einen Ich-Bezug können dagegen nur 20 \% der Probanden der Untersuchungs- sowie $16 \%$ der Probanden der Kontrollgruppe herstellen. Bei vielen Probanden wird die Antwort so formuliert, dass man die Notenschrift brauche. Selbst von den insgesamt 45 Schülern, die außerschulisch Instrumentalunterricht im ersten bis dritten Unterrichtsjahr erhalten, stellen nur $22 \%$ einen Ich-Bezug her.

\subsubsection{Subtest I Hörendes Vergleichen von Rhythmen}

Beim Vergleichen der fünf Rhythmuspaare erreichen die Probanden einen Mittelwert von 4.0 (UG) bzw. 3.6 (KG) von insgesamt 5 möglichen Punkten. Der prozentuale Anteil an richtigen Lösungen pro Item ist in beiden Gruppen relativ ähnlich (s. Tab. 4-30). Item I 3 ist das zweitschwerste Item dieses Subtests. Das Rhythmuspaar besteht aus Rhythmus 2a und Rhythmus 20 (s. Tab. 3-12). Rhythmus 2a ist gleichfalls Inhalt des Items B 2. Dieses Item gehört im Vor- und Nachtest zu den schweren Items. Im Vortest werden für Item B 2 die meisten Ersatzrhythmen gespielt. Deshalb verwundert es nicht, dass Item I 3 nur von zwei Dritteln der Probanden bewältigt wird.

Tab. 4-30: Anteil an richtigen Lösungen im ST I, VT und NT, getrennt nach UG und KG

\begin{tabular}{|c|c|c|c|c|c|c|c|c|c|c|}
\hline \multirow{3}{*}{$\begin{array}{c}\text { ST I, Vergleichendes } \\
\text { Hören }\end{array}$} & \multicolumn{3}{|c|}{ UG, $\mathbf{n}=82$} & \multirow[b]{2}{*}{ Item } & \multirow[b]{2}{*}{ Item } & \multicolumn{3}{|c|}{ KG, $\mathbf{n}=\mathbf{6 7}$} & \multirow[b]{2}{*}{ Item } & \multirow[b]{2}{*}{ Item } \\
\hline & Item & Item & Item & & & Item & Item & Item & & \\
\hline & 1 & 2 & 3 & 4 & 5 & 1 & 2 & 3 & 4 & 5 \\
\hline Vortest & $87 \%$ & $91 \%$ & $43 \%$ & $66 \%$ & $59 \%$ & $75 \%$ & $78 \%$ & $43 \%$ & $64 \%$ & $43 \%$ \\
\hline Nachtest & $94 \%$ & $95 \%$ & $67 \%$ & $88 \%$ & $57 \%$ & $85 \%$ & $90 \%$ & $66 \%$ & $76 \%$ & $45 \%$ \\
\hline
\end{tabular}

Das Item I 5 ist in diesem Subtest das schwerste Item. Dieses Rhythmuspaar besteht aus dem Zusatz-Item b 6 sowie einer Variation davon. Item b 6 wird sowohl im Vor- als auch im Nachtest am schlechtesten von allen nachzuspielenden Rhythmen reproduziert (s. Tab. 4-8).

Auch für den zusätzlichen Subtest I Hörendes Vergleichen von Rhythmen werden Korrelationen zu den anderen Subtests sowie zum Gesamtpunktwert berechnet. Dies hat folgenden Grund: Es wurde für diese Studie von vornherein nur nach kriteriumsorientierten und musizierpraktisch ausgerichteten Tests geschaut. Rezeptionsorientierte Tests kamen nicht in Betracht. Es wird nun betrachtet, wie groß Zusammenhänge zwischen Hörfähigkeit (Subtest I) und tatsächlicher Musizierfähigkeit (z. B. Subtest G) bzw. dem Gesamtpunktwert des Tests sind (s. Tab. 4-31). Korrelationen größer als $r=.30$ zeigen sich zwischen Subtest I und den Subtests C und E sowie dem Gesamtpunktwert. Alle anderen Korrelationswerte fallen schwächer $r=.30$ aus. Dies legt nahe, dass von 
den relativ guten Hörfähigkeiten im Subtest I nicht auf ebenso gut entwickelte Fähigkeiten in den musizierpraktischen Subtests wie z. B. die Subtests A, B, D und E geschlossen werden kann.

Tab. 4-31: Korrelation zwischen ST I und anderen Subtests sowie dem GPW im NT

\begin{tabular}{ccccccccc}
\hline Subtest & A & B & C & D & E & F & GPW & G \\
\hline I & .24 & .31 & .31 & .18 & .32 & .17 & .34 & .27 \\
\hline
\end{tabular}

\subsection{Ergebnisse in Bezug auf das Testmodell}

Für die Prüfung der Übereinstimmung des Testmodells mit dem Theoriemodell werden zuerst die tatsächlichen Itemschwierigkeiten im Nachtest mit den geplanten Itemschwierigkeiten verglichen. Dafür werden alle Items eines Subtests nach ansteigender Itemschwierigkeit platziert. Eine Übereinstimmung mit den geplanten Itemschwierigkeiten trifft nur in groben Zügen zu (s. Tab. 4-32). Für die Probanden der Untersuchungsgruppe sind vier Items zu leicht, drei davon im Subtest E. Zwei Items im Subtest A sind zu schwer. Für die Probanden der Kontrollgruppe sind etwa zwei Drittel der Items zu schwer. Die schwachen Leistungen im Subtest $C$ sind durch Nichterlernen der Rhythmussprache in den Klassen K 5 und K 6 jedoch erklärbar. Keines der Items ist zu leicht.

Tab. 4-32: Vergleich der tatsächlichen Itemschwierigkeiten im NT mit dem Modell

\begin{tabular}{c|cc|c|c|c|cc|cc|c|cc|cc|}
\hline Stufen & \multicolumn{4}{c}{ UG, $\mathbf{n}=\mathbf{8 2}$} & \multicolumn{1}{c}{ geplante Itemschwierigkeit } & \multicolumn{4}{c}{ KG, $\mathbf{n}=\mathbf{6 7}$} \\
\hline $\mathrm{F}$ & .57 & .55 & .51 & .26 & & .33 & .33 & .01 & .01 & & .27 & .25 & .15 & .10 \\
$\mathrm{E}$ & .73 & .66 & .49 & .41 & & .33 & .33 & .33 & .01 & & .48 & .34 & .33 & .13 \\
$\mathrm{D}$ & .43 & .43 & .39 & .41 & & .33 & .33 & .33 & .33 & & .40 & .21 & .34 & .25 \\
$\mathrm{C}$ & .72 & .44 & .55 & .55 & & .66 & .33 & .33 & .33 & & .28 & .22 & .24 & .12 \\
$\mathrm{~B}$ & .74 & .68 & .48 & .41 & & .66 & .66 & .33 & .33 & & .58 & .57 & .40 & .31 \\
$\mathrm{~A}$ & .71 & .62 & .60 & .41 & & .66 & .66 & .66 & .33 & & .60 & .55 & .55 & .28 \\
\hline
\end{tabular}

Auch die tatsächlichen Mittelwerte der Subtests A bis F im Vor- und Nachtest sind mit den erwarteten Mittelwerten zu vergleichen (vgl. Abb. 3-15). Die Mittelwerte im Vortest bleiben weit unter dem (für den Nachtest) erwarteten Mittelwerten zurück (s. Abb. 4-66). Jedoch weisen die Mittelwerte der Subtests A, B und D in beiden Gruppen schon beachtliche Werte auf. In der Kontrollgruppe fällt ebenfalls ein bereits höherer Mittelwert für den Subtest E auf. Dies wurde im Abschnitt 4.3.5 bereist kommentiert. Die geringen Mittelwerte in den Subtests C, E und F stehen im Einklang mit der curricular erst in dieser Klassenstufe beginnenden Einführung in die Notenschrift (ST E und F) bzw. mit dem curricular nicht geforderten Erlernen einer Rhythmussprache (ST C). 


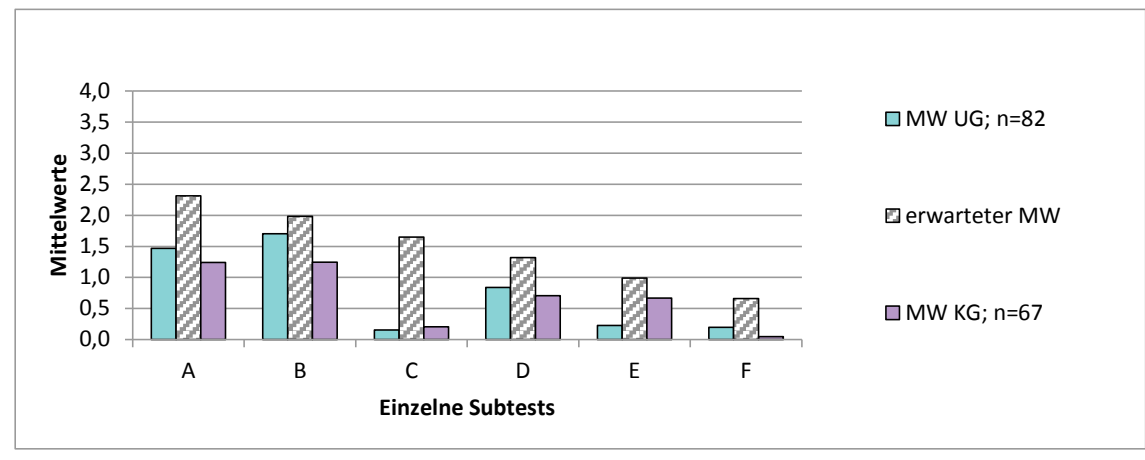

Abb. 4-66: Mittelwerte der einzelnen Subtests im VT, getrennt nach UG und KG

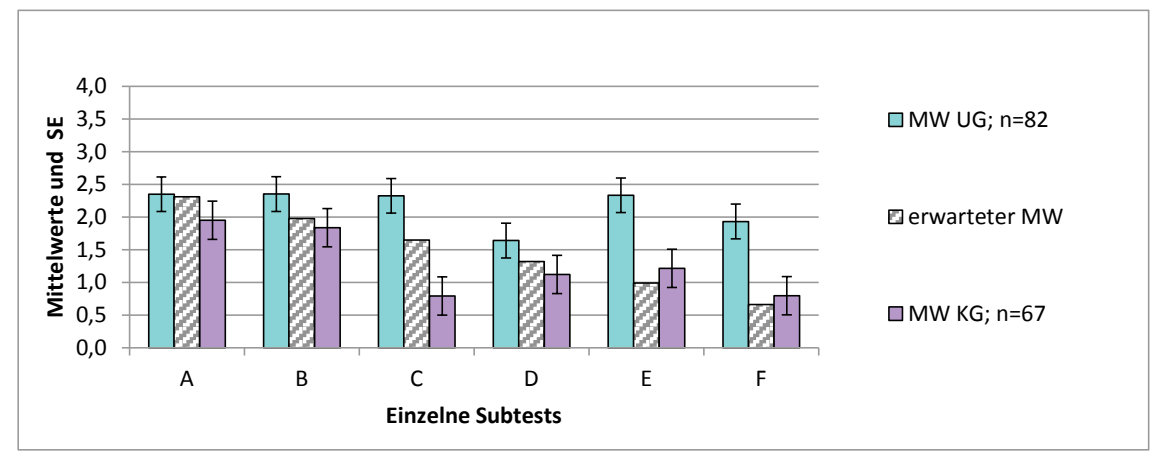

Abb. 4-67: Mittelwerte der einzelnen Subtests im NT, getrennt nach UG und KG

Im Nachtest werden fast alle erwarteten Mittelwerte erreicht bzw. überboten (s. Abb. 4-67). Die Mittelwerte in der Kontrollgruppe gehen bis auf kleine Abweichungen konform mit dem Pyramidenmodell. Ausnahme bildet der Mittelwert für Subtest C. Grund dafür sind das Nichterlernen einer Rhythmussprache in der den Klassen K 5 und K 6 sowie die Abschwächung des Leistungspotenzials durch das Nichtüberwinden des figuralen Typus als Fehlerquelle in Untertest fc (s. Abschnitt 4.3.1). Trotz der (erfreulicherweise) leicht erhöhten Mittelwerte in den Subtests E und F bilden alle erreichten Mittelwerte eine Zunahme der Schwierigkeit der Subtests gemäß des Pyramidenmodells zum Aufbauen rhythmischer Fähigkeiten bzw. dessen Operationalisierung ab.

Die Mittelwerte in der Untersuchungsgruppe bleiben relativ stabil auf einem Niveau um den Punktwert 2. Dabei fällt auf, dass die Ergebnisse im Subtest A nur um ein Geringes den erwarteten Mittelwert übersteigen. Im Subtest D liegt der erreichte Mittelwert ebenfalls sehr nah am erwarteten Mittelwert und bildet damit im negativen Sinne eine Ausnahme. Zugespitzt wird diese Tatsache durch die extrem hohen Mittelwerte in den Subtests E und F. Es ist ablesbar, dass die erreichten Mittelwerte in der Untersuchungsgruppe nicht einer Schwierigkeitszunahme folgen. Dies stellt das Pyramidenmodells zum Aufbauen rhythmischer Fähigkeiten bzw. dessen Operationalisierung in Frage und wird zu diskutieren sein. 


\subsection{Ergebnisse zur Testgütesicherung}

Mit den 82 Probanden in der Untersuchungsgruppe und den 67 Probanden in der Kontrollgruppe, einer einseitig gerichteten Hypothese sowie einer zu erreichenden Effektstärke in Höhe von $d=0.5$ wird eine Teststärke von 0.92 sowie für den t-Test ein Wert für Critical $t$ in Höhe von 1.6 berechnet (s. Anlage C 1).

Die Rohdaten der 24 einzelnen Items im Nachtest weisen im Qui-Quadrat-Test eine Normalverteilung auf. Die Histogramme und SPSS-Ausgaben werden in Anlage C 2 dargestellt.

Die Itemschwierigkeit liegt für alle Probanden im Nachtest zwischen 0.19 und 0.66 (s. Tab. 4-32), die Trennschärfe zwischen 0.34 und 0.67 (s. Anlage C 3).

\section{Objektivität}

In Bezug auf die Durchführungsobjektivität wurden die angegebenen Faktoren eingehalten. Bedingung für die Teilnahme der Schulen an der Studie war ein in die Schule integrierter Hort. So war es möglich, die Schüler am Nachmittag problemlos zum Test abzuholen. In einigen Schulen gab es zu bestimmten Zeiten ein erhöhtes Lärmpotential. Die Lage der Räume, in welchen der Test an den einzelnen Schulen stattfand, war nicht an jeder Schule zu jeder Tageszeit optimal zu nennen. Erstaunlicher Weise hatte das aber eher die Versuchsleiterin als die Probanden gestört, sie wirkten nicht abgelenkt. Die zeitlichen Strukturen des Tagesablaufes unterschieden sich in den Horten. Obwohl sowohl die Horterzieher als auch die Probanden die genauen Testzeiten kannten, mussten Kinder mitunter aus einem Ganztagsangebot geholt werden und fielen sozusagen Hals über Kopf in den Test. Dies trat in beiden Gruppen auf.

Beim schriftlichen Testverfahren (Subtest F) mit allen Klassen konnte nicht ausgeschlossen werden, dass es klassenspezifische Unterschiede gab, die in der Eigenart jeder Klasse begründet waren (Disziplin, Mitarbeit, Motivation, Aufmerksamkeit und Konzentration, Ehrlichkeit). Bedingt wurde dies dadurch, dass die Einführung in die Aufgabenformate durch verschieden hohe Aktivität, unterschiedliche Mitarbeit und ein weit gefächertes kreatives Potenzial geprägt war. Diese Phase der Testeinführung ließ sich nur bedingt vereinheitlichen. Einige Ergebnisse ließen Schlüsse zu, wer (trotz Sichtabdeckung) voneinander abgeschrieben haben könnte. Dies betraf aber nur einen äußerst geringen Anteil der Schüler.

Im Folgenden werden einige Anmerkungen zum Gelingen des Videotestverfahrens festgehalten. Im Subtest $B$ Rhythmen nachspielen sind die Erklärungen für die Call-Response-Aufgabenstellung gemessen an anderen Erklärungen zeitlich am umfangreichsten (s. Anlage B 1). Das CallResponse-Prinzip wird auch demonstriert. Trotzdem gab es vereinzelt Probanden, die erst nach einigen Items das Spielen in diesem Prinzip verinnerlichten. Deshalb war es sinnvoll gewesen, ein sogenanntes Start-Item einzubauen. 
Subtest D folgt unmittelbar auf Subtest B. Das klare Signal an die Probanden ist hierbei, nicht mehr den gleichen, sondern nun einen anderen Rhythmus als den Call-Rhythmus zu spielen. Die Bemerkung, dass sich der ausgedachte Takt von „,meinem Takt, dem langweiligsten Takt der Welt, bestehend aus vier Viertelnoten" unterscheiden müsse, erheiterte viele Probanden. Die Reaktionen gingen von Lächeln bis Lachen. Besonders im Nachtest war erkennbar, dass sich viele Probanden sehr auf diese Aufgabe freuten.

Die Auswertungsobjektivität wird über einen Algorithmus als Prüfwerkzeug für die Bewertung der Rhythmen aus den Subtests B, C, D und E abgesichert. Der Weg zur endgültigen Version führte über viele Zwischenversionen. Je nach Ausweitung bzw. Eingrenzung der Toleranzbereiche stieg oder sank die Itemschwierigkeit für alle Items. Die Optimierung orientierte sich stets an ursprünglichen Entscheidungen, die nur hörend von der Autorin getroffen wurden. In Version 10 fand die Optimierung diesbezüglich ihre größte Übereinstimmung. Obwohl der Algorithmus nur an der Hörempfindung der Versuchsleiterin orientiert ist, kann dennoch davon ausgegangen werden, dass alle Rhythmen den gleichen Wertungsmaßstäben unterworfen waren. Diese Form der Auswertung darf damit als nachvollziehbar, wiederholbar, überprüfbar und als objektiv gelten.

Für Item B 1 wird eine repräsentative Ansicht für ausgewertete Rhythmen gegeben (s. Abb. 3-23). Die farbige Hervorhebung der Zellen entspricht den Toleranzabweichungen. Die Schwere dieser Abweichungen entscheidet über die Zuordnung zu den Toleranzbereichen und Fehlerkategorien (s. Anlage B 3). Um auch die Rhythmen aus Subtest E, die mit freier Tempowahl musiziert werden, mit dem Algorithmus bewerten zu können, werden bei der Auswertung zuerst die tatsächlichen Tempi berechnet. Für jeden Rhythmus wird das Tempo als zusätzlicher Faktor in die Berechnung eingebettet.

Durch die Entwicklung und den Einsatz des Algorithmus können Fehlerkategorien gebildet werden. Sie entstehen aus der Beobachtung der im Algorithmus bedingten Formatierungen und gehen über das allein durch Zuhören Analysierbare hinaus. Zusätzlich können durch den Einsatz des Algorithmus Lösungskategorien gebildet werden. Diese analysieren das Spektrum von den korrekten bis hin zu extrem falschen oder sogar fehlenden Lösungen.

\section{Reliabilität}

Die Prüfung der Reliabilität des entwickelten Testes erfolgt über die Testhalbierung und die Berechnung der internen Konsistenz (s. Abschnitt 3.5.4). Die für die Testhalbierung gebildeten Testhälften haben eine mittlere Itemschwierigkeit von 0.44 bzw. 0.45. Die Mittelwerte der beiden Testhälften liegen bei 5.21 und 5.39. Die Werte für Cronbach's Alpha liegen bei 0.79 und 0.81. Die interne Konsistenz beträgt für den Gesamttest 0.89 (s. Anlage C 4). 


\section{Validität}

Die Inhaltsvalidität wurde durch die Anbindung der Entwicklung der Items an die Theorie Gordons und vergleichbare Inhalte verschiedener Curricula deutscher Bundesländer (insbesondere des sächsischen Lehrplans) bestmöglich realisiert. Für die Kriteriumsvalidität werden die Ergebnisse der 149 Probanden im Vortest in die Gruppen known group = Musikschüler (mit mindestens einem Jahr Instrumentalunterricht) und andere Schüler eingeteilt. Die Gruppe Musikschüler besteht aus insgesamt 32 Schülern, welche das zweite oder dritte Jahr Instrumentalunterricht erhalten (s. Abb. 4-6). Alle anderen Schüler umfassen eine Gruppe von 117 Probanden, die kein Instrument erlernt haben oder erst im ersten Unterrichtsjahr sind oder den Instrumentalunterricht wieder abgebrochen haben. Die Musikschüler erzielen im Vortest in allen Subtests deutlich bessere Ergebnisse (s. Abb. 4-68). (Die Ergebnisse für diese Methode, jedoch auf die Daten des Nachtests angewendet, finden sich in Anlage C 4 f.)

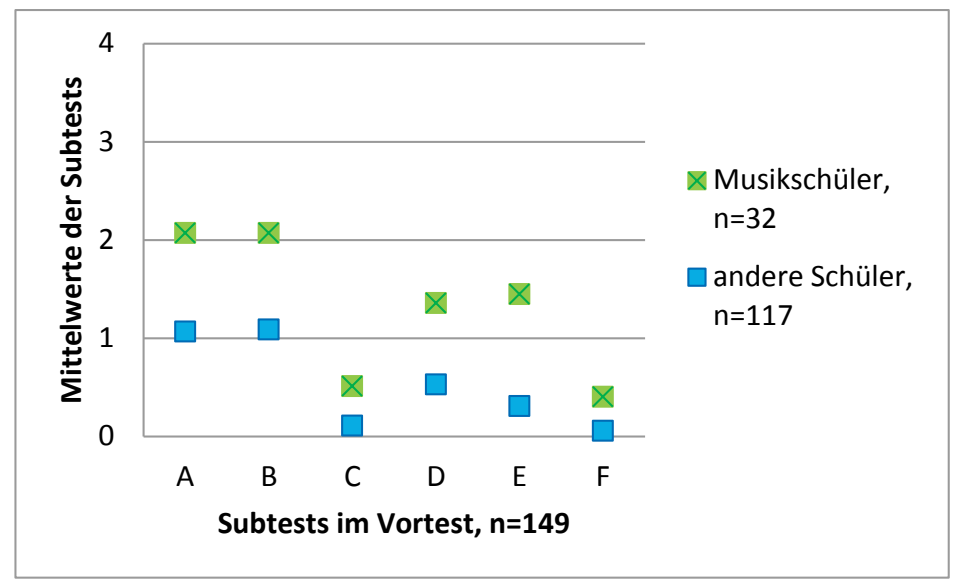

Abb. 4-68: Darstellung der Ergebnisse nach der Methode known groups

Eine zweite Methode berechnet die Korrelationen zwischen den Mittelwerten aller 149 Probanden in der zusätzlichen Itemgruppe G Play together (s. Abschnitt 3.4.2) sowie den Subtests A bis F und dem Gesamtpunktwert (GPW) des Tests. Sie werden im Abschnitt 4.4.1 dargestellt.

\section{Nebenkriterium Ökonomie}

Zum Nebenkriterium Ökonomie kann gesagt werden, dass das Gruppenverfahren (schriftlich) aufgrund seiner Einsatzmöglichkeit in einer gesamten Klasse und mit einer Dauer von ca. 30 Minuten sehr effizient war. Die Testbogen ließen sich schnell auswerten, da relativ wenige Kriterien zu berücksichtigen waren. Das Einzelverfahren erwies sich mit einer Dauer von max. 20 Minuten je Proband ebenfalls als effizient. Allerdings bedurfte die gründliche Auswertung eines Audio-Files eines erheblichen Zeitaufwandes, der pro File bei etwa 90 Minuten lag. Dies entstand vor allem durch das allmähliche und schrittweise Erarbeiten des bestmöglichen Auswertungsmodus basierend 
auf millisekundengenauer Messung der Rhythmusschläge und anschließender Berechnung im entwickelten Algorithmus.

\subsection{Ergebnisse in Bezug auf das Treatment}

Die Lehrkräfte der Klassen der Untersuchungsgruppe konnten bis auf LK-U 6 das Treatment in ihren Klassen gut durchführen (s. Abschnitt 3.2). Alle Lernsequenzen wurden in der angegebenen Reihenfolge umgesetzt und im abgesteckten Zeitrahmen absolviert. Die Lehrkräfte, die wieder eine 3. Klasse im darauffolgenden Schuljahr unterrichteten, setzten den Rhythmus-Lehrgang erneut ein. Es kam die Rückmeldung, dass die Lehrkräfte den Rhythmus-Lehrgang mit diesen neuen 3. Klassen über einen längeren Zeitraum als 20 Unterrichtswochen ausdehnen würden.

Die Empfehlungen für die Zusammensetzungen der Stammgruppen nach der Lernausgangslage im Vortest wurden umgesetzt. Die Schülerinnen und Schüler arbeiteten gern in ihren Vierergruppen. Die meisten waren mit ihrer zusätzlichen Aufgabe in der Gruppe (Chef, Räumer, Schreiber, Streitschlichter) zufrieden. Das Räumen der Tische und Bereitstellen der Instrumentenkörbe ging sehr schnell vonstatten. Das Wechseln von Stamm- und Expertengruppen fiel den Schülerinnen und Schülern insofern schwer, da in jeder Lernsequenz neue Expertengruppen entstanden. Die Stimmen wurden in jedem neuen Lerninhalt nach Ermessen der Gruppenmitglieder anders untereinander verteilt. Ein Schüler bzw. eine Schülerin konnte in Lernsequenz 1 zur Expertengruppe A, in Lernsequenz 2 zur Expertengruppe C zählen. Dies bedurfte zu Beginn jeder neuen Lernsequenz einer gewissen Neuorientierung innerhalb der Gruppenstrukturen. Hier könnten für die Expertengruppen Notenständer mit Hinweisen zur entsprechenden Stimmgruppe schnellere Orientierung schaffen.

Das methodische Arbeiten in den einzelnen Bausteinen (Warm up 1,Warm up 2, Workshop) bereitete Anlaufschwierigkeiten. Durch die einmalige Fortbildung zum Rhythmus-Lehrgang waren nicht alle Lehrkräfte sofort in der Lage, die vielen Hinweise zu berücksichtigen. Dazu zählten z. B. das Vermeiden einer verbalen Einflussnahme auf das Bewegen im Warm up 1, das fließende Rhythmusspiel in den Call-Response-Übungen im Warm up 2 sowie das methodisch richtige Erfinden eines Verbotenen Rhythmus.

Die Lehrkräfte meldeten zurück, dass die Schüler sehr gespannt auf die Musiktitel für das Warm up 1 waren. Manche Musikausschnitte wurden zu Lieblingstiteln, die von den Schülern ausdrücklich zum wiederholten Hören gewünscht wurden. Einigen Lehrkräften fiel es schwer zuzulassen, dass jedes Kind sich nach eigenem Vermögen in die Aufgabe einbrachte und von sich aus lernen sollte. Eine Lehrkraft versuchte sogar, korrigierend auf die Schüler einzugehen. Die Übung in der hospitierten Stunde glich eher dem Einstudieren einer Choreografie. Dies verfehlte die Intention des Warm up 1. Allen Lehrkräften (auch anfänglich der Versuchsleiterin) bereitete es Schwierigkeiten, in den Musikausschnitten zuerst das Metrum mit dem Körper/ den wippenden Füßen 
und dann erst den Grundschlag mit den Händen darzustellen. Als Abwandlung wurde von einigen Lehrkräften in den ersten beiden Lernsequenzen die Reihenfolge gewechselt. Es wurde zuerst der Grundschlag, dann erst das Metrum dargestellt. Eine Lehrkraft fand es schwer, den Impuls des Aufden-Ballen-Gehens auf Zählzeit 4 zu beginnen, um dann auf Zählzeit 1 aufzusetzen. Der Impuls begann bei ihr erst auf Zählzeit 1. Nach dem Besprechen der von Gordon gewollten Bewegung stellte sie dies um.

Die Lehrkräfte erklärten, dass es ihnen zu Beginn des Rhythmus-Lehrgangs im Warm up 2 sehr schwer fiel, ohne Vorlage abwechslungsreiche Rhythmen korrekt im Takt bleibend vorzuspielen. Eine Lehrkraft meinte rückblickend, dass besonders bei den Warm up 's eigene Sicherheit notwendig sei. Von den Lehrkräften wurde rückgemeldet, dass es unter den Schüler als hohe Auszeichnung galt, den Verbotenen Rhythmus auswählen zu dürfen. Bei zwei Lehrkräften fehlte zu Beginn das Bewusstsein dafür, dass dieser Rhythmus nicht von vielen Schülern als Puzzle, sondern nur von einem Schüler gebildet werden sollte. Für diesen einen Schüler bedeutete es, Gelegenheit zu erhalten, aus seinem Hörvokabular zu schöpfen, den Rhythmus im Taktrahmen zu bilden und ihn anschließend mit seinen Mitschülern zu üben. Eine andere Lehrkraft stellte ebenfalls bei dieser Methode nicht alle angefertigten Notenlaminate zur Verfügung, so dass die Freiheit in Bezug auf die Kombinationen aus Achtel-, Viertel- und halbe Noten beim Erfinden eines Rhythmus eingeschränkt wurde. Dies wurde besprochen und geändert.

In den Workshops traten keine nennenswerten Probleme auf. Die entstandenen Spielstücke wurden in Anlage D 6 dokumentiert. Die aus Klassenstufe 4 aufgegriffenen Lehrplanziele bezüglich des gleichzeitigen Spielens verschiedener Rhythmusbausteine bereiteten im Treatment keine Schwierigkeiten. Hier wurden die Schülerinnen und Schüler beim vierstimmigen Musizieren der Rhythmicals und Spielstücke von der Lehrkraft durch das Dirigieren unterstützt. Die Ergebnisse im zusätzlichen Subtest G Play together dokumentieren jedoch eindrücklich in den Items G 2 und G 4, dass das selbstständige zweistimmige Musizieren nur von wenigen Probanden bewältigt wurde (s. Abb. 4-65).

Das Ausfüllen der großen A2-Kopien als Tafelbild war für einige Lehrkräfte gewöhnungsbedürftig. Hier könnten dunkel unterlegte Felder in der Kopiervorlage Abhilfe schaffen. Die anderen weiterführenden Arbeitsmaterialien wurden gut genutzt. Die Lehrkräfte gaben positives Feedback für die klar strukturierten Anweisungen und Planungen im Handbuch. Die dazugehörigen CDs waren gut einsatzbar.

Die Verwendung der Strichnotation gelang den Probanden gut. Das Bezeichnen der verschiedenen Striche mit waagerechter und senkrechter bzw. langer und kurzer Strich gelang nicht reibungslos. Nachdem mit der Einführung der halben Note (in Lernsequenz 4) ein noch längerer Strich hinzukam, führte dies mitunter zu sprachlicher Verwirrung. Die Bezeichnungen mittellanger oder normal langer Strich für Viertelnoten waren ebenfalls nicht optimal. So gut sich die Striche beim 
schreibenden Gebrauch bewährten, so sperrig gestaltete sich der sprachliche Gebrauch. Eine Lehrkraft schlug vor, die Bezeichnungen für die Striche mit den Rhythmussilben zu koppeln, z. B. taund ti-Striche. Für die Viertelpause wurde in der Strichnotation kein Zeichen verwendet. Ob dies schlussendlich sinnvoll war, müsste auch in weiteren Erprobungen getestet werden. Festzustellen war, dass die Ergebnisse für das Verwenden des Pausensymbols und der Silbe für Pause in den Items E 4 (s. Abb. 4-41) und F 4 (s. Abb. 4-13 sowie Abb. 4-14) sowie in den Items ec 4 (s. Abb. 4-52) und fc 4 (s. Abb. 4-55 sowie Abb. 4-54) im Nachtest teilweise deutlich schwächer ausfielen.

Zum Lesen von Rhythmen konnten durch die Hospitationen im Unterricht kaum Aussagen getroffen werden. Die Aufzeichnungen in den Arbeitsheften der Schüler zeigten, dass das Schreiben der Rhythmen in Strichnotation und traditioneller Notation keine gehäuft auffallenden Schwierigkeiten bereitete. Hin und wieder waren klassenübergreifend folgende Fehler aufgefallen:

- Viertelnote: Die Strichnotation für zwei oder mehr Viertel erfolgte fehlinterpretiert als durchgehender Strich, nicht als mehrere einzelne kurze Striche.

- Halbe Note: Die Strichnotation für die halbe Note wurde mitunter um etwa ein Viertel zu kurz, nicht über zwei Grundschläge gehend gezeichnet.

- Synkope: Das Setzen des Striches für die Viertelnote der Synkope mitten auf den Trennstrich der benachbarten Grundschläge gelang erst beim zweiten Anlauf.

- Notenköpfe: Mitunter wurden in Lernsequenz 2 die Notenköpfe noch nicht ausgemalt, in den folgenden Lernsequenzen war der Fehler so gut wie nicht mehr aufgetreten; bei wenigen Schülern ähnelte die Kombination aus Notenkopf und Notenhals eher einem kleinen gedruckten $d$.

- Viertelpause: Sie war das schwerste darzustellende Symbol innerhalb des Lehrgangs und wurde von ca. einem Viertel der Schüler noch sehr unkenntlich gezeichnet.

- Fähnchen der Achtelpause: Ab und an gab es Notationen in den Schüler-Arbeitsheften, bei denen Hals und Fähnchen eher einem Hirtenstab ähnelten, weil sie vermutlich in einem Arbeitsgang gezeichnet wurden. 


\section{Diskussion und Schlussfolgerungen}

Einleitend werden in diesem Kapitel die Abweichungen in Bezug auf das Forschungsdesign benannt und diskutiert. Im Anschluss werden die in Abschnitt 0 gestellten Fragen beantwortet und die erzielten Ergebnisse diskutiert (s. Abschnitt 5.2). Es schließt sich die Beurteilung zu Testentwicklung, Testmodell sowie Testgüte an (s. Abschnitt 5.3). Gedanken zur möglichen Überarbeitung des Treatments werden vorgestellt (s. Abschnitt 5.4). Das Kapitel endet mit Schlussfolgerungen und einem Ausblick auf nachfolgende Studien (s. Abschnitt 0).

\subsection{Abweichungen vom Untersuchungsdesign}

Im Untersuchungszeitraum erhielten alle Klassen der Untersuchungs- und Kontrollgruppe die regulären zwei Stunden Musikunterricht. Dabei kam es zu drei Abweichungen. Der Musikunterricht in der Klasse U 6 konnte durch eine Erkrankung der LK-H für mehrere Wochen nicht fortgesetzt werden. Deshalb wurden die Probanden aus einer 3. Klasse, welche Unterricht bei der Autorin erhielten, ebenfalls den Vortest durchlaufen hatten und das Treatment erhielten, als Klasse U 7 eingewechselt (s. Abschnitt 4.1.1). Die Klassen U 4 und U 5 erhielten den Musikunterricht als Doppelstunde. Auswirkungen lassen sich aus den angegebenen Daten sowie den Ergebnissen in den einzelnen Subtests nicht ablesen (s. Tab. 4-22). Die Mittelwerte der Gesamtpunktwertung entsprechen in etwa denen der anderen Klassen der Untersuchungsgruppe.

Die dritte und wesentlichste Abweichung vom Untersuchungsdesign entstand in den Klassen K 1 und K 2. Diese beiden Klassen erhielten zusätzlich 14-tägig eine Stunde Unterricht im Flötenspiel. Für diese zusätzliche Stunde war die Klasse jeweils in zwei Hälften geteilt. Dieser Faktor schien sich positiv auf die Gesamtpunktwertung auszuwirken. Die Probanden der Klasse K 2 errangen innerhalb der Kontrollgruppe den besten Mittelwert in der Gesamtpunktwertung (s. Tab. 4-22).

Durch die zweite Erhebung ist es möglich (s. Abschnitt 4.1.4), die Störung dieses experimentellen Aufbaus zumindest in Bezug auf die Leistungen im Subtest F zu beurteilen. Verglichen werden dazu die Ergebnisse aus Subtest F sowohl von allen Schülern und Schülerinnen der Haupterhebung (in diesem Falle nicht begrenzt auf die 149 Probanden, sondern erweitert auf alle über 200 Schülerinnen und Schüler dieser 12 Klassen; s. Abb. 4-61) mit den Ergebnissen der Schülerinnen und Schüler aus den Klassen der zweiten Erhebung, in diesem Falle nur Klassenstufe 3 (s. Abb. 4-63, KS 3).

Die Anteile an richtigen Lösungen für Subtest F fallen bei den Schülern und Schülerinnen der Untersuchungs- und Kontrollgruppe insgesamt deutlich höher als bei den Schülerinnen und Schülern der zweiten Erhebung aus. Die Ergebnisse der Klassen K 1 und K 2, welche den zusätzlichen Flötenunterricht erhielten (s. Abb. 4-62), fallen deutlich besser als die Ergebnisse vieler Klassen der zweiten Erhebung aus (s. Abb. 4-64, links). Jedoch stehen sie in Korrelation zu den Leistungen 
anderer dritter Klassen der zweiten Erhebung, in denen ebenfalls eine Rhythmussprache erlernt wurde. Somit kann vermutet werden, dass die zusätzliche Förderung durch den 14-tägigen Flötenunterricht innerhalb der Kontrollgruppe zu einem Leistungsvorsprung in der Gesamtpunktwertung führte. Nachweislich im Subtest F sind die Leistungen aber durchaus vergleichbar mit den Leistungen anderer Klassen, in denen im regulären Musikunterricht eine Rhythmussprache erlernt und angewendet wurde. Es lässt sich darüber hinaus belegen, dass in den Klassen der Kontrollgruppe eine gute Lehr- und Lernarbeit verrichtet wurde. Insbesondere die Klassen K 1, K 2 und K 3 haben deutlich höhere Mittelwerte im Subtest F als viele anderen Klassen in der Klassenstufe 3 sowie einige Klassen in Klassenstufe 4 (s. Abb. 4-62 sowie Abb. 4-63).

\subsection{Diskussion der Testergebnisse}

Die Diskussion der Testergebnisse wird in die Bereiche Vor- und Nachtestergebnisse (Frage 1 und 2) sowie vergleichende Darstellung der Differenzen aus Vor- und Nachtestergebnissen untergliedert (s. Abschnitt 5.2.2, Frage 3). Die Ergebnisse aus den zusätzlichen Untertests e und f werden in den Unterkapiteln zu Subtest E und F kommentiert (s. Abschnitt 5.2.1). Die Ergebnisse der zusätzlichen Subtests G, H und I werden gesondert diskutiert (s. Abschnitt 5.2.2).

\subsubsection{Stand der Entwicklung zu Beginn und am Ende der Klassenstufe 3}

Die Vor- und Nachtestergebnisse für alle Probanden sind im Kapitel 4 dargestellt. Sie dienen der Beantwortung der eingangs gestellten Fragen:

(1) Welcher Stand der Entwicklung rhythmischer Kompetenz ist bei Grundschülern zu Beginn der Klassenstufe 3 zu verzeichnen?

(2) Welcher Stand der Entwicklung rhythmischer Kompetenz ist bei Grundschülern am Ende der Klassenstufe 3 zu verzeichnen?

Der Entwicklungsstand zu Beginn der Klassenstufe 3 sowie nach Abschluss des Untersuchungszeitraumes (nahe am Ende der Klassenstufe 3) wird jeweils zusammen für jeden Subtest und in der Reihenfolge A, B, D sowie E, F und C aufgeführt.

\section{Subtest A}

Im Subtest A Metrum und Grundschlag darstellen können im Vortest 40 \% der Probanden Grundschlag und Metrum des Liedes Bruder Jacob (Item A 1 und A 2; s. Abb. 4-9) richtig darstellen. Zählt man noch die Ergebnisse aus der Kategorie Lösung fast richtig mit kleinen Abstrichen hinzu, erhöht sich dieser Anteil auf etwa 60 \% (s. Abb. 4-22). Das Metrum zum Menuett G-Dur (Item A 3) können $36 \%$ (mit Kategorie Lösung fast richtig mit kleinen Abstrichen etwa $50 \%$ ) sowie das Metrum zum Popmusik-Beispiel (Item A 4) 21 \% (bzw. 35 \%) der Probanden richtig darstellen. Damit fällt den Probanden die Darstellung des geraden Metrums im 4/4-Takt zu einem Musikbei- 
spiel aus dem Popbereich am schwersten. Dies ist insofern verwunderlich, da doch gerade Popmusik zu den am häufigsten konsumierten Genres gehört.

Im Nachtest können über 60 \% der Probanden Grundschlag und Metrum des Liedes Bruder Jacob (Item A 1 und A 2; s. Abb. 4-10) richtig darstellen. Zählt man noch die Ergebnisse aus der Kategorie Lösung fast richtig mit kleinen Abstrichen hinzu, erhöht sich dieser Anteil auf etwa 80 \% bzw. $75 \%$ (s. Abb. 4-22). Damit gelingt das Musizieren des Grundschlages etwas besser als das Musizieren des Metrums. Das Metrum zum Menuett G-Dur (Item A 3) können ca. 60 \% (bzw. ca. 70 \%) sowie das Metrum zum Popmusik-Beispiel (Item A 4) ca. 35 \% (bzw. ca. 45 \%) der Probanden richtig darstellen. Item A 4 ist immer noch am schwersten für die Probanden zu lösen. Es fällt den Schülerinnen und Schülern auch am Ende der Klassenstufe 3 schwer, die Zeitspanne im 4/4Takt des Popmusikbeispiels zu ermessen und abzuwarten.

Im Subtest A gibt es vor jedem Item einen gleichlangen Spielabschnitt mit einer pantomimischen Hilfestellung im Video. Dies soll ermöglichen, die Aufgabenstellung intuitiv zu erfassen und in das jeweilige Tempo des Musikstücks hineinzukommen. Etwa die Hälfte der Probanden kann diese Hilfestellung nutzen, im Item A 3 sogar 67 \% der Probanden (Menuett G-Dur). Diese Ergebnisse sind insofern erstaunlich, da in der Fachliteratur die Auffassung vorherrscht, dass das Imitieren von diesen als relativ leicht eingeschätzten Handlungen besonders mühelos zu vollziehen sei. (vgl. Fuchs, 2010, S. 22-23). Das Imitieren gilt als die häufigste Form des Lernens in der Kindheit sowie als relativ leichte Form des Lernens aufgrund der Zugehörigkeit „zur Familie der ReizReaktionsmodell, mit denen auch das Lernen bei Tieren am häufigsten erklärt wird“" (Spychiger, 2015, S. 53). Das Imitieren des Darstellens von Grundschlag und Metrum scheint demnach schwieriger zu sein als angenommen. Eventuell sind jedoch auch folgende Hinderungsgründe zu bedenken: Das Medium Video entspricht nicht einer realen Situation, wie sie im Unterricht gegeben ist. Die zu imitierende Person ist nur auf dem Bildschirm zu sehen. Es könnte auch sein, dass die Handhabung des Triangels zu viel Aufmerksamkeit entzogen hat (s. Abschnitt 4.3.2) oder das Spielen des Metrums zu selten im Unterricht der involvierten Lehrkräfte stattfand.

Das gewinnbringende Nutzen der pantomimischen Hilfestellung steigt im Nachtest für Item A 1 von $44 \%$ auf $65 \%$, für Item A 2 von $46 \%$ auf $68 \%$, für Item A 3 von $67 \%$ auf $75 \%$ sowie für Item A 4 von $48 \%$ auf $73 \%$ an und erreicht damit einen Anteil von etwa zwei Dritteln der Probanden. Interessanterweise wenden sich einige Probanden im Nachtest bewusst von dieser Hilfestellung ab und schauen betont zur Seite. Dies zeigt, dass diese Probanden selbstbewusst und selbstständig die Aufgabe lösen und sich vom bloßen Imitieren distanzieren wollen. Dennoch kann etwa ein Drittel der Probanden noch nicht von dieser Hilfestellung profitieren. Dies legt nahe, Schülerinnen und Schülern der Klassenstufe 3 und auch 4 häufig Gelegenheit zum Imitieren und Lernen durch Imitation zu geben (s. auch Abschnitt 5.4). 


\section{Subtest B}

Im Subtest B Rhythmen nachspielen können im Vortest 60 \% bzw. 45 \% der Probanden die leichten Rhythmen aus Viertel- und Achtelnoten (Item B 1 und B 3; s. Abb. 4-9) fehlerfrei nachspielen. Zählt man noch die Ergebnisse aus der Kategorie Lösung fast richtig mit kleinen Abstrichen hinzu, erhöht sich dieser Anteil auf etwa $80 \%$ bei jedem der beiden Items (s. Abb. 4-31). Damit wird Upitis Aussage bestätigt, dass die Fähigkeiten zum Nachmachen rhythmischer Figuren bei Kindern bis zum zehnten Lebensjahr stärker ausgebildet sind als die metrischen Fähigkeiten (vgl. Upitis, 1987, S. 54). Etwa ein Viertel der Probanden kann die Rhythmen mit punktierter Viertelnote bzw. mit Synkope korrekt nachspielen, fast richtig mit leichten Abstrichen sogar die Hälfte der Probanden (Item B 2 und B 4).

Im Nachtest können über $60 \%$ der Probanden die beiden leichten Rhythmen aus Viertel- und Achtelnoten (Item B 1 und B 3; s. Abb. 4-10) fehlerfrei nachspielen. Zählt man noch die Ergebnisse aus der Kategorie Lösung fast richtig mit kleinen Abstrichen hinzu, erhöht sich dieser Anteil auf annähernd 90 \% (s. Abb. 4-31). Etwa $40 \%$ der Probanden können nun auch die Rhythmen mit Synkope bzw. mit der punktierten Viertelnote (Item B 2 und B 4) fehlerfrei nachspielen. Mit den Ergebnissen aus der Kategorie Lösung fast richtig mit kleinen Abstrichen erhöht sich dieser Anteil auf etwa $60 \%$ bis $75 \%$. Damit halten sich nun, anders als zu Beginn des Untersuchungszeitraumes, die Fähigkeiten zum Nachmachen rhythmischer Figuren sowie die metrischen Fähigkeiten in etwa die Waage (vgl. Upitis, 1987, S. 54).

Zu den häufigsten Fehlerquellen in Bezug auf die Rubrik Rhythmus an sich zählen im Vortest das Spielen eines falschen, jedoch gut erkennbaren anderen Rhythmus (Kat. 62; s. Abb. 4-28) sowie in Bezug auf den Einsatz und das Tempo des Rhythmus das zu frühe bzw. zu späte Einsetzen als auch das Spielen in leicht bis stark schwankenden Tempo (s. Abb. 4-29 und Abb. 4-30). Zu schnelles und zu langsames Rhythmusspiel halten sich die Waage. Das ersatzweise Spielen eines falschen, jedoch gut erkennbaren anderen Rhythmus (Kat. 62; s. Abb. 4-28) fällt in insgesamt 49 Fällen besonders auf. Der eigentliche Rhythmus kann scheinbar noch nicht richtig erfasst werden. Am häufigsten wird dies bei Item B 2 in 29 Fällen beobachtet (s. Tab. 4-9). Dies bestätigt die Beobachtung, dass sich eine „Tendenz zur Vereinfachung von rhythmischen Strukturen zeigt ..., wenn unregelmäßige Rhythmussequenzen reproduziert werden sollen“ (Beck, 2002 ${ }^{4}$, S. 460). Zwar handelt es sich im Falle von Item B 2 nicht um eine unregelmäßige Rhythmussequenz, jedoch ist die Folge von punktiertem Viertelschlag und nachfolgendem Achtelschlag sowie einem Abschluss auf Viertelpause auf der vierten Zählzeit für die Probanden anscheinend sehr ungewohnt. Die Strategien der Vereinfachung fallen sehr unterschiedlich aus, vom Spielen eines nur leicht abgewandelten bis hin zum Spielen eines ganz anderen Rhythmus.

In Bezug auf die Fehlerquellen in der Rubrik Rhythmus an sich verschwindet im Nachtest das Spielen eines falschen, jedoch gut erkennbaren anderen Rhythmus (Kat. 62) bis auf einen einzigen 
Fall. Dies belegt, dass sich die Fähigkeit zum Erfassen auch komplizierterer Rhythmen (über vier Grundschläge) innerhalb des Untersuchungszeitraumes von 20 Unterrichtswochen deutlich entwickelt hat. In Bezug auf den Einsatz und das Tempo des Rhythmus zählen im Nachtest zu den häufigsten Fehlerquellen immer noch das zu frühe oder zu späte Einsetzen sowie das Spielen in leicht bis stark schwankenden Tempo (s. Abb. 4-29 und Abb. 4-30). Das zu schnelle Rhythmusspiel ist im Nachtest deutlich häufiger anzutreffen als das zu langsame.

Die erreichten Mittelwerte der Items im Subtest B wurden mit dem Mittelwert eines zusätzlich gespielten, doppelt so langen Rhythmus über acht Grundschläge aus der Studie von Minkenberg (1991) verglichen. Nur etwa $10 \%$ der Probanden sind im Vortest in der Lage, im Call-Response mit dem Playback im Tempo von 86 bpm diesen langen Rhythmus korrekt zu reproduzieren (s. Tab. 4-8). Dabei besteht dieser lange Rhythmus aus einer Kombination der Items B 1 und B 3, welche einzeln zu $60 \%$ und $45 \%$ korrekt reproduziert werden können. Es scheint die Aussage zu bestätigen, dass beim Hörenden rhythmische Figuren durch das Bilden von chunks (Wahrnehmungseinheiten) entstehen. Die Fähigkeit, immer größere Gruppen von Elementen zusammenzufassen, wächst im Umgang mit geeignetem Stimulusmaterial kontinuierlich über die Jahre hinweg (vgl. Bruhn, 2002 ${ }^{4}$, S. 291). Im Nachtest sind bereits bis zu $20 \%$ der Probanden in der Lage, diesen langen Rhythmus korrekt zu reproduzieren (s. Tab. 4-8), doch muss die Aussage wiederholt bestätigt werden, dass das Bilden von chunks (Wahrnehmungseinheiten) zwar verbessert werden konnte, jedoch nicht an die Fähigkeiten zum Reproduzieren von kürzeren Rhythmen über vier Grundschläge heranreicht. Die Fähigkeit, immer größere Gruppen von Elementen zusammenzufassen, wächst im Umgang mit geeignetem Stimulusmaterial kontinuierlich über die Jahre hinweg (vgl. Bruhn, $2002^{4}$, S. 291), sie hat vermutlich am Ende der Klassenstufe 3 noch nicht ihren Höhepunkt erreicht.

\section{Subtest D}

Im Subtest D können im Vortest etwa 20 \% der Probanden einen Rhythmus abwandeln und diesen korrekt wiederholen (Items D 1 und D 2; s. Abb. 4-9). Im zweiten Durchgang gelingt das Abwandeln (Item D 3) bereits $25 \%$ der Probanden, das Wiederholen (Item D 4) dagegen nur 13\% der Probanden. Zählt man noch die Ergebnisse aus der Kategorie Lösung fast richtig mit kleinen Abstrichen hinzu, erhöhen sich diese Anteile um bis zu 25 \% (s. Abb. 4-37). Gemessen an den erhöhten Anforderungen dieser kreativen Aufgabenstellung fallen diese Ergebnisse recht positiv aus. Deutlich erkennbar ist die noch schwach ausgeprägte Fähigkeit zum Wiederholen des abgewandelten Rhythmus. Dies spricht dafür, dass die abgewandelten Rhythmen eher aus einem impliziten Wissensschatz hervorgebracht werden. Es bekräftigt die Erkenntnisse, dass der Musizierende für den Prozess der Improvisation durchaus auch aus dem sogenannten Unterbewussten passende Ideen generiert (vgl. Lehmann, 2011³ , S. 346). Das Repertoire der abgewandelten Rhythmen umfasst bereits 18 verschiedene Rhythmen. Darunter befinden sich zum größten Teil Rhythmen aus 
einfachen Kombinationen aus Viertel-, Achtel- und Halbeschlägen sowie in zwei Fällen Rhythmen mit Synkopen (Rhythmus 7b und 7c; s. Abb. 4-38).

Im Nachtest können in beiden Durchgängen etwa $40 \%$ der Probanden einen Rhythmus abwandeln (Item D 1 und D 3) sowie ca. 30 \% diesen auch wiederholen (Item D 2 und D 4; s. Abb. 4-10). Zählt man noch die Ergebnisse aus der Kategorie Lösung fast richtig mit kleinen Abstrichen hinzu, erhöhen sich diese Anteile um bis zu 27 \% (s. Abb. 4-37). Die Vielfalt der richtig gespielten abgewandelten Rhythmen steigt in der Anzahl von 18 auf 29 Rhythmen. Neben einfachen Kombinationen aus Viertel-, Achtel- und Halbeschlägen finden sich auch Rhythmen mit Synkope, punktierter Viertel mit nachfolgender Achtel sowie punktierter Achtel mit nachfolgender Sechzehntel (s. Abb. 4-39).

$\mathrm{Zu}$ den häufigsten Fehlerquellen im Vortest zählen in Bezug auf das Aufgabenverständnis für Item D 1 und D 3 das unzulässige Nachspielen des Calls sowie für Item D 2 und D 4 das Spielen eines neuen statt des zu wiederholenden Rhythmus (s. Abb. 4-34). Innerhalb der richtig abgewandelten und richtig wiederholten Rhythmen sind in Bezug auf den Einsatz und das Tempo des Rhythmus vor allem zu späte Einsätze sowie leichte bis starke Temposchwankungen zu beobachten (s. Abb. 4-35, Abb. 4-36). Dies liefert Indizien dafür, dass das Nachdenken über die Wahl des abgewandelten Rhythmus zu Zeitverzögerungen im Einsatz führt. Diese führen in Bezug auf das Tempo zu weiteren Schwankungen und Abweichungen. Im Nachtest bleibt die häufigste Fehlerquelle in Bezug auf den Aufgabenverständnis für Item D 2 und D 4 das Spielen eines neuen anstelle des abgewandelten Rhythmus (s. Abb. 4-34). In Bezug auf den Einsatz und das Tempo des Rhythmus nimmt der Anteil an korrekten Lösungen deutlich zu (s. Abb. 4-35 sowie Abb. 4-36).

\section{Subtest E}

Im Subtest E Rhythmen lesen und musizieren nach Noten gelingt im Vortest einem Anteil von $9 \%$ bis $25 \%$ der Probanden das fehlerfreie Musizieren vom Blatt (s. Abb. 4-9). Item E 1 und E 2 gehören dabei zu den eher leichten Items, Item E 3 und E 4 zu den schweren. Interessanterweise ist Item E 4, welches eine Viertelpause enthält, leichter als Item E 3 wiederzugeben. Zählt man die Ergebnisse aus der Kategorie Lösung fast richtig mit kleinen Abstrichen hinzu, erhöhen sich die Anteile um jeweils bis zu $5 \%$ (s. Abb. 4-46). Innerhalb der Gruppe der 32 Instrumentalschüler im zweiten oder dritten Unterrichtsjahr ist eine sehr große Leistungsspanne in diesem Subtest zu beobachten. Acht Schülern gelingt keines der vier Items, sechs Schülern gelingen bereits alle vier Items (s. Abschnitt 4.3.5).

Im Nachtest erzielen über die Hälfte der Probanden richtige Ergebnisse im Item E 1 und Item E 2. Ein Drittel der Probanden erzielen richtige Ergebnisse in den Items E 3 und E 4 (s. Abb. 4-10). Zählt man noch die Ergebnisse aus der Kategorie Lösung fast richtig mit kleinen Abstrichen hinzu, erhöhen sich diese Anteile mitunter um mehr als $10 \%$ (s. Abb. 4-46). Dies ist ein erstaunlich gutes 
Resultat. Am schwersten fällt den Probanden der Kontrollgruppe immer noch der Rhythmus im Item E 3, mit der mittig im Rhythmus liegenden Achtelgruppe (s. Abb. 4-41). Innerhalb der Gruppe der 32 Instrumentalschüler im zweiten oder dritten Unterrichtsjahr stellt sich nun die Leistungsspanne ausgeglichener dar. Nur zwei Schülern gelingt keines der vier Items, elf Schülern gelingen bereits alle vier Items (s. Abschnitt 4.3.5). Möglicherweise profitierten diese Probanden in Bezug auf das Lesen von Rhythmen von den Inhalten und Methoden des Musikunterrichts in der Schule. Diese Annahme legt der deutliche Zugewinn des Mittelwertes in diesem Subtest nach der Methode known groups im Subtest E des Nachtestes nahe (s. Abb. 4-68 und Anlage C 5 d).

$\mathrm{Zu}$ den häufigsten Fehlern in Bezug auf den Rhythmus an sich zählen im Vortest ein Schlag zu viel am Ende des Rhythmus (Kat. 52), Achtelpaare, die um einen Achtelschlag erweitert und triolisch gespielt werden (Kat. 55) sowie das schlichtweg falsche Spiel (Kat.61), aber auch das Spielen eines falschen, jedoch gut erkennbaren anderen Rhythmus (Kat. 62; s. Abb. 4-44). Im Nachtest kommt das Spielen eines Schlages zu wenig am Ende des Rhythmus (Kat. 52) hinzu. Es ändern sich die Anteile in den Fehlerkategorien. Neben dem deutlichen Zuwachs des korrekten Spielens der Rhythmen nimmt auch das Spielen eines falschen, jedoch gut erkennbaren anderen Rhythmus zu. Letzteres verweist auf das bekannte Phänomen, dass das Lesen von Notentext von Erfahrungen mit bestimmten Musiken geprägt wird und zu Erwartungshaltungen führt. Diese können unter Umständen bewirken, dass vom Musizierenden der Notentext gemäß den eigenen Erwartungen abgeändert wird (vgl. Lehmann $2002^{4}$, S. 488). Dieses Spielen eines anderen Rhythmus ist im Vortest in nur elf Fällen sowie im Nachtest in 36 Fällen zu beobachten, insbesondere beim schwersten Item E 3 (s. Tab. 4-15).

Zu den häufigsten Fehlern in Bezug auf das Tempo des Rhythmus gehört im Vortest das Spielen in einem leicht bis stark schwankendem Tempo (Kat. 60; s. Abb. 4-45). Diese Unsicherheit lässt vermuten, dass das Bilden von chunks (Lehmann \& Chaffin, 2011³ , S. 358) bzw. die Auge-HandSpanne (ebd., S. 366) noch nicht genügend entwickelt sind. Das beobachtete und grafisch dokumentierte regelmäßig stark schwankende Tempo (s. Abb. 4-43) belegt vor allem die ungenügende Augen-Hand-Spanne. Es wird von Grundschlag zu Grundschlag gelesen und anschließend umgesetzt. Die Achtelschläge gelingen zwar als kurze Schläge, danach wird aber eine erneute Lesephase eingeschoben. Eine treffendere Bezeichnung für diese Temposchwankungen könnte demzufolge lesebedingt stark schwankendes Tempo heißen. Im Nachtest gehört wiederum das Spielen in einem leicht bis stark schwankendem Tempo zu den häufigsten Fehlern bei jedoch deutlich erhöhten Anteilen an Lösungen in korrekt gespieltem Tempo. Dies spricht für eine Weiterentwicklung in Bezug auf das Bilden von chunks (Lehmann \& Chaffin, 2011³, S. 358) bzw. die Auge-Hand-Spanne (ebd., S. 366). Das beobachtete und grafisch dokumentierte regelmäßig stark schwankende Tempo ist in den relativen Anteilen stark zurückgegangen, fällt aber am stärksten im schwersten Item E 3 und besonders bei den Probanden der Kontrollgruppe auf. 
Die Möglichkeit, das Tempo zum Musizieren der notierten Rhythmen frei zu wählen, wird mit einer Bandbreite von 39 bpm bis 123 bpm genutzt. Knapp $80 \%$ der richtig gespielten Rhythmen sind im mittleren Tempobereich zwischen $71 \mathrm{bpm}$ bis $100 \mathrm{bpm}$ angesiedelt, gut $10 \%$ im langsameren sowie knapp $10 \%$ im schnelleren Tempobereich (s. Abb. 4-47). Der hohe Anteil an korrekten Lösungen im mittleren Tempobereich kann dadurch bedingt sein, dass die Items aus Subtest B und $\mathrm{D}$, welche beide mit einem Playback im Tempo von $86 \mathrm{bpm}$ durchgeführt werden, ein ungefähres Tempo vorgeben. Da sich Subtest E unmittelbar an das Video anschließt, könnte das Tempo intuitiv beibehalten worden sein. Die bei Minkenberg zitierte günstigste Tempospanne für das Reproduzieren von Rhythmen mit 112 bpm bis 136 bpm (Christensen, 1938, S. 44, zitiert nach Minkenberg, 1991, S. 37) bzw. der Hinweis, nicht unter 80 bpm zu gehen (Tee, 1983, ohne Seitenangabe sowie Pflederer, 1986, S. 29, beide zitiert nach ebd.; s. Abschnitt 3.4.4), kann für das Lesen und Musizieren für Rhythmen nicht bestätigt werden. Im Nachtest erweitert sich die Bandbreite für das gewählte Tempo auf 37 bpm bis 133 bpm (s. Abb. 4-48). Genau $88 \%$ der richtig gespielten Rhythmen sind im mittleren Tempobereich zwischen 71 bpm bis 100 bpm angesiedelt, knapp $5 \%$ im langsameren sowie etwa $7 \%$ im schnelleren Tempobereich. Damit sind die Anteile in den Randbereichen geringer geworden. Die erstaunliche Spanne, besonders in Bezug auf die extrem langsamen Tempi unter 50 bpm, signalisiert, auch im Rhythmus-Lehrgang eine größere Tempobandbreite anzubieten (s. Abschnitt 5.4).

Es wird beobachtet, dass bei einigen Probanden das absolute Tempo der einzelnen Items in starkem Maße ansteigt bzw. abfällt. Für jeden einzelnen Probanden werden deshalb Temposteigerungen bzw. -verluste zwischen den korrekt gespielten einzelnen Items nur bis zu einer Differenz von 15 bpm als noch akzeptabel gewertet. Ein im absoluten Tempo pro Rhythmus so stark schwankendes Rhythmusspiel wäre nicht ensemblefähig (s. Abschnitt 4.3.5). Größere Differenzen werden als Fehlerkategorie 35 abgewertet (s. Abb. 4-45).

Im parallel geführten Untertest $e$ Rhythmen lesen und musizieren nach Zeichen ist im Vortest zu beobachten, dass bis zu doppelt so große Anteile an richtigen Lösungen erbracht werden können wie im Subtest E beim Musizieren nach Noten (s. Tab. 4-27). Dies spricht dafür, dass die Hinzunahme einer ikonischen Notation, wie im Treatment geschehen, eine gewinnbringende Entscheidung ist. Die indirekte Information über die Balken- bzw. Strichlängen ermöglicht, implizites Vorwissen zu aktivieren und eine Brücke zur symbolischen Ebene der traditionellen Notenschrift zu schlagen. Es ist eine Bekräftigung für das im Treatment realisierte Prinzip der dreifachen Wissensdarbietung (Bruner, 1974, S. 48-53; s. Abschnitt 3.3.5). Im Nachtest ist zu beobachten, dass nur noch vereinzelt leicht höhere Anteile an richtigen Lösungen beim Rhythmen lesen und musizieren nach Zeichen erbracht werden können als beim Musizieren nach Noten (s. Tab. 4-28). Dies spricht dafür, dass die Hinzunahme einer ikonischen Notation im Treatment für die ersten Lernphasen im Rhythmus-Lehrgang sinnvoll ist, danach aber weggelassen werden kann. Die Mittlerfunktion hat 
nach den 20 Unterrichtswochen nur noch eine Berechtigung, wenn neue rhythmische Sachverhalte eingeführt werden.

\section{Subtest F}

Im Subtest F Noten musizieren und aufschreiben mit Noten werden im Vortest nur von einigen wenigen Probanden richtige Ergebnisse mit Anteilen zwischen $2 \%$ und $5 \%$ erzielt (s. Abb. 4-9). Zählt man noch die Ergebnisse aus der Kategorie Lösung fast richtig mit kleinen Abstrichen hinzu, erhöhen sich diese Anteile lediglich auf etwa 10 \% (s. Abb. 4-15). In der Kategorie Lösung fast richtig mit kleinen Abstrichen ist das Notieren im figuralen Typus eingeordnet. Lösungen in dieser Fehlerkategorie weisen beim kritischen Element (Bamberger, 1991, S. 46) das Symbol für einen Achtel- statt für einen Viertelschlag auf. Korrekte sowie fast richtige Ergebnisse entstammen zum größten Teil aus der Gruppe der Schülerinnen und Schüler mit außerschulischem Instrumentalunterricht im zweiten oder dritten Unterrichtsjahr. Selbst 23 dieser insgesamt 32 Schüler gelingt im Vortest jedoch noch keines der vier Items. Dies lässt vermuten, dass im Instrumentalunterricht wenig Augenmerk auf das Notieren von Rhythmen gelegt wird. Damit wird die Mahnung bestätigt, „alle Aspekte des musikalischen Wissens, auch die des Lesens und Schreibens von Musik, durch Übung zu entwickeln, um eine optimale Koordination zwischen den verschiedenen musikalischen Bereichen zu erreichen und eine optimale Leistungsfähigkeit zu entwickeln“ (Davidson und Scripp, 1990, zitiert nach Gembris, $2013^{4}$, S. 246). Häufige Fehler treten aufgrund der geringen Anzahl von angebotenen Lösungen nicht zutage.

Im Nachtest werden von vielen Probanden mit und ohne Instrumentalunterricht richtige Ergebnisse mit Anteilen zwischen $18 \%$ und $40 \%$ erzielt (s. Abb. 4-10). Dies ist ein erfreuliches Ergebnis, besonders mit Blick auf die entsprechenden Ergebnisse aus der 2. Erhebung, bei der die Anteile an korrekten Lösungen deutlich geringer ausfallen (s. Abschnitt 4.5). Zählt man noch die Ergebnisse aus der Kategorie Lösung fast richtig mit kleinen Abstrichen hinzu, erhöhen sich diese Anteile auf etwa $40 \%$ bis $60 \%$ (s. Abb. 4-15). Von den 32 Instrumentalschülern im zweiten oder dritten Unterrichtsjahr gelingt nun drei Vierteln von ihnen mindestens eines der vier Items, zehn Schülern bereits alle vier Items. Möglicherweise haben diese Probanden in Bezug auf das Schreiben von Rhythmen von den Inhalten und Methoden des Musikunterrichts in der Schule profitiert. Dies belegt der deutliche Zugewinn des Mittelwertes im Subtest F des Nachtestes nach der Methode known groups nahe (s. Abb. 4-68 und Anlage C 5 d).

Im parallel geführten Untertest $f$ Rhythmen musizieren und aufschreiben mit Zeichen ist im Vortest zu beobachten, dass alle beobachteten Typen des Darstellens von Rhythmen mit Zeichen nicht übereinstimmend, zählend, figural, metrisch - von den Probanden genutzt werden (Bamberger, 1982, 1991; Hildebrandt, 1987; Upitis, 1987; Werner, 2016; s. Tab. 4-23). Die als korrekt zu wertenden Lösungen im metrischen Typus nehmen einen Anteil zwischen $5 \%$ und $18 \%$ ein, die Lösungen im figuralen Typus zwischen $18 \%$ und $39 \%$. Dies belegt, dass das Darstellen von 
Rhythmen mit Zeichen auch ohne ausdrückliche Unterweisung bei Kindern durch Akkulturation in Symbolsysteme angelegt ist (Gardner, 1993, zitiert nach Gembris, 20134 , S. 240). Die Anzahl der korrekten Lösungen beim Aufschreiben von Rhythmen mit Zeichen im metrischen Typus fallen um ein Vielfaches höher aus als beim Aufschreiben mit Noten (s. Tab. 4-24). Dies ist eine doppelte Bekräftigung für das im Treatment realisierte Prinzip der dreifachen Wissensdarbietung (Bruner, 1974, S. 48-53; s. Abschnitt 3.3.5). Im Nachtest ist zu beobachten, dass immer noch alle beobachteten Typen des Darstellens von Rhythmen mit Zeichen genutzt werden. Die als korrekt zu wertenden Lösungen im metrischen Typus nehmen einen Anteil zwischen $16 \%$ und $47 \%$ ein, die Lösungen im figuralen Typus zwischen $7 \%$ und $30 \%$ (s. Tab. 4-25). Der Anteil an Lösungen im metrischen Typus ist damit deutlich angestiegen. Vergleicht man jedoch den Anteil an korrekten Lösungen im metrischen Typus mit Zeichen mit den jeweiligen Anteilen an korrekten Lösungen im Subtest $\mathrm{F}$ mit Noten, so ist nur noch in wenigen Fällen ein Vorsprung zu verzeichnen (s. Tab. 4-26). Die Arbeit mit ikonischer Notation verschafft zu diesem Zeitpunkt keinen Lernvorteil mehr.

\section{Subtest C}

Im Subtest C Rhythmussprache anwenden werden im Vortest nur von wenigen Probanden Lösungen angeboten. Dies verwundert nicht, da das Erlernen einer Rhythmussprache prinzipiell im Freistaat Sachsen (wie auch in den meisten anderen Bundesländern) nicht Lehrplaninhalt ist. Richtige Lösungen werden von Probanden aus der Gruppe von Schülern mit außerschulischem Instrumentalunterricht sowie aus den Klassen K 1 und K 2 erbracht. Letztere hatten bereits mehrere Wochen vor dem Vortest begonnen, eine Rhythmussprache zu erlernen. Der Anteil an korrekten Lösungen liegt zwischen $5 \%$ und $13 \%$ im lesenden (Item C 1 und $\mathrm{C} 2$ ) sowie bei etwa $3 \%$ im schreibenden Bereich (Item C 3 und C 4; s. Abb. 4-9). Zählt man noch die Ergebnisse aus der Kategorie Lösung fast richtig mit kleinen Abstrichen hinzu, erhöht sich dieser Anteil auf etwa $20 \%$ im lesenden sowie auf fast $10 \%$ im schreibenden Bereich (ec 1 und ec 3 s. Abb. 4-53; fc 2 und fc 3 s. Abb. 4-56). In der Gruppe der insgesamt 32 Schülerinnen und Schüler, die bereits zwei bis drei Jahre Instrumentalunterricht erhalten, gelingt 22 Probanden keines der vier Items. Diese Beobachtung zeigt, dass im Instrumentalunterricht das Erlernen einer Rhythmussprache vermutlich eine zu vernachlässigende Größe spielt. Alle zehn Instrumentalschüler, welche in den Items teilweise bzw. vollständig korrekte Leistungen erbringen, besuchten zusätzlich zum Instrumentalunterricht den Theorieunterricht ihrer Musikschule (s. Abschnitt 4.3.6).

Im Nachtest kann eine große Gruppe von Probanden Lösungen erbringen, da sowohl alle Klassen der Untersuchungsgruppe und erfreulicherweise die Klassen K 1, K 2, K 3 und K 4 eine Rhythmussprache erlernten. Kenntnisse und Fähigkeiten im Umgang mit Rhythmussilben besitzt gut ein Drittel der Probanden. Die Anteile an richtigen Lösungen liegen zwischen $34 \%$ und $52 \%$ (s. Abb. 4-10). Zählt man noch die Ergebnisse aus der Kategorie Lösung fast richtig mit kleinen Abstrichen hinzu, erhöhen sich diese Anteile auf etwa $50 \%$ bis $60 \%$ im lesenden sowie auf über 
$50 \%$ im schreibenden Bereich (ec 1 und ec 3 s. Abb. 4-53; fc 2 und fc 3 s. Abb. 4-56). In der Gruppe der 32 Instrumentalschüler gelingt vier Schülern keines der vier Items, zwölf Schülern gelingen alle vier Items. Viele dieser Schüler konnten von den Inhalten des regulären Musikunterrichts sowie des Rhythmus-Lehrgangs profitieren. Dies legt der deutliche Zugewinn des Mittelwertes im Subtest C des Nachtestes der Musikschüler gegenüber den anderen Schülern sowohl in der absoluten Höhe als auch in der Differenz zum Mittelwert nach der Methode known groups nahe (s. Abb. 4-68 und Anlage C 5 d).

Für die Items, die dem Untertest ec Lesen und musizieren von Rhythmen nach Noten und sprechen von Rhythmussilben entstammen, wird beobachtet, dass es einige Ergebnisse gibt, bei denen ein anderer Rhythmus als der notierte gespielt wird (s. Abb. 4-51). Dies bestätigt ebenfalls die Erkenntnis, wie bereits im Subtest E erwähnt, dass das Lesen von Notentext von Erfahrungen mit bestimmten Musiken geprägt wird und zu Erwartungshaltungen führt. Diese können unter Umständen bewirken, dass vom Musizierenden der Notentext gemäß den eigenen Erwartungen abgeändert wird (vgl. Lehmann $2002^{4}$, S. 488). Insgesamt muss festgestellt werden, dass das laute Mitsprechen der Rhythmussilben zwar einem Teil der Probanden hilft, die Rhythmen gleichmäßiger zu musizieren, jedoch andere Probanden eher irritiert. Die arithmetisch gemittelte Anzahl der richtigen Lösungen steigt im Nachtest nur im Item C 2 (ec 3) und nur in der Kontrollgruppe leicht an (s. Abb. 4-52).

Für die Items, die dem Untertest $f c$ Rhythmen musizieren und aufschreiben mit Rhythmussilben entstammen, wird beobachtet, dass häufig einmalig eine Silbe fehlerhaft notiert wird (s. Abb. 4-55). Dies betrifft stets die Silbe für einen Viertelschlag, der auf zwei Achtelschläge folgt (s. kritisches Element Abb. 2-13). Damit ist eine Analogie zum Notieren im figuralen Typus, jedoch mit Silben statt mit Zeichen oder Noten festzustellen (s. Abb. 4-14). Dies ist bedeutend häufiger in der Kontrollgruppe zu beobachten.

\subsubsection{Ergebnisse aus den zusätzlichen Subtests G, H und I}

Die Ergebnisse der Itemgruppe G Play together lassen erkennen, dass es Schülern und Schülerinnen in der Klassenstufe 3 relativ leicht fällt, in einem zweistimmig angelegten Musizierteil sowohl zuerst mit der eigenen Stimme (Item G 1) als auch als zweiter Spieler (Item G 3) pünktlich einzusetzen. Beim zweistimmigen Rhythmusspiel in den Items G 2 und G 4 wird sehr häufig der Rhythmus des Kindes im Video übernommen. Dies belegt, dass das zweistimmige Spielen von Rhythmen einen hohen Grad der Verinnerlichung des eigenen Rhythmus bedarf (mentale Repräsentation). Es muss das Wirken der Spiegelneuronen unterdrückt werden, bei gleichzeitigem Achten auf den Mitspielenden im Interesse eines koordinierten Zusammenspiels.

Die Leistungsdifferenz zwischen beiden Gruppen kann in dem Subtest G mit einem Wert von $d=0.18$ zugunsten der Untersuchungsgruppe beziffert werden, dies nähert sich dem Wert für einen kleinem Effekt an (s. Anlage C 5 f-g). Die erzielten Leistungen stehen in einer Korrelation von 
$r=.61$ zum Gesamtpunktwert (s. Tab. 4-29). Mit dem Ansteigen der Leistungen in den sechs verschiedenen Subtests (A-F) steigen auch die Voraussetzungen für ein gemeinsames Rhythmusspielen zwischen zwei oder mehr Musizierenden. Somit wird parallel zur Förderung rhythmischer Kompetenz in den sechs dargestellten Aufgabenstellungen (s. Abb. 2-7) eine wichtige Grundlage für das gelingende gemeinsame Musizieren im Musikunterricht der Grundschule gelegt. Damit wird belegt, dass die Begriffserweiterung für rhythmische Kompetenz von den bisherigen sechs Stufen A bis F (Jank, 2013², S. 129) um die Stufe G Zusammenspiel und Koordination mit einem Mitmusizierenden (im Test als Play together) als sinnvoll und einen Bogen spannend zwischen dem individuellen Aspekt rhythmischer Kompetenz in den Stufen A-F sowie dem sozialen Aspekt des Musizierens betrachtet werden kann (s. Abb. 2-7).

Die Ergebnisse des Subtests H Wozu Notenschrift? belegen, dass die Probanden sehr wohl wissen, wozu Notenschrift genutzt werden kann. Jedoch stellt nur ein kleiner Teil der Befragten einen Bezug zur eigenen Verwendung von Notenschrift beim Musizieren her, was die Frage „Wozu kann die Notenschrift für einen Grundschüler, z. B. dich, wichtig sein?" nahe legen soll. Möglicherweise ist dies dem häufig anzutreffenden methodischen Ansatz geschuldet, erst das Lesen und dann das Schreiben von Noten zu nutzen. Wie eingangs bei Bamberger geschildert, löste in ihrer Arbeit mit Kindern das gedankliche Festhalten eines Rhythmus den Anreiz für den Umgang mit Notation aus (bei Bamberger mit Zeichen; s. Abschnitt 2.2.5). Im Musiknotieren wider das Vergessen liegt möglicherweise ein überzeugenderes Potenzial als im Lesen von Musik, welche andere Menschen notiert haben. Zusätzlich könnte möglicherweise ein zu schnelles Auswendigspielen oder das Imitieren der Spielaktion des Lehrers das Bewusstsein für die Notwendigkeit des Lesens von Notiertem überlagern. Im Treatment liegt in den Lernsequenzen 1 und 4 der Schwerpunkt auf dem Lesen und Übertragen in Strichnotation sowie in traditionelle Notenschrift. In den Lernsequenzen 2 und 5 liegt der Schwerpunkt beim Aufschreiben der selbst erfundenen Rhythmen zum Entwickeln eines Spielstückes (s. Abschnitt 3.3.2). Dennoch ist das Bewusstsein für eine persönliche Bedeutung der Fähigkeiten zum Umgang mit Notenschrift bei den Probanden der Untersuchungsgruppe nur unerheblich höher als in der Kontrollgruppe (s. Abschnitt 4.6.4). Für den Musikunterricht in der Grundschule hieße das, beiden Funktionen, dem Schreiben und dem Lesen, beim Gebrauch von Notation im Unterrichtsgeschehen mehr Raum zu geben und zu vor allem zu reflektieren.

Die Ergebnisse des Subtests I Vergleichendes Hören belegen zwei Sachverhalte: Zum einen fallen die Entwicklungssprünge in Bezug auf das hörende Unterscheiden nach 20 Unterrichtswochen relativ gering aus (s. Tab. 4-30). Dies kann darauf zurückzuführen sein, dass es leichter ist, etwas Neues zu erlernen als bereits Erlerntes zu vervollkommnen (vgl. Lehmann \& Oerter, 2011 S. 118). Zum anderen lässt sich zeigen, dass die Fähigkeit zum Hören und Vergleichen von Rhythmuspaaren sowie die Fähigkeiten in den anderen sechs Subtests nicht sehr hoch korrelieren. 
Mit einem Korrelationswert von $r=.34$ ist der Zusammenhang nicht sehr stark ausgeprägt (s. Tab. 4-31).

\subsubsection{Vergleich der Mittelwertunterschiede und Effektstärken}

Die Mittelwerte aus Vor- und Nachtest sowie deren Differenzen wurden im Kapitel 4 dargestellt. Mit diesen Werten soll nun die eingangs gestellte Hypothese verifiziert werden:

(3) Welcher messbare Vorsprung beim Erwerb rhythmischer Kompetenz resultiert aus der Teilnahme am Treatment gegenüber der Teilnahme am regulären Musikunterricht?

Hypothese

$\mathrm{H}_{0}$ Durch die Teilnahme am Treatment, welches in den regulären Musikunterricht integriert ist, gewinnen Schüler der Klassenstufe 3 einen Lernzuwachs an rhythmischer Kompetenz mit einer eher kleinen Effektstärke $(d<0.5)$ gegenüber Schülern, welche regulären Musikunterricht erhalten.

$\mathrm{H}_{1}$ Durch die Teilnahme am Treatment, welches in den regulären Musikunterricht integriert ist, gewinnen Schüler der Klassenstufe 3 einen Lernzuwachs an rhythmischer Kompetenz mit einer mittleren Effektstärke $(d \geq 0.5)$ gegenüber Schülern, welche nur regulären Musikunterricht erhalten.

$\overline{\mathrm{x}}_{\mathrm{UG}}>\overline{\mathrm{x}}_{\mathrm{KG}}$ und $\mathrm{d} \geq 0.5$

Der Entwicklungsstand zu Beginn der Klassenstufe 3 ist im Vortest in beiden Gruppen (UG / KG) mit Mittelwerten um 4.0 für die Gesamtpunktwertung relativ ähnlich ausgeprägt. Am Ende der Klassenstufe 3 (Nachtest) differieren die Entwicklungsstände in der Gesamtpunktwertung sowie in den Subtests C, E und F sehr stark (s. Die Differenz der Mittelwerte der Gesamtpunktewertung aus Vor- und Nachtest fällt bei ihnen mehr als doppelt so hoch wie in der Kontrollgruppe aus. Die berechnete Effektstärke $d$ weist mit einem Wert von 1.36 einen sehr großen Effekt aus (s. Tab. 4-19).

Tab. 4-18). Die großen Entwicklungssprünge in den Mittelwerten der Probanden der Untersuchungsgruppe schlagen sich in diesen Subtests in mittleren und großen Effektstärken nieder (s. Anlage C 5 c). In den anderen drei Subtests A, B und D finden auch Entwicklungen statt. Jedoch sind die Unterschiede zwischen beiden Gruppen nur geringfügig. Dies spiegelt sich im Subtest D in einer kleinen Effektstärke wider, in den Subtests A und B kann kein Effekt nachgewiesen werden.

Da die Effektstärken für drei Subtests sowie für die Gesamtpunktwertung deutlich über $d=0.5$ liegen, muss Hypothese $\mathrm{H}_{0}$ verworfen und Hypothese $\mathrm{H}_{1}$ bestätigt werden. Es wird damit nachgewiesen, dass es einen deutlich messbaren Vorsprung durch die Teilnahme am Treatment gibt. Dieser Vorsprung entspricht in Bezug auf die Gesamtpunktwertung einer Effektstärke $d$ in Höhe von 1.36 und somit einem sehr großen Effekt. Zusätzlich lässt sich belegen, dass es über alle Aus- 
gangspositionen in den Mittelwerten der Subtests A-F hinweg größere Entwicklungssprünge bei den Probanden der Untersuchungsgruppe gibt (s. Abb. 4-59).

Die verschieden hohen Effektstärken lassen eine besondere Beziehung der sechs Subtests zueinander vermuten. Die Mittelwerte der Subtests A, B und D sowie C, E und F korrelieren jeweils am stärksten untereinander (s. Tab. 4-20). Möglicherweise handelt es sich bei den zugrunde liegenden operativen Fähigkeiten um verschieden stark förderbare Aspekte rhythmischer Kompetenz. In den Subtests A, B und D sind bereits im Vortest gut entwickelte Fähigkeiten zu beobachten (s. Abb. 4-9). Im Nachtest muss festgestellt werden, dass der Zuwachs an Fähigkeiten in diesen Subtests eher gering ausfällt (s. Anlage C 5 d). Dafür wird in den Subtests C, E und F ein enorm großer Zuwachs verzeichnet. Die Weiterentwicklung und Förderung der Fähigkeiten in diesen sechs Subtests unterscheidet sich nur in den Subtests, in denen mindestens eine kleine Effektstärke durch das Treatment erzielt wird.

Die Teilnahme am Treatment zeigt in Bezug auf die Weiterentwicklung der Fähigkeiten in den Subtests A Grundschlag und Metrum darstellen sowie B Rhythmen nachspielen keine messbare Wirkung. Dies verwundert, da jede Stunde mit einem Lernsequenzanteil ein Warm up 1 / 2 enthält. Jedoch entfalten diese Übungen in den anderen Subtests ihre Wirkung. Dass die Fähigkeit, einen Rhythmus abzuwandeln (Subtest D), bei den Probanden der Untersuchungsgruppe mit Treatment im Nachtest besser als bei den Probanden der Kontrollgruppe ohne Treatment entwickelt ist, scheint auf den Aufbau eines Rhythmusrepertoires im Subtest B zu fußen. Dies belegt das bessere Abschneiden der Probanden der Untersuchungsgruppe beim Wiederholen des abgewandelten Rhythmus. Diese wird sehr wahrscheinlich durch die andere Qualität der mentalen Repräsentationen der Rhythmen ermöglicht (vgl. Gruhn, 2003, S. 110).

Die schwerere Zugänglichkeit für eine Förderung der Subtests A und B kann aber auch ihren Ursprung im Beginnen des entsprechenden Lernens haben. So scheint es schwieriger zu sein, Fähigkeiten zu vervollkommnen bzw. zu verfeinern als neue Fähigkeiten aufzubauen. Dieser Zusammenhang von Übung und Leistung wird von Lehmann \& Oerter einer mathematischen Potenzfunktion gleichgesetzt. Anfänglich kann mit relativ wenig Übung relativ viel Fortschritt erzielt werden. Bei der Vervollkommnung der angelegten Fähigkeiten muss für immer kleiner werdende Leistungsverbesserungen immer mehr Übung aufgewandt werden (vgl. Lehmann \& Oerter, 201133, S. 118).

Es ist aber auch nicht auszuschließen, das für die schwerer förderbaren Fähigkeiten in den Subtests A und B eventuell ein natürlich begrenztes aptitude (Gordon, 2012 ${ }^{8}$, S. 43-57; TappertSüberkrüb, 1999, S. 78) bzw. ein nicht mehr geöffnetes optimales Förderfenster verantwortlich sein könnte. Wenn dies der Fall wäre, müsste die Förderung dieser Fähigkeiten im Musikunterricht deutlich früher einsetzen, also bereits in den Klassenstufen 1 und 2. Dagegen spricht jedoch die Feststellung, dass Kinder bis zum 10. Lebensjahr musikalische Grundkompetenzen ausbilden (Se- 
rafine, 1988, zitiert nach Gembris, $2013^{4}$, S. 257) und sich innerhalb des Untersuchungszeitraumes von 20 Unterrichtswochen die Fähigkeiten messbar weiterentwickelten (s. Abb. 4-22 und Abb. 4-26). Diese Entwicklungen sind jedoch unabhängig von der Zugehörigkeit in Untersuchungs- bzw. Kontrollgruppe. Wichtig scheint demnach also prinzipiell eine musikalische Förderung sowohl im Musikunterricht der Grundschule, in diesem Falle im Rahmen von zwei Musikstunden pro Woche, als auch im privaten Bereich in der Beschäftigung mit Musik nach den eigenen Vorlieben.

Die Beobachtungen zu den unterschiedlichen Auswirkungen einer Förderung durch regulären Musikunterricht im Allgemeinen als auch durch den Rhythmus-Lehrgang im Besonderen auf die Stufen A bis F lassen die Frage zu, ob das zugrunde gelegte Pyramidenmodell für die Entwicklung rhythmischer Kompetenz in der Klassenstufe 3 tragfähig ist. Dies wird im folgenden Abschnitt zur Beurteilung des Testmodells eingehender betrachtet.

\subsection{Beurteilung von Testentwicklung, Testmodell und Testgüte}

\section{Beurteilung der Testentwicklung}

Für die Testentwicklung kann festgestellt werden, dass das Gesamtpaket aus Einzelverfahren mit mündlichem Test (Video und Musizierteil mit Musiziervorlagen) sowie aus Gruppenverfahren mit schriftlichem Test von den Probanden in beiden Gruppen gut angenommen wurde. Bewährt haben sich die Start-Items in den Subtests A und B. Ebenso erwiesen sich die Hilfsmittel Demonstration (Subtests A und B) sowie grafische Veranschaulichung (Subtests A und D) als hilfreich. Die pantomimische Unterstützung in den Items von Subtest A ermöglichte ein intuitives und auf Imitation angelegtes Erfassen der Aufgabestellung, deren begriffliche Grundlagen verbal in dieser Altersstufe nicht gegeben sind. Lediglich Item A 3 ließ erkennen, dass der Übergang innerhalb eines Subtests von Items mit Demonstration (a 5, A 1 und A 2) zu Items nur mit verbaler Erklärung und grafischer Anschauung (A 3 und A 4) nicht klar genug herausgearbeitet war. Das Musizieren zum Playback funktionierte prinzipiell sehr gut.

Es konnte mit diesem Messinstrument bei den 149 Probanden sowohl das Ausgangsniveau als auch ein fortgeschrittenes Niveau nach 20 Unterrichtswochen analysiert werden. Drei Probanden erzielten im Nachtest keinen Wertungspunkt, nur ein Proband erreichte die volle Punktzahl in der Gesamtpunktwertung. Damit ist der Test nicht zu leicht konstruiert und kann auch in den Randbereichen messen.

Für die Rohdaten des Nachtestes kann eine Normalverteilung nachgewiesen werden. Dennoch werden in den Histogrammen Schiefe (Vortest) sowie Gipfel (Nachtest) abgebildet (s. Anlage C 2). Das Histogramm im Vortest bildet eine Rechtsschiefe ab (positive Schiefe). Dies ist ein Anzeichen dafür, dass der Test zu Beginn der Klassenstufe 3 für die meisten Probanden zu schwer ist (vgl. 
Bühner, 2006, S. 51). Die Daten im Nachtest bilden eine zwei- bzw. mehrgipflige Verteilungen ab. Eine Interpretation des Statistikentwicklers StatSoft lautet:

„Die Multimodalität einer Verteilung von Stichprobenwerten ist oft ein starker Hinweis darauf, dass die jeweilige Variable in der Grundgesamtheit nicht normalverteilt ist. Die mehrgipflige Verteilungsform kann wichtige Informationen über die Natur der untersuchten Variablen (d. h. die gemessene Qualität) liefern. Um ein Beispiel zu nennen, wenn die Variable eine berichtete Vorliebe oder Neigung darstellt, dann könnte die Multimodalität auf eine Polarisierung hinweisen. Die Multimodalität zeigt jedoch oft an, dass die Stichprobe inhomogen ist und die Beobachtungswerte zu mehreren sich gegenseitig überlappenden Verteilungen gehören.“ (vgl. https://www.statsoft.de/glossary/M/MultimodalDistribution.htm. [Zuletzt aufgerufen am 22.08.16.]).

Möglicherweise könnte rhythmische Kompetenz als Teil einer allgemeinen musikalischen Kompetenz einem angeborenem aptitude (Gordon, $2012^{8}$, S. 43-57; Tappert-Süberkrüb, 1999, S. 78) stärker verhaftet sein, als von der Autorin angenommen. Dies würde Gordons Theorie der Verfestigung mit dem 9. Lebensjahr bestätigen. In diesem Alter befanden sich bereits die meisten Schülerinnen und Schüler (s. Abb. 4-4). Im Umkehrschluss würde der zum Einsatz gekommene Test damit in gewissem Sinne Begabung testen. Der Einsatz eines Paralleltests - z. B. eines Musikalitätstests - wäre aufschlussreich.

Es wäre aber auch möglich, dass das Ziehen der Stichproben als Klumpenstichproben (viele Probanden aus der jeweils gleichen Klasse) kombiniert mit einer persönlichen und freiwilligen Zustimmungserklärung diese Gipfelausformung beeinflusste. Dieser Umstand könnte eine mögliche Störvariable Vorliebe / Abneigung zum Fach Musik eingebracht haben. Vermutlich haben sich zur Teilnahme am Test nur Schülerinnen und Schüler bereit erklärt, die das Fach Musik mögen bzw. ihm nicht abgeneigt gegenüber stehen.

\section{Beurteilung des Testmodells}

Für das Testmodell ist folgender Nachweis zu erbringen:

(4) In welchem Maße bilden die erhobenen Daten das Modell der Pyramide des Aufbauens rhythmischer Fähigkeiten ab? Die Itemschwierigkeiten des Leistungstestes (Nachtest) sollen auf jeder höheren Stufe ansteigen und folgender Gleichung entsprechen:

$$
\bar{x}_{S T_{-} A}<\bar{x}_{S T_{-} B}<\bar{x}_{S T_{-} C}<\bar{x}_{S T_{-} D}<\bar{x}_{S T_{-} E}<\bar{x}_{S T_{-} F}
$$

Das Modell (s. Abb. 3-15) wird mit den erreichten Mittelwerten nur teilweise abgebildet. In der Untersuchungsgruppe fallen alle Werte zu hoch aus. Besonders trifft dies auf die Ergebnisse in den Subtests E, F und C zu (s. Abb. 4-67). In der Kontrollgruppe fallen die Mittelwerte für Subtest A, B und D leicht zu niedrig aus. Für Subtest $C$ fallen sie ausgesprochen niedrig aus, was besonders auf die fehlenden Leistungen der Klassen K 5 und K 6 zurückzuführen ist, welche keine Rhythmussprache erlernten. Die Mittelwerte für Subtest E und F fallen leicht zu hoch aus. Dennoch bilden 
die erreichten Mittelwerte in der Kontrollgruppe insgesamt das Pyramidenmodell mit abfallenden Mittelwerten annähernd ab. Die erreichten Mittelwerte in der Untersuchungsgruppe entsprechen mit Ausnahme des Wertes für Subtest D eher einem ausgeglichenen Niveau. Dies widerspricht dem Gedanken an das Ansteigen der Schwierigkeit von Stufe zu Stufe gemäß dem Pyramidenmodell.

Das Herausfallen der Mittelwerte für Subtest D aus diesem ansonsten ausgeglichenen Niveau der Mittelwerte der Probanden der Untersuchungsgruppe im Nachtest kann verschiedene Gründe haben. Zum einen sind die Items in diesem Subtest von einem hohen Improvisationscharakter bei trotzdem gefordertem pünktlichem Rhythmuseinsatz geprägt. Beides zusammen bildet eine sehr große Herausforderung. Zum anderen wird im Abschnitt 5.4 dargestellt, dass die für diesen Subtest benötigten Fähigkeiten im Rhythmus-Lehrgang deutlich weniger gefördert werden können als andere Fähigkeiten. Dies ist der Tatsache geschuldet, dass diese kreative Fähigkeit sehr schwer im Klassenverband förderbar ist. So darf gefragt werden, ob Gordons prinzipielle Einordnung des kreativen Umgangs mit Rhythmen nicht doch eher in die nächst höhere Stufe des Übertragenden Lernens gehört (s. Gordon, $2012^{8}$, s. 98; Tappert-Süberkrüb, 1999, S. 79) und damit die Spitze des Pyramidenmodells bilden müsste. Insgesamt sind jedoch die erwarteten Mittelwerte für diesen Subtest leicht übertroffen worden (s. Abb. 4-67), der Lernzuwachs in beiden Gruppen unterscheidet sich um eine kleine Effektstärke (s.

Tab. 4-12).

Die Interpretation der unterschiedlichen Mittelwerte in beiden Gruppen im Nachtest legt folgenden Schluss nahe: Die Mittelwerte in der Kontrollgruppe entsprechen annähernd dem ansteigenden Niveau des Pyramidenmodells und repräsentieren damit den natürlicher Weise gegebenen Entwicklungsstand von Schülern in der Klassenstufe 3. Die Mittelwerte in der Untersuchungsgruppe zeigen ein annähernd gleich stark ausgebildetes Leistungsbild, zumindest in den Subtests A, B, C, E und F. Sie bilden damit eine homogen geförderte Kompetenz ab und repräsentieren das Potenzial einer gelungenen Förderung rhythmischer Kompetenz aus einer Kombination von Komponenten aus der Music Learning Theory Gordons $\left(2012^{8}\right)$, der dreifachen Wissensdarbietung nach Bruner (1974), dem Konzept der natürlichen Differenzierung nach Krauthausen und Scherer (2010) sowie einem Wechsel der Sozialformen zwischen Plenum und Gruppenarbeit.

Obwohl es im Grunde genommen erfreulich ist, dass einige Mittelwerte zu hoch ausfallen, bleibt jedoch im Interesse einer Vergewisserung für die Testgestaltung nach Gründen dafür zu fragen. Die unerwartet hohen Mittelwerte in den Subtests E, F und C in der Untersuchungsgruppe scheinen auf der grundlegend anderen Art und Weise der Vermittlung der Rhythmussprache zu beruhen. Diese ist durch das Erlernen im Call-Response-Prinzip im Warm up 2 sowie die kontinuierliche Anwendung in den Workshops geprägt. Belegt wird dies durch zwei vertiefende Beobachtungen.

Die Mittelwerte der einzelnen Items im Subtest E Rhythmen lesen und musizieren fallen bei den Probanden der Kontrollgruppe sehr unterschiedlich aus, besonders Item E 3 fällt aus dem Rahmen (s. Abb. 4-41), obwohl dieses Item nur aus einer simplen Folge aus Achtel- und Viertelschlägen 
besteht. Bei den Probanden der Untersuchungsgruppe folgen die Mittelwerte aller vier Items einer konstant zunehmenden Itemschwierigkeit im Subtest E. Durch das Erlernen der Rhythmussprache in o. g. Weise führen unterschiedliche Anordnungen von Achtel- und Viertelschlägen im Rhythmus zwar ebenfalls zu Niveauunterschieden, diese sind aber relativ ausgeglichen. Im Subtest $C$ Rhythmussprache anwenden werden die Probanden aufgefordert, die gleichen Rhythmen (wie im Subtest E) noch einmal zu lesen und zu musizieren und die Silben der Rhythmussprache mitzusprechen. Nun steigt die Leistung für das Item ec 3 (analog zu Item E 3) in der Kontrollgruppe sprunghaft an (s. Abb. 4-52). Dies belegt, dass dieses Item nicht in dem Maße schwieriger ist, wie die Ergebnisse für Item E 3 in der Kontrollgruppe suggerieren. Dies scheint ein Indiz dafür zu sein, dass die Probanden der Kontrollgruppe die Rhythmussprache zwar erlernt haben (mit Ausnahme der Probanden in den Klassen K 5 und K 6), aber nicht simultan unterstützend im Musiziervollzug nutzen. Erst durch die ausdrückliche Aufforderung wird dies bewusst getan und kann, wie dargestellt, zu besseren Leistungen führen. Bei den Probanden der Untersuchungsgruppe scheint das simultane Nutzen der Rhythmussprache im Musiziervollzug aber der Fall zu sein, vermutlich gibt es deshalb kaum Steigerungen in den Leistungen im Untertest ec im Vergleich zu Subtest E.

Im Subtest $F$ Rhythmen musizieren und aufschreiben mit Noten können bei den Lösungen der Probanden der Untersuchungsgruppe kaum Fälle des figuralen Typus (Kat. 41) nachgewiesen werden. In der Kontrollgruppe wird dagegen ein hoher Anteil an Lösungen im figuralen Typus erbracht (s. Abb. 4-14). Die Art und Weise des Erlernens und Festigens der Rhythmussprache im Rhythmus-Lehrgang ermöglicht ein Verständnis über die einzelnen Silben hinaus auf ein metrisches Eingebettetsein aller Schläge eines Rhythmus. Dies führt zu Notationsleistungen im erwünschten metrischen Typus und kann für die Lösungen der Probanden der Untersuchungsgruppe auch in den parallel geführten Untertests fc sowie f nachgewiesen werden (s. Abb. 4-55 sowie Tab. 4-26).

Diese besondere Art und Weise des Erlernens der Rhythmussprache in Kombination mit den bereits benannten anderen Faktoren (dreifache Wissensdarbietung, natürliche Differenzierung, wechselnde Arbeit in Plenum und Gruppen) wird als Ursache für die sehr guten Leistungen in den Subtests E, F und C sowie deren überzeugenden Effektstärken gewertet. Dies legt ebenfalls nahe, das Pyramidenmodell anders aufzubauen. Als Abfolge für zu realisierende Lehrschritte ist das Modell nach Jank (2013 ${ }^{5}$, S. 129) sehr gut geeignet. Als Modell eines Entwicklungstandes zu Beginn der Klassenstufe 3 sollten die Stufen C und D ausgetauscht werden (s. Abb. 5-1). Diesen Tausch legen die deutlich ausgeprägten Fähigkeiten im Subtest D im Vortest (s. Abb. 4-8), der Nachweis der unterschiedlichen Lernstände nach der Methode known groups (auch bei Musikschülern kaum Vorkenntnisse zu Rhythmussilben; s. Abb. 4-68), die Korrelationswerte der Mittelwerte im Nachtest (s. Tab. 4-20), die Werte der Differenzen aus den Subtests im Vor- und Nachtest sowie die Effektstärken nahe (s. Anlage C 5 c-d). 


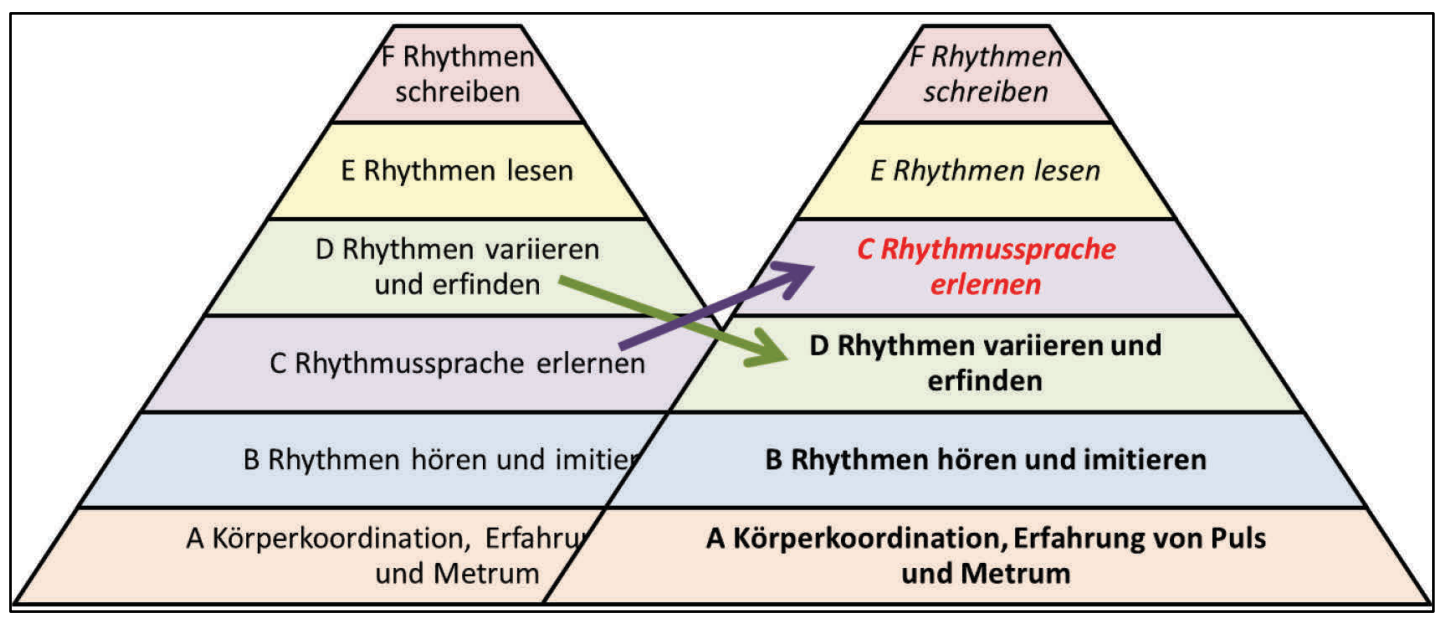

Abb. 5-1: Vorschlag für eine Veränderung des Modells zur Förderung rhythmischer Kompetenz

Damit kann für die Subtests A, B und D ausgesagt werden, dass zu Beginn der Klassenstufe 3 bereits deutlich messbare Fähigkeiten vorhanden sind und diese sowohl auf inzidentelles Lernen (beiläufiges Lernen in der Freizeit, vgl. Oerter, 1997, zitiert nach Oerter \& Bruhn, 1998, S. 333), informelles Lernen (zwangloses Lernen, vgl. Gruhn, 2006, S. 37; Tappert-Süberkrüb, 1999, S. 78) als auch schulisches Lernen im Musikunterricht der vorangehenden Klassenstufen 1 und 2 sowie außerschulischen Instrumentalunterricht zurückzuführen ist.

Für die Subtests C, E und F kann ausgesagt werden, dass zu Beginn der Klassenstufe 3 Fähigkeiten eher schwach ausgeprägt sind und vornehmlich von Schülerinnen und Schülern erbracht werden, die bereits über ein oder zwei Jahre außerschulischen Instrumentalunterricht erhalten. Die Entwicklung von Fähigkeiten in diesen drei Subtests ist nur durch formales Lernen zu erwerben. Dieses Lernen hat seine Verortung sowohl im Musikunterricht in der Grundschule als auch im außerschulischen Instrumentalunterricht. Damit würden die unteren drei Stufen elementare rhythmische Fähigkeiten und die oberen drei Stufen kognitiv förderbare Fähigkeiten abbilden.

\section{Beurteilung der Testgüte}

Folgender Nachweis ist zu erbringen:

(5) In welchem Maße hält der entwickelte Test die Gütekriterien ein?

Mit den 82 Probanden in der Untersuchungs- und den 67 Probanden in der Kontrollgruppe kann mit einer mittleren Effektstärke in Höhe von $d=0.5$ eine Teststärke (Power: $1-\beta$ err prob) von 0.92 erzielt werden (s. Anlage C 1). Dieser Wert darf als zufriedenstellend betrachtet werden (Bortz \& Döring, 2006 ${ }^{4}$, S. 501).

Die Itemschwierigkeiten liegen im anvisierten Bereich (s. Tab. 3-14, Tab. 4-32 sowie Anlage $\mathrm{C} 3$ b). Das schwerste Item ist Item F 4 mit einem Wert von 0.19 , das leichteste Item ist Item B 1 mit einem Wert von 0.66. Eine mittlere Itemschwierigkeit mit Werten zwischen 0.33 und 0.65 
bilden insgesamt 20 von 24 Items ab und können damit prinzipiell eine höhere Trennschärfe aufweisen. Die anderen vier Items eigenen sich für das Messen im Randbereichen eines Merkmals (Bühner, 2006, S. 99). Die Trennschärfen der Items liegen ebenfalls im anvisierten Bereich mit Werten über 0.2 (s. Anlage C 3 b). Die Werte für die Trennschärfe liegen zwischen 0.34 und 0.66, damit wird die Grenze zum kritischen Bereich nicht berührt (vgl. Lienert \& Raatz, 1998 ${ }^{6}$, S. 78).

Die Durchführungsobjektivität wurde mit Abstrichen in Bezug auf die örtlichen Bedingungen eingehalten. Dazu trug in hohem Maße das eingesetzte Video bei. Die Auswertungsobjektivität darf besonders durch die Entwicklung und den Einsatz des Prüf-Algorithmus als objektiv betrachtet werden (s. Abschnitt 3.5.2 und 0). Zur Interpretationsobjektivität fehlen vergleichbare Daten-sätze. Lediglich im Falle der Unterscheidung von Leistungsniveaus in Bezug auf rhythmische Kompetenz bei gleichaltrigen Mädchen und Jungen (Wagner, 1970, S. 27) sowie für ein zusätzliches Item im Subtest B (Item b 6) können Vergleiche angestellt werden (s. Anlage 2 a-e).

Die Reliabilität wird sowohl in Bezug auf die interne Konsistenz (Cronbach's Alpha 0.89) als auch auf die Testhalbierung (Teil 1: Cronbach's Alpha 0.79, Teil 2: 0.81; s. Abschnitt 0) nachgewiesen. Die Methode der known groups sowie die Korrelationen zwischen dem zusätzlichen Subtest $G$ Play together und dem Gesamtpunktwert des Testes legen nahe, mit einem validen Messinstrument gemessen zu haben (s. Abschnitt 0). Die Korrelationen des Gesamtpunktwertes zu den beiden zusätzlichen Subtests fallen mit $r=0.61$ zugunsten des musizierpraktischen Subtests G sowie mit $r=0.34$ zum Nachteil des rezeptionsorientierten Subtests I aus (s. Tab. 4-29, Tab. 4-31). Die Autorin liest daraus eine Bestätigung für den Einsatz eines kriteriumsorientierten und musizierpraktisch ausgerichteten Tests ab.

\subsection{Beurteilung der Treatmententwicklung}

Der Rhythmus-Lehrgang als Kernstück des Treatments beweist seine Stärken insbesondere in den herausragenden Ergebnissen in den Subtests C, E und F. Große bzw. mittelgroße Effekte können mit Werten in Höhe von $d=1.15,1.10$ sowie 0.77 nachgewiesen werden (s. Anlage $\mathrm{C} 4 \mathrm{c}$ ). Für die Subtests A und B werden Werte in Höhe von $d=0.17$ und 0.06 erzielt. Sie dokumentieren ausbleibende Effekte in Bezug auf Unterschiede in den Entwicklungen der Probanden beider Gruppen. Für Subtest D wird ein kleiner Effekt in Höhe von $\mathrm{d}=0.31$ gemessen. Für den Gesamtpunktwert liegt die Effektstärke bei einem Wert von $d=1.36$ (s. Tab. 4-19). Für das Treatment gilt es zu fragen, ob alle Bausteine ihre beabsichtigte Funktion erfüllen. Diese Frage wird unter der Prämisse gestellt, keine Änderung zuzulassen, die eine Verlängerung der einzelnen Lernsequenzanteile zur Folge hätte, da dies die anderen Lernbereiche zurückdrängen würde.

\section{Warm up 1}

Die Fähigkeit aus Stufe $A$ Grundschlag und Metrum darstellen wird besonders im Warm up 1 aufgegriffen. Die Übungen erbringen die ihnen innewohnende Intention nicht in Form von entspre- 
chenden Leistungen im adäquaten Subtests A. Die Leistungen der beiden Gruppen unterscheiden sich kaum voneinander (s. Abb. 4-18). Die Erfahrungen der Erprobungsphase, wenig Einfluss auf die Ergebnisse in diesem Bereich zu haben (s. Abschnitt 3.3.8 sowie Tab. 4-4 und Tab. 4-5), werden in der Hauptuntersuchung bestätigt. Dennoch wird in diesem Baustein ein wesentlicher Beitrag dafür geleistet, ein inneres Grundschlaggefühl zu entwickeln (vgl. Auhagen, 2011³, S. 443). Dies kommt den überdurchschnittlich guten Ergebnissen in den Subtests E (Tempostabilität, s. Abb. 4-30) und F zugute (Notation im metrischen Typus, s. Abb. 4-14).

Für Veränderung bestehen im Warm up 1 drei Möglichkeiten:

- Die Musikbeispiele wurden erst nach der Erprobungsphase ausgewählt und deshalb nicht geprüft. Einige Beispiele scheinen zu schwierig für die Altersgruppe zu sein. Sie könnten ausgetauscht werden.

- Vor- und Nachtest haben erwiesen, dass eine große Anzahl der Probanden erst einmal das Imitieren der Bewegungen zum Darstellen von Metrum und Grundschlag erlernen muss (s. Abschnitt 4.3.2). Der methodische Hinweis, nicht zu schnell bei dieser Übung eine Vorbildfunktion einzunehmen, muss deshalb hinterfragt werden und eher andersherum gelten, nämlich länger Unterstützung für das imitatorische Lernen anzubieten.

- Das Warm up 1 könnte auch aus dem Rhythmus-Lehrgang entfernt werden. Dagegen sprechen aber Rückmeldungen der Lehrkräfte und Schüler, nach denen diese einleitende Übung sehr viel Freude gemacht und Neugier auf die jeweiligen Musikstücke geweckt hatte (s. Abschnitt 4.9).

Um die erwähnte Schwierigkeit des Imitierens von Grundschlag und Metrum der Schülerinnen und Schülern vom Vorbild der Lehrkraft zu fördern (s. Abschnitt 4.3.2 und 5.2.1), bietet sich möglicherweise Folgendes zusätzlich an: Die von Gordon gewünschte Bewegungsübung weist für Schüler der Klassenstufe 3 nach einigen Wiederholungen keinen Beobachtungsreiz mehr auf. Wenn statt des gleichförmigen Betonens des Metrums mit dem Absetzen des Fußballens sowie des Grundschlages durch Patschen der Hände auf dem Oberschenkel, wechselnde Körpergesten und tänzerisch anmutende Bewegungen durchgeführt werden, erhöht dies deutlich den Reiz der kontinuierlichen Beobachtung und Nachahmung der Bewegungen der Lehrkraft. Das genaue Beobachten wird dabei als Grundlage für das Imitieren gesehen.

\section{Warm up 2}

Die Realisierung der Stufe $B$ Rhythmen nachspielen im Warm up 2 ist das ideale Medium zum Erlernen der Rhythmussprache (Subtest C) und zum Aufbauen eines Rhythmusrepertoires. Dies spiegelt sich u.a. in der Sicherheit der Probanden der Untersuchungsgruppe beim Anwenden der Silben im Untertest ec (s. Abb. 4-52), aber auch bei der geringen Anzahl an Lösungen im figuralen Typus im Untertest fc wider (s. Abb. 4-55). Darüber hinaus beeinflusst das Warm up 2 die größere Bandbreite an Rhythmen für Subtest D (s. Abb. 4-39) sowie die besser entwickelte Fähigkeit zum 
Wiederholen des abgewandelten Rhythmus in diesem Subtest (s. Abb. 4-33). Zu überdenken wäre eine größere Bandbreite an Tempi in den Rhythmus-Backgrounds. Diese liegen im RhythmusLehrgang zwischen 98 bpm und 104 bpm. Da die Probanden eine größere Bandbreite beim Musizieren im Subtest E zeigen (s. Abb. 4-48), sollten auch langsamere Tempi angeboten werden. Möglicherweise könnte dies dem beobachteten Trend des zu schnellen Spielens von Rhythmen entgegenwirken (s. Abb. 4-30) und ein Einlassen auf langsamere Tempi fördern. Für die Ergebnisse im Subtest B kann die Teilnahme am Treatment keinen größeren Lerneffekt als ohne Treatment ausweisen (s. Tab. 4-6, Tab. 4-7). Dennoch ist das Warm up 2 wesentlicher und unverzichtbarer Bestandteil zum Aufbauen eines Rhythmusrepertoires und dem Erlernen einer Rhythmussprache. Es kann deshalb nicht eliminiert werden.

Die Stufe D Rhythmen bewusst abwandeln wird im Treatment nur über die Methode des Verbotenen Rhythmus im Warm up 2 und das zweimalige Suchen nach Rhythmen für die Spielstücke 1 und $2 \mathrm{im}$ Workshop realisiert (s. Anlage D 6). Dennoch haben diese wenigen Gelegenheiten ausgereicht, um den geplanten Mittelwert zu erreichen. Den Probanden fällt das Wiederholen ihres abgewandelten Rhythmus deutlich leichter als den Probanden der Kontrollgruppe. Eine mentale Repräsentation des Gespielten scheint vorhanden zu sein. Die Teilnahme am Treatment verschafft den Probanden der Untersuchungsgruppe einen Vorsprung in Höhe eines kleinen Effekts (s. Tab. 4-11,

Tab. 4-12). Möglicherweise könnte eine noch stärkere Förderung und damit eine Angleichung an die erzielten Mittewerte der anderen Subtests erzielt werden, wenn in irgendeiner Form im Warm up 2 auch das Antworten auf einen Call mit einem abgewandelten Rhythmus eingebettet wäre. Dies könnte aber jeweils nur individuell von einzelnen Schülerinnen und Schülern geschehen und bräuchte eine gezielte Gestik, damit Lehrkraft und Schüler ohne Reden wissen, wer diesen abgewandelten Rhythmus spielen soll (vgl. Gordon, 2001, S. 118). Möglicherweise ist dieses Arbeiten innerhalb von 20 Unterrichtswochen noch verfrüht, da zuerst einmal Grundlagen in Bezug auf das Rhythmusrepertoire und die verschiedenen Rhythmussilben gelegt werden müssen.

\section{Workshop}

Durch die im Workshop realisierten Stufen E Rhythmen lesen und musizieren sowie F Rhythmen musizieren und aufschreiben werden hervorragende Ergebnisse von den Probanden der Untersuchungsgruppe erzielt (s. Tab. 4-13, Tab. 4-14, Tab. 4-2, Tab. 4-3). Das Treatment braucht diesbezüglich nicht verändert zu werden. Die Hinzunahme einer ikonischen Ebene nach dem Konzept der dreifachen Wissensdarbietung und dem Konzept der natürlichen Differenzierung erweist sich als erfolgreich. Lediglich Silbe und Symbol für Pause sollten eher und häufiger Verwendung finden, um zu mehr Anwendungssicherheit zu führen.

\section{Lehrplanziele aus Klassenstufe 4}


Die vorweg genommenen Lehrplanziele aus Klassenstufe 4 werden erfolgreich implementiert (vgl. SMK, 2009, S. 16; s. Anlage A 1). Ohne diese Änderung wäre das Konzept des RhythmusLehrgangs insbesondere in Bezug auf das gleichzeitige Spielen verschiedener Rhythmen nicht aufgegangen. Das Erarbeiten eigener Rhythmen unter der Verwendung vielfältiger Rhythmusbausteine ist wesentlicher Bestandteil für die Übung Verbotener Rhythmus sowie für das Entstehen der beiden Spielstücke. Das Zusammenspielen verschiedener Rhythmen (vgl. ebd., S. 16) fördert insgesamt die rhythmische Kompetenz und die Fähigkeiten im zusätzlichen Subtest G Play together. Dieses mehrstimmige Zusammenspiel ist eine wesentliche Aufgabe beim gemeinsamen Musizieren im Musikunterricht.

\subsection{Schlussfolgerungen}

Die eingangs angeführte Kritik von Lehrkräften an weiterführenden Schulen in Bezug auf sehr unterschiedlich ausgebildete Fähigkeiten im Umgang mit Notation in Klassenstufe 5 (s. Kap. 1) wird insbesondere durch die Daten der 2. Erhebung dieser Studie in weiten Teilen bestätigt (s. Abschnitt 4.5). In vielen Klassen verschiedener Grundschulen (Klassenstufe 3 und 4) bestehen kaum nennenswerten Fähigkeiten in Bezug auf das Aufschreiben von Rhythmen mit Noten. Dies wird aber dadurch relativiert, dass es in etwa einem Drittel der Klassen über dem erwarteten Mittelwert liegende Ergebnisse gibt (s. Abb. 4-64). In diesen Klassen wurde eine Rhythmussprache erlernt. Auch die Ergebnisse der Hauptuntersuchung zeigen, dass mit dem Erlernen einer Rhythmussprache vielfältige Lernprozesse positiv beeinflusst werden. So verwundert es nicht, dass der Mittelwert des Subtests C Rhythmussprache anwenden am höchsten mit dem Mittelwert der Gesamtpunktwertung korreliert (s. Abschnitt 4.4.1) und die höchste Effektstärke aller Subtests erreicht wird (s. Anlage C 5 c).

Es kann durch diese Studie belegt werden, dass die Fähigkeiten im Umgang mit Notation bereits zu Beginn der Klassenstufe 3 einen natürlichen Entwicklungsstand erreicht haben, der das Lesen von Rhythmen nach ikonischer Notation sowie das Schreiben von Rhythmen mit selbst erfundenen Zeichen im figuralen Typus umfasst (s. Abschnitt 4.6.1 und 4.6.2). Der Einsatz des RhythmusLehrgangs im regulären Musikunterricht der Untersuchungsgruppe kann diese Fähigkeiten in besonderem Maße weiterentwickeln und fördern. Am Ende des 20 Unterrichtswochen umfassenden Treatments weisen die Leistungsunterschiede zwischen beiden Gruppen in vier der sechs Subtests kleine, mittlere und große Effekte auf (s. Anlage C 5 c).

Es lässt sich zeigen, dass das Pyramidenmodell nach Jank (2013노 S. 129), welches mit den Stufen A bis F eine ansteigende Schwierigkeit der verschiedenen Fähigkeiten andeutet, als Lehrkonzept tragfähig ist. Jedoch kann es nicht für beide Gruppen als theoretisches Konstrukt für den Entwicklungsstand am Ende der Klassenstufe 3 bestätigt werden (s. Abschnitt 5.2.3). Die erreichten Mittelwerte der Probanden der Kontrollgruppe bilden das ansteigende Niveau relativ gut ab. Das gemessene Leistungsspektrum scheint einem natürlichen Entwicklungsstand am Ende der Klassen- 
stufe 3 zu entsprechen. Die erreichten Mittelwerte der Probanden der Untersuchungsgruppe widersprechen dem Pyramidenmodell. Eine Zunahme der Itemschwierigkeiten von Subtest zu Subtest bzw. Stufe zu Stufe lässt sich nicht belegen. Die erreichten Mittelwerte entsprechen mit Ausnahme des Wertes für Subtest D einem ausgewogenen Leistungsniveau, welches auf eine umfassende Förderung in allen Stufen zurückzuführen ist (s. Abb. 4-67). Als Folge dieser Beobachtung wird im Pyramidenmodell ein Tausch der Stufen C und D begründet und empfohlen (s. Abb. 5-1).

Das ausgeglichene Leistungsspektrum der Probanden der Untersuchungsgruppe repräsentiert das Potenzial einer Förderung rhythmischer Kompetenz aus einer Kombination von Komponenten aus der Music Learning Theory Gordons $\left(2012^{8}\right)$, der dreifachen Wissensdarbietung nach Bruner (1974), dem Konzept der natürlichen Differenzierung nach Krauthausen und Scherer (2010) sowie einem Wechsel der Sozialformen zwischen Plenum und Gruppenarbeit (s. Abschnitt 3.3). Herzstück dieser gelungenen Förderung ist die im Treatment praktizierte Einführung und Anwendung einer Rhythmussprache. Diese wird kontinuierlich im Call-Response-Prinzip des Warm up 2 erlernt und fördert über das grundlegende Erlernen der Silben hinaus das Empfinden für das metrische Gefüge, in welchem ein Rhythmus eingebettet ist. Dies schlägt sich messbar in einer stärker entwickelten Fähigkeit im Lesen und Musizieren von Rhythmen nieder (s. Abschnitt 4.3.5 sowie 4.3.6). Ebenso fördert es das erwünschte Notieren von Rhythmen im metrischen Typus (s. Abschnitt 4.3.1 sowie 4.3.6). Durch diese Art des Erlernen der Rhythmussprache wird die mentale Repräsentation der Rhythmen gefördert, was sich in der deutlich besser entwickelten Fähigkeit wiederspiegelt, einen abgewandelten Rhythmus auch bewusst wiederholen zu können (s. Abschnitt 4.3.4).

Die Ergebnisse dieser Studie können an andere Studien angebunden werden. Im Vortest kann bestätigt werden, dass die Fähigkeiten zum Nachmachen rhythmischer Figuren (Subtest B) von Kindern bis zum zehnten Lebensjahr stärker ausgebildet sind als die metrischen Fähigkeiten (Subtest A) (vgl. Upitis, 1987, S. 54 sowie Abb. 4-8). Im Nachtest lässt sich dies nicht mehr bestätigen, zu diesem Zeitpunkt halten sich beide Fähigkeiten in etwa die Waage (s. Abb. 4-10). Beim Nachspielen von Rhythmen (Subtest B) wird im Vortest relativ häufig nicht der gleiche, sondern ein falscher, aber gut erkennbarer anderer Rhythmus wiedergegeben (s. Tab. 4-9). Dies bestätigt die Beobachtung, dass sich eine „Tendenz zur Vereinfachung von rhythmischen Strukturen zeigt ..., wenn unregelmäßige Rhythmussequenzen reproduziert werden sollen“ (Beck, 2002 ${ }^{4}$, S. 460). Die Strategien der Vereinfachung fallen sehr verschieden aus, vom Spielen eines nur leicht abgewandelten bis hin zum Spielen eines ganz anderen Rhythmus (s. Tab. 4-10). Im Nachtest verschwindet diese Fehlerkategorie.

Im Subtest $D$ Rhythmen abwandeln fällt auf, dass die eigentliche Improvisationsleistung schon recht gut gelingt, jedoch das Wiederholen dieses Rhythmus eine große Herausforderung darstellt (s. Abb. 4-33). Dies spricht dafür, dass die abgewandelten Rhythmen eher aus einem impliziten Wissensschatz hervorgebracht werden bzw. dass der Spielende für den Prozess der Improvisation 
durchaus auch aus dem sogenannten Unterbewussten passende Ideen generiert (vgl. Lehmann, 2011 $1^{3}$, S. 346).

Im Subtest $E$ Rhythmen lesen und musizieren wird in der Rubrik Tempo des Rhythmus eine besondere Art und Weise des stark schwankenden Tempospiels beobachtet (s. Abb. 4-45). Die Temposchwankungen weisen eine Regelmäßigkeit auf und lassen darauf schließen, dass möglicherweise von Grundschlag zu Grundschlag gelesen und musiziert wird. Diese Fehlerkategorie kann als regelmäßig bzw. lesebedingt stark schwankendes Tempo bezeichnet werden (s. Abb. 4-43, Zeile 1). Es wird vermutet, dass das Bilden von chunks (Lehmann \& Chaffin, 2011 ${ }^{3}$, S. 358) bzw. die AugeHand-Spanne (ebd., S. 366) noch nicht genügend entwickelt sind. Das laute Mitsprechen der Rhythmussilben im Untertest ec (Subtest C) führt zwar prinzipiell nur in wenigen Fällen zu einer Verbesserung des Lesens und Musizierens von Rhythmen (s. Abb. 4-52), doch scheint es für den auffallend geringeren Anteil an Lösungen im regelmäßig bzw. lesebedingt stark schwankendes Tempo bei den Probanden der Untersuchungsgruppe zu sein.

Die Lösungen im Subtest $F$ Rhythmen musizieren und aufschreiben mit Noten können den bereits erforschten Typen des Darstellens von Rhythmen mit Zeichen gleichgestellt werden. Dazu zählen der nicht übereinstimmende, zählende, figurale und metrische Typus (s. Abb. 4-15; Bamberger, 1982, 1991; Hildebrandt, 1987; Upitis, 1987; Werner, 2016). Ohne spezielle Förderung bleiben viele als fast richtig einzuschätzende Lösungen dem figuralen Typus verhaftet (s. Abb. 4-14). Beim Aufschreiben der Rhythmen mit Rhythmussilben im Untertest $f_{c}$ (Subtest C) notiert der größte Teil der Probanden der Kontrollgruppe ebenfalls im figuralen Typus (s. Tab. 4-25).

Ein Blick auf die Schüler, die außerschulisch seit ein zwei bis zwei Jahren Instrumentalunterricht erhalten, gibt interessante Rückschlüsse auf deren Fähigkeiten in Bezug auf rhythmische Kompetenz. Für diese 32 Schülerinnen und Schüler kann speziell im Vortest mit der Methode known groups festgestellt werden, dass deren rhythmische Kompetenz messbar über dem Niveau der anderen Probanden liegt (s. Abb. 4-68). Auffallend sind jedoch die nicht allzu großen Leistungsunterschiede in den Subtests $\mathrm{C}$ und $\mathrm{F}$ gegenüber Schülern ohne Instrumentalunterricht. Bei genauer Betrachtung zum Subtests $C$ Rhythmussprache anwenden kann festgestellt werden, dass nur die Schüler, die neben dem Instrumentalunterricht auch Unterricht in Musiklehre erhalten, Erfahrungen im Umgang mit Rhythmussilben besitzen. Im Instrumentalunterricht scheint dieses Denk- und Übungsmittel (Losert, 2011) kaum Anwendung zu finden (s. Abschnitt 4.3.6). Im Subtest $F$ Rhythmen musizieren und aufschreiben ist zu beobachten, dass zwei Drittel dieser 32 Schüler und Schülerinnen kein Item lösen können (s. Abschnitt 4.3.1). Das Notieren von Rhythmen spielt demzufolge im Instrumentalunterricht keine wesentliche Rolle. Dies unterstreicht die Mahnung, „alle Aspekte des musikalischen Wissens, auch die des Lesens und Schreibens von Musik, durch Übung zu entwickeln, um eine optimale Koordination zwischen den verschiedenen musikalischen Bereichen zu erreichen und eine optimale Leistungsfähigkeit zu entwickeln“ (Davidson \& Scripp, 
1990, zitiert nach Gembris, $2013^{4}$, S. 246). Im Nachtest steigen die Mittelwerte in der Gruppe der Instrumentalschüler besonders in den Subtests E, F und C stark an (s. Anlage C 5 f). Dies legt nahe, dass diese Schülerinnen und Schüler, sowohl vom außerunterrichtlichen Instrumentalunterricht als auch vom Umgang mit Notenschrift und Rhythmussprache im regulären Musikunterricht sowie speziell in der Untersuchungsgruppe vom Rhythmus-Lehrgang stark profitiert haben.

\section{Zusammenfassung}

Zusammenfassend kann gesagt werden, dass in Bezug auf rhythmische Kompetenz bereits zu Beginn der Klassenstufe 3 deutlich messbare Fähigkeiten besonders beim Nachspielen von Rhythmen, beim Darstellen von Grundschlag und Metrum sowie beim Abwandeln von Rhythmen vorhanden sind. Das Musizieren nach ikonischer Notation sowie das Aufschreiben von Rhythmen mit selbst ausgedachten Zeichen im figuralen Typus gelingen bereits einem großen Teil der Probanden. Der figurale Typus scheint beim Notieren von Rhythmen für diese Altersgruppe typisch zu sein. Über Fähigkeiten beim Lesen und Schreiben von Rhythmen mit Noten verfügen hauptsächlich Schülerinnen und Schülern mit außerschulischem Instrumentalunterricht. Fähigkeiten zum Anwenden einer Rhythmussprache sind fast ausschließlich bei Schülern mit zusätzlichem Unterricht in Musiklehre auszumachen.

Durch den regulären Musikunterricht in der Kontrollgruppe sowie den regulären Musikunterricht mit implementiertem Treatment in der Untersuchungsgruppe werden die Leistungen in allen Subtests gefördert. Das Pyramidenmodell mit einer ansteigenden Schwierigkeit in den einzelnen Stufen wird im Nachtest mit den erreichten Mittelwerten der Probanden der Kontrollgruppe abgebildet. In der Untersuchungsgruppe fallen die erreichten Mittelwerte im Nachtest in allen Subtests nahezu ausgeglichen aus. Das Pyramidenmodell wird nicht bestätigt. Besonders auffallend ist in der Untersuchungsgruppe beim Notieren von Rhythmen der hohe Anteil an Leistungen im erwünschten metrischen Typus sowie beim Musizieren nach Noten die insgesamt deutlich höheren Anteile an korrekten Leistungen sowie die seltener auftretende Fehlerquelle regelmäßig bzw. lesebezogen stark schwankendes Tempo (Kat. 60). Als Grund dafür wird die besondere Art und Weise des Erlernens einer Rhythmussprache gesehen. Dies belegt im Subtest C Rhythmussprache anwenden die höchste in einem Subtest erreichte Effektstärke mit einem Wert von $d=1.15$. Gleichzeitig weist der Mittelwert des Subtests C die höchsten Korrelationen zum Gesamtpunktwert mit einem Wert von $r=.84$ auf. Mit dem entwickelten Rhythmus-Lehrgang als Teil eines 20 Unterrichtswochen umfassenden Treatments ist es möglich, den vorhandenen Entwicklungsstand rhythmischer Kompetenz umfassend zu fördern und eine erfolgreiche Einführung in den Umgang mit Rhythmusnotation zu initiieren. Mit der Zunahme von Fähigkeiten in allen sechs Subtests durch diese umfassende Förderung rhythmischer Kompetenz, steigt die Fähigkeit zum zweistimmigen Rhythmusspiel. 


\section{Ausblick}

Für weiterführende Arbeiten sollen einige Gedanken aufgezeigt werden. Zum Subtest $F$ Rhythmen musizieren und aufschreiben mit Noten erfolgte eine in dieser Arbeit dokumentierte 2. Erhebung mit mehr als 500 teilnehmenden Schülerinnen und Schülern aus den Klassenstufen 3 und 4. Es konnten mehr Erkenntnisse gewonnen werden, als im Rahmen dieser Studie dargestellt wurden. Sie sind in folgendem Artikel veröffentlicht:

Werner, C. (2016). Wie notieren Grundschülerinnen und Grundschüler musizierte Rhythmen? Eine empirische Untersuchung von Typen der Darstellung von Rhythmen mit Zeichen. Beiträge empirischer Musikpädagogik, 7, 1-38.

Andere Beobachtungen in verschiedenen Subtests konnten ebenfalls nicht vertiefender betrachtet werden. Zu ihnen zählt das Phänomen der Nachschwingkraft und die Frage, ob das fehlerhafte Spielen von Grundschlag und Metrum eher einem verfrühten oder einem verspäteten Muster folgt (vgl. Auhagen, 2011³, S. 445). Im Subtest E Rhythmen lesen und musizieren könnte noch eingehender die Frage verfolgt werden, inwiefern das laute Sprechen von Rhythmussilben die Tempi der vier Items aneinander angleichen hilft und größere Tempoanstiege (Kat. 35) zwischen korrekt gespielten Rhythmen vermeiden hilft.

Darüber hinaus wäre auch vorstellbar, aus den gewonnenen Daten der einzelnen Subtests Leistungsprofile zu erstellen. Aus ihnen könnten eventuell spezielle Typen des Musiklernens abgeleitet werden. Ebenfalls wäre eine vertiefende Betrachtung der Lernerfolge der beiden Gruppen Musikschüler und andere Schüler, wie sie in der Methode known groups gebildet wurden, von Interesse.

Da laut Curricula in Klassenstufe 4 die Erweiterung des Umgangs mit Notenschrift auf den tonalen Bereich erweitert wird, wären zu diesem Bereich analoge Erhebungen wünschenswert.

Der entwickelte Test ließe durch eine Ausweitung auf drei Messzeitpunkte noch präzisere Erkenntnisse zu. Im Rahmen dieser Studie waren allerdings die Kapazitäten der Datenerhebung und auswertung vollends ausgeschöpft. Der Algorithmus erfüllte eine wichtige Aufgabe im Rahmen einer objektiven Bewertung der Daten. Er diente zur Prüfung von Toleranzabweichungen und damit dem Unterscheiden der zu bewertenden Musizierleistungen in richtige oder fehlerhafte Ergebnisse. Ihm liegen aber nur die subjektiven Hörerfahrungen einer Person zugrunde. In Zusammenarbeit mehrerer Beurteiler und vor allem in Kombination mit Mathematikern und Statistikern könnte dieser Algorithmus effektiv weiterentwickelt werden. Statistische Verfahren wie Faktoren- bzw. Mehrebenanalyse kamen nicht zum Einsatz. Mit ihnen könnten sowohl die 24 Items als auch die Leistungen der einzelnen Probanden in ihren Klassen und Gruppen differenzierter geprüft werden. 


\section{Literaturverzeichnis}

A

Abel-Struth, S. $\left(2005^{2}\right)$. Grundriss der Musikpädagogik. Schott Musikpädagogik. Mainz, London: Schott.

Altenmüller, E. (2006). Hirnphysiologische Grundlagen des Übens. In U. Mahlert \& E. Altenmüller (Hrsg.), Handbuch Üben. Grundlagen, Konzepte, Methoden . Wiesbaden: Breitkopf \& Härtel.

Altenmüller, E., \& Jabusch, H.-C. (2016). Zur Hirnphysiologie des Übens: ein Update. Musikphysiologie und Musikermedizin, 23(2), 51-65.

Andreas, R. (2002 ${ }^{4}$ a). Improvisation. In H. Bruhn, R. Oerter \& H. Rösing (Hrsg.), Musikpsychologie. Ein Handbuch. (4. Aufl.) (rowohlts enzyklopädie, S. 506-514). Reinbek bei Hamburg: Rowohlt.

Andreas, R. (2002 b). Kreativität. In H. Bruhn, R. Oerter \& H. Rösing (Hrsg.), Musikpsychologie. Ein Handbuch. (4. Aufl.) (rowohlts enzyklopädie, S. 520-529). Reinbek bei Hamburg: Rowohlt.

Atteslander, P. (2010). Methoden der empirischen Sozialforschung (13. Aufl.). ESV basics. Berlin: Schmidt.

Auhagen, W. (2011 ${ }^{3}$ ). Rhythmus und Timing. (3. Aufl.) In H. Bruhn, R. Kopiez \& A. C. Lehmann (Hrsg.), Musikpsychologie. Das neue Handbuch. Orig.-Ausg. (S. 437-457). Reinbek bei Hamburg: Rowohlt.

B

Bähr, J. (2007). Klassenmusizieren. In W. Jank (Hrsg.), Musik-Didaktik. Praxishandbuch für die Sekundarstufe I und II. 2. Aufl. (S. 159-167). Berlin: Cornelsen Scriptor.

Bamberger, J. (1982). Revisiting chidren's cescriptions of simple rhythms: A function for reflection-in-action. In S. Strauss (Hrsg.), U-shaped behavioral growth (S. 191-226). New York: Academic Press.

Bamberger, J. S. (1991). The mind behind the musical ear: How children develop musical intelligence. Cambridge, Mass: Harvard University Press.

Bastian, H. G. (2001). Kinder optimal fördern - mit Musik: Intelligenz, Sozialverhalten und gute Schulleistungen durch Musikerziehung. Mainz: Schott Musik International Atlantis MusikbuchVerlag. 
Beck, K. (2002 $)$. Rhythmus und Timing. In H. Bruhn, R. Oerter \& H. Rösing (Hrsg.), Musikpsychologie. Ein Handbuch. (4. Aufl.) (rowohlts enzyklopädie, S. 459-466). Reinbek bei Hamburg: Rowohlt.

Bluestine, E. (2000). The ways children learn music: An introduction and practical guide to music learning theory (2. Aufl.). Chicago, IL: Gia Publications.

Bochmann, R. \& Kirchmann, R. (2006). Kooperatives Lernen in der Grundschule: Zusammen arbeiten - Aktive Kinder lernen mehr. Essen: Neue-Dt.-Schule-Verl.-Ges.

Bortz, J. \& Döring, N. (2006 ${ }^{4}$ ). Forschungsmethoden und Evaluation: Für Human- und Sozialwissenschaftler (4. Aufl.). Berlin [u.a.]: Springer.

Bovet, G. \& Huwendiek, V. (Hrsg.) (2008). Leitfaden Schulpraxis: Pädagogik und Psychologie für den Lehrerberuf (5. Aufl.). Berlin: Cornelsen Scriptor.

Bruhn, H. (2002. Rhythmus in Wahrnehmung und musikbezogener Handlung. In H. Bruhn, R. Oerter \& H. Rösing (Hrsg.), Musikpsychologie. Ein Handbuch. (4. Aufl.) (rowohlts enzyklopädie, S. 291-299). Reinbek bei Hamburg: Rowohlt.

Bruner, J. S. (1974). Entwurf einer Unterrichtstheorie. Sprache und Lernen: Bd. 5. Berlin, Düsseldorf: Berlin Verlag Arnold Spitz.

Brüning, L. \& Saum, T. (2007). Erfolgreich unterrichten durch kooperatives Lernen: Strategien zur Schüleraktivierung (3. Aufl.). Essen: Neue-Dt.-Schule-Verl.-Ges.

Bühner, M. (2006). Einführung in die Test- und Fragebogenkonstruktion (2. Aufl.). ps Psychologie. München: Pearson Studium.

\section{D}

Dartsch, M. (2006). Üben im Vorschul-und Grundschulalter. In U. Mahlert \& E. Altenmüller (Hrsg.), Handbuch Üben. Grundlagen, Konzepte, Methoden . Wiesbaden: Breitkopf \& Härtel.

Dartsch, M. (2010). Mensch, Musik und Bildung: Grundlagen einer Didaktik der Musikalischen Früherziehung. Wiesbaden, Leipzig [u.a.]: Breitkopf \& Härtel.

Detterbeck, M. \& Schmidt-Oberländer, G. (2011). MusiX: Das Kursbuch 1. Für den Unterricht an allgemeinbildenden Schulen. Lehrerband, Kapitel 1-4. Rum/Innsbruck ; Esslingen: Helbling.

$\mathbf{E}$

Ernst, A. (2006). Didaktik des Übens. In U. Mahlert \& E. Altenmüller (Hrsg.), Handbuch Üben. Grundlagen, Konzepte, Methoden (S. 98-116). Wiesbaden: Breitkopf \& Härtel.

Evelein, F. (2009). Musik unterrichten mit kooperativen Lernmethoden: Praxisanregungen, Grundlageninfos, Materialien \& Hörbeispiele. Mülheim an der Ruhr: Verlag an der Ruhr. 
Filz, R. \& Heidecker, B. (2008). Rhythmus für Kids: Eine spielerische Entdeckungsreise mit allen Sinnen für das Gruppenmusizieren. Wien [u.a.]: Universal Ed.

Fischinger, T. \& Kopiez, R. (2011). Wirkungsphänomene des Rhythmus. In H. Bruhn, R. Kopiez \& A. C. Lehmann (Hrsg.), Musikpsychologie. Das neue Handbuch. (3. Aufl.) (S. 458-475). Reinbek bei Hamburg: Rowohlt.

Flämig, M. (2000). Didaktische Analyse als Kern der Begründung von Musikunterricht. Musik in der Schule (4), 8-9.

Frühauf, J., Kopiez, R. \& Platz, F. (2013). Music on the timing grid: The influence of microtiming on the perceived groove quality of a simple drum pattern performance. Musicae Scientiae (17), 246-260.

Fuchs, M. (2004). Musik lernen im Anfangsunterricht auf der Basis neuer Musiklerntheorien: Auszug aus dem Forschungsbericht. Verfügbar unter: http://home.ph-freiburg.de/fuchsfr/ [Zuletzt aufgerufen am 23.02.12].

Fuchs, M. (2006). Was soll Musikunterricht in der Grundschule leisten? In M. Fuchs (Hrsg.), Welchen Musikunterricht braucht die Grundschule? Konzeptionelle und unterrichtsspezifische Beiträge zu einem nachhaltigen Musikunterricht. Musikwissenschaft / Musikpädagogik in der blauen Eule: Bd. 75 (S. 43-56). Essen: Verl. Die Blaue Eule.

Fuchs, M. (2010). Musik in der Grundschule neu denken - neu gestalten (1. Aufl.). Esslingen: Helbling.

Fuchs, M. (Hrsg.) (2015). Musikdidaktik Grundschule: Theoretische Grundlagen und Praxisvorschläge (1. Aufl.). Innsbruck, Esslingen, Bern-Belp: Helbling.

G

Gallus, H. U. (2003). You must feel the beat: Körperkoordination und Gewichtserfahrung als Grundlage gemeinsamen Musizierens, AfS-Magazin 15 06/2003. Verfügbar unter: http://www.afsmusik.de/magazin/2003/6/03-you_must_feel_the_beat.pdf [Zuletzt aufgerufen am 17.5.2013].

Gembris, H. (2013 ${ }^{4}$. Grundlagen musikalischer Begabung und Entwicklung (4. Aufl.). Forum Musikpädagogik: Bd. 20. Augsburg: Wißner.

Gies, S. (2001). Alte Eisen der Musikpädagogik?: Von Curwen bis Gordon: zur Aktualität historischer Konzeptionen. Musik \& Bildung (3), 1-8.

Gies, S., Jank, W. \& Nimczik, O. (2001). Musik lernen: Zur Neukonzeption des Musikunterrichts in den allgemein bildenden Schulen. Diskussion Musikpädagogik (9), 6-24. 
Giordani, D. \& Brodesser, Y. (2009). Notation. http://trommel-schule.eu/Notation.htm. [Zuletzt aufgerufen am 22.08.2016]

Gnahs, D. (2010). Kompetenzen - Erwerb, Erfassung, Instrumente (1. Aufl.). Bielefeld: Bertelsmann.

Gordon, E. (2001). Reference handbook for using learning sequence activities (4. Aufl.). Jump right in. The music curriculum. Chicago: Gia Publications.

Gordon, E. E. $\left(2012^{8}\right)$. Learning Sequences in Music: A Contemporary Music Learning Theory (8. Aufl.). Chicago: Gia Publications.

Gramß, K. (2007). Boomwhackers elementar 1: 9 Spielstücke zum Singen, Spielen und Tanzen mit Boomwhackers und anderen Instrumenten für die 3. bis 6. Jahrgangsstufe (1. Aufl.). : Bd. 1. Rum/Innsbruck [u.a.]: Helbling.

Gruhn, W. (1999). Wie denkt, hört und lernt der ungeschulte Kopf? Diskussion Musikpädagogik (2), 60-74.

Gruhn, W. (2003). Lernziel Musik: Perspektiven einer neuen theoretischen Grundlegung des Musikunterrichts (1. Aufl.). Olms Forum: Bd. 5. Hildesheim, Zürich, New York: Georg Olms Verlag.

Gruhn, W. (2006). Musiklernen in der Grundschule. In M. Fuchs (Hrsg.), Welchen Musikunterricht braucht die Grundschule? Konzeptionelle und unterrichtsspezifische Beiträge zu einem nachhaltigen Musikunterricht. Musikwissenschaft / Musikpädagogik in der blauen Eule: Bd. 75 (S. 3141). Essen: Verl. Die Blaue Eule.

Gruhn, W. (2010). Anfänge des Musiklernens: Eine lerntheoretische und entwicklungspsychologische Einführung. Olms-Forum: Bd. 7. Hildesheim [u.a.]: Olms.

\section{H}

Hasselhorn, J. (2015). Messbarkeit musikpraktischer Kompetenzen von Schülerinnen und Schülern: Entwicklung und empirische Validierung eines Kompetenzmodells. Perspektiven musikpädagogischer Forschung: Bd. 2. Münster [u.a.]: Waxmann.

Hattie, J. (2013). Lernen sichtbar machen (1. Aufl.) (Beywl, W. \& Zierer, K., Hrsg.). Baltmannsweiler: Schneider-Verl. Hohengehren.

Helmke, A. (2010). Unterrichtsqualität und Lehrerprofessionalität: Diagnose, Evaluation und Verbesserung des Unterrichts (2. Aufl.). Seelze-Velber: Kallmeyer.

Herrmann, M. (2009). Notenlernen mit Trommelspaß: Rhythmen und Notenwerte lernen, so leicht und unterhaltsam, dass es jeder lernen und lehren kann (1. Aufl.). Offenburg: Mildenberger. 
Hildebrandt, C. (1987). Structural-Developmental Research in Music: Conservation and Representation. In P. e. a. Peery (Ed.), Music and child development (pp. 80-95).

Hirsch, F. (1987). Das große Wörterbuch der Musik(2. Aufl., 1 Band). Berlin: Verlag Neue Musik.

Hortien, R., Kivi, A., Stange, C. \& Urban, U. (2007). Wünsche der weiterführenden Schulen an den Musikunterricht in der Grundschule. Diskussion Musikpädagogik (34), 8-11.

Jank, W. (Hrsg.) (2007). Musik-Didaktik: Praxishandbuch für die Sekundarstufe I und II (2. Aufl.). Berlin: Cornelsen Scriptor.

Jank, W. (Hrsg.) $\left(2013^{5}\right)$. Musik-Didaktik: Praxishandbuch für die Sekundarstufe I und II (5. Aufl.). Berlin: Cornelsen Scriptor.

Jungbluth, A. \& Hafen, R. (1997). Musik-Screening für Kinder: Teil I (5-8;6 Jahre) und II (8;7 -11 Jahre).Unveröffentlicht. Anfrage an Prof. Roland Hafen, Universität Vechta, möglich.

$\mathbf{K}$

Kaiser, H. J. (2001). Kompetent, aber wann?: Über die Bestimmung von "musikalischer Kompetenz" in Prozessen ihres Erwerbs. Musik \& Bildung (3), 5-10.

Klafki, W. (1958). Didaktische Analyse als Kern der Unterrichtsvorbereitung. Die deutsche Schule (10), 450-471.

Klafki, W. $\left(2007^{6}\right)$. Neue Studien zur Bildungstheorie und Didaktik: Zeitgemässe Allgemeinbildung und kritisch-konstruktive Didaktik (6. Aufl.). Beltz Bibliothek. Weinheim, Basel: Beltz.

Knigge, J. \& Lehmann-Wermser, A. (2008). Bildungsstandards für das Fach Musik: Eine Zwischenbilanz. ZfkM; Sonderedition: Bildungsstandards und Kompetenzmodelle für das Fach Musik?, 2008, 60-98.

Knigge, J. \& Lehmann-Wermser, A. (2009). Kompetenzorientierung im Musikunterricht: Einige Anmerkungen zu einem Perspektivwechsel. Musik \& Unterricht (94) S. 56-60. Langfassung der CD-Rom S. 1-12.

Knigge, J. (2010). Modellbasierte Entwicklung und Analyse von Testaufgaben zur Erfassung der Kompetenz "Musik wahrnehmen und kontextualisieren" (1. Aufl.). Universität Bremen. Verfügbar unter: http://nbn-resolving.de/urn:nbn:de:gbv:46-diss000120066. [Zuletzt aufgerufen am 22.08.2016.]

Knigge, J. (2014). Der Kompetenzbegriff in der Musikpädagogik: Verwendung Kritik Perspektiven. In J. Vogt, F. Heß \& M. Brenk (Hrsg.), (Grund-)Begriffe musikpädagogischen Nachdenkens. 
Entstehung, Bedeutung, Gebrauch : Sitzungsbericht 2013 der Wissenschaftlichen Sozietät Musikpädagogik. 1. Aufl. (Wissenschaftliche Musikpädagogik, S. 105-135). Berlin: Lit.

Kormann, A. (1985). Tests in der Musikpsychologie. In H. Bruhn, R. Oerter \& H. Rösing (Hrsg.), Musikpsychologie. Ein Handbuch in Schlüsselbegriffen. 1. Aufl. (S. 502-509). München, Wien, Baltimore: Urban \& Schwarzenberg.

Krauthausen, G. \& Scherer, P. (2010). Umgang mit Heterogenität: Natürliche Differenzierung im Mathematikunterricht der Grundschule. Handreichungen des Programms SINUS an Grundschulen. Kiel: IPN Leibniz-Institut f. d. Pädagogik d. Naturwissenschaften an d. Universität Kiel.

Küntzel, B. (2006). Die Persönlichkeit des Schülers im Zentrum des Musikunterrichts. In M. Fuchs (Hrsg.), Welchen Musikunterricht braucht die Grundschule? Konzeptionelle und unterrichtsspezifische Beiträge zu einem nachhaltigen Musikunterricht. Musikwissenschaft / Musikpädagogik in der blauen Eule: Bd. 75 (S. 57-65). Essen: Verl. Die Blaue Eule.

$\mathbf{L}$

Lehmann, A. C. $\left(2002^{4}\right)$. Vomblattspiel und Erkennen musikalischer Formen. In H. Bruhn, R. Oerter \& H. Rösing (Hrsg.), Musikpsychologie. Ein Handbuch. (4. Aufl.) (rowohlts enzyklopädie, S. 486-492). Reinbek bei Hamburg: Rowohlt.

Lehmann, A. C. \& Chaffin, R. $\left(2011^{3}\right)$. Erinnern und Wiedererkennen:: Auswendig- und Vomblattspiel. In H. Bruhn, R. Kopiez \& A. C. Lehmann (Hrsg.), Musikpsychologie. Das neue Handbuch. (3. Aufl.) (S. 354-373). Reinbek bei Hamburg: Rowohlt.

Lehmann, A. C. \& Oerter, R. (201133). Lernen, Übung und Expertisierung. In H. Bruhn, R. Kopiez \& A. C. Lehmann (Hrsg.), Musikpsychologie. Das neue Handbuch. (3. Aufl.) (S. 105-128). Reinbek bei Hamburg: Rowohlt.

Lienert, G. A. \& Raatz, U. (1998). Testaufbau und Testanalyse (6. Aufl.). Weinheim: Beltz, Psychologie Verl.-Union.

Losert, M. (2011). Die didaktische Konzeption der Tonika-Do-Methode: Geschichte, Erklärungen, Methoden. Forum Musikpädagogik, Bd. 100: Bd. 95. Augsburg: Wißner.

$\mathbf{M}$

Maschke, H. (2008). Spaß beim Singen (1. Aufl.). Lehrer-Bücherei : Grundschule. Berlin: Cornelsen Scriptor.

McPherson, G. \& Gabrielsson, A. (2002). From Sound to Sign. In: Richard Parncutt und Gary E. McPherson (Hrsg.): The science and psychology and music performance: creative strategies for teaching and learning. Oxford: Oxford University Press, S. 99-115. 
McPherson, G. (2005). From child to musician: skill development during the beginning stages of learning an instrument. In: Psychology of Music 33 (1/2005), S. 5-35.

McPherson, G. \& Mills, J. (2006). Musical Literacy. In: Gary E. McPherson (Hrsg.): The Child As Musician. A handbook of musical development. Oxford: Oxford University Press.

Ministerium für Bildung, Wissenschaft, Jugend und Kultur Rheinland-Pfalz (2009). Rahmenplan Grundschule: Teilrahmenplan Musik. Neuleiningen: MF Druckservice.

Minkenberg, H. (1991). Das Musikerleben von Kindern im Alter von fünf bis zehn Jahren: Eine Längsschnittuntersuchung als Basis für die Erforschung von abweichender Musikrezeption. Studien zur Musik: Bd. 4. Frankfurt am Main, Bern, New York, Paris: P. Lang.

$\mathbf{N}$

Niessen, A., Lehmann-Wermser, A., Knigge, J. \& Lehmann, A. (2008). Entwurf eines Kompetenzmodells 'Musik wahrnehmen und kontextualisieren'. ZfkM, Sonderedition: Bildungsstandards und Kompetenzmodelle für das Fach Musik?

$\mathbf{0}$

Oerter, R. (2002). Handlungstheoretische Fundierung. In H. Bruhn, R. Oerter \& H. Rösing (Hrsg.), Musikpsychologie. Ein Handbuch. (4. Aufl.) (rowohlts enzyklopädie, S. 253-267). Reinbek bei Hamburg: Rowohlt.

Oerter, R. \& Bruhn, H. (1998). Musizieren. In H. Bruhn \& H. Rösing (Hrsg.), Musikwissenschaft. Ein Grundkurs (rowohlts enzyklopädie, S. 330-348). Reinbek bei Hamburg: Rowohlt.

$\mathbf{P}$

Patho, K. \& Schnabel, A. (2007). Der neue Musikus: Ein Musikbuch für die Grundschule (1. Aufl.). Berlin, Berlin: Volk-und-Wissen-Verl; Cornelsen.

Platz, F., Kopiez, R. \& Lehmann, M. (2012). Statistische Poweranalyse als Weg zu einer ,kraftvolleren' Musikpsychologie im 21. Jahrhundert. In W. Auhagen, C. Bullerjahn \& H. Höge (Hrsg.), Musikpsychologie. Jahrbuch der Deutschen Gesellschaft für Musikpsychologie. Populäre Musik (S. 165-179). Göttingen [u.a.]: Hogrefe.

$\mathbf{R}$

Richter, C. (2006). Über die notwendige Balance zwischen individuellem, ,natürlichen'; und von anderen verordnetem, ,künstlichem 'Lernen. In M. Fuchs (Hrsg.), Welchen Musikunterricht braucht die Grundschule? Konzeptionelle und unterrichtsspezifische Beiträge zu einem nachhaltigen Musikunterricht. Musikwissenschaft / Musikpädagogik in der blauen Eule: Bd. 75 (S. 67-82). Essen: Verl. Die Blaue Eule. 
Rost, D. H. (2007). Interpretation und Bewertung pädagogisch-psychologischer Studien: Eine Einführung (2. Aufl.). Weinheim und Basel: Beltz.

Rüdiger, W. (2006). Üben im Ensemble. In U. Mahlert \& E. Altenmüller (Hrsg.), Handbuch Üben. Grundlagen, Konzepte, Methoden . Wiesbaden: Breitkopf \& Härtel.

$\mathbf{S}$

Sächsisches Ministerium für Kultus (2008). Verwaltungsvorschrift des Sächsischen Staatsministeriums für Kultus über Sponsoring, Werbung, Spenden, Erhebungen, Wettbewerbe und den Warenverkauf an Schulen. (VwV Sponsoring, Spenden und Erhebungen an Schulen). Az.: 21$6499.21 / 116$.

Sächsisches Staatsministerium für Kultus (2009). Lehrplan Grundschule: Musik. Dresden: Digital- und Offsetdruckerei.

Schütz, V. (1996). Welchen Musikunterricht brauchen wir? Teil 1: Klärung einiger Voraussetzungen. afs-Magazin (1), 3-8.

Schütz, V. (1997). Welchen Musikunterricht brauchen wir? Teil 2: Perspektiven eines brauchbaren Musikunterrichts. afs-Magazin (3), 3-10.

Seel, N. M. (2003). Psychologie des Lernens: Lehrbuch für Pädagogen und Psychologen (2. Aufl.). München, Basel: E. Reinhardt.

Spitzer, M. (2002). Musik im Kopf: Hören, Musizieren, Verstehen und Erleben im neuronalen Netzwerk (1. Aufl.). Stuttgart [u.a.]: Schattauer.

Spychiger, M. B. (2005). Perspektiven musikalischen Lernens. Verfügbar unter: http://wiki.doebe.li/pub/Music/Bwsc4Literatur/Sep05Spychiger_BeitragDGM.pdf [Zuletzt aufgerufen am 27.07.16].

Spychiger, M. B. (2006). Musikalische Fähigkeiten bilden sich nicht von selbst. In M. Fuchs (Hrsg.), Welchen Musikunterricht braucht die Grundschule? Konzeptionelle und unterrichtsspezifische Beiträge zu einem nachhaltigen Musikunterricht. Musikwissenschaft / Musikpädagogik in der blauen Eule: Bd. 75 (S. 11-29). Essen: Verl. Die Blaue Eule.

Spychiger, M. (2015). Lernpsychologische Perspektiven für eine grundschulspezifische Musikdidaktik. In M. Fuchs (Hrsg.), Musikdidaktik Grundschule. Theoretische Grundlagen und Praxisvorschläge. 1. Aufl. (S. 50-71). Innsbruck, Esslingen, Bern-Belp: Helbling.

Süberkrüb, A. (2005). Musiklernen: 'Verstehen und Geschehen': "Didaktische Interpretation von Musik" und "Music Learning Theory" als Grundlage für vieldimensionales Musiklernen. Saarbrücken: Pfau. 
Tappert-Süberkrüb, A. (1999). "Music Learning Theory": Edwin Gordons Theorie des Musiklernens. Diskussion Musikpädagogik (2), 75-97.

$\mathbf{U}$

Upitis, R. (1987). Children's understanding of rhythm: The relationship between development and music training. Psychomusicology, pp. 41-60.

V

Volst, R. (2000). Wie man sich musikalisch Gehör verschaffen kann: Eine exemplarische Anleitung. 1-48. Verfügbar unter: http://members.chello.at/yvonnefink/website/paedagogik.pdf [Zuletzt aufgerufen am 25.01.17].

W

Wagner, R. (1970). Untersuchungen zur Entwicklung der Musikalität: Ein Musikleistungstest. Erziehung und Psychologie: Bd. 53. München, Basel: Ernst Reinhardt Verlag.

Weber, R. (2006). Musik begreifen - Musiklehre - Musiktheorie. In S. Helms, R. Schneider \& R. Weber (Hrsg.), Handbuch des Musikunterrichts. Primarstufe. 3. Aufl. (Handbuch des Musikunterrichts, S. 265-276). Kassel: Bosse.

Weinert, F. E. (2001). Leistungsmessungen in Schulen (2. Aufl.). Beltz Pädagogik. Weinheim [u.a.]: Beltz-Verl.

Werner, C. (2016). Wie notieren Grundschülerinnen und Grundschüler musizierte Rhythmen? Eine empirische Untersuchung von Typen der Darstellung von Rhythmen mit Zeichen. emBeiträge empirischer Musikpädagogik, 7(1), 1-38. 
Anlagen

7 Anlagen 


\section{Anlage A Forschungsstand und Curricula}

\section{A 1 Sächsischer LP-Auszug}

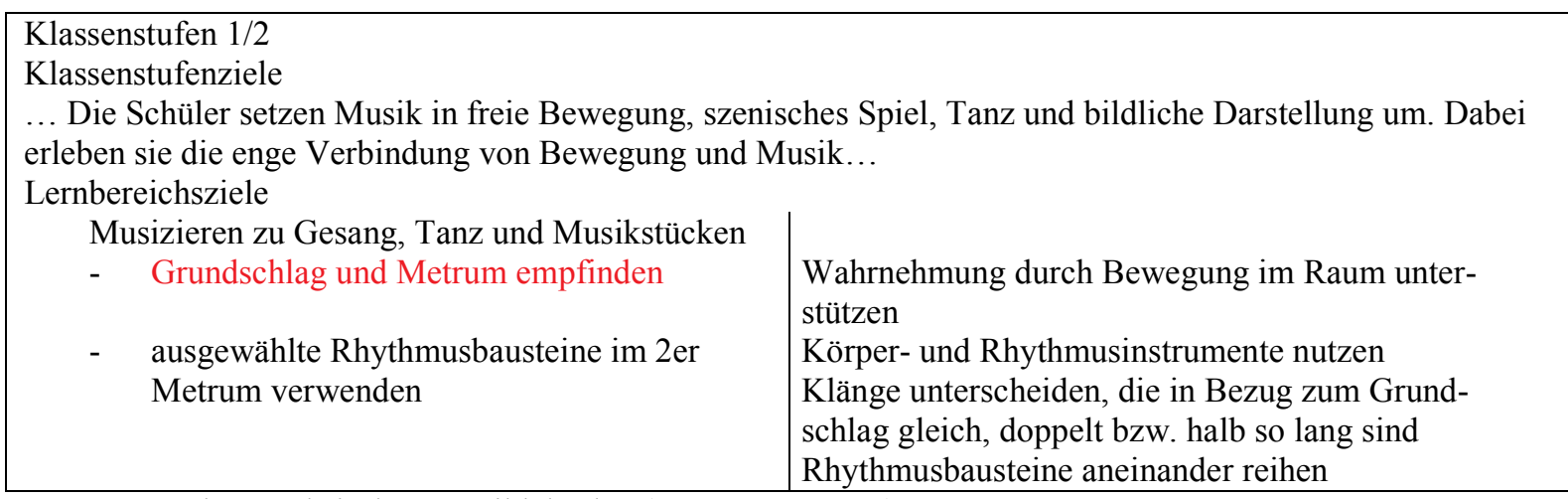

Auszug aus dem sächsischen Musiklehrplan (SMK, 2009, S. 7)

\section{Klassenstufe 3}

Klassenstufenziele

... Die Schüler erwerben erste Erfahrungen im Umgang mit dem traditionellen Notenbild in Bezug auf Rhythmus- und Tonhöhennotation...

Lernbereichsziele

Musizieren zu Gesang, Tanz und Musikstücken

- $\quad$ vielfältige Rhythmusbausteine im 2er und 3er Metrum verwenden

verschiedene Rhythmusbausteine aneinander reihen

Unterstützung durch Bewegung

- Notation von Rhythmusbausteinen verwenden

verschiedene Notationsformen für die Erarbei-

tung und Übung nutzen

eigenen Notationen anregen

- $\quad$ einfache Liedbegleitungen auf Melodiein-

(Stabspiele, Bassklangstäbe, Keyboards) strumenten spielen

Verständnis für Notation von Tonhöhen für aufwärts und abwärts, Wiederholung, Schritt, Sprung anbahnen

Auszug aus dem sächsischen Musiklehrplan (SMK, 2009, S. 11)

\section{Klassenstufe 4}

Klassenstufenziele

... Die Schüler vertiefen ihre Erfahrungen im Umgang mit dem traditionellen Notenbild in Bezug auf Rhythmus- und Tonhöhennotation...

Lernbereichsziele

Musizieren zu Gesang, Tanz und Musikstücken

- $\quad$ Repertoire an Rhythmusbausteinen im 2er und 3 er Metrum erweitern

- $\quad$ eigene Rhythmen unter Verwendung vielfältiger Rhythmusbausteine erarbeiten

verschiedene Rhythmusbausteine aneinander reihen

verschiedene Rhythmusbausteine gleichzeitig spielen

2er- und 3er-Metrum verbinden, z. B. 4er-, 6erMetrum

- $\quad$ Lieder mit Melodieinstrumenten begleiten eine zweite Stimme spielen

- Notation von Rhythmusbausteinen verwenden

Auszug aus dem sächsischen Musiklehrplan (SMK, 2009, S. 16) 


\section{A 2 Relevante Items anderer Leistungstests}

\section{A 2a Wagner (1970)}

Untertest II, Rhythmus-Auffassung $(\mathrm{n}=5000)$

Wagner führte in den 1970er Jahren mit 5000 sechs- bis elfjährigen Kindern eine „Untersuchung zur Entwicklung der Musikalität mit Hilfe eines Musikleistungstests“ durch. Sieben Items prüften im Einzelverfahren mit dem Probanden das Hören von Unterschieden zwischen sowie das Nachklatschen von Rhythmen.

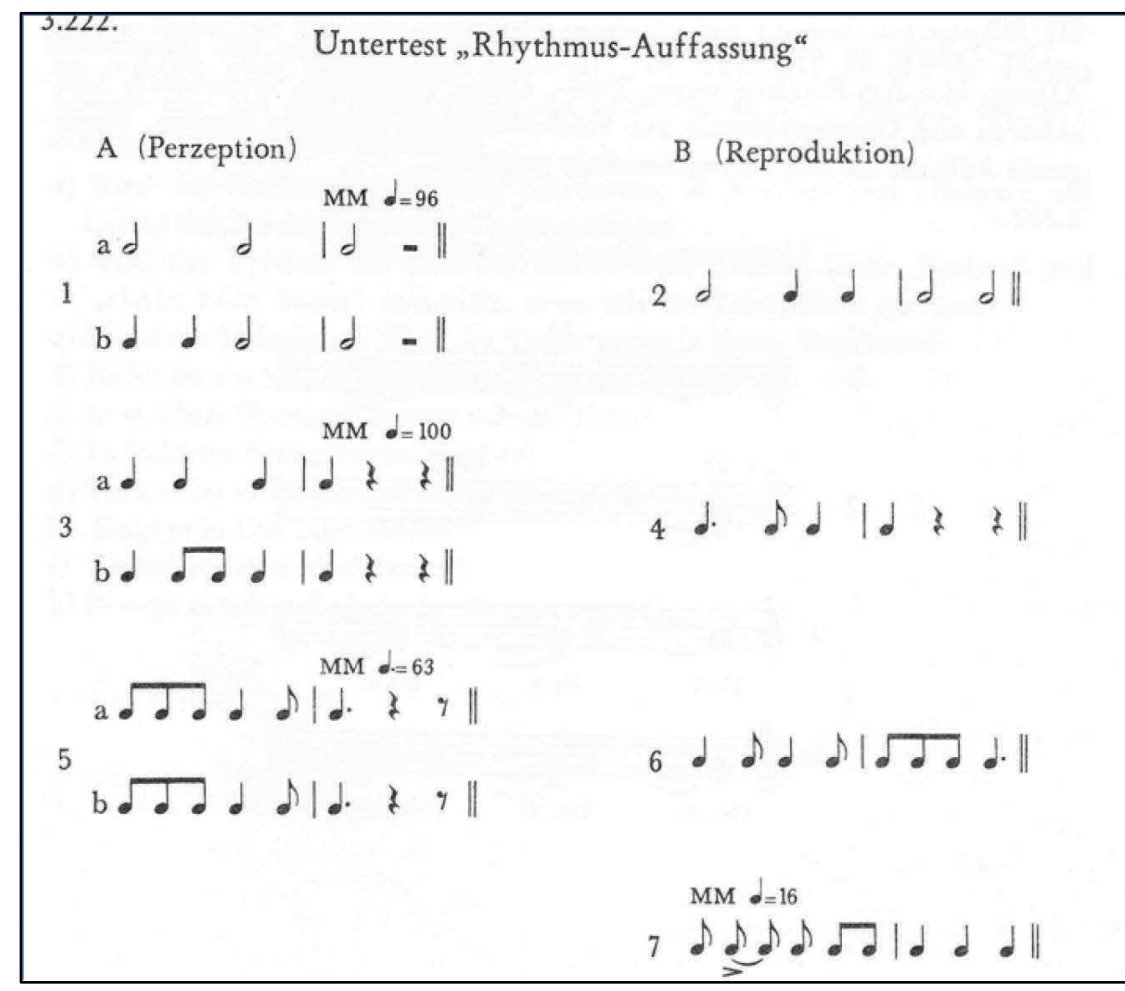

Itemgruppe im Untertest ,RhythmusAuffassung' (Wagner, 1970, S. 17)

Aufgabenstellung

„Im Untertest ,Rhythmus-Erfassung“ handelt es sich um Hören und Wiedergabe tonlos vorgeklopfter Rhythmen." (Wagner, S. 17) Die Aufgaben werden vom Tonband in den angegebenen Zeiten geboten. Bei den Höraufgaben der A-Serie dauert die Pause zwischen den Aufgaben a und b so lange wie die jeweilige Aufgabe a. Die VP antwortet auf das Aufgabenpaar mit ,gleich“ oder „verschieden..." u.ä. Unmittelbar auf die Höraufgabe erfolgt die Reproduktionsaufgabe... (ebd., S. 15) „Bandaufnahme nicht erforderlich (ebd. S. 17).“

Aufzeichnung der Probandenleistung

keine

Wertung

„Richtige Antworten werden mit ... mit x, falsche Antworten mit - ... festgehalten. Im Untertest I und II wird der VP bei falscher Reproduktion der B-Aufgaben nochmals die Aufgabe vorgespielt. Die VP kann wiederholen. Gelingt die Reproduktion beim zweiten Versuch, so gilt die Aufgabe als richtig gelöst mit dem Vermerk $\mathrm{x}_{1}$ (= beim 2- Mal richtig) (Lerneffekt!). Mißlingt der 2. Versuch, folgt Eintrag als Fehlleistung (-). $\mathrm{x}$ und $\mathrm{x}_{1}$ gelten je als 1 Punkt.“

Ergebnisse für die interessierende Altersgruppe der 8 bis 9jährigen Drittklässler

„Die Jahrgangsleistungen lassen bemerkenswerte Einblicke in den Stand der jeweiligen Entwicklung zu. Auffallend ist die durchweg bessere Leistung der Mädchen im Vergleich zu Buben. Der Niveauunterschied liegt bei $10 \%$ oder, anders ausgedrückt, die Mädchen sind 1 bis $1 \frac{1}{2}$ Jahre den Buben voraus (ebd., S. 27).“

„Für die 8jährigen ... [gab es viele] Unsicherheiten ab II 4 (ebd.).“ 
„Die 9jährigen ... Alle Höraufgaben von II werden klar gelöst (ebd.).“

„Bei den 10jährigen ... Weitere Leistungsverbesserungen in II 1-6 (ebd.).“

„Die rhythmische Empfindung verläuft in ihrer Entwicklung ähnlich der melodischen zwischen 8 und 10 Jahren besonders schnell. Die Leistungen liegen wegen der beiden letzten Aufgaben niedriger als in der Melodieauffassung. Der gerade Takt wird bei den 7jährigen leichter erfasst. Vermutlich ist er von Kinderreimen her geläufiger... Das Gespür für den Dreiertakt ist bei den Mädchen stärker ausgeprägt (ebd., S. 33)

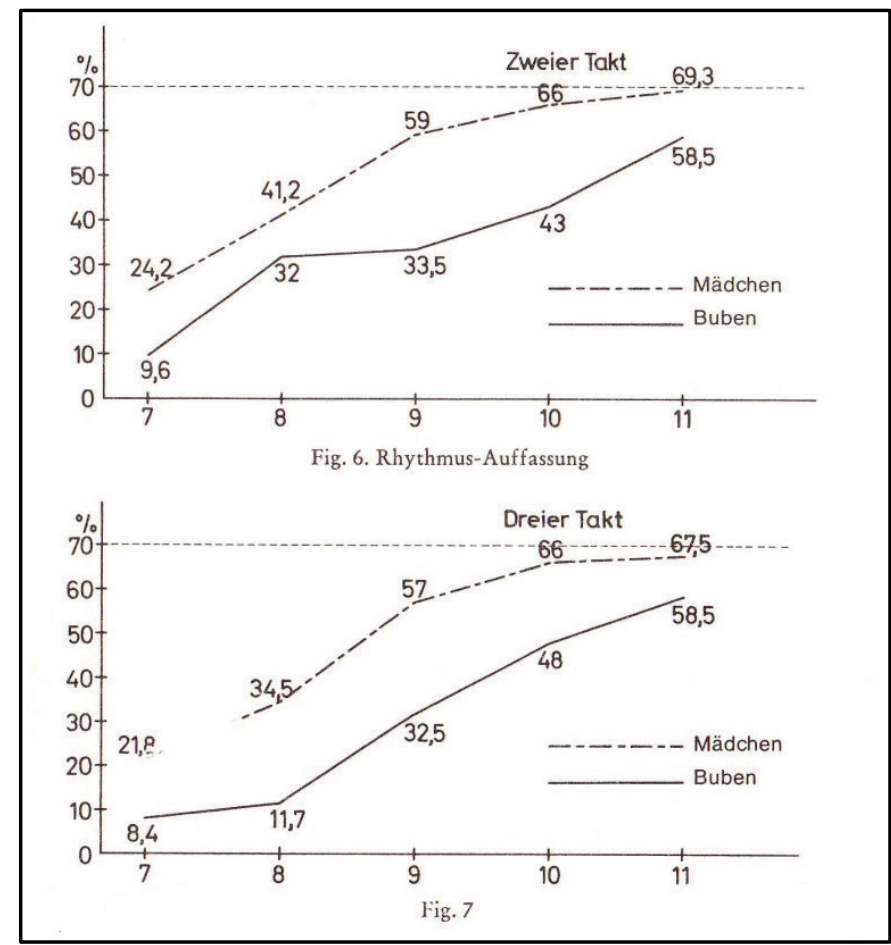

Grafiken der Rhythmenauswertung in Wagner, 1970, S. 33 


\section{A $2 b$ Minkenberg (1991)}

Rhythmustest ( $\mathrm{n}=33$ bzw. 38)

Minkenberg führte eine Längsschnittuntersuchung zum „Musikerleben von Kindern im Alter von fünf bis zehn Jahren" mit mehr als 50 Probanden in den 1980er Jahren durch. Im Bereich rhythmisch-metrischer Kompetenz wurden ebenfalls das Hören von Unterschieden sowie das Nachklatschen von Rhythmen (jeweils im Einzelverfahren) geprüft. Die Rhythmen sind jedoch wesentlich komplexer als bei Wagner.

\begin{tabular}{|c|c|}
\hline K 1 & $\left.\frac{4}{4} \cdot\right\lrcorner \curvearrowleft \cdot|\cdot, \cdot \cdot \cdot|$ \\
\hline K 2 & 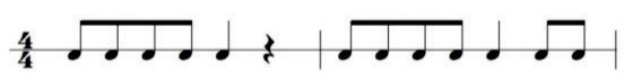 \\
\hline K 3 & 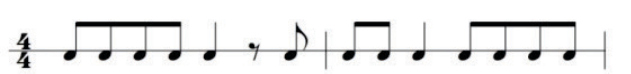 \\
\hline K 4 & 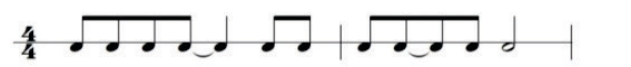 \\
\hline K 5 & 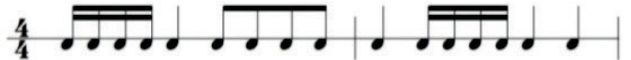 \\
\hline
\end{tabular}

im Tempo MM = 68 vorgeklatscht (S. 72)
Rhythmen des Rhythmustests bei Minkenberg (S. 72)

\section{Aufgabenstellung}

„Die fünf verschiedenen Rhythmen wurden den Versuchspersonen vom Versuchsleiter im Tempo Viertel $=68 \mathrm{MM}$ vorgeklatscht. Dieses Tempo entspricht ungefähr dem Ruhepuls. Es erwies sich in allen Testaufgaben in den Vorversuchen als bestes Tempo für rhythmische Reaktionen (vgl. Christensen, 1938, S. 444f.)

Der Verfasser wählte zweitaktige Rhythmen aus, um gezielt die Entwicklung der Fähigkeit von Kindern, längere Zeitgestalten zu behalten, untersuchen zu können. Ein weiterer Grund für die Auswahl zweitaktiger Rhythmen war die Tatsache, daß sich in anderen Untersuchungen längerer Rhythmen im Vergleich zu kurzen Rhythmen als leichter reproduzierbar erwiesen hatten (vgl. Davidson, Colley, 1987, S. 104.) Die von Kindern geklatschten Rhythmen wurden vom Versuchsleiter mit einem Cassettenrecorder aufgenommen und später ausgewertet. Die Rhythmen wurden jeweils nur einmal vorgestellt und nur in wenigen Ausnahmefällen auf Wunsch der Kinder wiederholt Minkenberg, 1991, S. 72).“

\section{Aufzeichnung der Probandenleistung}

ja, auf Kassette

\section{Wertung}

„Bei diesem Test sollten die Vpn die ... aufgeführten Rhythmen K 1 bis K 5 nachklatschen. Damit sollte ermittelt werden, ob die Reproduktionen der Vpn langsamer oder schneller als die Vorlage ausgeführt wurden, ob sie accelerierend bzw. ritardierend nachvollzogen wurden, und welcher Teil des Rhythmusbeispiels richtig geklatscht wurde.“ (S. 73) „Da die Versuchspersonen häufig nur Teile des vorgegebenen Rhythmus reproduzierten, wurden alle richtig wiedergegebenen Teilproduktionen in die Auswertung mit einbezogen."(ebd.)

Ergebnisse für die interessierende Altersgruppe der 8 bis 9jährigen Drittklässler 
Die in der Tabelle dargestellten Ergebnisse der Kinder zwischen sieben und acht Jahren bzw. zwischen acht und zehn Jahren wurden dem Fließtext S. 201-203 sowie S. 229-232 entnommen. Grafiken sind nicht im Original enthalten.

\begin{tabular}{|l|l|l|l|l|}
\hline & \multicolumn{2}{|l|}{7 bis 8 Jahre, $\mathrm{n}=33$} & \multicolumn{2}{l|}{8 bis 10 Jahre, $\mathrm{n}=38$} \\
\hline & $\begin{array}{l}\text { ganzer Rhythmus } \\
\text { fehlerfrei repro- } \\
\text { duziert }\end{array}$ & $\begin{array}{l}\text { erster Takt richtig } \\
\text { reproduziert }\end{array}$ & $\begin{array}{l}\text { ganzer Rhythmus } \\
\text { reproduziert }\end{array}$ & $\begin{array}{l}\text { erster Takt richtig } \\
\text { reproduziert }\end{array}$ \\
\hline K1 & $12 \%$ & $33 \%$ & $54 \%$ & $18 \%$ \\
\hline K2 & $15,2 \%$ & $24,2 \%$ & $64 \%$ & $26 \%$ \\
\hline K3 & $0 \%$ & $21,2 \%$ & $13 \%$ & $10 \%$ \\
\hline K4 & $0 \%$ & $9,1 \%$ & $28 \%$ & k.A. \\
\hline K5 & $6,1 \%$ & k.A. & $0 \%$ & $8 \%$ \\
\hline
\end{tabular}




\section{A 2c Jungbluth \& Hafen (1997)}

Rhythmus nachspielen ( $\mathrm{n}=161)$ aus Rubrik I, 5-8;6 Jahre

Dieser Subtest gehört in den Teil „Einzelverfahren“ und besteht in der Rubrik II aus nachfolgend abgebildeten Rhythmen:

1.

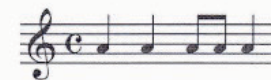

3.

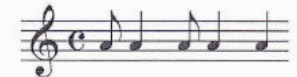

5.

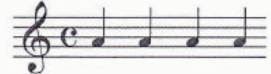

7.

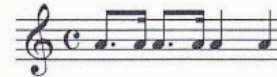

9.

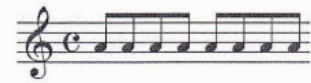

2.

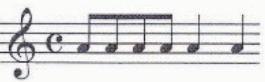

4.

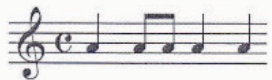

6.

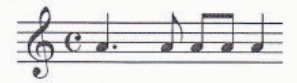

8.

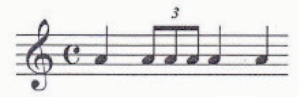

10.

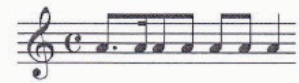

Jungbluth \& Hafen, 1997, Musik-Screening für Kinder I, S. 2

\section{Aufgabenstellung}

Die Rhythmen wurden von CD vorgespielt und von den Probanden auf einem Keyboard auf dem Ton a nachgespielt.

\section{Aufzeichnung der Probandenleistung}

keine

\section{Wertung}

„Bei richtigem Nachspiel aller rhythmischen Vorgaben werden unter, richtig ${ }^{6}$ zwei Punkte eingetragen. Wurde ein Ton weniger oder mehr gespielt, gibt es ausschließlich in der entsprechenden Spalte einen Punkt. Bei (gröberen) Fehlern im Nachspiel gibt es keinen Punkt.

Höchstpunktzahl: 20 Punkte“ (Auswertungshinweise, ohne Seitenzahl).

Ergebnisse für die interessierende Altersgruppe der 8 bis 9jährigen Drittklässler

Ergebnisse sind nicht vergleichbar, da nur Häufigkeiten der Summen für den interessierenden Rhythmus angegeben wurden. Ergebnisse beziehen sich auf 161 Probanden in der Rubrik I, 5-8;6 Jahre

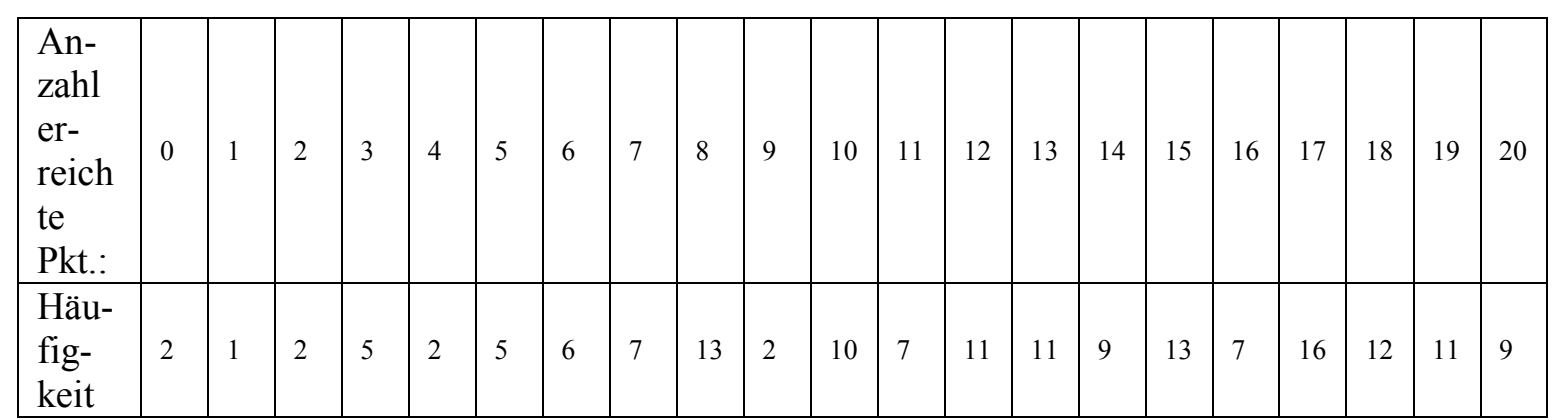

Metrum ausführen $(\mathrm{n}=161)$

Neben den ähnlich wie bei Wagner und Minkenberg angelegten Items zum Hören von Unterschieden (Gruppenverfahren) sowie zum Nachklatschen von Rhythmen (Einzelverfahren) gibt es in diesem Screening auch zwei Itemgruppen zum metrischen Bereich. Die erste Itemgruppe „Metrum ausführen“" (Einzelverfahren) fokussiert auf das Empfinden des Pulses bzw. Grundschlages eines ausgewählten Marsches. 


\section{Aufgabenstellung}

Die Probanden werden aufgefordert, beim Erklingen eines Marsches (1) aufzustehen und genauso schnell zu marschieren, wie die Musik spielt. Anschließend erklingt die Musik noch einmal, nun soll der Proband (2) stehen bleiben und klatschen. Als drittes wird die Musik noch einmal erklingen und der Proband gebeten, wiederum zu (3) klatschen und dies auch noch weiter zu tun, wenn die Musik schon aufgehört hat. Erst beim gerufenen Wort „Halt“ ist damit aufzuhören. Die dritte Aufgabe wird leicht verändert wiederholt (4) angeboten. Es wird nur ein Musikbeispiel herangezogen. (vgl. Jungbluth \& Hafen, 1997, S. 3)

\section{Aufzeichnung der Probandenleistung}

keine

\section{Wertung}

„Für die vier Bewegungsaufgaben können jeweils 2 Punkte verteilt werden.

Kriterien sind für die ganzkörperliche Bewegung (1, nach Musik, marschieren $\left.{ }^{\circ}\right)$ das vollständige oder teilweise Einhalten des der Musik zugrundeliegenden Pulses in Tempo und zeitlicher Geschlossenheit der Bewegung.

Beim ersten ,teilkörperlichen` Bewegen (2) geht es um temporechtes und gleichmäßiges Klatschen.

Bei Zahl (3) wird gezählt, wie oft ab der (imaginären), Eins` der abgeschalteten Musik bis zum ,Halt' (=die ,Eins‘ des vierten stummen Taktes) geklatscht wird. Es müssen somit 13 Klatscher erklingen $=2$ Punkte. Bei 12 und 14 Klatschern gibt es noch 1 Punkt, sonst 0 Punkte.

,Zahl' (4) wird entsprechend bewertet. Diesmal ergibt die Zahl 17 (=die ,Eins' des fünften stummen Taktes) 2 Punkte, 16 und 16 ergeben 1 Punkt, alle weiteren Zahlen 0 Punkte.“

Höchstpunktzahl: 8 Punkte (Auswertungshinweise, ohne Seitenzahl)

\section{Ergebnisse für die interessierende Altersgruppe der 8 bis 9jährigen Drittklässler}

Ergebnisse sind nicht vergleichbar, da nur Häufigkeiten der Summen für den interessierenden Subtest angegeben wurden. Warum bei Anzahl der erreichten Punkte die Rubrik 8 Punkte leer blieb ist aus den Unterlagen nicht ersichtlich.

\begin{tabular}{|l|l|l|l|l|l|}
\hline $\begin{array}{l}\text { Anzahl der } \\
\text { erreichten } \\
\text { Punkte }\end{array}$ & 0 & 2 & 4 & 6 & 8 \\
\hline Häufigkeit & 14 & 18 & 58 & 71 & k.A. in den Unterlagen \\
\hline
\end{tabular}

\section{Metrum vergleichen}

Die zweite Itemgruppe Metrum vergleichen (Gruppenverfahren) erweist sich bei näherer Betrachtung als Vergleich verschiedener Tempi. Es wird deshalb nur die Aufgabenstellung genannt.

\section{Aufgabenstellung}

„Du hörst langsame und schnelle Töne von Klanghölzern gespielt. Diese klingen langsam $(\mathrm{MM}=60)$, diese viel schneller $(\mathrm{MM}=160)$. Du hörst die Klanghölzer wieder zweimal gespielt. Zuerst spielen die blauen danach die roten Töne. Sind die blauen Töne langsam, kommt das Kreuz in das zweite Kästchen. Sind die roten Töne langsam, kommt das Kreuz in das zweite Kästchen. Spielen die blauen und roten Töne gleich schnell, kommt das Kreuz in das dritte Kästchen“ (Jungbluth \& Hafen, 1997, S. 16). 


\section{A 2d McPherson (2005)}

\section{Vom-Blatt-Spiel / Sight-reading $(\mathrm{n}=124)$}

McPherson (2005) führte die Langzeituntersuchung „From child to musician: skill development during the beginning stages of learning an instrument" mit 124 Schülern eines Schulmodells ähnlich dem Klassenmusizieren durch. Diese Studie ist in die Bereiche „Spiel geübter Stücke“, „Vom-Blatt-Spiel“, „Spiel aus dem Gedächtnis“, „Spiel nach Gehör“ sowie „Improvisieren“ unterteilt (McPherson, 2005, S. 14). Mit Hilfe der „Watkins-Farnum Performance Scale Exercises“ wird die Fähigkeit des Notenlesens im rhythmisch-metrischen und tonalen Bereich gleichzeitig getestet.

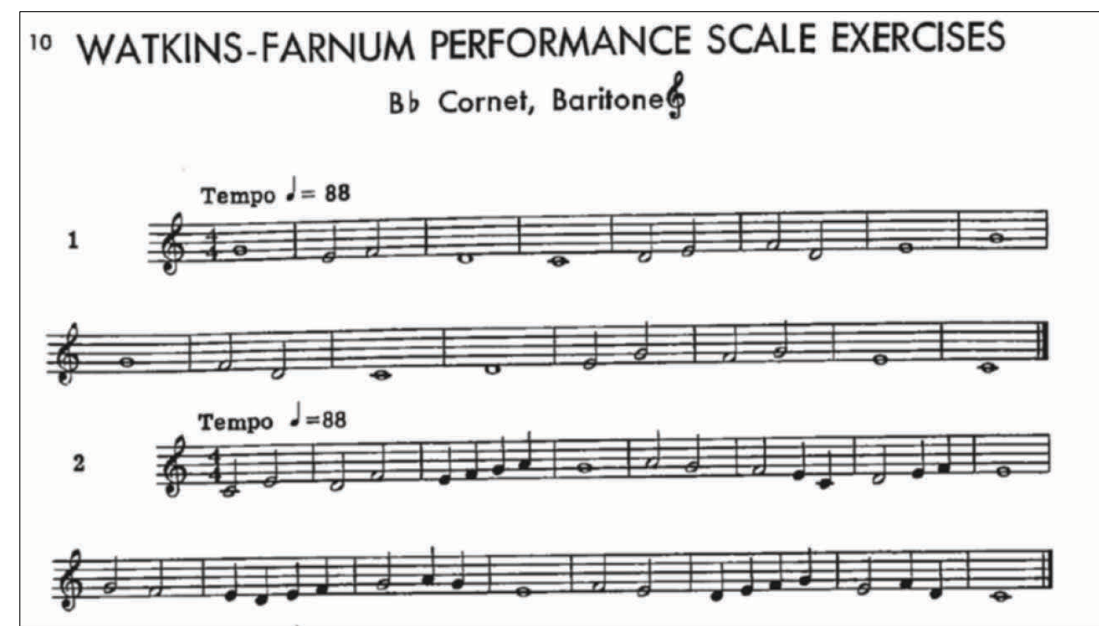

Beispiel der „Sight-read“Items für Baritonstimme; Quelle: z. B. unter http://www.union.k12.pa.u s/cms/lib6/PA01001828/C entricity/Domain $/ 122 /$ Winds $\% 20$ and $\%$ 20Mallet\%20Audition.pdf, zuletzt aufgerufen am 11.11 .2014

\section{Aufgabenstellung}

Die notierten Melodien sind auf dem erlernten Melodieinstrument im „Vom-Blatt-Spiel“ akkurat wiederzugeben. „Sight-read: Accurately reproducing music from notation that has not been previously seen or heard (McPherson, 2005, S. 9)."

Aufzeichnung der Probandenleistung

keine

\section{Wertung}

Die Auswertung dieser Itemgruppe wurde so gestaltet, dass der Versuchsleiter während des Testprozesses in einer Vorlage ankreuzte, wenn es in einem der 16 Takte einen Fehler gab (S. 11).

Ergebnisse für die interessierende Altersgruppe der 8 bis 9jährigen Drittklässler nicht vergleichbar durch tonale Bindung der Items 


\section{A 2e Hasselhorn (2015)}

\section{Rhythmusreproduktion $(\mathrm{n}=445)$}

Ergänzend soll die Dissertation von Hasselhorn zum Thema „Messbarkeit musikpraktischer Kompetenzen von Schülerinnen und Schülern“ mit einbezogen werden. Hasselhorn (2015) entwickelte ein computerbasiertes Setup, mit welchem er ausschließlich musikpraktische Kompetenzen in den Subtests „Gesang“, „Instrumentales Musizieren“ sowie „Rhythmusproduktion“ testete. Die Studie basiert auf den Ergebnissen von 445 Probanden der Jahrgangsstufe 9. Im Bereich „Rhythmusproduktion" spielten die Probanden auf einem speziell ausgestatteten Tablet mithilfe einer App, deren zweigeteiltes Display zwei unterschiedlich hohe Töne erzeugen ließ. Aufgaben zu Metrum und Grundschlag wurden über 6 bzw. 9 Items realisiert. Dies fand unter Hinzunehme von Vorspiel oder Notenbild bzw. beidem statt. Es fand eine Aufzeichnung der Ergebnisse statt, die im Nachhinein von verschiedenen Ratern nach festgelegten Ratings (ebd., S. 97-100) bewertet wurde.

\section{Aufgabenstellung}

„Diese [neun] Patterns sollten von den Schülern jeweils zu achttaktigen Playbacks gespielt werden. Als Variationsmöglichkeiten gab es hier verschiedene Tempi für die Playbacks, sowie als Hilfen während der Vorbereitung auf die Aufgaben das Präsentieren eines Notenbeispiels, das Vorspielen des Patterns oder eine Kombination aus beidem.“ (ebd., S. 65)

Gespielt wurden die Rhythmen von den Probanden auf einer eigens dafür entwickelten App auf einem Tablet.

\section{Aufzeichnung der Probandenleistung}

ja, digital

\section{Rating}

Auf der Basis der HTR-G selbst erstellte Skala zur Bewertung von Schüler-Rhythmusleistungen: HTR-R (Abschrift Tab. 6.4, Hasselhorn, 2015, S. 98)

\begin{tabular}{|c|l|}
\hline Rating & Beschreibung \\
\hline 1 & Der Schüler spielt den Rhythmus ganz richtig in nahezu oder völlig korrektem Tempo. \\
\hline 2 & Der Schüler spielt den Rhythmus fast richtig in nahezu oder völlig korrektem Tempo. \\
\hline 3 & Der Schüler spielt den Rhythmus mit einigen Fehlern, größtenteils im korrekten Tempo. \\
\hline 4 & Der Schüler spielt den Rhythmus mit einigen Fehlern in einem deutlich falschen Tempo. \\
\hline 5 & $\begin{array}{l}\text { Der Schüler ist erkennbar bemüht, die Aufgabe zu bearbeiten, spielt aber einen falschen } \\
\text { Rhythmus in einem falschen Tempo. }\end{array}$ \\
\hline 6 & Der Schüler spielt entweder gar nicht oder etwas, was nichts mit der Aufgabe zu tun hat. \\
\hline
\end{tabular}

Abweichungstoleranzen zwischen Rating 1 und 2 (oder anderen) werden nicht definiert. Der Schwerpunkt wird auf eine gute statistische Übereinstimmung der Bewertungen durch die Rater gelegt.

\section{Ergebnisse für die interessierende Altersgruppe der 8 bis 9jährigen Drittklässler}

liegen nicht vor, da die Probanden der Jahrgangsstufe 9 entstammen

\section{Rhythmusreproduktion / Metrumsaufgaben $(n=445)$}

\section{Aufgabenstellung}

„Außerdem wurden Playbacks in unterschiedlichen Musikstilen verwendet. Die Metrumsaufgaben wurden zu den gleichen Playbacks ausgeführt. Hier sollte ausschließlich das Metrum der Aufnahmen mitgespielt werden: in binärem Varianten (4/4-Takt) auf Viertel-, in den ternären Varianten (6/8-Takt) auf Achtelebene. Auf der Zählzeit 1 sollte dabei immer der tiefere Rhythmuston, auf den anderen Zählzeiten jeweils der höhere Rhythmuston gespielt werden.“ (ebd., S. 66)

\section{Aufzeichnung der Probandenleistung}

ja, digital 
Anlagen

Ergebnisse für die interessierende Altersgruppe der 8 bis 9jährigen Drittklässler liegen nicht vor, da die Probanden der Jahrgangsstufe 9 entstammen 


\section{Anlage B Material und Methoden der Testgestaltung}

\section{B 1 Testdauer mündlicher und schriftlicher Test}

Die sekundengenaue Abfolge der einzelnen Items im Videotest wurde in Tabellenform dargestellt.

Die Erteilung der Aufgabenstellung sowie der Demonstration sind unter der Abkürzung „E“ dargestellt. Die reine Aktionszeit der Probanden beläuft sich im Video (Gesamtlänge $15 \mathrm{~min} 4 \mathrm{sec}$ ) auf 8 Minuten und 30 Sekunden.

\begin{tabular}{|l|l|l|l|l|l|l|l|}
\hline Items & & & Start & & A 1 & & A 2 \\
\hline Inhalt & $\begin{array}{l}\text { Begrü- } \\
\text { Bung }\end{array}$ & E & $\begin{array}{l}\text { Rhythmus } \\
\text { Bruder } \\
\text { Jacob }\end{array}$ & E & $\begin{array}{l}\text { Grundschlag } \\
\text { Bruder Jacob }\end{array}$ & E & $\begin{array}{l}\text { Metrum } \\
\text { Bruder } \\
\text { Jacob }\end{array}$ \\
\hline Tempo & & & MM 106 & & MM 106 & & MM 106 \\
\hline Beginn & $00: 00$ & $00: 04$ & $01: 04$ & $01: 54$ & $02: 48$ & $03: 38$ & $04: 20$ \\
\hline Dauer & $00: 04$ & $01: 00$ & $00: 50$ & $00: 54$ & $00: 50$ & $00: 44$ & $00: 48$ \\
\hline
\end{tabular}

\begin{tabular}{|l|l|l|l|l|l|l|l|}
\hline & A 3 & & A 4 & & B 1 - 4 & & D 1 - 4 \\
\hline E & $\begin{array}{l}\text { Metrum Me- } \\
\text { nuett }\end{array}$ & E & $\begin{array}{l}\text { Metrum Pop- } \\
\text { song }\end{array}$ & E & $\begin{array}{l}\text { Call- } \\
\text { Response }\end{array}$ & E & $\begin{array}{l}\text { Call- } \\
\text { Answer }\end{array}$ \\
\hline & MM 96 & & MM 132 & & MM 86 & & MM 86 \\
\hline 05:08 & $05: 38$ & $06: 14$ & $06: 44$ & $07: 40$ & $09: 06$ & $10: 26$ & $11: 14$ \\
\hline 00:30 & $00: 36$ & $00: 30$ & $00: 56$ & $01: 26$ & $01: 20$ & $00: 48$ & $00: 56$ \\
\hline
\end{tabular}

\begin{tabular}{|c|c|c|c|c|c|c|c|c|c|}
\hline \multirow[b]{2}{*}{$\begin{array}{l}\text { E + } \\
\text { Übung } \\
\text { Rhyth- } \\
\text { mus }\end{array}$} & \multicolumn{3}{|c|}{ zusätzlicher Subtests } & \multirow[b]{2}{*}{$\begin{array}{l}\text { Verab- } \\
\text { schie- } \\
\text { dung }\end{array}$} & \multirow[b]{2}{*}{$\begin{array}{l}\text { Ende } \\
\text { Vi- } \\
\text { deo }\end{array}$} & \multirow[b]{2}{*}{$\mathrm{E}$} & \multirow{2}{*}{\multicolumn{2}{|c|}{$\begin{array}{ll}\text { e 1 - 4 } & \text { E 1 - 4 } \\
\text { nach } & \\
\text { ikoni- } & \text { nach } \\
\text { scher } & \text { Rhythmus- } \\
\text { Notation } & \text { notation } \\
\text { spielen } & \text { spielen } \\
\end{array}$}} & \multirow[b]{2}{*}{$\begin{array}{l}\text { ec } 1-4 \\
\text { nach Rhyth- } \\
\text { musnotation } \\
\text { spielen und } \\
\text { gleichzeitig } \\
\text { Rhythmus- } \\
\text { sprache spre- } \\
\text { chen }\end{array}$} \\
\hline & $\begin{array}{l}\text { Play } \\
\text { to- } \\
\text { gether } \\
1, \text { „G“ }\end{array}$ & $\begin{array}{l}\text { E+ } \\
\text { Übung } \\
\text { Rhyth- } \\
\text { mus }\end{array}$ & $\begin{array}{l}\text { Play } \\
\text { to- } \\
\text { gether } \\
2 \text { „G“ }\end{array}$ & & & & & & \\
\hline & \begin{tabular}{|l|} 
MM \\
86
\end{tabular} & & $\begin{array}{l}\text { MM } \\
86\end{array}$ & & & & & & \\
\hline 12:00 & $12: 56$ & $13: 28$ & $14: 24$ & $14: 56$ & $\begin{array}{l}15: 0 \\
4 \\
\end{array}$ & ca. & \multicolumn{3}{|c|}{ ca. 3 bis 4 Minuten } \\
\hline 00:56 & $00: 32$ & $00: 56$ & $00: 32$ & 00:08 & & $\begin{array}{l}\text { 01:0 } \\
0\end{array}$ & \multicolumn{3}{|c|}{ 01:00 Spielzeit } \\
\hline
\end{tabular}

$\mathrm{E}=$ Erklärung 
Im Folgenden ist die Abfolge der einzelnen Items des schriftlichen Tests in Tabellenform zu sehen. Die Erteilung der Aufgabenstellung sowie der Demonstration sind unter der Abkürzung „E“ dargestellt. Die reine Aktionszeit der Probanden beläuft sich auf etwa 18 Minuten bei einer ungefähren Dauer von 28 Minuten.

\begin{tabular}{|l|l|l|l|l|l|l|l|l|l|l|l|}
\hline Ablauf & & & Subtest H & & f 1 & F 1 & cf 1 & & f2 & F 2 & $\begin{array}{l}\text { cf } \\
2\end{array}$ \\
\hline Inhalt & $\begin{array}{l}\text { Begrüßung und } \\
\text { Vorbereitung }\end{array}$ & E & $\begin{array}{l}\text { Wozu } \\
\text { brauchst du } \\
\ldots ?\end{array}$ & E & Rh 1 & E & Rh 2 \\
\hline Tempo & & & & & MM 86 & & MM 86 \\
\hline Beginn & $0: 00$ & $02: 10$ & $02: 40$ & $04: 20$ & $09: 50$ & $13: 20$ & $13: 50$ \\
\hline Dauer & $2: 10$ & $00: 30$ & $01: 40$ & $05: 30$ & $03: 30$ & $0: 30$ & $03: 30$ \\
\hline
\end{tabular}

\begin{tabular}{|l|l|l|l|l|l|l|l|l|l|l|}
\hline & f 3 & F 3 & cf 3 & & f 4 & F 4 & cf 4 & & Subtest I & \\
\hline E & Rh 3 & E & Rh 4 & & E & $\begin{array}{l}\text { Vergleichendes } \\
\text { Hören }\end{array}$ & $\begin{array}{l}\text { Verabschiedung und } \\
\text { einsammeln der Materi- } \\
\text { alien }\end{array}$ \\
\hline & MM 86 & & MM 86 & & MM 86 & \\
\hline $17: 20$ & $17: 50$ & $19: 30$ & $20: 00$ & $23: 30$ & $25: 00$ & $28: 00$ \\
\hline $0: 30$ & $02: 40$ & $0: 30$ & $03: 30$ & $1: 30$ & $03: 00$ & \\
\hline
\end{tabular}




\section{B 2 Toleranzbereiche der Rhythmenauswertung}

An dieser Stelle wird die Bewertung der Rhythmen anhand der drei Faktoren des Algorithmus für Item B 1 (Rhythmus 1a) beispielhaft dargestellt.

\begin{tabular}{|c|c|c|c|c|c|c|c|c|c|c|c|c|c|c|c|c|c|c|c|c|c|}
\hline & Pos. & Dau. & Pos. & Dau. & Pos. & Dau. & Pos. & Dau. & Pos. & Dau. & Pos. & Diff. $d$. & Dau. & & Einso & $t z, R$ & ythm & $S, T E$ & npo & & \\
\hline & 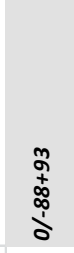 & $\begin{array}{l}+/- \\
95 ; \\
694: \\
599- \\
789\end{array}$ & $\begin{array}{l}\infty \\
0 \\
0 \\
0 \\
0 \\
\dot{\sigma} \\
0 \\
\ddot{\tilde{~}} \\
\infty \\
\infty \\
1 \\
+\end{array}$ & $\begin{array}{l}\text { +/- } \\
95 \\
694: \\
599- \\
789\end{array}$ & 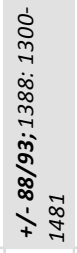 & $\begin{array}{l}+/- \\
75 \\
347: \\
272- \\
422\end{array}$ & 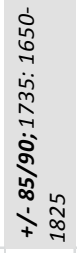 & $\begin{array}{l}\text { +/- } \\
75 ; \\
347: \\
272- \\
422\end{array}$ & 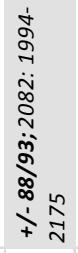 & $\begin{array}{l}\text { +/- } \\
95 ; \\
694: \\
599- \\
789\end{array}$ & 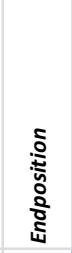 & 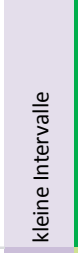 & 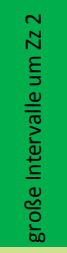 & 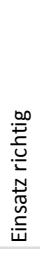 & 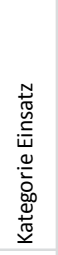 & 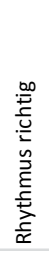 & 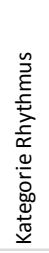 & 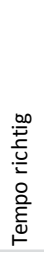 & 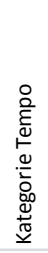 & 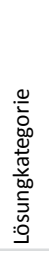 & 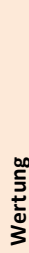 \\
\hline Proband & 1 & & 2 & & 3 & & $u$ & & 4 & & & 85 & 155 & & & & & & & & \\
\hline 4 & -41 & 694 & 653 & 729 & 1382 & 321 & 1703 & 360 & 2063 & 713 & 2776 & -39 & -35 & 1 & 0 & 1 & 0 & 1 & 0 & A & 1 \\
\hline 74 & -50 & 678 & 628 & 649 & 1277 & 359 & 1636 & 346 & 1982 & 794 & 2776 & 13 & 29 & 1 & 0 & 1 & 0 & 0 & 10 & B & 0 \\
\hline 221 & 62 & 560 & 622 & 650 & 1272 & $327^{\circ}$ & 1599 & 321 & 1920 & 856 & 2776 & 6 & -90 & 1 & 0 & 1 & 0 & 0 & 11 & B & 0 \\
\hline 193 & 23 & 756 & 779 & 758 & 1537 & $310^{\prime \prime}$ & 1847 & 341 & 2188 & 588 & 2776 & -31 & -2 & 1 & 0 & 1 & 0 & 0 & 20 & B & 0 \\
\hline 187 & 5 & 731 & 736 & 748 & 1484 & 296 & 1780 & 319 & 2099 & 677 & 2776 & -23 & -17 & 1 & 0 & 1 & 0 & 0 & 32 & B & 0 \\
\hline 209 & -14 & 831 & 817 & 507 & 1324 & $318^{\circ}$ & 1642 & 386 & 2028 & 748 & 2776 & -68 & 324 & 1 & 0 & 1 & 0 & 0 & 33 & B & 0 \\
\hline 227 & -139 & 691 & 552 & 355 & 907 & $289^{\prime \prime}$ & 1196 & 635 & 1831 & 945 & 2776 & -346 & 336 & 0 & 100 & 1 & 0 & 0 & 60 & C & 0 \\
\hline 177 & 543 & 656 & 1199 & 515 & 1714 & $416^{\prime \prime}$ & 2130 & 326 & 2456 & 320 & 2776 & 90 & 141 & 0 & 222 & 0 & 70 & 0 & 90 & $\mathrm{D}$ & 0 \\
\hline
\end{tabular}

\begin{tabular}{|c|c|c|c|c|c|}
\hline Bereiche & erweit. TB- EwB & \multicolumn{3}{|c|}{ Toleranzbereich - TB } & erweit. TB- EwB \\
\hline Toleranz & - & -88 & & 93 & + \\
\hline \multirow{4}{*}{$\begin{array}{c}\text { Toleranzspiel- } \\
\text { räume }\end{array}$} & -176 bis -89 & -88 & 0 & 93 & 94 bis 186 \\
\hline & 518 bis 605 & 606 & 694 & 787 & 788 bis 880 \\
\hline & 1212 bis 1299 & 1300 & 1388 & 1481 & 1482 bis 1574 \\
\hline & 1906 bis 1993 & 1994 & 2082 & 2175 & 2176 bis 2268 \\
\hline Kategorie Einsatz & 100 & \multicolumn{3}{|c|}{0} & 200 \\
\hline Kategorie Tempo & $10,11,30,31,33$ & \multicolumn{3}{|c|}{0} & $20,22,30,32,33$ \\
\hline
\end{tabular}

\begin{tabular}{|c|c|c|c|c|c|}
\hline Bereiche & erweit. TB- EwB & \multicolumn{3}{|c|}{ Toleranzbereich - TB } & erweit. TB- EwB \\
\hline Toleranz & - & -85 & $\begin{array}{c}\text { exa ste } \\
\text { Posi Ion }\end{array}$ & 90 & + \\
\hline \multirow{4}{*}{$\begin{array}{c}\text { Toleranzspiel- } \\
\text { räume }\end{array}$} & 177 bis 261 & 262 & 347 & 437 & 438 bis 527 \\
\hline & 871 bis 955 & 956 & 1041 & 1131 & 1042 bis 1221 \\
\hline & 1565 bis 1655 & 1650 & 1735 & 1825 & 1735 bis 1915 \\
\hline & 2259 bis 2343 & 2344 & 2429 & 2519 & 2430 bis 2609 \\
\hline Kategorie Einsatz & 100 & \multicolumn{3}{|c|}{0} & 200 \\
\hline Kategorie Tempo & $10,11,30,31,33$ & \multicolumn{3}{|c|}{0} & $20,22,30,32,33$ \\
\hline
\end{tabular}




\section{Beispiel Position Hauptschläge und Nebenschläge}

Alle Schläge der Rhythmen müssen auf den Hauptschlägen den Werten der exakten Position (maximal -88 ms früher bzw. + 93 ms später) bzw. auf den Nebenschlägen (maximal - 85 ms früher bzw. + 90 ms später) entsprechen. Nur für die gemessenen Positionen der Rhythmen der Probanden 4 und 212 trifft dies zu. Der Rhythmus von Proband 4 weist auch keine anderen Auffälligkeiten in Bezug auf Dauern sowie die Differenzen der Dauern auf. Er wird als richtig gewertet. Der Rhythmus von Proband 212 weist eine Auffälligkeit auf Dauer 2 auf.

\section{Beispiel Toleranzbereich für die Dauern verschiedener Schlaglängen}

Alle Viertelschläge in diesem Rhythmus dürfen eine exakte Dauer von 694 Millisekunden nur um 95 Millisekunden über- bzw. unterschreiten (siehe Tabelle darunter). Die exakte Dauer der zwei Achtelschläge von 347 Millisekunden darf nur um 75 Millisekunden über- bzw. unterschritten werden. Nur für die berechneten Dauern der Schläge der Probanden 4 und 187 trifft dies zu.

\begin{tabular}{|c|c|c|c|c|c|c|}
\hline $\begin{array}{c}\text { Extrem- } \\
\text { bereich } \\
\text { - ExB }\end{array}$ & $\begin{array}{c}\text { erweit. } \\
\text { TB- EwB }\end{array}$ & \multicolumn{2}{|c|}{ Toleranzbereich - TB } & $\begin{array}{c}\text { erweit. } \\
\text { TB- EwB }\end{array}$ & $\begin{array}{c}\text { Extrem- } \\
\text { bereich } \\
- \text { ExB }\end{array}$ \\
\hline & & - & Exakte & + & & \\
\hline-75 & -75 & -75 & & 75 & 75 & 75 \\
\hline 122 & 197 & 272 & 347 & 422 & 497 & 572 \\
\hline-95 & -95 & -95 & & 95 & 95 & 95 \\
\hline 409 & 504 & 599 & 694 & 789 & 884 & 979 \\
\hline-75 & -75 & -75 & & 75 & 75 & 75 \\
\hline 816 & 891 & 966 & 1041 & 1116 & 1191 & 1266 \\
\hline
\end{tabular}

\section{Beispiel Toleranzbereich der Differenzen der Dauern gleicher Schlaglängen}

Die zwei Viertelschläge am Anfang des Rhythmus dürfen in ihrer Dauer nur um 155 Millisekunden differieren. Die zwei Achtelschläge in der Mitte des Rhythmus dürfen sich in ihrer Dauer nur um 85 Millisekunden unterscheiden (siehe Tabelle darunter). Die berechneten Differenzen der Probanden 55, 209, 227 und 177 überschreiten diesen Toleranzbereich.

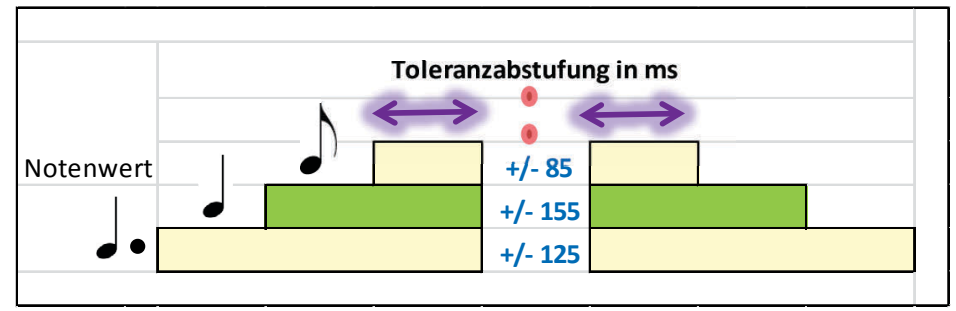




\section{Fallbeispiele}

Wie bereits erwähnt kann nur der Rhythmus des Probanden 4 als richtig gewertet werden. Alle anderen Rhythmen wiesen in Bezug auf Positionen, Dauern oder / und Differenzen der Dauern Überschreitungen der Toleranzbereiche auf.

Proband 74 spielt die Schläge auf den Positionen 3, 3u sowie 4 zu früh (hellblaue Formatierung). Der Rhythmus wird accelerierend gespielt und erhält die Fehlerkategorie 10.

Proband 221 spielt die Schläge auf den Positionen 3, 3u sowie 4 zu früh (hellblaue Formatierung). Zusätzlich ist die Dauer zwischen Position 1 und 2 zu kurz. Der Rhythmus wird sprunghaft schneller gespielt und erhält die Fehlerkategorie 11.

Proband 193 spielt die Schläge auf den Positionen 3, 3u sowie 4 zu spät (hellrote Formatierung). Der Rhythmus wird ritardierend gespielt und erhält die Fehlerkategorie 20.

Proband 207 spielt den Schlag auf Positionen 1 zu spät (hellrote Formatierung), Fehlerkategorie 100 für zu späten Einsatz des Rhythmus. Zusätzlich ist die Dauer zwischen Position 2 und 3 zu kurz. Der Rhythmus erhält in Bezug auf das Tempo die Fehlerkategorie 30, alle Positionen richtig, Fehler nur bei Dauern und Differenzen. (Position 1 Einsatz des Rhythmus wird separat gewertet.)

Proband 212 spielt den Rhythmus auf allen Positionen richtig. Nur die Dauer zwischen Position 2 und 3 ist zu kurz. Der Rhythmus erhält in Bezug auf das Tempo die Fehlerkategorie 30, alle Positionen richtig, Fehler nur bei Dauern und Differenzen.

Proband 55 spielt die Schläge auf den Positionen 3 sowie $3 u$ zu früh (hellblaue Formatierung), auf Position 4 wird der Toleranzbereich wieder eingehalten. Der Rhythmus wird auf einigen Positionen im negativ erweiterten Toleranzbereich gespielt, die Endposition liegt jedoch im Toleranzbereich. Der Rhythmus erhält die Fehlerkategorie 31.

Proband 187 spielt den Schlag auf Position 3 zu spät (hellrote Formatierung), auf Position 3u sowie 4 wird der Toleranzbereich wieder eingehalten. Der Rhythmus wird auf einigen Positionen im positiv erweiterten Toleranzbereich gespielt, die Endposition liegt jedoch im Toleranzbereich. Der Rhythmus erhält die Fehlerkategorie 32.

Proband 209 spielt den Schlag auf Position 2 zu spät (hellrote Formatierung) und den Schlag auf Position $3 \mathrm{u}$ zu früh (hellblaue Formatierung). Auf den anderen Positionen wird der Toleranzbereich eingehalten. Damit entsteht eine Kombination aus negativem und positiv erweitertem Toleranzbereich (hellblau und hellrot). Der Rhythmus erhält die Fehlerkategorie 33.

Proband 227 spielt die Schläge auf Position 2, 3, 3u sowie 4 leicht zu spät bzw. viel zu spät (hellund dunkelblaue Formatierung). Mehrere Dauern liegen im erweiterten sowie eine Dauer im Extrembereich. Der Rhythmus erhält die Fehlerkategorie 60.

Proband 177 spielt fast alle Schläge viel zu spät (dunkelrote Formatierung). Der Rhythmus beginnt extrem zu spät und ist damit nicht mehr bewertbar. Er erhält die Fehlerkategorie 90. 


\section{B 3 Ausgewählte Fehlerkategorien im Subtest B}

Es werden hier für den Subtest B die ausführlichen Fehlerkategorien. Diese finden auch in den Subtests D und E Anwendung.

Rubrik Rhythmus an sich (Subtest B, D, E, ec)

\begin{tabular}{|l|c|}
\hline Rhythmus erkennbar & 0 \\
\hline Rhythmus erkennbar, fehlender Schlag am Ende & 51 \\
\hline Rhythmus falsch (kann den Positionen im Takt nicht sinnvoll zugeordnet werden) & 61 \\
\hline Rhythmus falsch, jedoch ein anderer Rhythmus gut erkennbar & 62 \\
\hline Rhythmus beginnt mehr als Achtelschlag zu früh / zu spät und wird nicht gewertet & 70 \\
\hline kein Response & 80 \\
\hline
\end{tabular}

Rubrik Einsatz des Rhythmus (Subtest B, D)

\begin{tabular}{|l|l|c|}
\hline extrem zu früh & früher als -264 & 111 \\
\hline viel zu früh & -177 bis $-264 \mathrm{~ms}$ & 101 \\
\hline zu früh & -88 bis $-176 \mathrm{~ms}$ & 100 \\
\hline im Toleranzbereich & zwischen -88 und $93 \mathrm{~ms}$ & 0 \\
\hline zu spät & 94 bis $186 \mathrm{~ms}$ & 200 \\
\hline viel zu spät & 187 bis $279 \mathrm{~ms}$ & 202 \\
\hline extrem zu spät & später als $279 \mathrm{~ms}$ & 222 \\
\hline nicht bestimmbar & durch Überlagerung mit Call oder keine Rhythmusspiel & 90 \\
\hline
\end{tabular}

Rubrik Tempo des Rhythmus (Subtest B, D, E, ec)

\begin{tabular}{|c|c|}
\hline korrekt: innerhalb des Toleranzbereichs auf allen Positionen und bei allen Dauern & 0 \\
\hline \multicolumn{2}{|l|}{ leicht schwankend, Endposition über/unter dem Toleranzbereich sowie ... } \\
\hline $\begin{array}{l}\text { kontinuierlich schneller werdend: eine oder mehrere Positionen im negativ erweiterten Toleranzbe- } \\
\text { reich (hellblau); Anzahl der aufeinanderfolgend richtigen Dauern }=x-1\end{array}$ & 10 \\
\hline $\begin{array}{l}\text { sprunghaft schneller werdend: eine oder mehrere Positionen im negativ erweiterten Toleranzbereich } \\
\text { (hellblau) und eine deutlich zu kurze Dauermeist vor der ersten Fehlposition oder früher, Anzahl der } \\
\text { aufeinanderfolgend richtigen Dauern beliebig }\end{array}$ & 11 \\
\hline $\begin{array}{l}\text { kontinuierlich langsamer werdend: eine oder mehrere Positionen im positiv erweiterten Toleranzbe- } \\
\text { reich (hellrot); Anzahl der aufeinanderfolgend richtigen Dauern }=x-1\end{array}$ & 20 \\
\hline $\begin{array}{l}\text { extrem langsamer werdend: eine oder mehrere Positionen im positiv erweiterten Toleranzbereich } \\
\text { (hellrot) und eine deutlich zu lange Dauer meist vor der ersten Fehlposition oder früher, Anzahl der } \\
\text { aufeinanderfolgend richtigen Dauern beliebig }\end{array}$ & 22 \\
\hline \multicolumn{2}{|l|}{ leicht schwankend, Endposition im Toleranzbereich sowie ... } \\
\hline alle Positionen richtig, Fehler nur bei Dauern oder Differenzen & 30 \\
\hline $\begin{array}{l}\text { einige bis alle Positionen im negativ erweiterten Toleranzbereich (hellblau), auch Fehler bei Dauern } \\
\text { oder Differenzen }\end{array}$ & 31 \\
\hline $\begin{array}{l}\text { einige bis alle Positionen im positiv erweiterten Toleranzbereich (hellrot), auch Fehler bei Dauern } \\
\text { oder Differenzen }\end{array}$ & 32 \\
\hline \multicolumn{2}{|l|}{ unregelmäßig, Endposition im erweiterten Toleranzbereich sowie ... } \\
\hline $\begin{array}{l}\text { Kombination aus Toleranzbereich sowie erweitertem Toleranzbereich auf mindestens drei Positio- } \\
\text { nen im Schema falsch-richtig-falsch }\end{array}$ & 33 \\
\hline Kombination aus negativ und positiv erweiterten Toleranzbereich (hellblau und hellrot) & 33 \\
\hline \multicolumn{2}{|l|}{ stark unregelmäßig } \\
\hline ein oder mehrere Werte im Extrembereich & 60 \\
\hline $\begin{array}{l}\text { Tempo nicht bestimmbar; aufgrund vorgelagerter Fehler (Kat. 61, } 70 \text { bzw. } 80 \text { in der Rubrik Rhyth- } \\
\text { mus an sich) }\end{array}$ & 90 \\
\hline Tempo nicht bestimmbar; aufgrund vorgelagerter Fehler (Kat. 62 in der Rubrik Rhythmus an sich) & 91 \\
\hline
\end{tabular}




\section{Beispiele für konkrete Auswertungen}

Für den Umgang mit den verschiedenen Fehlerkategorien werden hier Beispiele gegeben. Es wird Bezug zur wiederholt dargestellten Abb. Abb. 3-23 genommen. Zur besseren Lesbarkeit wurde sie hier um die Angabe der Fehlerkategorien erneut abgebildet.

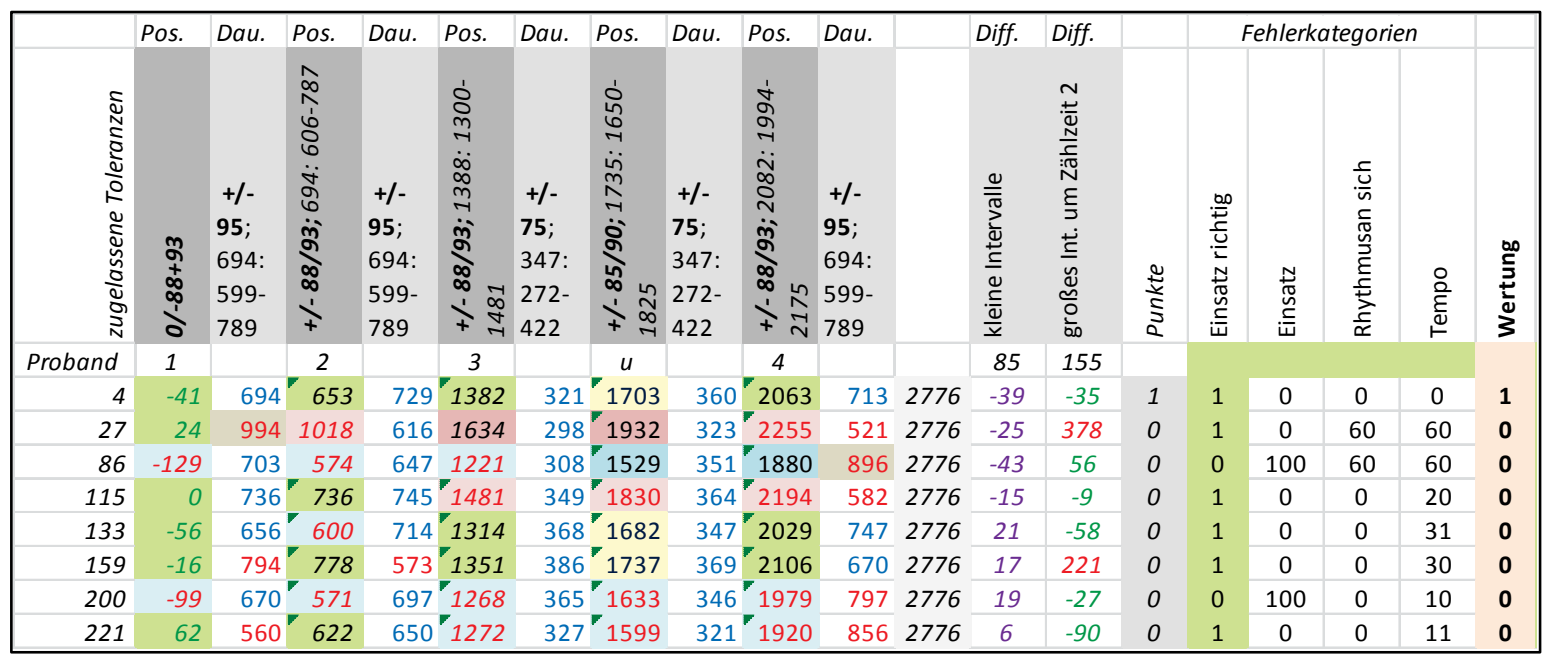

Proband 4 spielt Item B 1 in allen Positionen, Dauern sowie Differenzen der Dauern korrekt. Es gibt keine zu berücksichtigende Fehlerkategorie.

Proband 27 spielt Item B 1 so, dass nur der Schlag auf die erste Position richtig gespielt wurde, alle anderen Positionen liegen im erweiterten Toleranzbereich oder im Extrembereich. Ebenfalls liegt der Wert für die Dauer von Schlag 1 im Extrembereich. Dem Rhythmus wird in der Rubrik Tempo die Fehlerkategorie 60 ein oder mehrere Werte aus dem Extrembereich sowie in der Rubrik Rhythmus an sich die Fehlerkategorie 60 Rhythmus entstellt zugeordnet.

Proband 115 spielt Item B 1 so, dass die letzten drei Schläge ihre Positionen im positiv erweiterten Toleranzbereich haben. Dieser Rhythmus wird stark ritardierend gespielt. Die letzte Dauer ist deshalb zu kurz und liegt im erweiterten Toleranzbereich. Dem Rhythmus wird in der Rubrik Tempo die Fehlerkategorie 20 kontinuierlich langsamer werdend, eine oder mehrere Positionen im positiv erweiterten Toleranzbereich (hellrot); Anzahl der aufeinanderfolgend richtigen Dauern $=x-1$ zugeordnet.

Proband 159 spielt Item B 1 so, dass alle Schläge auf den richtigen Positionen liegen. Zwei Dauern und eine Differenz der Dauern liegen im erweiterten Toleranzbereich. Dem Rhythmus wird in der Rubrik Tempo die Fehlerkategorie 30 leicht schwankend, Endposition im Toleranzbereich und alle Positionen richtig, Fehler nur bei Dauern oder Differenzen zugeordnet.

Proband 221 spielt Item B 1 so, dass die letzten drei Schläge ihre Positionen im negativ erweiterten Toleranzbereich hatten. Dieser Rhythmus wurde stark accelerierend gespielt. Zwei Dauern waren im erweiterten Toleranzbereich. Dem Rhythmus wurde die Fehlerkategorie 11 sprunghaft schneller werdend und eine deutlich zu kurze Dauer, meist vor der ersten Fehlposition oder früher, Anzahl der aufeinanderfolgend richtigen Dauern beliebig zugeordnet. 


\section{B 4 Testbogen}

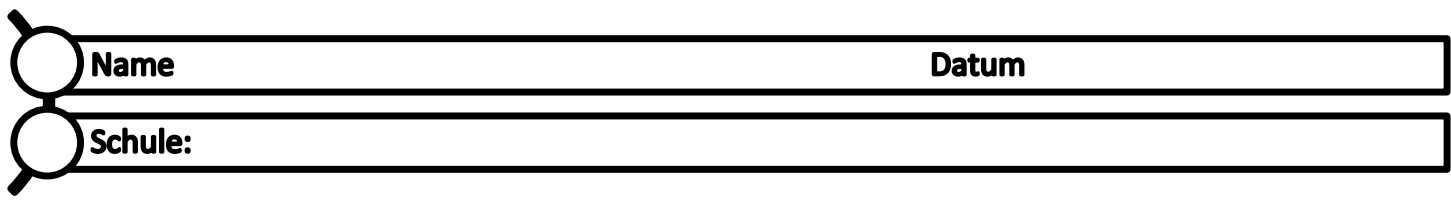

Schüler in der Grundschule lernen ab der 3. Klasse die Notenschrift kennen.

Wozu kann die Notenschrift für einen Grundschüler, z. B. dich, wichtig sein?

Schreibe deine Gedanken auf.

Du hörst jeweils zwei Rhythmen. Kreuze an, ob diese beiden Rhythmen gleich oder verschieden sind. Wenn du dir nicht sicher bist, kreuze an „Weiß es nicht.“

Achtung! Rhythmus E ist doppelt so lang wie die anderen Rhythmen.

\begin{tabular}{|ccccccc|}
\hline A & $\circ$ & gleich & $\circ$ & verschieden & $\circ$ & Weiß es nicht. \\
B & $\circ$ & gleich & $\circ$ & verschieden & $\circ$ & Weiß es nicht. \\
C & $\circ$ & gleich & $\circ$ & verschieden & $\circ$ & Weiß es nicht. \\
D & $\circ$ & gleich & $\circ$ & verschieden & $\circ$ & Weiß es nicht. \\
E & $\circ$ & gleich & $\circ$ & verschieden & $\circ$ & Weiß es nicht. \\
\hline
\end{tabular}

Über viele Jahre haben die Menschen keine Notenschrift gekannt. Sie haben Lieder, Tänze und Musikwerke durch gemeinsames Musizieren von einer Generation zur nächsten Generation weitergegeben. Erst vor tausend Jahren begannen die Menschen, Musik aufzuschreiben. Diese Schrift, die sogenannte Notenschrift, hat sich dabei immer wieder verändert.

Nun sollst du in einer Schrift, die du dir ausdenkst oder die du kennst, vier Rhythmen aufschreiben.

Wir musizieren gemeinsam diese Rhythmen. Du schreibst sie auf. Verwende dazu im weißen Rahmen Zeichen, Punkte, Linien. Verwende in den grauen Rahmen Rhythmussprache oder Notenschrift, wenn du es schon gelernt hast. 


\begin{tabular}{|l|l|}
\hline Rhythmus 1 Selbst ausgedachte Notation \\
\multicolumn{2}{|c|}{} \\
\hline Rhythmussprache & Notenschrift \\
\hline
\end{tabular}

\begin{tabular}{|l|l|}
\hline Rhythmus $2 \quad$ selbst ausgedachte Notation \\
\hline \\
\hline Rhythmussprache & Notenschrift \\
\hline
\end{tabular}

\begin{tabular}{|l|l|}
\hline Rhythmus 3 selbst ausgedachte Notation \\
\\
\hline Rhythmussprache & Notenschrift \\
\hline & \\
\hline
\end{tabular}

\begin{tabular}{|l|l|}
\hline Rhythmus $4 \quad$ selbst ausgedachte Notation \\
\\
\hline Rhythmussprache & Notenschrift \\
\hline & \\
\hline
\end{tabular}

Dankeschön fürs Mitmachen! 


\section{B 5 Musiziervorlagen}

Untertest e „Lesen und Musizieren von Rhythmen nach Zeichen“
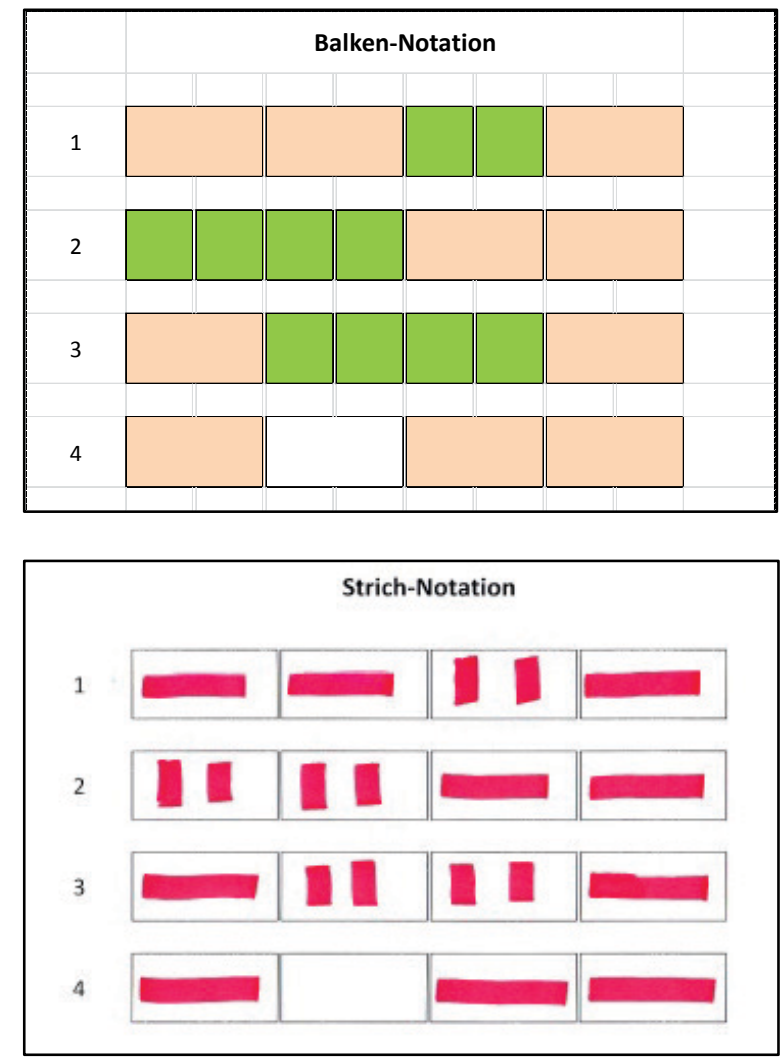

Untertest E „Lesen und Musizieren von Rhythmen nach Noten“ sowie ec „Lesen und Musizieren von Rhythmen nach Noten und sprechen von Rhythmussilben“
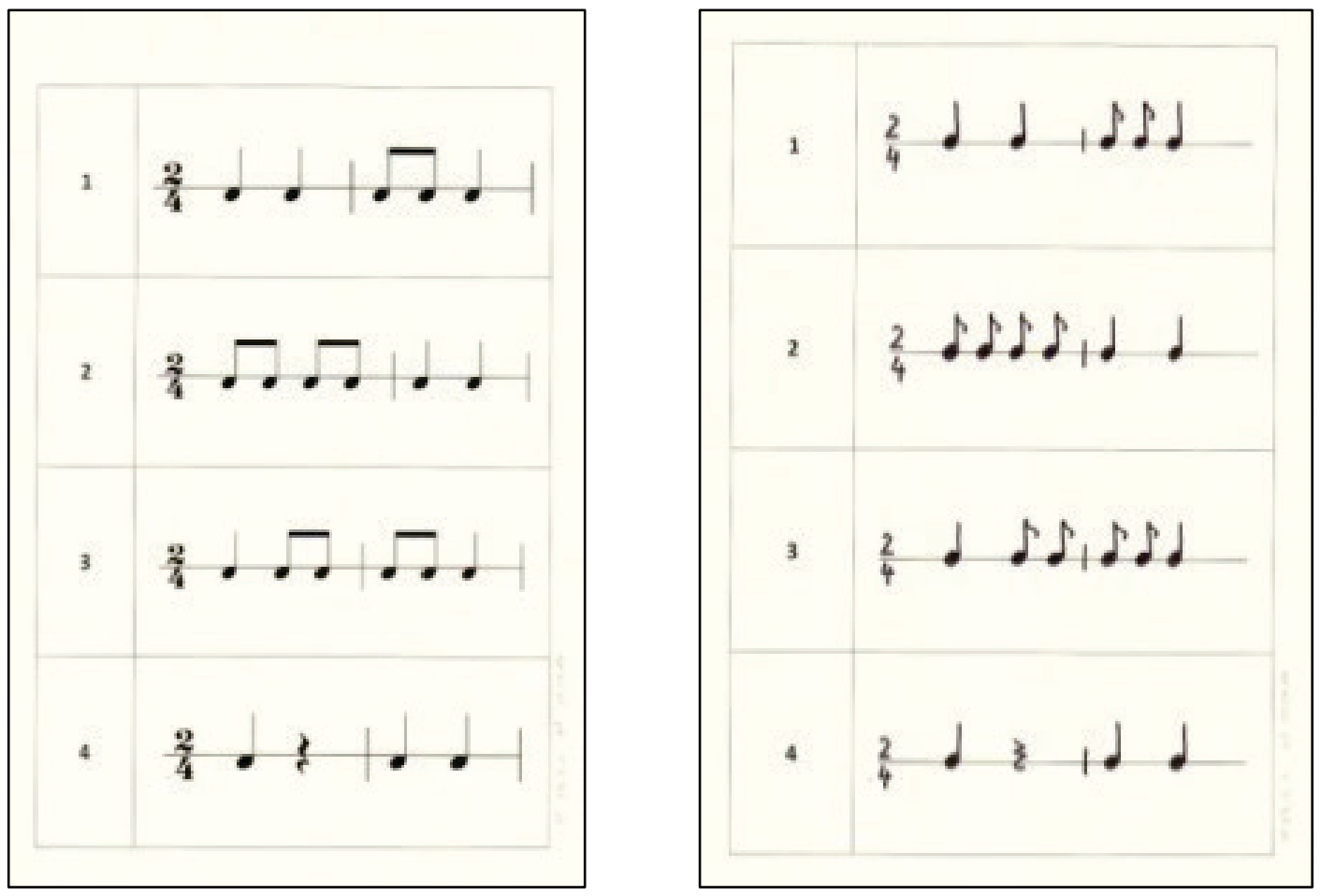


\section{Anlage C Testerhebung}

\section{1 Teststärke (Power) und kritischer Wert t (Critical t)}

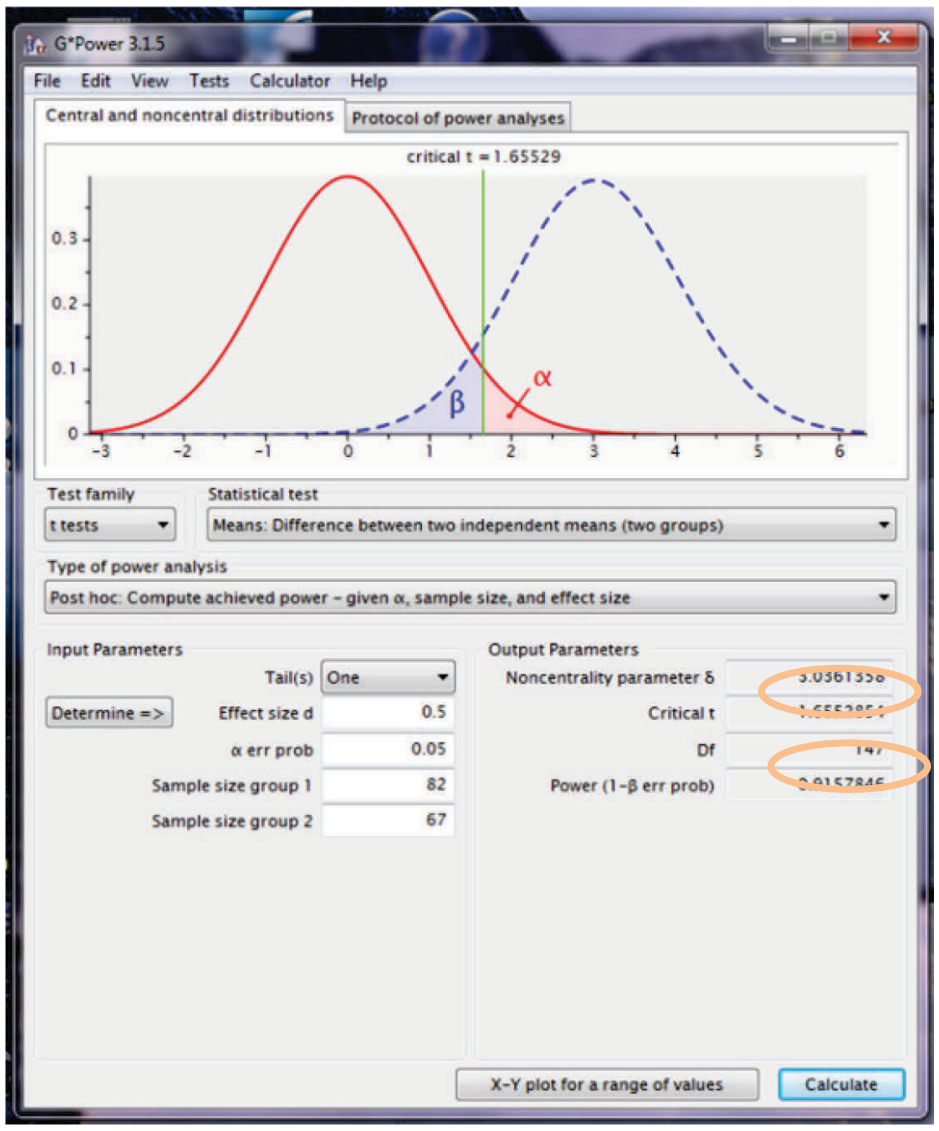

\section{2 Histogramme zur Normalverteilung der Daten}

Vortest, $\mathrm{n}=149$

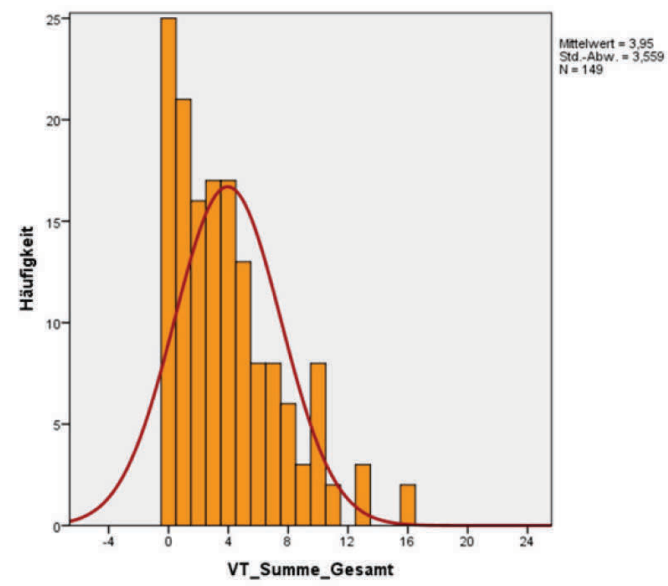


Nachtest, $\mathrm{n}=149$

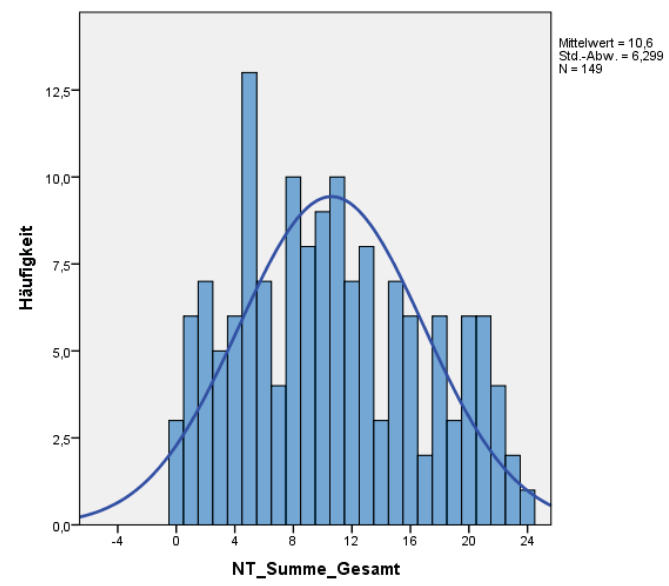

\begin{tabular}{|l|r|}
\hline \multicolumn{2}{|c|}{ Statistik für Test } \\
\hline & NT_Summe_Gesamt \\
\hline Chi-Quadrat & $32,711^{\mathrm{a}}$ \\
\hline df & \\
\hline $\begin{array}{l}\text { Asympto- } \\
\text { tische } \\
\text { Signifikanz }\end{array}$ \\
\hline $\begin{array}{l}\text { a. Bei 0 Zellen (0,0\%) werden weniger als } \\
5 \text { Häufigkeiten erwartet. Die kleinste } \\
\text { erwartete Zellenhäufigkeit ist 6,0. }\end{array}$ \\
\hline
\end{tabular}

Nachtest, UG, $\mathrm{n}=82$

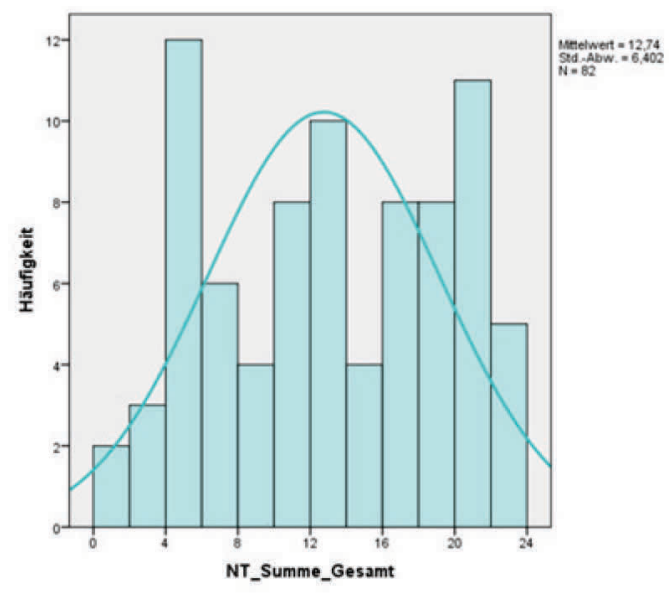

\begin{tabular}{|l|r|}
\hline \multicolumn{2}{|c|}{ Statistik für Test } \\
\hline & NT_Summe_Gesamt \\
\hline Chi-Quadrat & $29,220^{\mathrm{a}}$ \\
\hline df & \\
\hline $\begin{array}{l}\text { Asympto- } \\
\text { tische } \\
\text { Signifikanz }\end{array}$ & \\
\hline $\begin{array}{l}\text { a. Bei 24 Zellen (100,0\%) werden weniger } \\
\text { als 5 Häufigkeiten erwartet. Die kleinste } \\
\text { erwartete Zellenhaüfigkeit ist 3,4. }\end{array}$ \\
\hline
\end{tabular}

Nachtest,KG, n $=67$

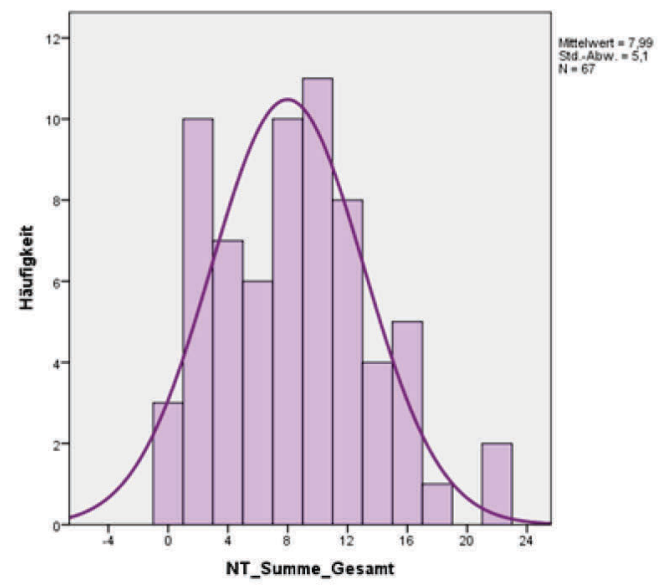

\begin{tabular}{|l|r|}
\hline \multicolumn{2}{|c|}{ Statistik für Test } \\
\hline & NT Summe Gesamt \\
\hline Chi-Quadrat & $21,194^{\mathrm{a}}$ \\
\hline $\mathrm{df}$ & \\
\hline $\begin{array}{l}\text { Asympto- } \\
\text { tische } \\
\text { Signifikanz }\end{array}$ \\
\hline $\begin{array}{l}\text { a. Bei 19 Zellen (100,0\%) werden weniger } \\
\text { als 5 Häufigkeiten erwartet. Die kleinste } \\
\text { erwartete Zellenhäufigkeit ist 3,5. }\end{array}$ \\
\hline
\end{tabular}




\section{3 Itemstatistiken}

a) Itemstatistiken für 28 Items vor der Auswahl der Items für Subtest C

\begin{tabular}{cccccccc}
\hline Item & MW & SD & N & $\begin{array}{c}\text { Skalenmit- } \\
\text { telwert, wenn } \\
\text { Item weggelassen }\end{array}$ & $\begin{array}{c}\text { Skalenvarianz, } \\
\text { wenn Item } \\
\text { weggelassen }\end{array}$ & $\begin{array}{c}\text { Korrigierte Item- } \\
\text { Skala-Korrelation } \\
\text { (Itemschwierigkeit) }\end{array}$ & $\begin{array}{c}\text { Cronbach's Al- } \\
\text { pha, wenn Item } \\
\text { weggelassen }\end{array}$ \\
\hline NT_A01 &, 64 &, 482 & 149 & 11,13 & 46,847 &, 518 &, 902 \\
NT_A02 &, 61 &, 489 & 149 & 11,16 & 47,433 &, 420 &, 904 \\
NT_A03 &, 58 &, 496 & 149 & 11,19 & 47,144 &, 457 &, 903 \\
NT_A04 &, 36 &, 480 & 149 & 11,42 & 47,528 &, 414 &, 904 \\
NT_B01 &, 66 &, 474 & 149 & 11,11 & 47,718 &, 391 &, 904 \\
NT_B02 &, 41 &, 493 & 149 & 11,36 & 47,246 &, 444 &, 903 \\
NT_B03 &, 64 &, 482 & 149 & 11,13 & 47,671 &, 390 &, 904 \\
NT_B04 &, 40 &, 492 & 149 & 11,37 & 46,802 &, 514 &, 902 \\
NT_D01 &, 40 &, 491 & 149 & 11,38 & 47,952 &, 340 &, 905 \\
NT_D02 &, 33 &, 471 & 149 & 11,44 & 47,965 &, 355 &, 905 \\
NT_D03 &, 39 &, 489 & 149 & 11,38 & 47,332 &, 435 &, 903 \\
NT_D04 &, 34 &, 476 & 149 & 11,43 & 47,314 &, 452 &, 903 \\
NT_E01 &, 62 &, 488 & 149 & 11,15 & 46,875 &, 508 &, 902 \\
NT_E02 &, 52 &, 501 & 149 & 11,26 & 45,975 &, 629 &, 900 \\
NT_E03 &, 33 &, 471 & 149 & 11,44 & 46,316 &, 618 &, 900 \\
NT_E04 &, 38 &, 486 & 149 & 11,40 & 47,889 &, 353 &, 905 \\
NT_ec1 &, 52 &, 501 & 149 & 11,25 & 46,458 &, 555 &, 901 \\
NT_ec2 &, 35 &, 478 & 149 & 11,42 & 47,043 &, 492 &, 902 \\
NT_ec3 &, 34 &, 476 & 149 & 11,43 & 45,882 &, 681 &, 899 \\
NT_ec4 &, 25 &, 433 & 149 & 11,52 & 48,265 &, 340 &, 905 \\
NT_F01 &, 42 &, 495 & 149 & 11,36 & 46,893 &, 497 &, 902 \\
NT_F02 &, 38 &, 488 & 149 & 11,39 & 46,347 &, 590 &, 901 \\
NT_F03 &, 40 &, 491 & 149 & 11,38 & 46,385 &, 580 &, 901 \\
NT_F04 &, 19 &, 392 & 149 & 11,58 & 48,218 &, 391 &, 904 \\
NT_fc1 &, 41 &, 493 & 149 & 11,36 & 46,989 &, 483 &, 903 \\
NT_fc2 &, 36 &, 480 & 149 & 11,42 & 46,082 &, 642 &, 900 \\
NT_fc3 &, 41 &, 493 & 149 & 11,36 & 46,489 &, 560 &, 901 \\
NT_fc4 &, 16 &, 369 & 149 & 11,61 & 48,374 &, 388 &, 904 \\
\hline & & & & & & & \\
\hline
\end{tabular}


b) Itemstatistiken für 24 Items nach der Auswahl der Items für Subtest C

\begin{tabular}{cccccccc}
\hline Item & MW & SD & N & $\begin{array}{c}\text { Skalenmit- } \\
\text { telwert, wenn } \\
\text { Item weggelassen }\end{array}$ & $\begin{array}{c}\text { Skalenvarianz, } \\
\text { wenn Item } \\
\text { weggelassen }\end{array}$ & $\begin{array}{c}\text { Korrigierte Item- } \\
\text { Skala-Korrelation } \\
\text { Itemschwierigkeit) }\end{array}$ & $\begin{array}{c}\text { Cronbach's Al- } \\
\text { pha, wenn Item } \\
\text { weggelassen }\end{array}$ \\
\hline NT_A01 &, 64 &, 482 & 149 & 9,97 & 36,303 &, 540 &, 891 \\
NT_A02 &, 61 &, 489 & 149 & 9,99 & 36,750 &, 452 &, 893 \\
NT_A03 &, 58 &, 496 & 149 & 10,03 & 36,580 &, 475 &, 893 \\
NT_A04 &, 36 &, 480 & 149 & 10,25 & 36,985 &, 421 &, 894 \\
NT_B01 &, 66 &, 474 & 149 & 9,94 & 37,152 &, 398 &, 894 \\
NT_B02 &, 41 &, 493 & 149 & 10,19 & 36,725 &, 452 &, 893 \\
NT_B03 &, 64 &, 482 & 149 & 9,97 & 37,060 &, 405 &, 894 \\
NT_B04 &, 40 &, 492 & 149 & 10,20 & 36,338 &, 521 &, 891 \\
NT_ec1 &, 52 &, 501 & 149 & 10,08 & 36,156 &, 542 &, 891 \\
NT_ec3 &, 34 &, 476 & 149 & 10,26 & 35,654 &, 667 &, 888 \\
NT_fc2 &, 36 &, 480 & 149 & 10,25 & 35,864 &, 622 &, 889 \\
NT_fc3 &, 41 &, 493 & 149 & 10,19 & 36,293 &, 528 &, 891 \\
NT_D01 &, 40 &, 491 & 149 & 10,21 & 37,355 &, 346 &, 896 \\
NT_D02 &, 33 &, 471 & 149 & 10,28 & 37,417 &, 353 &, 895 \\
NT_D03 &, 39 &, 489 & 149 & 10,21 & 36,832 &, 438 &, 893 \\
NT_D04 &, 34 &, 476 & 149 & 10,26 & 36,884 &, 443 &, 893 \\
NT_E01 &, 62 &, 488 & 149 & 9,99 & 36,446 &, 508 &, 892 \\
NT_E02 &, 52 &, 501 & 149 & 10,09 & 35,702 &, 621 &, 889 \\
NT_E03 &, 33 &, 471 & 149 & 10,28 & 35,971 &, 615 &, 889 \\
NT_E04 &, 38 &, 486 & 149 & 10,23 & 37,407 &, 341 &, 896 \\
NT_F01 &, 42 &, 495 & 149 & 10,19 & 36,708 &, 454 &, 893 \\
NT_F02 &, 38 &, 488 & 149 & 10,22 & 35,998 &, 587 &, 890 \\
NT_F03 &, 40 &, 491 & 149 & 10,21 & 36,085 &, 568 &, 890 \\
NT_F04 &, 19 &, 392 & 149 & 10,42 & 37,745 &, 369 &, \\
\hline & & & & & & & \\
\hline
\end{tabular}




\section{4 Interne Konsistenz und Reliabilität im Nachtest}

a) Cronbach's Alpha

\begin{tabular}{|c|c|c|c|}
\hline \multicolumn{4}{|c|}{ Zusammenfassung der Fallverarbeitung } \\
\hline & & $\mathrm{N}$ & $\%$ \\
\hline \multirow[t]{3}{*}{ Fälle } & Gültig & 149 & 100,0 \\
\hline & Ausgeschlossen $^{\mathrm{a}}$ & 0 & 0,0 \\
\hline & Gesamt & 149 & 100,0 \\
\hline
\end{tabular}

\begin{tabular}{|c|c|c|c|}
\hline Mittelwert & Varianz & $\begin{array}{l}\text { Standarda } \\
\text { b- } \\
\text { weichung }\end{array}$ & $\begin{array}{l}\text { Anzahl } \\
\text { der Items }\end{array}$ \\
\hline 10,60 & 39,673 & 6,299 & 24 \\
\hline
\end{tabular}

a. Listenweise Löschung auf der Grundlage aller Variablen in der Prozedur.

\begin{tabular}{|l|l|}
\hline \multicolumn{2}{|l|}{ Reliabilitätsstatistiken } \\
\hline $\begin{array}{l}\text { Cronbachs } \\
\text { Alpha }\end{array}$ & $\begin{array}{l}\text { Anzahl der } \\
\text { Items }\end{array}$ \\
\hline, 896 & 24 \\
\hline
\end{tabular}

b) Übersicht über Itemschwierigkeit (p) und die Paarbildung für die Testhalbierung-Reliabilitätsberechnung

\begin{tabular}{|c|c|c|c|c|}
\hline Subtest & $\mathrm{p}$ & $\mathrm{p}$ & $\mathrm{p}$ & $\mathrm{p}$ \\
\hline \multirow{2}{*}{ ST F } & NT_F01 & NT_F02 & NT_F03 & NT_F04 \\
\hline & ,42 & ,38 & ,40 & ,19 \\
\hline \multirow{2}{*}{ ST E } & NT_E01 & NT_E02 & NT_E03 & NT_E04 \\
\hline & ,62 &, 52 &, 33 & ,38 \\
\hline \multirow{2}{*}{ ST D } & NT_D01 & NT_D02 & NT_D03 & NT_D04 \\
\hline & ,40 & ,33 & ,39 &, 34 \\
\hline \multirow{2}{*}{ ST C } & NT_ec1 & NT_ec3 & NT fc2 & NT fc3 \\
\hline &, 52 & ,34 &, 36 & ,41 \\
\hline \multirow{2}{*}{ ST B } & NT_B01 & NT_B02 & NT_B03 & NT_B04 \\
\hline &, 66 &, 41 &, 64 &, 40 \\
\hline \multirow{2}{*}{ St A } & NT_A01 & NT_A02 & NT_A03 & NT A04 \\
\hline & $\overline{6}, 64$ & $\overline{6}, 61$ & $\overline{58}$ &, 36 \\
\hline
\end{tabular}

c) Reliabilitätsstatistiken zur Testhalbierung

\begin{tabular}{|c|c|c|c|}
\hline \multicolumn{4}{|c|}{ Reliabilitätsstatistiken } \\
\hline \multirow{5}{*}{ Cronbachs Alpha } & \multirow{2}{*}{ Teil 1} & Wert &, 800 \\
\hline & & Anzahl der Items & $12^{\mathrm{a}}$ \\
\hline & \multirow{2}{*}{ Teil 2} & Wert &, 821 \\
\hline & & Anzahl der Items & $12^{\mathrm{b}}$ \\
\hline & \multicolumn{2}{|c|}{ Gesamtzahl der Items } & 24 \\
\hline \multicolumn{3}{|c|}{ Korrelation zwischen Formen } &, 830 \\
\hline Spearman-Brown- & \multicolumn{2}{|c|}{ Gleiche Länge } & ,907 \\
\hline Koeffizient & \multicolumn{2}{|c|}{ ungleiche Länge } & ,907 \\
\hline \multicolumn{3}{|c|}{ Guttmans Split-Half-Koeffizient } & ,906 \\
\hline \multicolumn{4}{|c|}{$\begin{array}{l}\text { a. Die Items sind: NT_A01, NT_A04, NT_B01, NT_B04, NT_ec1, NT_fc2, NT_D01, } \\
\text { NT_D02, NT_E01, NT_E03, NT_F01, NT_F04. } \\
\text { b. Die Items sind: NT_A02, NT_A03, NT_B02, NT_B03, NT_ec3, NT_fc3, NT_D03, } \\
\text { NT_D04, NT_E02, NT_E04, NT_F02, NT_F03. }\end{array}$} \\
\hline
\end{tabular}


d) Skala-Statistiken der Testhalbierung

\begin{tabular}{|c|c|c|c|c|}
\hline \\
\hline \multicolumn{5}{|c|}{\begin{tabular}{l|l|l} 
& \\
& Mittelwert & Varianz \\
\end{tabular}} \\
\hline Teil 1 & \multicolumn{4}{|c|}{\begin{tabular}{|l|l} 
Mittelwert & Varianz \\
5021
\end{tabular}} \\
\hline Teil 2 & 5,39 & 11,564 & 3,401 & $12^{\mathrm{b}}$ \\
\hline Beide Teile & 10,60 & 39,673 & 6,299 & 24 \\
\hline
\end{tabular}

e) Item-Statistiken der Testhalbierung

\begin{tabular}{|l|l|l|l|l|l|l|l|}
\hline \multicolumn{2}{|l|}{ Auswertung der Itemstatistiken } \\
\hline
\end{tabular}

f) Wiederholung der Methode known groups zum Zeitpunkt des Nachtests

Bezugnehmend auf die Methode known groups im Unterkapitel 0 Validität soll an dieser Stelle die Methode wiederholend eingesetzt werden, um den Lernfortschritt besonders in den Subtests C und $\mathrm{F}$ zu dokumentieren.

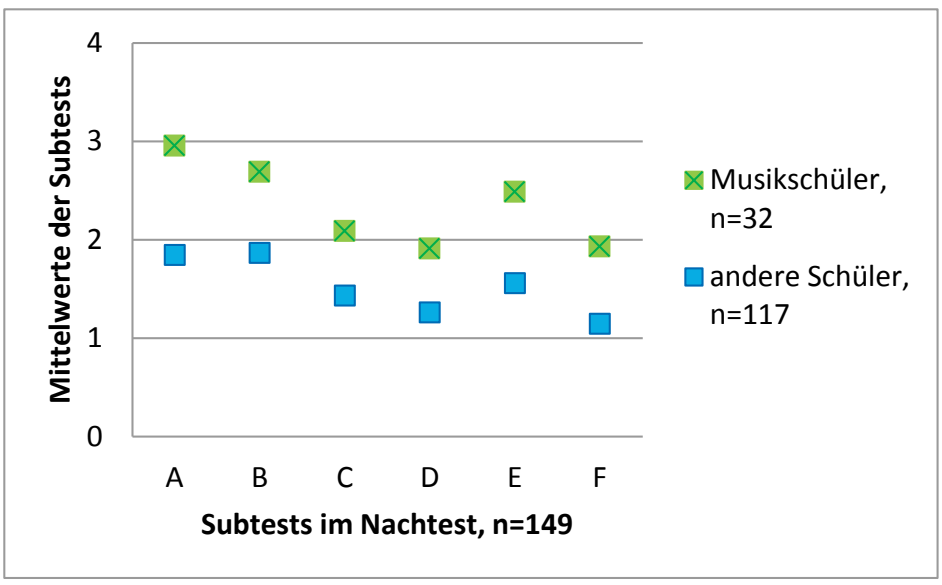




\section{5 Mittelwerte, t-Test-Werte, Effektstärken und Differenzen der MW im VT und NT}

a) Mittelwerte für ST A-F sowie GPW, getrennt nach UG und KG

\begin{tabular}{|c|c|c|c|c|}
\hline \multicolumn{5}{|c|}{ Gruppenstatistiken } \\
\hline UG_KG & $\mathrm{N}$ & Mittelwert & $\begin{array}{c}\text { Standardabw } \\
\text { eichung }\end{array}$ & $\begin{array}{l}\text { Standardfehl } \\
\text { er des } \\
\text { Mittelwertes }\end{array}$ \\
\hline \multirow{2}{*}{$\begin{array}{r}\text { Diff_SubT_A } 1_{2} \\
\end{array}$} & 82 & 0,90 & 1,193 & 0,132 \\
\hline & 67 & 0,70 & 1,168 & 0,143 \\
\hline \multirow{2}{*}{ Diff_SubT_B 1} & 82 & 0,61 & 1,235 & 0,136 \\
\hline & 67 & 0,54 & 1,159 & 0,142 \\
\hline \multirow{2}{*}{ Diff_SubT_C } & 82 & 2,07 & 1,464 & 0,162 \\
\hline & 67 & 0,57 & 1,076 & 0,131 \\
\hline \multirow{2}{*}{ Diff_SubT_D } & 82 & 0,85 & 1,362 & 0,150 \\
\hline & 67 & 0,45 & 1,247 & 0,152 \\
\hline \multirow{2}{*}{ Diff_SubT_E 1} & 82 & 1,82 & 1,398 & 0,154 \\
\hline & 67 & 0,39 & 1,167 & 0,143 \\
\hline \multirow{2}{*}{ Diff_SubT_F 1} & 82 & 1,67 & 1,458 & 0,161 \\
\hline & 67 & 0,70 & 0,938 & 0,115 \\
\hline \multirow{2}{*}{$\begin{array}{c}\text { Diff_Summe_1 } \\
\text { Gesamt }\end{array}$} & 82 & 8,73 & 4,503 & 0,497 \\
\hline & 67 & 4,10 & 3,090 & 0,378 \\
\hline
\end{tabular}

b) t-Test-Werte für ST A-F sowie GPW

\begin{tabular}{|c|c|c|c|c|c|c|c|c|c|c|}
\hline \multicolumn{11}{|c|}{ Test bei unabhängigen Stichproben } \\
\hline & & \multicolumn{2}{|c|}{ Varianzgleichheit } & \multicolumn{7}{|c|}{ T-Test für die Mittelwertgleichheit } \\
\hline & & \multirow[t]{2}{*}{$\mathrm{F}$} & \multirow[t]{2}{*}{ Signifikanz } & \multirow[t]{2}{*}{$\mathrm{T}$} & \multirow[t]{2}{*}{ df } & \multirow[t]{2}{*}{$\begin{array}{l}\text { Sig. (2- } \\
\text { seitig) }\end{array}$} & \multirow[t]{2}{*}{\begin{tabular}{|c|} 
Mittlere \\
Differen \\
$z$
\end{tabular}} & \multirow[t]{2}{*}{$\begin{array}{c}\text { d-fehler } \\
\text { der } \\
\text { Differen }\end{array}$} & \multicolumn{2}{|c|}{$\begin{array}{c}95 \% \\
\text { Konfidenzinterval } \\
\text { I der Differenz }\end{array}$} \\
\hline & & & & & & & & & Untere & Obere \\
\hline \multirow[t]{2}{*}{ Diff_SubT_A } & Varianzen sind gleich & 0,006 & 0,939 & 1,033 & 147 & 0,303 & 0,201 & 0,195 & $-0,184$ & 0,586 \\
\hline & Varianzen sind nicht gleich & & & 1,035 & 142,242 & 0,303 & 0,201 & 0,194 & $-0,183$ & 0,585 \\
\hline \multirow[t]{2}{*}{ Diff_SubT_B } & Varianzen sind gleich & 0,215 & 0,643 & 0,366 & 147 & 0,715 & 0,072 & 0,198 & $-0,319$ & 0,463 \\
\hline & Varianzen sind nicht gleich & & & 0,369 & 144,159 & 0,713 & 0,072 & 0,197 & $-0,316$ & 0,461 \\
\hline \multirow[t]{2}{*}{ Diff_SubT_C } & Varianzen sind gleich & 7,616 & 0,007 & 7,012 & 147 & 0,000 & 1,506 & 0,215 & 1,082 & 1,930 \\
\hline & Varianzen sind nicht gleich & & & 7,227 & 145,482 & 0,000 & 1,506 & 0,208 & 1,094 & 1,918 \\
\hline \multirow[t]{2}{*}{ Diff_SubT_D } & Varianzen sind gleich & 0,012 & 0,913 & 1,879 & 147 & 0,062 & 0,406 & 0,216 & $-0,021$ & 0,833 \\
\hline & Varianzen sind nicht gleich & & & 1,896 & 145,075 & 0,060 & 0,406 & 0,214 & $-0,017$ & 0,829 \\
\hline \multirow[t]{2}{*}{ Diff_SubT_E } & Varianzen sind gleich & 4,014 & 0,047 & 6,678 & 147,000 & 0,000 & 1,429 & 0,214 & 1,006 & 1,852 \\
\hline & Varianzen sind nicht gleich & & & 6,800 & 146,922 & 0,000 & 1,429 & 0,210 & 1,014 & 1,844 \\
\hline \multirow[t]{2}{*}{ Diff_SubT_F } & Varianzen sind gleich & 22,043 & 0,000 & 4,704 & 147,000 & 0,000 & 0,969 & 0,206 & 0,562 & 1,376 \\
\hline & Varianzen sind nicht gleich & & & 4,906 & 139,810 & 0,000 & 0,969 & 0,198 & 0,579 & 1,360 \\
\hline \multirow{2}{*}{$\begin{array}{c}\text { Diff_Summe_Ge } \\
\text { samt }\end{array}$} & Varianzen sind gleich & 12,998 & 0,000 & 7,146 & 147 & 0,000 & 4,627 & 0,647 & 3,348 & 5,907 \\
\hline & Varianzen sind nicht gleich & & & 7,412 & 142,990 & 0,000 & 4,627 & 0,624 & 3,393 & 5,861 \\
\hline
\end{tabular}


Anlagen

c) Effektstärken für Subtest A-F sowie GPW im Vergleich von UG und KG

\begin{tabular}{|c|c|c|c|c|c|}
\hline Subtest & $\mathrm{T}$ & Sign. (2seit.) & $\begin{array}{c}\text { Vergleich mit } \\
\text { critical t }\end{array}$ & Effektstärke d & Effekt \\
\hline Subtest A & 1,033 & 0,303 & $<1,66$ & $\mathbf{0 , 1 7}$ & - \\
\hline Subtest B & 0,366 & 0,715 & $<1,66$ & $\mathbf{0 , 0 6}$ & - \\
\hline Subtest C & 7,012 & 0,000 & $>1,66$ & $\mathbf{1 , 1 5}$ & groß \\
\hline Subtest D & 1,879 & 0,062 & $>1,66$ & $\mathbf{0 , 3 1}$ & klein \\
\hline Subtest E & 6,678 & 0,000 & $>1,66$ & $\mathbf{1 , 1 0}$ & groß \\
\hline Subtest F & 4,704 & 0,000 & $>1,66$ & $\mathbf{0 , 7 7}$ & mittel \\
\hline GPW & 7,146 & 0,000 & $>1,66$ & $\mathbf{1 , 3 6}$ & groß \\
\hline
\end{tabular}

d) Differenzen der Mittelwerte aus Vor- und Nachtest

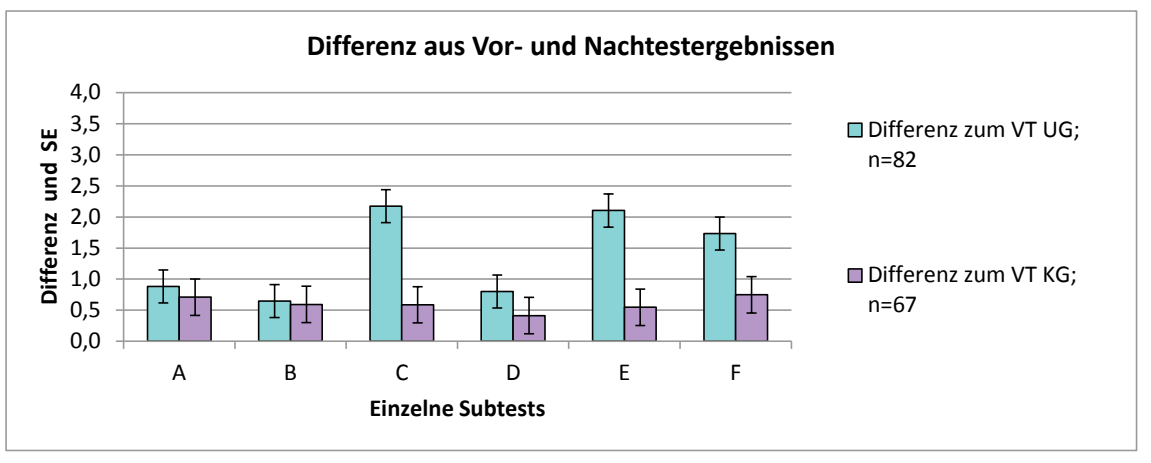

e) Mittelwerte für Untertest ec, fc, e, f und ST G

\begin{tabular}{|c|c|c|c|c|c|}
\hline \multicolumn{6}{|c|}{ Gruppenstatistiken } \\
\hline UG_KG & & $\mathrm{N}$ & Mittelwert & $\begin{array}{c}\text { Standardabw } \\
\text { eichung }\end{array}$ & $\begin{array}{l}\text { Standardfehl } \\
\text { er des } \\
\text { Mittelwertes }\end{array}$ \\
\hline \multirow[t]{2}{*}{ Diff_SubT_ec } & 1 & 82 & 1,83 & 1,294 & 0,143 \\
\hline & 2 & 67 & 0,36 & 1,124 & 0,137 \\
\hline \multirow[t]{2}{*}{ Diff_subT_fc } & 1 & 82 & 1,74 & 1,447 & 0,160 \\
\hline & 2 & 67 & 0,61 & 1,100 & 0,134 \\
\hline \multirow[t]{2}{*}{ Diff_SubT_ee } & 1 & 82 & 1,46 & 1,344 & 0,148 \\
\hline & 2 & 67 & 0,42 & 1,529 & 0,187 \\
\hline \multirow[t]{2}{*}{ Diff_SubT_ff } & 1 & 82 & 1,23 & 1,477 & 0,163 \\
\hline & 2 & 67 & 0,61 & 1,314 & 0,160 \\
\hline \multirow[t]{2}{*}{ Diff_Play_together } & $F_{1}$ & 82 & 0,46 & 0,919 & 0,101 \\
\hline & 2 & 67 & 0,31 & 0,656 & 0,080 \\
\hline
\end{tabular}


f) t-Test-Werte für Untertest ec, fc, e, f und ST G

\begin{tabular}{|c|c|c|c|c|c|c|c|c|c|c|}
\hline \multicolumn{11}{|c|}{ Test bei unabhängigen Stichproben } \\
\hline & & \multicolumn{2}{|c|}{ Varianzgleichheit } & \multicolumn{7}{|c|}{ T-Test für die Mittelwertgleichheit } \\
\hline & & \multirow[b]{2}{*}{$\mathrm{F}$} & \multirow[b]{2}{*}{$\begin{array}{c}\text { Signifik } \\
\text { anz }\end{array}$} & \multirow[b]{2}{*}{$\mathrm{T}$} & \multirow[b]{2}{*}{ df } & \multirow[b]{2}{*}{$\begin{array}{l}\text { Sig. (2- } \\
\text { seitig) }\end{array}$} & \multirow{2}{*}{\begin{tabular}{|c|} 
Mittlere \\
Differen \\
$z$ \\
\end{tabular}} & \multirow{2}{*}{$\begin{array}{l}\text { Standar } \\
\text { dfehler } \\
\text { der } \\
\text { Differen }\end{array}$} & \multicolumn{2}{|c|}{ Konfidenzintervall } \\
\hline & & & & & & & & & Untere & Obere \\
\hline \multirow[t]{2}{*}{ Diff_SubT_ec } & $\begin{array}{l}\text { Varianzen } \\
\text { sind gleich }\end{array}$ & 5,614 & 0,019 & 7,318 & 147 & 0,000 & 1,471 & 0,201 & 1,074 & 1,868 \\
\hline & $\begin{array}{l}\text { Varianzen } \\
\text { sind nicht }\end{array}$ & & & 7,423 & 146,423 & 0,000 & 1,471 & 0,198 & 1,079 & 1,863 \\
\hline \multirow[t]{2}{*}{ Diff_subT_fc } & $\begin{array}{l}\text { Varianzen } \\
\text { sind gleich }\end{array}$ & 12,718 & 0,000 & 5,276 & 147 & 0,000 & 1,132 & 0,215 & 0,708 & 1,556 \\
\hline & $\begin{array}{l}\text { Varianzen } \\
\text { sind nicht }\end{array}$ & & & 5,421 & 146,293 & 0,000 & 1,132 & 0,209 & 0,719 & 1,545 \\
\hline \multirow[t]{2}{*}{ Diff_SubT_ee } & $\begin{array}{l}\text { Varianzen } \\
\text { sind aleich }\end{array}$ & 0,450 & 0,503 & 4,439 & 147 & 0,000 & 1,046 & 0,236 & 0,580 & 1,511 \\
\hline & $\begin{array}{l}\text { Varianzen } \\
\text { sind nicht }\end{array}$ & & & 4,382 & 132,602 & 0,000 & 1,046 & 0,239 & 0,574 & 1,517 \\
\hline \multirow[t]{2}{*}{ Diff_SubT_ff } & $\begin{array}{l}\text { Varianzen } \\
\text { sind aleich }\end{array}$ & 1,036 & 0,310 & 2,677 & 147 & 0,008 & 0,620 & 0,232 & 0,162 & 1,077 \\
\hline & $\begin{array}{l}\text { Varianzen } \\
\text { sind nicht }\end{array}$ & & & 2,709 & 145,904 & 0,008 & 0,620 & 0,229 & 0,168 & 1,072 \\
\hline \multirow[t]{2}{*}{$\begin{array}{l}\text { Diff_Play_tog } \\
\text { ether }\end{array}$} & $\begin{array}{l}\text { Varianzen } \\
\text { sind gleich }\end{array}$ & 7,901 & 0,006 & 1,122 & 147 & 0,264 & 0,150 & 0,134 & $-0,114$ & 0,414 \\
\hline & $\begin{array}{l}\text { Varianzen } \\
\text { sind nicht }\end{array}$ & & & 1,160 & 144,555 & 0,248 & 0,150 & 0,129 & $-0,106$ & 0,406 \\
\hline
\end{tabular}

g) Effektstärken für Untertest ec, fc, e, f und ST G im Vergleich von UG und KG

\begin{tabular}{|l|c|c|c|c|c|}
\hline & T & Sign. (2seit.) & $\begin{array}{c}\text { Vergleich mit } \\
\text { critical t }\end{array}$ & $\begin{array}{c}\text { Effekt- } \\
\text { stärke d }\end{array}$ & Effekt \\
\hline Diff_SubT_ec & 7,318 & 0,000 & $>1,66$ & 1,21 & groß \\
\hline Diff_subT_fc & 5,276 & 0,000 & $>1,66$ & 0,87 & groß \\
\hline Diff_SubT_ee & 4,439 & 0,000 & $>1,66$ & 0,73 & mittel \\
\hline Diff_SubT_ff & 2,677 & 0,008 & $>1,66$ & 0,44 & klein \\
\hline Diff_Play_together & 1,122 & 0,264 & $<1,66$ & 0,18 & - \\
\hline
\end{tabular}

\section{6 Übersicht über Ergebnisse in den Untertests ec und fc im Vor- und Nachtest}

\begin{tabular}{|c|c|c|c|c|c|c|c|c|}
\hline \multirow{2}{*}{ Vortest } & \multicolumn{4}{|c|}{ UG } & \multicolumn{4}{|c|}{ KG } \\
\cline { 2 - 9 } & Item 1 & Item 2 & Item 3 & Item 4 & Item 1 & Item 2 & Item 3 & Item 4 \\
\hline E (Noten) & $\mathbf{1 7 \%}$ & $\mathbf{1 6 \%}$ & $\mathbf{9 \%}$ & $\mathbf{6 \%}$ & $\mathbf{3 4 \%}$ & $\mathbf{2 7 \%}$ & $\mathbf{9 \%}$ & $\mathbf{1 9 \%}$ \\
\hline ec (+ Silben) & $\mathbf{7 \%}$ & $\mathbf{1 \%}$ & $\mathbf{4 \%}$ & $\mathbf{0 \%}$ & $\mathbf{1 9 \%}$ & $\mathbf{1 3 \%}$ & $\mathbf{6 \%}$ & $\mathbf{9 \%}$ \\
\hline
\end{tabular}

\begin{tabular}{|c|c|c|c|c|c|c|c|c|}
\hline \multirow{2}{*}{ Nachtest } & \multicolumn{4}{|c|}{ UG } & \multicolumn{4}{|c|}{ KG } \\
\cline { 2 - 9 } & Item 1 & Item 2 & Item 3 & Item 4 & Item 1 & Item 2 & Item 3 & Item 4 \\
\hline Untertest E (mit Noten) & $\mathbf{7 3 \%}$ & $\mathbf{6 6} \%$ & $\mathbf{4 9 \%}$ & $\mathbf{4 1} \%$ & $\mathbf{4 8 \%}$ & $\mathbf{3 4} \%$ & $\mathbf{1 3 \%}$ & $\mathbf{3 3 \%}$ \\
\hline Untertest ec (+ Rhythmussilben) & $\mathbf{7 2 \%}$ & $\mathbf{4 8} \%$ & $\mathbf{4 4} \%$ & $\mathbf{3 3} \%$ & $\mathbf{2 8} \%$ & $\mathbf{1 9 \%}$ & $\mathbf{2 2 \%}$ & $\mathbf{1 5 \%}$ \\
\hline
\end{tabular}

\begin{tabular}{|c|c|c|c|c|c|c|c|c|}
\hline \multirow{2}{*}{ Vortest } & \multicolumn{4}{|c|}{ UG } & \multicolumn{4}{|c|}{ KG } \\
\cline { 2 - 9 } & Item 1 & Item 2 & Item 3 & Item 4 & Item 1 & Item 2 & Item 3 & Item 4 \\
\hline F (Noten) & $\mathbf{6 \%}$ & $\mathbf{5 \%}$ & $\mathbf{7 \%}$ & $\mathbf{2 \%}$ & $\mathbf{1 \%}$ & $\mathbf{1 \%}$ & $\mathbf{3 \%}$ & $\mathbf{1 \%}$ \\
\hline fc (+ Silben) & $\mathbf{5 \%}$ & $\mathbf{4 \%}$ & $\mathbf{4 \%}$ & $\mathbf{0 \%}$ & $\mathbf{3 \%}$ & $\mathbf{3 \%}$ & $\mathbf{1 \%}$ & $\mathbf{0 \%}$ \\
\hline
\end{tabular}

\begin{tabular}{|c|c|c|c|c|c|c|c|c|}
\hline \multirow{2}{*}{ Nachtest } & \multicolumn{4}{|c|}{ UG } & \multicolumn{4}{|c|}{ KG } \\
\cline { 2 - 9 } & Item 1 & Item 2 & Item 3 & Item 4 & Item 1 & Item 2 & Item 3 & Item 4 \\
\hline F (Noten) & $\mathbf{5 4 \%}$ & $\mathbf{5 7 \%}$ & $\mathbf{5 1} \%$ & $\mathbf{2 6 \%}$ & $\mathbf{2 7} \%$ & $\mathbf{1 5} \%$ & $\mathbf{2 5} \%$ & $\mathbf{1 0} \%$ \\
\hline fc (+ Silben) & $\mathbf{5 5 \%}$ & $\mathbf{5 5} \%$ & $\mathbf{5 5} \%$ & $\mathbf{2 2} \%$ & $\mathbf{2 4} \%$ & $\mathbf{1 2 \%}$ & $\mathbf{2 4} \%$ & $\mathbf{9 \%}$ \\
\hline
\end{tabular}


Anlage D zur Treatmentgestaltung und - durchführung

D 1 Ablauf des Untersuchungszeitraums und Hospitationstermine

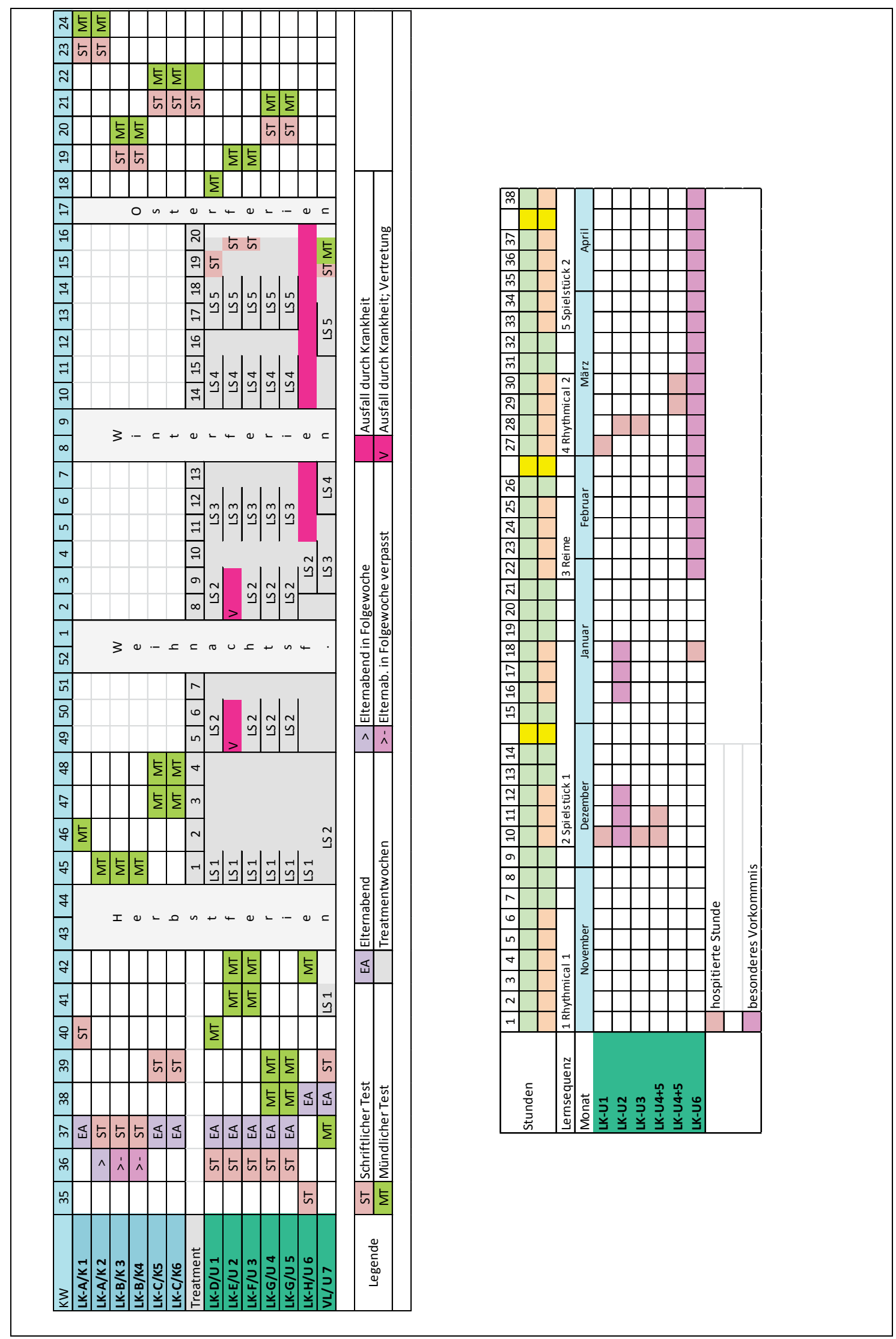

links: Gesamtablauf; rechts: Übersicht über die Lage der Hospitationen innerhalb des Treatments 


\section{2 Inhalte der einzelnen Stunden der Lernsequenzen 1 bis 5}

\begin{tabular}{|c|c|c|}
\hline Lernsequenz & Stunde & Workshop-Inhalt \\
\hline \multirow{4}{*}{$\begin{array}{l}\text { 1: } \\
\text { Rhythmical } 1\end{array}$} & 1 & Einführung in die Gruppenarbeit und in das erste Rhythmical \\
\hline & 2 & Rhythmical zur Background-Musik einstimmig sprechen \\
\hline & 3 & Einführung in die Strichnotation \\
\hline & 4 & Rhythmical aufbauend musizieren \\
\hline \multirow{6}{*}{$\begin{array}{l}\text { 2: } \\
\text { Spielstück } 1\end{array}$} & 5 & Vielfältige Rhythmen finden und in Strichnotation fixieren \\
\hline & 6 & $\begin{array}{l}\text { Einführung der Rhythmussprache, Wiederholung der erfundenen Rhyth- } \\
\text { men }\end{array}$ \\
\hline & 7 & Rhythmen auf geeigneten Rhythmusinstrumenten spielen \\
\hline & 8 & Verteilung der Stimmen, Ablauf des Spielstücks festlegen \\
\hline & 9 & Notenschrift kennen lernen, Spielstück festigen \\
\hline & 10 & Präsentation des Spielstücks durch die Gruppen \\
\hline \multirow{4}{*}{$\begin{array}{l}\text { 3: } \\
\text { Abzählreime }\end{array}$} & 11 & Abzählverse lesen und auf Gruppen aufteilen \\
\hline & 12 & Abzählverse gemeinsam rhythmisch sprechen \\
\hline & 13 & In Abzählversen Rhythmus, Grundschlag und Metrum hervorheben \\
\hline & 14 & Abzählverse zum Playback vortragen \\
\hline \multirow{4}{*}{$\begin{array}{l}\text { 4: } \\
\text { Rhythmical } 2\end{array}$} & 15 & Sätze erarbeiten und in Strichnotation übertragen (für 2 Stimmen) \\
\hline & 16 & Rhythmical einstimmig erarbeiten, Strichnotation fortsetzen \\
\hline & 17 & Rhythmical aufbauend musizieren \\
\hline & 18 & Strichnotation in Notenschrift übertragen \\
\hline \multirow{6}{*}{$\begin{array}{l}\text { 5: } \\
\text { Spielstück } 2\end{array}$} & 19 & Vielfältige Rhythmen finden und in Strichnotation fixieren \\
\hline & 20 & Rhythmen auf geeigneten Rhythmusinstrumente spielen \\
\hline & 21 & Verteilung der Stimmen, Ablauf des Spielstücks festlegen \\
\hline & 22 & Spielstück festigen \\
\hline & 23 & Präsentation des Spielstücks durch die Gruppen \\
\hline & 24 & Rückblick auf den Lehrgang \\
\hline
\end{tabular}


D 3 Hörbeispiele für Warm up 1

\begin{tabular}{|c|c|c|c|c|}
\hline $\begin{array}{l}\text { Lernsequenz / } \\
\text { Stunde }\end{array}$ & Komponist / Interpret & Titel & Metrum & Länge \\
\hline \multirow{2}{*}{$1,1+2$} & unbekannt & „Katz und Maus“ & gerade & $0: 54$ \\
\hline & C. Saint Saèns & „Elefant“ & ungerade & $1: 28$ \\
\hline \multirow{2}{*}{$1,3+4$} & Avicii & „Wake me up“ & gerade & $1: 16$ \\
\hline & J.S. Bach & „Badinerie“ & gerade & $0: 35$ \\
\hline \multirow{2}{*}{$2,1+2$} & W.A. Mozart & „Schlittenfahrt“ & gerade & 1:09 \\
\hline & P.I. Tschaikowski & „Blumenwalzer“ & ungerade & 1:05 \\
\hline \multirow{2}{*}{$2,3+4$} & $\begin{array}{l}\text { J. Pachelbel/Hand In Hand } \\
\text { For Children e.V. }\end{array}$ & „Children“ & gerade & $1: 19$ \\
\hline & F. Sinatra/trad. & ,Jingle bells“ & gerade & 1:19 \\
\hline \multirow{2}{*}{$2,5+6$} & Gospel Passengers/trad. & „Good News“" & gerade & $0: 53$ \\
\hline & P.I. Tschaikowski & „Klavierkonzert“ & ungerade & $0: 56$ \\
\hline \multirow{2}{*}{$3,1+2$} & B. Hering, M. Wester & „Fächertanz“ & gerade & $0: 55$ \\
\hline & Unbekannt & „Agir Hayley“ & unregelmäßig & 1:00 \\
\hline \multirow{2}{*}{$3,3+4$} & P. Budde & „Wichi Tai Tai““ & gerade & 1:09 \\
\hline & C. Candela & „Von allein“ & gerade & $1: 18$ \\
\hline \multirow{2}{*}{$4,1+2$} & Valente & „Gagliarda napolitana“ & & $1: 00$ \\
\hline & Mahler & „Sinfonie Nr. 1, 3. Satz“ & gerade & $1: 17$ \\
\hline \multirow{2}{*}{$4,3+4$} & Take That & „Love Ain’t Here Anymore“ & ungerade & $1: 20$ \\
\hline & Carpentier & „Te Deum“ & gerade & $1: 11$ \\
\hline \multirow{2}{*}{$5,1+2$} & L. Mozart & „Kindersinfonie Allegro“ & gerade & 1:06 \\
\hline & L. Mozart & „Kindersinfonie Menuett“ & ungerade & 1:03 \\
\hline \multirow{2}{*}{$5,3+4$} & $\mathrm{P} ! \mathrm{NK}$ & „Try“ & gerade & $1: 22$ \\
\hline & L. Clio & „Couldn’t Care Less“ & gerade & $1: 11$ \\
\hline \multirow{2}{*}{$5,5+6$} & N. Matteis & $\begin{array}{l}\text { "Ground after the Scotch } \\
\text { Humor" }\end{array}$ & ungerade & $1: 18$ \\
\hline & D. Garret & „Viva La Vida“ & gerade & $1: 26$ \\
\hline
\end{tabular}




\section{4 Schüler-Arbeitsheft Vorlage}

Vorlage zum Notieren ausgewählter Rhythmusbausteine / Notizen zur Aufgabe der Gruppenmitglieder

\begin{tabular}{|c|c|c|}
\hline Uier findest du die Rhythmussilben im \\
Überblick.
\end{tabular}

Rhythmical 1 und 2

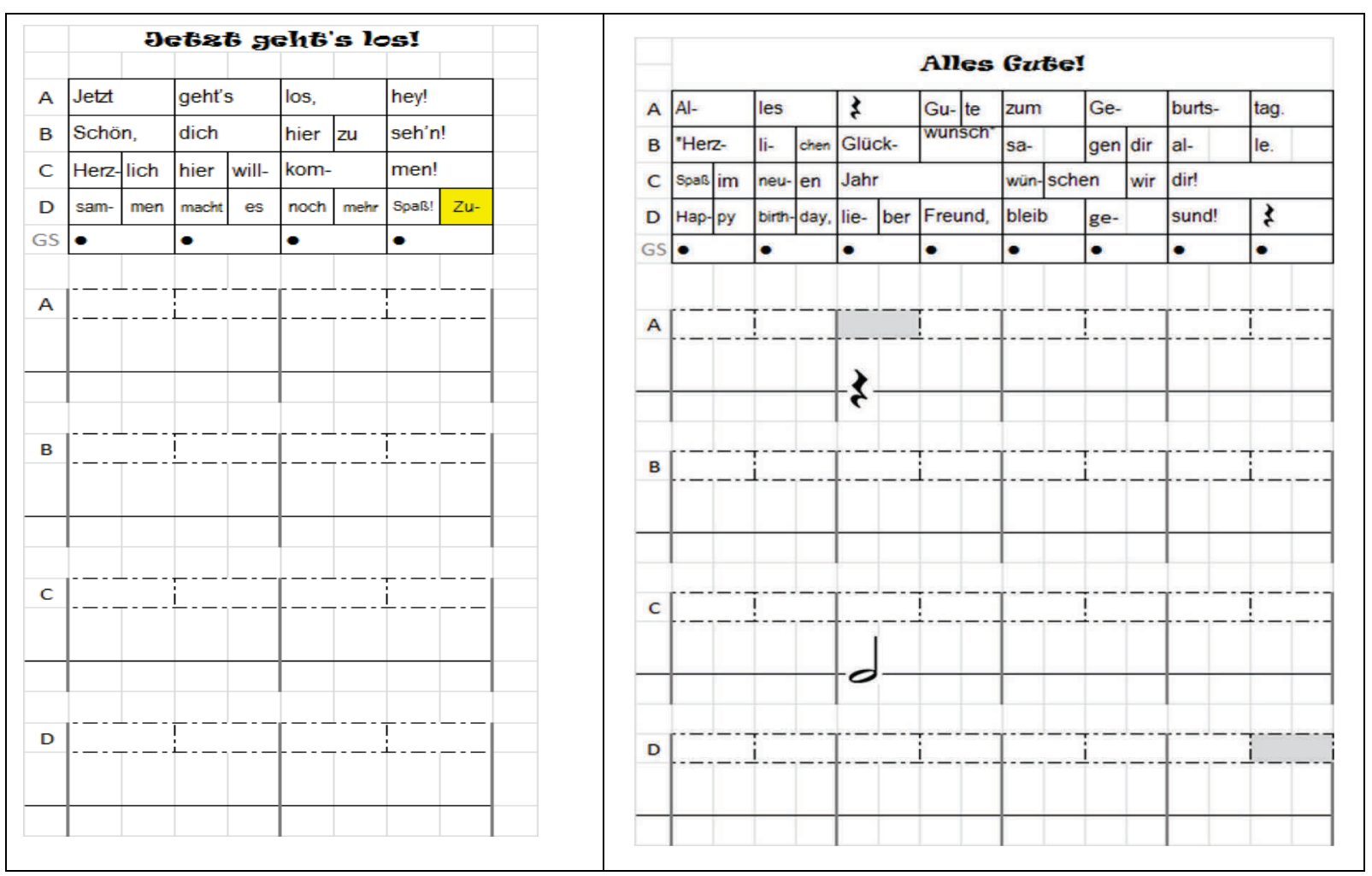


Spielstück 1 und 2

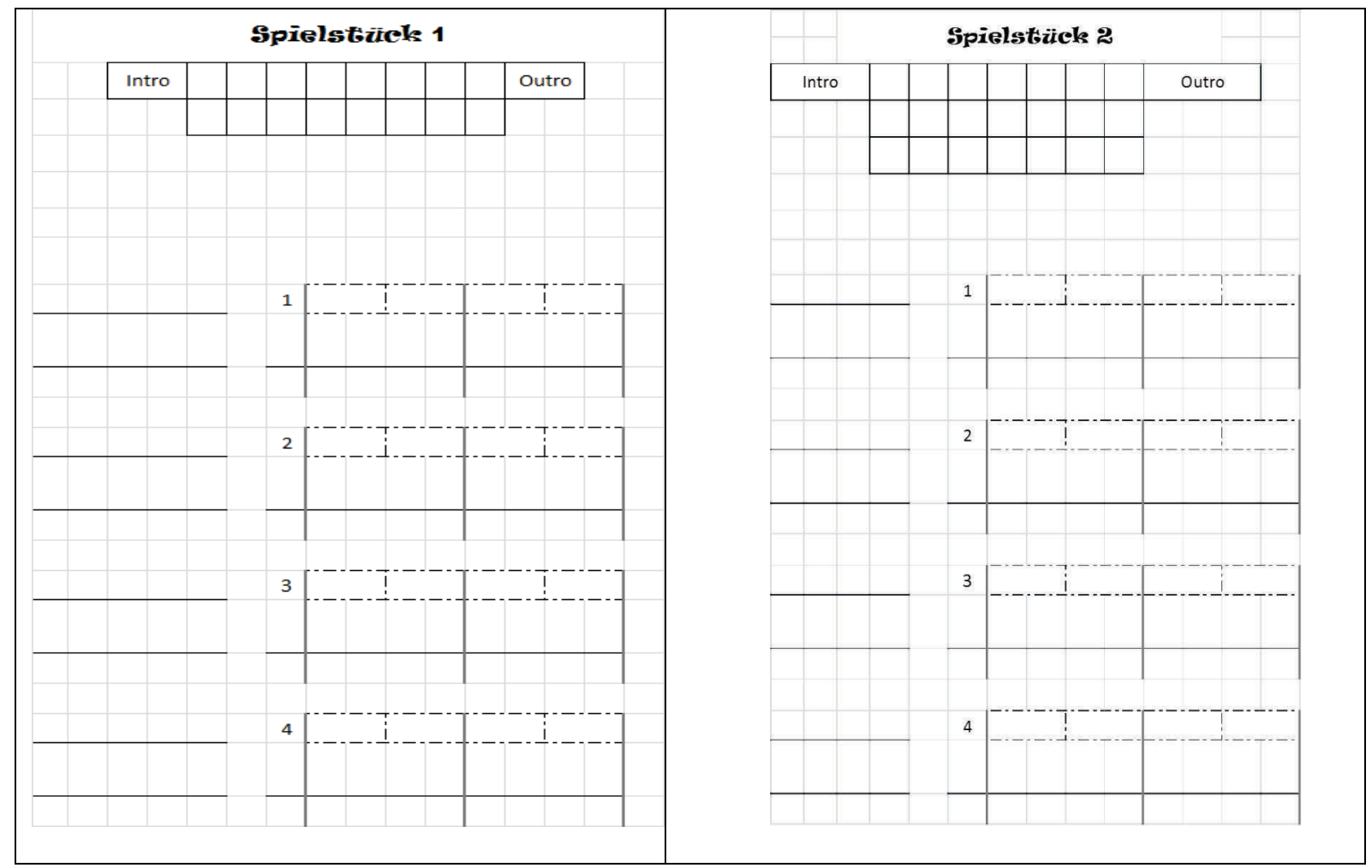

Doppelseite für die Arbeit an Abzählreimen

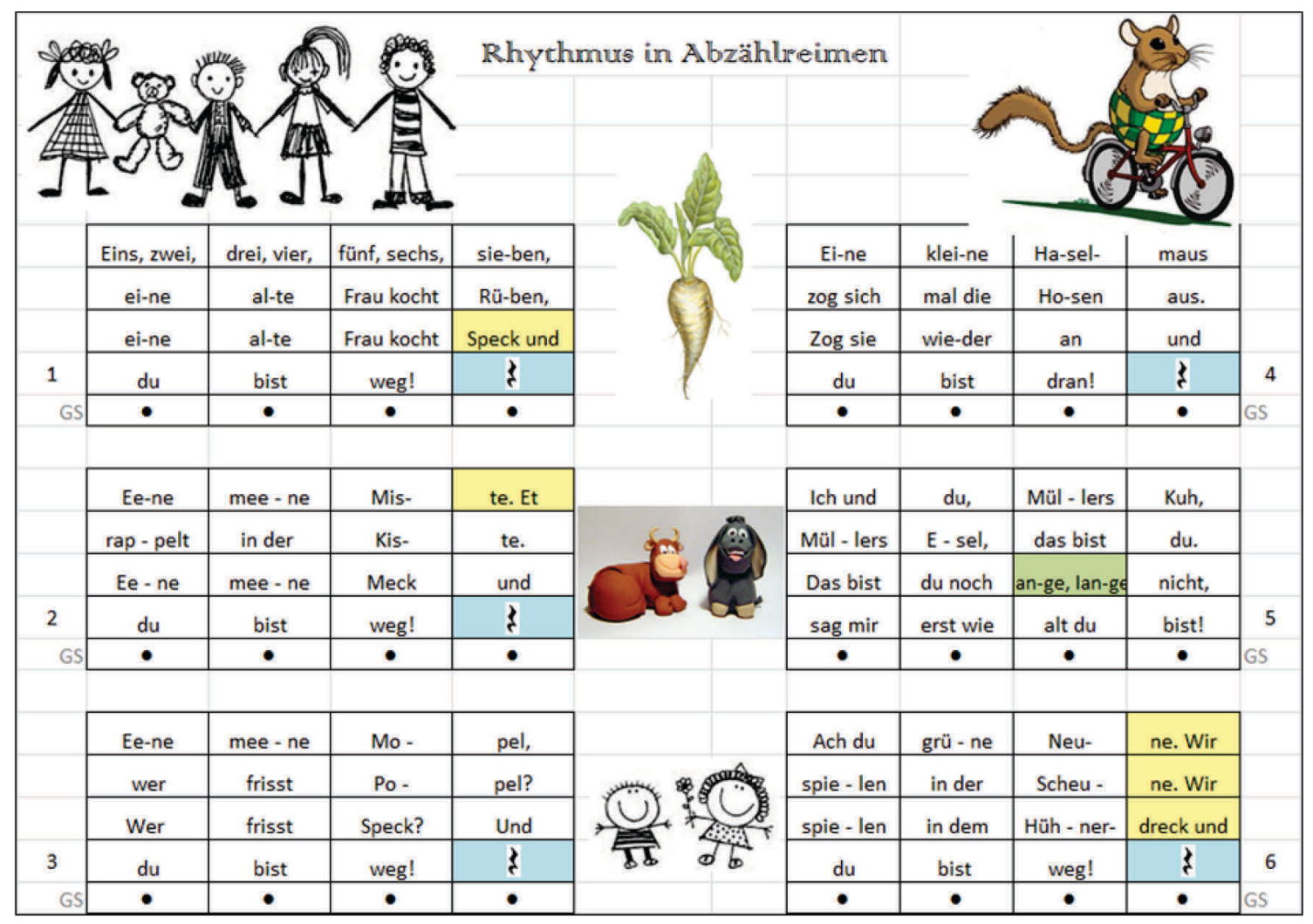


D 5 Styles für Warm up 2

\begin{tabular}{|l|l|l|l|l|}
\hline LS & Komponist / Interpret & Style & $\begin{array}{l}\text { Tempo in } \\
\text { bpm }\end{array}$ & $\begin{array}{l}\text { Länge in } \\
\text { min:sec }\end{array}$ \\
\hline 1 & Rhythmus-Background & Danse/Saturday Night & 100 & $1: 11$ \\
\hline 2 & \multirow{3}{*}{$\begin{array}{l}\text { Titel: ,It's good“ } \\
\text { Komponist: K. Gramß } \\
\text { Arrangements: C. Werner }\end{array}$} & Pop\&Rock/Beat Gtr Pop & 98,104 & $1: 43 ; 1: 45$ \\
\cline { 3 - 5 } & & Latin/Rock Cha Cha & 108 & $2: 05$ \\
\cline { 3 - 5 } & & Ballad/Chillout & 104 & $2: 33$ \\
\cline { 3 - 5 } & & Danse/Chart R\&B & 104 & $2: 33$ \\
\hline 5 & & & &
\end{tabular}

\section{6 Entstandene Spielstücke}

Kopien des Spielstückes 1 in den sechs Untersuchungsklassen

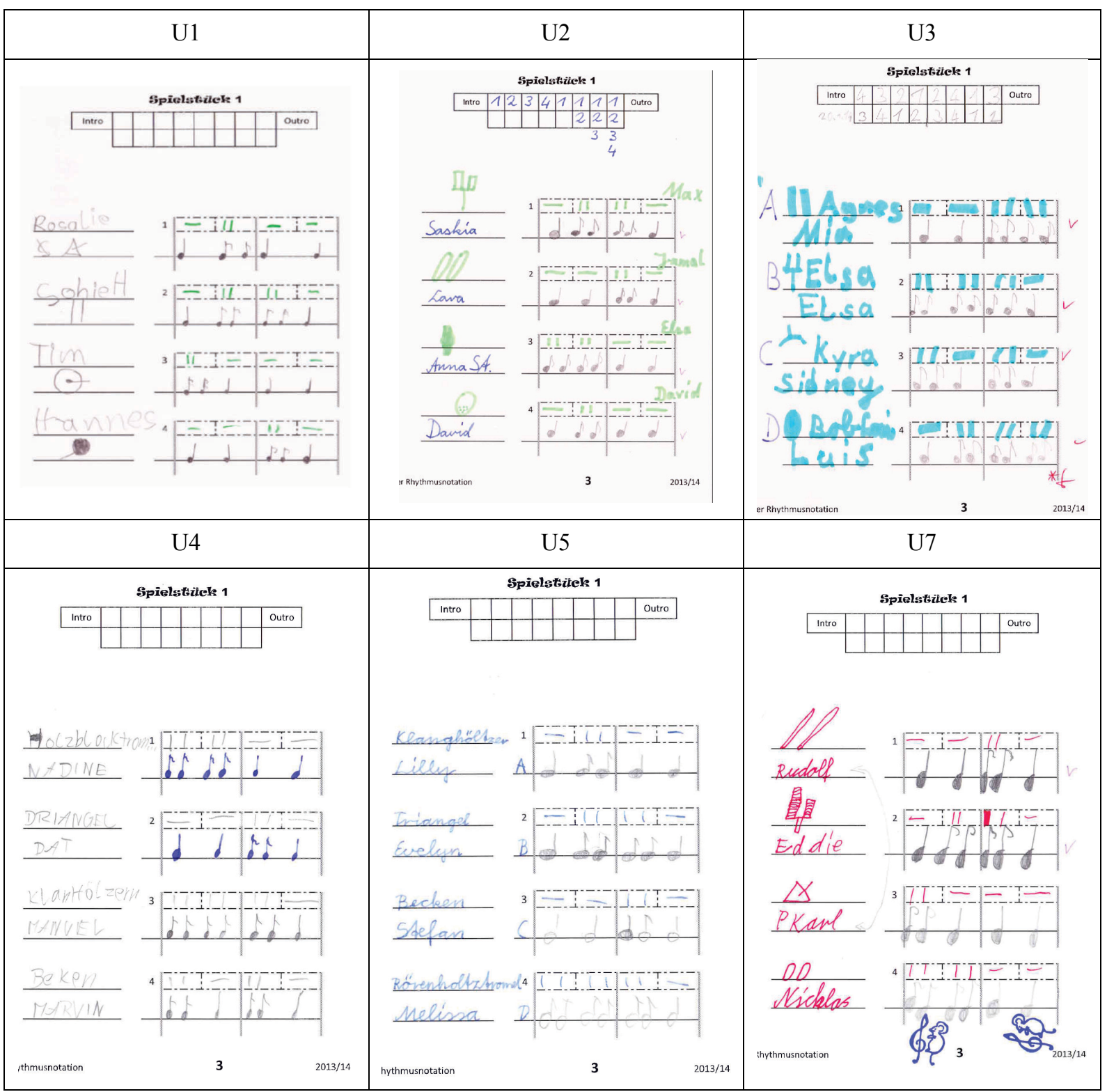


Kopien der Spielstückes 2 in den sechs Untersuchungsklassen

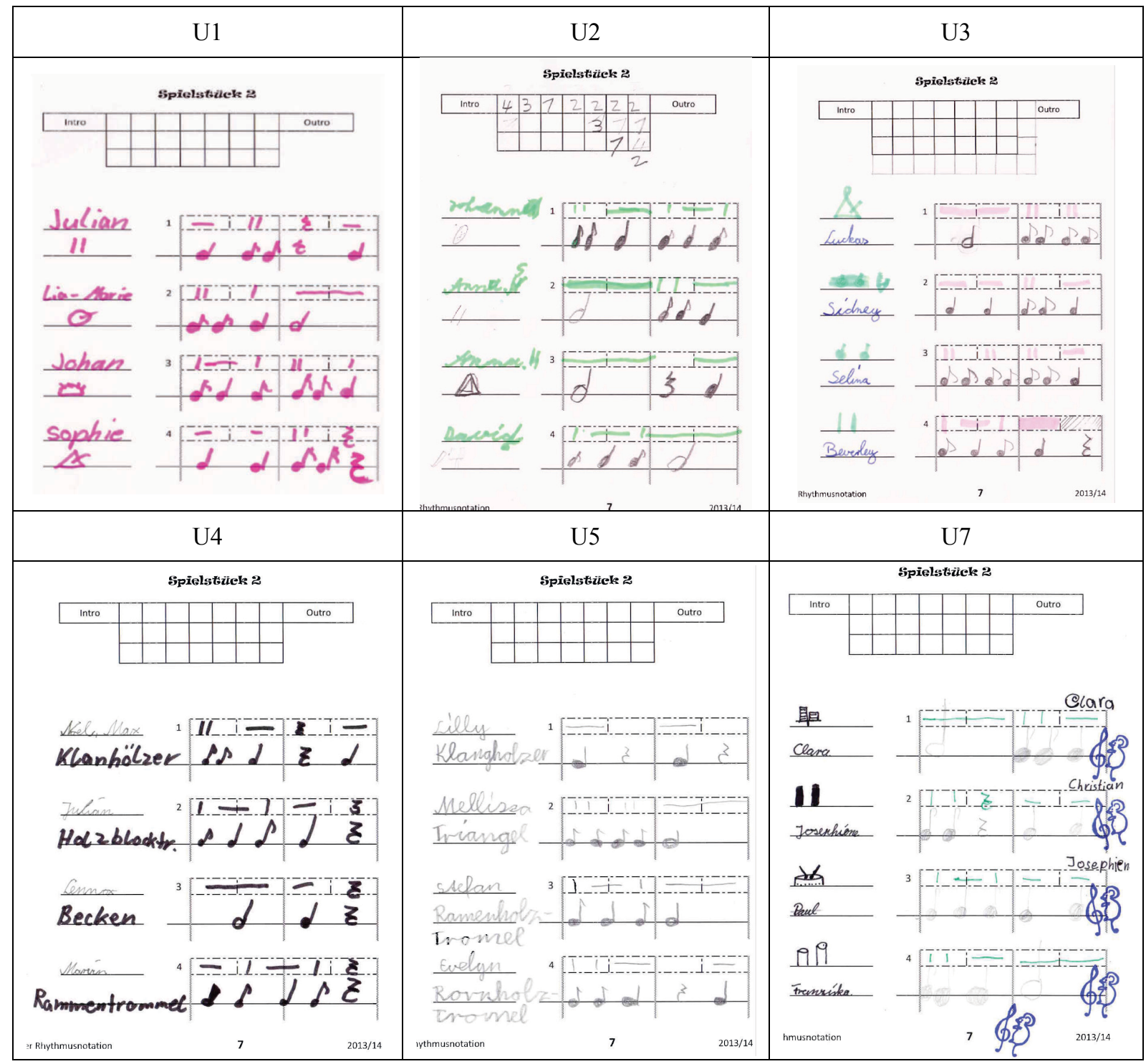





\section{Anlage E Vorgegebene und gespielte Rhythmen im Test}

\begin{tabular}{|c|c|c|c|c|}
\hline \multicolumn{2}{|c|}{ Rhythmus-Nummer und -Notenbild } & \multirow{2}{*}{$\begin{array}{l}\text { ST B } \\
\text { B } 1\end{array}$} & \multirow{2}{*}{$\begin{array}{l}\mathrm{ST} \\
\mathrm{E} / \mathrm{C}^{1} \\
\mathrm{E} / \mathrm{C}^{1} 1\end{array}$} & \multirow{2}{*}{$\begin{array}{l}\mathrm{ST} \\
\mathrm{F} / \mathrm{C}^{2} \\
\mathrm{~F} / \mathrm{C}^{2} 1\end{array}$} \\
\hline $1 \mathrm{a}$ & $\cdots+$ & & & \\
\hline $1 b$ & $\frac{2}{4} \cdot|\cdot ?+|$ & & & \\
\hline $1 \mathrm{c}$ & $\frac{2}{4} \cdot+|\cdot . \cdot|$ & & & \\
\hline $1 \mathrm{~d}$ & $\frac{2}{\mathbf{4}} \cdot \downarrow|\cdots \cdot+|$ & & & \\
\hline 1e & $\frac{2}{4} \cdot \cdot|\sqrt{-3-} \cdot d|$ & & & \\
\hline $1 \mathrm{f}$ & $\frac{\mathbf{2}}{\mathbf{4}} \cdot|+|$ y & & & \\
\hline $2 \mathrm{a}$ & 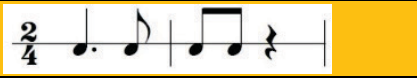 & B 2 & & \\
\hline $2 b$ & $\frac{2}{4} \cdot d|\cdot \cdot d|$ & & & \\
\hline $2 \mathrm{c}$ & $\frac{2}{4} \cdot \rho|\cdot \cdot \cdot|$ & & & \\
\hline $2 \mathrm{~d}$ & $\frac{2}{4} \cdot \cdot \cdot|\cdot| \cdot \mid$ & & & \\
\hline $2 \mathrm{e}$ & 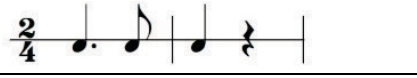 & & & \\
\hline $2 \mathrm{f}$ & $\frac{2}{4} \cdot d|? \cdot 0|$ & & & \\
\hline $3 a$ & $\frac{2}{4} \cdot \cdots \cdot \cdot \mid$ & B 3 & $\mathrm{E} / \mathrm{C}^{1} 2$ & $\mathrm{~F} / \mathrm{C}^{2} 3$ \\
\hline $3 b$ & $\frac{2}{4} \cdots \cdot|\cdot|$ & & & \\
\hline $3 c$ & $\frac{2}{4} \cdot \cdots+?$ & & & \\
\hline $3 \mathrm{~d}$ & 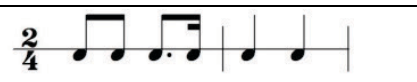 & & & \\
\hline & 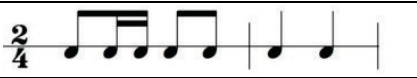 & & & \\
\hline
\end{tabular}

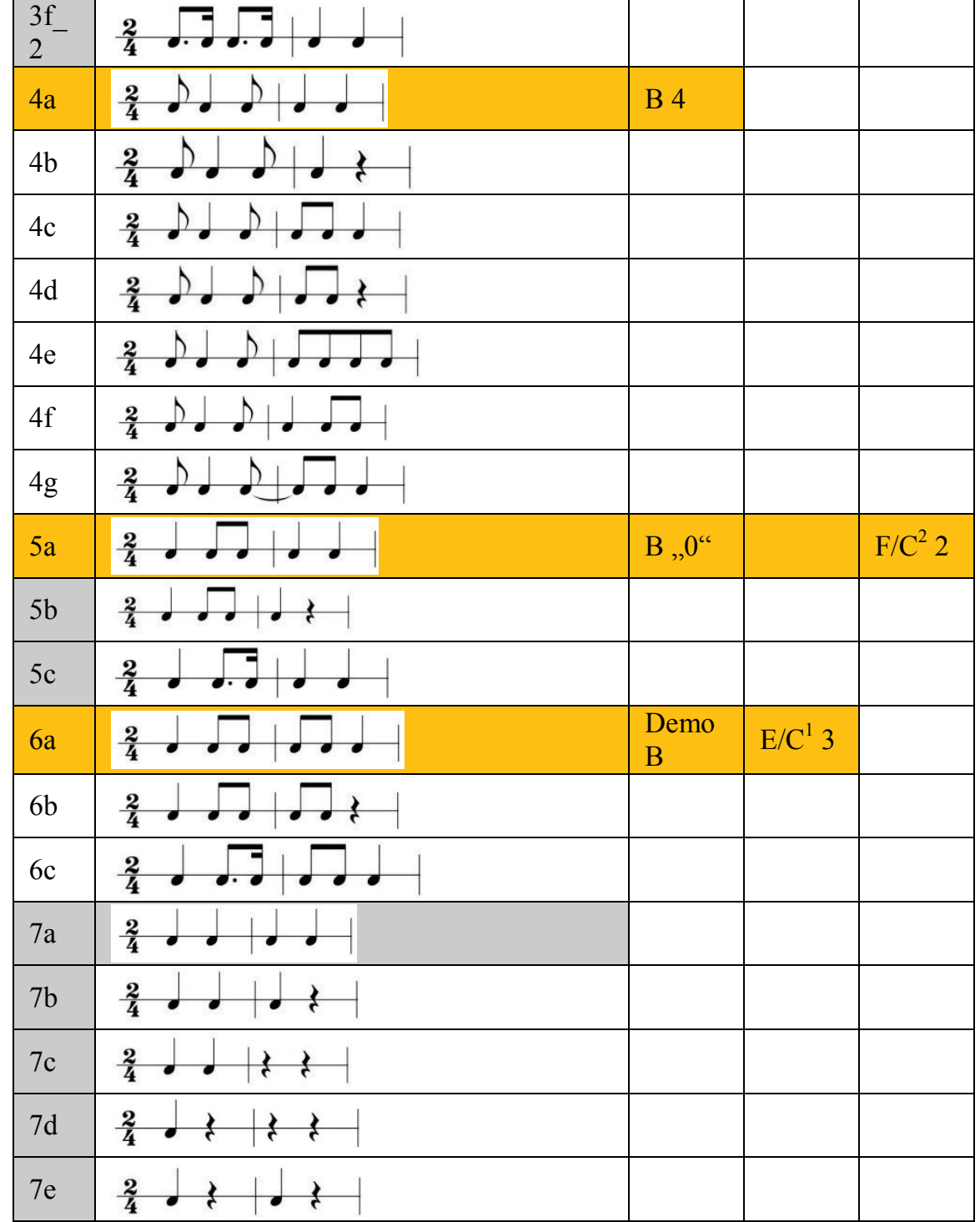




\begin{tabular}{|c|c|c|c|}
\hline $7 \mathrm{f}$ & $\frac{2}{4} \cdot \cdot|? \cdot|$ & & \\
\hline $7 \mathrm{~g}$ & $\frac{2}{4} \cdot ?|\cdot|$ & $\mathrm{E} / \mathrm{C}^{1} 4$ & \\
\hline $7 \mathrm{~h}$ & $\frac{2}{4} \cdot ?|z \cdot|$ & & \\
\hline $8 \mathrm{a}$ & $\frac{2}{4} \quad \cdots \cdot|\cdot|$ & & \\
\hline $8 \mathrm{~b}$ & $\frac{2}{4} \cdot \cdots \cdot \cdot ! \mid$ & & \\
\hline $8 \mathrm{c}$ & 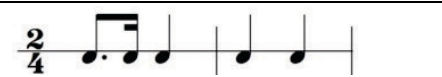 & & \\
\hline $8 \mathrm{~d}$ & $\left.\frac{2}{4} \cdot.\right] \cdot|+|$ & & \\
\hline $9 \mathrm{a}$ & $\frac{2}{4} \cdots \cdot|\cdots|$ & & \\
\hline $9 \mathrm{~b}$ & $\frac{2}{4}, \cdots \mid \neg ?$ & & \\
\hline $9 \mathrm{c}$ & $\frac{2}{4} \cdot \cdots \cdot \cdots \cdot \mid$ & & \\
\hline 9d & $\frac{2}{4} \curvearrowleft \Gamma|\sigma|$ & & \\
\hline $9 \mathrm{e}$ & 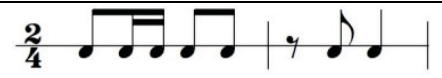 & & \\
\hline $10 \mathrm{a}$ & $\frac{2}{4} \cdot|\cdot| \cdots \mid$ & & \\
\hline $10 \mathrm{~b}$ & $\frac{2}{4} \cdot d|y \cdot \cdots|$ & & \\
\hline $10 \mathrm{c}$ & $\frac{2}{4} \cdot \cdot|\cdot, \cdot|$ & & \\
\hline $10 \mathrm{~d}$ & $\frac{2}{4} \cdot:|\cdots|$ & & $\mathrm{F} / \mathrm{C}^{2} 2$ \\
\hline $10 \mathrm{e}$ & $\frac{2}{4} \cdot \cdot \mid \cdot \cdots \cdot$ & & \\
\hline 11 & $\frac{2}{4} \ldots|\cdots|$ & & \\
\hline $12 \mathrm{a}$ & $\frac{2}{4} \cdots \cdots \mid \cdots$ & & \\
\hline
\end{tabular}

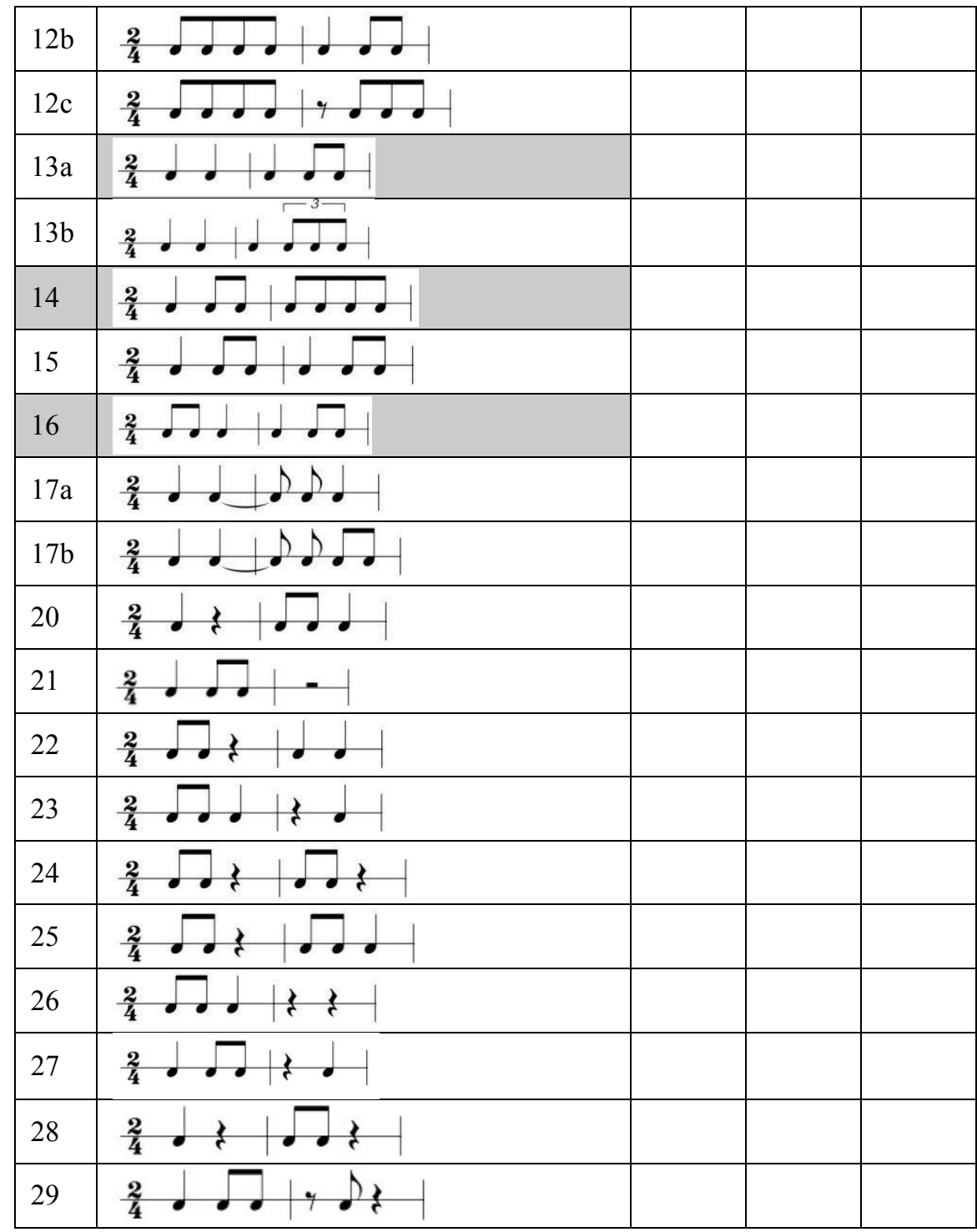


Anlagen

\section{Eidesstattliche Erklärung}

Ich versichere, dass ich die von mir vorgelegte Dissertation selbstständig und ohne unzulässige Hilfe angefertigt, die benutzten Quellen und Hilfsmittel vollständig angegeben und die Stellen der Arbeit - einschließlich Tabellen, Noten und Abbildungen -, die anderen Werken im Wortlaut oder dem Sinn nach entnommen sind, in jedem Einzelfall als Entlehnungen kenntlich gemacht habe; dass diese Dissertation noch keiner anderen Hochschule zur Prüfung vorgelegen hat; dass sie, gegebenenfalls abgesehen von einer durch die Promotionskommission auf Vorschlag des Betreuers der Dissertation genehmigten Publikation, noch nicht - auch nicht teilweise - veröffentlicht worden ist. Die Promotionsordnung der Hochschule für Musik Carl Maria von Weber Dresden ist mir bekannt.

Dresden, den 24.05.17 